\title{
Absolute and relative quantification of proteins in large protein-RNA assemblies by mass spectrometry
}

\author{
Dissertation \\ for the award of the degree \\ "Doctor rerum naturalium" (Dr. rer. nat.) \\ Division of Mathematics and Natural Sciences \\ of the Georg-August-Universität Göttingen
}

submitted by

Carla Schmidt

from Wolfenbüttel

Göttingen 2010 


\section{Thesis committee}

Dr. Henning Urlaub (Reviewer)

Prof. Dr. Ralf Ficner (Reviewer)

Prof. Dr. Ivo Feußner

Prof. Dr. Ulf Diederichsen
Bioanalytical Mass Spectrometry Group, Max Planck Institute for Biophysical Chemistry, Göttingen

Department of Molecular Structural Biology, Georg-August-Universität, Göttingen

Department of Plant Biochemistry, Georg-August-Universität, Göttingen

Department of Organic and Biomolecular Chemistry,

Georg-August-Universität, Göttingen

Date of the oral examination: June 8, 2010 


\section{Affidavit}

I hereby declare that my doctoral thesis entitled "Absolute and relative quantification of proteins in large protein-RNA assemblies by mass spectrometry" has been written independently and with no other sources and aids then quoted.

Carla Schmidt

May 14, 2010

Göttingen 



\section{Table of contents}

1 Summary 1

2 Introduction 3

2.1 Mass spectrometry-based protein identification and quantification 3

2.1.1 MS-based protein identification (Proteomics) 3

$\begin{array}{ll}2.1 .2 \text { Quantification by mass spectrometry } & 7\end{array}$

$\begin{array}{lr}\text { 2.1.3 Absolute quantification by mass spectrometry } & 10\end{array}$

$\begin{array}{ll}\text { 2.1.4 Relative quantification by mass spectrometry } & 13\end{array}$

$\begin{array}{ll}\text { 2.1.5 Quantification by mass spectrometry to analyze dynamic protein } & 19\end{array}$

$\begin{array}{ll}\text { 2.1.6 Quantifying mass spectrometric measurements } & 20\end{array}$

2.2 The spliceosome $\quad 25$

2.2.1 Eukaryotic pre-mRNA splicing $\quad 25$

2.2.1.1 Structure of eukaryotic pre-mRNAs $\quad 25$

2.2.1.2 The biochemical splice reaction $\quad 25$

2.2.1.3 The catalytic machinery $\quad 27$

$\begin{array}{ll}\text { 2.2.2 Components of the spliceosome } & 27\end{array}$

2.2.2.1 The U snRNPs $\quad 27$

2.2.2.2 non-snRNP components $\quad 29$

2.2.3 The stepwise assembly of the spliceosome $\quad 31$

2.3 Aim of this study 33

3 Materials and Methods $\quad 35$

3.1 Materials $\quad 35$

$\begin{array}{ll}\text { 3.1.1 Chemicals } & 35\end{array}$

3.1.2 Enzymes and Enzyme inhibitors 36

3.1.3 Nucleotides 36

3.1.4 DNA oligonucleotides 36

$\begin{array}{ll}3.1 .5 \text { Plasmids } & 37\end{array}$

$\begin{array}{ll}3.1 .6 \text { Bacteria strains } & 37\end{array}$

$\begin{array}{ll}\text { 3.1.7 Cell lines } & 37\end{array}$

$\begin{array}{ll}3.1 .8 \text { Cell culture } & 37\end{array}$

3.1.9 Commercial reaction kits and buffers $\quad 38$

3.1.10 Commonly used buffers 38

3.1.11 Standard peptides $\quad 39$

$\begin{array}{ll}\text { 3.1.12 Chromatography materials and consumables } & 39\end{array}$

$\begin{array}{ll}3.1 .13 \text { Instruments, Equipment } & 39\end{array}$

$\begin{array}{ll}3.2 \text { Methods } & 40\end{array}$

3.2.1 Molecular biology methods $\quad 40$

3.2.1.1 Concentration determination of nucleic acids 40

3.2.1.2 Phenol-Chloroform-Isoamylalcohol (PCl) extraction $\quad 40$

3.2.1.3 Agarose gel electrophoresis of DNA 41

3.2.1.4 In vitro transcription $\quad 41$

3.2.1.5 DNA amplification $\quad 42$

3.2.1.6 Restriction digestion of DNA 43

3.2.1.7 Proteinase K digestion $\quad 43$

3.2.1.8 Denaturing polyacrylamide gel electrophoresis 43

3.2.1.9 Silver staining of RNA 44 
3.2.1.10 Native gel electrophoresis of RNA complexes 44

3.2.1.11 Generation of pre-mRNA mutants (deletion of 5'ss and BPS) 44

3.2.2 Protein biochemical methods $\quad 45$

3.2.2.1 Protein concentration determination $\quad 45$

3.2.2.2 Polyacrylamide gel electrophoresis (PAGE) 46

3.2.2.3 Colloidal Coomassie staining of proteins 46

3.2.3 Cell culture, nuclear extract, purification of spliceosomal complexes 46

3.2.3.1 Metabolic Labeling of HeLa S3 cells (SILAC labeling) 46

3.2.3.2 Preparation of splicing active HeLa cell nuclear extract $\quad 47$

$\begin{array}{ll}\text { 3.2.3.3 In vitro splicing } & 47\end{array}$

3.2.3.4 Purification of human Prp19/CDC5L complex 48

3.2.3.5 Purification of human tri-snRNP (U4/U6.U5) 48

3.2.3.6 Overexpression of MS2-MBP fusion protein 48

3.2.3.7 Purification of human B and C complexes $\quad 49$

3.2.3.8 Investigating the protein composition during the spliceosomal 49 assembly

3.2.4 Mass spectrometry methods $\quad 51$

3.2.4.1 In-gel hydrolysis of proteins $\quad 51$

$\begin{array}{ll}\text { 3.2.4.2 Extraction of peptides } & 51\end{array}$

3.2.4.3 iTRAQ labeling of extracted peptides for relative quantification $\quad 52$

3.2.4.4 In-solution hydrolysis of hPrp19/CDC5L complex 52

3.2.4.5 Comparison of hydrolysis protocols and selection of standard 53 peptides (AQUA peptides) for absolute quantification

3.2.4.6 Internal standardization with the selected standard peptides $\quad 54$

3.2.4.7 Absolute quantification by LC-offline MALDI-ToF/ToF-MS (Peak 55 Area)

3.2.4.8 Absolute quantification by LC-online MS-MS/MS (Extracted lon 56 Chromatograms, XIC)

3.2.4.9 Absolute quantification by Multiple Reaction Monitoring 57

3.2.4.10 Relative quantification by LC-online MS-MS/MS (iTRAQ 58 quantification)

3.2.4.11 Relative quantification by LC-online MS-MS/MS (SILAC 59 quantification)

3.2.4.12 Statistical analysis

\section{Results}

4.1 Determination of the protein stoichiometry within the hPrp19/CDC5L complex by absolute quantification (AQUA)

4.1.1 Proteomic analysis of the hPrp19/CDC5L complex

4.1.2 Comparison of different protocols for in-solution hydrolysis

4.1.3 Selection of standard peptides for absolute quantification

4.1.4 Absolute quantification by LC-offline MALDI-ToF/ToF-MS (peak area)

4.1.5 Absolute quantification by LC-ESI-MS (extracted ion chromatograms)

4.1.6 Absolute quantification by Multiple Reaction Monitoring (MRM)

4.1.7 Comparison of results for absolute quantification in determining the Stoichiometry of the hPrp19/CDC5L complex

4.2 Relative quantification by iTRAQ-labeling of in-gel digested proteins 80

4.2.1 Optimization of iTRAQ-labeling of in-gel digested proteins 80

4.2.2 Relative quantification of different amounts of spliceosomal tri-snRNP 82 proteins - a feasibility study

4.3 Relative quantification of spliceosomal B and C complexes - a comparative study

4.3.1 Purification of spliceosomal B and C complexes

4.3.2 Proteomic analysis of spliceosomal B and C complexes - spectral count 87

4.3.3 iTRAQ labeling of spliceosomal B and C complexes for relative quantification 93

4.3.4 SILAC quantification of spliceosomal B and C complexes 101

4.3.5 Relative quantification of spliceosomal B and C complexes 109

4.3.5.1 Proteins common to B and C complexes 109 
4.3.5.2 Proteins predominantly associated with B complex

4.3.5.3 Proteins predominantly associated with $\mathrm{C}$ complex

4.3.6 Comparison of the three quantification methods - ITRAQ, SILAC, and spectral count

4.4 Protein assembly time line for spliceosomes by relative quantification $\quad 118$

$\begin{array}{ll}\text { 4.4.1 Generation of splicing inactive pre-mRNAs } & 118\end{array}$

$\begin{array}{ll}\text { 4.4.2 Triple SILAC to monitor dynamic protein changes } & 123\end{array}$

$\begin{array}{ll}\text { 4.4.3 Time-dependent protein assembly on splicing-active and splicing-inactive } & 126\end{array}$ pre-mRNAs

4.4.3.1 Experimental setup $\quad 126$

4.4.3.2 Normalization of the data 128

4.4.3.3 Protein assembly on splicing-active and splicing-inactive pre- $\quad 129$ mRNAs

4.4.4 Direct comparison of the protein assembly on splicing-active PM5 pre-mRNA 134 and splicing-inactive 5'ss deleted PM5 pre-mRNA

4.4.4.1 Experimental setup 134

4.4.4.2 Normalization of the data $\quad 135$

4.4.4.3 Protein assembly on PM5 pre-mRNA versus 5'ss-deleted PM5 pre- 136 mRNA

\section{Discussion}

5.1 Determination of the protein stoichiometry within the hPrp19/CDC5L complex by absolute quantification (AQUA)

5.2 Relative quantification by iTRAQ-labeling of in-gel digested proteins

5.3 Relative quantification of spliceosomal B and C complexes - a comparative study

5.3.1 Methodical considerations

5.3.2 Functional considerations

5.4 Protein assembly time line for spliceosomes by relative quantification

\section{References}

\section{Appendix}

Additional information

List of abbreviations

Acknowledgements

Curriculum Vitae 


\section{List of figures}

2.1 Protein identification by mass spectrometry 4

2.2 $\quad$ Principle of quantification by stable isotope labeling 8

2.3 Example MS spectrum of an endogenous and the corresponding standard 11 peptide for quantification

2.4 Isotope labeled amino acids used for cell growth in SILAC labeling 15

$2.5 \quad$ Chemical labeling reagents 16

$\begin{array}{lll}2.6 & \text { Structure of the 4-plex iTRAQ reagent } & 17\end{array}$

2.7 Example of Extracted ion chromatograms (XICs) to read out the signal 23 intensities for quantification

$\begin{array}{lll}2.8 & \text { MRM workflow } & 24\end{array}$

2.9 Schematic representation of the splicing process 26

2.10 The stepwise assembly of the spliceosome 32

3.1 Generation of 5'ss, BPS and BPS-ACTGA deleted PM5 pre-mRNA 45

3.2 In-house manufactured gel cutting device 51

4.1 1D-PAGE of the hPrp19/CDC5L complex 61

4.2 Miscleaved peptides obtained by hydrolysis in the presence of acetonitrile 65 are significantly longer than miscleaved peptides obtained by hydrolysis in the presence of urea

4.3 Analysis of AD-002 and CTNNBL1 sequences for presence of standard 67 peptides

$\begin{array}{lll}4.4 & \text { Solubility of standard peptides } & 68\end{array}$

4.5 Peak overlap is frequently observed in MALDI mass spectrometry 70

$\begin{array}{lll}4.6 & \text { Specificity of the designed MRM transitions } & 73\end{array}$

4.7 Example of the MRM transitions for an endogenous and the corresponding 74 standard peptide (LGLLGLPAPK derived from CDC5L protein)

4.8 Peptide ratios obtained from MRM analysis of the hPrp19/CDC5L complex 75

$\begin{array}{lll}\text { 4.9 PRL } 1 \text { sequence } & 77\end{array}$

4.10 Protein stoichiometry within the hPrp19/CDC5L complex 79

4.11 Workflow for iTRAQ-labeling of in-gel digested proteins 81 
4.12 Separation of different amounts of spliceosomal tri-snRNP proteins by gel electrophoresis

4.13 Example MS/MS spectrum of an ITRAQ labeled peptide

4.14 Example MS/MS spectrum of an ITRAQ labeled peptide

4.15 Purification of spliceosomal B and C complexes for ITRAQ and SILAC quantification

4.16 The proteomes of spliceosomal B and C complexes

4.17 Labeling efficiency achieved for iTRAQ labeling in two independent replicates

4.18 Example MS/MS spectrum for iTRAQ quantification of spliceosomal B and C complexes

4.19 Splicing kinetics, complex formation and RNA composition of B and C complexes as obtained using light and heavy SILAC nuclear extracts

4.20 Example MS spectrum for SILAC quantification of spliceosomal B and C complexes

4.21 Relative abundances of the $S m$ proteins in the $B$ and $C$ complexes determined by iTRAQ, SILAC, and spectral count

4.22 Relative protein abundances of the U2 and U5 snRNP specific proteins obtained by iTRAQ, SILAC, and spectral

4.23 Relative protein abundances of the U1, U4/U6, U4/U6.U5 snRNP specific proteins, and LSm proteins obtained by ITRAQ, SILAC, and spectral count

4.24 Protein ratios for hPrp19/CDC5L complex proteins obtained by iTRAQ, SILAC, and spectral count

4.25 Protein ratios of step 2 factors obtained by iTRAQ, SILAC, and spectral count

4.26 Scatter plot of SILAC and ITRAQ protein ratios

4.27 Sequence of the PM5 plasmid

4.28 The splicing kinetics and the spliceosomal complex formation using PM5 pre-mRNA and 5'ss- and BPS-deleted PM5 pre-mRNA

4.29 The splicing kinetics and the spliceosomal complex formation using PM5 pre-mRNA and BPS and BPS-ACTGA deleted PM5 pre-mRNAs

4.30 Splicing kinetics and spliceosomal complex formation using light, medium and heavy SILAC nuclear extracts

4.31 Comparison of the protein content within light, medium and heavy SILAC nuclear extracts 
4.32 Experimental setup to monitor the time-dependent protein assembly during pre-mRNA splicing

4.33 Coomassie stained gels of combined samples from assembly studies on PM5 pre-mRNA, 5'ss-deleted PM5 pre-mRNA and BPS-ACTGA-deleted PM5 pre-mRNA

4.34 Normalization of the obtained protein ratios on the ribosomal proteins

4.35 Assembly of U1 snRNP specific proteins on PM5 pre-mRNA, 5'ss-deleted

PM5 pre-mRNA, and BPS-ACTGA-deleted PM5 pre-mRNA over 30 minutes

4.36 Assembly of U2 snRNP specific proteins on PM5 pre-mRNA, 5'ss-deleted PM5 pre-mRNA and BPS-ACTGA-deleted PM5 pre-mRNA monitored over 30 minutes

4.37 Assembly of hPrp19/CDC5L complex proteins on PM5 pre-mRNA, 5'ssdeleted PM5 pre-mRNA and BPS-ACTGA-deleted PM5 pre-mRNA monitored over 30 minutes

4.38 Assembly of step 2 factors on PM5 pre-mRNA, 5'ss-deleted PM5 premRNA, and BPS-ACTGA-deleted PM5 pre-mRNA monitored over 30 minutes

4.39 Experimental setup to compare the protein compositions of the sets of proteins assembled on PM5 and 5'ss-deleted PM5 pre-mRNA at different time points during pre-mRNA splicing

4.40 Coomassie stained gel of combined samples from comparison of the protein assembly on PM5 and 5'ss-deleted PM5 pre-mRNA at different time points during pre-mRNA splicing

4.41 Normalization of the protein ratios found for the cap binding proteins

4.42 Direct comparison of protein assembly on the PM5 pre-mRNA compared with corresponding assembly on the 5'ss-deleted PM5 pre-mRNA for U1 snRNP specific proteins and Sm proteins

4.43 Protein assembly of the hPrp19/CDC5L complex and the step 2 factors on the PM5 pre-mRNA compared with corresponding assembly on the 5'ssdeleted PM5 pre-mRNA

5.1 Endogenous counterparts of the selected standard peptides for CDC5L protein are located in highly structured regions of CDC5L

A.1 Miscleaved peptides generated in the presence of urea show higher peptide scores for the peptide sequences selected for quantification

A.2 Incompletely cleaved peptides obtained by hydrolysis in the presence of acetonitrile are significantly longer than incompletely cleaved peptides obtained by hydrolysis in the presence of urea 


\section{List of tables}

2.1 Most important and popular MS-based quantification methods in proteomics

2.2 Composition of the human U snRNPs

3.1 Oligonucleotides used in this study

3.2 Isotope composition of amino acids used for different SILAC media

3.3 Used SILAC nuclear extracts for investigation of the protein assembly on premRNAs durin 30 min

3.4 Used SILAC nuclear extracts for direct comparison of the protein assembly on PM5 pre-mRNA and on a splicing inactive pre-mRNA during 30 min

4.1 Comparison of different hydrolysis protocols

4.2 Comparison of different hydrolysis protocols

4.3 Selected standard peptides

4.4 Relative protein stoichiometries within the hPrp19/CDC5L complex determined by LC-offline MALDI-ToF/ToF-MS

4.5 Relative protein stoichiometries within the hPrp19/CDC5L complex determined by LC-ESI-MS

4.6 Relative protein stoichiometries within the hPrp19/CDC5L complex as determined by MRM analyses

4.7 Summary of the protein stoichiometries obtained from different MS techniques

4.8 Protein ratios for the relative quantification of different amounts of spliceosomal tri-snRNP proteins

4.9 Spectral count for proteomic analysis of spliceosomal B and C complexes

4.10 Relative quantification of spliceosomal B and C complexes by iTRAQ

4.11 Relative quantification of spliceosomal B and C complexes by SILAC

A.1 Additional proteins identified in the hPrp19/CDC5L complex by LC-MS/MS

A.2 Spectral Count for peptides seleceted for quantification and their miscleaved versions

A.3 Three MRM transitions for each endogenous and standard peptide were designed

A.4 Absolute amounts of proteins of the hPrp19/CDC5L complex

A.5 Proteins identified in the first replicate of the relative quantification of spliceosomal $B$ and $C$ complexes by iTRAQ 
A.6 Proteins identified in the second replicate of the relative quantification of 186 spliceosomal B and C complexes by iTRAQ

A.7 Proteins that were found to be enriched in light (L), medium (M) or heavy $(H) \quad 198$ nuclear extracts, respectively

A.8 List of ribosomal proteins used for normalization 199

A.9 List of normalization factors calculated for the different assembly studies 199 


\section{Summary}

The vast majority of cellular processes is driven by protein complexes. These emerge as multimeric protein assemblies or are complexed with protein ligands such as RNA or DNA molecules. To understand the details of the underlying cellular processes, an analysis of protein complexes is therefore a prerequisite. The analysis involves identification of the protein components, determination of protein stoichiometries, relative quantification of the components in different complex states, and the study of protein-protein and protein-ligand interactions. Several mass spectrometry-based techniques have been developed to tackle these problems and greatly facilitating the high throughput analysis of a variety of protein complexes. We have applied these methods to the analysis of the spliceosome. The spliceosome is a protein-RNA machinery that catalyzes the excision of introns and the ligation of exons during eukaryotic pre-mRNA splicing. It passes through sequential assembly states that dramatically differ in their RNA and protein composition. Mass spectrometry-based quantification methods are thus ideally suited to answer these questions. In this study, absolute and relative quantification by mass spectrometry was used to characterize different spliceosomal complexes or subcomplexes, in terms of their protein composition and protein stoichiometry. The aim of this study was to generate a complete as possible picture of the dynamic protein changes of the spliceosome during its assembly pathway.

One spliceosomal subcomplex, the hPrp19/CDC5L complex, consists of seven individual proteins (hPrp19, Hsp70, CTNNBL1, PRL1, CDC5L, AD-002, and SPF27) and plays a crucial role in the assembly of a catalytically active spliceosome. The exact protein stoichiometries within this particular protein complex have not yet been investigated. We therefore set up a mass spectrometry-based quantification method and used the hPrp19/CDC5L complex to implement the methodology of absolute quantification (AQUA) with the help of synthetic standard peptides in combination with multiple reaction monitoring (MRM). Several conditions for complete hydrolysis of the protein complex were evaluated and found to be crucial for accurately determining the protein stoichiometries within this particular protein complex. In addition, the suitability of different standard peptides (AQUA peptides) was tested and different mass spectrometry techniques, to read out signal intensities for absolute quantification, were compared. The analyses revealed that the denaturing conditions used during protein hydrolysis were most crucial in obtaining a robust and reliable quantification of the different subcomponents of the hPrp19/CDC5L complex. Taking all independent experimental approaches into account, the hPrp19/CDC5L complex 
consists of four copies of hPrp19, two copies of CDC5L, and one copy each of SPF27, PRL1, and CTNNBL1.

In a different series of investigation, we set out to make a high throughput relative quantification of two distinct spliceosomal complexes, namely the pre-catalytic and the catalytically active spliceosomes (spliceosomal B and C complexes). For this purpose, a relative quantification approach involving ITRAQ (isobaric tags for relative and absolute quantification) labeling of in-gel digested proteins was optimized and applied to the two complexes. The results were compared to relative quantification by metabolic labeling (SILAC; stable isotope labeling with amino acids in cell culture) and semi-quantitative spectral count from proteomic analysis of the B and C complexes. Thus, three independent relative quantification data sets were generated allowing for an in depth comparison of the methodologies involved. We found an overall good agreement for the used quantification methods. Although limits for relative quantification by spectral count were observed for some proteins. Several proteins were found to be pre-dominantly associated with spliceosomal B or $\mathrm{C}$ complexes, and only few proteins were found to be present in equal amounts within the two complexes. The high dynamics of the spliceosome during its assembly pathway was thus clearly demonstrated. Furthermore, the study revealed a novel $\mathrm{C}$ complex association of a DEAD box helicase, the DDX34 protein.

In a final scene of investigation, we analyzed the dynamic protein changes during the premRNA splicing process. To this end, the protein compositions on splicing active and splicing inactive pre-mRNAs at different time points were compared by stable isotope labeling with amino acids in cell culture (SILAC). Splicing inactive pre-mRNAs were generated by deletion of the pre-mRNA's 5' splice site (5'ss) or the branchpoint site (BPS) to prevent the formation of a catalytically active spliceosome. Two approaches were then followed to monitor the spliceosomal assembly kinetics: (i) The time dependent protein assembly on the splicing active or splicing inactive pre-mRNAs, respectively, and (ii) the direct comparison of the assembled protein composition on a splicing active to a splicing inactive pre-mRNA at different time points during pre-mRNA splicing. The kinetics of the protein assembly on the different pre-mRNAs was analyzed for spliceosomal protein groups that are affected by deletion either of the 5'ss or the BPS. The results demonstrate that proteins specific for the pre-catalytic A complex could also be found on splicing inactive pre-mRNAs at a very early time point, whereas proteins, specific for the catalytically active spliceosome, show striking differences in their assembly characteristics on the different pre-mRNAs. The thus generated timelines for the assembly of whole groups of spliceosomal proteins during spliceosomal assembly and splicing catalysis are extremely helpful in understanding the dynamic process of pre-mRNA splicing. 


\section{Introduction}

Proteins are involved in all cellular processes. They emerge as protein or protein-ligand complexes (e.g. complexed with RNA/DNA molecules, sugars, lipids, cofactors etc.) to form the catalytic core for different cellular actions. These cellular actions are completed either by the proteins alone or by the ligands that are arranged by the proteins within the catalytic core. The identification of active proteins within protein and protein-ligand complexes is therefore of great importance to understand different cellular processes. In the past, two fundamental breakthroughs facilitated protein identification within unknown protein mixtures enormously: (i) complete sequencing of the genomes, and (ii) the invention of two fundamental mass spectrometry approaches. As a result, mass spectrometry-based protein identification have become a popular and indispensable technique during the last years.

\subsection{Mass spectrometry-based protein identification and quantification}

\subsubsection{MS-based protein identification (Proteomics)}

By definition, the proteome is the "entire set of proteins expressed by a genome, cell, tissue or organism. More specifically, it is the set of expressed proteins in a given type of cells or an organism at a given time under defined conditions" (Marc Wilkins, 1994). Proteomics thus deals with the identification of proteins. An early breakthrough was the development of the Edman degradation enabling for $\mathrm{N}$-terminal sequencing of unknown proteins by chemical cleavage in a step-wise manner (Edman, 1949). This discovery was rewarded in 1958 with the Nobel Prize in chemistry to Frederik Sanger for sequencing Insulin. Automation of Edman sequencing made it to a powerful technique for protein identification. However, this technique is very time-consuming, requires very homogenous samples, and a free amino terminus. Edman sequencing is limited to 20 - 30 amino acids and requires an intact $\mathrm{N}$-terminus. To circumvent this problem, the proteins are hydrolyzed by endoproteinases and the generated peptides are then sequenced instead of whole proteins. Therefore, generated peptide mixtures need to be purified by reversed phase (RP) high pressure liquid chromatography (HPLC). This requires large amounts of samples, which poses a problem.

The invention of two soft ionization techniques, namely Matrix-assisted laser desorption/ionization (MALDI; Karas et al., 1987; Karas and Hillenkamp, 1988; Tanaka et al., 1988) and electrospray ionization (ESI; Fenn et al., 1989), allowed the analysis of intact proteins and peptides by mass spectrometry (MS). This invention, together with the 


\section{4 | INTRODUCTION}

knowledge of the genome sequence, allows protein identification by mass spectrometry using the following workflow: (i) hydrolysis of proteins by endoproteinases into peptides, (ii) MS analysis of the peptides providing mass-to-charge ratios $(\mathrm{m} / \mathrm{z})$ of the intact peptides (MS experiment), (iii) selection of precursor ions (peptides) for tandem mass spectrometry (MS/MS), (iv) fragmentation of the selected precursor yielding the peptide sequence (MS/MS experiment), and ( $v$ ) identification of the peptide and finally the protein by comparing the peptide mass and the obtained peptide sequence with in silico hydrolyzed proteins in a database (Figure 2.1).
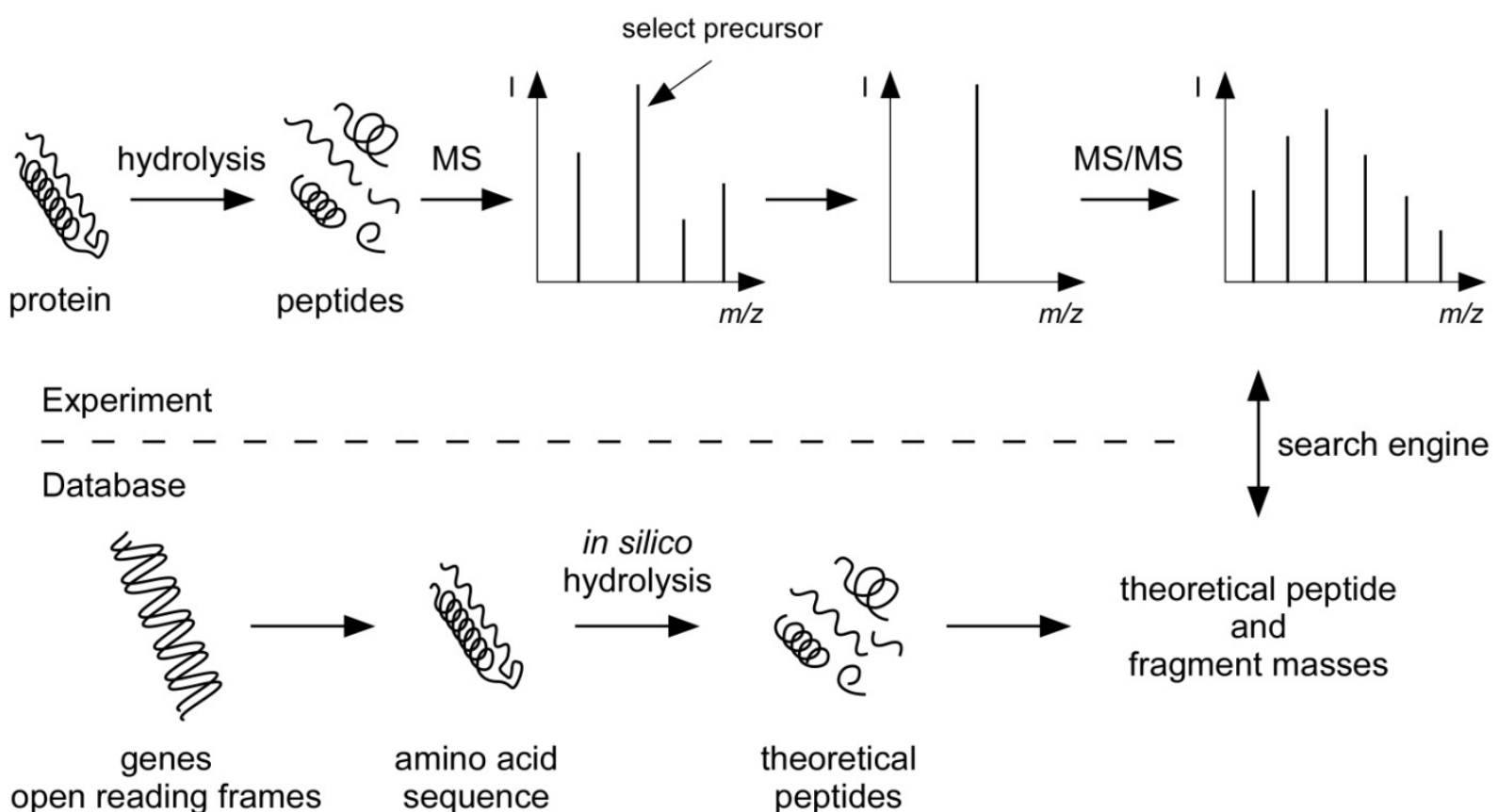

open reading frames sequence peptides

Figure 2.1: Protein identification by mass spectrometry. Proteins are hydrolyzed by specific endoproteinases and the mass-to-charge ratios of the generated peptides are determined by mass spectrometry. Precursor ions are selected for fragmentation (MS/MS) and the protein is identified by comparing the experimentally determined peptide and fragment masses with theoretical peptide and fragment masses in a database.

The more proteins are present in a sample the more peptides are generated by hydrolysis. As even state-of-the-art mass spectrometers can only analyze a limited number of peptides at a given time, reduction of the sample complexity is one of the prerequisites for a successful proteome analysis. Coupling of reversed-phase liquid chromatography (RP-LC) and mass spectrometry is a powerful tool to reduce the sample complexity. Peptides are separated during chromatography and eluted peptides are subsequently analyzed in the mass spectrometer. However, sample reduction solely by RP-LC is still not sufficient for highly complex samples. There are two general strategies to further reduce sample complexity before the actual RP-LC: (i) additional separation of the generated peptides after hydrolysis of the proteins, and (ii) separation of the proteins before hydrolysis. 
that the time-consuming protein separation step is bypassed, i.e. additional steps for sample preparation are omitted. Due to the high complexity of the generated peptide mixture, multidimensional peptide separation, which comprises the coupling of different separation techniques, is required. For this purpose, two dimensional liquid chromatography (2D-LC) using strong cation-exchange (SCX) chromatography in the first and RP-LC in the second dimension, has been shown to provide optimal results. Washburn et al., 2001 successfully introduced the multidimensional protein identification technology (MuD-PIT), where generated peptides are separated on a biphasic microcapillary column packed with SCX and RP material and are subsequently eluted into the mass spectrometer (Washburn et al., 2001). A different 2D-LC approach using RP in both dimensions but at different pH values has been described (Gilar et al., 2005). The peptides are separated at high pH (10) in the first, followed by orthogonal separation at low $\mathrm{pH}(2)$ in the second dimension (Gilar et al., 2005). The advantage of this set-up is to avoid SCX, whose resolution for peptides is relatively low and which involves the use of buffers with high salt concentrations that affect ionization of the sample. An alternative approach is the separation of generated peptides by isoelectric focusing (IEF) on immobilized pH gradient (IPG) strips. The IPG strips are cut into pieces, peptides are eluted and subsequently analyzed by reversed-phase liquid chromatography coupled mass spectrometry (Krijgsveld et al., 2006).

Separation of proteins Fractionation leads to a first reduction of sample complexity. The fractions are then applied to protein hydrolysis by specific endoproteinases. There are two different strategies for protein separation. Either, the proteins are separated by liquid chromatography and eluted fractions are collected for further sample preparation or, most popular, the proteins are separated by gel electrophoresis and excised proteins are hydrolyzed in-gel. Liquid chromatography-based protein separation is mostly performed by size exclusion chromatography (SEC) or ion exchange chromatography (IEC). The latter technique can be divided into cation and anion exchange chromatography depending on the chromatographic material used. Furthermore, the proteins can be separated according to their hydrophobicity using hydrophobic interaction chromatography (HIC) or hydrophilic interaction chromatography (HILIC). All these methods fractionate the sample in groups of proteins of similar size, charge or hydrophobicity / hydrophilicity, respectively. For protein separation by gel electrophoresis, several one and two dimensional methods are available (e.g. 1D (Laemmli, 1970), 2D (Klose, 1975; O'Farrell, 1975), Blue Native (Schagger et al., 1988; Schagger and von Jagow, 1991), and 16BAC (Hartinger et al., 1996; Macfarlane, 1989) gel electrophoresis). The combination of IEF and sodium dodecylsulfate (SDS) polyacrylamide gel electrophoresis (PAGE) is the most common approach, allowing for a resolution of up to 10,000 protein spots per gel (Klose, 1999). However, this approach is 


\section{6 | INTRODUCTION}

limited to the very basic and acidic proteins and precipitation of the sample is sometimes observed. When working in experimental systems investigating samples with moderate complexity (e.g. purified cell compartments, purified protein complexes) 1D-SDS-PAGE (Laemmli, 1970) is the simplest and most adequate alternative.

Mass spectrometry

As mentioned above, MALDI and ESI allow the determination of the molecular weight of intact proteins and peptides by mass spectrometry. NanoLC uses very low flow rates and is thus well suited for coupling to an ESI mass spectrometer. Consequently, the sample complexity is reduced and eluted peptides are immediately ionized and analyzed in the mass spectrometer. State-of-the-art mass spectrometers determine the mass-to-charge $(\mathrm{m} / \mathrm{z})$ ratio of peptides within a mixture (MS experiment) and isolate peptides (precursors) for subsequent fragmentation within the mass spectrometer (MS/MS experiment). Mass analysis of the fragment ions and the accurate monoisotopic peptide mass allows the identification of the corresponding protein by comparison to in silico hydrolyzed proteins in a database. For this purpose, search engines that compare experimentally determined peptide and fragment masses with in silico generated masses are employed (Figure 1).

Most of the mass spectrometers use data-dependent-acquisition (DDA). A DDA duty cycle consists of two steps: (i) a MS scan over a certain mass range (typically $350-1500 \mathrm{~m} / \mathrm{z}$ ) to detect masses and charge states of the peptides (MS experiment), and (ii) selection of peptide precursors and subsequent fragmentation (MS/MS experiment). While the mass spectrometer is occupied with MS/MS experiments, usually no MS scans can be performed (except for Orbitrap-FT mass analyzer). Consequently, instruments with a short duty cycle can sequence more peptides in a given time. In general, ion-trap instruments have shorter duty cycles than Qq-ToF instruments, whereas the quality of MS/MS spectra from Qq-ToF instruments is better, so that more MS/MS spectra can be correctly assigned to peptide sequences (Elias et al., 2005). However, the sensitivity of a mass spectrometer is not only dependent on the acquisition speed, but also on the mass accuracy (i.e. the accuracy, with which the precursor and fragment masses are determined) and the peak resolution. The mass resolution is the ratio of the peak's mass and its width. The peak width is usually taken as the full width at half maximum intensity (FWHM). The more accurate the peptide and fragment masses are determined, the better can the peptide be assigned to the correct protein during database search. A high resolution of peaks allows to distinguish between coeluting peaks of similar mass and leads to more data information. Currently, a mass accuracy of $0.2 \mathrm{ppm}$ and a resolution of 100,000 is routinely achieved and is sufficient for most purposes. The recent development of a new, highly sensitive and fast mass spectrometer, 
the LTQ-Orbitrap-FT (Hu et al., 2005), greatly expedited the number of proteins identified by single proteome studies (Graumann et al., 2008; Shi et al., 2007).

\subsubsection{Quantification by mass spectrometry}

The development of highly sensitive and fast mass spectrometers leads to the analysis of post-translational modifications and protein quantification. During the last decade numerous MS-based quantification techniques have emerged and there is a clear trend in proteomic studies towards MS-based quantification (Bantscheff et al., 2007; Ong et al., 2003; Ong and Mann, 2005; Wilm, 2009).

Every peptide contains a certain amount of stable heavy isotopes $\left({ }^{13} \mathrm{C},{ }^{15} \mathrm{~N},{ }^{18} \mathrm{O}\right.$, and $\left.{ }^{2} \mathrm{D}\right)$ at their natural abundances. The isotope pattern of a peptide (also called the isotopic envelope) thus reflects abundance of stable isotopes within the peptide. Artificial incorporation of heavy stable isotopes (i.e. labeling with stable isotopes) induces a mass shift of the peptide's peak (including its isotopic envelope) in the mass spectrum. As mass spectrometry is not a quantitative method per se, incorporation of stable isotopes, such as ${ }^{13} \mathrm{C},{ }^{15} \mathrm{~N},{ }^{18} \mathrm{O}$ or ${ }^{2} \mathrm{D}$, can be used for a relative comparison of peptides or proteins from samples to be quantified. Importantly, differently labeled peptides show the same behavior in the mass spectrometer, but feature a mass shift in the MS or MS/MS spectrum according to the incorporated stable isotopes. The peak's signal intensity of the differently labeled peptides derived from various samples reflects the relative quantities of the particular peptide and therefore of the particular protein in the different samples (see Figure 2.2 for an overview).

Incorporation of stable isotopes is achieved by chemical, metabolic, and enzymatic labeling. Chemical labeling is performed on the protein or peptide level, whereas metabolic labeling takes place during cell growth and enzymatic labeling during protein hydrolysis. Performing metabolic labeling guarantees a nearly $100 \%$ labeling efficiency in cell culture, whereas labeling efficiency using chemical labels is typically below $100 \%$ and should be carefully checked during data analysis. Relative quantification is used to compare peptides and finally proteins from different cell states or different protein assemblies. Usually, proteins or peptides from different conditions are differently labeled, pooled in equal amounts and analyzed by LC-MS/MS. In the event of absolute quantification, the sample is spiked with synthetic standard peptides in known amounts during hydrolysis of the proteins or before LCMS/MS analysis. Absolute quantification is thus used to determine the absolute amount (mass or mole) of proteins in a mixture. To date, there are various methods for relative and absolute quantification available (see Table 2.1 for a summary of the most important and popular methods). 


\section{8 | INTRODUCTION}

Nonetheless, contrary to introduction of stable isotopes, several label-free quantification approaches, such as spectral count (Old et al., 2005), emPAl (Ishihama et al., 2005) and non-directed LC-MS/MS (Silva et al., 2006), have been reported for relative and absolute MS-based quantification.

A

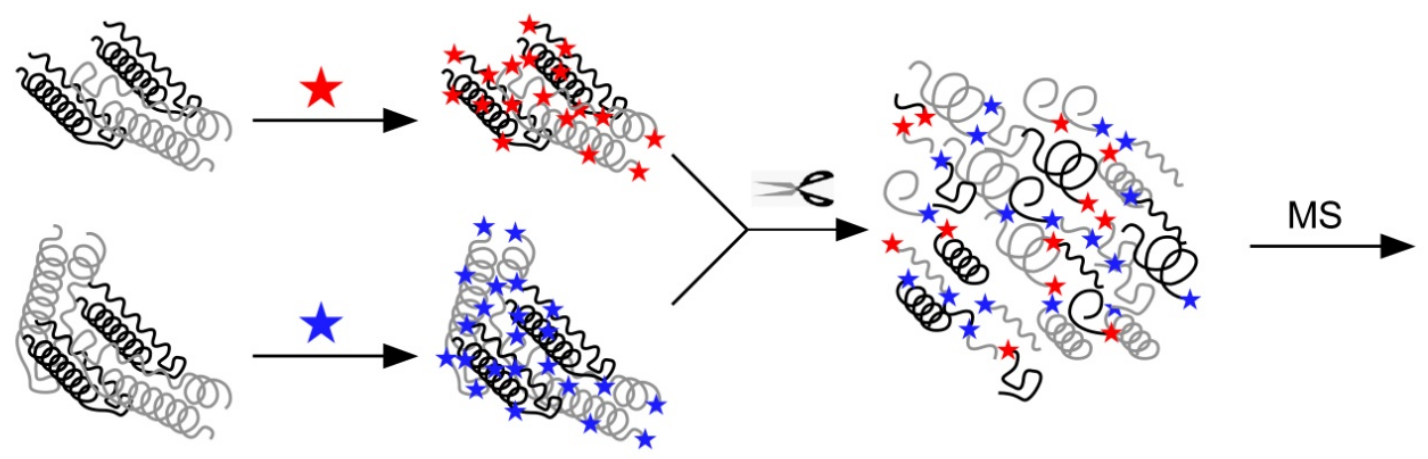

B
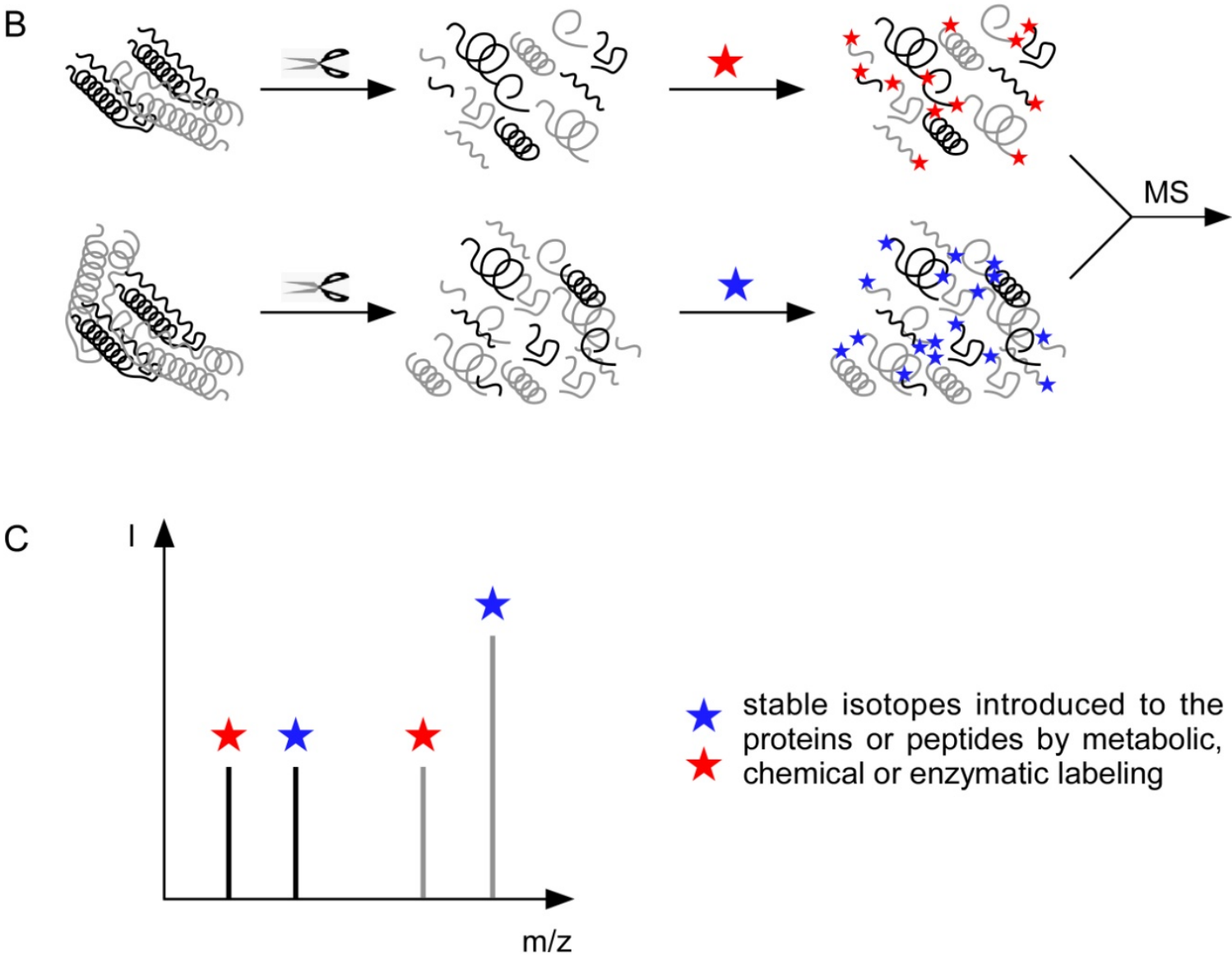

stable isotopes introduced to the proteins or peptides by metabolic, chemical or enzymatic labeling

Figure 2.2: Principle of quantification by stable isotope labeling. Stable isotopes are introduced on the protein (A) or peptide (B) level. (A) Proteins from different samples are labeled with stable isotopes by metabolic or chemical labeling. After pooling, the proteins are hydrolyzed with a specific endoproteinase and generated peptides are subsequently analyzed by MS. (B) Proteins from different samples are hydrolyzed in parallel and stable isotopes are introduced to the generated peptides by chemical labeling. Labeled peptides are pooled and analyzed by MS. (C) Differently labeled peptides show same behavior in the mass spectrometer, but feature a distinct mass shift in the MS according to the incorporated stable isotopes. 
Table 2.1: Most important and popular MS-based quantification methods in proteomics.

\begin{tabular}{|c|c|c|c|c|c|}
\hline Method & Principle & Quantification & Advantages & Disadvantages & References \\
\hline AQUA & $\begin{array}{l}\text { addition of stable } \\
\text { isotope labeled } \\
\text { standard pep- } \\
\text { tides }\end{array}$ & absolute & $\begin{array}{l}\text { absolute quanti- } \\
\text { fication of pro- } \\
\text { teins/peptides in } \\
\text { complex mixtu- } \\
\text { res }\end{array}$ & $\begin{array}{l}\text { complete hydroly- } \\
\text { sis of the proteins is } \\
\text { required, solubility } \\
\text { of standard pep- } \\
\text { tides }\end{array}$ & $\begin{array}{l}\text { Desidero and } \\
\text { Kai, 1983; Gerber } \\
\text { et al., 2003; } \\
\text { Kirkpatrick et al., } \\
2005\end{array}$ \\
\hline $\begin{array}{l}{ }^{15} \mathrm{~N}- \\
\text { labeling }\end{array}$ & $\begin{array}{l}\text { metabolic labe- } \\
\text { ling during cell } \\
\text { growth }\end{array}$ & relative & $\begin{array}{l}\text { complete intro- } \\
\text { duction of stable } \\
\text { isotopes (labe- } \\
\text { ling efficiency } \\
\text { nearly } 100 \% \text { ) }\end{array}$ & $\begin{array}{l}\text { complex data ana- } \\
\text { lysis, extremely en- } \\
\text { riched nitrogen is } \\
\text { needed }\end{array}$ & Oda et al., 1999 \\
\hline SILAC & $\begin{array}{l}\text { metabolic labe- } \\
\text { ling during cell } \\
\text { growth }\end{array}$ & relative & $\begin{array}{l}\text { complete intro- } \\
\text { duction of stable } \\
\text { isotopes (labe- } \\
\text { ling efficiency } \\
\text { nearly } 100 \% \text { ) }\end{array}$ & no labeling in tissue & Ong et al., 2002 \\
\hline ICAT & $\begin{array}{l}\text { chemical labe- } \\
\text { ling, cysteine } \\
\text { specific }\end{array}$ & relative & $\begin{array}{l}\text { enrichment of } \\
\text { labeled peptides } \\
\text { (reduced sample } \\
\text { complexity) }\end{array}$ & $\begin{array}{l}\text { no quantification of } \\
\text { proteins containing } \\
\text { no cysteine, partial } \\
\text { separation of } \\
\text { differently labeled } \\
\text { peptides during LC }\end{array}$ & Gygi et al., 1999 \\
\hline iTRAQ & $\begin{array}{l}\text { chemical labe- } \\
\text { ling with isobaric } \\
\text { tags, amine spe- } \\
\text { cific, quantifica- } \\
\text { tion in MS/MS }\end{array}$ & relative & $\begin{array}{l}\text { multiplex (up to } \\
\text { eight samples), } \\
\text { quantification of } \\
\text { proteins in tissue }\end{array}$ & $\begin{array}{l}\text { labeling efficiency } \\
\text { needs to be } \\
\text { checked, specific } \\
\text { software for data } \\
\text { analysis is required }\end{array}$ & Ross et al., 2004 \\
\hline TMT & $\begin{array}{l}\text { chemical labe- } \\
\text { ling with isobaric } \\
\text { tags, amine spe- } \\
\text { cific, quantifica- } \\
\text { tion in MS/MS }\end{array}$ & relative & $\begin{array}{l}\text { multiplex (up to } \\
\text { six samples), } \\
\text { quantification of } \\
\text { proteins in tissue }\end{array}$ & $\begin{array}{l}\text { labeling efficiency } \\
\text { needs to be } \\
\text { checked, specific } \\
\text { software for data } \\
\text { analysis is required }\end{array}$ & $\begin{array}{l}\text { Thompson et al., } \\
2003\end{array}$ \\
\hline $\begin{array}{l}\text { dimethyl } \\
\text { labeling }\end{array}$ & $\begin{array}{l}\text { introduction of } \\
\text { stable isotopes } \\
\text { by dimethylation } \\
\text { of N-termini and } \\
\text { lysine side } \\
\text { chains }\end{array}$ & relative & $\begin{array}{l}\text { cheap reagents, } \\
\text { nearly } 100 \% \\
\text { labeling effici- } \\
\text { ency, triplex }\end{array}$ & $\begin{array}{l}\text { specific software for } \\
\text { data analysis is } \\
\text { required }\end{array}$ & $\begin{array}{l}\text { Hsu et al., 2003; } \\
\text { Boersema et al., } \\
2008\end{array}$ \\
\hline $\begin{array}{l}{ }^{18} \mathrm{O}- \\
\text { labeling }\end{array}$ & $\begin{array}{l}\text { introduction of } \\
{ }^{18} \mathrm{O} \text { during enzy- } \\
\text { matic hydrolysis }\end{array}$ & relative & $\begin{array}{l}\text { cheap reagents, } \\
\text { simple labeling } \\
\text { protocol }\end{array}$ & $\begin{array}{l}\text { incomplete labeling } \\
\text { complicates data } \\
\text { analysis enormous- } \\
\text { ly }\end{array}$ & $\begin{array}{l}\text { Mirgorodskaya et } \\
\text { al., 2000; } \\
\text { Reynolds et al., } \\
2002 \text {; Yao et al., } \\
2001\end{array}$ \\
\hline $\begin{array}{l}\text { spectral } \\
\text { count }\end{array}$ & $\begin{array}{l}\text { quantification on } \\
\text { the number of }\end{array}$ & relative & $\begin{array}{l}\text { no labeling } \\
\text { required }\end{array}$ & semi-quantitative & Liu et al., 2004 \\
\hline
\end{tabular}
acquired MS/MS spectra 


\subsubsection{Absolute quantification by mass spectrometry}

Determination of the absolute amount of proteins in proteomic studies is often derived from a measurement of the absolute peptide concentration. This is achieved by either providing standard peptides or by label-free approaches. In addition, a procedure for absolute quantification of intact proteins using labeled standard proteins has recently been described (Waanders et al., 2007).

Absolute quantification using stable-isotope labeled standard peptides The use of synthetic standard peptides for absolute quantification was originally described in 1983, where enzymatically deuterium labeled standard peptides were used for absolute quantification of enkephalin in thalamus tissue (Desiderio and Kai, 1983). This approach has been refined by Gygi and co-workers, and is now commonly known as AQUA (absolute quantification; Gerber et al., 2003; Kirkpatrick et al., 2005). Heavy isotope labeled standard peptides (also termed AQUA peptides) are synthesized by incorporation of one ${ }^{13} \mathrm{C}$ - and/or ${ }^{15} \mathrm{~N}$-labeled amino acid. As a result, the standard and the endogenous peptide share the same physicochemical properties, including chromatographic co-elution, ionization efficiency, and fragmentation pattern during MS/MS experiments. Importantly, the endogenous and the standard peptide can be distinguished by a distinct mass shift in the MS spectrum caused by the incorporated heavy labeled amino acid. The endogenous and the corresponding standard peptide thus present a peak pair consisting of a light peak (the non-labeled endogenous peptide) and a heavy peak (belonging to the standard peptide harboring incorporated stable isotopes; see Figure 2.3 for an example). The signal intensities of the light (endogenous) and the heavy (standard) peptides reflect the relative amounts. Because the concentration of the standard peptide is known, the absolute amount of the endogenous peptide and finally the protein can thus be determined. Absolute quantification using standard peptides is often applied to measure the level of particular peptide modification (e.g. phosphorylation (Gerber et al., 2003), ubiquitinylation (Kirkpatrick et al., 2005)) or to analyze and validate biomarkers in clinical studies (Pan et al., 2005). The selection of standard peptides is often empirically (Bantscheff et al., 2007), i.e. the choice of synthetic standard peptides results from the analysis of endogenous peptides generated from the proteins under investigation. There are several aspects that have to be taken into account when selecting standard peptides, e.g. possible modification of amino acid residues (e.g. oxidation of methionine), chromatographic elution, ionization efficiency etc. A study about prediction of frequently detected tryptic peptides in a given proteomic platform (so-called proteotypic peptides) might help when selecting standard peptides for absolute quantification (Mallick et al., 2007). 


\section{ILLGGYQSR*}

1016.58

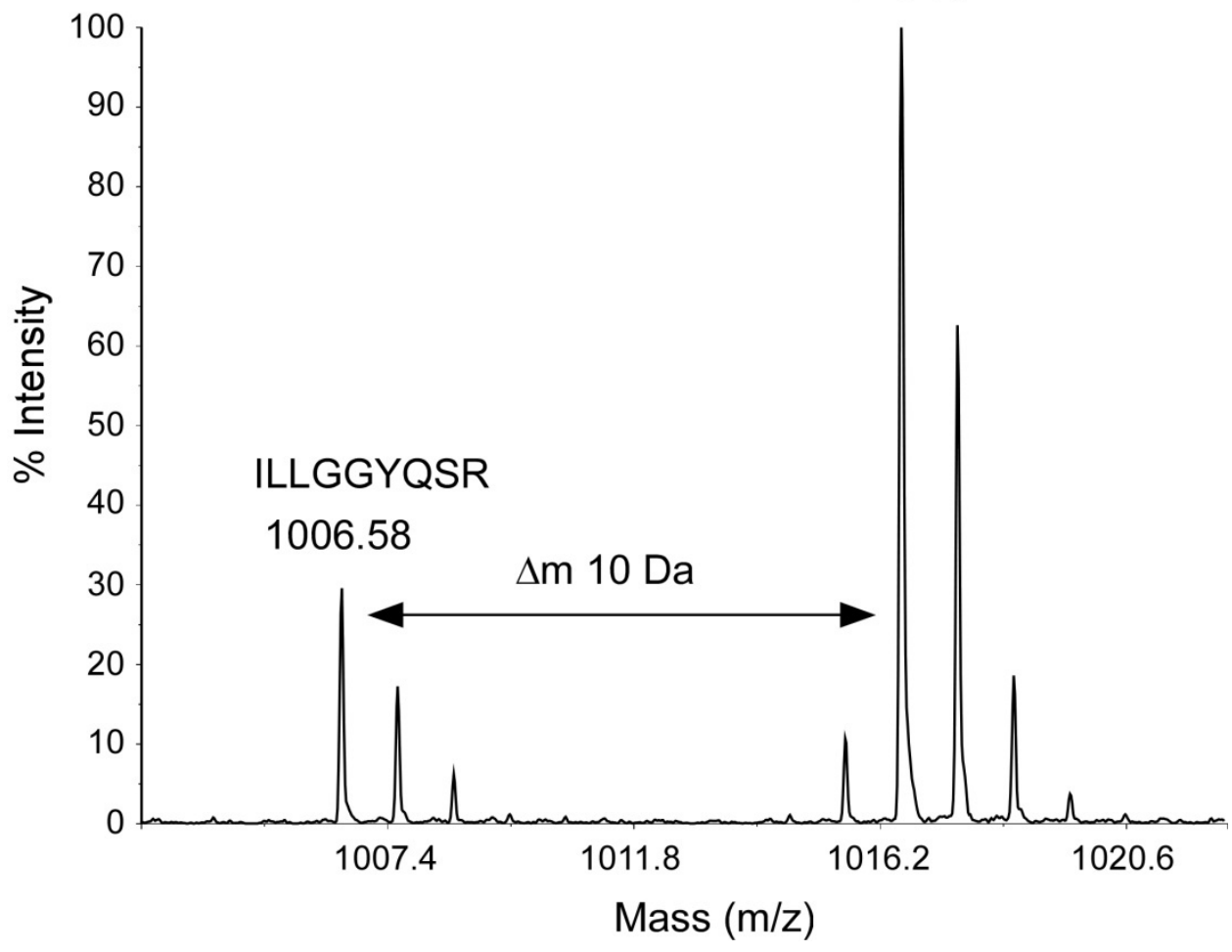

Figure 2.3: Example MS spectrum of an endogenous and the corresponding standard peptide for quantification. MS spectrum of ILLGGYQSR (CDC5L protein) and the corresponding synthetic standard peptide. The endogenous and the standard peptide show according to the incorporated stable isotope labeled amino acid (arginine) a distinct mass shift (10 Da). The spectrum was acquired by nanoLC-offline MALDI-ToF/ToF-MS.

There are however limitations for the absolute quantification using standard peptides. First, the addition of standard peptides to a proteome digest provides quantification of only single or few proteins of the sample. For reliable absolute quantification of a protein in a mixture, several standard peptides per protein have to be first selected and then provided during quantification to achieve more than one reference value per protein. Furthermore, incomplete protein hydrolysis is one of the most critical issue in the event of absolute quantification as it dramatically affects the final result.

To increase confidence in quantification experiments, addition of multiple peptides for each protein to be quantified is recommended. This can be simplified by using labeled standard proteins, which provide multiple peptides for absolute quantification after their hydrolysis. To this end, heavily labeled amino acids are incorporated into entire proteins resulting in heavily labeled standard proteins, which are then added to the sample under investigation. After protein hydrolysis of the endogenous and the standard proteins, standard peptides for all generated endogenous peptides are available. Several approaches using labeled standard peptides have recently been introduced (PSAQ, Protein Standard Absolute Quantification (Brun et al., 2007); Absolute SILAC (Hanke et al., 2008); FLEXIQuant, Full-length expressed 
stable isotope-labelled proteins for quantification (Singh et al., 2009)). Very similar is the use of artificial QconCAT proteins, which are assembled from different standard peptide sequences (concatenated signature peptides encoded by QconCAT genes; Pratt et al., 2006). During hydrolysis of QconCAT proteins several standard peptides belonging to diverse proteins are generated allowing the quantification of different proteins in a sample.

Label-free approaches for absolute quantification

Label-free quantification is a method that determines relative or absolute protein amounts without using stable isotope containing compounds. The advantage of label-free quantification is that time-consuming steps of introducing labels to proteins or peptides are not required and that there are no costs for expensive labeling reagents. Furthermore, there is no limit as to the number of experiments to be compared and several peptides per protein are available for quantification. Mass spectral complexity is not increased as in the case of differently labeled samples what provides a higher analytical depth. Unfortunately, label-free approaches are least accurate among the mass spectrometric quantification techniques and they require a high reproducibility at each step, i.e. all experiments need to be accurately reproduced to achieve reliable quantification (Bantscheff et al., 2007).

One possibility to determine absolute protein amounts without labeling of peptides or proteins is to make use of the number of observed and theoretically observable peptides. The protein abundance index (PAI) is then calculated by dividing the number of observed tryptic peptides by the number of theoretically observable peptides from a particular protein and gives an estimate for absolute protein amounts in a complex mixture (Rappsilber et al., 2002). For absolute quantification, the PAl was later converted to an exponentially modified form (emPAl), which is proportional to the protein content in a protein mixture. Ishihama et al. have shown that the emPAl-abundances from the actual values are within $63 \%$ on average. Nonetheless, emPAl values are easily calculated but provide only a rough estimate of the absolute protein amounts (Ishihama et al., 2005).

Incomplete digestion is a critical issue for absolute quantification. One way to deal with this is to average the quantities of the three most abundant peptides of every protein. It is generally assumed that some parts of the protein are completely digested and thus the three most abundant peptides reflect the protein concentration. The protein mixture is spiked with an intact standard protein and after hydrolysis the average MS signal response of the standard protein is used to calculate an universal signal response factor (ion counts/mole of protein) for the particular experimental setup. This factor can then be used to determine the concentration of the analyzed proteins within the mixture (Silva et al., 2006). 
Top-down quantification of SILAC-labeled proteins

Top-down is the analysis of intact proteins instead of generated peptides. To date, only one study about top-down absolute quantification has been released. Waanders et al., 2007 introduced the quantification of intact SILAC-labeled proteins. During the SILAC (stable isotope labeling using amino acids in cell culture, see below) method, cells are grown in media containing different isotope labeled amino acids. When using heavy and light lysine and arginine for SILAC labeling, intact proteins do not interfere with peaks of different charge states between 10 and $200 \mathrm{kDa}$. The authors have shown that two SILAC proteins (light and heavily labeled) can be quantified with an average standard deviation of $6 \%$ (Waanders et al., 2007).

Absolute quantification to determine the protein stoichiometry within protein complexes

By absolutely quantifying proteins in a purified protein complex, the protein stoichiometry of the quantified proteins can be established. In recent years, only few studies addressed the protein stoichiometries within protein complexes using absolute quantification. Two studies, combining chemical labeling of endogenous and standard peptides, were recently introduced. Hochleitner et al., 2005 determined the protein stoichiometry of the spliceosomal U1 small nuclear ribonucleoprotein complex and Holzmann et al., 2009 determined the stoichiometry of the MP1-p14 complex. A different approach has been designed for affinitytag purified protein complexes (Wepf et al., 2009). An amino acid sequence serving as standard peptide is embedded in the affinity tag and is released after tryptic digestion. The protein is then quantified by adding a stable isotope labeled reference peptide of the tag and the other proteins are quantified by correlational quantification to the tagged protein. This approach benefits from the fact that one stable isotope labeled standard peptide can be used for quantification of different proteins, but requires the protein of interest to be present as tagged protein in the sample.

\subsubsection{Relative quantification by mass spectrometry}

Several different approaches and techniques are available for relative MS-based quantification. Most of these approaches are based on the introduction of stable isotopes. This is performed by metabolic, chemical or enzymatic labeling. In addition, some label-free methods (e.g. spectral count) are available. As described for absolute quantification (see above), differently labeled peptides with the same physicochemical properties can be distinguished by a mass difference according to the introduced heavy stable isotopes. Relative quantification is then achieved by comparison of the peaks' signal intensities from differently labeled peptides (MS). In case of isobaric labeling reagents, i.e. labeling reagents that differ in their isotope composition but have the same mass, signal intensities from different reporter ions, which are released during fragmentation of the labeling reagent are 
used for relative quantification (iTRAQ and TMT, see below). The most important and popular techniques for relative MS-based quantification will be discussed in detail in the following paragraphs.

\section{Metabolic labeling}

The earliest time point to introduce stable isotopes into proteins is during cell growth (see also Table 2.1). This will reduce errors as samples to be quantified can be combined at a very early step during sample processing (Ong and Mann, 2005). Metabolic labeling followed by quantification was initially described for bacteria using ${ }^{15} \mathrm{~N}$ enriched cell culture medium (Oda et al., 1999). Subsequently, mammalian cells (Conrads et al., 2001) and even small organisms such as C.elegans and D.melanogaster (Krijgsveld et al., 2003) have been fully labeled with ${ }^{15} \mathrm{~N}$. ${ }^{15} \mathrm{~N}$-labeling achieved complete incorporation of ${ }^{15} \mathrm{~N}$ to all amino acids within the cells/organisms, thus providing a high number of peptides suitable for quantification. However, the mass difference between the labeled and unlabeled peptide depends on the number of nitrogen atoms within the amino acid sequence of the particular peptide. This complicates data analysis enormously, making ${ }^{15} \mathrm{~N}$-labeling not to the method of choice. In addition, highly enriched ${ }^{15} \mathrm{~N}$ labeled sources are required to avoid complicated isotopic distribution from partially labeled peptides (Ong and Mann, 2005).

As a simpler method, stable isotope labeling by amino acids in cell culture (SILAC), was introduced by Mann and co-workers (Ong et al., 2002). In this approach, cells are grown in cell culture medium containing ${ }^{13} \mathrm{C}$-, ${ }^{15} \mathrm{~N}$ - and ${ }^{2} \mathrm{D}$-labeled L-lysine and L-arginine. Incorporation of isotope-labeled lysine and arginine ensures a defined mass difference between the differently labeled peptides when using trypsin for proteolytic hydrolysis in proteome studies. As trypsin specifically cleaves proteins C-terminal of arginine and lysine (Olsen et al., 2004) the mass difference of differentially labeled peptides is defined by the isotope incorporation into lysine and arginine provided during cell growth. Using different combinations of stable isotope labeled lysine and arginine allows quantification of up to three samples in one MS measurement (see Figure 2.4 for an overview of differently labeled lysines and arginines). The introduction of the SILAC method led to a high number of quantitative studies during the last years. It was also successfully combined with quantification of post-translational changes in different systems (e.g. Oellerich et al., 2009; Olsen et al., 2010; Pan et al., 2009a). In addition, development of a new software (MaxQuant; Cox and Mann, 2008) facilitated data analysis and made SILAC to a very powerful technique for quantitative proteome studies. Since labeling occurs in cell culture, quantification of proteins in tissue is not possible. However, the production of a SILAC mouse using a "heavy diet" has recently been described (Kruger et al., 2008). Nonetheless, labeling of animals is high priced, requires a big effort and is not in all cases achievable. 
A
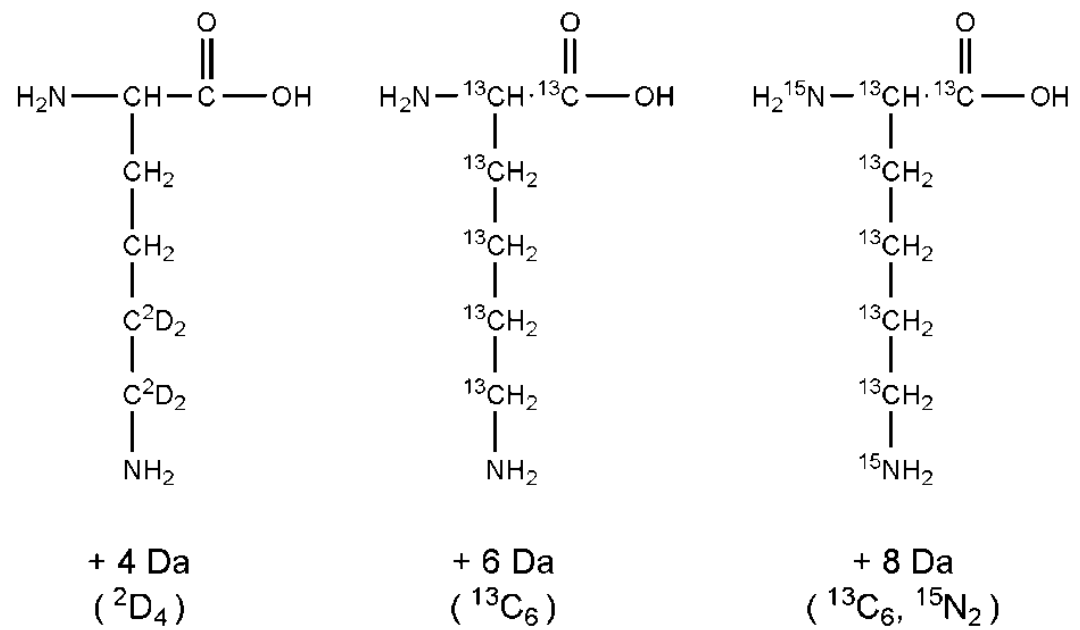

B
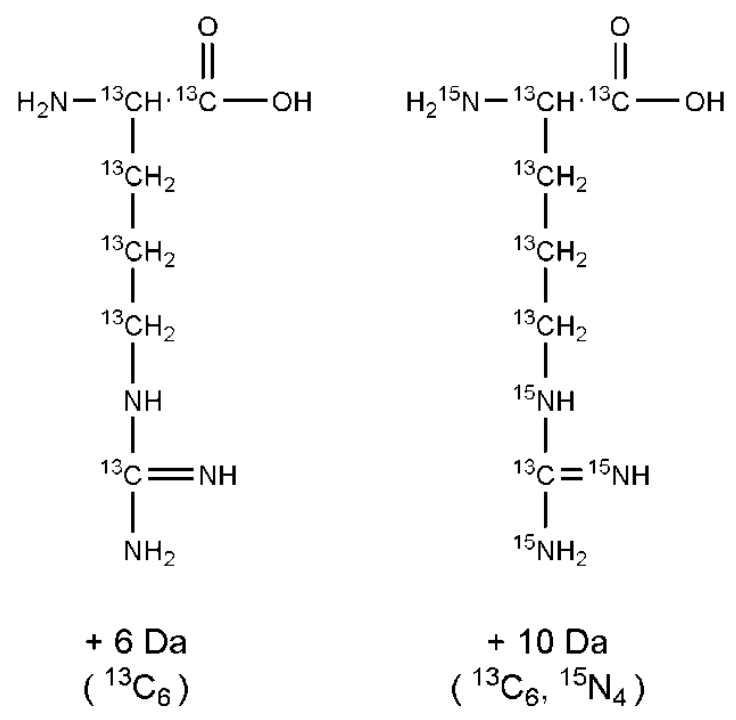

Figure 2.4: Isotope labeled amino acids used for cell growth in SILAC labeling. (A) Introduction of ${ }^{13} \mathrm{C},{ }^{15} \mathrm{~N}$ and ${ }^{2} \mathrm{D}$ to lysine generates a mass difference of 4,6 , and $8 \mathrm{Da}$ between the labeled and the non-labeled lysine. (B) Introduction of ${ }^{13} \mathrm{C}$ and ${ }^{15} \mathrm{~N}$ generates a mass difference of 6 and 10 Da between labeled and non-labeled arginine.

Chemical labeling

During chemical labeling, the peptides or proteins are labeled with isotope containing reagents. At this, advantage is taken of the chemical reactivity of amino acid side chains or the peptide's N-terminus (for an overview see Table 2.1). The first reagent for chemical labeling was the isotope-coded affinity tag (ICAT; Gygi et al., 1999). ICAT is a cysteine specific reagent consisting of a cysteine-reactive iodoacetyl group, a linker containing either zero (light ICAT) or eight (heavy ICAT) deuterium atoms and a biotin group for affinity purification of cysteine-derivatized peptides. Light and heavy ICAT-labeled protein samples are then combined and subsequently hydrolyzed. The advantage of ICAT is the reduced sample complexity after affinity purification, which allows protein quantification in complex samples. Complications arose with proteins containing none or only few cysteine 
residues as these are excluded from quantification. Further, the presence of deuterium atoms led to partial separation by chromatography and the size of the label affected fragmentation in the mass spectrometer. Therefore, an improved reagent (cICAT) using ${ }^{13} \mathrm{C}$ labeling of the linker (resulting in a mass difference of $9 \mathrm{Da}$ ) and containing an acid-cleavable biotin group was developed (Figure $2.5 \mathrm{~A}$ ).

Another approach is the isotope-coded protein label (ICPL; Schmidt et al., 2005), which is based on the N-hyroxysuccinimide (NHS) chemistry targeting the epsilon-amino group of lysine residues in proteins (Figure $2.5 \mathrm{~B}$ ). N-nicotinoyloxy-succinimide is used in a light (d0) and a heavy (d4) form allowing for relative quantification of two different samples. Further improve by incorporation of ${ }^{13} \mathrm{C}$ atoms to the nicotinoyl group resulted in a quadruplex reagent.

A<smiles>[13CH3]SNC(=O)CCC(=O)NCCCCNC(=O)CI</smiles>

B

C<smiles>O=C(ON1C(=O)CCC1=O)c1cccnc1</smiles><smiles>CC1CCCC(C)N1CC(=O)NCCC(=O)ON1C(=O)CCC1=O</smiles>

$\mathbf{D}$<smiles>COC1=NCCN1</smiles>

C-terminal<smiles>O=C1CCC(=O)O1</smiles>

$\mathrm{N}$-terminal

Figure 2.5: Chemical labeling reagents. (A) CICAT reagent. The exact structure of the cleavable biotin tag has not been publicly disclosed. (B) N-nicotinoyloxy-succinimide used for ICPL quantification. (C) Structure of the TMT reagent. (D) C-terminal and N-terminal reagents for IPTL. 2-Methoxy-4,5-dihydro-1H-imidazole (C-terminal) and succunic anhydride (N-terminal).

A different approach, based also on NHS chemistry, is iTRAQ (isobaric tags for relative and absolute quantification; Ross et al., 2004). iTRAQ reagents are amine specific and label lysine side chains and amino termini of peptides. They are multiplexing reagents, i.e. due to the isotope composition of the reagents several samples can be compared relative to another in one experiment. iTRAQ 4-plex and 8-plex reagents are available allowing relative quantification of four and eight samples in one experiment. They consist of an amine reactive 
group, a balance group and a reporter group (Figure 2.6). iTRAQ reagents are isobaric, i.e. all reagents have the same mass and all labeled peptides have a mass tag of $144.1 \mathrm{Da}$ in case of the 4-plex iTRAQ reagents. Upon fragmentation in the MS/MS experiment, all isobaric tags release a marker ion (so-called reporter ions) of 114.1, 115.1, 116.1 and 117.1 $\mathrm{Da}$, respectively, and a neutral fragment $(28,29,30,31 \mathrm{Da}$, respectively), which is not detected in the mass spectrometer (see Figure 2.6 for a detailed description of the 4-plex iTRAQ reagents). In contrast to the methods described above, iTRAQ-labeled peptides do not show a mass difference in the MS. Quantification of differently labeled peptides is therefore only achieved upon fragmentation. The described ITRAQ reagents were further modified so that labeling of eight samples can be performed simultaneously. iTRAQ has several advantages: (i) iTRAQ-labeling is multiplexing, i.e. up to four or eight samples can be compared in one quantification experiment. (ii) Since all labeled peptides have the same mass, the signal intensity in the MS is enhanced. (iii) The signal intensity of the fragment ions is enhanced as well because the mass tag is completely cleaved during fragmentation. (iv) iTRAQ labeling offers the opportunity to create an internal standard that contains a mixture of all samples, so that more than four or eight samples can be quantified in relation to the internal standard.

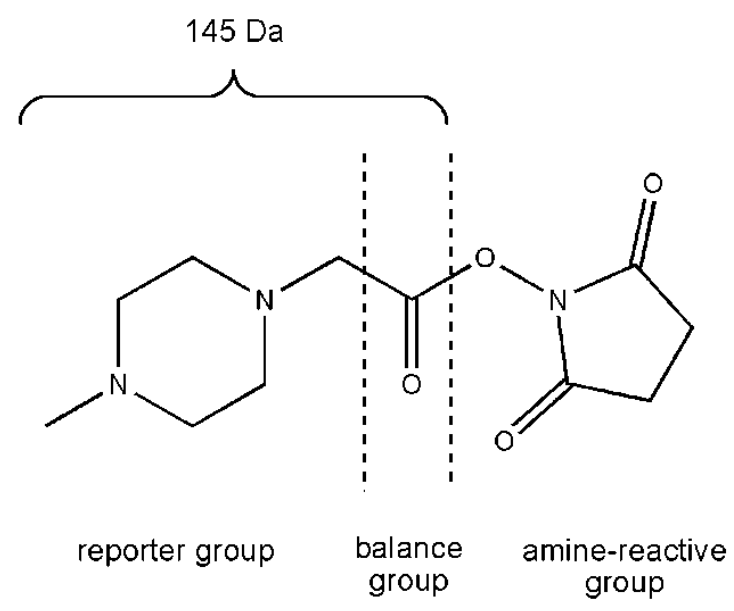

$\begin{array}{lll}\text { iTRAQ-114: } & { }^{13} \mathrm{C}_{1} & { }^{13} \mathrm{C}_{1},{ }^{18} \mathrm{O}_{1} \\ \text { iTRAQ-115: } & { }^{13} \mathrm{C}_{2} & { }^{12} \mathrm{C}_{1},{ }^{18} \mathrm{O}_{1} \\ \text { iTRAQ-116: } & { }^{13} \mathrm{C}_{2},{ }^{15} \mathrm{~N}_{1} & { }^{13} \mathrm{C}_{1},{ }^{16} \mathrm{O}_{1} \\ \text { iTRAQ-117: } & { }^{13} \mathrm{C}_{3},{ }^{15} \mathrm{~N}_{1} & { }^{12} \mathrm{C}_{1},{ }^{16} \mathrm{O}_{1}\end{array}$

Figure 2.6: Structure of the 4-plex iTRAQ reagent. The reagent consists of an amine-reactive group, a balance group and a reporter group. The isobaric tags have a mass of $145 \mathrm{Da}$ resulting in a mass difference of $144.1 \mathrm{Da}$ for all differently labeled peptides. Upon fragmentation, the reporter group and a neutral fragment (balance group) are released. Due to the isotope composition of the reporter and the balance group, the generated reporter ions show different masses for the different iTRAQ reagents.

A very similar approach is the labeling with TMTs (tandem mass tags; Thompson et al., 2003). The chemical structure resembles the one of ITRAQ reagents and the quantification 
procedure is the same (Figure $2.5 \mathrm{C}$ ). Using TMTs, up to six samples can be compared in one quantification experiment.

A different MS/MS-based quantification approach is the isobaric peptide termini labeling (IPTL; Koehler et al., 2009). Labeling of both peptide termini with tetradeuterated and nondeuterated reagents leads to isobaric peptides of the samples to be compared. After Lys-C hydrolysis of the proteins the peptides' C-termini are labeled with 2-methoxy-4,5-dihydro- $1 \mathrm{H}$ imidazole $(\mathrm{MDHI})$ or tetradeuterated $\mathrm{MDHI}-\mathrm{d} 4$ whereas the $\mathrm{N}$-termini are labeled tetradeuterated succinic anhydride (SA-d4) or SA, respectively (Figure 2.5 D). Fragmentation during $M S / M S$ causes the cleavage of the $\mathrm{N}$-terminal succinic anhydride resulting in fragment ion pairs that contain either the C-terminal MDHI- or MDHI-d4-label. The advantage of this approach is the generation of several quantification data points for each peptide and the opportunity to use mass spectrometers with limited capabilities in the low molecular mass range.

Dimethyl labeling of peptides at the $\mathrm{N}$-terminus and epsilon-amino groups of lysine residues has recently been introduced (Hsu et al., 2003). The use of differentially ${ }^{2} \mathrm{D}$ - and ${ }^{13} \mathrm{C}$-labeled formaldehyde and sodium cyanoborohydride allows triplex labeling (Boersema et al., 2008). Dimethylation of amino groups causes a mass increase of 28,32 , and $34 \mathrm{Da}$, respectively, resulting in a mass difference of 4 and $6 \mathrm{Da}$ between the differently dimethylated peptides. This labeling method has several advantages: (i) It uses inexpensive reagents and is thus a cost-effective labeling technique in comparison to other stable isotope-reagents. (ii) It is a reliable method providing a $100 \%$ labeling efficiency in almost all cases. (iii) Different labeling protocols (in-solution, online and on-column) for different amounts of sample have been described allowing for automation and high-throughput proteomics (Boersema et al., 2009). (iv) No reaction byproducts have been observed. (v) As dimethyl labeling is based on a simple chemical reaction, a large number of samples can be quantified by this method.

Enzymatic labeling Isotopic labels can also be incorporated during enzymatic proteolysis (Mirgorodskaya et al., 2000; Reynolds et al., 2002; Yao et al., 2001). Proteolytic hydrolysis in "heavy" $\left(\mathrm{H}_{2}{ }^{18} \mathrm{O}\right)$ and "normal" $\left(\mathrm{H}_{2}{ }^{16} \mathrm{O}\right)$ water leads to introduction of two ${ }^{18} \mathrm{O}$ atoms at the peptide's C-terminus resulting in a mass shift of 4 Da between the labeled $\left({ }^{18} \mathrm{O}\right)$ and non-labeled $\left({ }^{16} \mathrm{O}\right)$ peptide. Very common is also enzymatic labeling after proteolysis in a second incubation step with the protease. Suitable enzymes are Trypsin and Glu-C as they introduce two ${ }^{18} \mathrm{O}$ atoms to the $\mathrm{C}$-terminus resulting in a sufficient mass shift for differentiation of isotopomers (4 Da). Asp- $\mathrm{N}$ and other enzymes introduce only one ${ }^{18} \mathrm{O}$ atom (Rao et al., 2005) and should therefore be avoided. Advantageous over chemical labeling is that side reactions do not occur. A critical point is acid- and base-catalyzed back-exchange at extreme $\mathrm{pH}$ values (Schnolzer et al., 1996), whereas mild acidic conditions during ESI- 
and MALDI-MS analysis guarantee stability of the introduced label. However, incomplete labeling (i.e. incorporation of only one ${ }^{18} \mathrm{O}$ atom) complicates data analysis and requires correction of overlapping isotopic patterns (Johnson and Muddiman, 2004; RamosFernandez et al., 2007).

Label-free relative quantification

The observation that the more of a particular protein is present in a sample the more tandem MS spectra are collected during MS analysis led to the assumption that there is a correlation between number of spectra and the protein amount. Spectral count has therefore been applied for relative quantification in different studies (Gilchrist et al., 2006; Washburn et al., 2001). Liu et al., 2004 have analyzed this correlation more in detail and found a linear correlation over 2 orders of magnitude between the number of spectra and the relative protein abundance whereas no correlation between relative protein amounts and peptide count or sequence coverage has been observed. Spectral count is less accurate for small changes between proteins, but was shown to be very accurate for large changes allowing a measure how protein changes overall (Liu et al., 2004). However, dynamic exclusion of ions that already have been selected for fragmentation during MS analysis is disadvantageous for accurate quantification (Old et al., 2005). Spectral counting is a very attractive approach but neglects that no physical property of a peptide is measured. Furthermore, the assumption that the response for every protein is the same is misleading (Bantscheff et al., 2007). Due to different amino acid sequence and different properties of the generated peptides (e.g. chromatographic behavior) the number of spectra detectable varies for different proteins.

Certainly, all methods described for absolute quantification can also be applied for relative quantification by absolutely quantifying proteins in different samples and compare the absolute protein amounts in a relative manner.

\subsubsection{Quantification by mass spectrometry to analyze dynamic protein transitions}

Relative MS-based quantification can not only be used to compare two or multiple different cell stages, complexes etc., it can also be applied to monitor protein dynamics or protein assembly of multi-protein components. To date, only few studies about dynamic protein changes were published. All these studies use metabolic labeling and take advantage of relative peptide ratios at different time points to display protein changes over time.

Williamson and co-workers developed a method called pulse-chase monitored by quantitative mass spectrometry (PC/QMS; Talkington et al., 2005; Williamson, 2005). They analyzed the assembly of the $30 \mathrm{~S}$ ribosomal subunit using 16 rRNA and ${ }^{15} \mathrm{~N}$-labeled $30 \mathrm{~S}$ proteins as starting products. At various time points, protein binding was chased with excess 
of unlabeled proteins and completely formed $30 \mathrm{~S}$ subunits were purified. The ratios of labeled to unlabeled peptides for each protein was then plotted as a function of time providing a progress curve for the binding of this specific protein during the assembly event. This method enabled a detailed and quantitative kinetic characterization of this specific assembly process.

Compared to ${ }^{15} \mathrm{~N}$-labeling, SILAC shows several advantages (see above). It is therefore not surprising that SILAC approaches were recently introduced to monitor protein changes. To this end, cells were grown in media supplemented with stable isotope labeled amino acids and differently labeled cells were treated with e.g. inhibitors or inducers. Triple SILAC allows the comparison of three different time points in one experiment. Performing multiple experiments using the zero time point as a reference allowed construction of dynamic profiles for single proteins. This method was successfully applied to analyze the nucleolar proteome dynamics (Andersen et al., 2005; Lam et al., 2007), endoplasmic reticulum stress response (Mintz et al., 2008), and protein turnover rates in intact animals (Doherty et al., 2005).

Pulsed SILAC (pSILAC) is a novel SILAC variant where cells cultivated in normal medium are pulse-labeled by transferring the cells in medium containing stable isotope labeled amino acids. Newly synthesized proteins are labeled with heavy stable isotopes whereas preexisting proteins present before labeling remain in the light form. Protein changes upon treatment of the cells can thus be monitored. By this method, changes in protein synthesis induced by microRNAs (Selbach et al., 2008) and translational regulation of cellular iron homeostasis (Schwanhausser et al., 2009) have been successfully analyzed.

\subsubsection{Quantifying mass spectrometric measurements}

A mass spectrometer consists of an ion source, a mass analyzer that measures the mass-tocharge ratio $(\mathrm{m} / \mathrm{z})$ of the generated ions, and a detector that detects the number of ions at each $\mathrm{m} / \mathrm{z}$ value. Two suitable techniques for the ionization of peptides and proteins are ESI and MALDI (see above). The analytes are ionized out of a solution (ESI) or out of a crystalline matrix via laser pulses (MALDI). Whereas these two techniques are set, a wider range of mass analyzers is available. Important parameters are sensitivity, resolution, mass accuracy, and the ability to generate information-rich mass spectra. The basic types of mass analyzers currently used in proteomic studies are time-of-flight (ToF), ion trap, quadrupole, Fourier transform ion cyclotron resonance (FT ICR), and the Orbitrap mass analyzers. MALDI is usually coupled to ToF analyzers whereas all other mass analyzers are commonly coupled to an ESI source. In addition, hybrid mass analyzers, such as hybrid quadrupole ToF 
(Qq-ToF) mass analyzers, are available. Here, precursor ions for MS/MS experiments are selected in the first quadrupole, fragmented in a collision cell, and the fragment ion masses are analyzed in the ToF. These instruments are commonly coupled to an ESI source and have high sensitivity, resolution, mass accuracy, and - most important - generate informationrich fragment spectra.

Quantitative information can be obtained from MS or MS/MS signals (see above). The advantage of quantification from MS spectra is that several spectra are available and ion intensities are high. However, very low and very strong signals are problematic. Low signals are hard to distinguish from background noise and very strong signals can saturate the detector, what in turn limits precision of the measurement. The latter is more often observed in ToF and Qq-ToF instruments compared to ion traps as the latter can control the number of ions before detection. Using fragment ion intensities (MS/MS) for quantification, detector saturation and interference with background ions can be neglected. Low intensities are rather a problem as poor ion statistics may result in less robust quantification. However, limits to quantification of complex samples can often be attributed to interference of co-eluting components of similar masses.

Different specific mass spectrometric scanning modes are used to read out signal intensities during quantitative analysis. The commonly used and most powerful techniques will be described in the following paragraphs.

MALDI-ToF-MS Technical advances have enhanced the application of MALDI mass spectrometry for proteomics but also for quantitative studies. The investigation of tandem time-of-flight instruments (ToF-ToF) allows the fragmentation of precursors and thus unambiguously assigns the species to be quantified (Bienvenut et al., 2002). Decoupled MS and MS/MS analysis allows for data-dependent MS/MS analysis, and the manner of sample preparation reserves most of the sample for repeated analysis. Furthermore, the generation of predominant singly charged ions during MALDI simplifies data analysis (Pan et al., 2009b). During MALDI-ToF-MS quantitative information is often obtained from the area under the peaks to be quantified. However, LC-offline allows the generation of extracted ion chromatograms (XICs, see below) over the whole chromatographic timescale for the peptide of interest. However, in a MALDI spectrum there is often a large discrepancy between ion intensities and analyte concentration on the MALDI target. Ionization of the peptides occurs via proton transfer from the acidic matrix and the ionization efficiency is therefore dependent on the proton affinity of the different peptides. The presence of a peptide with a very high proton affinity can consequently influence the intensity of other ions through ion suppression effects (Knochenmuss et al., 2000). For this reason, peptides below a mass of $3 \mathrm{kDa}$ can only be quantified using standard peptides, which have the same chemical structure and thus 
show the same behavior during the ionization process (see Figure 2.3 for an example of a spectrum of an endogenous and a standard peptide acquired by MALDI-ToF-MS). Peptides or proteins larger than $3 \mathrm{kDa}$ have such a high proton affinity that ion suppression effects are very unlikely (Wilm, 2009).

Extracted ion chromatograms (XICs) Electrospray ionization is well suited for quantitative measurements if the flow rates are $100 \mathrm{nl} / \mathrm{min}$ or lower. In this case, spectral intensities correspond to the analyte concentrations very well. For higher flow rates the electrospray is unsteady and ion intensities become irregular and do not reflect molecular concentrations (Wilm, 2009). In addition, ion suppression has been observed for higher flow rates (Schmidt et al., 2003). The peptide's signal in the MS analysis can be plotted over time while the peptide is eluting from the chromatography column, i.e. so-called extracted ion chromatograms (XICs) for defined peptides can be generated. The XIC signal is related to the relative amount for the same peptide at the same experimental conditions and can therefore be used for comparison of the same peptide in different samples. XICs are usually generated from samples analyzed by LC-ESI-MS whereas generation of XICs from LC-offline MALDI-MS analyses is also possible (see above). Using high mass accuracy mass spectrometers, greater than two-fold changes of a peptide can be measured. As one peptide in a complex mixture is not always selected for fragmentation in different MS runs (Kuster et al., 2005) it is critical to find and quantify the correct peptide in the different analyses to be compared. Development of required software and normalization of the runs to be compared by spiked-in calibrants can overcome this problem. The great advantage of XIC-based quantification is that no labeling strategy is used and almost every different MS analyses can be compared as long as they were performed under the same conditions. However, this requires a very high reproducibility during sample preparation, chromatography, and MS analysis. One alternative to circumvent this problem is the use of stable isotope labeled standard peptides in known amounts. As the labeled and the non-labeled peptide show the same behavior during chromatography and MS generated XICs from both peptides show the same chromatographic retention time. The area under the XICs can then be used for absolute or relative quantification (see Figure 2.7 for an example of XICs of a labeled and a non-labeled peptide). 
A

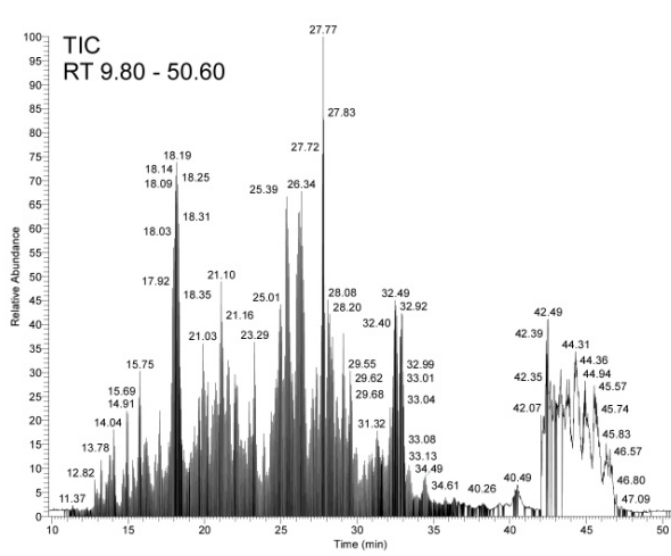

B

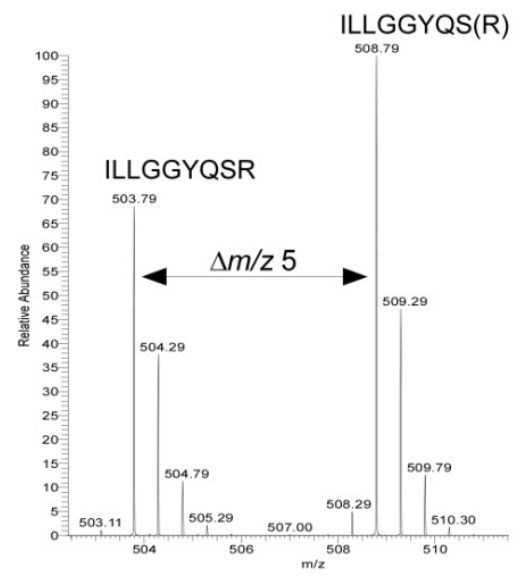

D

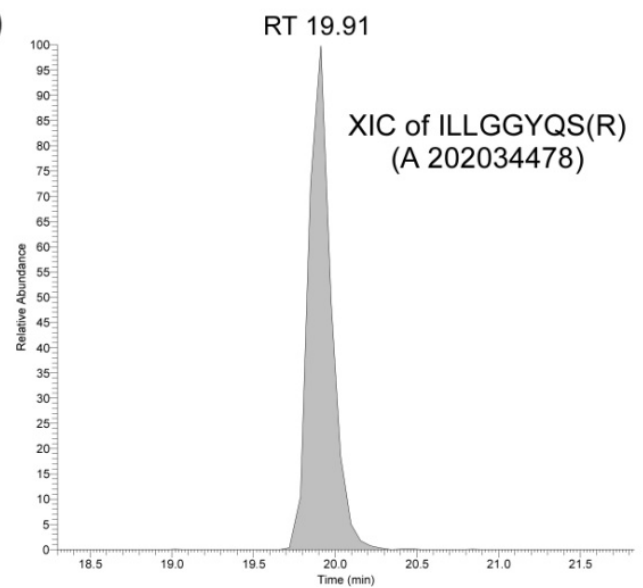

Figure 2.7: Example of Extracted ion chromatograms (XICs) to read out the signal intensities for quantification. (A) Total ion count of the hydrolyzed hPrp19/CDC5L complex. (B) MS spectrum of ILLGGYQSR (CDC5L protein) and the stable isotope labeled standard peptide. According to the incorporated stable isotope labeled amino acid (arginine) a mass difference of $5 \mathrm{~m} / \mathrm{z}$ between the doubly charged peptides is observed. (C) Extracted ion chromatogram of ILLGGYQSR. (D) Extracted ion chromatogram of the standard peptide ILLGGYQS(R). The endogenous and the standard peptide show the same retention time. The peak area of the signals can be used for quantification.

\section{Multiple Reaction Monitoring (MRM)}

A quadrupole mass analyzer can be operated as a mass filter allowing only one specific $m / z$ to pass the quadrupole. This feature is utilized to detect the specific transition from a given precursor to a user-defined fragment ion (selected reaction monitoring, SRM) in a triple quadrupole mass spectrometer. To this end, the precursor mass is selected in quadrupole Q1, whereupon (after fragmentation in q2) the user-defined fragment ion is detected in Q3 (SRM transition). This technique has been extended to the detection of multiple fragment ions per precursor and is then called multiple reaction monitoring (MRM; Anderson and Hunter, 2006; Kuhn et al., 2004; Stahl-Zeng et al., 2007, Figure 2.8). The MRM signal is quantitative over 4-5 orders of magnitude (Wolf-Yadlin et al., 2007) and can be used for relative and for absolute quantification as outlined in section 2.1.3. One run, in which all MRM transitions are monitored once, is called a duty cycle and its length is dependent on the dwell time (i.e. the time to accumulate ions in the quadrupole), the 


\section{4 | INTRODUCTION}

number of precursors, and the number of MRM transitions per precursor. The duty cycle is repeated consistently during the MRM analysis. It should be repeated several times while the peptide is eluting from the chromatography column to achieve a certain number of data points, which are required to record a sufficient MRM signal. The length of the duty cycle is therefore not unlimited and needs to be adjusted for every analysis. MRM is a very sensitive and, as two mass filters are connected in series, a very specific method. For this reason, it is not only a quantification technique but also very well suited for targeted proteomics, such as biomarker verification and validation.

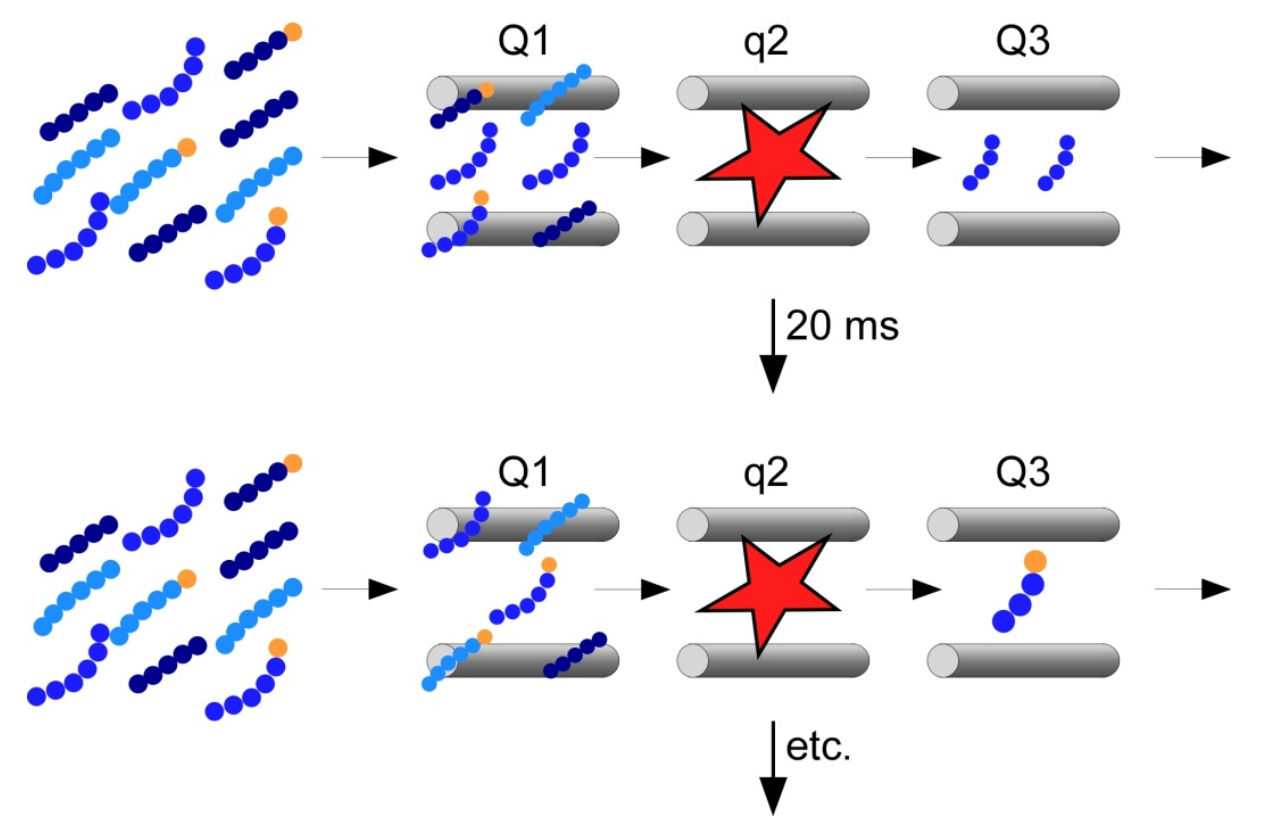

Figure 2.8: MRM workflow. A peptide mixture containing endogenous and stable isotope labeled standard peptides is analyzed by MRM in a triple quadrupole mass analyzer. In quadrupole 1 (Q1) a defined precursor ion is selected and, upon fragmentation in q2, a specific fragment ion of the selected precursor is detected in Q3. After a defined time (e.g. $20 \mathrm{~ms}$ ) the next precursor is selected for MRM analysis. One duty cycle comprises the analysis of all MRM transitions scheduled for the peptide sample to be analyzed. The obtained signal for the MRM transitions is quantitative and can be used for relative and absolute quantification of the peptides.

Parallel fragmentation $\left(M S^{E}\right)$

quantify peptides is parallel fragmentation (also known as $\mathrm{MS}^{\mathrm{E}}$; Silva et al., 2006). Here, all precursors, entering the mass spectrometer at a particular time, are fragmented without selecting an individual precursor ion. For this purpose, the mass spectrometer switches continuously between MS and MS/MS mode, thus delivering an almost complete data set of the sample. Fragment ions are assigned retrospectively to their precursors by their identical time profiles. However, this requires a well-resolved chromatographic system and the assignment of fragment ions to their precursor might fail due to fragment ions that are generated by several precursors simultaneously. Therefore, the $\mathrm{MS}^{\mathrm{E}}$ scanning method is performed on Qq-ToF mass spectrometers that might compensate probable mis- 
assignments by their high mass resolution in MS/MS. Once the experimental data are obtained, the data set can be interrogated for a specific ion and a set of specific fragment ions (pseudo-MRM; Niggeweg et al., 2006). This interrogation is not as specific as a real MRM experiment but has been proven for direct, label-free quantification (Wilm, 2009).

\subsection{The spliceosome}

\subsubsection{Eukaryotic pre-mRNA splicing}

Gene expression is the transcription of DNA in messenger RNA (mRNA) and the translation of mRNA into proteins. Most eukaryotic genes are expressed as precursor mRNAs (premRNAs) consisting of coding sequences (exons) and non-coding sequences (introns). One of the major steps during gene expression is therefore the excision of introns and the ligation of adjacent exons to yield mature mRNA (pre-mRNA splicing).

\subsubsection{Structure of eukaryotic pre-mRNAs}

Eukaryotic pre-mRNAs consist of exons and introns. The introns are defined by very short, conserved sequences at the 5'exon/intron and 3'intron/exon junctions (5' and 3' splice sites, respectively) as well as the branch point site (BPS), which is located 18-40 nucleotides upstream of the 3' splice site (3'ss) and involves a conserved adenosine (branch point adenosine). In addition, pre-mRNAs of higher eukaryotes usually contain a polypyrimidine tract consisting of 10-15 pyrimidines located between the BPS and the 3'ss (Figure $2.9 \mathrm{~A}$ ). These cis-acting elements are conserved in yeast but are degenerated in higher eukaryotes with only GU (5'ss) and AG (3'ss) at the intron ends being invariable. Two types of spliceosomes that catalyze pre-mRNA splicing co-exist in most eukaryotes. There is a U2dependent spliceosome, which catalyzes splicing of U2-type introns and which is found in all eukaryotes (major spliceosome). In addition, there is a U12-dependent spliceosome (the minor spliceosome), which is only present in a subset of eukaryotes.

\subsubsection{The biochemical splice reaction}

The splicing process follows two subsequent transesterification reactions (Figure $2.9 \mathrm{~B}$ ): First, the 2' hydroxyl group of the branch point adenosine attacks the phosphodiester bond at the 5' splice site (5'ss). This results in a 2'-5' phosphodiester bond formed between the branch point adenosine and the first nucleotide of the intron, resulting in an intron-exon 2 lariat and a free exon 1. In the second step of splicing, the 3' hydroxyl group of the free exon 1 attacks the phosphodiester bond of the 3' splice site (3'ss). Exon 1 and exon 2 are thus 
ligated and the intron lariat is released (Burge et al., 1999; Green, 1991; Moore et al., 1993; Moore and Sharp, 1993; Nilsen, 1998).

A

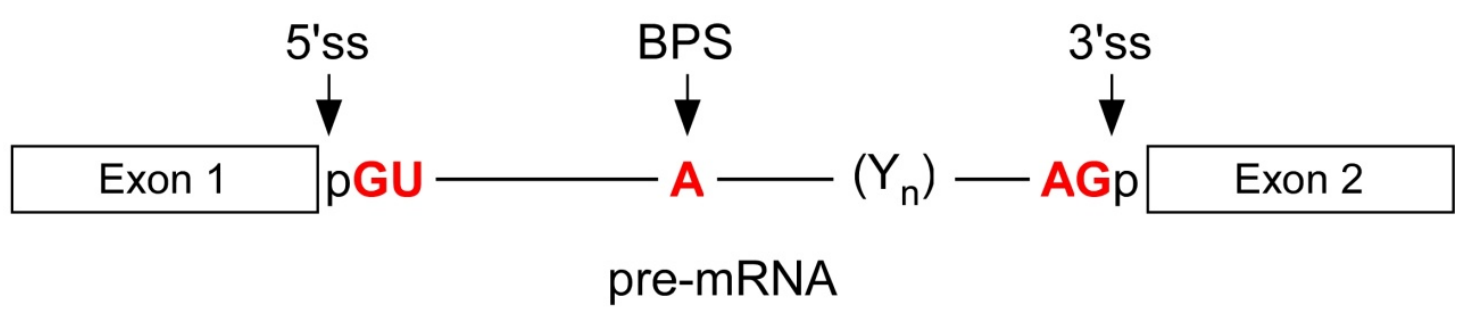

B
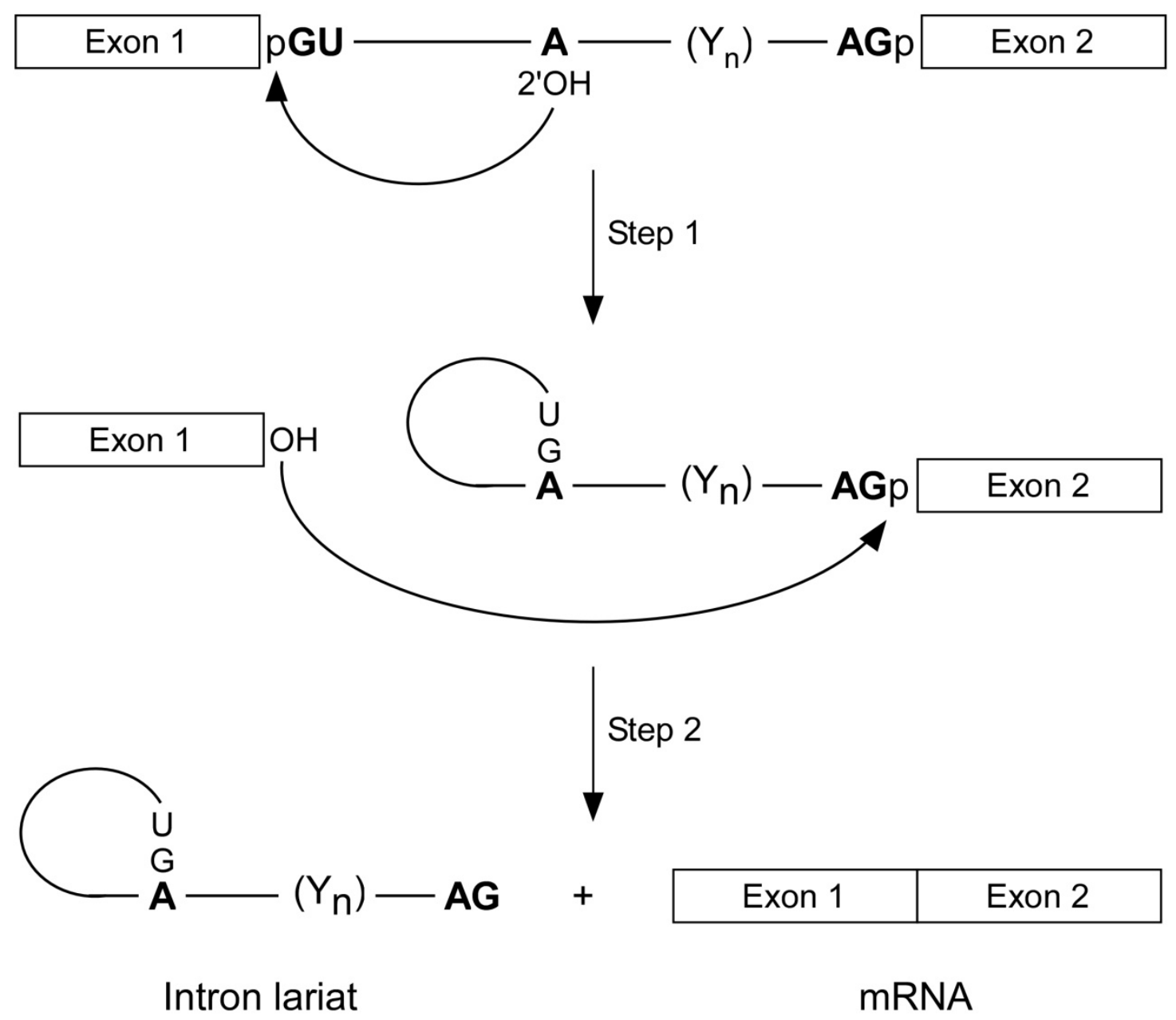

Figure 2.9: Schematic representation of the splicing process. (A) pre-mRNAs in higher eukaryotes consist of exons and interjacent introns. The introns are defined by a 5' and a 3' splice site, a branch point site containing a conserved adenosine and in most cases a polypyrimidine tract $\left(Y_{n}\right)$. Conserved nucleotides of U2-dependent introns in higher eukaryotes are highlighted in bold red. Note that conserved nucleotides of U12-dependent introns differ from U2-dependent introns. (B) The two-step mechanism of splicing. See text for details. 


\subsubsection{The catalytic machinery}

The spliceosome is responsible for the recognition and base pairing of the 5' and 3' splice site and for catalysis of the excision of the defined introns and the ligation of adjacent exons. It assembled in a stepwise fashion on each intron that is processed. The catalytic site of the spliceosome is formed by an extensive RNA-RNA, RNA-protein and protein-protein network. The RNA network is designed by several inter- and intra-snRNA interactions, as well as interactions between snRNAs and the pre-mRNA (reviewed by Nilsen, 1998). The spliceosome is highly dynamic and undergoes several structural rearrangements during formation of an active spliceosome. These structural changes are facilitated by proteins of the DExD/H-box protein family, which are able to rearrange RNP and RNA-RNA interactions while hydrolyzing ATP (reviewed by Schwer, 2001; Staley and Guthrie, 1998). Due to the extensive RNA network, which is designed during spliceosomal activation, the spliceosome resembles an RNA enzyme with active sites comprised of RNA. However, the catalytically active structure requires the assembly of numerous spliceosomal proteins.

\subsubsection{Components of the spliceosome}

\subsubsection{The U snRNPs}

A large number of trans-acting factors interact with the pre-mRNA during pre-mRNA splicing. The major spliceosome assembles from U1, U2, U4/U6 and U5 snRNPs and several nonsnRNP proteins. All $U$ snRNPs consist of a specific uridine-rich RNA (U snRNA) and a set of particle-specific proteins (Table 2.2). The sequence of the $U$ snRNAs is highly conserved evolutionary (Guthrie and Patterson, 1988) and all U snRNAs (except U6 snRNA) posses a conserved Sm binding site (Liautard et al., 1982). U1, U2, U4 and U5 snRNAs are transcribed by RNA polymerase II and transported to the cytoplasm, where seven Sm proteins are loaded onto the Sm site of the RNAs. They are further modified by addition of a $\mathrm{m}_{3} \mathrm{G}(2,2,7$-trimethylguanosine) cap. U6 snRNA is transcribed by RNA polymerase III and carries a gamma-monomethyl phosphate cap (Kunkel et al., 1986; Singh and Reddy, 1989). In addition, all snRNAs are post-transcriptionally modified by pseudouridines, 6methyladenosines and 2'-O-methylation at the ribose residues (Massenet et al., 1998). They are involved in RNA-RNA and RNA-protein interactions and thus play an important role in the formation of a catalytically active spliceosome (see above).

Common to all U snRNPs (except U6) are the seven Sm proteins (E, F, G, D1, D2, D3, and B/B'). They form a ring-shaped heptamer and bind the $U$ snRNA at the Sm site via a Sm motif to form the Sm core RNPs (Raker et al., 1999; Raker et al., 1996; Urlaub et al., 2001). 
U6 snRNA does not contain a Sm binding site but associates with a group of related proteins, called Sm-like proteins (LSm2-LSm8). They also form a heptameric ring and bind the U6 snRNA at the 3' end (Achsel et al., 1999; Vidal et al., 1999). In addition to Sm and LSm proteins, all $U$ snRNPs contain a specific set of proteins, which contribute to the functionality of the $U$ snRNPs during pre-mRNA splicing. Biochemical and immunological procedures allowed for purification of the snRNPs (Kastner and Luhrmann, 1999).

The 12S U1 snRNP contains beside the Sm proteins three specific proteins: The U1-70K, $\mathrm{U} 1-\mathrm{A}$ and $\mathrm{U} 1-\mathrm{C}$ proteins. From these, the U1-70K and the U1-A bind directly to the snRNA (Patton et al., 1989; Patton and Pederson, 1988; Query et al., 1989; Scherly et al., 1989; Urlaub et al., 2000), whereas the U1-C protein only binds to the U1 snRNP in the presence of the Sm core and U1-70K (Nelissen et al., 1994). The U1-C protein is important for splicing activity, as it directly contacts the pre-mRNA near the 5'ss stabilizing snRNA-pre-mRNA interactions (Heinrichs et al., 1990; Pomeranz Krummel et al., 2009).

The U2 snRNP includes several proteins. It was first described as $12 S$ U2 snRNP consisting of the U2 snRNA, the Sm proteins and two other proteins, namely A' and B', and was later found to be present as splicing active 17S U2 snRNP including two further heteromeric splicing factors, called SF3a and SF3b (Behrens et al., 1993; Brosi et al., 1993; Will et al., 2002). SF3a is composed of three proteins with apparent molecular weight of 120,66 and $60 \mathrm{kDa}$, whereas SF3b consist of seven proteins with molecular weight of 10, 15 (SF3b14a and b), 49, 130, 145, $155 \mathrm{kDa}$. Almost all SF3a and SF3b proteins contact the pre-mRNA near the branch point site and are thus essential for the spliceosomal assembly (Gozani et al., 1996; Kramer et al., 1999). In addition to SF3a and SF3b, several other proteins (e.g. hPrp5, SPF45 and CHERP) were identified in immunoaffinity purified U2 snRNP (Will et al., 2002).

The 20S U5 snRNP contains eight U5 specific proteins with an apparent molecular weight of 15, 40, 52, 100, 102, 116, 200, and $220 \mathrm{kDa}$ (Bach et al., 1989). A couple of these proteins are involved in structural rearrangements, mostly in the first step of splicing (Staley and Guthrie, 1998). A stable RNA-free sub-complex of U5-220K, $-200 \mathrm{~K},-116 \mathrm{~K}$, and $-40 \mathrm{~K}$ has been isolated (Achsel et al., 1998) and protein interactions with the U5 snRNA could only be detected for the $220 \mathrm{~K}$ protein (Urlaub et al., 2000). This specific protein plays an important role during the splicing process, as it contacts the 5'ss of the pre-mRNA and is thought to align the 5' and 3' splice sites after the first step of splicing before the second step (Collins and Guthrie, 1999). The U5 snRNP is recruited to the spliceosome after tri-snRNP formation with U4/U6 and is remodeled during its activation, at which hPrp19/CDC5L and related proteins associate with the U5 snRNP to form the 35S U5 snRNP (Makarov et al., 2002). 
The 13S U4/U6 di-snRNP consists of two snRNAs (U4 and U6), Sm proteins and LSm proteins. It forms a stable heterodimer as a result of base pairing between U4 and U6 snRNAs. There are five specific proteins within the U4/U6 di-snRNP: the $15.5 \mathrm{~K}, 20 \mathrm{~K}(\mathrm{CypH})$, $60 \mathrm{~K}, 61 \mathrm{~K}$, and $90 \mathrm{~K}$ proteins.

Under physiological conditions the 13S U4/U6 di-snRNP and the $20 \mathrm{~S}$ U5 snRNP form the 25S tri-snRNP (U4/U6.U5; Behrens and Luhrmann, 1991; Black and Pinto, 1989), which is integrated into the spliceosome during B complex formation (see below). The U4/U6.U5 involves all U5 and U4/U6 specific proteins except U5-52K, which dissociates during its formation (Laggerbauer et al., 2005). It further contains three tri-snRNP specific proteins $(27 \mathrm{~K}, 65 \mathrm{~K}$, and $110 \mathrm{~K})$ which are required for integration into the spliceosome (Makarova et al., 2001).

\subsubsection{2 non-snRNP components}

There are several non-snRNP protein components that bind to the spliceosome during its assembly pathway and play an important role during pre-mRNA splicing. One group of essential splicing factors are the SR proteins (reviewed by Manley and Tacke, 1996; Sanford et al., 2003). SR proteins involve a various number of C-terminal SR dipeptides (serine/arginine-rich (SR) domain) and one or two RNA recognition motifs (RRMs) at the Nterminus (Birney et al., 1993; Graveley, 2000). The RRMs allow interaction with the premRNA whereas the SR domain is responsible for protein-protein interactions. The SR proteins are thus able to function as bridge between the pre-mRNA and protein splicing factors.

Another group of proteins binding to the pre-mRNA are the heterogenous ribonucleoproteins (hnRNPs; reviewed by Dreyfuss et al., 1993). They are among the most abundant proteins in the nucleus and more than 20 major hnRNP proteins and several isoforms have been identified to date. The hnRNP proteins are designated alphabetically, starting from low molecular weight (A1 (34 kDa) to $U(120 \mathrm{kDa}))$. All hnRNP proteins contain one or more RNA recognition motifs (RRM) and auxiliary domains that mediate protein-protein interactions. Although the precise function of hnRNP proteins is not clear, several hnRNP proteins have been shown to regulate splicing. The hnRNP C proteins have been shown to inhibit cleavage at the 5'ss (Choi et al., 1986), hnRNP I/PTB has been shown to be essential for splicing (Patton et al., 1991) and hnRNP A1 effects a switch from a 5'ss to an upstream splice site in pre-mRNAs that contain multiple splice sites (Mayeda and Krainer, 1992). 
Table 2.2: Composition of the human U snRNPs. U1, U2, U4 and U5 snRNPs contain Sm proteins, whereas the U6 snRNP contains LSm proteins. All U snRNPs consist of additional snRNP specific proteins.

\begin{tabular}{|c|c|c|c|c|c|c|c|c|}
\hline Proteins & Name & $\begin{array}{c}\text { apparent } \\
\text { MW [kDa] }\end{array}$ & 12S U1 & $12 S \mathrm{U} 2$ & $17 S$ U2 & 20S U5 & $\begin{array}{c}\text { 13S } \\
\text { U4/U6 }\end{array}$ & $\begin{array}{c}25 S \\
\text { U4/U6.U5 }\end{array}$ \\
\hline \multirow{7}{*}{$\begin{array}{c}\mathrm{Sm} \\
\text { proteins }\end{array}$} & $\mathrm{SmB} / \mathrm{B}^{\prime}$ & $28 / 29$ & $\downarrow$ & $\bullet$ & $\bullet$ & $\bullet$ & $\checkmark$ & $\leftrightarrow$ \\
\hline & SmD1 & 16 & $\bullet$ & $\bullet$ & $\bullet$ & $\bullet$ & $\bullet$ & $\leftrightarrow$ \\
\hline & SmD2 & 16.5 & $\bullet$ & $\bullet$ & $\bullet$ & $\bullet$ & $\bullet$ & $\leftrightarrow$ \\
\hline & SmD3 & 18 & $\bullet$ & $\bullet$ & $\bullet$ & $\bullet$ & 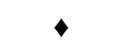 & $\leftrightarrow$ \\
\hline & SmE & 12 & $\bullet$ & $\bullet$ & $\bullet$ & $\bullet$ & $\bullet$ & $\leftrightarrow$ \\
\hline & $\mathrm{SmF}$ & 11 & $\bullet$ & $\bullet$ & $\bullet$ & $\bullet$ & $\bullet$ & $\leftrightarrow$ \\
\hline & SmG & 9 & $\bullet$ & 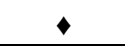 & $\bullet$ & $\bullet$ & 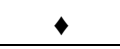 & $\leftrightarrow$ \\
\hline \multirow{7}{*}{$\begin{array}{c}\mathrm{LSm} \\
\text { proteins }\end{array}$} & LSm2 & 10 & & & & & $\bullet$ & $\bullet$ \\
\hline & LSm3 & 15 & & & & & $\bullet$ & $\bullet$ \\
\hline & LSm4 & 15 & & & & & 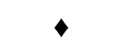 & $\bullet$ \\
\hline & LSm5 & 10 & & & & & 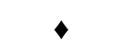 & 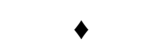 \\
\hline & LSm6 & 8 & & & & & $\bullet$ & $\bullet$ \\
\hline & LSm7 & 13 & & & & & $\bullet$ & $\downarrow$ \\
\hline & LSm8 & 13 & & & & & $\checkmark$ & 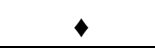 \\
\hline \multirow{3}{*}{ U1 snRNP } & $70 \mathrm{~K}$ & 70 & $\downarrow$ & & & & & \\
\hline & $A$ & 34 & $\bullet$ & & & & & \\
\hline & C & 22 & 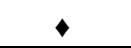 & & & & & \\
\hline \multirow{12}{*}{ U2 snRNP } & $A^{\prime}$ & 31 & & $\checkmark$ & 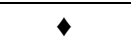 & & & \\
\hline & $\mathrm{B}^{\prime \prime}$ & 28.5 & & 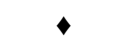 & $\bullet$ & & & \\
\hline & SF3a120 & 110 & & & $\bullet$ & & & \\
\hline & SF3a66 & 66 & & & $\bullet$ & & & \\
\hline & SF3a60 & 60 & & & $\bullet$ & & & \\
\hline & SF3b155 & 160 & & & $\bullet$ & & & \\
\hline & SF3b145 & 150 & & & $\bullet$ & & & \\
\hline & SF3b130 & 120 & & & $\bullet$ & & & \\
\hline & SF3b49 & 53 & & & $\bullet$ & & & \\
\hline & SF3b14a/p14 & 15 & & & $\bullet$ & & & \\
\hline & SF3b14b & 15 & & & $\bullet$ & & & \\
\hline & SF3b10 & 9 & & & $\bullet$ & & & \\
\hline \multirow{7}{*}{ U2-related } & hPrp5 & 140 & & & $\bullet$ & & & \\
\hline & SR140 & 140 & & & $\bullet$ & & & \\
\hline & CHERP & 130 & & & $\bullet$ & & & \\
\hline & hPrp43 & 90 & & & $\bullet$ & & & \\
\hline & SPF45 & 50 & & & $\bullet$ & & & \\
\hline & SPF31 & 33 & & & $\bullet$ & & & \\
\hline & SPF30 & 31 & & & $\bullet$ & & & \\
\hline \multirow{8}{*}{ U5 snRNP } & $220 K$ & 220 & & & & 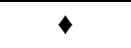 & & 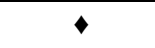 \\
\hline & $200 \mathrm{~K}$ & 200 & & & & $\bullet$ & & $\bullet$ \\
\hline & $116 \mathrm{~K}$ & 116 & & & & $\bullet$ & & $\bullet$ \\
\hline & $102 K$ & 120 & & & & $\bullet$ & & $\bullet$ \\
\hline & $100 \mathrm{~K}$ & 100 & & & & $\bullet$ & & $\bullet$ \\
\hline & $52 \mathrm{~K}$ & 52 & & & & $\bullet$ & & \\
\hline & $40 \mathrm{~K}$ & 40 & & & & $\bullet$ & & $\bullet$ \\
\hline & $15 \mathrm{~K}$ & 15 & & & & $\bullet$ & & 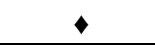 \\
\hline \multirow{5}{*}{$\begin{array}{l}\text { U4/U6 } \\
\text { snRNP }\end{array}$} & $90 \mathrm{~K}$ & 90 & & & & & $\checkmark$ & $\downarrow$ \\
\hline & $61 \mathrm{~K}$ & 61 & & & & & $\bullet$ & $\bullet$ \\
\hline & $60 \mathrm{~K}$ & 60 & & & & & $\bullet$ & $\bullet$ \\
\hline & 20K/СypH & 20 & & & & & $\bullet$ & $\bullet$ \\
\hline & $15.5 \mathrm{~K}$ & 15.5 & & & & & $\bullet$ & $\bullet$ \\
\hline \multirow{3}{*}{$\begin{array}{l}\text { U4/U6.U5 } \\
\text { snRNP }\end{array}$} & $110 \mathrm{~K}$ & 110 & & & & & & 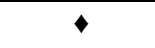 \\
\hline & $65 \mathrm{~K}$ & 65 & & & & & & 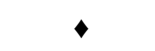 \\
\hline & $27 \mathrm{~K}$ & 27 & & & & & & $\bullet$ \\
\hline
\end{tabular}


The hPrp19/CDC5L complex is a non-snRNP protein complex, which binds to the spliceosome during its activation. It consists of seven proteins (CDC5L, Hsp70, CTNNBL1, PRL1, hPrp19, AD-002, and SPF27; Ajuh et al., 2000; Makarova et al., 2004) and associates with additional related proteins with the U5 snRNP to form the remodeled $35 S$ U5 (Makarova et al., 2004). It thus plays a crucial role in the assembly of a catalytically active spliceosome, presumably by stabilizing the RNA interaction network in the catalytic core (Ajuh et al., 2000). The architecture and the protein stoichiometry of this particular protein complex in yeast and human has been recently analyzed (Grote et al., 2010; Tarn et al., 1994). All studies revealed that the hPrp19 protein is present as a tetramer within this particular protein complex.

Several splicing factors belong to the DExD/H-box protein family. These proteins are able to rearrange RNP and RNA-RNA interactions and are therefore required for structural rearrangements during the spliceosomal assembly. Some of them are U snRNP specific proteins (see Table 2.2), whereas others are non-snRNP specific. There are eight DExD/H box proteins that are important for pre-mRNA splicing: hPrp5, U5-200K, U5-100K, UAP56, hPrp2, hPrp16, hPrp22, and hPrp43 (Schwer, 2001). However, the precise function has only been established for few of these proteins.

Numerous additional proteins have been detected, predominantly by mass spectrometry, within the different spliceosomal transition states (Behzadnia et al., 2007; Bessonov et al., 2008; Deckert et al., 2006; Hartmuth et al., 2002). From these, Prp16, Prp17, Prp18, Prp22, and Slu7 (reviewed by Umen and Guthrie, 1995) have been reported to bind the spliceosome after the first step of splicing to function at the second step of splicing (Figure 2.9). Additionally, several factors specific for the catalytically active spliceosome (see below) have been identified (Bessonov et al., 2008).

\subsubsection{The stepwise assembly of the spliceosome}

Pre-mRNA splicing is a highly dynamic process, which involves the assembly of the $U$ snRNPs and additional non-snRNP splicing factors, the dissociation of these factors upon structural changes, the completion of the two transesterification steps, and finally the release of the generated mRNA. The spliceosome assembles on the pre-mRNA in a stepwise manner passing through different functional intermediate states (Figure 2.10).

In the first assembly step, the U1 snRNP binds to the 5'ss of the pre-mRNA by base pairing of $\mathrm{U} 1$ snRNA and the pre-mRNA. Formation of the thus formed $\mathrm{E}$ complex (the early complex) is mediated by interactions of U1-C and the 5'ss (Heinrichs et al., 1990; Will et al., 1996). Recruitment of U2 snRNP leads then to formation of the A complex (the pre- 
spliceosome). At this stage, the U2 snRNA base pairs with the pre-mRNA in the branch point site region bulging out the branch point adenosine, which is thus enabled to carry out the nucleophilic attack of the first step of splicing (Query et al., 1994). Binding of U2 is promoted by splicing factors U2AF and SF3a and SF3b (Gozani et al., 1996; Valcarcel et al., 1996). Upon integration of the U4/U6.U5 tri-snRNP, which is mediated by tri-snRNP specific proteins $110 \mathrm{~K}$ and $65 \mathrm{~K}$ (Makarova et al., 2001), the pre-catalytic spliceosome (B complex) is developed. Structural RNA and protein rearrangements within the B complex induce the dissociation of $U 1$ and $U 4$ snRNPs. Dissociation of $U 1$ and $U 4$, as well as remodeling of $U 5$ by the hPrp19/CDC5L complex (see above), generate the activated spliceosome $\left(B^{*}\right)$, in which the first catalytic step of splicing occurs. The complex that forms during this process is the catalytically active $C$ complex, which performs the second step of splicing. The final step is the release of the generated mRNA, dissociation of the post-spliceosomal intron complex, and the reconstitution of the splicing factors.

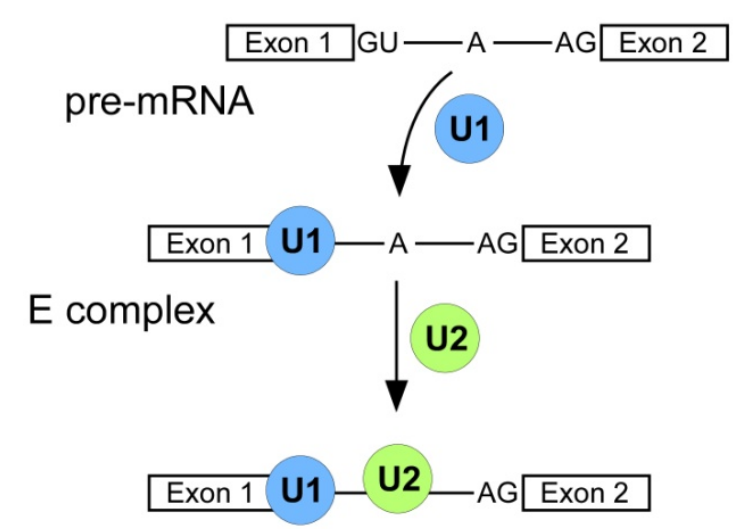

A complex

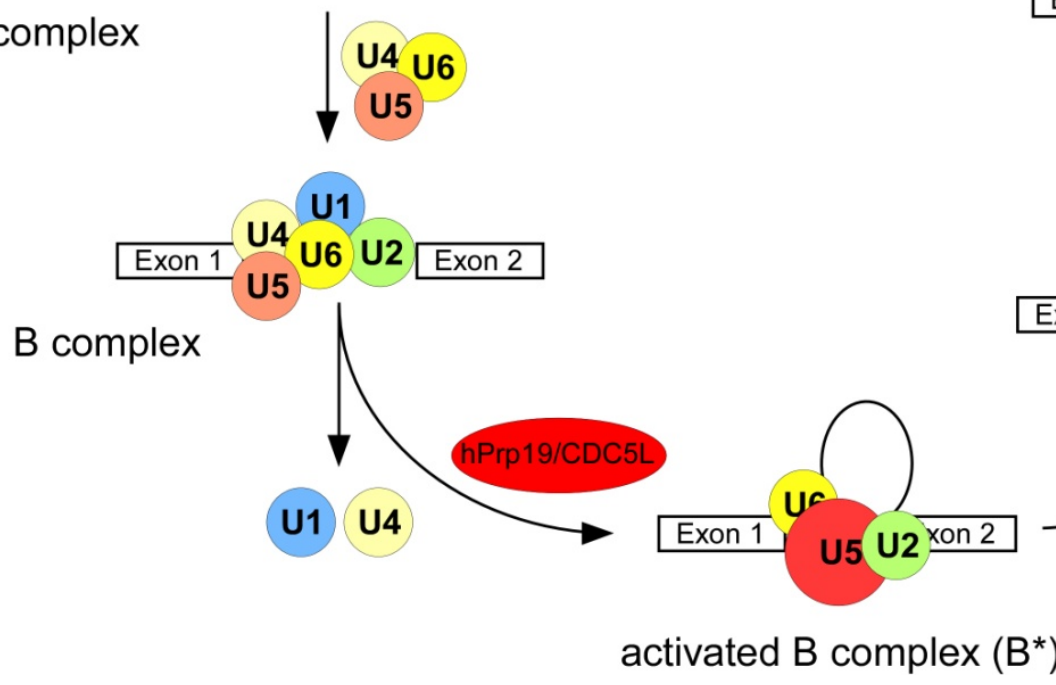

Figure 2.10: The stepwise assembly of the spliceosome. In the first step, U1 snRNP binds to the 5'ss forming the E complex followed by binding of U2 snRNP (A complex formation). Recruitment of the preassembled trisnRNP (U4/U6.U5) leads to formation of the pre-catalytic B complex. Upon structural rearrangements U1 and U4 snRNPs dissociate and incorporation of the hPrp19/CDC5L complex leads to remodeling of U5 generating the activated $B$ complex. The first step of splicing occurs in this intermediate assembly yielding the $C$ complex, in which the second step of splicing is carried out. The generated mRNA and the post-spliceosomal complex are released and the splicing factors are reconstituted. 
Different strategies for affinity purification of the functional intermediates during the spliceosomal assembly have been developed: (i) affinity purification of spliceosomal intermediates using peptide antibodies specific for particular proteins, (ii) isolation of spliceosomal complexes using tobramycin-tagged pre-mRNA, and (iii) affinity selection of spliceosomal complexes using MS2-tagged pre-mRNA and a MS2-MBP fusion protein. From these, the latter has proven to be most suitable. The method has been used to purify $A$ complexes (Behzadnia et al., 2007), B complexes (Deckert et al., 2006), C complexes (Bessonov et al., 2008) and activated B complexes (B*) from yeast (Fabrizio et al., 2009). Mass spectrometric analyses of the purified complexes revealed a complex and highly dynamic proteome of the spliceosome in its different functional states.

In addition to the stepwise assembly model of the spliceosome, a second model comprising the recruitment of a pre-assembled spliceosome followed by structural rearrangements on the pre-mRNA, exists. Strongest evidence of a pre-assembled spliceosome gave the isolation of a 45S RNP complex in yeast consisting of all spliceosomal snRNPs, which is functional in extracts (Stevens et al., 2002). Furthermore, a 200 S RNP consisting of all spliceosomal snRNPs was isolated from HeLa nuclear extracts and was shown to assemble on RNA containing a 5'ss (Malca et al., 2003). The two models are not necessarily contradictory. As the spliceosomal assembly intermediates (A, B, $B^{*}$ and $C$ complexes) are purified under relatively stringent conditions, they reflect distinct stabilization states of the spliceosomal snRNPs (Will and Luhrmann, 2005). The stepwise assembly model therefore represents a proper model showing at which time during splicing the different $U$ snRNPs are stably associated with the pre-mRNA.

\subsection{Aim of this study}

The spliceosome assembles from different uridine-rich small nuclear ribonucleoprotein particles (U snRNPs) and additional non-snRNP proteins. The RNA and protein composition of the involved $U$ snRNPs has been well characterized in the recent past. Therefore, the identification of the non-snRNP splicing factors in the splicing intermediates is of great interest. In recent studies, mass spectrometry allowed identification of several proteins within different spliceosomal complexes. Changes in the protein abundance between these assembly states will provide an insight into the functionality of the different protein components. As protein abundances cannot be determined by simple mass spectrometric analyses, quantification of the complexes' proteins is required to detect differences within the different spliceosomal intermediates. 


\section{4 | INTRODUCTION}

In the course of investigating different intermediates of in vitro spliceosomal assembly, problems have arisen that require quantification of proteins within different spliceosomal complexes. Therefore, the aim of this work was the establishment of robust mass spectrometry-based quantification methods for the quantitative analysis of proteins in the different spliceosomal complexes to tackle the following problems: (i) Determination of the protein stoichiometry within the spliceosomal hPrp19/CDC5L complex, which is recruited to the spliceosome during its activation, by absolute quantification of the proteins within this particular complex. (ii) Relative comparison of the pre-catalytic and the catalytically active spliceosomal complexes (i.e. B and C complexes) to show differences in their protein composition and in this manner to determine proteins, which are necessary for splicing activity. (iii) Monitoring the dynamic protein changes of spliceosomal proteins during the assembly on the pre-mRNA by relative quantification.

To achieve the aim, different mass spectrometry-based quantification strategies, namely AQUA, ITRAQ and SILAC, were to be set up. At the same time, sample preparation and data analysis had to be implemented. For absolute quantification, the complete hydrolysis of proteins needed to be achieved and the suitability of synthetic standard peptides and different methods to read out the quantitative signals were to be tested to achieve most accurate results for reliable quantification. For relative comparison of different protein complexes, a workflow comprising iTRAQ labeling of in-gel digested proteins had to be established. This method was then set out to be compared to other quantification techniques, such as SILAC or spectral count, which is a simple and often applied but only semiquantitative quantification method. In view of protein changes during the spliceosomal assembly pathway, metabolic labeling (SILAC) was to be applied to display the protein dynamics of the spliceosome during pre-mRNA splicing. An experimental setup allowing the construction of assembly time lines needed to be implemented. To this end, different premRNAs lacking the 5'ss and the BPS, respectively, were to be generated and tested for their splicing behavior. These were then used to compare the protein assembly on different premRNAs to understand the dynamic process of pre-mRNA splicing. 


\section{Materials and methods}

\subsection{Materials}

\subsubsection{Chemicals}

Acetic acid

Acetonitrile, LiChrosolv

Agarose (low melting point)

Agarose, Ulra Pure

Ammonium bicarbonate

Ammonium peroxodisulfate (APS)

Ammonium sulfate

Boric acid

Bovine Serum Albumin (BSA)

Bromophenol blue (sodium salt)

Calcium chloride dihydrate

Chloroform

Cleland's Reagent (DTT, for MS analyses)

Coomassie Brilliant Blue G-250

Creatine phsophate

$\alpha$-Cyano-4-hydroxycinnamic acid

Dimethylsulfoxide (DMSO)

di-Potassium hydrogen phosphate trihydrate

Dithioerythrol (DTE)

Dithiothreitol (DTT)

Dodecyl sulfate sodium salt (SDS)

Ethanol

Ethidium bromide solution $10 \mathrm{mg} / \mathrm{ml}$

Ethylenediaminetetraacetic acid (EDTA, disodium salt)

Formaldehyde

Formamide

Formic acid

Glycerol

Glycine

Glycogen

Heparin

4-(2-hydroxyethyl)-1-piperazinethanesulfonic acid (HEPES)

lodoacetamide

Magnesium chloride

Maltose

Methanol

Methanol, LiChrosolv

N,N,N',N'-Tetramethylethylendiamid (TEMED)

$\mathrm{N}, \mathrm{N}$-Dimethylformamide

ortho-Phosphoric acid

Phenol/Chloroform/Isoamylalcohol (PCl)

Phenylmethylsulfonyl fluoride (PMSF)

Potassium chloride

Potassium dihydrogen phosphate

RapiGest

Rotiphorese Gel 40 (38\% Acrylamide, 2\% Bis-Acrylamide)
Merck, Darmstadt

Merck, Darmstadt

Invitrogen, USA

Invitrogen, USA

Fluka, Switzerland

Merck, Darmstadt

Merck, Darmstadt

Merck, Darmstadt

New England Biolabs, USA

Merck, Darmstadt

Merck, Darmstadt

Merck, Darmstadt

Calbiochem, USA

Fluka, Switzerland

Sigma-Aldrich, Steinheim

Sigma-Aldrich, Steinheim

Roth, Karlsruhe

Merck, Darmstadt

Roth, Karlsruhe

Roth, Karlsruhe

Merck, Darmstadt

Merck, Darmstadt

Roth, Karlsruhe

Roth, Karlsruhe

Merck, Darmstadt

Merck, Darmstadt

Fluka, Switzerland

Merck, Darmstadt

Merck, Darmstadt

Merck, Darmstadt

Sigma-Aldrich, Steinheim

Merck, Darmstadt

Sigma-Aldrich, Steinheim

Sigma-Aldrich, Steinheim

Merck, Darmstadt

Merck, Darmstadt

Merck, Darmstadt

Sigma-Aldrich, Steinheim

Merck, Darmstadt

Merck, Darmstadt

Roth, Karlsruhe

Roche, Mannheim

Merck, Darmstadt

Merck, Darmstadt

Waters, UK

Roth, Karlsruhe 
Silver nitrate

Sodium acetate

Sodium carbonate

Sodium chloride

Sodium dihydrogen phosphate monohydrate

Trifluoric acid

Tris-(hydroxymethyl) aminoethane (Tris)

Urea

Water, LiChrosolv

Xylene Cyanol FF

\subsubsection{Enzymes and enzyme inhibitors}

BamH I

LysC

Proteinase Inhibitor Cocktail Complete, EDTA free

Proteinase $\mathrm{K}$

RNasin $(40 \mathrm{U} / \mu \mathrm{l})$

RQ DNAse I

SP6 polymerase

T4 polynucleotide kinase

T4 DNA ligase

Trypsin

Trypsin

Turbo Pfu DNA polymerase

\subsubsection{Nucleotides}

adenosine 5'-triphosphate (rATP, $100 \mathrm{mM}$ )

cytidine 5'-triphosphate (rCTP, $100 \mathrm{mM}$ )

guanosine 5'-triphosphate (rGTP, $100 \mathrm{mM}$ )

uridine 5'-triphosphate (rUTP, $100 \mathrm{mM}$ )

Easy Tides Uridine 5'-triphosphate, $\left[\alpha{ }^{32} \mathrm{P}\right]$

2'-deoxyadenosine-5'-triphosphate (dATP, $100 \mathrm{mM}$ )

2'-deoxycytidine-5'-triphosphate (dCTP, $100 \mathrm{mM}$ )

2'-deoxyguanosine-5'-triphosphate (dGTP, $100 \mathrm{mM}$ )

2'-deoxythymidine-5'-triphosphate (dTTP, $100 \mathrm{mM}$ )

m7G(5')ppp(5')G cap
Merck, Darmstadt

Merck, Darmstadt

Merck, Darmstadt

Merck, Darmstadt

Merck, Darmstadt

Fluka, Switzerland

Roth, Karlsruhe

Merck, Darmstadt

Merck, Darmstadt

Fluka, Switzerland

New England Biolabs, USA

Roche, Mannheim

Roche, Mannheim

Sigma-Aldrich, Steinheim

Promega, Mannheim

Promega, Mannheim

Promega, Mannheim

New England Biolabs, USA

New England Biolabs, USA

Roche, Mannheim

Promega, Mannheim

Stratagene, Heidelberg

\subsubsection{DNA oligonucleotides}

Table 3.1: Oligonucleotides used in this study

\begin{tabular}{|l|l|l|}
\hline Name & Sequence (5' $\rightarrow$ 3') & Description \\
\hline M6 & GGCGGTCTCGTC & RNAseH digestion \\
\hline M12 & CTCGTCGGCAGC & RNAseH digestion \\
\hline PM5-5'ss-del_for & ATCAAGCTTACAAGACAGCTTT & $\begin{array}{l}\text { deletion of the 5'ss within the } \\
\text { PM5 plasmid }\end{array}$ \\
\hline PM5-5'ss-del_rev & TGGCGGCGGTCTCGTCG & $\begin{array}{l}\text { deletion of the 5'ss within the } \\
\text { PM5 plasmid }\end{array}$ \\
\hline PM5-BPS-del_for & AAATACATAAGAATCAGGTAGTG & $\begin{array}{l}\text { deletion of the BPS within the } \\
\text { PM5 plasmid }\end{array}$ \\
\hline
\end{tabular}




\begin{tabular}{|l|l|l|}
\hline PM5-BPS-del_rev & CTGTGTTTTTTTGCTACTTTTTTT & $\begin{array}{l}\text { deletion of the BPS within the } \\
\text { PM5 plasmid }\end{array}$ \\
\hline $\begin{array}{l}\text { PM5-BPS_ACTGA- } \\
\text { del_for }\end{array}$ & TAGAACACTACCTGATTCTTATG & $\begin{array}{l}\text { deletion of duplicate BPS } \\
\text { sequence within the PM5 } \\
\text { plasmid }\end{array}$ \\
\hline $\begin{array}{l}\text { PM5-BPS_ACTGA- } \\
\text { del_for }\end{array}$ & CACACTCCACACACATTCCA & $\begin{array}{l}\text { deletion of duplicate BPS } \\
\text { sequence within the PM5 } \\
\text { plasmid }\end{array}$ \\
\hline
\end{tabular}

M6 and M12 oligos were purchased from IBA (Göttingen) and all other oligonucleotides were purchased from Eurofins MWG Operon (Ebersberg).

\subsubsection{Plasmids}

PM5

pMS2-MBP

\subsubsection{Bacteria strains}

The following Escherichia coli strains were used in this study:

BL21-Rosetta

$\mathrm{DH} 5 \alpha$

\subsubsection{Cell lines}

HeLa S3 cells

\subsubsection{Cell culture}

Ampicillin

Chloramphenicol

DMEM High Glucose w/o Arginine, w/o Lysine

Foetal Bovine Serum, dialyzed

IPTG (Isopropyl-b-D-1-thiogalactopyranoside)

L-Arginine

L-Arigine, ${ }^{13} \mathrm{C}_{6}$

L-Arigine, ${ }^{13} \mathrm{C}_{6},{ }^{15} \mathrm{~N}_{4}$

LB Medium

LB-Agar

L-Lysine

L-Lysine, ${ }^{13} \mathrm{C}_{6}$

L-Lysine, ${ }^{13} \mathrm{C}_{6},{ }^{15} \mathrm{~N}_{2}$

L-Lysine, ${ }^{2} \mathrm{D}_{4}$

$100 \times$ Penicillin/Streptomycin Concentrate
Anderson and Moore, 1997

Das et al., 2000
Sigma-Aldrich, Steinheim

Roth, Karlsruhe

PAA Laboratories, Cölbe PAA Laboratories, Cölbe Roth, Karlsruhe

Sigma-Aldrich, Steinheim

Eurisotop, Saarbrücken

Eurisotop, Saarbrücken

MP Biomedicals, USA

MP Biomedicals, USA

Sigma-Aldrich, Steinheim

Eurisotop, Saarbrücken

Eurisotop, Saarbrücken

Eurisotop, Saarbrücken

PAA Laboratories, Cölbe 


\subsubsection{Commercial reaction kits and buffers}

Bradford solution, Bio-Rad Protein Assay

Buffer 4

$10 \times$ cloned Pfu DNA Polymerase Buffer

Invitrogen PureLink HiPure Plasmid Filter Maxiprep kit

iTRAQ reagent Multi-Plex Kit

NucleoSpin Extract II kit

NuPAGE Antioxidant

NuPAGE LDS Sample Buffer $(4 \times)$

NuPAGE MOPS SDS Running Buffer $(10 \times)$

NuPAGE Sample Reducing Agent $(10 \times)$

10 PNK Buffer

QIAprep Spin Miniprep kit

T4 DNA Ligase Buffer

Transcription Optimized $5 \times$ Buffer

Triethylammonium bicarbonate buffer, $1 \mathrm{M}, \mathrm{pH} 8.5$
Bio-Rad, München

New England Biolabs, USA

Stratagene, Heidelberg

Invitrogen, USA

ABSciex, Darmstadt

Machery-Nagel

Invitrogen, USA

Invitrogen, USA

Invitrogen, USA

Invitrogen, USA

New England Biolabs, USA

Qiagen, Hilden

New England Biolabs, USA

Promega, Mannheim

Sigma-Aldrich, Steinheim

\subsubsection{Commonly used buffers}

$10 \times D^{-}$Buffer

20 mM HEPES pH 7.9

$1.5 \mathrm{mM} \mathrm{MgCl}_{2}$

$0.2 \mathrm{mM}$ EDTA pH 8.0

$1 \times$ PBS

$130 \mathrm{mM} \mathrm{NaCl}$

$20{\mathrm{mM} \mathrm{K}-\mathrm{PO}_{4}}$

$\underline{\text { Roeder C buffer }}$

$25 \%$ (v/v) Glycerol

$20 \mathrm{mM}$ HEPES

$420 \mathrm{mM} \mathrm{NaCl}$

$1.5 \mathrm{mM} \mathrm{MgCl}_{2}$

$0.2 \mathrm{mM}$ EDTA $\underline{\text { Na-P buffer }}$

$5 \mathrm{mM} \mathrm{Na}_{2} \mathrm{HPO}_{4}$

$2 \times$ PK-Buffer

$200 \mathrm{mM}$ Tris pH 7.5

25 mM EDTA pH 8.0

$2 \%(\mathrm{~m} / \mathrm{v})$ SDS

$\underline{\text { Roeder D buffer }}$

$10 \%$ (v/v) Glycerol

$20 \mathrm{mM}$ HEPES

$100 \mathrm{mM} \mathrm{KCl}$

$1.5 \mathrm{mM} \mathrm{MgCl}_{2}$

$0.2 \mathrm{mM}$ EDTA

$0.5 \mathrm{mM}$ DTT

$0.5 \mathrm{mM}$ PMSF

\section{$1 \times \mathrm{TBE}, \mathrm{pH} 8.3$}

$0.1 \mathrm{M}$ Boric acid

$0.1 \mathrm{M}$ Tris

$0.1 \mathrm{M}$ EDTA 


\subsubsection{Standard peptides}

[Glu ${ }^{1}$ ]-Fibrinopeptide $B$

EAAAA-LVEEET $\left({ }^{13} \mathrm{C}_{6}{ }^{15} \mathrm{~N}_{4}-\mathrm{R}\right)$

FVDILG $\left({ }^{13} \mathrm{C}_{6}{ }^{15} \mathrm{~N}-\mathrm{L}\right) \mathrm{R}$

HYTFASGSPDN $\left({ }^{13} \mathrm{C}_{6}{ }^{15} \mathrm{~N}-\mathrm{I}\right) \mathrm{K}$

ILLGGYQS $\left({ }^{13} \mathrm{C}_{6}{ }^{15} \mathrm{~N}_{4}-\mathrm{R}\right)$

LGLLGLPAP $\left({ }^{13} \mathrm{C}_{6}{ }^{15} \mathrm{~N}_{2}-\mathrm{K}\right)$

NVVV $\left({ }^{13} \mathrm{C}_{9}{ }^{15} \mathrm{~N}-\mathrm{F}\right) \mathrm{DK}$

TGYN $\left({ }^{13} \mathrm{C}_{9}{ }^{15} \mathrm{~N}-\mathrm{F}\right) \mathrm{QR}$

TIVQLENEIYQ- $\left({ }^{13} \mathrm{C}_{6}{ }^{15} \mathrm{~N}-\mathrm{I}\right) \mathrm{K}$

TLQLDNNFEV $\left({ }^{13} \mathrm{C}_{6}{ }^{15} \mathrm{~N}_{2}-\mathrm{K}\right)$

TVPEELVKPEELS $\left({ }^{13} \mathrm{C}_{6}{ }^{15} \mathrm{~N}_{2}-\mathrm{K}\right)$

YADLL $\left({ }^{13} \mathrm{C}_{6}{ }^{15} \mathrm{~N}-\mathrm{L}\right) \mathrm{EK}$

\subsubsection{Chromatography materials and consumables}

Amylose Resin

anti-FLAG-M2-Agarose beads

Bio-Spin Disposable Chromatography Columns

FLAG peptide

Heparin Sepharose 6Fast Flow

m-Precolumn ${ }^{\mathrm{TM}}$ Cartridge, Acclaim PepMap100 C18,

$300 \mu \mathrm{m}$ i.d. $\times 5 \mathrm{~mm}$

Nucleosil 100-5 C18, $5 \mu \mathrm{m}$

NuPAGE Novex Bis-Tris Mini Gels

PepMap C18, $300 \mu \mathrm{m}, 5 \mu \mathrm{m}$

PepMap C18, $75 \mu \mathrm{m}, 15 \mathrm{~cm}$

Reprosil-Pur Basic C18-HD, $5 \mu \mathrm{m}$

\subsubsection{Instruments, Equipment}

Blank Opti-TOF, $123 \mathrm{~mm} \times 81 \mathrm{~mm}$, MALDI target

CAP-LC system

HP1100 series chromatography system

LTQ-Orbitrap XL

4800 MALDI TOF/TOF Analyzer

Phosphorimager Typhoon 8600

Probot, robotic spotting device

Q-ToF Ultima mass spectrometer 4000 QTRAP mass spectrometer Scintillation counter LS1701/TRI-CARB 2100TR

Sorvall SA-600 rotor

Sorvall SS-34 rotor

Tempo 1D chromatography system

Ultimate chromatography system

Ultracentrifuges

XCell Sure Lock Mini Cell
Sigma-Aldrich, Steinheim

Sigma-Genosys, USA

Thermo Fisher Scientific, UIm

Thermo Fisher Scientific, UIm

Sigma-Genosys, USA

Sigma-Genosys, USA

Thermo Fisher Scientific, UIm Thermo Fisher Scientific, UIm Thermo Fisher Scientific, UIm Sigma-Genosys, USA

Sigma-Genosys, USA

Thermo Fisher Scientific, UIm

New England Biolabs, USA

Sigma-Aldrich, Steinheim

Bio-Rad, München

Sigma-Aldrich, Steinheim

GE Healthcare, UK

LC Packings, Netherlands

Machery-Nagel, Düren

Invitrogen, USA

Dionex, Idstein

Dionex, Idstein

Maisch, Ammerbuch

ABSciex, Darmstadt

Waters, UK

Agilent, USA

Thermo Scientific, Dreieich

ABSciex, Darmstadt

Molecular Dynamics,

Switzerland

Dionex, Idstein

Waters, UK

ABSciex, Darmstadt

Beckmann/Packard, USA

Kendro, USA

Kendro, USA

ABSciex, Darmstadt

Dionex, Idstein

Sorvall/Beckmann

Invitrogen, USA 


\subsection{Methods}

\subsubsection{Molecular biology methods}

\subsubsection{Concentration determination of nucleic acids}

To determine the concentration of nucleic acids, the extinction in aqueous solution was measured at a wavelength of $260 \mathrm{~nm}$ in comparison to a reference (buffer). Following equations were used to calculate the concentration (Sambrook et al., 1989):

$1 \mathrm{OD}_{260}=50 \mu \mathrm{g} / \mathrm{ml}$ double stranded DNA $=0.15 \mathrm{mM}$ (in nucleotides)

$1 \mathrm{OD}_{260}=33 \mu \mathrm{g} / \mathrm{ml}$ single stranded DNA $=0.10 \mathrm{mM}$ (in nucleotides)

$1 \mathrm{OD}_{260}=40 \mu \mathrm{g} / \mathrm{ml}$ single stranded RNA $=0.11 \mathrm{mM}$ (in nucleotides)

The concentration of $\alpha-\left[{ }^{32} \mathrm{P}\right]$ UTP-labeled RNA was determined by the ratio of $\alpha-\left[{ }^{32} \mathrm{P}\right] \mathrm{UTP}$ and ${ }^{31} \mathrm{P}$-UTP, the isotope concentration of $\alpha-\left[{ }^{32} \mathrm{P}\right]$ UTP, the absolute number of uridines in the transcript and an instrument constant using the following equations:

$$
\begin{aligned}
& \text { mixing ratio }=\frac{\left[{ }^{31} \mathrm{P}-\mathrm{UTP}\right]}{\left[\alpha-\left[{ }^{32} \mathrm{P}\right] \mathrm{UTP}\right]} \\
& \frac{\text { isotope concentration }\left(\frac{\mathrm{Ci}}{\mathrm{mmol}}\right)}{\text { mixing ratio }} \times \# \text { Uridines = radioactivity }\left(\frac{\mathrm{Ci}}{\mathrm{mmol}}\right) \\
& \text { specific activity }\left(\frac{\mathrm{dpm}}{\mathrm{mmol}}\right)=\text { radioactivity }\left(\frac{\mathrm{Ci}}{\mathrm{mmol}}\right) \times 2.2 \cdot 10^{6}\left(\frac{\mathrm{dpm}}{\mathrm{Ci}}\right) \\
& \text { specific activity }\left(\frac{\mathrm{cpm}}{\mathrm{mmol}}\right)=\text { specific activity }\left(\frac{\mathrm{dpm}}{\mathrm{mmol}}\right) \div 2
\end{aligned}
$$

\subsubsection{Phenol-Chloroform-Isoamylalcohol (PCl) extraction}

Phenol-chloroform-isoamylalcohol ( $\mathrm{PCl})$ extraction was used to purify and separate proteins and nucleic acids. The sample was mixed with 1 vol. of PCl and $1 \mu \mathrm{l}$ of $10 \mu \mathrm{g} / \mu \mathrm{l}$ glycogen and vigorously agitated on a vortex. Aqueous and organic phases were separated by centrifugation for $5 \mathrm{~min}$ at room temperature $(13,000 \mathrm{rpm})$. The aqueous phase, containing nucleic acids, was transferred to a new tube. Proteins (organic phase) were precipitated with 5 vol. $100 \%(\mathrm{v} / \mathrm{v})$ acetone at $-20{ }^{\circ} \mathrm{C}$ for at least $2 \mathrm{~h}$. Nucleic acids (aqueous phase) were further purified by addition of 1 vol. of chloroform. After mixing and centrifugation as above, 
the nucleic acids were precipitated from the aqueous phase in $3 \mathrm{vol}$. of $100 \%(\mathrm{v} / \mathrm{v})$ ethanol and $1 / 10$ vol. of $0.3 \mathrm{M}$ sodium acetate $\mathrm{pH} 5.3$ for at least $2 \mathrm{~h}$ at $-20^{\circ} \mathrm{C}$. Precipitated proteins or nucleic acids were collected by centrifugation for $30 \mathrm{~min}$ at $4{ }^{\circ} \mathrm{C}(13,000 \mathrm{rpm})$. Proteins or nucleic acids were washed with $80 \%(\mathrm{v} / \mathrm{v})$ ethanol, collected by centrifugation (see above) and dried in a vacuum centrifuge.

\section{Phenol-Chloroform-Isoamylalcohol (PCl):}

$50 \%(v / v)$ Phenol

$48 \%(\mathrm{v} / \mathrm{v})$ Chloroform

$2 \%(\mathrm{v} / \mathrm{v})$ Isoamylalcohol

\subsubsection{Agarose gel electrophoresis of DNA}

Agarose gel electrophoresis was used to analyze and purify DNA. Gels were prepared with $1 \%(\mathrm{w} / \mathrm{v})$ agarose and $0.5 \mathrm{\mu g} / \mathrm{ml}$ ethidium bromide to visualize DNA by UV light. DNA samples were diluted with $5 \times$ DNA loading dye and allowed to migrate horizontally at $120 \mathrm{~V}$ using $1 \times$ TBE as running buffer until the dye migrated through the gel. DNA was visualized under a UV light at a wavelength of $365 \mathrm{~nm}$.

\section{Gel solution:}

$1 \%(w / v)$ Agarose

$1 \times \mathrm{TBE}$

$0.5 \mu \mathrm{g} / \mathrm{ml}$ Ethidium bromide

\section{DNA loading dye:}

$30 \%(w / v)$ Glycerol

$0.25 \%(w / v)$ Bromophenol blue

\subsubsection{In vitro transcription}

Pre-mRNAs were synthesized by in vitro transcription using SP6 polymerase and linearized plasmid DNA as template. The reaction was incubated at $40^{\circ} \mathrm{C}$ for $3-4 \mathrm{~h}$. Template DNA was subsequently digested with $1 \mathrm{U} R Q 1 \mathrm{DNAse} / \mu \mathrm{g}$ template DNA at $37{ }^{\circ} \mathrm{C}$ for $20 \mathrm{~min}$. RNA transcripts were purified by gel purification using $5 \%$ polyacrylamide gels containing $8 \mathrm{M}$ urea. Unlabeled RNA was visualized by UV-shadowing $(254 \mathrm{~nm})$ and $\alpha-\left[{ }^{32} \mathrm{P}\right] \mathrm{UTP}$-labeled RNA was visualized by exposure to an X-ray film (1 $\mathrm{min})$. Bands were excised from the gel and extracted by incubation with RNA extraction buffer overnight. Extracted RNA was further purified by $\mathrm{PCl}$ extraction and ethanol precipitation. The purified RNA was resuspended with RNAse-free water. 


\section{Preparation of capped PM5-pre-mRNA:}

$$
\alpha-\left[{ }^{32} P\right] U T P \text {-labeled }
$$

$5 \times$ transcription buffer

$0.1 \mathrm{M}$ ATP

$0.1 \mathrm{M}$ UTP

$0.1 \mathrm{M}$ CTP

$0.01 \mathrm{M}$ GTP

$152 \mathrm{mM} \mathrm{m}^{7}$ GpppG cap

${ }^{32} \mathrm{P}-\alpha \mathrm{UTP}(3000 \mathrm{Ci} / \mathrm{mmol})$

$1 \mathrm{M} \mathrm{MgCl} 2$

1M DTT

$10 \mathrm{mg} / \mathrm{ml} \mathrm{BSA}$

$40 \mathrm{U} / \mu \mathrm{l}$ RNAsin

SP6 polymerase

DNA template

The volume was adjusted to (RNAse-free water)
$10.00 \mu \mathrm{l}$

$3.75 \mu \mathrm{l}$

$3.75 \mu \mathrm{l}$

$3.75 \mu \mathrm{l}$

$6.50 \mu \mathrm{l}$

$1.64 \mu \mathrm{l}$

$5.00 \mu \mathrm{l}$

$1.05 \mu \mathrm{l}$

$0.50 \mu \mathrm{l}$

$0.50 \mu \mathrm{l}$

$1.25 \mu \mathrm{l}$

$5.33 \mu \mathrm{l}$

$5.00 \mu \mathrm{g}$

$50 \mu \mathrm{l}$

\section{non-labeled}

$30.00 \mu \mathrm{l}$

$11.25 \mu \mathrm{l}$

$11.25 \mu \mathrm{l}$

$11.25 \mu \mathrm{l}$

$19.50 \mu \mathrm{l}$

$4.92 \mu \mathrm{l}$

$3.15 \mu \mathrm{l}$

$1.50 \mu \mathrm{l}$

$1.50 \mu \mathrm{l}$

$3.75 \mu \mathrm{l}$

$16.00 \mu \mathrm{l}$

$15.00 \mu \mathrm{g}$

$150 \mu \mathrm{l}$

$8 \mathrm{M}$ urea-5 \% Polyacrylamide

Gel Solution (100 ml):

$12.5 \mathrm{ml}$ Rotiphorese Gel 40

$42 \mathrm{~g}$ Urea

$10 \mathrm{ml} 10 \times$ TBE

Adjust to $100 \mathrm{ml}$ (RNAse free water)

Per $100 \mathrm{ml}$ gel solution:

$10 \mu \mathrm{ITEMED}$

$100 \mu 10 \%(w / v)$ APS

\subsubsection{DNA amplification}

$100 \mu \mathrm{l}$ chemical competent $\mathrm{dH} 5 \alpha$ cells were transfected with $100 \mathrm{ng}$ of plasmid DNA by the heat shock method according to standard protocols (Sambrook et al., 1989). To this end, competent bacteria were thawed on ice and mixed with plasmid DNA. After incubation on ice for $30 \mathrm{~min}$, cells were heat shocked at $42^{\circ} \mathrm{C}$ for $90 \mathrm{~s}$ and subsequently cooled on ice for $2 \mathrm{~min} .1 \mathrm{ml}$ of LB medium was added followed by incubation at $37{ }^{\circ} \mathrm{C}$ for $1 \mathrm{~h}$. To select transformed bacteria, cells were plated on agar plates containing the appropriate antibiotic and incubated at $37{ }^{\circ} \mathrm{C}$ overnight. Single clones were selected and grown at $37{ }^{\circ} \mathrm{C}$ in LB medium. DNA was recovered using QIAprep Spin Miniprep kit or Invitrogen PureLink HiPure Plasmid Filter Maxiprep kit. 


\subsubsection{Restriction digestion of DNA}

Restriction digestion was used to generate desired ends of template DNA for in vitro transcription from plasmid DNA. $200 \mu \mathrm{g}$ of plasmid DNA, $100 \mathrm{U}$ of BamHI, $140 \mu \mathrm{l}$ of $10 \times$ Buffer 4 were adjusted to a final volume of $1400 \mu$ with RNAse-free water. The mixture was incubated at $37{ }^{\circ} \mathrm{C}$ overnight and linearized DNA was recovered by $\mathrm{PCl}$ extraction. Linearization was assayed by agarose gel electrophoresis.

\subsubsection{Proteinase K digestion}

Proteinase $\mathrm{K}$ digestion was performed to improve RNA recovery from splicing reactions. $125 \mu \mathrm{l}$ of proteinase $\mathrm{K}$ mix were added to one $20 \mu \mathrm{l}$ aliquot of splice reaction followed by incubation for $1 \mathrm{~h}$ at $37{ }^{\circ} \mathrm{C}$. The volume was adjusted to a final volume of $200 \mu \mathrm{l}$ with $35 \mu \mathrm{l}$ $1 \times \mathrm{D}^{-}$buffer and $20 \mu \mathrm{l}$ of $10 \%(\mathrm{v} / \mathrm{v})$ SDS. The RNA was recovered by $\mathrm{PCl}$ extraction and ethanol precipitation.

\section{Proteinase $\mathrm{K}$ mix:}

$62.5 \mu \mathrm{l} 2 \times$ PK buffer

$1 \mu \mathrm{l} 10 \mathrm{mg} / \mathrm{ml}$ Glycogen

$57.5 \mu \mathrm{RNAse}$-free water

$4 \mu \mathrm{l} 10 \mathrm{mg} / \mathrm{ml}$ Proteinase $\mathrm{K}$

\subsubsection{Denaturing polyacrylamide gel electrophoresis}

Denaturing gel electrophoresis was used to analyze snRNA and pre-mRNA splicing products. RNA samples were dissolved in RNA sample buffer, heated for $3-5$ min at $96{ }^{\circ} \mathrm{C}$ and loaded onto $0.5 \mathrm{~mm}$ thick $10 \%$ polyacrylamide gels (37.5:1 acrylamide to bis-acrylamide ratio) containing $8 \mathrm{M}$ urea. Electrophoresis was performed vertically at $20 \mathrm{~W}$ using $1 \times \mathrm{TBE}$ as running buffer.

$8 \mathrm{M}$ urea-10\% Polyacrylamide Gel Solution $(100 \mathrm{ml})$ :

$25.0 \mathrm{ml}$ Rotiphorese Gel 40

$42 \mathrm{~g}$ Urea

$10 \mathrm{ml} 10 \times$ TBE

Adjust to $100 \mathrm{ml}$ (RNAse free water)

Per $100 \mathrm{ml}$ gel solution:

$10 \mu$ TEMED

$100 \mu 10 \%(w / v)$ APS

\section{RNA Sample Buffer:}

$80 \%$ (w/v) Formamide

1 mM EDTA

$0.05 \%(w / v)$ Bromophenol blue

$0.05 \%$ (w/v) Xylene cyanol 


\subsubsection{Silver staining of RNA}

RNA was visualized by silver staining according to Schoenle et al., 1984. Briefly, RNA was fixed in $40 \%(\mathrm{v} / \mathrm{v})$ methanol/10 \% (v/v) acetic acid for at least $30 \mathrm{~min}$ and washed twice with $10 \%(\mathrm{v} / \mathrm{v})$ ethanol/5\% (v/v) acetic acid for $15 \mathrm{~min}$. The gel was briefly rinsed with water and incubated with $12 \mathrm{mM}$ silver nitrate for $30 \mathrm{~min}$. After 3 short rinsings with water, the gel was incubated with $280 \mathrm{mM}$ sodium carbonate/0.0185 \% (v/v) formaldehyde until the desired staining intensity was reached. Staining was then stopped immediately with $5 \%(\mathrm{v} / \mathrm{v})$ acetic acid.

\subsubsection{Native gel electrophoresis of RNA complexes}

Splicing complex formation was analyzed by native gel electrophoresis. $0.7 \mu \mathrm{l}$ Heparin ( $5 \mathrm{mg} / \mathrm{ml}$, final concentration $170 \mathrm{mg} / \mathrm{ml}$ ) was added to $20 \mu \mathrm{l}$ aliquots of splicing complexes and heated for $1 \mathrm{~min}$ at $30^{\circ} \mathrm{C}$. Samples were diluted with $5 \times$ native loading dye and loaded onto $1.5 \%$ agarose gels. Electrophoresis was performed horizontally at $40 \mathrm{~V}$ for $15 \mathrm{~h}$ using $0.5 \%$ TBE as running buffer. The gels were fixed with $10 \%(\mathrm{v} / \mathrm{v})$ acetic acid/10 \% (v/v) methanol for $30 \mathrm{~min}$ and dried under vacuum at $60^{\circ} \mathrm{C}$ for $4.5 \mathrm{~h}$. Radioactively labeled RNA was visualized by exposing the dried gels to phosphorimager screens for approx. $1 \mathrm{hr}$ and scanning using Typhoon Phosphorlmager.

Agarose Gel Solution:

$1.5 \%(\mathrm{v} / \mathrm{v})$ Low Melting Point Agarose $0.5 \times \mathrm{TBE}$
Native loading dye:

$1 \times$ TBE

$30 \%$ Glycerol

$0.02 \%$ Bromophenol blue

\subsubsection{Generation of pre-mRNA mutants (deletion of 5'ss and BPS)}

5'splice site (5'ss)-, branch point site (BPS)- and BPS-ACTGA-deleted PM5 pre-mRNA was generated as described before (Dönmez, 2006; see also Figure 3.1 for an overview). PCR primers were used to exclude the 5'SS or BPS region within the PM5 plasmid. The PCR product was purified by agarose gel electrophoresis and recovered using the NucleoSpin Extract II kit (Machery-Nagel). The linear plasmid was kinased with T4 ploynucleotide kinase (T4 PNK) in the presence of $1 \mathrm{mM}$ ATP. The reaction was incubated for $1 \mathrm{~h}$ at $37^{\circ} \mathrm{C}$ and the kinased plasmid was purified using the NucleoSpin Extract II kit. The plasmid was then ligated with T4 DNA ligase for $30 \mathrm{~min}$ at $37^{\circ} \mathrm{C}$ followed by incubation at $16{ }^{\circ} \mathrm{C}$ overnight. The ligase was deactivated by incubation for $10 \mathrm{~min}$ at $65^{\circ} \mathrm{C}$. The ligated plasmid was purified by ethanol precipitation, dissolved in water and used for transformation of $\mathrm{dH} 5 \alpha$ cells. Amplified 
DNA was isolated using the QIAprep Spin Miniprep kit and cloned plasmids were sequenced (Seqlab Sequence Laboratories Göttingen $\mathrm{GmbH}$ ).

PCR reaction: $(100 \mu \mathrm{l})$

$0.5 \mu \mathrm{l}$ PM5 plasmid $(20 \mu \mathrm{g} / \mathrm{ml})$

$1 \mu \mathrm{l}$ Turbo Pfu

$1.6 \mu \mathrm{l}$ dNTPs (25 mM each)

$1 \mu \mathrm{l}$ forward primer $(100 \mu \mathrm{M})$

$1 \mu \mathrm{l}$ reverse primer $(100 \mu \mathrm{M})$

$10 \mu$ l cloned Pfu buffer

$7.5 \mu \mathrm{LMSO}$

$77.4 \mu \mathrm{H} \mathrm{H}_{2} \mathrm{O}$

\section{PCR program:}

\begin{tabular}{|c|c|c|c|}
\hline & & $\begin{array}{l}\text { 5'ss } \\
\text { deletion }\end{array}$ & $\begin{array}{c}\text { BPS / BPS-ACTGA } \\
\text { deletion }\end{array}$ \\
\hline \multirow[t]{2}{*}{ step 1} & $90 \mathrm{~s}$ & $95^{\circ} \mathrm{C}$ & $95^{\circ} \mathrm{C}$ \\
\hline & $30 \mathrm{~s}$ & $95^{\circ} \mathrm{C}$ & $95^{\circ} \mathrm{C}$ \\
\hline step 2 & $45 \mathrm{~s}$ & $53^{\circ} \mathrm{C}$ & $50^{\circ} \mathrm{C}$ \\
\hline (32 & $7 \mathrm{~min}$ & $68^{\circ} \mathrm{C}$ & $68^{\circ} \mathrm{C}$ \\
\hline step 3 & hold & $4^{\circ} \mathrm{C}$ & $4{ }^{\circ} \mathrm{C}$ \\
\hline
\end{tabular}

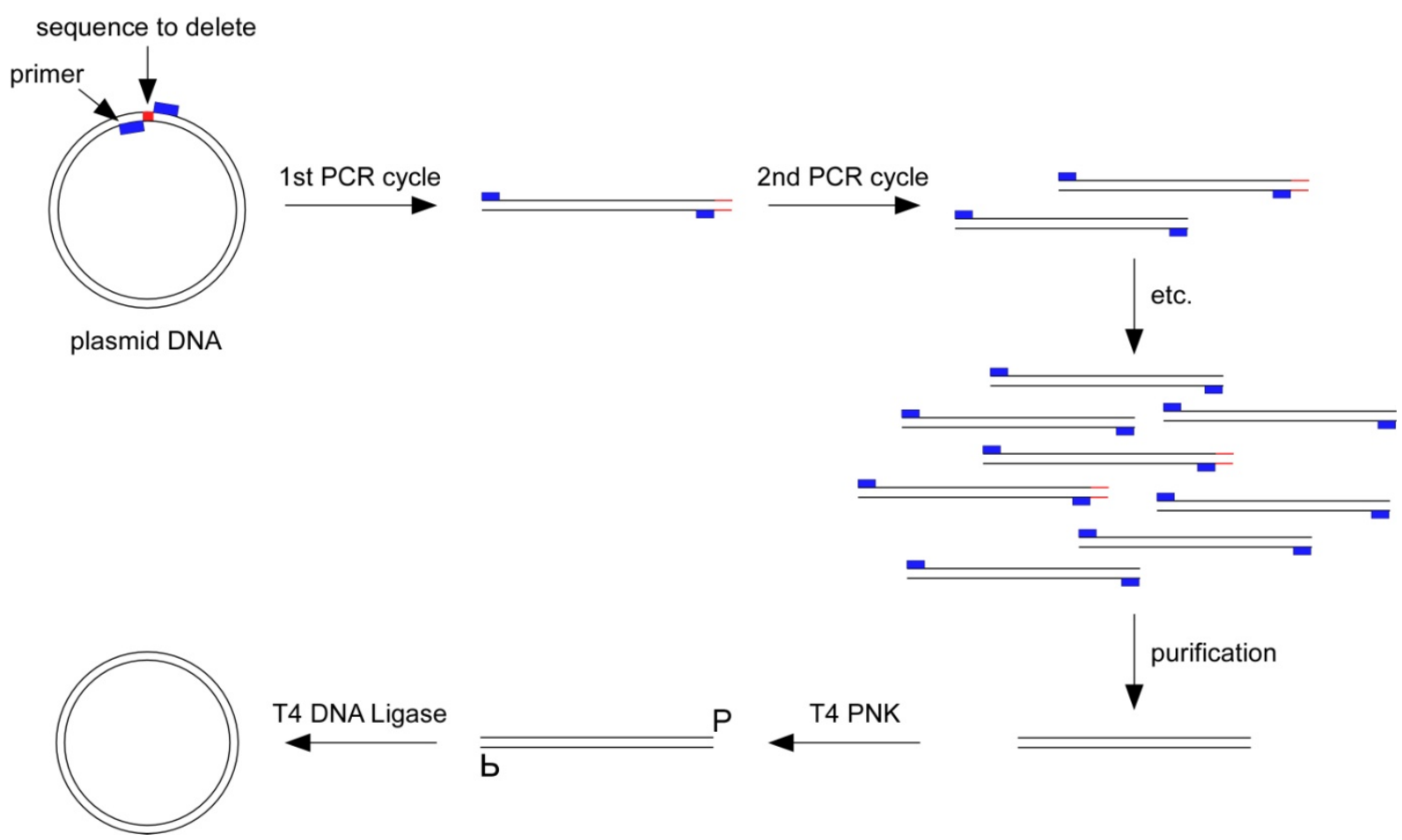

Figure 3.1: Generation of 5'ss, BPS and BPS-ACTGA deleted PM5 pre-mRNA. PCR primers were used to exclude the sequence to be deleted. After purification of the linear PCR product, the DNA was kinased using T4 PNK and subsequently ligazed with T4 DNA Ligase. The obtained plasmid DNA does not include the deleted nucleotide sequence.

\subsubsection{Protein biochemical methods}

\subsubsection{Protein concentration determination}

The concentration of proteins in solution was determined by using the Bradford Protein Assay (Bearden, 1978). The protein sample was diluted with water to a final volume of $800 \mu \mathrm{l}$ 
followed by addition of $200 \mu$ of Bradford solution (Bio-Rad Protein Assay) and incubation at room temperature for $10 \mathrm{~min}$. The absorbance at $595 \mathrm{~nm}$ was then measured and the protein concentration was determined by comparison to a standard dilution series of BSA $(0.2,0.4$, $0.6,0.8,1.0,1.2,1.4,1.6,1.8,2.0 \mu \mathrm{g})$.

\subsubsection{Polyacrylamide gel electrophoresis (PAGE)}

Protein samples were separated using the NuPAGE ${ }^{\circledR}$ system (Invitrogen) according to manufacturer's protocols. Briefly, precipitated and dried protein samples were dissolved in $4 \times$ sample buffer, $10 \times$ reducing agent and water. Dissolved samples were loaded onto 4$12 \%$ Bis-Tris pre-cast gels $(1.0 \mathrm{~mm} \times 10$ well) and gel electrophoresis was performed for approx. 50 min at $200 \mathrm{~V}$ using MOPS running buffer and antioxidant.

\section{Buffers and reagents:}

- NuPAGE LDS Sample Buffer $(4 \times)$

- NuPAGE Sample Reducing Agent $(10 \times)$

- NuPAGE MOPS SDS Running Buffer $(20 \times)$

- NuPAGE Antioxidant

\subsubsection{Colloidal Coomassie staining of proteins}

Separated proteins were stained using colloidal coomassie solution (Neuhoff et al., 1988). To this end, gels were incubated with staining solution overnight and background staining was removed by rinsing the gels with water.

\section{Colloidal Coomassie staining solution:}

$0.08 \%(\mathrm{~m} / \mathrm{v})$ Coomassie Brilliant Blue G 250

$20 \%(\mathrm{v} / \mathrm{v})$ Methanol

$1.6 \%(\mathrm{v} / \mathrm{v})$ ortho-Phosphoric acid

$8 \%(\mathrm{~m} / \mathrm{v})$ Ammonium sulfate

\subsubsection{Cell culture, nuclear extract, purification of spliceosomal complexes}

\subsubsection{Metabolic Labeling of HeLa S3 cells (SILAC labeling)}

HeLa S3 cells were grown in custom made DMEM medium containing normal (light) or stable isotope labeled (medium and heavy) L-arginine and L-lysine (see table 3.2). Cells were grown for at least six passages at smaller volumes and then expanded to $2.0 \mathrm{I}$ in spinner 
flasks $\left(0.5-1.0 \times 10^{6} \mathrm{cells} / \mathrm{ml}\right)$. The cells were transferred to a $2.5 \mathrm{I}$ fermenter and grown under standard conditions $\left(2.5-5.0 \times 10^{6} \mathrm{cells} / \mathrm{ml}\right)$.

SILAC medium:

$500 \mathrm{ml}$ DMEM w/o Arginine, w/o Lysine

$50 \mathrm{ml}$ dialyzed FBS

$5 \mathrm{ml} 100 \times$ Penicillin/Streptomycin

$50 \mathrm{mg} / \mathrm{l} \mathrm{L-Arginine}$

$50 \mathrm{mg} / \mathrm{l} \mathrm{L}-$ Lysine

Table 3.2: Isotope composition of amino acids used for different SILAC media. Due to the number of incorporated ${ }^{13} \mathrm{C},{ }^{15} \mathrm{~N}$ or ${ }^{2} \mathrm{D}$ atoms a mass difference between the light amino acid and the stable isotope labeled amino acid is obtained.

\begin{tabular}{|c|l|c|c|c|c|}
\cline { 3 - 6 } \multicolumn{2}{c|}{} & L-Arginine & $\Delta \mathbf{m}$ & L-Lysine & $\Delta \mathbf{m}$ \\
\hline \multirow{2}{*}{ duplex SILAC } & light & - & 0 & - & 0 \\
\cline { 2 - 6 } & heavy & ${ }^{13} \mathrm{C}_{6},{ }^{15} \mathrm{~N}_{4}$ & $+10 \mathrm{Da}$ & ${ }^{13} \mathrm{C}_{6}$ & $+6 \mathrm{Da}$ \\
\hline \multirow{3}{*}{ triple SILAC } & light & - & 0 & - & 0 \\
\cline { 2 - 6 } & medium & ${ }^{13} \mathrm{C}_{6}$ & $+6 \mathrm{Da}$ & ${ }^{2} \mathrm{D}_{4}$ & $+4 \mathrm{Da}$ \\
\cline { 2 - 6 } & heavy & ${ }^{13} \mathrm{C}_{6},{ }^{15} \mathrm{~N}_{4}$ & $+10 \mathrm{Da}$ & ${ }^{13} \mathrm{C}_{6},{ }^{15} \mathrm{~N}_{2}$ & $+8 \mathrm{Da}$ \\
\hline
\end{tabular}

\subsubsection{Preparation of splicing-active HeLa cell nuclear extract}

Cells from a fermenter were used to prepare nuclear extract according to Dignam et al., 1983. Briefly, cells were harvested by centrifugation for $5 \mathrm{~min}$ at $2000 \mathrm{rpm}$ (Cryofuge 6000i, Hereaus) and washed with ice-cold PBS. The cells were resuspended in 1.25 vol. MC buffer supplemented with $1 / 500$ vol. $0.25 \mathrm{M}$ DTE and 1/100 vol. EDTA free protease inhibitor cocktail, incubated on ice for $5 \mathrm{~min}$ and lyzed in a Dounce homogenizer (18 strokes) at $4{ }^{\circ} \mathrm{C}$. Nuclei were pelleted by centrifugation for $5 \mathrm{~min}$ at $18,000 \times \mathrm{g}$ (Sorvall SS34 rotor) and dounced (20 strokes) at $4{ }^{\circ} \mathrm{C}$ in 1.3 vol. of Roeder $\mathrm{C}$ buffer supplemented with $1 / 500$ vol. $0.25 \mathrm{M}$ DTE and 1/200 vol. 0.1 M PMSF. The mixture was stirred for $40 \mathrm{~min}$ at $4{ }^{\circ} \mathrm{C}$ followed by centrifugation for $30 \mathrm{~min}$ at $16,000 \mathrm{rpm}$ (Sorvall SS34 rotor). The supernatant was dialyzed three times for $2 \mathrm{~h}$ against 50 vol. of Roeder $\mathrm{D}$ buffer. The dialysate was centrifuged for 2 min at $9000 \times \mathrm{g}$ (Sorvall SA600 rotor) and aliquots of the supernatant were frozen in liquid nitrogen, stored at $-80{ }^{\circ} \mathrm{C}$ and tested for splicing activity.

\subsubsection{In vitro splicing}

In vitro splicing was performed using $\mathrm{m7G}\left(5^{\prime}\right) \mathrm{ppp}\left(5^{\prime}\right) \mathrm{G}$-capped, ${ }^{32} \mathrm{P}$-labeled and MS2-tagged pre-mRNA. Splicing reactions contained $50 \%(\mathrm{v} / \mathrm{v})$ HeLa nuclear extracts, $65 \mathrm{mM} \mathrm{KCl}, 3 \mathrm{mM}$ 
$\mathrm{MgCl}_{2}$, $2 \mathrm{mM}$ ATP, $20 \mathrm{mM}$ creatine phoshphate and $30 \mathrm{nM}$ pre-mRNA and were incubated for different time intervals at $30^{\circ} \mathrm{C}$. Assembled spliceosomal complexes were analyzed by native gel electrophoresis. RNA was recovered by $\mathrm{PCl}$ extraction and analyzed by denaturing gel electrophoresis.

\subsubsection{Purification of human Prp19/CDC5L complex}

hPrp19/CDC5L complex was isolated from AD-002-FLAG/HA-tagged HeLa nuclear extract. Briefly, HeLa S3 cell lines stably expressing FLAG/HA-tagged human AD-002 were generated according to general protocols. Nuclear extract from these cells was prepared according to Dignam et al., 1983. The hPrp19/CDC5L complex was affinity-purified from nuclear extract by using anti-FLAG-M2-Agarose beads and FLAG peptide. Isolated complexes were purified on a 5-20\% glycerol gradient. Protein tagging, stable cell line construction and hPrp19/CDC5L complex purification were performed by M. Grote (Dept. for Cellular Biochemistry; Grote et al., 2010).

\subsubsection{Purification of human tri-snRNP (U4/U6.U5)}

Total snRNPs were isolated from HeLa cell nuclear extract by immunoaffinity chromatography using monoclonal anti-m ${ }_{3}$ G-specific H20-antibody (Bach et al., 1990; Bringmann et al., 1983). Total snRNPs were separated on 10-30\% glycerol gradients. $1.5 \mathrm{ml}$ fractions were collected yielding the single snRNP components (12S U1 snRNP, 17S U2 snRNP, 20S U5 snRNP and 25S U4/U6.U5 snRNP) in different fractions. This was always verified by analyzing RNA and protein composition after $\mathrm{PCl}$ extraction by gel electrophoresis. All fractions were routinely frozen in liquid nitrogen and peak fractions containing only the U4/U6.U5 snRNP were used for the experiments described herein. Isolation of total snRNPs was performed on a routine basis by $\mathrm{P}$. Kempkes and $\mathrm{H}$. Kohansal (Dept. for Cellular Biochemistry) and purification of U4/U6.U5 snRNP was performed by M. Raabe (Bioanalytical Mass Spectrometry Group).

\subsubsection{Overexpression of MS2-MBP fusion protein}

The MS2-MBP fusion protein was used to affinity purify spliceosomal complexes assembled on MS2-tagged pre-mRNA. To overexpress the MS2-MBP protein, $50 \mu \mathrm{l}$ chemical competent BL21 cells were transfected with $50 \mathrm{ng}$ MS2-MBP fusion protein plasmid DNA by 
electroporation and subsequently transferred to LB medium containing ampicillin and chloramphenicol. After incubation at $37{ }^{\circ} \mathrm{C}$ overnight, cells were diluted with LB medium, grown at $37{ }^{\circ} \mathrm{C}$ until they reached an OD of 0.7 and induced with IPTG. When the cells reached an OD of 1.6 they were harvested by centrifugation for $30 \mathrm{~min}$ at $5000 \mathrm{rpm}$ (SL6000). Cells were washed with PBS and the cell pellet was resuspended in Tris- $\mathrm{HCl}$ pH 7.5 supplemented with $200 \mathrm{mM} \mathrm{NaCl}$ and protease inhibitor cocktail. The cells were solubilized by sonication and centrifuged for $15 \mathrm{~min}$ at $4000 \mathrm{rpm}$ (Megafuge) at $4{ }^{\circ} \mathrm{C}$. The supernatant was incubated overnight with amylose beads and the MS2-MBP fusion protein was eluted from the beads with $15 \mathrm{mM}$ maltose in Na-P buffer. The protein was further purified on Heparin beads using $20 \mathrm{mM}$ Hepes pH 7.9, $100 \mathrm{mM} \mathrm{KCl}, 15 \%$ (v/v) glycerol, 0.5 M DTT and 0.2 M PMSF as elution buffer. The protein concentration of the collected fractions was determined by the Bradford assay (section 3.2.2.1) and the fractions containing MS2-MBP fusion protein were identified by PAGE.

\subsubsection{Purification of human $B$ and $C$ complexes}

Spliceosomal B and C complexes were purified as described before (Bessonov et al., 2008; Deckert et al., 2006). Briefly, ${ }^{32}$ P-labeled pre-mRNA was incubated with a 20 -fold molar excess of MS2-MBP fusion protein and used without further purification in a standard splicing reaction. This contained $10 \mathrm{nM}$ (B complex) or $30 \mathrm{nM}$ (C complex) pre-mRNA and was incubated at $30^{\circ} \mathrm{C}$ for $6 \mathrm{~min}(\mathrm{~B})$ or $180 \mathrm{~min}(\mathrm{C})$. A 30-fold molar excess of M6 and M12 DNA oligonucleotides was added and the reaction was incubated for further 2 min (B) or 20 min (C). Assembled complexes were separated on 10-30\% glycerol gradients and 40-45 S gradient fractions were affinity purified on amylose beads. Spliceosomal B and C complexes were purified in collaboration with S. Bessonov and J. Deckert (Dept. for Cellular Biochemistry).

\subsubsection{Investigating the protein composition during the spliceosomal assembly}

To investigate the time dependent protein assembly of spliceosomal proteins, two different approaches were followed: (i) The time dependent assembly of proteins on different premRNAs, and (ii) the direct comparison of the time dependent protein assembly on PM5 premRNA and on a splicing-inactive pre-mRNA.

To analyze the time dependent assembly of proteins on the tagged pre-mRNA during $30 \mathrm{~min}$,

${ }^{32} \mathrm{P}$-labeled pre-mRNA was first incubated with a 20 -fold molar excess of MS2-MBP fusion protein. Several standard splicing reactions, each containing 20 pmol pre-mRNA, were then 
assembled and incubated at $30^{\circ} \mathrm{C}$ for different time intervals using triple (i.e. light, medium and heavy) SILAC extracts (as outlined in detail in Table 3.3). Assembled complexes were affinity purified on amylose beads and the concentration of samples from different time points to be compared (Table 3.3) was determined according to the radioactivity of the used premRNA. Samples from different time points to be compared were pooled in equal molar amounts and proteins were precipitated with ethanol.

To directly compare the protein assembly on PM5 pre-mRNA and on a splicing-inactive premRNA, ${ }^{32}$ P-labeled pre-mRNA was incubated with a 20-fold molar excess of MS2-MBP fusion protein. Standard splicing reactions were incubated at $30{ }^{\circ} \mathrm{C}$ for different time intervals during $30 \mathrm{~min}$ using duplex SILAC extracts (Table 3.4). Assembled complexes from different time points were affinity purified on amylose beads and the concentration was determined according to the radioactivity of the pre-mRNAs. Samples from the same time point but assembled on different pre-mRNAs were pooled in equal molar amounts and proteins were subsequently precipitated with ethanol.

Precipitated proteins were separated by PAGE, hydrolyzed with trypsin as described in section 3.2.4.1 except that $100 \mathrm{mM}$ ammonium bicarbonate buffer was used instead of $50 \mathrm{mM}$ TEAB and analyzed by LC-MS/MS.

Table 3.3: Used SILAC nuclear extracts for investigation of the protein assembly on pre-mRNAs during $30 \mathrm{~min}$. The concentration of samples from different time points to be compared was determined according to the radioactivity contained within the used pre-mRNAs and samples were then pooled in equal molar amounts (Pool 1, Pool 2, Pool 3).

\begin{tabular}{|c|c|c|c|c|c|c|c|c|c|}
\hline time & $\mathbf{0}^{\prime}$ & $\mathbf{2}^{\prime}$ & $\mathbf{5}^{\prime}$ & $\mathbf{0}^{\prime}$ & $\mathbf{1 0}$ & $\mathbf{1 5}$ & $\mathbf{0}^{\prime}$ & $\mathbf{2 0}$ & $\mathbf{3 0}$ \\
\hline NE & light & medium & heavy & light & medium & heavy & light & medium & heavy \\
\hline \multicolumn{3}{|c|}{ Pool 1 } & \multicolumn{3}{c|}{ Pool 2 } \\
\hline
\end{tabular}

Table 3.4: Used SILAC nuclear extracts for direct comparison of the protein assembly on PM5 pre-mRNA and on a splicing-inactive pre-mRNA during $\mathbf{3 0} \mathrm{min}$. The concentration of samples from different time points to be compared was determined according to the radioactivity contained within the used pre-mRNAs and samples were then pooled in equal molar amounts (Pools 1 - 8).

\begin{tabular}{|c|c|c|c|}
\hline time & PM5 pre-mRNA & Splicing-inactive pre-mRNA & \\
\hline $0^{\prime}$ & heavy NE & light NE & $\rightarrow$ Pool 1 \\
\hline $2^{\prime}$ & heavy NE & light NE & $\rightarrow$ Pool 2 \\
\hline $5^{\prime}$ & heavy NE & light NE & $\rightarrow$ Pool 3 \\
\hline $10^{\prime}$ & heavy NE & light NE & $\rightarrow$ Pool 4 \\
\hline $15^{\prime}$ & heavy NE & light NE & $\rightarrow$ Pool 5 \\
\hline $20^{\prime}$ & heavy NE & light NE & $\rightarrow$ Pool 6 \\
\hline $25^{\prime}$ & heavy NE & light NE & $\rightarrow$ Pool 7 \\
\hline $30^{\prime}$ & heavy NE & light NE & $\rightarrow$ Pool 8 \\
\hline
\end{tabular}




\subsubsection{Mass spectrometry methods}

\subsubsection{In-gel hydrolysis of proteins}

In-gel hydrolysis was performed as previously described with few modifications (Shevchenko et al., 1996). All incubation steps were carried out at $26^{\circ} \mathrm{C}$ in a thermomixer at $1050 \mathrm{rpm}$ for 15 min unless otherwise stated. The solutions were removed after incubation steps. Gel slices were cut from entire gel lanes using an in-house manufactured gel cutting device (see Figure 3.2). Gel slices were cut into small pieces and washed with $150 \mu$ water, followed by dehydration with $150 \mu \mathrm{l}$ acetonitrile. Gel pieces were dried in a vacuum centrifuge and proteins were reduced by addition of $100 \mu \mathrm{l} 100 \mathrm{mM}$ DTT (in $50 \mathrm{mM}$ triethylammonium bicarbonate buffer (TEAB)) and incubation at $56^{\circ} \mathrm{C}$ for $50 \mathrm{~min}$. After dehydration with $150 \mu \mathrm{l}$ acetonitrile, reduced cysteine residues were alkylated by addition of $100 \mu \mathrm{l} 60 \mathrm{mM}$ iodoacetamide (in $50 \mathrm{mM}$ TEAB) and incubation at $26^{\circ} \mathrm{C}$ for $20 \mathrm{~min}$. The gel pieces were incubated with $150 \mu \mathrm{l}$ of $50 \mathrm{mM}$ TEAB for $15 \mathrm{~min}$, followed by addition of $150 \mu \mathrm{l}$ acetonitrile. After dehydration with $150 \mu \mathrm{l}$ acetonitrile, the gel pieces were dried in a vacuum centrifuge and rehydrated on ice with buffer 1 . The gel pieces were covered with buffer 2 and tryptic digestion was carried out overnight at $37^{\circ} \mathrm{C}$.

\section{Buffer 1:}

$50 \mu \mathrm{l} 50 \mathrm{mM}$ TEAB

$50 \mu \mathrm{l}$ water

$15 \mu \mathrm{l} 0.1 \mu \mathrm{g} / \mu \mathrm{l}$ Trypsin (Roche)

\section{Buffer 2:}

$50 \mu \mathrm{l} 50 \mathrm{mM}$ TEAB

$50 \mu \mathrm{l}$ water
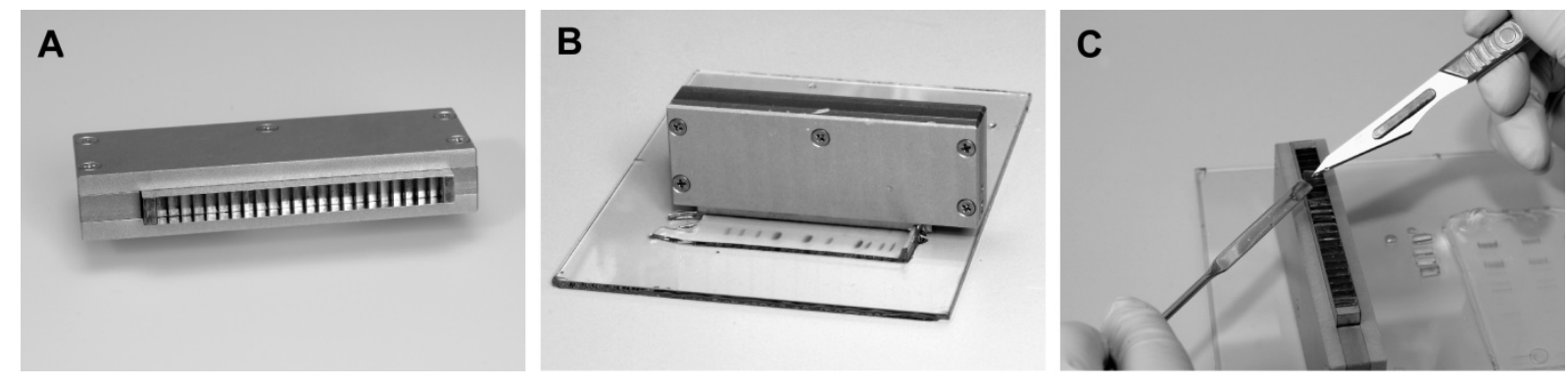

Figure 3.2: In-house manufactured gel cutting device. (A) Side view of the gel cutting device. Entire lanes of NuPAGE pre-cast gels are cut into 23 slices of equal size. (B) Gel cutting device attached to a NuPAGE pre-cast gel. (C) Cutting gel slices into small pieces.

\subsubsection{Extraction of peptides}

Extraction of peptides from in-gel hydrolysis was performed as described before (Shevchenko et al., 1996). All incubation steps were carried out at $37{ }^{\circ} \mathrm{C}$ in a thermomixer at 
$1050 \mathrm{rpm}$ for $15 \mathrm{~min}$. Briefly, gel pieces were incubated with $50 \mu \mathrm{l}$ water, followed by addition of $50 \mu \mathrm{l}$ acetonitrile. The supernatant containing tryptic peptides was removed and collected in a new eppendorf tube. In a second extraction step, $50 \mu \mathrm{l}$ of $5 \%(\mathrm{v} / \mathrm{v})$ formic acid (FA) were added, followed by addition of $50 \mu \mathrm{l}$ acetonitrile. The supernatant was removed and pooled with the first supernatant. In a third extraction step, $50 \mu \mathrm{l}$ of acetonitrile were added to the gel pieces to ensure complete extraction of peptides. The supernatant was collected and pooled with the other supernatants. Supernatants were evaporated to dryness in a vacuum centrifuge and the pellet could be stored for prolonged periods at $-20{ }^{\circ} \mathrm{C}$.

\subsubsection{3 iTRAQ labeling of extracted peptides for relative quantification}

For iTRAQ labeling the extracted peptides were dissolved in $20 \mu 100$ mM TEAB buffer. Internal standards were prepared by mixing $5 \mu$ aliquots of samples to be compared and obtained from different gel lanes, resulting in a final volume of $15 \mu \mathrm{l}$ (if 2 samples were compared $5 \mu$ TEAB buffer was added). ITRAQ reagents were reconstituted at room temperature in $70 \mu \mathrm{l}$ ethanol per vial. $5 \mu \mathrm{l}$ of iTRAQ reagents were added to each sample and incubated at room temperature for $1 \mathrm{~h}$ by gentle mixing. Internal standards were labeled with iTRAQ reagent 114 and corresponding samples to be compared were labeled with iTRAQ reagents 115,116 and 117 , respectively. After the reaction, the remaining reagent was quenched by addition of $5 \mu \mathrm{l}$ of $50 \mathrm{mM}$ glycine and incubation at room temperature for $30 \mathrm{~min}$ by gentle mixing. Samples to be compared, i.e. peptides labeled with iTRAQ reagents 115, 116, and 117, and belonging internal standards (labeled with ITRAQ reagent 114) were pooled and dried in a SpeedVacc.

\subsubsection{In-solution hydrolysis of hPrp19/CDC5L complex}

$5 \mu \mathrm{g}$ of purified hPrp19/CDC5L complex was diluted with $25 \mathrm{mM}$ Tris- $\mathrm{HCl} \mathrm{pH} 7.9$ to a final volume of $200 \mu \mathrm{l}$. $20 \mu \mathrm{l}$ of $0.5 \mathrm{M}$ sodium acetate buffer $\mathrm{pH} 5.3$ and $600 \mu \mathrm{l}$ of ice-cold $100 \%$ $(\mathrm{v} / \mathrm{v})$ ethanol were added and the sample was incubated for at least $2 \mathrm{~h}$ at $-20{ }^{\circ} \mathrm{C}$. After centrifugation (30 min, $4{ }^{\circ} \mathrm{C}, 13,300 \mathrm{rpm}$ ) the pellet was washed with $80 \%(\mathrm{v} / \mathrm{v})$ ice-cold ethanol followed again by centrifugation (see above). The pellet was dried in a vacuum centrifuge for approximately $5 \mathrm{~min}$. The different in-solution protocols were performed at room temperature with gentle mixing.

(i) In-solution hydrolysis in the presence of urea Hydrolysis of the protein complex in the presence of urea was performed as described before with a few modifications (Gruhler et al., 2005). The protein pellet from ethanol precipitation was dissolved in $20 \mu \mathrm{l} 8 \mathrm{M}$ urea (in 25 
$\mathrm{mM}$ Tris- $\mathrm{HCl} \mathrm{pH} \mathrm{7.9)} \mathrm{and} \mathrm{incubated} \mathrm{at} \mathrm{room} \mathrm{temperature} \mathrm{for} 15 \mathrm{~min}$. Proteins were reduced by addition of $20 \mu \mathrm{l} 10 \mathrm{mM}$ DTT (in $8 \mathrm{M}$ urea/25 mM Tris- $\mathrm{HCl} \mathrm{pH} 7.9$ ) and incubation for $30 \mathrm{~min}$. Alkylation of cysteine residues was subsequently performed by addition of $20 \mu \mathrm{l}$ $60 \mathrm{mM}$ iodoacetamide (in $8 \mathrm{M}$ urea/25 mM Tris- $\mathrm{HCl} \mathrm{pH} \mathrm{7.9)} \mathrm{and} \mathrm{incubation} \mathrm{for} 30 \mathrm{~min}$. Lys-C $(0.6 \mu \mathrm{g})$ was added followed by incubation for $3 \mathrm{~h}$ at room temperature. Before tryptic digestion, the sample was diluted to $2 \mathrm{M}$ urea with $100 \mathrm{mM}$ ammonium bicarbonate $\mathrm{pH}$ 7.9. Trypsin $(1 \mu \mathrm{g})$ was added and proteolysis was continued overnight at room temperature. Aliquots of the hydrolyzed protein complex were stored at $-80^{\circ} \mathrm{C}$.

(ii) In-solution digestion in the presence of acetonitrile The protein pellet from ethanol precipitation was dissolved in $50 \mu \mathrm{l} 80 \%(\mathrm{v} / \mathrm{v})$ acetonitrile/20 mM ammonium bicarbonate $\mathrm{pH} 7.9$ and incubated at room temperature for $15 \mathrm{~min}$. Proteins were reduced by addition of $50 \mu 10 \mathrm{mM}$ DTT (in $80 \%$ (v/v) acetonitrile/20 mM ammonium bicarbonate $\mathrm{pH}$ 7.9) and incubation for $30 \mathrm{~min}$. Alkylation of cysteine residues was subsequently performed by addition of $50 \mu \mathrm{l} 60 \mathrm{mM}$ iodoacetamide (in $80 \%$ (v/v) acetonitrile/20 mM ammonium bicarbonate $\mathrm{pH}$ 7.9) and incubation for $30 \mathrm{~min}$. Lys-C and Trypsin digestion was then performed as described above except dilution of the used buffer. Aliquots of the hydrolyzed protein complex were stored at $-80^{\circ} \mathrm{C}$.

(iii) In-solution digestion in the presence of RapiGest The protein pellet from ethanol precipitation was dissolved in $10 \mu \mathrm{l} 1 \%$ RapiGest/25 mM ammonium bicarbonate $\mathrm{pH}$ 8.5. Proteins were reduced by addition of $10 \mu \mathrm{l} 50 \mathrm{mM}$ DTT (in $25 \mathrm{mM}$ ammonium bicarbonate) and incubation for $1 \mathrm{~h}$ at $37^{\circ} \mathrm{C}$. Alkylation was subsequently performed by addition of $10 \mu \mathrm{l}$ $100 \mathrm{mM}$ iodoacetamide (in $25 \mathrm{mM}$ ammonium bicarbonate) and incubation for $1 \mathrm{hr}$ at $37^{\circ} \mathrm{C}$. $0.25 \mu \mathrm{g}$ Trypsin in $70 \mu \mathrm{l}$ ammonium bicarbonate were added for proteolysis followed by incubation overnight at $37{ }^{\circ} \mathrm{C}$. To decompose RapiGest $20 \mu \mathrm{l} \%$ (v/v) trifluoroacetic acid (TFA) were added followed by incubation for $2 \mathrm{~h}$ at $37^{\circ} \mathrm{C}$. The sample was centrifuged for 30 $\min$ at $13,000 \mathrm{rpm}$. The supernatant was transferred to another tube and dried in a SpeedVacc.

3.2.4.5 Comparison of hydrolysis protocols and selection of standard peptides (AQUA peptides) for absolute quantification

Prior to performing AQUA experiments, the hydrolyzed protein complex was analyzed qualitatively by high-resolution mass spectrometry to determine completeness of digestion and to identify peptide sequences suitable for absolute quantification. Aliquots were analyzed both by nanoLC-ESI-MS/MS on a hybrid Linear Ion Trap-Orbitrap mass spectrometer (LTQOrbitrap XL, Thermo Scientific) and by offline nanoLC-MALDI-MS/MS on a Tandem-ToF 
mass spectrometer (4800 MALDI TOF/TOF Analyzer, Applied Biosystems/MDS Sciex). Peak lists were generated from raw data using Mascot Daemon (Matrix Science) or the 4000 Series Explorer Remote Client Software (Applied Biosystems/MDS Sciex). The lists were searched against an NCBI non-redundant database (2007, Oct $8^{\text {th }} ; 5539442$ sequences) by using Mascot v2.2.04. For offline nanoLC-MALDI-MS/MS the mass accuracy filter used was $150 \mathrm{ppm}$ for precursor ions and 0.2 Da for product ions. For the Orbitrap mass spectrometer this was $5 \mathrm{ppm}$ for precursor and $0.5 \mathrm{Da}$ for product ions. Peptides with none or maximally two missed cleavage sites were defined as tryptic peptides. Carbamidomethylation of cysteines, oxidation of methionine residues and carbamylation of lysine residues and $\mathrm{N}$ terminal carbamylation for hydrolysis in the presence of urea were allowed as variable modifications.

Up to three peptide sequences per protein were selected from qualitative analysis of the hPrp19/CDC5L complex according to their intensities in MS and MS/MS spectra and the detailed requirements for AQUA peptides as suggested by the manufacturer (SigmaGenosys and Thermo Fisher Scientific; see below). Where possible, proteotypic peptides (Mallick et al., 2007) were selected. The following is a list of important criteria for standard peptides:

(i) The peptides must resolve well by HPLC; (ii) The peptides must not be too hydrophobic. (A peptide is classified as hydrophobic if more than $50 \%$ of its amino acids were either lle, Leu, Val, Phe, Trp or Met.); (iii) The peptides must not be too hydrophilic; (iv) The peptides must ionize well to ensure detection in the mass spectrometer; (v) The peptides must not contain chemically reactive amino acids (Cys, Met, Trp); (vi) The peptides must not contain chemically unstable sequences (N-terminal Asn, N-terminal Gln, Asp-Gly); (vii) The peptides' length must be limited to 15 amino acids; (viii) The peptides must contain amino acids that are well suited for labeling with stable isotopes (e.g. Arg, Lys, Phe, Ile).

The different hydrolysis protocols were compared for sequence coverage of the proteins observed, for the number of missed cleavages and the peptide score observed for the endogenous counterparts of the peptides selected for AQUA quantification.

\subsubsection{Internal standardization with the selected standard peptides}

The following peptides were obtained from Sigma-Genosys: TVPEELVKPEELS $\left({ }^{13} \mathrm{C}_{6}{ }^{15} \mathrm{~N}_{2}-\mathrm{K}\right)$, TLQLDNNFEV $\left({ }^{13} \mathrm{C}_{6}{ }^{15} \mathrm{~N}_{2}-\mathrm{K}\right)$, ILLGGYQS $\left({ }^{13} \mathrm{C}_{6}{ }^{15} \mathrm{~N}_{4}-\mathrm{R}\right)$, LGLLGLPAP $\left({ }^{13} \mathrm{C}_{6}{ }^{15} \mathrm{~N}_{2}-\mathrm{K}\right)$ and EAAAALVEEET $\left({ }^{13} \mathrm{C}_{6}{ }^{15} \mathrm{~N}_{4}-\mathrm{R}\right)$. They were delivered as $1 \mathrm{nmol}$ lyophilized peptide and were dissolved in $20 \mu \mathrm{l}$ dimethyl formamide by extensive vortexing and sonication in a sonication bath. 
Dissolved peptides were diluted with $180 \mu \mathrm{l}$ of $20 \%(\mathrm{v} / \mathrm{v})$ acetonitrile/0.1\% (v/v) FA, resulting in a concentration of $5 \mathrm{pmol} / \mu \mathrm{l}$. Small aliquots of the dissolved peptides were stored at $-20^{\circ} \mathrm{C}$.

The following peptides were obtained from Thermo Fisher Scientific: FVDILG $\left({ }^{13} \mathrm{C}_{6}{ }^{15} \mathrm{~N}-\mathrm{L}\right) \mathrm{R}$, HYTFASGSPDN $\left({ }^{13} \mathrm{C}_{6}{ }^{15} \mathrm{~N}-\mathrm{I}\right) \mathrm{K}, \quad \mathrm{NVVV}\left({ }^{13} \mathrm{C}_{9}{ }^{15} \mathrm{~N}-\mathrm{F}\right) \mathrm{DK}, \quad$ YADLL $\left({ }^{13} \mathrm{C}_{6}{ }^{15} \mathrm{~N}-\mathrm{L}\right) \mathrm{EK}, \quad$ TIVQLENEIYQ$\left({ }^{13} \mathrm{C}_{6}{ }^{15} \mathrm{~N}-\mathrm{I}\right) \mathrm{K}$ and TGYN $\left({ }^{13} \mathrm{C}_{9}{ }^{15} \mathrm{~N}-\mathrm{F}\right) \mathrm{QR}$. They were delivered in $5 \%(\mathrm{v} / \mathrm{v})$ acetonitrile at a concentration of $5 \mathrm{pmol} / \mu \mathrm{l}$. After extensive vortexing and sonication, aliquots were stored at $-20{ }^{\circ} \mathrm{C}$. Before use, peptides were dried in a vacuum centrifuge and redissolved in $100 \%$ acetonitrile followed by extensive vortexing and sonication as above to ensure complete solubilization.

Dissolved (Sigma Genosys) and redissolved (Thermo Fisher Scientific) peptides were diluted 2-fold with the appropriate loading buffer (see sections that deal with MS analyses) and again vortexed and sonicated. Peptide mixtures with concentrations of 100, 50, and $25 \mathrm{fmol} / \mu \mathrm{l}$ of each peptide were prepared. Aliquots from in-solution hydrolysis of the hPrp19/CDC5L complex containing $250 \mathrm{ng}$ were diluted with loading buffer and peptide mixture to give final amounts of $\sim 70 \mathrm{ng}, 35 \mathrm{ng}$ and $17.5 \mathrm{ng}$ of protein complex and $100 \mathrm{fmol}$, $50 \mathrm{fmol}$ and $25 \mathrm{fmol}$ of standard peptides per injection (injection volume $5 \mu \mathrm{l}$ ).

\subsubsection{Absolute quantification by LC-offline MALDI-ToF/ToF-MS (Peak Area)}

The sample containing endogenous peptides of the hPrp19/CDC5L complex and standard peptides was separated offline by reversed-phase nanoflow chromatography (Ultimate, Dionex) using $3.5 \%(\mathrm{v} / \mathrm{v})$ acetonitrile/0.1 \% (v/v) TFA as loading buffer, $0.1 \%(\mathrm{v} / \mathrm{v})$ TFA as mobile phase $A$ and $60 \%(\mathrm{v} / \mathrm{v})$ acetonitrile/0.1 \% (v/v) TFA as mobile phase $B$. The peptides were loaded on a trap column $\left(\mu\right.$-Precolumn ${ }^{\text {TM }}$ Cartridge, Acclaim PepMap100 C18, $300 \mu \mathrm{m}$ i.d. $\times 5 \mathrm{~mm}$, LC Packings) at a flow rate of $5 \mu \mathrm{l} / \mathrm{min}$ and separated at a flow rate of $300 \mathrm{nl} / \mathrm{min}$ on an analytical capillary C18 column packed in-house $(15 \mathrm{~cm}, 360 \mu \mathrm{m}$ o.d., $75 \mu \mathrm{m}$ i.d., Nucleosil 100-5 C18), with a gradient of 10-60\% buffer B over $60 \mathrm{~min}$. Separated peptides were mixed with $\alpha$-cyano-4-hydroxycinnamic acid $(10 \mathrm{mg} / \mathrm{ml}$ in $70 \%(\mathrm{v} / \mathrm{v})$ acetonitrile/0.1\% (v/v) TFA) containing $10 \mathrm{fmol} / \mu \mathrm{l}$ Glu-fibrinogen peptide as internal standard and delivered at a flow rate of $0.9 \mu \mathrm{l} / \mathrm{min}$. Fractions were spotted every $15 \mathrm{~s}$ onto blank stainless steel MALDI targets (Applied Biosystems/MDS Sciex) using a robotic spotting device (Probot, Dionex). The spotted peptide fractions were analyzed by MALDI-Tandem-ToF mass spectrometry (4800 MALDI TOF/TOF Analyser, Applied Biosystems/MDS Sciex) in positive-ion mode. MS spectra were generated with a total of 1000 shots. Up to 20 of the most intense peptide precursors per spot were selected for subsequent MS/MS analysis. For MS/MS a maximum 
of 5000 shots were accumulated per precursor using dynamic stop criteria depending on spectral quality. MS/MS spectra were acquired using a collision energy (potential difference between source acceleration voltage and collision cell) of $1 \mathrm{kV}$, with air at a pressure of $1 \times 10^{-6}$ torr as collision gas.

The peptides were quantified by their peak areas obtained from single mass spectra using Data Explorer software (Applied Biosystems/Sciex MDS, Foster City). The ratios of the peak areas for the endogenous and the corresponding standard peptides were calculated manually. Protein stoichiometries were determined by comparing the peptide ratios obtained for different proteins.

\subsubsection{Absolute quantification by LC-online MS-MS/MS (Extracted lon Chromatograms, XIC)}

The sample containing endogenous peptides of the hPrp19/CDC5L complex and standard peptides was independently analyzed by online reversed-phase nanoflow chromatography (HP 1100 series, Agilent; mobile phase A, $0.1 \%$ (v/v) FA; mobile phase B, $95 \%$ (v/v) acetonitrile/0.1\% ( $/ / \mathrm{v})$ FA coupled to a hybrid Linear Ion Trap-Orbitrap mass spectrometer (Thermo Scientific). To this end, the peptides were loaded on a manually packed trap column (1.5 cm, $360 \mu$ m o.d., $75 \mu \mathrm{m}$ i.d., Nucleosil 100-5 C18, Macherey-Nagel) and separated with a flow rate of $300 \mathrm{nl} / \mathrm{min}$ on an analytical C18 capillary column $(30 \mathrm{~cm}, 360 \mu \mathrm{m}$ o.d., $75 \mu \mathrm{m}$ i.d., Nucleosil 100-5 C18) with a gradient of 0-38 \% mobile phase B over 30 min. Eluted peptides were analyzed directly in the mass spectrometer (LTQ-Orbitrap XL, Thermo Scientific). The LTQ-Orbitrap was operated in data-dependent mode. Survey full scan MS spectra were acquired in the orbitrap $(\mathrm{m} / \mathrm{z} 350-1600)$ with a resolution of 30,000 at $\mathrm{m} / \mathrm{z} 400$ and an automatic gain control target at $10^{6}$. The five most intense ions were selected for CID (collision induced dissociation) MS/MS fragmentation and detection in the linear ion trap with previously selected ions dynamically excluded for $90 \mathrm{~s}$. Singly charged ions as well as ions with unrecognized charge state were also excluded. Internal calibration of the orbitrap was performed by using the lock mass option (lock mass: $\mathrm{m} / \mathrm{z}$ 445.120025; Olsen et al., 2005).

The peptides were quantified by generating extracted ion chromatograms (XICs) of the endogenous and the corresponding standard peptide. XICs were generated from the single MS spectra collected over time using the Qual Browser feature of Xcalibur software suite (Thermo Scientific) with a mass tolerance of $5 \mathrm{ppm}$ and a mass precision of $0.001 \mathrm{amu}$. Signals in the XICs were inspected by eye to make sure that the endogenous and the standard peptides showed the same retention time. The peptide ratios of the endogenous and the corresponding standard peptides were further calculated in a spread sheet (Excel) 
from the peak area of the extracted ions. The ratios calculated were used to determine the stoichiometry of the proteins within the complex.

\subsubsection{Absolute quantification by Multiple Reaction Monitoring}

MRM mass spectrometry was carried out on a hybrid Triple Quadrupole/Linear lon Trap mass spectrometer (4000 QTRAP LC-MS/MS System, Applied Biosystems/MDS Sciex). MRM transitions for each peptide were first designed by nanoSpray direct-infusion mass spectrometry. To this end, each standard peptide was diluted with $40 \%(\mathrm{v} / \mathrm{v})$ acetonitrile/0.1\% (v/v) FA to a final concentration of $200 \mathrm{fmol} / \mu \mathrm{l}$ and analyzed by Enhanced Resolution (ER) single MS and Enhanced Product lon (EPI) MS/MS scans to first establish the exact molecular weight and the fragmentation pattern. For each peptide the monoisotopic $\mathrm{m} / \mathrm{z}$ of the doubly charged precursor was then chosen as Q1 mass, while the three most abundant fragments with an $\mathrm{m} / \mathrm{z}$ above that of the doubly charged precursor were chosen as Q3 masses. Q1 and Q3 were both set to unit resolution (0.7 FWHM). For each MRM transition the declustering potential (DP), entrance potential (EP), collision energy (CE), and collision cell exit potential (CXP) were finally optimized by ramping the parameters and choosing the values displaying highest signal intensity. MRM transitions for the corresponding endogenous peptides were obtained by Q1/Q3 mass transition using the expected mass differential from the standard peptides.

Samples containing endogenous peptides together with the standard peptides were separated by reversed-phase nanoflow chromatography (Tempo 1D, Applied Biosystems/MDS Sciex) using $2 \%$ (v/v) acetonitrile/0.1 \% (v/v) FA as mobile phase $A$ and $98 \%(\mathrm{v} / \mathrm{v})$ acetonitrile/0.1 \% (v/v) FA as mobile phase B. The peptides were loaded on a trap column (Dionex PepMap C18, $300 \mu \mathrm{m}, 5 \mathrm{~mm}$ ) at a flow rate of $20 \mu \mathrm{l} / \mathrm{min}$ and washed with loading buffer $(2 \%(\mathrm{v} / \mathrm{v})$ acetonitrile/0.5 \% (v/v) FA) for 5 minutes. The peptides were separated on a capillary column (Dionex PepMap C18, $75 \mu \mathrm{m}, 15 \mathrm{~cm}$ ) at a flow rate of $300 \mathrm{nl} / \mathrm{min}$ with a gradient of $5-40 \%$ mobile phase B over $30 \mathrm{~min}$. Eluted peptides were analyzed directly in the 4000 QTRAP mass spectrometer. For each standard peptide and each endogenous peptide three MRM transitions were monitored with a dwell time of $20 \mathrm{~ms}$ per transition. The peptide ratios were obtained by automatic integration of peak areas for each endogenous peptide MRM transition and its AQUA counterpart using MultiQuant 1.0 Software (Applied Biosystems/MDS Sciex). The peptide ratios thus obtained were then used to determine the protein stoichiometry within the complex. 


\subsubsection{Relative quantification by LC-online MS-MS/MS (iTRAQ quantification)}

Samples were analyzed on a CAP-LC system coupled to Q-ToF Ultima mass spectrometer (Waters, Manchester, UK). To this end, peptides were dissolved in $10 \%$ acetonitrile/0.15\% FA and separated online by reversed phase chromatography using $0.1 \%(v / v)$ FA as mobile phase $A$ and $80 \%(\mathrm{v} / \mathrm{v})$ acetonitrile/0.15 \% (v/v) FA as mobile phase B. The peptides were loaded on a trap column ( $\mu$-Precolumn ${ }^{\mathrm{TM}}$ Cartridge, Acclaim PepMap100 C18, $300 \mu \mathrm{m}$ i.d. $\times$ $5 \mathrm{~mm}$, LC Packings) and separated with a flow rate of $200 \mathrm{nl} / \mathrm{min}$ on an analytical column $\left(\mathrm{C}_{18}\right.$, Reprosil, Maisch, Germany; packed in-house) with a gradient of $7-40 \%$ mobile phase $B$ over $50 \mathrm{~min}$. Eluted peptides were directly analyzed in the Q-ToF mass spectrometer in a data dependent manner. MS scans were acquired for $1 \mathrm{~s}$ followed by three MS/MS spectra for $3 \mathrm{~s}$ each with an ion mass window set to $2.5 \mathrm{Da}$. MS to MS/MS switch was set to 15 counts/s, and MS/MS to MS was set to an intensity below a threshold of 2 counts/s. Charge state recognition was used to estimate the collision energy for the selected precursors. Scan time and interscan time were set to $0.9 \mathrm{~s}$ and $0.1 \mathrm{~s}$, respectively. Data analysis was performed using MassLynx v4.0 software. Peak lists were generated from rawdata using the following settings: smooth window 4.00 , number of smooth 2 , smooth mode Savitzky-Golay, percentage of peak height to calculate centroid spectra: $80 \%$ with no baseline subtraction. Generated peak lists were searched against NCBI non-redundant database (2007, Oct $8^{\text {th }} ; 5539442$ sequences) using Mascot v.2.2.04 as search engine. Mass accuracy was set to $0.2 \mathrm{Da}$ for the parent and fragment ions. Peptides with no or maximal two missed cleavage sites were defined as tryptic peptides. Carbamidomethylation of cysteines and oxidation of methionine residues were allowed as variable modifications.

Non-normalized peptide ratios for ITRAQ quantification were obtained from Mascot v2.2.04 for unique peptides with a minimum peptide score of 20 . Proteins were quantified from the main bands by calculating the mean ratio after manual removal of outliers. Data normalization was performed on proteins known to be present in a 1:1 ratio (5' pre-mRNA cap binding proteins CBP20 and CBP80 for comparison of B and C complexes). Obtained protein ratios were further validated by three independent procedures: (i) calculation of the labeling efficiency for each protein in each band, (ii) using same amounts of non-modified trypsin (Roche) resulting in a 1:1 ratio for autoproteolytic Trypsin peptides, and (iii) analyzing peak intensities of the reporter ions for the internal standards (iTRAQ-114) of low scoring peptides $($ Intensity $($ iTRAQ-114) $=1 / 3$ Intensity $($ iTRAQ-115) $+1 / 3$ Intensity $($ iTRAQ-116) + 1/3 Intensity(iTRAQ-117)). 


\subsubsection{Relative quantification by LC-online MS-MS/MS (SILAC quantification)}

The samples from in-gel digestion were dissolved in $18 \mu \mathrm{l} 5 \%(\mathrm{v} / \mathrm{v})$ acetonitrile/1 \% (v/v) FA. Aliquots of $5 \mu \mathrm{l}$ were separated by reversed-phase nanoflow chromatography (HP 1100 series, Agilent) and eluted peptides were analyzed directly on a LTQ-Orbitrap XL (Thermo Scientific). For detailed information on experimental conditions see the sections that deal with absolute quantification (3.2.4.6).

Raw data were analyzed using MaxQuant software. Generated peak lists were searched against IPI human decoy database. Peptides with no or maximal two missed cleavage sites were defined as tryptic peptides. Carbamidomethylation of cysteines and oxidation of methionine residues were allowed as variable modifications. Depending on the nuclear extract used (see section 3.2.3.8, Table A and B), stable isotope lysine and arginine residues were set as fixed modifications.

\subsubsection{Statistical analysis}

Statistical analyses were performed using software $\mathrm{R}$ version 2.8.0.

To analyze differences in the peptides length and peptide scores, boxplots were generated and the variances were tested for heterogeneity. Differences of mean values were tested for statistical significance by applying Welch's two sample t-test.

To analyze the correlation between protein ratios obtained by ITRAQ and SILAC, the protein ratios were $\log _{2}$ transformed and plotted in a scatter plot. The correlation was tested according to Pearson. 


\section{Results}

\subsection{Determination of the protein stoichiometry within the hPrp19/CDC5L complex by absolute quantification (AQUA)}

The hPrp19/CDC5L complex consists of CDC5L, HSP70, CTNNBL1, PRL1, hPrp19, AD-002, and SPF27 proteins. Previous studies of the yeast homologue of the hPrp19/CDC5L complex revealed that Prp19 forms a tetramer within this complex. For this reason, the stoichiometry within the human complex was analyzed. The protein stoichiometry within protein complexes can be determined by absolute quantification of the complex's proteins. For this purpose absolute protein amounts have to be determined and compared in a relative manner. In proteomic studies absolute protein amounts are often deduced by the peptide concentrations. Here, stable isotope labeled standard peptides were used to determine the concentration of endogenous peptides generated from the hPrp19/CDC5L complex (AQUA). Different mass spectrometric techniques have been applied to obtain signal intensities for endogenous and standard peptides which reflect the relative abundance between these two peptide species. By addition of known amounts of standard peptides absolute amounts of the endogenous peptides and finally the proteins can be determined. The workflow for absolute quantification of the hPrp19/CDC5L complex thus comprised (i) proteomic analysis of the hPrp19/CDC5L complex, (ii) optimization of the hydrolysis conditions of the proteins, (iii) selection of suitable standard peptides for absolute quantification, (iv) synthesis of selected standard peptides by incorporation of stable isotopes, ( $v$ ) addition of defined amounts of the standard peptides to the hydrolyzed protein complex, (vi) determination of the abundance ratios between the labeled standard peptides and the corresponding endogenous peptides by mass spectrometry, and (vii) comparison of the determined ratios to obtain the protein stoichiometry within the hPrp19/CDC5L complex.

\subsubsection{Proteomic analysis of the hPrp19/CDC5L complex}

The hPrp19/CDC5L complex was affinity purified from nuclear extract of HeLa cells expressing FLAG/HA-tagged AD-002. A homogeneous preparation of the complex was obtained after glycerol gradient centrifugation. The protein composition of the purified complex in the corresponding fraction was visualized by 1D-PAGE and Coomassie staining of the proteins (Figure 4.1). The proteins were identified by LC-MS/MS of the generated peptides after in-gel digestion of the visible protein bands and, in parallel, by LC-MS/MS of 
generated peptides after in-solution hydrolysis (see below) of the gradient fraction containing the purified complex. Consistent with previous studies (Grote et al., 2010; Makarova et al., 2004), mass spectrometric analyses revealed that the hPrp19/CDC5L complex consists of seven proteins, namely CDC5L, Hsp70, CTNNBL1, PRL1, hPrp19, AD-002, and SPF27. Additional proteins could be detected but were represented with only few peptides and not in all biological replicates (for further information on additionally identified proteins see Table A1 in the Appendix). For Hsp70, different isoforms have been identified by MS analysis.

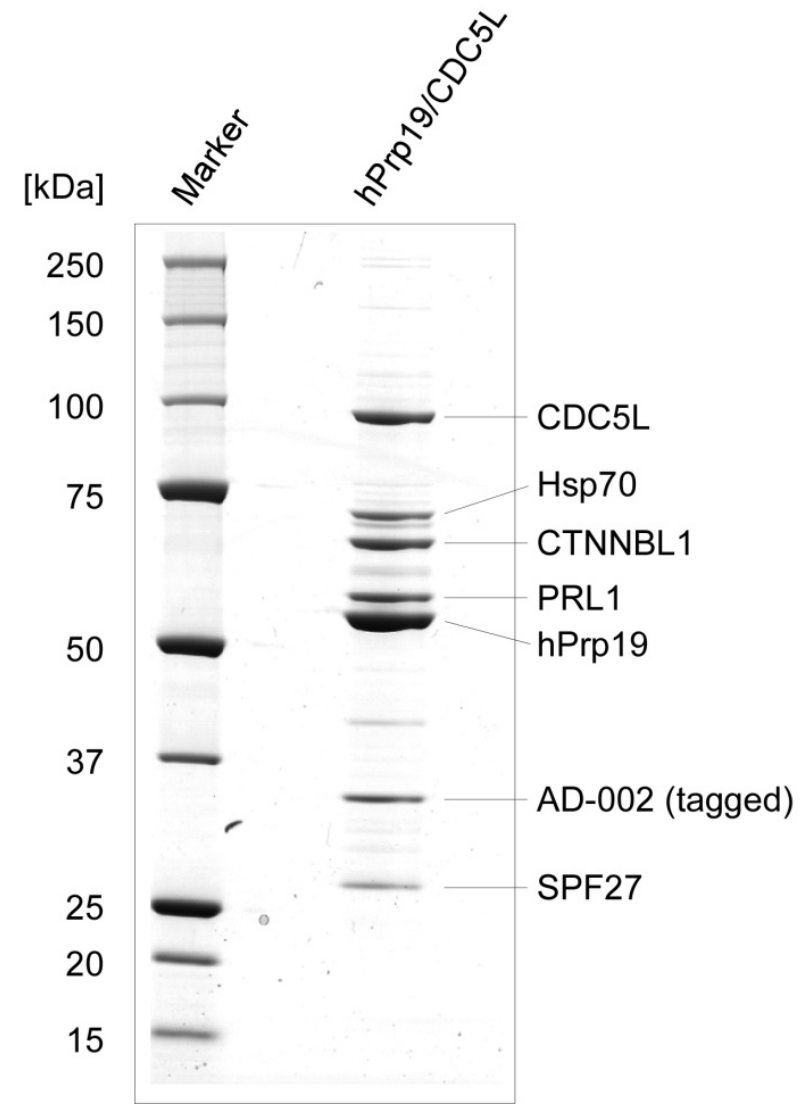

\begin{abstract}
Figure 4.1: 1D-PAGE of the hPrp19/CDC5L complex. The protein composition of the affinity purified complex was visualized by 1D-PAGE and Coomassie staining. Proteomic analysis, i.e. qualitative analysis, revealed that the protein complex consists of seven proteins. In-gel digestion and MS analysis allowed assignment of the proteins to different protein
\end{abstract} bands.

\title{
4.1.2 Comparison of different protocols for in-solution hydrolysis
}

There are two major prerequisites for absolute quantification using isotope labeled standard peptides: (i) the complete hydrolysis of the proteins to be investigated, and (ii) complete dissolving of the synthetic standard peptides with which the sample is spiked. In this study, three different sets of conditions for enzymatic hydrolysis of the proteins of the hPrp19/CDC5L complex were compared and evaluated: (i) in the presence of urea, (i) in the presence of acetonitrile, and (iii) in the presence of RapiGest (Waters, Manchester). The different hydrolysis conditions were compared by LC-ESI-MS/MS and LC-offline MALDIToF/ToF analysis. To this end, a total amount of $100 \mathrm{ng}$ of hydrolyzed hPrp19/CDC5L complex was analyzed in two to three replicates. As criteria for complete hydrolysis we used 
the sequence coverage of the proteins and the presence of missed cleavage site containing peptides within the endogenous quantification proxy (see below).

After hydrolysis in the presence of urea and by the use of LC-ESI-MS/MS we identified all seven proteins of the hPrp19/CDC5L complex with a sequence coverage of $40-70 \%$ (Tables 4.1 and $4.2 \mathrm{~A}$ ). After hydrolysis in the presence of acetonitrile the proteins were identified with a sequence coverage of $42-85 \%$ (Tables 4.1 and $4.2 \mathrm{~A}$ ). However, after hydrolysis in the presence of RapiGest the proteins were only identified with a sequence coverage of 24$53 \%$ (Table 4.1). Since the sequence coverage in the presence of RapiGest was significantly lower compared to urea and acetonitrile, hydrolysis in the presence of RapiGest was not used in further experiments.

Table 4.1: Comparison of different hydrolysis protocols. The sequence coverage (SC), the number of unique peptide sequences (UPS), and the number of peptides containing missed cleavage sites (MC) is specified for the hydrolysis in the presence of RapiGest, urea and acetonitrile analyzed by LC-ESI-MS/MS. The obtained sequence coverage is significantly lower for hydrolysis in RapiGest compared to urea and acetonitrile. The parameters listed take all detected protein isoforms into account.

\begin{tabular}{|l|ccc|ccc|ccc|}
\cline { 2 - 10 } \multicolumn{1}{c|}{} & \multicolumn{3}{c|}{ RapiGest } & \multicolumn{3}{c|}{ 8M/2M Urea } & \multicolumn{3}{c|}{$80 \%$ (v/v) Acetonitrile } \\
\hline Protein & SC [\%] & \# UPS & \# MC & SC [\%] & \# UPS & \# MC & SC [\%] & \# UPS & \# MC \\
\hline CDC5L & 45 & 59 & 34 & 70 & 78 & 45 & 79 & 74 & 37 \\
Hsp70 & $36 / 19 / 24$ & $27 / 14 / 8$ & $9 / 0 / 5$ & $62 / 30 / 39$ & $44 / 25 / 16$ & $17 / 9 / 6$ & $54 / 22 / 41$ & $39 / 24 / 11$ & $14 / 5 / 1$ \\
CTNNBL1 & 32 & 37 & 20 & 45 & 53 & 35 & 49 & 50 & 29 \\
PRL1 & 49 & 27 & 6 & 69 & 42 & 16 & 72 & 36 & 5 \\
hPrp19 & 24 & 17 & 7 & 50 & 27 & 7 & 60 & 32 & 8 \\
AD-002 & 47 & 18 & 11 & 55 & 20 & 15 & 60 & 22 & 15 \\
SPF27 & 53 & 17 & 7 & 60 & 25 & 10 & 70 & 22 & 8 \\
\hline
\end{tabular}

Under all the hydrolysis conditions tested, the overall sequence coverage of the single proteins was higher when LC-ESI-MS/MS was applied as compared with LC-offline MALDIToF/ToF-MS (Table 4.2 A and B), presumably due to the higher sensitivity of the used ESI mass spectrometer in MS/MS (LTQ-Orbitrap XL). Comparing the hydrolysis in the presence of urea and acetonitrile, hydrolysis in the presence of acetonitrile resulted in an approximately 10\% higher sequence coverage for all proteins (Table $4.2 \mathrm{~A}$ and B). An exception is AD-002 which revealed a higher sequence coverage in only one replicate. In contrast, the sequence coverage of two proteins, namely CTNNBL1 and AD-002, was significantly lower (only $\sim 50 \%$ sequence coverage) as compared to other proteins of the 
hPrp19/CDC5L complex. This is due to the low number of tryptic cleavage sites within both protein sequences (see below).

Table 4.2: Comparison of different hydrolysis protocols. (A) The sequence coverage (SC), the number of unique peptide sequences (UPS), and the number of peptides containing missed cleavage sites (MC) is specified for three independent replicates after hydrolysis in $8 \mathrm{M} / 2 \mathrm{M}$ urea or $80 \%(\mathrm{v} / \mathrm{v})$ acetonitrile analyzed by LC-ESIMS/MS. (B) The sequence coverage, the number of unique peptide sequences, and the number of peptides containing missed cleavage sites is specified for two independent replicates after hydrolysis in $8 \mathrm{M} / 2 \mathrm{M}$ urea or $80 \%(\mathrm{v} / \mathrm{v})$ acetonitrile analyzed by LC-offline MALDI-MS/MS. The parameters listed take all detected protein isoforms into account.

\begin{tabular}{|c|c|c|c|c|c|c|c|c|c|}
\hline \multirow{3}{*}{$\mathbf{A}$} & \multicolumn{9}{|c|}{ LC-ESI-MS/MS (LTQ Orbitrap XL) } \\
\hline & \multicolumn{3}{|c|}{1} & \multicolumn{3}{|c|}{2} & \multicolumn{3}{|c|}{3} \\
\hline & SC [\%] & \# UPS & \# MC & SC [\%] & \# UPS & \# MC & SC [\%] & \# UPS & \# MC \\
\hline \multicolumn{10}{|c|}{$8 \mathrm{M} / 2 \mathrm{M}$ Urea } \\
\hline CDC5L & 65 & 71 & 42 & 75 & 86 & 46 & 77 & 80 & 43 \\
\hline Hsp70 & $58 / 32 / 27$ & $45 / 24 / 14$ & $19 / 12 / 4$ & $54 / 32$ & $38 / 22 / 9$ & $13 / 7 / 3$ & $50 / 37$ & $40 / 27$ & $15 / 9$ \\
\hline CTNNBL1 & 41 & 57 & 33 & 47 & 48 & 30 & 41 & 47 & 32 \\
\hline PRL1 & 48 & 37 & 17 & 69 & 41 & 15 & 68 & 38 & 12 \\
\hline hPrp19 & 40 & 28 & 10 & 59 & 35 & 10 & 60 & 29 & 8 \\
\hline AD-002 & 54 & 24 & 19 & 63 & 24 & 18 & 59 & 24 & 18 \\
\hline SPF27 & 56 & 23 & 10 & 60 & 28 & 13 & 71 & 26 & 10 \\
\hline \multicolumn{10}{|c|}{$80 \%(\mathrm{v} / \mathrm{v})$ Acetonitrile } \\
\hline CDC5L & 76 & 78 & 31 & 85 & 98 & 56 & 82 & 79 & 40 \\
\hline Hsp70 & $58 / 49 / 35$ & $40 / 27 / 14$ & $12 / 7 / 2$ & $52 / 34$ & $38 / 20 / 7$ & $14 / 5 / 0$ & $53 / 38$ & $41 / 25$ & $17 / 9$ \\
\hline CTNNBL1 & 51 & 52 & 28 & 50 & 57 & 34 & 42 & 51 & 31 \\
\hline PRL1 & 69 & 33 & 7 & 70 & 45 & 18 & 70 & 43 & 16 \\
\hline hPrp19 & 66 & 36 & 10 & 71 & 44 & 20 & 63 & 42 & 18 \\
\hline AD-002 & 55 & 22 & 15 & 55 & 22 & 15 & 49 & 20 & 13 \\
\hline SPF27 & 78 & 22 & 7 & 83 & 29 & 15 & 81 & 28 & 12 \\
\hline
\end{tabular}

\begin{tabular}{|c|c|c|c|c|c|c|}
\hline \multirow{3}{*}{ B } & \multicolumn{6}{|c|}{ LC-offline MALDI-MS/MS (4800 MALDI ToF/ToF Analyzer) } \\
\hline & \multicolumn{3}{|c|}{1} & \multicolumn{3}{|c|}{2} \\
\hline & SC [\%] & \# UPS & \# MC & SC [\%] & \# UPS & $\# \mathrm{MC}$ \\
\hline \multicolumn{7}{|c|}{ 8M/2M Urea } \\
\hline CDC5L & 33 & 29 & 14 & 43 & 32 & 12 \\
\hline Hsp70 & 21 & 12 & 4 & 28 & 15 & 5 \\
\hline CTNNBL1 & 23 & 21 & 9 & 33 & 27 & 17 \\
\hline PRL1 & 21 & 11 & 4 & 33 & 15 & 5 \\
\hline hPrp19 & 23 & 12 & 1 & 28 & 12 & 1 \\
\hline AD-002 & 18 & 5 & 3 & 27 & 7 & 5 \\
\hline SPF27 & 40 & 8 & 4 & 48 & 15 & 6 \\
\hline \multicolumn{7}{|c|}{$80 \%(v / v)$ Acetonitrile } \\
\hline CDC5L & 43 & 31 & 7 & 52 & 36 & 12 \\
\hline Hsp70 & $33 / 14 / 27$ & $18 / 6 / 7$ & $5 / 1 / 1$ & 37 & 17 & 3 \\
\hline CTNNBL1 & 33 & 21 & 9 & 35 & 25 & 13 \\
\hline PRL1 & 37 & 19 & 3 & 48 & 17 & 4 \\
\hline hPrp19 & 33 & 16 & 3 & 47 & 18 & 4 \\
\hline AD-002 & 29 & 8 & 5 & 29 & 5 & 3 \\
\hline SPF27 & 43 & 13 & 7 & 50 & 13 & 6 \\
\hline
\end{tabular}


Hydrolysis in the presence of urea always risks artifactual carbamylation of the generated peptides. This is because isocyanic acid is generated upon decomposition of urea which in turn can carbamylate the peptide's N-terminus. Carbamylated peptides show different properties in liquid chromatography and the mass spectrometer when compared to the nonmodified peptides. Consequently, the amount of the endogenous peptide available for quantification is reduced and does not reflect the actual concentration. Quantification of this specific peptide is then not possible. Performing the hydrolysis at room temperature reduced the amount of carbamylated peptides to $1-4 \%$ and possible carbamylation of peptides can then be neglected.

The two hydrolysis conditions, i.e. hydrolysis in the presence of urea and in the presence of acetonitrile, were also compared regarding peptides containing missed cleavage sites for the particular endoproteinase used in the hydrolysis. As we used a highly sensitive mass spectrometer (LTQ-Orbitrap XL) more peptides containing missed cleavage sites ( 10\%) were detected by LC-ESI-MS/MS as compared to LC-offline MALDI-ToF/ToF-MS (compare Tables 4.2 A and B). Peptides containing missed cleavage sites were more frequently detected when they were generated in the presence of urea as compared to acetonitrile (Table 4.2 A and B). They also showed higher peptide scores for the peptide sequences selected for quantification (see below) as compared to hydrolysis in the presence of acetonitrile, i.e. they were sequenced with a higher probability when generated from hydrolysis in the presence of urea (Figure A.1 in the Appendix). Importantly, some peptides were very frequently detected with missed cleavage site (e.g. EAAAALVEEETR/R and MK/ILLGGYQSR derived from proteins SPF27 and CDC5L, respectively). Close inspection of the missed cleavage site-containing peptides showed that in the presence of acetonitrile incompletely cleaved peptides are significantly longer than incompletely cleaved peptides generated in the presence of urea. To further investigate this observation, the peptide length (number of amino acids) of missed cleavage site-containing peptides obtained by the two different hydrolysis protocols was statistically analyzed using the software $\mathrm{R}$ (version 2.8.0). Differences of mean values for peptide length were tested for statistical significance by applying Welch's two sample t-test (Figure 4.2). A p-value of 0.035 confirms the observation that incompletely cleaved peptides in the presence of acetonitrile are longer when compared to incompletely cleaved peptides generated in urea. On average, missed cleavage sitecontaining peptides generated in the presence of acetonitrile are 14.1 amino acids long whereas missed cleavage site-containing peptides generated in the presence of urea are 12.8 amino acids long (the statistical output is shown in Figure A.2 in the Appendix). However, these peptides were only detected by LC-ESI-MS/MS and were much less frequently detected than their correctly cleaved counterparts (as determined by spectral count; for further information see Table A2 in the Appendix). 


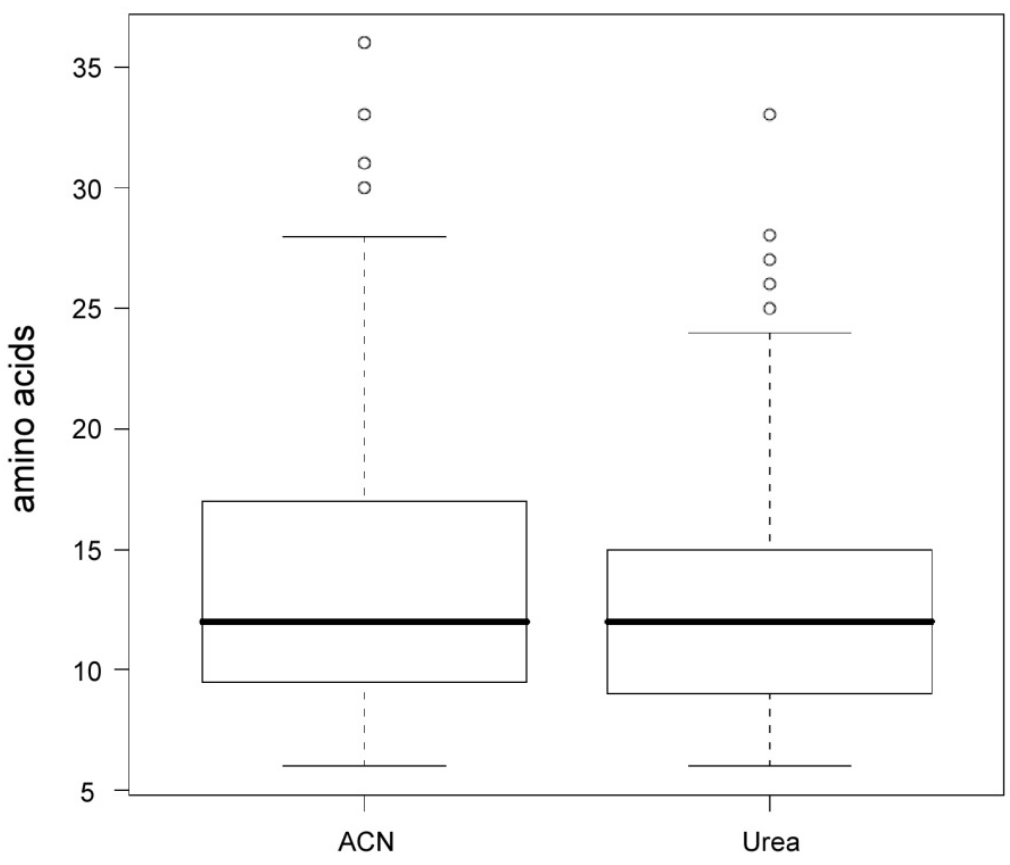

Figure 4.2: Miscleaved peptides obtained by hydrolysis in the presence of acetonitrile are significantly longer than miscleaved peptides obtained by hydrolysis in the presence of urea. Boxplot of peptide length (number of amino acids) for miscleaved peptides obtained by hydrolysis in the presence of acetonitrile and urea. On average, incompletey cleaved peptides generated in the presence of acetonitrile are 14.1 amino acids long, whereas incompletey cleaved peptides generated in urea are 12.8 amino acids long. The output of the statistical analysis is shown in Figure A.2 in the Appendix.

In summary, both protocols for hydrolysis of the hPrp19/CDC5L complex produced similar results in the database search. As described above, the effects of carbamylation and the generation of missed cleavage site-containing peptides can be neglected. Consequently, the hPrp19/CDC5L complex was analyzed after hydrolysis according to both protocols. 


\subsubsection{Selection of standard peptides for absolute quantification}

After optimization of hydrolysis conditions of the proteins to be investigated, the next step for absolute quantification involved the selection of suitable standard peptides. Sequenced endogenous peptides were evaluated whether they meet the selection criteria for standard peptides (see section 3.2.4.3). To this end, endogenous peptides were analyzed for their amino acid composition, length and hydrophobicity. The MS and MS/MS spectra of suitable peptides were inspected for sufficient intensities and a proper retention time in chromatography, i.e. elution of the different peptides in a well-separated range and no peak overlap with other peptides. Finally, the chosen peptides were checked to see whether they meet the criteria for proteotypic peptides (Mallick et al., 2007). The latter are peptides that are repeatedly and consistently identified for any given protein in a mixture and are thus suitable to function as standard peptides during absolute quantification. The selection of proteotypic peptides increases the possibility that the peptide of interest (here, the peptide selected for quantification) is identified (sequenced) during MS analysis and thus allows the unambiguous assignment of the identified (sequenced) peptide to the protein of interest.

Even though a high number of peptides derived from the hPrp19/CDC5 complex proteins could be identified by MS and MS/MS analysis, only a very few peptide sequences come into consideration as standard peptides for absolute quantification. For reliable absolute quantification it is recommended to select up to three standard peptides per protein. For most of the proteins, size and amino acid sequence limit the number of peptides that are suitable to serve as standard peptides. Small proteins do not generate a high number of peptides during hydrolysis, thus reducing the selection. The amino acid sequence further limits the selection of standard peptides as certain amino acids are unsuitable (e.g. chemically reactive amino acids). Figure 4.3 shows the protein sequences of AD-002 and CTNNBL1 and theoretically expected tryptic peptides that can be analyzed by mass spectrometry (> $700 \mathrm{Da}$ ). According to the above mentioned criteria, no peptide for AD-002 and only one peptide of CTNNBL1 could be selected for quantitative analysis.

The standard peptides were synthesized by incorporation of stable isotope labeled amino acids and synthetic peptides obtained were checked mass and sequence by MALDIToF/ToF-MS. Synthesized standard peptides were obtained by Sigma-Genosys or Thermo Fisher Scientific (for further information see section 3.2.4.4). Table 4.3 shows selected standard peptides for absolute quantification of the hPrp19/CDC5L complex. 
A AD-002 sequence

1

51

MTTAARPTFE PARGGRGKGE GDLSQLSKQY SSRDLPSHTK IKYRQTTQDA PEEVRNRDFR RELEERERAA 101

AREKNRDRPT REHTTSSSVS KKPRLDOIPA ANLDADDPLT DEEDEDFEEE SDDDDTAALL AELEKIKKER 151

AEEQARKEQE QKAEEERIRM ENILSGNPLL NLTGPSQPQA NFKVKRRWDD DVVFKNCAKG VDDQKKDKRF

VNDTLRSEFH KKFMEKYIK

\begin{tabular}{|c|c|c|c|}
\hline$[\mathrm{M}+\mathrm{H}]^{+}$ & Position & Peptide sequence & Remarks \\
\hline 1448.73 & $1-13$ & MTTAARPTFEPAR & proteotypic, contains methionine \\
\hline 1033.52 & $19-28$ & GEGDLSQLSK & not proteotypic \\
\hline 797.42 & $34-40$ & DLPSHTK & not proteotypic \\
\hline 1273.60 & $45-55$ & QTTQDAPEEVR & $\mathrm{N}$-terminal glutamine, not proteotypic \\
\hline 1062.51 & $82-91$ & EHTTSSSVSK & not proteotypic \\
\hline 4535.00 & $95-135$ & $\begin{array}{l}\text { LDQIPAANLDADDPLTDEEDE- } \\
\text { DFEEESDDDDTAALLAELEK }\end{array}$ & too long \\
\hline 703.34 & $141-146$ & AEEQAR & too short, not proteotypic \\
\hline 2583.32 & $160-183$ & MENILSGNPLLNLTGPSQPQANFK & too long \\
\hline 1023.48 & $188-195$ & WDDDVVFK & proteotypic, contains tryptophan \\
\hline
\end{tabular}

\section{CTNNBL1 sequence}

1 51

MVDGQVVALL VQNLERLDES VKEEADGVHN TLAIVENMAE FRPEMCTEGA QQGLLQWLLK RLKAKMPFDA 101

NKLYCSEVLA ILLQDNDENR ELLGELDGID VLLQQLSVFK RHNPSTAEEQ EMMENLFDSL CSCLMLSSNR

ERFLKGEGLQ LMNLMLREKK ISRSSALKVL DHAMIGPEGT DNCHKFVDIL GLRTIFPLFM KSPRKIKKVG 251

TTEKEHEEHV CSILASLLRN LRGQQRTRLL NKFTENDSEK VDRLMELHFK YLGAMQVADK KIEGEKHDMV 301

RRGEIIDNDT EEEFYLRRLD AGLFVLQHIC YIMAEICNAN VPQIRQRVHQ ILNMRGSSIK IVRHIIKEYA 351

ENIGDGRSPE FRENEQKRIL GLLENF

D

\begin{tabular}{|c|c|c|c|}
\hline$[\mathrm{M}+\mathrm{H}]^{+}$ & Position & Peptide sequence & Remarks \\
\hline 1783.97 & $1-16$ & MVDGQVVALLVQNLER & contains methionine, not proteotypic \\
\hline 4271.06 & $23-60$ & $\begin{array}{l}\text { EEADGVHNTLAIVENMAEFRPE- } \\
\text { MCTEGAQQGLLQWLLK }\end{array}$ & too long \\
\hline 822.38 & $66-72$ & MPFDANK & contains methionine, not proteotypic \\
\hline 2108.03 & $73-90$ & LYCSEVLAILLQDNDENR & too long, not proteotypic \\
\hline 2229.24 & $91-110$ & ELLGELDGIDVLLQQLSVFK & too long, not proteotypic \\
\hline 3316.40 & $112-140$ & $\begin{array}{l}\text { HNPSTAEEQEMMENLFDSLCS- } \\
\text { CLMLSSNR }\end{array}$ & too long \\
\hline 1374.72 & $146-157$ & GEGLQLMNLMLR & proteotypic, contains methionine \\
\hline 1836.84 & $169-185$ & VLDHAMIGPEGTDNCHK & proteotypic, contains methionine \\
\hline 932.56 & 186-193 & FVDILGLR & proteotypic \\
\hline 996.56 & 194-201 & TIFPLFM & proteotypic, contains methionine \\
\hline 1735.88 & $215-229$ & EHEEHVCSILASLLR & proteotypic, contains cysteine \\
\hline 969.42 & $243-250$ & FTENDSEK & not \\
\hline 917.49 & $254-260$ & LMELHFK & proteotypic, contains methionine \\
\hline 1095.55 & $261-270$ & YLGAMQVADK & contains methionine, not proteotypic \\
\hline 1842.84 & 283-297 & GEIIDNDTEEEFYLR & not proteotypic \\
\hline 3044.55 & 299-325 & $\begin{array}{l}\text { LDAGLFVLQHICYIMAEICNANV- } \\
\text { PQIR }\end{array}$ & too long \\
\hline 1010.56 & 335 & VHQILNMR & contains methionine, not proteotypic \\
\hline 1123.50 & 348-357 & EYAENIGDGR & not proteotypic \\
\hline
\end{tabular}

Figure 4.3: Analysis of AD-002 and CTNNBL1 sequences for presence of standard peptides. (A) AD-002 sequence. Proteotypic peptides are underlined. (B) Theoretically expected tryptic peptides > 700 kDa of AD-002 that could be detected by MS. Peptides were inspected according to the selection criteria of standard peptides. No peptide of AD-002 could be selected for quantitative analysis. (C) CTNNBL1 sequence. Proteotypic peptides are underlined. (D) Theoretically expected tryptic peptides $>700$ Da of CTNNBL1 that could be detected by MS. Only one peptide could be selected for quantitative analysis (FVDILGLR, highlighted in bold). 
Table 4.3: Selected standard peptides. Up to three peptide sequences were selected from proteomic analysis of the hPrp19/CDC5L complex. Owing to protein size and sequence the number of peptides that fulfilled the requirements to serve as standard peptides was limited. No peptide for AD-002 was available.

\begin{tabular}{llcccc}
\hline & & \multicolumn{2}{c}{ mass [Da] } & & \\
\cline { 3 - 4 } protein & peptide sequence & endogenous & standard & $\Delta$ m [Da] & proteotypic \\
\hline \multirow{2}{*}{ CDC5L } & ILLGGYQS(R) & 1005 & 1015 & 10 & \\
CDC5L & LGLLGLPAP(K) & 977 & 985 & 8 & \\
CDC5L & YADLL(L)EK & 963 & 970 & 7 & $\vdots$ \\
CTNNBL1 & FVDILG(L)R & 931 & 938 & 7 & \\
PRL1 & HYTFASGSPDN(I)K & 1435 & 1442 & 7 & \\
PRL1 & TGYN(F)QR & 884 & 894 & 10 & \\
hPrp19 & TLQLDNNFEV(K) & 1319 & 1327 & 8 & \\
hPrp19 & NVVV(F)DK & 819 & 829 & 10 & \\
SPF27 & EAAAALVEEET(R) & 1287 & 1297 & 10 & \\
SPF27 & TIVQLENEIYQ(I)K & 1589 & 1596 & 7 & \\
& & & & & \\
\hline
\end{tabular}

The chromatographic behavior of the synthetic standard peptides was investigated whether they reveal the same behavior as their endogenous counterparts. Surprisingly, standard peptides that were delivered in $5 \%(\mathrm{v} / \mathrm{v})$ acetonitrile and subsequently stored in smaller aliquots at $-20{ }^{\circ} \mathrm{C}$ did not reveal sufficient signal intensity in nanoLC. These peptides were only detectable in LC after drying the aliquots in a vacuum centrifuge and re-dissolving them in $100 \%$ (v/v) acetonitrile before use. Complete solubility of the standard peptides is a major prerequisite for reliable and accurate absolute quantification. During this study, we found that an initial LC analysis of peptides dissolved under different conditions gave an easy estimate on the solubility and chromatographiy behavior of the synthetic standard peptides.

A

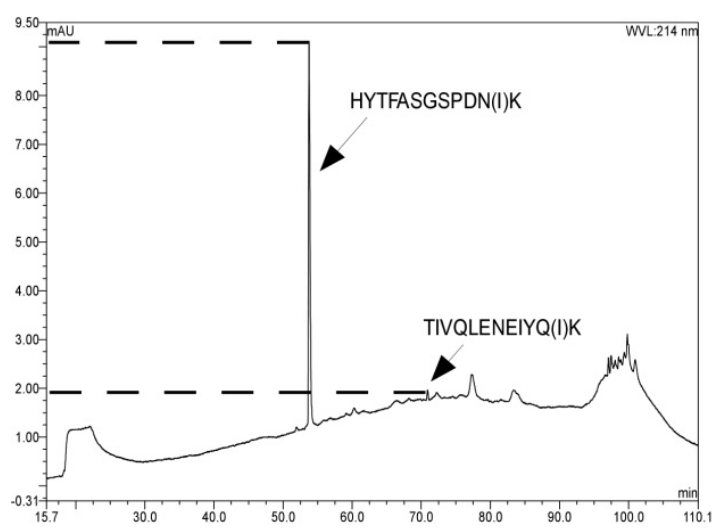

B

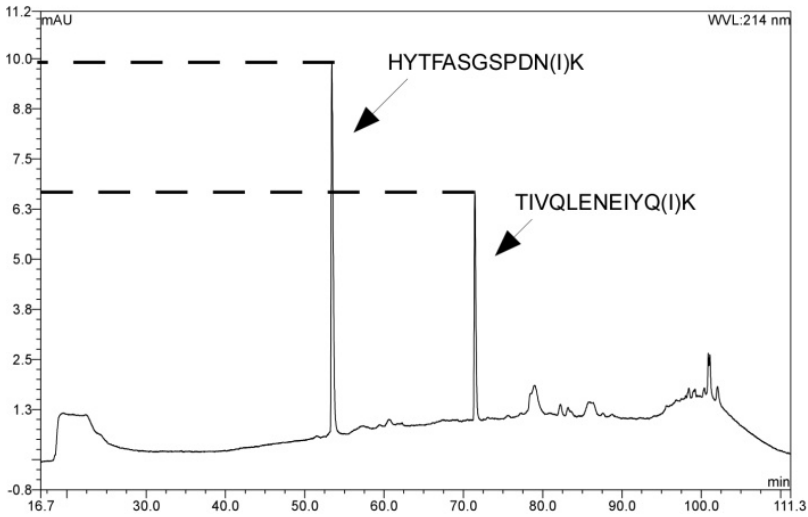

Figure 4.4: Solubility of standard peptides. After storage at $-20^{\circ} \mathrm{C}$, peptides delivered in $5 \%(\mathrm{v} / \mathrm{v})$ acetonitrile did not reveal sufficient signal intensity in LC. (A) The peptides HYTFASGSPDN(I)K and TIVQLENEIYQ(I)K were diluted with $3.5 \%(\mathrm{v} / \mathrm{v})$ acetonitrile/0.1 \% (v/v) TFA, $1.25 \mathrm{pmol}$ of each peptide were subjected to nanoLC and detected at $214 \mathrm{~nm}$. (B) The peptides were dried in a vacuum centrifuge and re-dissolved in $100 \%(\mathrm{v} / \mathrm{v})$ acetonitrile. They were diluted with $3.5 \%(\mathrm{v} / \mathrm{v})$ acetonitrile/0.1 \% (v/v) TFA and $1.25 \mathrm{pmol}$ of each peptide were subjected to nanoLC. 


\subsubsection{Absolute quantification by LC-offline MALDI-ToF/ToF-MS (peak area)}

In this part, different mass spectrometry techniques have been applied to obtain signal intensities for endogenous and standard peptides of the hPrp19/CDC5L complex proteins. The use of MALDI mass spectrometry features several advantages. First, the generation of singly charged ions facilitates data analysis and the peak area of the peptides can be easily obtained. Second, the coupling of nanoLC and MALDI-ToF/ToF-MS allows reinvestigation of the samples, i.e. low abundance precursors that are used for quantification can be detected. This means that the sample separated by nanoLC and spotted on a MALDI target can be manually reinvestigated if precursors have been missed during the analysis.

For absolute quantification, various amounts of hydrolyzed hPrp19/CDC5L complex were supplemented with equal amounts of all standard peptides (Table 4.3) and vice versa. Peak area ratios of endogenous and standard peptides were obtained from individual MS spectra (for an example spectrum see Figure 2.3 in the Introduction). Protein ratios were calculated from average peptide ratios of three technical replicates. Protein stoichiometries within the complex were determined by comparing protein ratios. They are displayed by the ratio of hPrp19 to each of the single proteins of the complex (Table 4.4).

For both hydrolysis conditions (i.e. urea and acetonitrile) the ratios indicate that hPrp19 is present in a higher stoichiometry than the other proteins. However, the values obtained for the two hydrolysis protocols are not consistent. Hydrolysis in the presence of urea yielded a stoichiometry of 2.5 for hPrp19 relative to CDC5L, PRL1 and CTNNBL1, and of 5.5 relative to SPF27. Hydrolysis in acetonitrile yielded a stoichiometry of 2:1 for hPrp19 relative to CDC5L and CTNNBL1, and of approximately 4:1 relative to PRL1 and SPF27 (Table 4.4).

Table 4.4: Relative protein stoichiometries within the hPrp19/CDC5L complex determined by LC-offline MALDI-ToF/ToF-MS. Average peptide ratios of three replicates were used to calculate protein ratios. Protein stoichiometries are displayed by the ratio of hPrp19 to the relavant protein showing the stoichiometry of hPrp19 within the hPrp19/CDC5L complex. Values in parantheses are not included in the statistics (apparent outliers).

\begin{tabular}{|c|c|c|c|c|c|c|}
\hline $\begin{array}{l}\text { Protein complex [ng] } \\
\text { standard peptides [fmol] }\end{array}$ & $\begin{array}{c}70 \\
100 \\
\end{array}$ & $\begin{array}{c}35 \\
100\end{array}$ & $\begin{array}{l}17.5 \\
100\end{array}$ & $\begin{array}{l}70 \\
50\end{array}$ & $\begin{array}{l}70 \\
25\end{array}$ & Average \\
\hline \multicolumn{7}{|c|}{$8 \mathrm{M} / 2 \mathrm{M}$ urea } \\
\hline $\begin{array}{l}\text { Protein stoichiometry } \\
\text { hPrp19/CDC5 } \\
\text { hPrp19/SPF27 } \\
\text { hPrp19/PRL1 } \\
\text { hPrp19/CTNNBL1 }\end{array}$ & $\begin{array}{l}2.48 \\
2.44 \\
4.35 \\
2.30 \\
\end{array}$ & $\begin{array}{l}2.24 \\
2.54 \\
4.76 \\
1.76\end{array}$ & $\begin{array}{l}2.38 \\
3.26 \\
6.00 \\
2.01\end{array}$ & $\begin{array}{l}2.86 \\
1.87 \\
6.75 \\
2.80\end{array}$ & $\begin{array}{c}2.74 \\
(0.67) \\
5.91 \\
2.58\end{array}$ & $\begin{array}{l}2.54 \pm 0.256 \\
2.53 \pm 0.571 \\
5.55 \pm 0.979 \\
2.29 \pm 0.419\end{array}$ \\
\hline \multicolumn{7}{|c|}{$80 \%(v / v)$ acetonitrile } \\
\hline $\begin{array}{l}\text { Protein stoichiometry } \\
\text { hPrp19/CDC5 } \\
\text { hPrp19/SPF27 } \\
\text { hPrp19/PRL1 } \\
\text { hPrp19/CTNNBL1 }\end{array}$ & $\begin{array}{l}1.76 \\
5.36 \\
3.79 \\
1.92 \\
\end{array}$ & $\begin{array}{l}1.64 \\
3.08 \\
4.28 \\
1.19 \\
\end{array}$ & $\begin{array}{l}1.86 \\
4.79 \\
4.79 \\
2.01 \\
\end{array}$ & $\begin{array}{c}2.01 \\
(1.96) \\
5.03 \\
2.69 \\
\end{array}$ & $\begin{array}{c}1.85 \\
(2.19) \\
6.15 \\
2.34 \\
\end{array}$ & $\begin{array}{l}1.82 \pm 0.136 \\
4.41 \pm 1.187 \\
4.81 \pm 0.890 \\
2.17 \pm 0.559\end{array}$ \\
\hline
\end{tabular}


One reason for this clear discrepancy might have been the frequently observed peak overlap in LC-offline MALDI-MS. In case of the selected peptide sequence derived from the protein CTNNBL1, peak overlap of the corresponding standard peptide was observed in all spectra. Since only one peptide for this protein is available for quantification (see Table 4.3), no further reference value was present and the protein could thus not be quantified reliably by this method. Figure 4.3 shows MALDI MS spectra of peptide FVDILGLR (generated from CTNNBL1) in three consecutive spots during LC-offline MALDI-ToF/ToF-MS (Figure 4.5 AC). Peak overlap was observed in the spot with highest intensity of the peptides. The calculated peptide ratio within this particular spot differs from the calculated peptide ratio (Figure $4.5 \mathrm{D}$ ) within the adjacent spots presumably due to peak overlap of the standard peptide.

A

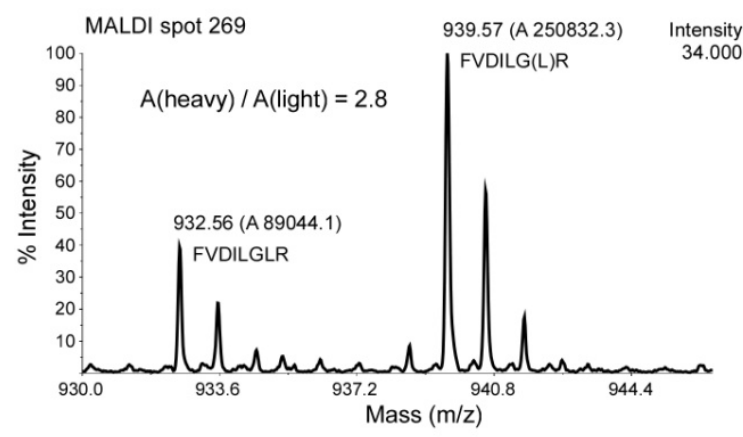

C

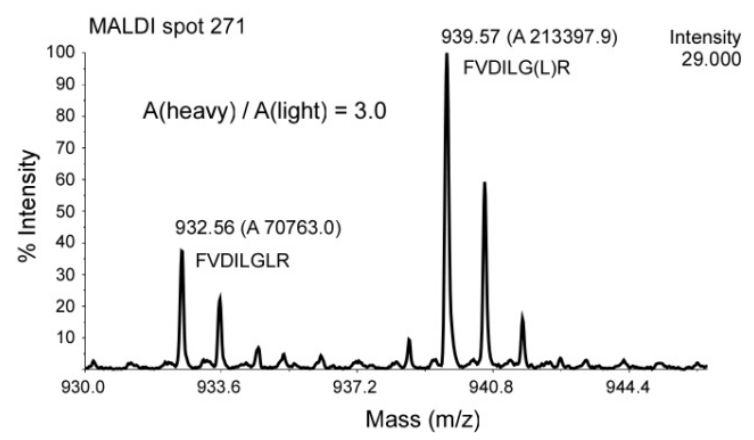

B

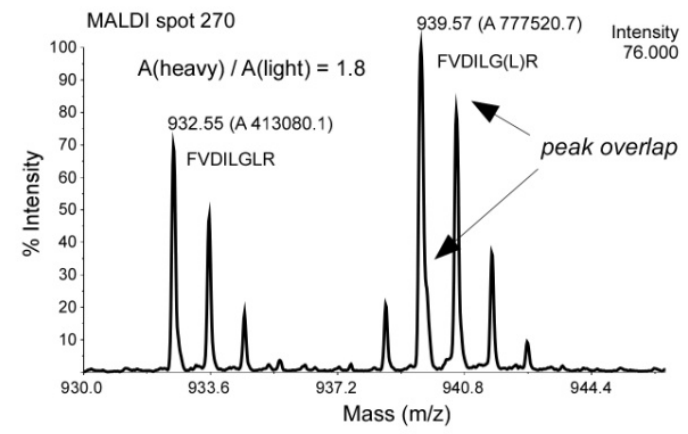

$\begin{array}{cc}\mathbf{m} / \mathbf{z} & \text { Intensity } \\ 932.56 & 1.000000000 \\ 933.56 & 0.548961681 \\ 934.56 & 0.169892478 \\ 935.56 & 0.038131043 \\ 936.56 & 0.006846962 \\ 937.56 & 0.001034910 \\ 938.56 & 0.000134187 \\ 939.56 & 0.000014219 \\ 940.56 & 0.000000468 \\ 941.56 & 0.000000008\end{array}$

Figure 4.5: Peak overlap is frequently observed in MALDI mass spectrometry. Upon closer inspection of the MALDI MS spectra for FVDILGLR and the corresponding standard peptide, peak overlap was observed in almost all analyses. (A-C) show MS spectra of FVDILGLR in three consecutive spots (269-271). (A) FVDILGLR and the corresponding standard peptide first appeared in spot 269. The isotopic patterns of both peptides are consistent with the calculated isotopic pattern on FVDILGLR (D). The calculated ration of the endogenous and the standard peptide is 2.8. (B) The peptides show highest intensity in spot 270 . The isotopic pattern of the standard peptide is different from the endogenous and the calculated isotopic patterns. In addition, the monoisotopic peak shows a shoulder. These findings lead to the conclusion that two peaks overlap. The calculated peptide ratio is 1.8 . (C) In the subsequent spot (271) the peptides show lower intensity but again show the theoretical isotopic pattern and a ratio of 3.0 comparable with spot 269. (D) Calculated intensities of the isotopic pattern of FVDILGLR.

In summary, quantification by LC-offline MALDI-TOF/TOF-MS does not show unambiguously interpretable protein stoichiometries within the hPrp19/CDC5L complex although the data do 
suggest that hPrp19 is present in more than one copy. It is surprising that although similar values for the sequence coverage have been observed for both hydrolysis protocols (i.e., urea and acetonitrile) the determined protein stoichiometries differ significantly. These data therefore indicate that the hydrolysis conditions are a critical parameter in the absolute quantification using standard peptides.

\subsubsection{Absolute quantification by LC-ESI-MS (extracted ion chromatograms)}

Since MALDI mass spectrometry did not reveal clear protein stoichiometries within the hPrp19/CDC5L complex, LC-ESI-MS was used as a complementary method. In this study, extracted ion chromatograms (XICs) were used to read out signal intensities for quantification. Various amounts of hydrolyzed hPrp19/CDC5L complex were supplemented with equal amounts of all standard peptides (Table 4.3) and vice versa. XICs were generated for endogenous and corresponding standard peptides for the single proteins and resulting peak areas were used to calculate peptide ratios (for an example see Figure 2.7 in the Introduction). Protein ratios were calculated from average peptide ratios from three replicates.

Protein ratios are displayed as the ratio of hPrp19 to other proteins of the complex for both hydrolysis conditions (i.e. hydrolysis in the presence of urea and acetonitrile, respectively). Hydrolysis in the presence of urea yielded a stoichiometry of approximately 3:1 for hPrp19 relative to CDC5L, SPF27, and CTNNBL1 and of 9:1 relative to PRL1. Hydrolysis in the presence of acetonitrile yielded a stoichiometry of $2: 1$ for hPrp19 relative to CDC5L, 4:1 relative to CTNNBL1, and approximately 5:1 relative to PRL1 and SPF27 (Table 4.5).

Table 4.5: Relative protein stoichiometries within the hPrp19/CDC5L complex determined by LC-ESI-MS. Average peptide ratios of three replicates were used to calculate protein ratios. Protein stoichiometries are displayed by the ratio of hPrp19 to the relavant protein showing the stoichiometry of $\mathrm{hPrp} 19$ within the hPrp19/CDC5L complex. Values in parantheses are not included in the statistics (apparent outliers).

\begin{tabular}{|c|c|c|c|c|c|c|}
\hline $\begin{array}{l}\text { Protein complex [ng] } \\
\text { standard peptides [fmol] }\end{array}$ & $\begin{array}{c}70 \\
100\end{array}$ & $\begin{array}{c}35 \\
100\end{array}$ & $\begin{array}{l}17.5 \\
100\end{array}$ & $\begin{array}{l}70 \\
50\end{array}$ & $\begin{array}{l}70 \\
25 \\
\end{array}$ & Average \\
\hline \multicolumn{7}{|c|}{$8 \mathrm{M} / 2 \mathrm{M}$ urea } \\
\hline Protein stoichiometry & & & & & & \\
\hline hPrp19/CDC5 & 3.87 & 3.61 & 3.62 & 3.39 & 3.42 & $3.58 \pm 0.193$ \\
\hline hPrp19/SPF27 & 3.66 & 2.66 & 2.80 & $(1.70)$ & $(1.04)$ & $3.04 \pm 0.541$ \\
\hline hPrp19/PRL1 & 7.59 & 8.11 & 10.03 & 9.33 & 9.79 & $8.97 \pm 1.069$ \\
\hline hPrp19/CTNNBL1 & $(6.52)$ & 3.8 & 3.57 & 2.99 & 3.06 & $3.36 \pm 0.393$ \\
\hline \multicolumn{7}{|c|}{$80 \%(v / v)$ acetonitrile } \\
\hline Protein stoichiometry & & & & & & \\
\hline hPrp19/CDC5 & 1.87 & 2.37 & 2.38 & 2.31 & 2.28 & $2.24 \pm 0.212$ \\
\hline hPrp19/SPF27 & 4.77 & 4.76 & 4.91 & $(8.67)$ & $(7.48)$ & $4.81 \pm 0.084$ \\
\hline hPrp19/PRL1 & 3.42 & 6.56 & 7.53 & 4.51 & 5.77 & $5.56 \pm 1.628$ \\
\hline hPrp19/CTNNBL1 & 3.05 & 4.44 & 4.71 & 4.12 & 3.51 & $3.97 \pm 0.680$ \\
\hline
\end{tabular}


The values yielded by the two different hydrolysis protocols are again inconsistent. In both hydrolysis procedures, the values for PRL1 protein differ significantly among the different analyses. PRL1 shows higher values for both hydrolysis protocols compared with the other proteins; these might be due to incomplete digestion or to interference with co-eluting peptides. Indeed, selected peptides for PRL1 co-eluted with other peptides in the analysis and thus affected the quantification.

As for the MALDI analysis, no clear protein stoichiometry could be determined by LC-ESI-MS using XIC signal intensities for quantification. A method which specifically identifies and quantifies endogenous and standard peptides is therefore required. One such method is multiple reaction monitoring (MRM) in which two quadrupoles of a triple quadrupole mass analyzer are operated as mass filters to detect the specific transition of a precursor ion to a specific fragment ion.

\subsubsection{Absolute quantification by Multiple Reaction Monitoring (MRM)}

The use of a triple quadrupole mass analyzer allows the detection of the specific transition from a given precursor to a user-defined fragment ion (single reaction monitoring). The precursor mass is selected in quadrupole Q1, fragmentation takes place in q2, and the fragment ion is detected in Q3. Multiple reaction monitoring (MRM) allows the detection of multiple fragment ions specific for one precursor. Signals from MRM transitions of endogenous and standard peptides are well suited for absolute quantification of the peptide/ protein under investigation. In previous studies, MRM with standard peptides has proven to be a suitable method for absolute quantification of proteins in a mixture (Abbatiello et al., 2008; Langenfeld et al., 2009). In LC-coupled MRM experiments, peak overlaps caused by co-eluting peptides can be neglected under defined conditions, namely by choosing several MRM transitions specific for a certain precursor. Operating the first and the third quadrupole as a mass filters guarantees that only the specific MRM transitions are monitored and coeluting peptides do not influence the quantitative signal.

For absolute quantification of the hPrp19/CDC5L complex, three MRM transitions for each selected peptide sequence were designed. In all cases, the doubly charged precursor was chosen as Q1 mass, and the three most intense y-type fragment ions with an $m / z$ above that of the precursor were chosen as Q3 masses. For the selected MRM transitions, the declustering potential (DP), entrance potential (EP), collision energy (CE), and collision cell exit potential (CXP) of the instrument were first optimized (for information about MRM transitions and optimized instrument parameters see Table A.3 in the Appendix) and then tested by analyzing the standard peptides and the endogenous peptides separately. For this 
purpose, $70 \mathrm{ng}$ of hydrolyzed hPrp19/CDC5L complex or $100 \mathrm{fmol}$ of standard peptides were separated by LC, and the MRM transitions were monitored using the optimized parameters. All MRM transitions were well separated and showed sufficient intensity for quantitative analysis (Figure 4.6). The analysis of the hydrolyzed hPrp19/ CDC5L complex showed no MRM transition for the heavy counterparts (Figure 4.6 A) and vice versa (Figure 4.6 B). We infer that the chosen MRM transitions are highly peptide-specific and can therefore be used for their investigation.

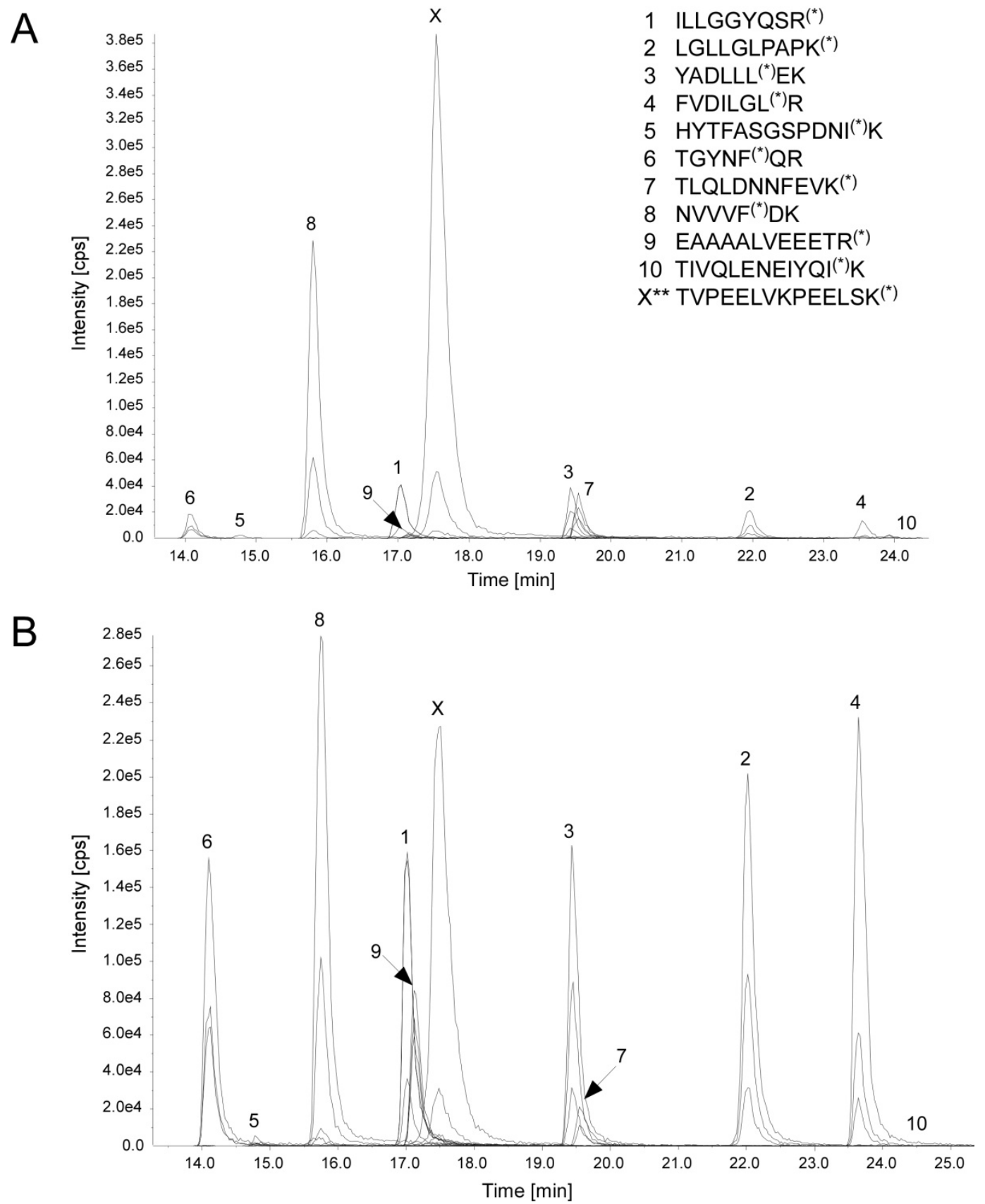

Figure 4.6: Specificity of the designed MRM transitions. Labeled standard and endogenous peptides were analyzed separately. The detected MRM transitions show sufficient intensity for quantitative analysis. (A) $70 \mathrm{ng}$ of hydrolyzed hPrp19/CDC5L complex were separated by LC and analyzed by MRM. Traces for MRM transitions of standard peptides are empty. (B) $100 \mathrm{fmol}$ of standard peptides were separated by LC and analyzed by MRM. Traces for MRM transitions of endogenous peptides are empty. ** TVPEELVKPEELSK was not used for absolute quantification. $\left(^{*}\right)$ labeled amino acid (standard peptides). 
As described for MALDI and ESI analyses, various amounts of hydrolyzed hPrp19/CDC5L complex were supplemented with equal amounts of all standard peptides and vice versa. In the different experiments, three MRM transitions were monitored for each standard and endogenous peptide. Figure 4.7 shows an example of the total of six MRM transitions for the coeluting standard and endogenous peptides (LGLLGLPAPK derived from CDC5L). Ratios between standard and endogenous peptides were obtained by integration of the peak areas of the corresponding transitions and the values were then used to calculate protein ratios from three technical replicates.

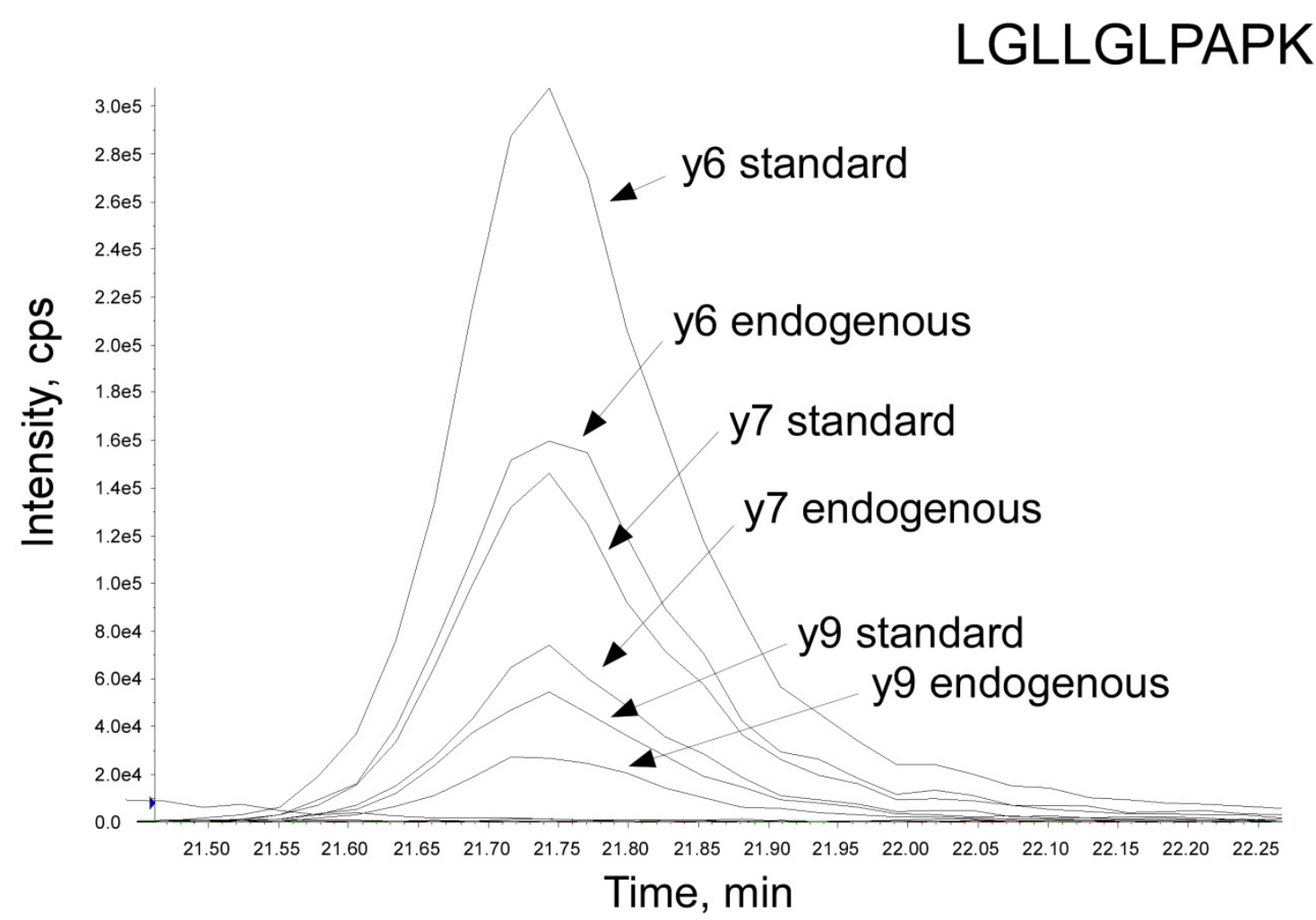

Figure 4.7: Example of the MRM transitions for an endogenous and the corresponding standard peptide (LGLLGLPAPK derived from CDC5L protein). The doubly charged precursor mass was selected as Q1 mass and the y6, y7, and y9 fragment ions were chosen as Q3 masses. Peptide ratios were calculated from integrated peak areas of the corresponding transitions.

The peptide ratios based on the three MRM transitions for the individual peptides are compared in bar Figure 4.8. Clearly, peptide ratios obtained for a given protein differed significantly when hydrolysis was performed in the presence of urea as compared with the corresponding ratios as obtained from hydrolysis in the presence of acetonitrile (hPrp19, SPF27, and PRL1, Figure 4.8 A). Peptide ratios obtained from hydrolysis in acetonitrile were more consistent for the same protein, i.e. peptide ratios obtained for peptides generated from the same protein show comparable values (Figure $4.8 \mathrm{~B}$ ). The standard deviations of the protein ratios derived after hydrolysis in the presence of acetonitrile were lower than the corresponding values after digestion in the presence of urea (Table 4.6). 


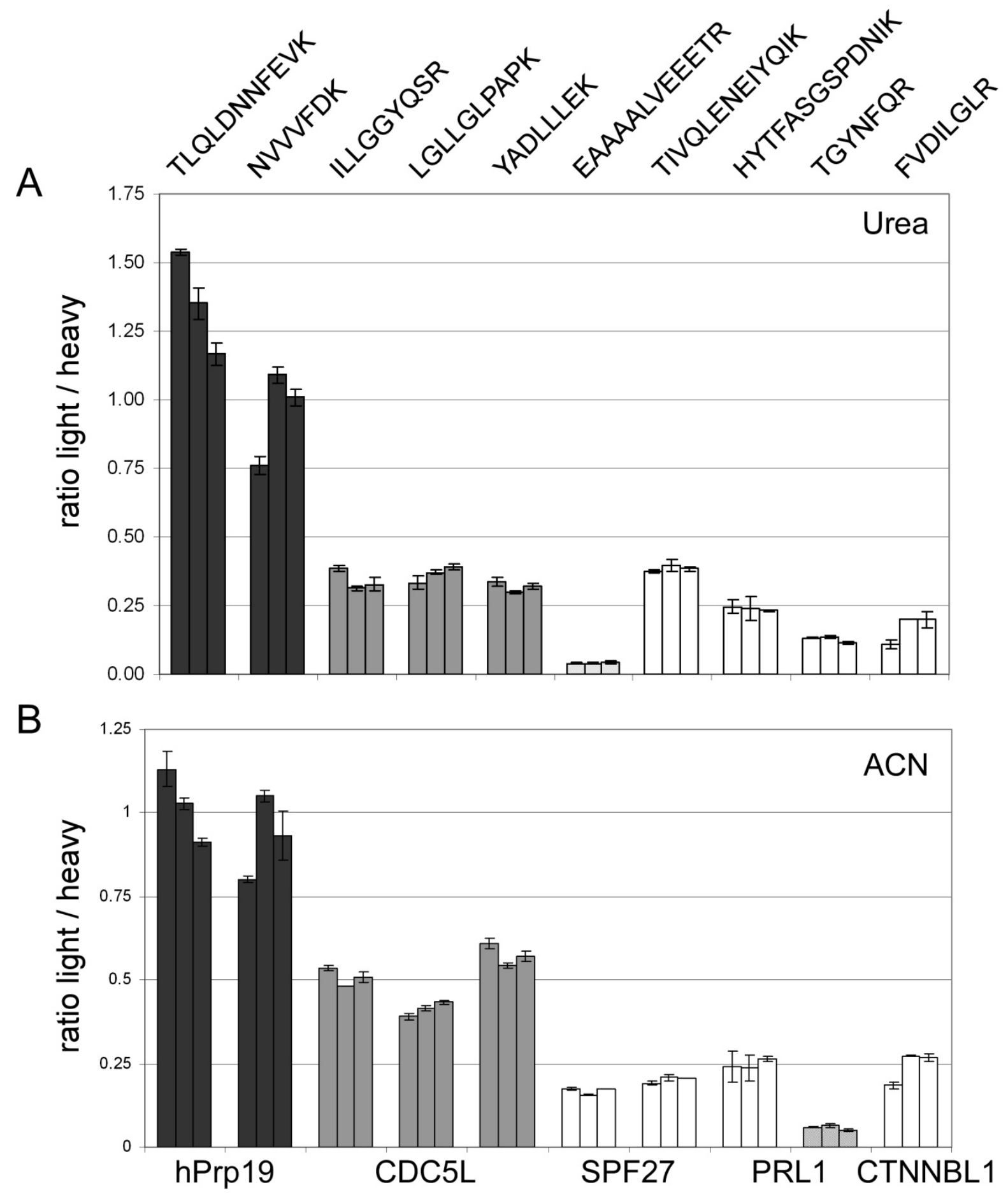

Figure 4.8: Peptide ratios obtained from MRM analysis of the hPrp19/CDC5L complex. Peptide ratios from three replicates of three different MRM transitions for each peptide are plotted in bar diagrams for hydrolysis in the presence of urea (A) or acetonitrile (B). (A) Peptide ratios obtained from hydrolysis in urea show no clear protein stoichiometry. (B) Peptide ratios obtained from hydrolysis in acetonitrile are more consistent than those obtained from hydrolysis in urea. 
Two peptides (EAAAALVEEETR derived from SPF27 after hydrolysis in the presence of urea, Figure 4.8 A; and TGYNFQR derived from PRL1 after hydrolysis in the presence of acetonitrile, Figure 4.8 B) consistently revealed a very low peptide ratio. The low value for this peptide is probably caused by low abundance of the endogenous peptide when compared with the isotopically labeled standard peptide suggesting that the endogenous peptide might be underrepresented. Indeed, both of these peptides were also hardly detectable in MALDI-TOF/TOF and ESI-LC-MS/MS experiments and could thus not be used for calculation of the protein ratio in the previous experiments. The low values are indeed consistent with the presence of a slightly longer form of the peptide EAAAALVEEETR (SPF27) containing a missed cleavage site after hydrolysis in the presence of urea as monitored in the initial analysis (see above). The adjacent protein sequence of this particular SPF27 peptide shows several tryptic C-terminal cleavage sites (EAAAALVEEETR/R/YR/) that might increase the possibility of missed cleavages in urea, but not in acetonitrile (compare peptide ratios for SPF27 in Figure $4.8 \mathrm{~A}$ and B). Conversely, the peptide derived from PRL1 (TGYNFQR), which shows a low value after hydrolysis using acetonitrile, does not have any additional adjacent tryptic sites. The nearest tryptic sites are 43 positions in the $\mathrm{N}$-terminal and 27 positions in the C-terminal direction. Thus, the selected peptide sequence is located in a protein region that contains only very few tryptic cleavage sites (Figure 4.9). A very long tryptic peptide containing a missed cleavage might have been generated in the presence of acetonitrile (consistently with the observation in early experiments that longer peptides with missed cleavages are generated preferentially when acetonitrile is used for digestion; see above, Figure 4.2), and its detection might be hampered in ESI and MALDI analyses. As the presence of missed cleavage site-containing peptides causes underrepresentation of the endogenous peptide to be quantified, both these peptides were excluded from the calculation of the protein stoichiometry of the hPrp19/CDC5L complex after hydrolysis in urea and acetonitrile, respectively. Since one of the peptides (TGYNFQR derived from PRL1) represents a proteotypic peptide (Table 4.3), the results for the missed cleavage sites of tryptic peptides in the presence of different denaturing agents highlight the need for thorough evaluation of experimental data before standard peptides for absolute quantification are selected. 


\section{PRL1 sequence}

1

MVEEVQKHSV HTLVFRSLKR THDMFVADNG KPVPLDEESH KRKMAIKLRN EYGPVLHMPT SKENLKEKGP 101

QNATDSYVHK QYPANQGQEV EYFVAGTHPY PPGPGVALTA DTKIQRMPSE SAAQSLAVAL PLQTKADANR 151

201

TAPSGSEYRH PGASDRPQPT AMNSIVMETG NTKNSALMAK KAPTMPKPQW HPPWKLYRVI SGHLGWVRCI 251

AVEPGNQWFV TGSADRTIKI WDLASGKLKL SLTGHISTVR GVIVSTRSPY LFSCGEDKQV KCWDLEYNKV 301

IRHYHGHLSA VYGLDLHPTI DVLVTCSRDS TARIWDVRTK ASVHTLSGHT NAVATVRCQA AEPQIITGSH 351 401

DTTIRLWDLV AGKTRVTLTN HKKSVRAVVL HPRHYTFASG SPDNIKQWKF PDGSFIQNLS GHNAIINTLT 451

VNSDGVLVSG ADNGTMHLWD WRTGYNFQRV HAAVQPGSLD SESGIFACAF DQSESRLLTA EADKTIKVYR 501

EDDTATEETH PVSWKPEIIK RKRF

Figure 4.9: PRL 1 sequence. The peptide TGYNFQR (underlined) is located in a protein region that contains no further tryptic cleavage sites. The nearest tryptic sites are 43 positions in N-terminal and 27 positions in Cterminal direction.

As described above, protein ratios were calculated from average peptide ratios of three technical replicates and three different MRM transitions for each peptide. The MRM experiments after hydrolysis of the hPrp19/CDC5L complex in the presence of urea revealed a stoichiometry of approximately $4: 1$ for hPrp19 relative to CDC5L and SPF27, 5:1 relative to PRL1, and 9:1 relative to CTNNBL1. Hydrolysis in the presence of acetonitrile and subsequent analysis by MRM resulted in a stoichiometry of $2: 1$ for $h P r p 19$ relative to CDC5L, 5:1 relative to SPF27, and 4:1 relative to PRL1 and CTNNBL1 (Table 4.6).

Table 4.6: Relative protein stoichiometries within the hPrp19/CDC5L complex as determined by MRM analyses. Peptide ratios were calculated from MRM signals of endogenous and standard peptides. Average peptide ratios from replicates from three different MRM transitions for each peptide were used to calculate protein ratios. Protein stoichiometries are displayed by the ratio of hPrp19 to the relevant protein showing the stoichiometry of hPrp19 within the hPrp19/CDC5L complex. Values in parentheses were omitted for calculation of the average values. For SPF27 no ratio could be determined for dilution of the standard peptides.

\begin{tabular}{|c|c|c|c|c|c|c|c|}
\hline $\begin{array}{l}\text { Protein complex [ng] } \\
\text { standard peptides [fmol] }\end{array}$ & $\begin{array}{c}70 \\
100 \\
\end{array}$ & $\begin{array}{c}70 \\
100 \\
\end{array}$ & $\begin{array}{c}35 \\
100 \\
\end{array}$ & $\begin{array}{l}17.5 \\
100 \\
\end{array}$ & $\begin{array}{l}70 \\
50\end{array}$ & $\begin{array}{l}70 \\
25 \\
\end{array}$ & Average \\
\hline \multicolumn{8}{|c|}{ 8M/2M urea } \\
\hline Protein stoichiometry & & & & & & & \\
\hline hPrp19/CDC5 & 3.54 & 3.37 & 3.46 & 3.73 & 3.09 & 3.70 & $3.48 \pm 0.236$ \\
\hline hPrp19/SPF27 & 3.32 & 2.99 & 5.44 & $(11.92)$ & l & / & $3.92 \pm 1.330$ \\
\hline hPrp19/PRL1 & 5.35 & 4.80 & 5.21 & 6.62 & 4.37 & 4.96 & $5.22 \pm 0.767$ \\
\hline hPrp19/CTNNBL1 & 10.00 & 6.78 & 13.03 & 9.03 & 6.39 & $(3.94)$ & $9.05 \pm 2.691$ \\
\hline \multicolumn{8}{|c|}{$80 \%(v / v)$ acetonitrile } \\
\hline Protein stoichiometry & & & & & & & \\
\hline hPrp19/CDC5 & 1.97 & 1.96 & 2.01 & 1.95 & 1.87 & 1.82 & $1.93 \pm 0.071$ \\
\hline hPrp19/SPF27 & 4.69 & 4.83 & 5.58 & 6.11 & I & I & $5.30 \pm 0.665$ \\
\hline hPrp19/PRL1 & 4.31 & 3.94 & 3.77 & 3.37 & 3.82 & 3.72 & $3.82 \pm 0.306$ \\
\hline hPrp19/CTNNBL1 & 4.24 & 4.03 & 3.74 & 3.59 & $(1.97)$ & $(2.33)$ & $3.90 \pm 0.106$ \\
\hline
\end{tabular}




\subsubsection{Comparison of results for absolute quantification in determining the} Stoichiometry of the hPrp19/CDC5L complex

A comparison of the different MS analysis approaches MALDI-ToF/ToF, LC-ESI-MS, and MRM (Table 4.7 and Figure 4.10) shows that the MRM method yielded the most consistent and reproducible results when judging the experimentally determined peptide and resulting protein ratios from the standard deviation. The large variability in MALDI-ToF/ToF-MS (peak area) or ESI-MS (XIC) experiments could be explained by co-eluting and thus contaminating peptides that cause peak overlap in the chromatograms and spectra as described above.

Although MRM experiments produce consistent values for the single proteins (as a result of the highly specific monitoring of the investigated peptides), the protein ratios obtained for hPrp19 vs. CDC5L still vary with the hydrolysis conditions used (Figure $4.10 \mathrm{~A}$ and $\mathrm{B}$ ). $\mathrm{A}$ stoichiometry of 1:1:1:2:4 (CTNNBL1/PRL1/SPF27/CDC5L/hPrp19) is calculated for these proteins within the hPrp19/CDC5L complex after hydrolysis in the presence of acetonitrile (Figure $4.10 \mathrm{~B}$ and Figure $4.8 \mathrm{~B}$ ) whereas the values obtained for the complex after hydrolysis in the presence of urea do not allow a clear determination of the stoichiometry (Figure 4.10 A and Figure 4.8 A). As no peptide of AD-002 could be selected for quantitative analysis (see above) AD-002 could not be quantified in this study. Furthermore, Hsp70, which was detected during proteomic analysis of the hPrp19/CDC5L complex (Figure 4.1), could not be quantified because the analysis revealed that Hsp70 is underrepresented in the protein complex analyzed under optimized conditions using standard peptides and LC-ESIMS and XICs (Table A.4 in the Appendix).

Table 4.7: Summary of the protein stoichiometries obtained from different MS techniques. Peptide ratios were obtained from peak areas, XICs and MRM transitions, respectively (Tables 4.3, 4.4, and 4.5). Protein ratios from different experiments were averaged and listed as the ratio of hPrp19 to the relevant protein showing the stoichiometry of hPrp19 within the hPrp19/CDC5L complex.

\begin{tabular}{|l|cc|cc|cc|}
\hline & \multicolumn{2}{|c|}{$\begin{array}{c}\text { LC-offline MALDI- } \\
\text { ToF/ToF-MS } \\
\text { (peak area) }\end{array}$} & \multicolumn{2}{|c|}{$\begin{array}{c}\text { LC-online ESI-MS/MS } \\
\text { (XIC) }\end{array}$} & \multicolumn{2}{c|}{$\begin{array}{c}\text { LC-online ESI-MS/MS } \\
\text { (MRM) }\end{array}$} \\
\hline Hydrolysis & \multicolumn{2}{|c|}{ urea } & ACN & urea & ACN & \multicolumn{2}{c|}{ urea } & ACN \\
\hline Protein stoichiometry & \multicolumn{2}{|c|}{} & & & & \\
hPrp19/CDC5 & $2.54 \pm 0.26$ & $1.82 \pm 0.14$ & $3.58 \pm 0.19$ & $2.24 \pm 0.21$ & $3.48 \pm 0.24$ & $1.93 \pm 0.07$ \\
hPrp19/SPF27 & $2.53 \pm 0.57$ & $4.41 \pm 1.19$ & $3.04 \pm 0.54$ & $4.81 \pm 0.08$ & $3.92 \pm 1.33$ & $5.30 \pm 0.67$ \\
hPrp19/PRL1 & $5.55 \pm 0.98$ & $4.81 \pm 0.89$ & $8.97 \pm 1.07$ & $5.56 \pm 1.63$ & $5.22 \pm 0.77$ & $3.82 \pm 0.31$ \\
hPrp19/CTNNBL1 & $2.29 \pm 0.42$ & $2.17 \pm 0.56$ & $3.36 \pm 0.39$ & $3.97 \pm 0.68$ & $9.05 \pm 2.69$ & $3.90 \pm 0.11$ \\
\hline
\end{tabular}




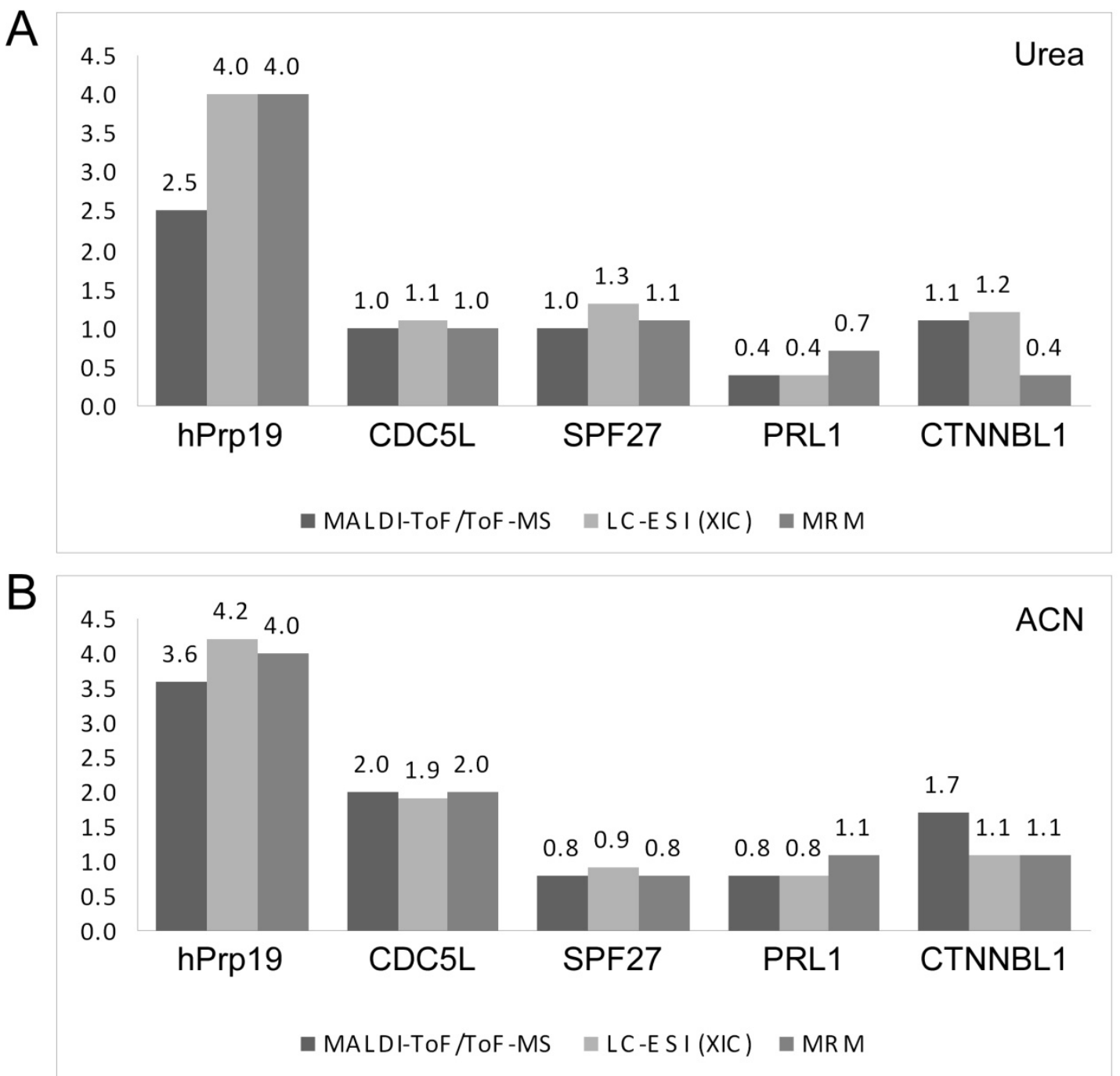

Figure 4.10: Protein stoichiometry within the hPrp19/CDC5L complex. The values are normalized to the number of copies of hPrp19 obtained by MRM analysis. For each protein, the stoichiometry is given for all three MS techniques tested. (A) Protein stoichiometry within the hPrp19/CDC5L complex obtained by hydrolysis in urea. The values obtained do not allow unambiguous determination of the stoichiometries. (B) Protein stoichiometry within the hPrp19/CDC5L complex obtained by hydrolysis in acetonitrile. A stoichiometry of 4:2:1:1:1 (hPrp19/CDC5L/SPF27/PRL1/CTNNBL1) is calculated for these proteins in the hPrp19/CDC5L complex.

In previous studies, the stoichiometry of Prp19p in the yeast nineteen complex (NTC, the yeast homologue of the hPrp19/CDC5L complex) was analyzed by electron microscopy and density gradient centrifugation (Ohi et al., 2005). Ohi et al., 2005 found that Prp19p forms a tetramer and that oligomerization is essential for its function in vivo. In addition, the authors also analyzed the protein-protein interactions of Prp19p and Cef1p (the yeast homologue of CDC5L) by the yeast two-hybrid assay. They found interactions between Prp19p and Cef1p and self-association of Prp19 but no self-interaction of CDC5L. Consistent with a similar study by Grillari et al., 2005, the possibility of dimerization of CDC5L was recently discussed by (Graub et al., 2008). Using cross-linking with glutaraldehyde and subsequent immunoblotting these authors demonstrated the presence of CDC5L homodimers. By 
mutation of CDC5L phosphorylation sites, they found that homodimerization is independent of phosphorylation, suggesting that CDC5L might be present as a homodimer. Our results are consistent with extensive biochemical studies characterizing the molecular architecture of the Prp19/CDC5L (Grote et al., 2010). In this study, four copies of Prp19 and one copy of the proteins PRL1, AD-002, and SPF27 were found by $\left[{ }^{14} \mathrm{C}\right]$-iodoacetamide labeling and SyproRuby staining of the denatured proteins of the complex and subsequent quantification of radioactivity and fluorescence, respectively. However, as observed here in the presence of urea, CDC5L was only present in one copy.

\subsection{Relative quantification by iTRAQ-labeling of in-gel digested proteins}

A robust mass spectrometry-based quantification of spliceosomal proteins for characterization of the spliceosome's protein composition at different intermediate states is required. Various methods for MS-based relative quantification exist (for an overview see Table 2.1 in the Introduction). ITRAQ reagents are chemical labels that specifically label amino termini and lysine side chains of peptides. ITRAQ quantification has some clear advantages: (i) iTRAQ reagents are multiplexing (i.e. up to four or eight samples can be compared in one experiment). (ii) As the ITRAQ reagents are amine specific, the peptides' $N$ termini and lysine side chains are labeled thus providing many quantification data points per protein. (iii) Differentially labeled peptides are isobaric and the intensity in MS is thus enhanced. (iv) The different mass tags are completely cleaved during fragmentation leading to an enhanced fragment ion intensity. (v) iTRAQ-labeling offers the opportunity to create an internal standard by mixing aliquots of all samples to be compared, allowing comparison of even more samples than four (four-plex iTRAQ) or eight (eight-plex iTRAQ) samples.

\subsubsection{Optimization of iTRAQ-labeling of in-gel digested proteins}

The ABSciex ITRAQ labeling protocol comprises in-solution hydrolysis of the proteins, subsequent labeling of the peptides with iTRAQ reagents, and final removal of excess reagent by strong cation exchange chromatography (SCX). Here, we optimized ITRAQ labeling for in-gel digested proteins. This alteration has the major advantage that sample complexity is reduced before hydrolysis and even small quantities can be quantified as sample loss during SCX is avoided. For this purpose, the buffers used during in-gel hydrolysis of the proteins and the amount of iTRAQ reagents have been adjusted to the ingel hydrolysis protocol. An additional reaction step to quench the excess of iTRAQ reagents by addition of glycine was included. Furthermore, an internal standard was prepared by 
mixing equal amounts of all samples to be compared and labeling with one of the ITRAQ reagents. This allows controlling of the labeling reaction because the reporter ion intensity of the internal standard is related to the intensities of the reporter ions of the differently labeled samples. The optimized workflow thus comprises (i) the separation of the samples to be compared by gel electrophoresis, (ii) cutting entire gel lanes into gel slices of equal size, (iii) in-gel digestion of the proteins and extraction of the peptides, (iv) preparation of an internal standard from samples to be compared (i.e. from peptides extracted from gel slices of the same molecular weight region within the different samples), (v) iTRAQ-labeling of the internal standard and the samples with the different ITRAQ reagents and subsequent quenching of iTRAQ reagent excess, (vi) pooling of the samples and their respective internal standard, (vii) LC-MS/MS analysis, and (viii) identification (database search) and quantification of the peptides and finally the proteins (Figure 4.11).

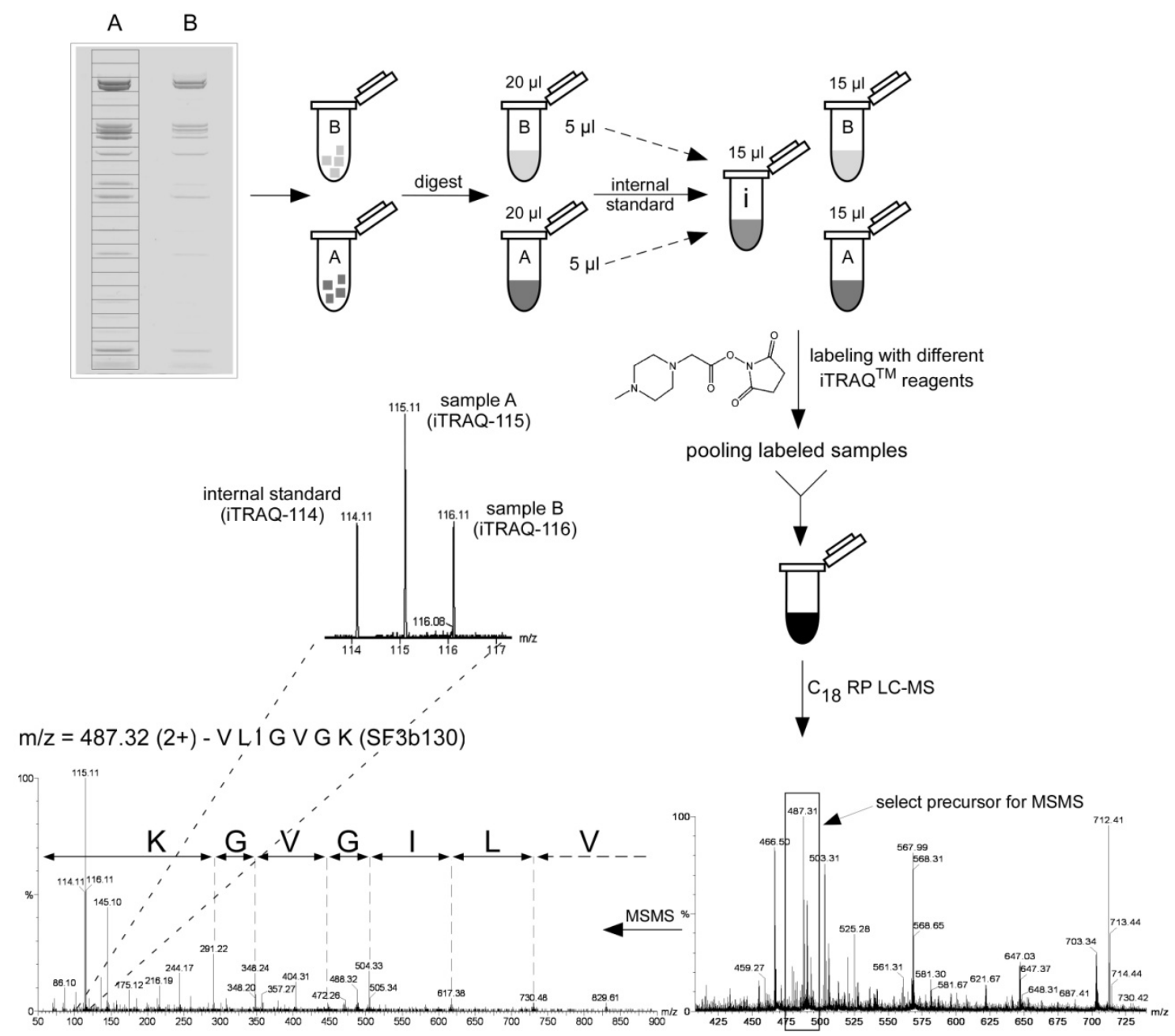

Figure 4.11: Workflow for iTRAQ-labeling of in-gel digested proteins. Entire gel lanes of samples to be analyzed and quantified are cut into gel slices of equal size and gel slices are manually cut into smaller pieces. Proteins are digested with trypsin within the gel and generated peptides are extracted. Extracted peptides are redissovled in $20 \mu \mathrm{l}$ TEAB and an internal standard is prepared by pooling $5 \mu \mathrm{l}$ of each sample. The internal standard and the samples to be compared are labeled with different iTRAQ reagents. After pooling, the samples are analyzed by LC-MS/MS and quantification is done by comparing the peak areas of individual reporter ions. 


\subsubsection{Relative quantification of different amounts of spliceosomal tri-snRNP proteins - a feasibility study}

To validate the established iTRAQ workflow, different amounts of spliceosomal tri-snRNP proteins were quantified using ITRAQ labeling. To this end, 5.0 and $2.5 \mu \mathrm{g}$ of purified human tri-snRNP were separated by gel electrophoresis (Figure 4.12) and entire gel lanes were cut into gel slices of equal size. In addition, an empty gel lane (blank) was cut and processed together with the other samples to show accuracy of the established iTRAQ protocol.

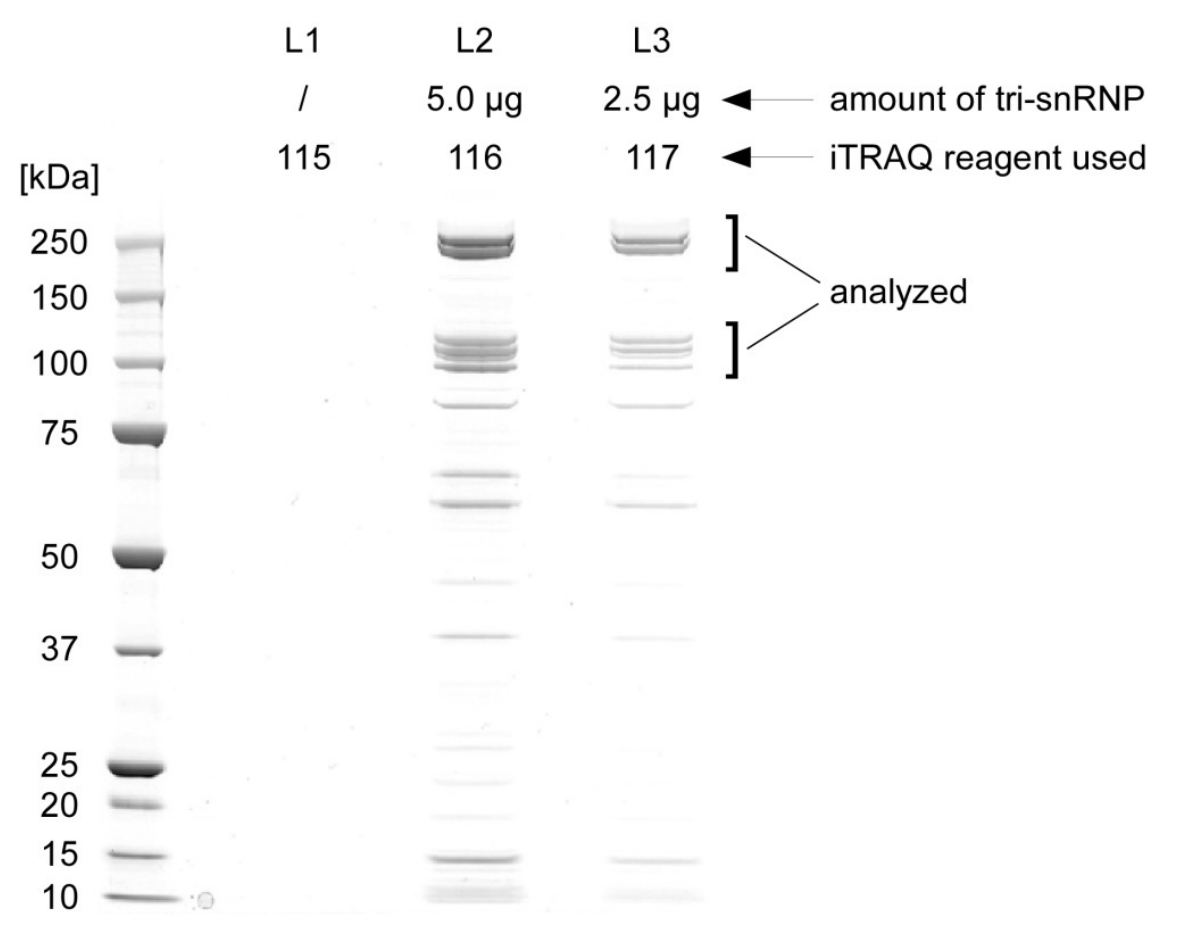

Figure 4.12: Separation of different amounts of spliceosomal tri-snRNP proteins by gel electrophoresis. $5.0 \mu \mathrm{g}$ and $2.5 \mu \mathrm{g}$ tri-snRNP were separated by gel electrophoresis and entire gel lanes were cut into slices of equal size. An empty gel lane (L1) was cut and processed with the other samples.

The proteins from two gel slices at different molecular weight (approximately $250 \mathrm{kDa}$ and $100 \mathrm{kDa})$, showing proper stained protein bands at both concentrations, were hydrolyzed with trypsin in-gel. An internal standard was prepared from the samples to be compared (i.e. L1, L2 (5.0 $\mu \mathrm{g}$ tri-snRNP) and L3 (2.5 $\mu \mathrm{g}$ tri-snRNP)) and the peptides of the different samples and the internal standard were labeled separately with ITRAQ reagents. The internal standard was labeled with ITRAQ reagent 114 and the different samples were labeled with iTRAQ reagents 115-117 (see also Figure 4.12). Excess iTRAQ reagents were quenched by adding glycine. Samples to be compared (i.e. L1-L3) and their corresponding internal standard were then pooled and subsequently analyzed by LC-MS/MS. Quantification was performed by comparing the peak areas of generated reporter ions after fragmentation. Figure 4.13 shows a typical MS/MS spectrum. 


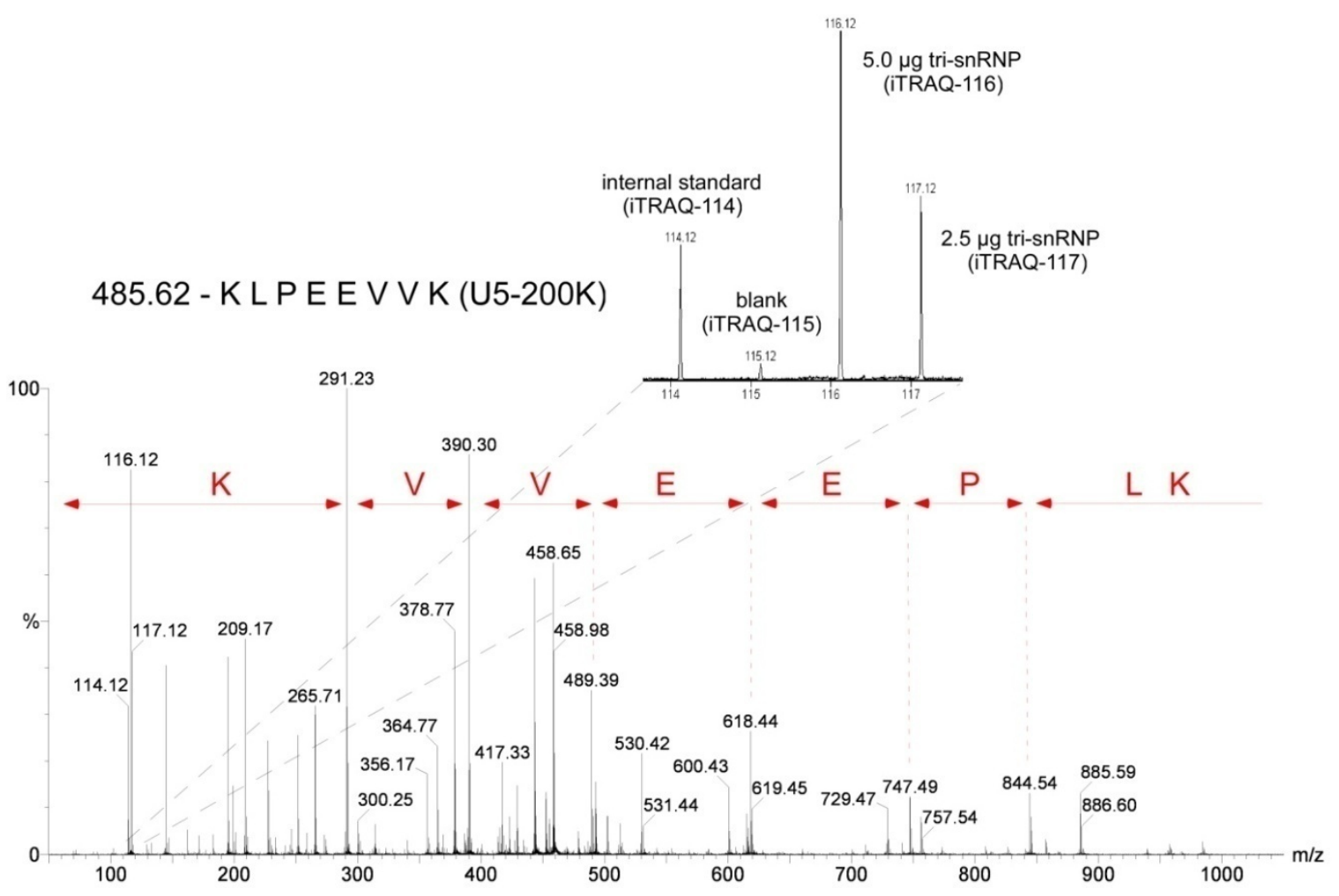

Figure 4.13: Example MS/MS spectrum of an iTRAQ labeled peptide. During MS/MS KLPEEVVK (U5-200K) was sequenced and the reporter ions were released. The reporter region $(\mathrm{m} / \mathrm{z} 114-117)$ is magnified and shows the different reporter ions that could be used for quantification.

The different tri-snRNP specific proteins within the two molecular weight regions were identified. For quantification, the protein ratios are calculated relatively to the internal standard, i.e. by dividing the peak area of reporter ions of the blank sample (iTRAQ-115), 5.0 $\mu \mathrm{g}$ (iTRAQ-116), and $2.5 \mu \mathrm{g}$ (iTRAQ-117) tri-snRNP, respectively, by the peak area of the internal standard (iTRAQ-114). In addition, the protein ratio comparing the different amounts of tri-snRNP was also calculated (iTRAQ-116/iTRAQ-117).

A protein ratio of approximately 2.5 for the different amounts of tri-snRNP (iTRAQ116/iTRAQ-117) was obtained for almost all proteins. The protein ratio of the blank sample (iTRAQ-115/iTRAQ-114) was in all cases approximately 0.1 or lower (Table 4.8). The obtained protein ratios are plotted in bar diagrams to visualize the relative protein amounts within the different gel lanes (Figure 4.14). As chemical labeling always risks being incomplete, the labeling efficiency (i.e. the percentage of labeled peptides) was calculated for all identified proteins. For this purpose, the obtained precursor masses (MS) and fragment ion masses (MS/MS) were searched against the database allowing for iTRAQ-labels as variable modifications. This yields labeled and non-labeled peptides and the labeling efficiency could be calculated from the number of identified iTRAQ-labeled peptides and the total number of identified peptides. In pilot experiments, the labeling efficiency was found to 


\section{4 | RESULTS}

be one of the major issues for reliable quantification, which was only achieved for labeling efficiencies $>90 \%$. For this proof of principle, a labeling efficiency of $92 \%$ or higher was obtained (Table 4.8).

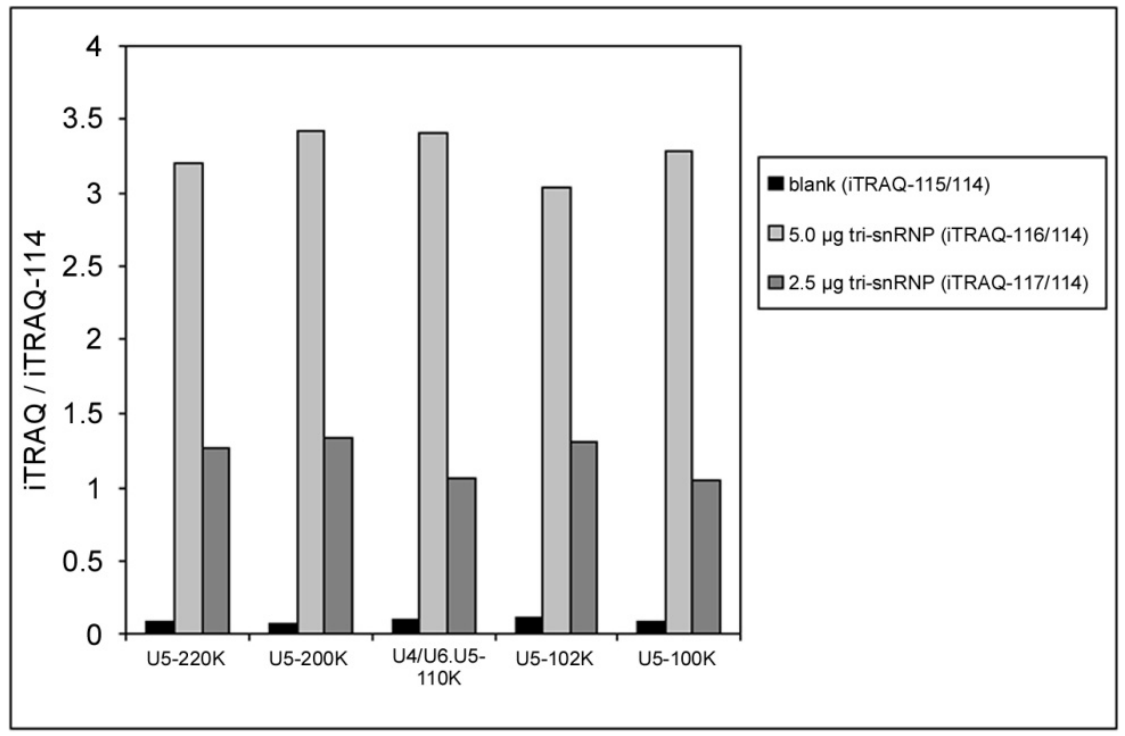

Figure 4.14: iTRAQ protein ratios for various identified tri-snRNP proteins. Ratios were calculated by dividing the peak area of reporter ions of the blank sample (iTRAQ-115), $5.0 \mu \mathrm{g}$ (iTRAQ-116), and $2.5 \mu \mathrm{g}$ (iTRAQ117) tri-snRNP, respectively, by the peak area of the internal standard (iTRAQ-114).

Table 4.8: Protein ratios for the relative quantification of different amounts of spliceosomal tri-snRNP proteins. iTRAQ ratios relative to the internal standard and one iTRAQ ratio comparing the different tri-snRNP amounts were calculated. The labeling efficiency is indicated in percent.

\begin{tabular}{|c|c|c|c|c|c|}
\hline \multirow{2}{*}{ Protein } & \multicolumn{4}{|c|}{ iTRAQ ratios } & \multirow{2}{*}{ labeling efficiency [\% } \\
\hline & $115 / 114$ & $116 / 114$ & $117 / 114$ & $116 / 117$ & \\
\hline U5-220K & 0.085 & 3.192 & 1.269 & 2.515 & 95.27 \\
\hline U5-200K & 0.073 & 3.413 & 1.341 & 2.545 & 94.15 \\
\hline U4/U6.U5-110K & 0.104 & 3.407 & 1.057 & 3.223 & 100.00 \\
\hline U5-102K & 0.106 & 3.036 & 1.300 & 2.335 & 97.27 \\
\hline U5-100K & 0.092 & 3.283 & 1.048 & 3.133 & 92.86 \\
\hline
\end{tabular}




\subsection{Relative quantification of spliceosomal B and C complexes - a comparative study}

The spliceosome is a highly dynamic protein-RNA machinery. During pre-mRNA splicing, it passes through different functional states that differ in their protein and RNA composition. In this study, the proteomes of the pre-catalytic and the catalytically active spliceosome (i.e. the spliceosomal $\mathrm{B}$ and $\mathrm{C}$ complexes) were analyzed by relative quantification to show differences in their protein abundances. To this end, affinity purified spliceosomal $\mathrm{B}$ and $\mathrm{C}$ complexes were labeled with ITRAQ reagents and analyzed by liquid chromatographycoupled mass spectrometry. In addition, B and C complexes prepared from metabolically labeled nuclear extracts were affinity purified and quantified by mass spectrometry (SILAC quantification). Protein ratios showing protein abundances within $\mathrm{B}$ and $\mathrm{C}$ complexes obtained by ITRAQ and SILAC quantification were then evaluated and compared. In addition, these results based on stable isotope labeling made an evaluation of semi-quantitative spectral count from proteomic analysis of the two spliceosomal complexes possible.

\subsubsection{Purification of spliceosomal $B$ and $C$ complexes}

Spliceosomal B and C complexes were purified from HeLa nuclear extract using MS2-tagged radioactively labeled PM5 pre-mRNA as described in previous studies (Bessonov et al., 2008; Deckert et al., 2006). Briefly, radioactively labeled PM5 pre-mRNA was pre-incubated with MS2-MBP fusion protein and spliceosomal complexes were allowed to assemble in nuclear extract in vitro. B complexes were assembled by kinetic control for 6 minutes, whereas $\mathrm{C}$ complexes were allowed to accumulate for $180 \mathrm{~min}$. Assembled complexes were separated by glycerol gradient centrifugation and B or $\mathrm{C}$ complexes were affinity purified from fractions that contained the corresponding complex. For proteomic analysis (spectral count) and iTRAQ quantification, equal amounts of affinity purified $B$ and $C$ complexes were separated by gel electrophoresis (Figure $4.15 \mathrm{~A}$ ). For SILAC quantification, B and C complexes were assembled in metabolically labeled light ( $\mathrm{C}$ complex) and heavy (B complex) nuclear extracts, respectively. Equal amounts of $B$ and $C$ complexes were then pooled and the proteins were separated by gel electrophoresis (Figure $4.15 \mathrm{~B}$ ). B and C complexes for ITRAQ analysis were purified and provided by $S$. Bessonov. Purification of $B$ and $C$ complexes for SILAC quantification was performed by J. Deckert. 
A

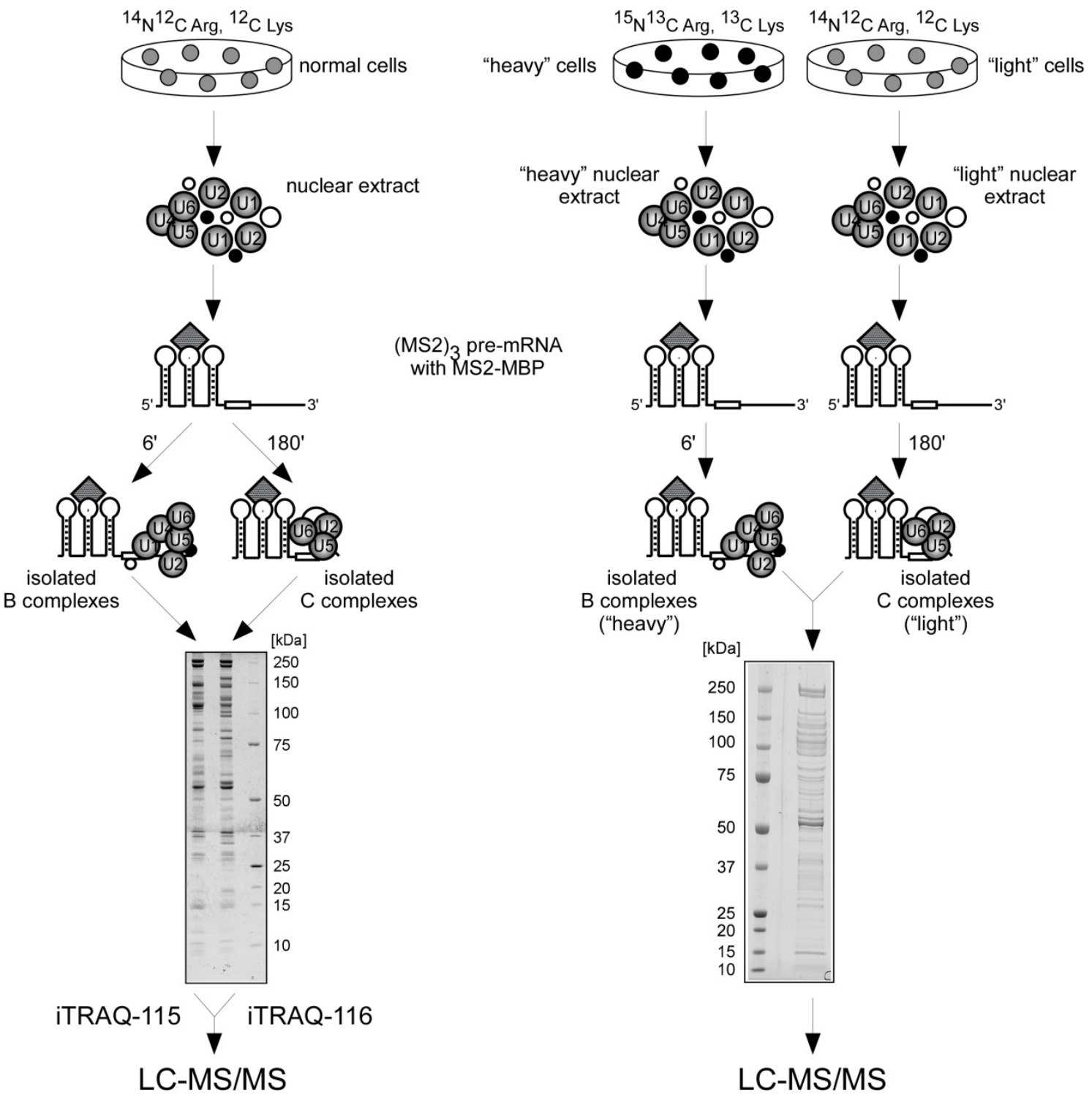

Figure 4.15: Purification of spliceosomal $B$ and $C$ complexes for ITRAQ and SILAC quantification. (A) B and $\mathrm{C}$ complexes were allowed to assemble on MS2-tagged pre-mRNA for 6 and 180 min, respectively. The complexes were isolated by gradient centrifugation and affinity purification and proteins were separated by gel electrophoresis. After in-gel digestion of the proteins, peptides generated from B complex were labeled with iTRAQ reagent 115 and peptides generated from $C$ complex proteins were labeled with iTRAQ reagent 116 . After pooling the samples were analyzed by LC-MS/MS. (B) B and C complexes were purified from light (C complex) and heavy (B complex) nuclear extract, respectively. Isolated complexes were pooled in equal amounts, the proteins were separated by gel electrophoresis and generated peptides were analyzed by LC-MS/MS. 


\subsubsection{Proteomic analysis of spliceosomal B and C complexes - spectral count}

The proteomes of spliceosomal B and C complexes have been analyzed in previous studies (Bessonov et al., 2008; Deckert et al., 2006). Approximately 150 proteins were identified within the two complexes. From these, several are snRNP specific whereas others are nonsnRNP specific proteins. Figure 4.16 shows the proteomes of $B$ and $C$ complexes separated by gel electrophoresis.

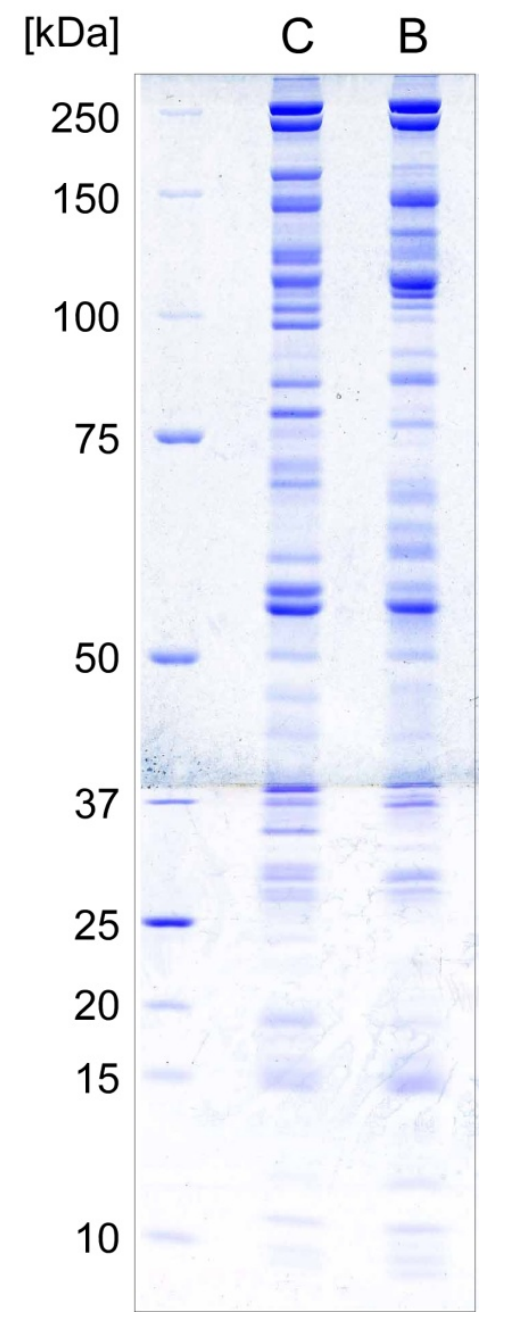

Figure 4.16: The proteomes of spliceosomal B and $C$ complexes. Equal amounts of $B$ and $C$ complexes were loaded onto the gel and the complexes' proteins were separated by gel electrophoresis.

A comparison of the protein composition of the precatalytic (B complex) versus the catalytic (C complex) spliceosome has been performed by Bessonov et al., 2008. For this purpose, the absolute number of peptides was used to determine protein abundances within B and C complexes. According to their abundance within the complexes, several snRNP specific and non-snRNP specific proteins have been assigned to be specific for $\mathrm{B}$ or $\mathrm{C}$ complexes.

The correlation between the relative protein abundance and the number of tandem MS spectra (spectral count), the number of identified peptides (peptide count), and the obtained sequence coverage has recently been analyzed (Liu et al., 2004). A linear correlation was found between the relative protein abundance and the number of acquired MS/MS spectra, but not between the protein amount and the number of identified peptides or the sequence coverage. The data set analyzed in Bessonov et al., 2008 was therefore reanalyzed using the software Scaffold 2 to achieve unweighted spectral count for relative comparison of spliceosomal B and C complexes from the proteomic analysis of the two complexes (Table 4.9). 
Table 4.9: Spectral count for proteomic analysis of spliceosomal B and C complexes. The number of acquired tandem MS spectra for two independent purifications of $B$ and $C$ complexes (B1, B2, C1, and C2, respectively) is given for all spliceosomal proteins identified in Bessonov et al., 2008. The average number of spectra from the two replicates and the resulting protein ratio showing the protein abundances within $\mathrm{B}$ and $\mathrm{C}$ complexes $(\mathrm{B} / \mathrm{C})$ were calculated. The proteins are grouped according to Bessonov et al., 2008. For proteins identified solely in B complexes no protein ratio could be calculated ("/"). For proteins that were only or predominantly identified in $\mathrm{C}$ complexes the calculated protein ratio is 0 or close to 0 .

\begin{tabular}{|c|c|c|c|c|c|c|c|c|c|}
\hline Protein & MW [kDa] & accession no. & B1 & B2 & B & C1 & $\mathrm{C} 2$ & C & B / C \\
\hline $\begin{array}{l}\text { U1 snRNP } \\
\text { U1-A } \\
\text { U1-C } \\
\text { U1-70K }\end{array}$ & $\begin{array}{l}31.3 \\
17.4 \\
51.6\end{array}$ & $\begin{array}{l}\text { gi|4759156 } \\
\text { gi|4507127 } \\
\text { gi|29568103 }\end{array}$ & $\begin{array}{l}6 \\
1 \\
4\end{array}$ & $\begin{array}{l}4 \\
0 \\
3\end{array}$ & $\begin{array}{c}5 \\
0.5 \\
3.5 \\
\end{array}$ & $\begin{array}{l}0 \\
0 \\
0\end{array}$ & $\begin{array}{l}0 \\
0 \\
0\end{array}$ & $\begin{array}{l}0 \\
0 \\
0\end{array}$ & $\begin{array}{l}1 \\
1 \\
1\end{array}$ \\
\hline $\begin{array}{l}\text { 17S U2 snRNP } \\
\text { U2A' } \\
\text { U2B" } \\
\text { SF3a120 } \\
\text { SF3a66 } \\
\text { SF3a60 } \\
\text { SF3b155 } \\
\text { SF3b145 } \\
\text { SF3b130 } \\
\text { SF3b49 } \\
\text { SF3b14a (p14) } \\
\text { SF3b14b } \\
\text { SF3b10 }\end{array}$ & $\begin{array}{c}28.4 \\
25.4 \\
88.9 \\
49.3 \\
58.5 \\
145.8 \\
100.2 \\
135.5 \\
44.4 \\
14.6 \\
12.4 \\
10.1 \\
\end{array}$ & $\begin{array}{l}\text { gi|50593002 } \\
\text { gi|} 4507123 \\
\text { gi|5032087 } \\
\text { gi|21361376 } \\
\text { gi|5803167 } \\
\text { gi|54112117 } \\
\text { gi|55749531 } \\
\text { gi|54112121 } \\
\text { gi|5032069 } \\
\text { gi||7706326 } \\
\text { gi|14249398 } \\
\text { gi| } 13775200\end{array}$ & \begin{tabular}{|c|}
12 \\
8 \\
33 \\
17 \\
30 \\
89 \\
41 \\
182 \\
1 \\
9 \\
13 \\
3 \\
\end{tabular} & \begin{tabular}{|c|}
30 \\
12 \\
31 \\
2 \\
18 \\
77 \\
25 \\
93 \\
3 \\
12 \\
7 \\
1 \\
\end{tabular} & \begin{tabular}{|c|}
21 \\
10 \\
32 \\
9.5 \\
24 \\
83 \\
33 \\
137.5 \\
2 \\
10.5 \\
10 \\
2 \\
\end{tabular} & $\begin{array}{c}17 \\
8 \\
5 \\
1 \\
7 \\
18 \\
7 \\
42 \\
1 \\
6 \\
7 \\
0 \\
\end{array}$ & $\begin{array}{c}17 \\
7 \\
13 \\
2 \\
2 \\
15 \\
4 \\
19 \\
1 \\
7 \\
1 \\
0 \\
\end{array}$ & \begin{tabular}{|c|}
17 \\
7.5 \\
9 \\
1.5 \\
4.5 \\
16.5 \\
5.5 \\
30.5 \\
1 \\
6.5 \\
4 \\
0 \\
\end{tabular} & $\begin{array}{l}1.24 \\
1.33 \\
3.56 \\
6.33 \\
5.33 \\
5.03 \\
6.00 \\
4.51 \\
2.00 \\
1.62 \\
2.50 \\
1 \\
\end{array}$ \\
\hline $\begin{array}{l}\text { 17S U2 related } \\
\text { U2AF65 } \\
\text { U2AF35 } \\
\text { hPRP43 } \\
\text { SPF45 } \\
\text { SR140 } \\
\text { CHERP } \\
\text { SF3b125 }\end{array}$ & $\begin{array}{c}53.5 \\
27.9 \\
90.9 \\
45.0 \\
118.2 \\
100.0 \\
103.0 \\
\end{array}$ & $\begin{array}{l}\text { gi|6005926 } \\
\text { gi||5803207 } \\
\text { gi|68509926 } \\
\text { gi|14249678 } \\
\text { gi|122937227 } \\
\text { gi|119226260 } \\
\text { gi||45446747 }\end{array}$ & \begin{tabular}{|c|}
1 \\
1 \\
36 \\
6 \\
8 \\
2 \\
10 \\
\end{tabular} & \begin{tabular}{|c|}
2 \\
1 \\
26 \\
3 \\
2 \\
6 \\
8 \\
\end{tabular} & $\begin{array}{c}1.5 \\
1 \\
31 \\
4.5 \\
5 \\
4 \\
9 \\
\end{array}$ & $\begin{array}{c}0 \\
0 \\
19 \\
0 \\
0 \\
0 \\
0 \\
\end{array}$ & $\begin{array}{c}0 \\
0 \\
19 \\
0 \\
0 \\
0 \\
0\end{array}$ & $\begin{array}{c}0 \\
0 \\
19 \\
0 \\
0 \\
0 \\
0\end{array}$ & $\begin{array}{c}1 \\
1 \\
1.63 \\
1 \\
1 \\
1 \\
1\end{array}$ \\
\hline $\begin{array}{l}\text { U5 snRNP } \\
220 \mathrm{~K} \\
200 \mathrm{~K} \\
116 \mathrm{~K} \\
40 \mathrm{~K} \\
102 \mathrm{~K} \\
15 \mathrm{~K} \\
100 \mathrm{~K} \\
52 \mathrm{~K}\end{array}$ & $\begin{array}{c}273.3 \\
244.5 \\
109.4 \\
39.3 \\
106.9 \\
16.8 \\
95.6 \\
37.6 \\
\end{array}$ & $\begin{array}{l}\text { gi|3661610 } \\
\text { gi|45861372 } \\
\text { gi|41152056 } \\
\text { gi|4758560 } \\
\text { gi|40807485 } \\
\text { gi|5729802 } \\
\text { gi|41327771 } \\
\text { gi|} 5174409\end{array}$ & \begin{tabular}{|c|}
227 \\
282 \\
109 \\
26 \\
138 \\
8 \\
59 \\
4 \\
\end{tabular} & \begin{tabular}{|c|}
184 \\
144 \\
94 \\
12 \\
39 \\
4 \\
17 \\
7 \\
\end{tabular} & \begin{tabular}{|c|}
205.5 \\
213 \\
101.5 \\
19 \\
88.5 \\
6 \\
38 \\
5.5 \\
\end{tabular} & $\begin{array}{c}158 \\
220 \\
100 \\
19 \\
36 \\
0 \\
29 \\
3\end{array}$ & $\begin{array}{c}80 \\
93 \\
79 \\
5 \\
8 \\
0 \\
8 \\
1\end{array}$ & $\begin{array}{c}119 \\
156.5 \\
89.5 \\
12 \\
22 \\
0 \\
18.5 \\
2 \\
\end{array}$ & $\begin{array}{c}1.73 \\
1.36 \\
1.13 \\
1.58 \\
4.02 \\
/ \\
2.05 \\
2.75 \\
\end{array}$ \\
\hline $\begin{array}{l}\text { U4/U6 snRNP } \\
90 \mathrm{~K} \\
60 \mathrm{~K} \\
20 \mathrm{~K} \\
61 \mathrm{~K} \\
15.5 \mathrm{~K}\end{array}$ & $\begin{array}{l}77.6 \\
58.4 \\
20.0 \\
55.4 \\
14.2 \\
\end{array}$ & $\begin{array}{l}\text { gi|4758556 } \\
\text { gi|45861374 } \\
\text { gi|5454154 } \\
\text { gi|40254869 } \\
\text { gi|4826860 }\end{array}$ & $\begin{array}{c}54 \\
45 \\
10 \\
47 \\
7\end{array}$ & $\begin{array}{l}33 \\
25 \\
17 \\
15 \\
10 \\
\end{array}$ & \begin{tabular}{c|}
43.5 \\
35 \\
13.5 \\
31 \\
8.5 \\
\end{tabular} & $\begin{array}{l}7 \\
6 \\
3 \\
8 \\
2\end{array}$ & $\begin{array}{l}3 \\
1 \\
2 \\
0 \\
0\end{array}$ & $\begin{array}{c}5 \\
3.5 \\
2.5 \\
4 \\
1\end{array}$ & $\begin{array}{c}8.70 \\
10.00 \\
5.40 \\
7.75 \\
8.50 \\
\end{array}$ \\
\hline $\begin{array}{l}\text { U4/U6.U5 snRNP } \\
\text { 110K } \\
65 \mathrm{~K} \\
\text { hPRP38 } \\
\text { TFIP11 }\end{array}$ & $\begin{array}{l}90.2 \\
65.4 \\
37.5 \\
96.8\end{array}$ & $\begin{array}{l}\text { gi|13926068 } \\
\text { gi|56550051 } \\
\text { gi|24762236 } \\
\text { gi|8393259 }\end{array}$ & $\begin{array}{c}44 \\
30 \\
19 \\
6\end{array}$ & $\begin{array}{c}32 \\
10 \\
8 \\
4\end{array}$ & \begin{tabular}{c|}
38 \\
20 \\
13.5 \\
5
\end{tabular} & $\begin{array}{c}10 \\
18 \\
4 \\
6\end{array}$ & $\begin{array}{l}4 \\
5 \\
0 \\
5\end{array}$ & $\begin{array}{c}7 \\
11.5 \\
2 \\
5.5\end{array}$ & $\begin{array}{l}5.43 \\
1.74 \\
6.75 \\
0.91\end{array}$ \\
\hline
\end{tabular}




\begin{tabular}{|c|c|c|c|c|c|c|c|c|c|}
\hline Protein & MW [kDa] & accession no. & B1 & B2 & B & $\mathrm{C} 1$ & $\mathrm{C} 2$ & C & B / C \\
\hline $\begin{array}{l}\text { LSm Proteins } \\
\text { LSM } 2 \\
\text { LSM } 3 \\
\text { LSM } 4 \\
\text { LSM } 6 \\
\text { LSM } 7 \\
\text { LSM } 8\end{array}$ & $\begin{array}{c}10.8 \\
11.8 \\
15.4 \\
9.1 \\
11.6 \\
10.4\end{array}$ & $\begin{array}{l}\text { gi|10863977 } \\
\text { gi|7657315 } \\
\text { gi|6912486 } \\
\text { gi|5919153 } \\
\text { gi|7706423 } \\
\text { gi|7706425 } \\
\end{array}$ & $\begin{array}{l}8 \\
2 \\
9 \\
9 \\
2 \\
6\end{array}$ & $\begin{array}{c}8 \\
1 \\
4 \\
14 \\
4 \\
7 \\
\end{array}$ & $\begin{array}{c}8 \\
1.5 \\
6.5 \\
11.5 \\
3 \\
6.5 \\
\end{array}$ & $\begin{array}{l}1 \\
1 \\
0 \\
1 \\
0 \\
1\end{array}$ & $\begin{array}{l}0 \\
0 \\
0 \\
0 \\
0 \\
0\end{array}$ & $\begin{array}{c}0.5 \\
0.5 \\
0 \\
0.5 \\
0 \\
0.5\end{array}$ & $\begin{array}{c}16.00 \\
3.00 \\
I \\
23.00 \\
/ \\
13.00 \\
\end{array}$ \\
\hline $\begin{array}{l}\text { Sm Proteins } \\
\text { B } \\
\text { D1 } \\
\text { D2 } \\
\text { D3 } \\
\text { E } \\
\text { F } \\
\text { G } \\
\end{array}$ & $\begin{array}{c}24.6 \\
13.3 \\
13.5 \\
13.9 \\
10.8 \\
9.7 \\
8.5 \\
\end{array}$ & $\begin{array}{l}\text { gi|4507125 } \\
\text { gi|5902102 } \\
\text { gi|29294624 } \\
\text { gi|4759160 } \\
\text { gi|4507129 } \\
\text { gi|4507131 } \\
\text { gi|} 4507133 \\
\end{array}$ & $\begin{array}{c}15 \\
16 \\
48 \\
30 \\
9 \\
16 \\
5 \\
\end{array}$ & $\begin{array}{c}20 \\
9 \\
21 \\
11 \\
8 \\
2 \\
6 \\
\end{array}$ & $\begin{array}{c}17.5 \\
12.5 \\
34.5 \\
20.5 \\
8.5 \\
9 \\
5.5 \\
\end{array}$ & $\begin{array}{c}15 \\
17 \\
42 \\
38 \\
9 \\
8 \\
2 \\
\end{array}$ & \begin{tabular}{c|}
14 \\
9 \\
17 \\
12 \\
9 \\
2 \\
5 \\
\end{tabular} & \begin{tabular}{|c|}
14.5 \\
13 \\
29.5 \\
25 \\
9 \\
5 \\
3.5 \\
\end{tabular} & $\begin{array}{l}1.21 \\
0.96 \\
1.17 \\
0.82 \\
0.94 \\
1.80 \\
1.57 \\
\end{array}$ \\
\hline $\begin{array}{l}\text { hPRP19/CDC5L complex } \\
\text { hPrp19 } \\
\text { CDC5L } \\
\text { SPF27 } \\
\text { PRL1 } \\
\text { Hsp70 } \\
\text { AD-002 } \\
\text { CTNNBL1 } \\
\text { Npw38BP } \\
\text { Npw38 }\end{array}$ & $\begin{array}{l}55.2 \\
92.2 \\
21.5 \\
57.2 \\
70.4 \\
26.6 \\
65.1 \\
70.0 \\
30.5 \\
\end{array}$ & $\begin{array}{l}\text { gi|7657381 } \\
\text { gi|11067747 } \\
\text { gi|} \mid 5031653 \\
\text { gi|4505895 } \\
\text { gi||5729877 } \\
\text { gi|7705475 } \\
\text { gi|18644734 } \\
\text { gi|7706501 } \\
\text { gi|74735456 }\end{array}$ & $\begin{array}{c}56 \\
36 \\
8 \\
22 \\
3 \\
1 \\
7 \\
8 \\
0 \\
\end{array}$ & $\begin{array}{c}14 \\
32 \\
9 \\
9 \\
3 \\
4 \\
4 \\
7 \\
2 \\
\end{array}$ & $\begin{array}{c}35 \\
34 \\
8.5 \\
15.5 \\
3 \\
2.5 \\
5.5 \\
7.5 \\
1 \\
\end{array}$ & $\begin{array}{c}85 \\
83 \\
9 \\
36 \\
14 \\
4 \\
5 \\
0 \\
0 \\
\end{array}$ & $\begin{array}{c}46 \\
86 \\
25 \\
26 \\
14 \\
9 \\
2 \\
0 \\
0 \\
\end{array}$ & $\begin{array}{c}65.5 \\
84.5 \\
17 \\
31 \\
14 \\
6.5 \\
3.5 \\
0 \\
0 \\
\end{array}$ & $\begin{array}{l}0.53 \\
0.40 \\
0.50 \\
0.50 \\
0.21 \\
0.38 \\
1.57 \\
1 \\
1\end{array}$ \\
\hline $\begin{array}{l}\text { hPRP19/CDC5L related } \\
\text { hSYF1 } \\
\text { CRNKL1 } \\
\text { hlsy1 } \\
\text { SKIP } \\
\text { RBM22 } \\
\text { Cyp-E } \\
\text { PPIL1 } \\
\text { KIAA0560 } \\
\text { G10 } \\
\end{array}$ & $\begin{array}{c}100.0 \\
100.6 \\
33.0 \\
51.1 \\
46.9 \\
33.4 \\
18.2 \\
171.3 \\
17.0 \\
\end{array}$ & $\begin{array}{l}\text { gi|55770906 } \\
\text { gi|30795220 } \\
\text { gi|20149304 } \\
\text { gi|6912676 } \\
\text { gi|8922328 } \\
\text { gi|5174637 } \\
\text { gi|7706339 } \\
\text { gi|38788372 } \\
\text { gi|32171175 }\end{array}$ & $\begin{array}{c}22 \\
52 \\
4 \\
43 \\
7 \\
4 \\
0 \\
30 \\
6 \\
\end{array}$ & $\begin{array}{c}31 \\
24 \\
2 \\
12 \\
12 \\
6 \\
0 \\
17 \\
7 \\
\end{array}$ & $\begin{array}{c}26.5 \\
38 \\
3 \\
27.5 \\
9.5 \\
5 \\
0 \\
23.5 \\
6.5 \\
\end{array}$ & $\begin{array}{c}65 \\
128 \\
19 \\
65 \\
31 \\
8 \\
33 \\
107 \\
15 \\
\end{array}$ & $\begin{array}{l}66 \\
75 \\
16 \\
46 \\
28 \\
17 \\
15 \\
72 \\
18 \\
\end{array}$ & $\begin{array}{c}65.5 \\
101.5 \\
17.5 \\
55.5 \\
29.5 \\
12.5 \\
24 \\
89.5 \\
16.5 \\
\end{array}$ & $\begin{array}{l}0.40 \\
0.37 \\
0.17 \\
0.50 \\
0.32 \\
0.40 \\
0.00 \\
0.26 \\
0.39 \\
\end{array}$ \\
\hline $\begin{array}{l}\text { hRES complex proteins } \\
\text { SNIP1 } \\
\text { MGC12135 } \\
\text { CGI-79 }\end{array}$ & $\begin{array}{l}45.8 \\
70.5 \\
39.7\end{array}$ & $\begin{array}{l}\text { gi|21314720 } \\
\text { gi|14249338 } \\
\text { gi|4929627 }\end{array}$ & $\begin{array}{c}10 \\
13 \\
4\end{array}$ & $\begin{array}{c}5 \\
13 \\
0\end{array}$ & $\begin{array}{c}7.5 \\
13 \\
2\end{array}$ & $\begin{array}{c}9 \\
10 \\
4\end{array}$ & $\begin{array}{c}5 \\
10 \\
1\end{array}$ & $\begin{array}{c}7 \\
10 \\
2.5\end{array}$ & $\begin{array}{l}1.07 \\
1.30 \\
0.80\end{array}$ \\
\hline $\begin{array}{l}\text { Proteins recruited to A } \\
\text { complex } \\
\text { RBM39 } \\
\text { p68 (DDX5) } \\
\text { ELAV-like } 1 \text { (HuR) } \\
\text { p72/DDX17 }\end{array}$ & $\begin{array}{l}59.4 \\
69.2 \\
36.1 \\
80.5\end{array}$ & $\begin{array}{l}\text { gi|4757926 } \\
\text { gi|4758138 } \\
\text { gi|38201714 } \\
\text { gi|3122595 }\end{array}$ & $\begin{array}{l}27 \\
13 \\
60 \\
13\end{array}$ & $\begin{array}{c}9 \\
4 \\
24 \\
3\end{array}$ & $\begin{array}{c}18 \\
8.5 \\
42 \\
8\end{array}$ & $\begin{array}{l}6 \\
6 \\
8 \\
4\end{array}$ & $\begin{array}{l}1 \\
2 \\
9 \\
0\end{array}$ & $\begin{array}{c}3.5 \\
4 \\
8.5 \\
2\end{array}$ & $\begin{array}{l}5.14 \\
2.13 \\
4.94 \\
4.00\end{array}$ \\
\hline $\begin{array}{l}\text { Proteins recruited to B } \\
\text { complex } \\
\text { MFAP1 } \\
\text { RED } \\
\text { hSmu-1 } \\
\text { PPIL2 } \\
\text { hPRP2 (DDX16) } \\
\text { hPRP4-Kinase }\end{array}$ & $\begin{array}{r}51.9 \\
65.6 \\
57.5 \\
59.5 \\
119.2 \\
117.1\end{array}$ & $\begin{array}{l}\text { gi|} 50726968 \\
\text { gi|10835234 } \\
\text { gi|8922679 } \\
\text { gi|7657473 } \\
\text { gi|4503293 } \\
\text { gi|89276756 }\end{array}$ & $\begin{array}{l}38 \\
41 \\
61 \\
15 \\
11 \\
24\end{array}$ & $\begin{array}{c}15 \\
16 \\
31 \\
6 \\
11 \\
1\end{array}$ & $\begin{array}{c}26.5 \\
28.5 \\
46 \\
10.5 \\
11 \\
12.5\end{array}$ & $\begin{array}{c}6 \\
2 \\
12 \\
9 \\
17 \\
33\end{array}$ & $\begin{array}{c}2 \\
0 \\
0 \\
10 \\
11 \\
1\end{array}$ & $\begin{array}{c}4 \\
1 \\
6 \\
9.5 \\
14 \\
17\end{array}$ & $\begin{array}{r}6.63 \\
28.50 \\
7.67 \\
1.11 \\
0.79 \\
0.74\end{array}$ \\
\hline
\end{tabular}




\begin{tabular}{|c|c|c|c|c|c|c|c|c|c|}
\hline Protein & MW [kDa] & accession no. & B1 & B2 & B & $\mathrm{C} 1$ & $\mathrm{C} 2$ & C & B / C \\
\hline THRAP3 & 108.6 & gi|4827040 & 26 & 12 & 19 & 9 & 1 & 5 & 3.80 \\
\hline PABP1 & 70.5 & gi|46367787 & 11 & 5 & 8 & 7 & 4 & 5.5 & 1.45 \\
\hline SKIV2L2 & 117.8 & gi|39930353 & 6 & 3 & 4.5 & 6 & 8 & 7 & 0.64 \\
\hline PABPN1 & 32.6 & gi|4758876 & 3 & 1 & 2 & 2 & 1 & 1.5 & 1.33 \\
\hline RNF113A & 38.8 & gi|5902158 & 1 & 5 & 3 & 3 & 1 & 2 & 1.50 \\
\hline NY-CO-10 & 53.8 & gi|64276486 & 5 & 3 & 4 & 2 & 7 & 4.5 & 0.89 \\
\hline KIAA1604 & 105.5 & gi|55749769 & 4 & 12 & 8 & 34 & 29 & 31.5 & 0.25 \\
\hline hsp27 & 22.8 & gi|4504517 & 2 & 1 & 1.5 & 1 & 4 & 2.5 & 0.60 \\
\hline GCFC & 104.7 & gi|22035565 & 0 & 0 & 0 & 2 & 2 & 2 & 0.00 \\
\hline UBL5 & 8.5 & gi|13236510 & 4 & 3 & 3.5 & 0 & 0 & 0 & l \\
\hline CCDC16 & 42.0 & gi|49472814 & 2 & 3 & 2.5 & 4 & 1 & 2.5 & 1.00 \\
\hline CCDC12 & 19.2 & gi|21389497 & 1 & 6 & 3.5 & 3 & 8 & 5.5 & 0.64 \\
\hline HsKin17 & 45.2 & gi|13124883 & 7 & 7 & 7 & 1 & 0 & 0.5 & 14.00 \\
\hline \multicolumn{10}{|l|}{ Step 2 factors } \\
\hline hPRP22 & 139.3 & gi|4826690 & 4 & 2 & 3 & 107 & 68 & 87.5 & 0.03 \\
\hline hPRP18 & 39.9 & gi|4506123 & 0 & 0 & 0 & 2 & 2 & 2 & 0.00 \\
\hline hPRP17 & 65.5 & gi|7706657 & 8 & 6 & 7 & 30 & 25 & 27.5 & 0.25 \\
\hline hPRP16 & 140.5 & gi|17999539 & 0 & 0 & 0 & 3 & 1 & 2 & 0.00 \\
\hline hSLU7 & 68.4 & gi|27477111 & 0 & 0 & 0 & 44 & 17 & 30.5 & 0.00 \\
\hline \multicolumn{10}{|l|}{$\begin{array}{l}\text { Proteins recruited to } \mathrm{C} \\
\text { complex }\end{array}$} \\
\hline Abstrakt & 69.8 & gi|21071032 & 1 & 0 & 0.5 & 64 & 54 & 59 & 0.01 \\
\hline GCIP p29 & 28.7 & gi|46371998 & 0 & 0 & 0 & 16 & 18 & 17 & 0.00 \\
\hline DDX35 & 78.9 & gi|20544129 & 3 & 0 & 1.5 & 22 & 20 & 21 & 0.07 \\
\hline Q9BRR8 & 103.3 & gi|74732921 & 0 & 0 & 0 & 6 & 10 & 8 & 0.00 \\
\hline c19orf29 (NY-REN-24) & 88.6 & gi|126723149 & 1 & 0 & 0.5 & 52 & 16 & 34 & 0.01 \\
\hline PPlase-like 3b & 18.6 & gi|19557636 & 0 & 0 & 0 & 10 & 6 & 8 & 0.00 \\
\hline PPWD1 & 73.6 & gi|24308049 & 0 & 0 & 0 & 33 & 15 & 24 & 0.00 \\
\hline MORG1 & 34.3 & gi|153791298 & 1 & 0 & 0.5 & 2 & 9 & 5.5 & 0.09 \\
\hline FRG1 & 29.2 & gi|4758404 & 0 & 0 & 0 & 2 & 8 & 5 & 0.00 \\
\hline NOSIP & 33.2 & gi|7705716 & 0 & 0 & 0 & 2 & 7 & 4.5 & 0.00 \\
\hline GPKOW & 52.1 & gi|15811782 & 0 & 1 & 0.5 & 4 & 1 & 2.5 & 0.20 \\
\hline C1orf55 & 39.3 & gi|148664216 & 0 & 0 & 0 & 24 & 8 & 16 & 0.00 \\
\hline FAM32A & 13.1 & gi|7661696 & 0 & 0 & 0 & 4 & 3 & 3.5 & 0.00 \\
\hline RACK1 (GNB2L1) & 35.1 & gi|5174447 & 0 & 0 & 0 & 0 & 1 & 0.5 & 0.00 \\
\hline Tip-49 & 50.2 & gi|4506753 & 0 & 0 & 0 & 3 & 1 & 2 & 0.00 \\
\hline \multicolumn{10}{|l|}{$\begin{array}{l}\text { Potential C complex } \\
\text { specific proteins }\end{array}$} \\
\hline PPIG & 88.5 & gi|42560244 & 1 & 0 & 0.5 & 8 & 3 & 5.5 & 0.09 \\
\hline FAM50A & 40.1 & gi|4758220 & 0 & 0 & 0 & 17 & 6 & 11.5 & 0.00 \\
\hline FAM50B & 38.6 & gi|6912326 & 0 & 0 & 0 & 12 & 0 & 6 & 0.00 \\
\hline C9orf78 & 33.7 & gi|7706557 & 0 & 0 & 0 & 6 & 4 & 5 & 0.00 \\
\hline C10orf4 & 37.5 & gi|24432067 & 0 & 0 & 0 & 3 & 5 & 4 & 0.00 \\
\hline CXorf56 & 25.6 & gi|11545813 & 0 & 0 & 0 & 17 & 16 & 16.5 & 0.00 \\
\hline DGCR14 & 52.4 & gi|13027630 & 0 & 0 & 0 & 5 & 11 & 8 & 0.00 \\
\hline CCDC130 & 44.7 & gi|13540614 & 0 & 0 & 0 & 4 & 1 & 2.5 & 0.00 \\
\hline TOE1 & 56.4 & gi|156564398 & 0 & 0 & 0 & 1 & 0 & 0.5 & 0.00 \\
\hline NKAP & 47.0 & gi|13375676 & 0 & 0 & 0 & 7 & 2 & 4.5 & 0.00 \\
\hline $\mathrm{ZCCHC} 10$ & 18.4 & gi|8923106 & 0 & 0 & 0 & 10 & 3 & 6.5 & 0.00 \\
\hline CDK10 & 35.4 & gi|16950647 & 0 & 0 & 0 & 7 & 0 & 3.5 & 0.00 \\
\hline TTC14 & 88.2 & gi|33457330 & 0 & 0 & 0 & 9 & 4 & 6.5 & 0.00 \\
\hline WDR70 & 73.2 & gi|8922301 & 0 & 0 & 0 & 1 & 2 & 1.5 & 0.00 \\
\hline NFKBIL1 & 43.1 & gi|26787991 & 0 & 0 & 0 & 0 & 3 & 1.5 & 0.00 \\
\hline
\end{tabular}




\begin{tabular}{|c|c|c|c|c|c|c|c|c|c|}
\hline Protein & MW [kDa] & accession no. & B1 & B2 & B & $\mathrm{C} 1$ & $\mathrm{C} 2$ & C & B / C \\
\hline $\begin{array}{l}\text { EJC/mRNP } \\
\text { elF4A3 } \\
\text { Magoh } \\
\text { Y14 }\end{array}$ & $\begin{array}{l}46.9 \\
17.2 \\
19.9\end{array}$ & $\begin{array}{l}\text { gi|7661920 } \\
\text { gi|4505087 } \\
\text { gi|4826972 }\end{array}$ & $\begin{array}{c}11 \\
1 \\
2\end{array}$ & $\begin{array}{c}12 \\
2 \\
2\end{array}$ & $\begin{array}{c}11.5 \\
1.5 \\
2\end{array}$ & $\begin{array}{l}51 \\
11 \\
8\end{array}$ & $\begin{array}{c}24 \\
4 \\
5\end{array}$ & $\begin{array}{l}37.5 \\
7.5 \\
6.5\end{array}$ & $\begin{array}{l}0.31 \\
0.20 \\
0.31\end{array}$ \\
\hline $\begin{array}{l}\text { Pinin } \\
\text { RNPS1 } \\
\text { Acinus } \\
\text { SAP18 } \\
\text { Aly/REF (THOC4) } \\
\text { UAP56 }\end{array}$ & $\begin{array}{c}81.6 \\
34.2 \\
151.8 \\
17.4 \\
26.9 \\
49.1 \\
\end{array}$ & $\begin{array}{l}\text { gi|33356174 } \\
\text { gi|6857826 } \\
\text { gi|7662238 } \\
\text { gi|5032067 } \\
\text { gi|55770864 } \\
\text { gi|18375623 } \\
\end{array}$ & $\begin{array}{c}5 \\
4 \\
23 \\
4 \\
5 \\
9\end{array}$ & $\begin{array}{c}1 \\
0 \\
14 \\
3 \\
0 \\
1\end{array}$ & $\begin{array}{c}3 \\
2 \\
18.5 \\
3.5 \\
2.5 \\
5 \\
\end{array}$ & $\begin{array}{c}2 \\
4 \\
15 \\
1 \\
4 \\
7\end{array}$ & $\begin{array}{l}2 \\
0 \\
9 \\
0 \\
2 \\
4\end{array}$ & $\begin{array}{c}2 \\
2 \\
12 \\
0.5 \\
3 \\
5.5\end{array}$ & $\begin{array}{l}1.50 \\
1.00 \\
1.54 \\
7.00 \\
0.83 \\
0.91\end{array}$ \\
\hline $\begin{array}{l}\text { TREX } \\
\text { THOC1 } \\
\text { THOC2 } \\
\text { THOC3 } \\
\text { KIAA0983 (THOC5) } \\
\text { WDR58 (THOC6) }\end{array}$ & $\begin{array}{c}75.6 \\
169.6 \\
38.8 \\
78.4 \\
37.4\end{array}$ & $\begin{array}{l}\text { gi|154448890 } \\
\text { gi|125656165 } \\
\text { gi|14150171 } \\
\text { gi|50959110 } \\
\text { gi|31543164 }\end{array}$ & $\begin{array}{l}3 \\
1 \\
0 \\
7 \\
0\end{array}$ & $\begin{array}{l}5 \\
1 \\
2 \\
3 \\
0\end{array}$ & $\begin{array}{l}4 \\
1 \\
1 \\
5 \\
0\end{array}$ & $\begin{array}{l}2 \\
3 \\
0 \\
5 \\
2\end{array}$ & $\begin{array}{l}5 \\
8 \\
0 \\
4 \\
4\end{array}$ & $\begin{array}{c}3.5 \\
5.5 \\
0 \\
4.5 \\
3\end{array}$ & $\begin{array}{l}1.14 \\
0.18 \\
/ \\
1.11 \\
0.00\end{array}$ \\
\hline $\begin{array}{l}\text { pre-mRNA / mRNA binding } \\
\text { proteins } \\
\text { CBP20 } \\
\text { CBP80 } \\
\text { NF45 } \\
\text { ZC3H18 } \\
\text { YB-1 } \\
\text { ELG } \\
\text { DDX3 } \\
\text { ASR2B } \\
\text { BCLAF1 } \\
\text { RBM7 } \\
\text { HSP70 }\end{array}$ & $\begin{array}{c}18.0 \\
91.8 \\
43.0 \\
104.0 \\
35.9 \\
38.9 \\
73.3 \\
100.0 \\
107.2 \\
30.5 \\
70.0 \\
\end{array}$ & $\begin{array}{l}\text { gi||110349727 } \\
\text { gi|4505343 } \\
\text { gi|24234747 } \\
\text { gi|31377595 } \\
\text { gi|34098946 } \\
\text { gi|8923771 } \\
\text { gi|87196351 } \\
\text { gi|33383233 } \\
\text { gi|7661958 } \\
\text { gi|4503293 } \\
\text { gi|} 5123454 \\
\end{array}$ & $\begin{array}{c}15 \\
69 \\
6 \\
2 \\
12 \\
6 \\
8 \\
14 \\
20 \\
1 \\
3 \\
\end{array}$ & $\begin{array}{c}7 \\
26 \\
8 \\
4 \\
1 \\
5 \\
3 \\
11 \\
3 \\
0 \\
3 \\
\end{array}$ & $\begin{array}{c}11 \\
47.5 \\
7 \\
3 \\
6.5 \\
5.5 \\
5.5 \\
12.5 \\
11.5 \\
0.5 \\
3 \\
\end{array}$ & $\begin{array}{c}23 \\
67 \\
2 \\
2 \\
7 \\
7 \\
2 \\
14 \\
3 \\
2 \\
14 \\
\end{array}$ & $\begin{array}{c}10 \\
36 \\
4 \\
3 \\
5 \\
6 \\
0 \\
5 \\
0 \\
4 \\
14 \\
\end{array}$ & $\begin{array}{c}16.5 \\
51.5 \\
3 \\
2.5 \\
6 \\
6.5 \\
1 \\
9.5 \\
1.5 \\
3 \\
14 \\
\end{array}$ & $\begin{array}{l}0.67 \\
0.92 \\
2.33 \\
1.20 \\
1.08 \\
0.85 \\
5.50 \\
1.32 \\
7.67 \\
0.17 \\
0.21 \\
\end{array}$ \\
\hline $\begin{array}{l}\text { Miscellaneous proteins } \\
\text { BAG2 } \\
\text { RBBP6 } \\
\text { RBM42 }\end{array}$ & $\begin{array}{c}23.4 \\
197.2 \\
50.3\end{array}$ & $\begin{array}{l}\text { gi|4757834 } \\
\text { gi|33620716 } \\
\text { gi|21359951 }\end{array}$ & $\begin{array}{l}0 \\
1 \\
1\end{array}$ & $\begin{array}{l}1 \\
0 \\
0\end{array}$ & $\begin{array}{l}0.5 \\
0.5 \\
0.5\end{array}$ & $\begin{array}{l}1 \\
1 \\
1\end{array}$ & $\begin{array}{l}2 \\
0 \\
0\end{array}$ & $\begin{array}{l}1.5 \\
0.5 \\
0.5\end{array}$ & $\begin{array}{l}0.33 \\
1.00 \\
1.00\end{array}$ \\
\hline $\begin{array}{l}\text { SR related proteins } \\
\text { SRm160 } \\
\text { SRm300 }\end{array}$ & $\begin{array}{l}102.5 \\
300.0\end{array}$ & $\begin{array}{l}\text { gi|42542379 } \\
\text { gi|4759098 }\end{array}$ & $\begin{array}{l}1 \\
0\end{array}$ & $\begin{array}{l}0 \\
7\end{array}$ & $\begin{array}{l}0.5 \\
3.5\end{array}$ & $\begin{array}{l}1 \\
6\end{array}$ & $\begin{array}{c}0 \\
29\end{array}$ & $\begin{array}{c}0.5 \\
17.5\end{array}$ & $\begin{array}{l}1.00 \\
0.20\end{array}$ \\
\hline $\begin{array}{l}\text { SR proteins } \\
\text { SF2/ASF } \\
\text { 9G8 } \\
\text { SRp20 } \\
\text { SRp30c } \\
\text { SRp38 } \\
\text { SRp40 } \\
\text { SRp46 } \\
\text { SRp55 } \\
\text { SC35 (SFRS2) } \\
\text { hTra-2 alpha } \\
\text { hTra-2 beta (SFRS10) }\end{array}$ & $\begin{array}{l}27.8 \\
27.4 \\
19.4 \\
25.5 \\
31.3 \\
31.3 \\
31.2 \\
39.6 \\
25.5 \\
32.7 \\
33.7 \\
\end{array}$ & $\begin{array}{l}\text { gi|5902076 } \\
\text { gi|72534660 } \\
\text { gi|4506901 } \\
\text { gi|4506903 } \\
\text { gi|5730079 } \\
\text { gi|3929378 } \\
\text { gi|15055543 } \\
\text { gi|20127499 } \\
\text { gi|47271443 } \\
\text { gi| } 9558733 \\
\text { gi|4759098 }\end{array}$ & $\begin{array}{c}11 \\
44 \\
10 \\
10 \\
22 \\
22 \\
0 \\
30 \\
0 \\
13 \\
21\end{array}$ & $\begin{array}{c}21 \\
25 \\
2 \\
11 \\
9 \\
5 \\
1 \\
6 \\
1 \\
6 \\
9 \\
\end{array}$ & $\begin{array}{c}16 \\
34.5 \\
6 \\
10.5 \\
15.5 \\
13.5 \\
0.5 \\
18 \\
0.5 \\
9.5 \\
15 \\
\end{array}$ & $\begin{array}{c}21 \\
36 \\
8 \\
10 \\
34 \\
15 \\
4 \\
24 \\
4 \\
6 \\
18 \\
\end{array}$ & $\begin{array}{c}15 \\
11 \\
4 \\
15 \\
15 \\
0 \\
0 \\
7 \\
0 \\
7 \\
5\end{array}$ & $\begin{array}{c}18 \\
23.5 \\
6 \\
12.5 \\
24.5 \\
7.5 \\
2 \\
15.5 \\
2 \\
6.5 \\
11.5 \\
\end{array}$ & $\begin{array}{l}0.89 \\
1.47 \\
1.00 \\
0.84 \\
0.63 \\
1.80 \\
0.25 \\
1.16 \\
0.25 \\
1.46 \\
1.30\end{array}$ \\
\hline $\begin{array}{l}\text { hnRNP } \\
\text { hnRNP A1 } \\
\text { hnRNP A3 } \\
\text { hnRNP AB } \\
\text { hnRNP A2/B1 }\end{array}$ & $\begin{array}{l}38.7 \\
39.6 \\
36.0 \\
37.4\end{array}$ & $\begin{array}{l}\text { gi|4504445 } \\
\text { gi|34740329 } \\
\text { gi|12803583 } \\
\text { gi|14043072 }\end{array}$ & $\begin{array}{c}12 \\
10 \\
4 \\
11\end{array}$ & $\begin{array}{l}2 \\
0 \\
1 \\
1\end{array}$ & $\begin{array}{c}7 \\
5 \\
2.5 \\
6\end{array}$ & $\begin{array}{l}1 \\
1 \\
0 \\
1\end{array}$ & $\begin{array}{l}2 \\
0 \\
0 \\
0\end{array}$ & $\begin{array}{c}1.5 \\
0.5 \\
0 \\
0.5\end{array}$ & $\begin{array}{c}4.67 \\
10.00 \\
/ \\
12.00\end{array}$ \\
\hline
\end{tabular}




\begin{tabular}{|l|c|l|c|c|c|c|c|c|c|}
\hline Protein & MW [kDa] & accession no. & B1 & B2 & B & C1 & C2 & C & B / C \\
\hline hnRNP C & 33.3 & gi|4758544 & 36 & 18 & 27 & 21 & 22 & 21.5 & 1.26 \\
hnRNP D & 38.4 & gi|14110420 & 3 & 0 & 1.5 & 1 & 0 & 0.5 & 3.00 \\
hnRNP F & 45.7 & gi|148470406 & 4 & 0 & 2 & 0 & 0 & 0 & $/$ \\
hnRNP G & 47.4 & gi|56699409 & 22 & 12 & 17 & 10 & 17 & 13.5 & 1.26 \\
hnRNP H1 & 49.1 & gi|5031753 & 5 & 0 & 2.5 & 3 & 1 & 2 & 1.25 \\
hnRNP H3 & 36.9 & gi|14141157 & 4 & 0 & 2 & 0 & 0 & 0 & $/$ \\
hnRNP K & 51.0 & gi|14165435 & 6 & 7 & 6.5 & 0 & 0 & 0 & $/$ \\
hnRNP M & 77.5 & gi|14141152 & 17 & 3 & 10 & 8 & 1 & 4.5 & 2.22 \\
hnRNP Q & 69.6 & gi|15809590 & 7 & 2 & 4.5 & 6 & 0 & 3 & 1.50 \\
hnRNP R & 70.9 & gi|5031755 & 15 & 0 & 7.5 & 11 & 0 & 5.5 & 1.36 \\
hnRNP U & 90.6 & gi|14141161 & 2 & 2 & 2 & 0 & 1 & 0.5 & 4.00 \\
PCBP1 & 37.5 & gi|5453854 & 24 & 7 & 15.5 & 4 & 2 & 3 & 5.17 \\
PCBP2 & 38.1 & gi|14141166 & 24 & 14 & 19 & 4 & 8 & 6 & 3.17 \\
RALY & 32.5 & gi|8051631 & 4 & 3 & 3.5 & 5 & 10 & 7.5 & 0.47 \\
\hline
\end{tabular}

The number of acquired tandem MS spectra (MS/MS spectra) for two independent purifications of $B$ and $C$ complexes (B1, B2, $C 1$ and $C 2$, respectively) are given for the spliceosomal proteins identified in Bessonov et al., 2008. For both complexes, the average number of spectra for the two purifications and the protein ratio $(B / C)$ was calculated. The protein ratio displays the protein abundance within the two complexes. A protein ratio of 1 indicates that the distinct protein is present in equal amounts within both complexes. Protein ratios $>1$ indicate a higher abundance for these proteins in spliceosomal B complexes, whereas protein ratios $<1$ indicate a higher abundance in $\mathrm{C}$ complexes. For proteins identified solely in B complexes no protein ratio could be calculated (indicated by "p"). For proteins that were solely or predominantly identified in $\mathrm{C}$ complexes the calculated protein ratio is 0 or close to 0 (Table 4.9).

This reanalysis shows that the overall protein assignment was basically correct. The calculated $B / C$ ratio allows for clear assignment of the proteins to $B$ or $C$ specific proteins. Several proteins show a high abundance in B or C complexes. All proteins grouped as potential $\mathrm{C}$ specific proteins and proteins recruited to $\mathrm{C}$ complexes show a protein ratio of 0 or close to 0 and almost all proteins grouped as $B$ specific proteins show protein ratios higher than one. However, some proteins assigned to be recruited to $B$ complexes show low protein ratios indicating their enrichment in C complexes (e.g. GCFC, hsp27 and KIAA1604, Table 4.9). Surprisingly, only few proteins (e.g. CBP80, U5-116K, U2-A') appear to be present in a 1:1 ratio within $B$ and $C$ complexes.

On the basis of semi-quantitative analysis, we then compared two quantification techniques that are based on stable isotope labeling: (i) Quantification by chemical labeling with iTRAQ reagents, and (ii) quantification of $B$ and $C$ complexes assembled in metabolically labeled nuclear extracts (SILAC quantification). 


\subsection{3 iTRAQ labeling of spliceosomal $B$ and $C$ complexes for relative quantification}

Spliceosomal B and C complexes were purified as described above (Figure 4.15 A). Equal amounts of both complexes were separated by gel electrophoresis (Figure 4.16) and entire lanes were cut into gel slices of equal size. ITRAQ labeling was then performed according to the optimized workflow described in section 4.2.1 (see also Figure 4.11). An internal standard was prepared for all samples to be compared (i.e. from peptides generated from gel slices of $B$ and $C$ complexes cut at the same molecular weight region). Peptides generated from $B$ complex proteins were labeled with iTRAQ reagent 115 and peptides generated from C complexes were labeled with ITRAQ reagent 116 . The internal standard was labeled with ITRAQ reagent 114 . The samples to be compared and the corresponding internal standard were pooled and the samples were subsequently analyzed by LC-MS/MS. For relative quantification of $B$ and $C$ complexes, two independent replicates were performed. Purified complexes were kindly provided by S. Bessonov.

Pilot experiments showed that the labeling efficiency is crucial for reliable quantification by iTRAQ labeling. To estimate the degree of ITRAQ labeling, first the labeling efficiency was calculated for all protein hits identified in the two replicates. To this end, the peptide and fragment masses were searched against the NCBInr database allowing iTRAQ labels as variable modification. This yielded the total number of peptides and the number of iTRAQlabeled peptides. The labeling efficiency was then calculated from the number of labeled peptides and the total number of peptides identified. Figure 4.17 shows the labeling efficiency achieved for the two independent replicates. A labeling efficiency of $95-100 \%$ was achieved for approximately 55 and $75 \%$ of the protein hits detected in the two replicates. Approximately 40 and $20 \%$ of the protein hits show a labeling efficiency between 80 and $95 \%$. A labeling efficiency of $80 \%$ or lower was calculated for less than $10 \%$ of the protein hits. A high labeling efficiency for iTRAQ labeling was thus achieved for both replicates and reliable quantification can therefore be expected. 


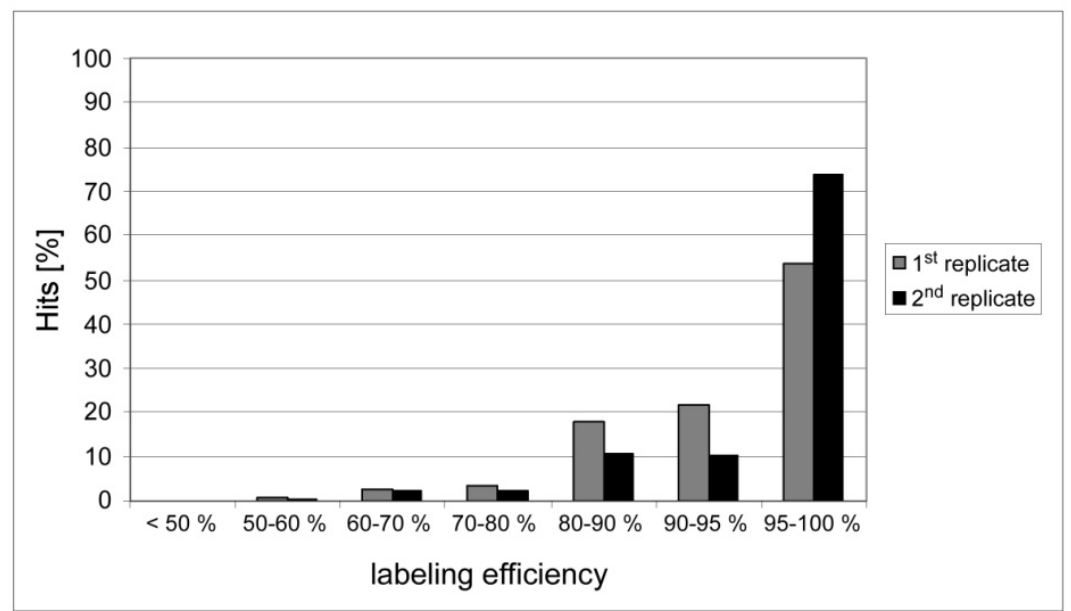

Figure 4.17: Labeling efficiency achieved for ITRAQ labeling in two independent replicates. The labeling efficiency was calculated from the number of peptides that were labeled with ITRAQ reagents and the total number of peptides that were identified for every protein. The number of protein hits is given in percent.

The proteins within $B$ and $C$ complexes were identified by searching peptide and fragment masses against the NCBInr database using Mascot as search engine. The samples were quantified by comparing the peak areas of iTRAQ reporter ions after fragmentation of the differently labeled peptides (i.e. peptides generated from B and C complexes). A typical spectrum is shown in Figure 4.18. Peptide ratios for all identified proteins were obtained from the Mascot search. Protein ratios were calculated from these after manual removal of outliers.

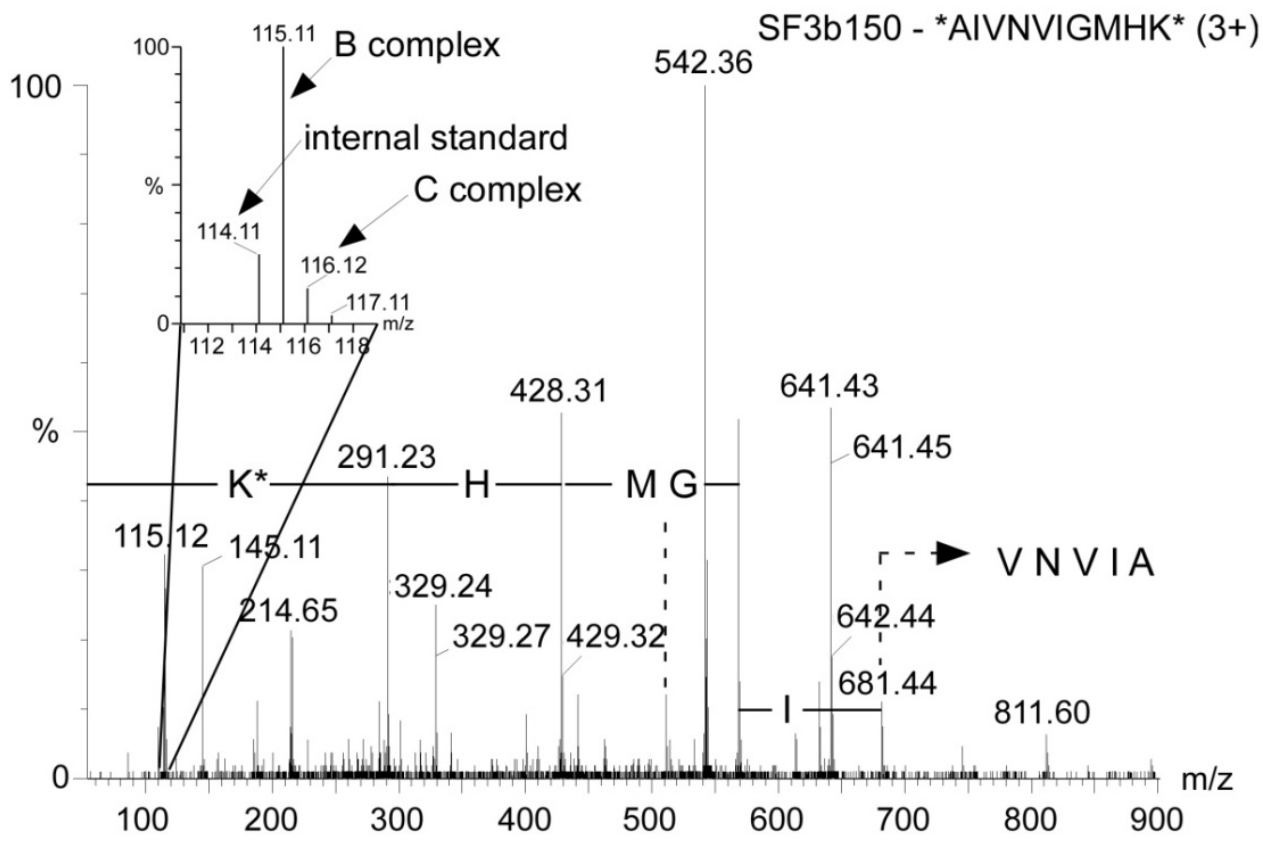

Figure 4.18: Example MS/MS spectrum for ITRAQ quantification of spliceosomal $B$ and $C$ complexes. The peptide AIVNVIGMHK (SF3b130) was labeled $\left({ }^{*}\right)$ with iTRAQ reagents at the N-terminus and at the C-terminal lysine. The iTRAQ reporter region $(\mathrm{m} / \mathrm{z} 114-117)$ is magnified showing the reporter ions. The peptide was quantified by comparing the area of $\mathrm{TTRAQ}$ reporter ions generated from $\mathrm{B}$ and $\mathrm{C}$ complexes after fragmentation. 
In total, 265 proteins were identified and quantified by ITRAQ from two independent replicates. From these, 186 proteins were detected in both replicates. (For the complete list of identified and quantified proteins in the two replicates see Tables A.5 and A.6 in the Appendix.) Spliceosomal proteins identified and quantified within the two replicates were combined in groups of proteins according to their particle or functional association (Bessonov et al., 2008; Deckert et al., 2006).

Calculated protein ratios were normalized on four proteins known to be present with the same abundance in B and C complexes. These are the two cap binding proteins CBP20 and CBP80 and the U5 snRNP specific proteins U5-220K and U5-200K. A normalization factor was calculated from protein ratios of these proteins and applied to all protein ratios obtained by ITRAQ. Table 4.10 shows the list of spliceosomal proteins quantified in $\mathrm{B}$ and $\mathrm{C}$ complexes. The protein ratio $(\mathrm{B} / \mathrm{C})$, the standard deviation of the calculated protein ratio, and the number of peptides used for quantification for the two replicates as well as the average values are given. 
Table 4.10: Relative quantification of spliceosomal B and C complexes by iTRAQ. The protein ratio (B/C), the standard deviation (StDev), and the number of peptides used to calculate the protein ratio (\#) are given for two replicates (iTRAQ 1 and 2). The mean protein ratio and the corresponding standard deviation were calculated from the two replicates (iTRAQ mean). Proteins without assigned value could not be quantified.

\begin{tabular}{|c|c|c|c|c|c|c|c|c|c|c|}
\hline \multirow{2}{*}{ Protein } & \multirow{2}{*}{$\begin{array}{c}\text { MW } \\
\text { [kDa] }\end{array}$} & \multirow{2}{*}{$\begin{array}{c}\text { accession } \\
\text { no. }\end{array}$} & \multicolumn{3}{|c|}{ iTRAQ 1} & \multicolumn{3}{|c|}{ iTRAQ 2} & \multicolumn{2}{|c|}{ iTRAQ mean } \\
\hline & & & $\mathrm{B} / \mathrm{C}$ & StDev & \# & $\mathrm{B} / \mathrm{C}$ & StDev & \# & $\mathrm{B} / \mathrm{C}$ & StDev \\
\hline $\begin{array}{l}\text { U1 snRNP } \\
\text { U1-A } \\
\text { U1-C } \\
\text { U1-70K }\end{array}$ & $\begin{array}{l}31.3 \\
17.4 \\
51.6 \\
\end{array}$ & $\begin{array}{l}\text { gi|4759156 } \\
\text { gi|4507127 } \\
\text { gi|29568103 }\end{array}$ & 3.24 & 1.42 & 12 & $\begin{array}{l}22.35 \\
7.08 \\
\end{array}$ & $\begin{array}{r}7.32 \\
1.10 \\
\end{array}$ & 5 & $\begin{array}{r}22.35 \\
5.16 \\
\end{array}$ & 2.71 \\
\hline $\begin{array}{l}\text { 17S U2 snRNP } \\
\text { U2A' } \\
\text { U2B" } \\
\text { SF3a120 } \\
\text { SF3a66 } \\
\text { SF3a60 } \\
\text { SF3b155 } \\
\text { SF3b145 } \\
\text { SF3b130 } \\
\text { SF3b49 } \\
\text { SF3b14a (p14) } \\
\text { SF3b14b } \\
\text { SF3b10 }\end{array}$ & \begin{tabular}{|c|}
28.4 \\
25.4 \\
88.9 \\
49.3 \\
58.5 \\
145.8 \\
100.2 \\
135.5 \\
44.4 \\
14.6 \\
12.4 \\
10.1
\end{tabular} & $\begin{array}{l}\text { gi|50593002 } \\
\text { gi|} 4507123 \\
\text { gi|5032087 } \\
\text { gi|21361376 } \\
\text { gi|5803167 } \\
\text { gi|54112117 } \\
\text { gi|55749531 } \\
\text { gi|54112121 } \\
\text { gi|5032069 } \\
\text { gi| } 7706326 \\
\text { gi||14249398 } \\
\text { gi||3775200 }\end{array}$ & $\begin{array}{l}0.48 \\
0.53 \\
2.66 \\
3.94 \\
4.91 \\
3.98 \\
4.63 \\
5.01 \\
5.67 \\
4.25 \\
3.75\end{array}$ & $\begin{array}{l}0.18 \\
0.25 \\
0.58 \\
0.71 \\
1.28 \\
2.36 \\
1.53 \\
1.85 \\
1.26 \\
1.31 \\
1.04\end{array}$ & $\begin{array}{c}23 \\
14 \\
26 \\
7 \\
24 \\
68 \\
21 \\
98 \\
2 \\
11 \\
10\end{array}$ & $\begin{array}{l}1.39 \\
1.74 \\
2.64 \\
8.09 \\
6.68 \\
3.66 \\
4.13 \\
3.52 \\
5.33 \\
4.37 \\
2.00\end{array}$ & $\begin{array}{l}0.20 \\
0.42 \\
0.44 \\
0.43 \\
0.65 \\
2.15 \\
0.93 \\
0.72 \\
2.56 \\
0.60 \\
0.25\end{array}$ & $\begin{array}{c}24 \\
12 \\
29 \\
6 \\
14 \\
118 \\
34 \\
49 \\
4 \\
14 \\
2\end{array}$ & $\begin{array}{l}0.93 \\
1.14 \\
2.65 \\
6.02 \\
5.80 \\
3.82 \\
4.38 \\
4.27 \\
5.50 \\
4.31 \\
2.87\end{array}$ & $\begin{array}{l}0.65 \\
0.86 \\
0.01 \\
2.93 \\
1.25 \\
0.22 \\
0.35 \\
1.05 \\
0.24 \\
0.08 \\
1.23\end{array}$ \\
\hline $\begin{array}{l}\text { 17S U2 related } \\
\text { U2AF65 } \\
\text { U2AF35 } \\
\text { hPRP43 } \\
\text { SPF45 } \\
\text { SR140 } \\
\text { CHERP } \\
\text { SF3b125 } \\
\end{array}$ & \begin{tabular}{|c|}
53.5 \\
27.9 \\
90.9 \\
45.0 \\
118.2 \\
100.0 \\
103.0 \\
\end{tabular} & \begin{tabular}{|l|} 
gi|6005926 \\
gi|5803207 \\
gi|68509926 \\
gi|14249678 \\
gi|122937227 \\
gi|119226260 \\
gi|45446747 \\
\end{tabular} & \begin{tabular}{|c|}
3.63 \\
5.59 \\
5.98 \\
5.38 \\
3.04 \\
5.36 \\
10.06 \\
\end{tabular} & $\begin{array}{l}0.80 \\
2.69 \\
2.21 \\
1.25 \\
0.17 \\
0.05 \\
4.64 \\
\end{array}$ & $\begin{array}{c}2 \\
8 \\
28 \\
5 \\
3 \\
2 \\
6 \\
\end{array}$ & $\begin{array}{l}2.59 \\
6.22 \\
4.82 \\
4.27 \\
6.19 \\
\end{array}$ & $\begin{array}{l}0.35 \\
0.84 \\
0.80 \\
1.06 \\
2.07 \\
\end{array}$ & $\begin{array}{c}37 \\
6 \\
5 \\
5 \\
6 \\
\end{array}$ & $\begin{array}{l}3.63 \\
5.59 \\
4.28 \\
5.80 \\
3.93 \\
4.82 \\
8.12 \\
\end{array}$ & $\begin{array}{l}2.39 \\
0.60 \\
1.26 \\
0.77 \\
2.73 \\
\end{array}$ \\
\hline $\begin{array}{l}\text { U5 snRNP } \\
220 \mathrm{~K} \\
200 \mathrm{~K} \\
116 \mathrm{~K} \\
40 \mathrm{~K} \\
102 \mathrm{~K} \\
15 \mathrm{~K} \\
100 \mathrm{~K} \\
52 \mathrm{~K} \\
\end{array}$ & \begin{tabular}{|c|}
273.3 \\
244.5 \\
109.4 \\
39.3 \\
106.9 \\
16.8 \\
95.6 \\
37.6 \\
\end{tabular} & $\begin{array}{l}\text { gi|3661610 } \\
\text { gi|45861372 } \\
\text { gi|41152056 } \\
\text { gi|4758560 } \\
\text { gi|40807485 } \\
\text { gi|5729802 } \\
\text { gi|41327771 } \\
\text { gi|5174409 }\end{array}$ & $\begin{array}{l}1.18 \\
1.05 \\
0.96 \\
1.03 \\
2.77 \\
7.03 \\
1.57 \\
1.06 \\
\end{array}$ & $\begin{array}{l}0.20 \\
0.24 \\
0.28 \\
0.18 \\
0.78 \\
1.46 \\
0.38 \\
0.57\end{array}$ & $\begin{array}{c}154 \\
211 \\
52 \\
20 \\
55 \\
3 \\
48 \\
3 \\
\end{array}$ & $\begin{array}{l}1.00 \\
1.06 \\
1.09 \\
1.33 \\
1.81 \\
4.73 \\
1.09 \\
3.50 \\
\end{array}$ & $\begin{array}{l}0.15 \\
0.20 \\
0.27 \\
0.18 \\
0.54 \\
0.45 \\
0.16 \\
1.17 \\
\end{array}$ & $\begin{array}{c}158 \\
168 \\
73 \\
16 \\
64 \\
3 \\
33 \\
5\end{array}$ & $\begin{array}{l}1.09 \\
1.05 \\
1.03 \\
1.18 \\
2.29 \\
5.88 \\
1.33 \\
2.28 \\
\end{array}$ & $\begin{array}{l}0.13 \\
0.01 \\
0.10 \\
0.22 \\
0.68 \\
1.63 \\
0.34 \\
1.72 \\
\end{array}$ \\
\hline $\begin{array}{l}\text { U4/U6 snRNP } \\
90 \mathrm{~K} \\
60 \mathrm{~K} \\
20 \mathrm{~K} \\
61 \mathrm{~K} \\
15.5 \mathrm{~K}\end{array}$ & $\begin{array}{l}77.6 \\
58.4 \\
20.0 \\
55.4 \\
14.2 \\
\end{array}$ & $\begin{array}{l}\text { gi|4758556 } \\
\text { gi|45861374 } \\
\text { gi|5454154 } \\
\text { gi|40254869 } \\
\text { gi|4826860 } \\
\end{array}$ & \begin{tabular}{|l|}
8.27 \\
8.18 \\
2.50 \\
7.43 \\
9.12 \\
\end{tabular} & $\begin{array}{l}3.88 \\
2.98 \\
0.33 \\
3.30 \\
2.96 \\
\end{array}$ & $\begin{array}{c}37 \\
26 \\
3 \\
26 \\
2\end{array}$ & $\begin{array}{c}4.37 \\
5.83 \\
2.55 \\
5.50 \\
16.96 \\
\end{array}$ & $\begin{array}{l}1.59 \\
0.85 \\
0.40 \\
1.46 \\
0.28 \\
\end{array}$ & $\begin{array}{c}39 \\
20 \\
4 \\
29 \\
2 \\
\end{array}$ & $\begin{array}{c}6.32 \\
7.00 \\
2.53 \\
6.46 \\
13.04 \\
\end{array}$ & $\begin{array}{l}2.75 \\
1.66 \\
0.04 \\
1.37 \\
5.54 \\
\end{array}$ \\
\hline $\begin{array}{l}\text { U4/U6.U5 snRNP } \\
\text { 110K } \\
65 \mathrm{~K} \\
\text { 27K (RY1) } \\
\text { hPRP38 } \\
\text { TFIP11 }\end{array}$ & $\begin{array}{l}90.2 \\
65.4 \\
18.9 \\
37.5 \\
96.8\end{array}$ & $\begin{array}{l}\text { gi|13926068 } \\
\text { gi||56550051 } \\
\text { gi|24307919 } \\
\text { gi|24762236 } \\
\text { gi|8393259 }\end{array}$ & $\begin{array}{l}4.95 \\
6.50\end{array}$ & $\begin{array}{l}1.96 \\
0.36\end{array}$ & $\begin{array}{l}23 \\
21\end{array}$ & $\begin{array}{l}3.60 \\
1.63\end{array}$ & $\begin{array}{l}1.09 \\
0.21\end{array}$ & $\begin{array}{l}19 \\
19\end{array}$ & $\begin{array}{l}4.05 \\
1.70\end{array}$ & $\begin{array}{l}0.63 \\
0.11\end{array}$ \\
\hline
\end{tabular}


RESULTS | 97

\begin{tabular}{|c|c|c|c|c|c|c|c|c|c|c|}
\hline \multirow{2}{*}{ Protein } & \multirow{2}{*}{$\begin{array}{c}\text { MW } \\
\text { [kDa] }\end{array}$} & \multirow{2}{*}{$\begin{array}{c}\text { accession } \\
\text { no. }\end{array}$} & \multicolumn{3}{|c|}{ iTRAQ 1} & \multicolumn{3}{|c|}{ ITRAQ 2} & \multicolumn{2}{|c|}{ iTRAQ mean } \\
\hline & & & $\mathrm{B} / \mathrm{C}$ & StDev & \# & $\mathrm{B} / \mathrm{C}$ & StDev & \# & $\mathrm{B} / \mathrm{C}$ & StDev \\
\hline $\begin{array}{l}\text { LSm Proteins } \\
\text { LSM } 2 \\
\text { LSM } 3 \\
\text { LSM } 4 \\
\text { LSM } 6 \\
\text { LSM } 7 \\
\text { LSM } 8\end{array}$ & $\begin{array}{c}10.8 \\
11.8 \\
15.4 \\
9.1 \\
11.6 \\
10.4\end{array}$ & $\begin{array}{l}\text { gi|10863977 } \\
\text { gi|7657315 } \\
\text { gi|6912486 } \\
\text { gi|5919153 } \\
\text { gi|7706423 } \\
\text { gi|7706425 }\end{array}$ & $\begin{array}{l}5.24 \\
3.45 \\
4.78 \\
5.64 \\
6.61 \\
3.55 \\
\end{array}$ & $\begin{array}{l}1.28 \\
\\
1.60 \\
2.12 \\
3.62 \\
1.53 \\
\end{array}$ & $\begin{array}{l}4 \\
1 \\
8 \\
4 \\
8 \\
3\end{array}$ & $\begin{array}{l}4.47 \\
3.21 \\
5.52 \\
6.16 \\
3.70 \\
4.14 \\
\end{array}$ & $\begin{array}{l}0.39 \\
\\
2.31 \\
1.02 \\
0.08 \\
0.70\end{array}$ & $\begin{array}{l}8 \\
1 \\
2 \\
3 \\
2 \\
2\end{array}$ & $\begin{array}{l}4.86 \\
3.33 \\
5.15 \\
5.90 \\
5.15 \\
3.85 \\
\end{array}$ & $\begin{array}{l}0.54 \\
0.17 \\
0.53 \\
0.36 \\
2.06 \\
0.41\end{array}$ \\
\hline $\begin{array}{l}\text { Sm Proteins } \\
\text { B } \\
\text { D1 } \\
\text { D2 } \\
\text { D3 } \\
\text { E } \\
\text { F } \\
\text { G }\end{array}$ & \begin{tabular}{|c|}
24.6 \\
13.3 \\
13.5 \\
13.9 \\
10.8 \\
9.7 \\
8.5 \\
\end{tabular} & $\begin{array}{l}\text { gi|4507125 } \\
\text { gi|} \mid 5902102 \\
\text { gi|29294624 } \\
\text { gi|4759160 } \\
\text { gi|4507129 } \\
\text { gi|4507131 } \\
\text { gi|4507133 }\end{array}$ & $\begin{array}{l}1.69 \\
1.39 \\
1.62 \\
1.40 \\
1.20 \\
2.91 \\
1.52\end{array}$ & $\begin{array}{l}0.28 \\
0.26 \\
0.66 \\
0.32 \\
0.17 \\
0.93 \\
0.44\end{array}$ & $\begin{array}{c}13 \\
6 \\
35 \\
32 \\
11 \\
2 \\
4\end{array}$ & $\begin{array}{l}1.70 \\
1.86 \\
1.88 \\
2.08 \\
2.03 \\
1.40 \\
1.19\end{array}$ & $\begin{array}{l}0.29 \\
0.51 \\
0.30 \\
0.44 \\
0.47 \\
0.37 \\
0.09\end{array}$ & $\begin{array}{c}15 \\
7 \\
22 \\
19 \\
7 \\
6 \\
5\end{array}$ & $\begin{array}{l}1.70 \\
1.62 \\
1.75 \\
1.74 \\
1.61 \\
2.15 \\
1.36 \\
\end{array}$ & $\begin{array}{l}0.01 \\
0.34 \\
0.18 \\
0.48 \\
0.59 \\
1.07 \\
0.23\end{array}$ \\
\hline $\begin{array}{l}\text { hPRP19/CDC5L } \\
\text { complex } \\
\text { hPrp19 } \\
\text { CDC5L } \\
\text { SPF27 } \\
\text { PRL1 } \\
\text { Hsp70 } \\
\text { AD-002 } \\
\text { CTNNBL1 } \\
\text { Npw38BP } \\
\text { Npw38 }\end{array}$ & $\begin{array}{l}55.2 \\
92.2 \\
21.5 \\
57.2 \\
70.4 \\
26.6 \\
65.1 \\
70.0 \\
30.5\end{array}$ & $\begin{array}{l}\text { gi|7657381 } \\
\text { gi|11067747 } \\
\text { gi|5031653 } \\
\text { gi|4505895 } \\
\text { gi|} \mid 5729877 \\
\text { gi|7705475 } \\
\text { gi||18644734 } \\
\text { gi|7706501 } \\
\text { gi|74735456 }\end{array}$ & $\begin{array}{l}0.62 \\
0.23 \\
0.53 \\
0.69 \\
0.25 \\
0.22 \\
1.44 \\
7.31\end{array}$ & $\begin{array}{l}0.36 \\
0.14 \\
0.13 \\
0.22 \\
0.14 \\
0.08 \\
0.46 \\
0.72\end{array}$ & $\begin{array}{l}61 \\
45 \\
18 \\
33 \\
15 \\
6 \\
4 \\
6\end{array}$ & $\begin{array}{l}0.57 \\
0.19 \\
0.55 \\
1.06 \\
1.19 \\
0.15 \\
2.29 \\
8.77 \\
7.68 \\
\end{array}$ & $\begin{array}{l}0.12 \\
0.12 \\
0.12 \\
0.22 \\
0.22 \\
0.08 \\
0.34 \\
2.32 \\
1.60 \\
\end{array}$ & $\begin{array}{c}62 \\
30 \\
15 \\
6 \\
10 \\
3 \\
11 \\
17 \\
3 \\
\end{array}$ & $\begin{array}{l}0.60 \\
0.21 \\
0.54 \\
0.87 \\
0.72 \\
0.18 \\
1.86 \\
8.04 \\
7.68 \\
\end{array}$ & $\begin{array}{l}0.04 \\
0.03 \\
0.01 \\
0.26 \\
0.67 \\
0.05 \\
0.60 \\
1.04\end{array}$ \\
\hline $\begin{array}{l}\text { hPRP19/CDC5L } \\
\text { related } \\
\text { hSYF1 } \\
\text { CRNKL1 } \\
\text { hlsy1 } \\
\text { SKIP } \\
\text { RBM22 }\end{array}$ & \begin{tabular}{|c|}
100.0 \\
100.6 \\
33.0 \\
51.1 \\
46.9 \\
\end{tabular} & $\begin{array}{l}\text { gi|55770906 } \\
\text { gi|30795220 } \\
\text { gi|20149304 } \\
\text { gi|6912676 } \\
\text { gi|8922328 }\end{array}$ & $\begin{array}{l}0.27 \\
0.47 \\
0.20 \\
0.58 \\
0.45 \\
\end{array}$ & $\begin{array}{l}0.09 \\
0.52 \\
0.07 \\
0.17 \\
0.13\end{array}$ & $\begin{array}{l}62 \\
88 \\
16 \\
58 \\
35\end{array}$ & $\begin{array}{l}0.38 \\
0.32 \\
0.61 \\
0.63 \\
0.53 \\
\end{array}$ & $\begin{array}{l}0.08 \\
0.07 \\
0.10 \\
0.10 \\
0.11\end{array}$ & $\begin{array}{l}65 \\
82 \\
17 \\
42 \\
39 \\
\end{array}$ & $\begin{array}{l}0.33 \\
0.39 \\
0.40 \\
0.60 \\
0.49\end{array}$ & $\begin{array}{l}0.08 \\
0.11 \\
0.28 \\
0.03 \\
0.06\end{array}$ \\
\hline $\begin{array}{l}\text { Cyp-E } \\
\text { PPIL1 } \\
\text { KIAA0560 } \\
\text { G10 }\end{array}$ & \begin{tabular}{|c|}
33.4 \\
18.2 \\
171.3 \\
17.0 \\
\end{tabular} & \begin{tabular}{|l|} 
gi| 5174637 \\
gi|7706339 \\
gi|38788372 \\
gi| 32171175 \\
\end{tabular} & $\begin{array}{l}0.13 \\
0.42 \\
0.09 \\
0.39 \\
\end{array}$ & $\begin{array}{l}0.05 \\
0.18 \\
0.05 \\
0.19\end{array}$ & $\begin{array}{c}8 \\
10 \\
96 \\
14\end{array}$ & $\begin{array}{l}0.58 \\
0.45 \\
0.39 \\
0.88 \\
\end{array}$ & $\begin{array}{l}0.14 \\
0.08 \\
0.13 \\
0.02 \\
\end{array}$ & $\begin{array}{c}5 \\
8 \\
186 \\
4 \\
\end{array}$ & $\begin{array}{l}0.36 \\
0.43 \\
0.24 \\
0.64 \\
\end{array}$ & $\begin{array}{l}0.32 \\
0.02 \\
0.21 \\
0.35\end{array}$ \\
\hline $\begin{array}{l}\text { hRES complex } \\
\text { proteins } \\
\text { SNIP1 } \\
\text { MGC12135 } \\
\text { CGI-79 }\end{array}$ & $\begin{array}{l}45.8 \\
70.5 \\
39.7\end{array}$ & $\begin{array}{l}\text { gi|21314720 } \\
\text { gi|14249338 } \\
\text { gi|4929627 }\end{array}$ & $\begin{array}{l}0.80 \\
1.97 \\
0.76\end{array}$ & $\begin{array}{l}0.11 \\
0.71 \\
0.11\end{array}$ & $\begin{array}{c}10 \\
10 \\
3\end{array}$ & $\begin{array}{l}1.66 \\
0.94 \\
1.36\end{array}$ & $\begin{array}{l}0.15 \\
0.38 \\
0.10\end{array}$ & $\begin{array}{c}2 \\
10 \\
2\end{array}$ & $\begin{array}{l}1.23 \\
1.46 \\
1.06 \\
\end{array}$ & $\begin{array}{l}0.61 \\
0.73 \\
0.42\end{array}$ \\
\hline $\begin{array}{l}\text { Proteins } \\
\text { recruited to A } \\
\text { complex } \\
\text { RBM39 } \\
\text { p68 (DDX5) } \\
\text { ELAV-like } 1 \text { (HuR) } \\
\text { p72/DDX17 }\end{array}$ & $\begin{array}{l}59.4 \\
69.2 \\
36.1 \\
80.5\end{array}$ & $\begin{array}{l}\text { gi|4757926 } \\
\text { gi|4758138 } \\
\text { gi|38201714 } \\
\text { gi|3122595 }\end{array}$ & $\begin{array}{l}4.08 \\
1.52 \\
4.76 \\
1.07\end{array}$ & $\begin{array}{l}0.89 \\
0.55 \\
2.29\end{array}$ & $\begin{array}{c}17 \\
8 \\
27 \\
1\end{array}$ & $\begin{array}{l}4.07 \\
2.30 \\
5.65 \\
2.30\end{array}$ & $\begin{array}{l}0.73 \\
0.45 \\
0.82 \\
0.73\end{array}$ & $\begin{array}{c}13 \\
15 \\
18 \\
2\end{array}$ & $\begin{array}{l}4.07 \\
1.91 \\
5.20 \\
1.69\end{array}$ & $\begin{array}{l}0.01 \\
0.55 \\
0.63 \\
0.87\end{array}$ \\
\hline
\end{tabular}




\begin{tabular}{|c|c|c|c|c|c|c|c|c|c|c|}
\hline \multirow{2}{*}{ Protein } & \multirow{2}{*}{$\begin{array}{c}\text { MW } \\
{[k D a]}\end{array}$} & \multirow{2}{*}{$\begin{array}{c}\text { accession } \\
\text { no. }\end{array}$} & \multicolumn{3}{|c|}{ ITRAQ 1} & \multicolumn{3}{|c|}{ ITRAQ 2} & \multicolumn{2}{|c|}{ ITRAQ mean } \\
\hline & & & $B / C$ & StDev & \# & $\mathrm{B} / \mathrm{C}$ & StDev & $\#$ & $\mathrm{~B} / \mathrm{C}$ & StDev \\
\hline \multicolumn{11}{|l|}{$\begin{array}{l}\text { Proteins } \\
\text { recruited to B } \\
\text { complex }\end{array}$} \\
\hline MFAP1 & 51.9 & gi|50726968 & 3.54 & 1.00 & 38 & 4.77 & 0.56 & 11 & 4.15 & 0.87 \\
\hline RED & 65.6 & gi|10835234 & 4.64 & 2.17 & 23 & 7.21 & 2.03 & 20 & 5.92 & 1.82 \\
\hline hSmu-1 & 57.5 & gi|8922679 & 5.67 & 2.36 & 44 & 4.00 & 0.65 & 4 & 4.83 & 1.18 \\
\hline PPIL2 & 59.5 & gi|7657473 & 1.33 & 0.88 & 19 & 2.96 & 0.74 & 32 & 2.15 & 1.15 \\
\hline hPRP2 (DDX16) & $\mid 119.2$ & gi|4503293 & 0.54 & 0.16 & 19 & 2.10 & 0.29 & 20 & 1.32 & 1.10 \\
\hline hPRP4-Kinase & 117.1 & gi|89276756 & 0.59 & 0.19 & 25 & 0.37 & 0.07 & 18 & 0.48 & 0.15 \\
\hline THRAP3 & 108.6 & gi|4827040 & 2.04 & 0.67 & 30 & 1.19 & 0.08 & 4 & 1.62 & 0.60 \\
\hline PABP1 & 70.5 & gi|46367787 & 1.70 & 0.48 & 8 & 4.72 & 2.46 & 4 & 3.21 & 2.13 \\
\hline SKIV2L2 & 117.8 & gi|39930353 & 1.13 & 0.20 & 12 & 1.15 & 0.19 & 8 & 1.14 & 0.01 \\
\hline PABPN1 & 32.6 & gi|4758876 & 1.21 & 0.09 & 3 & 0.79 & 0.00 & 2 & 1.00 & 0.29 \\
\hline RNF113A & 38.8 & gi|5902158 & 0.43 & 0.15 & 2 & 1.20 & 0.13 & 3 & 0.81 & 0.55 \\
\hline NY-CO-10 & 53.8 & gi|64276486 & 0.63 & 0.22 & 12 & 0.68 & 0.05 & 2 & 0.66 & 0.03 \\
\hline KIAA1604 & 105.5 & gi|55749769 & 0.35 & 0.15 & 25 & 0.46 & 0.23 & 27 & 0.40 & 0.08 \\
\hline hsp27 & 22.8 & gi|4504517 & 1.02 & & 1 & 0.47 & 0.02 & 2 & 0.74 & 0.39 \\
\hline GCFC & 104.7 & gi|22035565 & 2.12 & 0.42 & 16 & 1.36 & 0.26 & 24 & 1.74 & 0.54 \\
\hline UBL5 & 8.5 & gi|13236510 & 17.04 & & 1 & 10.08 & 6.92 & 2 & 13.56 & 4.92 \\
\hline CCDC16 & 42.0 & gi|49472814 & 0.93 & 0.37 & 11 & 1.38 & 0.25 & 22 & 1.16 & 0.31 \\
\hline CCDC12 & 19.2 & gi|21389497 & 0.53 & 0.17 & 6 & 0.51 & 0.05 & 4 & 0.52 & 0.02 \\
\hline HsKin17 & 45.2 & gi|13124883 & 3.10 & 1.36 & 6 & 2.99 & 057 & 8 & 3.04 & 0.08 \\
\hline \multicolumn{11}{|l|}{ Step 2 factors } \\
\hline hPRP22 & 139.3 & gi|4826690 & 0.13 & 0.12 & 55 & 0.29 & 015 & 89 & 0.21 & 0.11 \\
\hline hPRP18 & 39.9 & gi|4506123 & 0.38 & & 1 & 0.39 & 0.18 & 4 & 0.39 & 0.01 \\
\hline hPRP17 & 65.5 & gi|7706657 & 0.19 & 0.08 & 32 & 0.45 & 0.15 & 57 & 0.32 & 0.19 \\
\hline hPRP16 & 140.5 & gi|17999539 & & & & 0.43 & 0.11 & 12 & 0.43 & \\
\hline hSLU7 & 68.4 & gi|27477111 & 0.10 & 0.10 & 19 & 0.20 & $0 .($ & 38 & 0.15 & 0.07 \\
\hline \multicolumn{11}{|l|}{$\begin{array}{l}\text { Proteins } \\
\text { recruited to C } \\
\text { complex }\end{array}$} \\
\hline Abstrakt & 69.8 & gi|21071032 & 0.08 & 0.06 & 48 & 0.17 & 0.14 & 90 & 0.12 & 0.06 \\
\hline GCIP p29 & 28.7 & gi|46371998 & 0.18 & 0.14 & 11 & 0.18 & 0.07 & 7 & 0.18 & 0.00 \\
\hline DDX35 & 78.9 & gi|20544129 & 0.32 & 0.17 & 28 & 0.17 & 0.07 & 22 & 0.25 & 0.11 \\
\hline $\begin{array}{l}\text { Q9BRR8 } \\
\text { c19orf29 (N) }\end{array}$ & 103.3 & gi|74732921 & 0.40 & 0.09 & 3 & 0.19 & 0.07 & 9 & 0.29 & 0.15 \\
\hline REN-24) & 88.6 & gi|126723149 & 0.17 & 0.13 & 21 & 0.17 & 0.08 & 35 & 0.17 & 0.00 \\
\hline PPlase-like 3b & 18.6 & gi|19557636 & 0.17 & 0.14 & 8 & 0.31 & 0.19 & 3 & 0.24 & 0.10 \\
\hline PPWD1 & 73.6 & gi|24308049 & 0.10 & 0.12 & 39 & 0.20 & 0.09 & 53 & 0.15 & 0.07 \\
\hline MORG1 & 34.3 & gi|153791298 & 0.10 & & 1 & 0.69 & 0.09 & 3 & 0.39 & 0.42 \\
\hline FRG1 & 29.2 & gi|4758404 & & & & 0.58 & 0.17 & 6 & 0.58 & \\
\hline NOSIP & 33.2 & gi|7705716 & 0.11 & 0.08 & 9 & 0.19 & 0.09 & 3 & 0.15 & 0.05 \\
\hline GPKOW & 52.1 & gi|15811782 & 0.66 & 0.21 & 6 & & & & 0.66 & \\
\hline C1orf55 & 39.3 & gi|148664216 & 0.08 & 0.04 & 19 & 0.13 & 0.06 & 14 & 0.10 & 0.03 \\
\hline FAM32A & 13.1 & gi|7661696 & 0.11 & 0.06 & 2 & & & & 0.11 & \\
\hline RACK1 & 35.1 & gi|5174447 & & & & & & & & \\
\hline Тір-49 & 50.2 & gi|4506753 & & & & & & & & \\
\hline \multicolumn{11}{|l|}{$\begin{array}{l}\text { Potential C } \\
\text { complex specific } \\
\text { proteins }\end{array}$} \\
\hline PPIG & 88.5 & gi|42560244 & 0.20 & 0.09 & 6 & & & & 0.20 & \\
\hline FAM50A & 40.1 & gi|4758220 & 0.15 & 0.08 & 15 & 0.29 & 0.12 & 5 & 0.22 & 0.10 \\
\hline FAM50B & 38.6 & gi| 6912326 & 0.13 & 0.03 & 2 & 0.36 & 0.05 & 3 & 0.25 & 0.16 \\
\hline
\end{tabular}




\begin{tabular}{|c|c|c|c|c|c|c|c|c|c|c|}
\hline \multirow{2}{*}{ Protein } & \multirow{2}{*}{$\begin{array}{c}\text { MW } \\
{[\mathrm{kDa}]}\end{array}$} & \multirow{2}{*}{$\begin{array}{l}\text { accession } \\
\text { no. }\end{array}$} & \multicolumn{3}{|c|}{ iTRAQ 1} & \multicolumn{3}{|c|}{ iTRAQ 2} & \multicolumn{2}{|c|}{ iTRAQ mean } \\
\hline & & & $\mathrm{B} / \mathrm{C}$ & StDev & \# & $B / C$ & StDev & \# & $B / C$ & StDev \\
\hline C9orf78 & 33.7 & gi|7706557 & 0.14 & 0.05 & 6 & 0.26 & 0.01 & 2 & 0.20 & 0.09 \\
\hline C10orf4 & 37.5 & gi|24432067 & 0.10 & 0.06 & 2 & 0.11 & 0.05 & 6 & 0.10 & 0.01 \\
\hline CXorf56 & 25.6 & gi|11545813 & 0.08 & 0.08 & 12 & 0.24 & 0.13 & 11 & 0.16 & 0.11 \\
\hline DGCR14 & 52.4 & gi|13027630 & 0.09 & 0.03 & 3 & 0.29 & 0.16 & 8 & 0.19 & 0.14 \\
\hline CCDC130 & 44.7 & gi|13540614 & & & & & & & & \\
\hline TOE1 & 56.4 & gi|156564398 & 0.84 & 0.37 & 3 & & & & 0.84 & \\
\hline NKAP & 47.0 & gi|13375676 & 0.22 & 0.11 & 6 & & & & 0.22 & \\
\hline $\mathrm{ZCCHC} 10$ & 18.4 & gi|8923106 & 0.66 & 0.71 & 2 & & & & 0.66 & \\
\hline CDK10 & 35.4 & gi|16950647 & 0.20 & 0.09 & 3 & 0.24 & 0.10 & 4 & 0.22 & 0.03 \\
\hline TTC14 & 88.2 & gi|33457330 & 0.22 & 0.14 & 8 & 0.32 & 0.10 & 11 & 0.27 & 0.07 \\
\hline WDR70 & 73.2 & gi|8922301 & & & & 1.19 & 0.18 & 5 & 1.19 & \\
\hline NFKBIL1 & 43.1 & gi|26787991 & & & & & & & & \\
\hline JUP & 81.6 & gi|12056468 & & & & & & & & \\
\hline \multicolumn{11}{|l|}{ EJC/mRNP } \\
\hline elF4A3 & 46.9 & gi|7661920 & 0.15 & 0.07 & 31 & & & & 0.15 & \\
\hline Magoh & 17.2 & gi|4505087 & 0.15 & 0.06 & 10 & 0.20 & 0.05 & 5 & 0.18 & 0.04 \\
\hline Y14 & 19.9 & gi|4826972 & 0.40 & 0.14 & 7 & 0.11 & 0.03 & 2 & 0.25 & 0.20 \\
\hline Pinin & 81.6 & gi|33356174 & 2.11 & 0.58 & 5 & 1.89 & 0.06 & 3 & 2.00 & 0.15 \\
\hline RNPS1 & 34.2 & gi|6857826 & 0.63 & 0.17 & 2 & 2.09 & & 1 & 1.36 & 1.04 \\
\hline Acinus & 151.8 & gi|7662238 & 1.35 & 0.42 & 13 & 1.84 & 0.38 & 17 & 1.60 & 0.34 \\
\hline SAP18 & 17.4 & gi|5032067 & 1.37 & 0.21 & 6 & 1.19 & 0.17 & 6 & 1.28 & 0.13 \\
\hline Aly/REF (THOC4) & 26.9 & gi|55770864 & 1.11 & 0.27 & 4 & & & & 1.11 & \\
\hline UAP56 & 49.1 & gi|18375623 & 2.01 & 0.44 & 11 & 4.54 & 1.00 & 3 & 3.28 & 1.79 \\
\hline \multicolumn{11}{|l|}{ TREX } \\
\hline THOC1 & 75.6 & gi|154448890 & 1.13 & 0.42 & 4 & 1.77 & 0.17 & 3 & 1.45 & 0.45 \\
\hline THOC2 & 169.6 & gi|125656165 & 0.62 & 0.17 & 13 & 1.54 & 0.26 & 7 & 1.08 & 0.65 \\
\hline THOC3 & 38.8 & gi|14150171 & & & & & & & & \\
\hline $\begin{array}{l}\text { KIAA0983 } \\
\text { (THOC5) }\end{array}$ & 78.4 & gi|50959110 & 1.44 & 0.46 & 5 & 1.35 & 0.26 & 5 & 1.40 & 0.07 \\
\hline WDR58 (THOC6) & 37.4 & gi|31543164 & 0.51 & 0.25 & 2 & 1.50 & & 1 & 1.00 & 0.70 \\
\hline \multicolumn{11}{|l|}{$\begin{array}{l}\text { pre-mRNA/mRNA } \\
\text { binding proteins }\end{array}$} \\
\hline CBP20 & 18.0 & gi|110349727 & 0.71 & 0.16 & 8 & 0.93 & 0.16 & 10 & 0.82 & 0.16 \\
\hline СВР80 & 91.8 & gi|4505343 & 1.41 & 0.41 & 33 & 1.01 & 0.12 & 32 & 1.21 & 0.28 \\
\hline NF45 & 43.0 & gi|24234747 & & & & 1.94 & 0.32 & 4 & 1.94 & \\
\hline ZC3H18 & 104.0 & gi|31377595 & 0.71 & 0.42 & 11 & 1.68 & 0.36 & 27 & 1.19 & 0.68 \\
\hline YB-1 & 35.9 & gi|34098946 & 1.55 & 0.69 & 9 & 2.27 & 0.02 & 2 & 1.91 & 0.51 \\
\hline ELG & 38.9 & gi|8923771 & 3.11 & 0.54 & 5 & 1.08 & & 1 & 2.10 & 1.44 \\
\hline DDX3 & 73.3 & gi|87196351 & 1.05 & 0.16 & 2 & & & & 1.05 & \\
\hline ASR2B & 100.0 & gi|33383233 & 1.00 & 0.27 & 15 & 1.55 & 0.20 & 32 & 1.28 & 0.39 \\
\hline BCLAF1 & 107.2 & gi|7661958 & 1.61 & 0.24 & 23 & 2.23 & 0.31 & 6 & 1.92 & 0.44 \\
\hline DBPA & 40.1 & gi|20070160 & & & & & & & & \\
\hline RBM7 & 30.5 & gi|4503293 & 1.14 & & 1 & & & & 1.14 & \\
\hline HSP70 & 70.0 & gi|5123454 & 0.25 & 0.14 & 15 & 1.19 & 0.22 & 10 & 0.72 & 0.67 \\
\hline $\begin{array}{l}\text { Miscelleneous } \\
\text { proteins }\end{array}$ & & & & & & & & & & \\
\hline BAG2 & 23.4 & gi|4757834 & & & & 0.50 & & 1 & 0.50 & \\
\hline RBBP6 & 197.2 & gi|33620716 & 1.38 & & 1 & & & & 1.38 & \\
\hline RBM42 & 50.3 & gi|21359951 & & & & & & & & \\
\hline
\end{tabular}




\begin{tabular}{|c|c|c|c|c|c|c|c|c|c|c|}
\hline \multirow{2}{*}{ Protein } & \multirow{2}{*}{$\begin{array}{c}\text { MW } \\
{[k D a]}\end{array}$} & \multirow{2}{*}{$\begin{array}{c}\text { accession } \\
\text { no. }\end{array}$} & \multicolumn{3}{|c|}{ iTRAQ 1} & \multicolumn{3}{|c|}{ ITRAQ 2} & \multicolumn{2}{|c|}{ iTRAQ mean } \\
\hline & & & $\mathrm{B} / \mathrm{C}$ & StDev & $\#$ & $\mathrm{~B} / \mathrm{C}$ & StDev & $\#$ & $\mathrm{~B} / \mathrm{C}$ & StDev \\
\hline $\begin{array}{l}\text { SR related } \\
\text { proteins }\end{array}$ & & & & & & & & & & \\
\hline SRm160 & 102.5 & gi|42542379 & 1.12 & 0.16 & 5 & & & & 1.12 & \\
\hline SRm300 & 300.0 & gi|4759098 & 0.31 & 0.13 & 7 & 1.63 & 0.85 & 4 & 0.97 & 0.93 \\
\hline SR proteins & & & & & & & & & & \\
\hline SF2/ASF & 27.8 & gi|5902076 & 0.80 & 0.19 & 14 & 2.46 & 0.37 & 8 & 1.63 & 1.17 \\
\hline $9 \mathrm{G} 8$ & 27.4 & gi|72534660 & 1.16 & 0.32 & 24 & 2.58 & 0.38 & 9 & 1.87 & 1.00 \\
\hline SRp20 & 19.4 & gi|4506901 & 1.65 & 0.44 & 8 & 2.53 & & 1 & 2.09 & 0.62 \\
\hline SRp30c & 25.5 & gi|4506903 & 0.99 & 0.20 & 16 & 0.53 & & 1 & 0.76 & 0.33 \\
\hline SRp38 & 31.3 & gi|5730079 & 0.91 & 0.52 & 15 & 1.44 & 0.32 & 7 & 1.18 & 0.38 \\
\hline SRp40 & 31.3 & gi|3929378 & 0.85 & 0.07 & 4 & 1.83 & 0.26 & 5 & 1.34 & 0.70 \\
\hline SRp46 & 31.2 & gi|15055543 & 1.85 & 1.08 & 2 & & & & 1.85 & \\
\hline SRp55 & 39.6 & gi|20127499 & 1.35 & 0.23 & 17 & 0.88 & 0.18 & 7 & 1.11 & 0.33 \\
\hline SRp75 & 56.8 & gi|21361282 & & & & 1.16 & 0.17 & 2 & 1.16 & \\
\hline SC35 (SFRS2) & 25.5 & gi|47271443 & & & & & & & & \\
\hline hTra-2 alpha & 32.7 & gi|9558733 & 1.44 & 0.70 & 9 & 2.80 & 0.47 & 2 & 2.12 & 0.97 \\
\hline hTra-2 beta & 33.7 & gi|4759098 & 1.37 & 1.30 & 22 & 1.22 & 0.37 & 9 & 1.30 & 0.10 \\
\hline hnRNP & & & & & & & & & & \\
\hline hnRNP A1 & 38.7 & gi|4504445 & 3.44 & 1.81 & 6 & 5.21 & 0.13 & 2 & 4.32 & 1.25 \\
\hline hnRNP A3 & 39.6 & gi|34740329 & 4.62 & 1.12 & 6 & 5.14 & & 1 & 4.88 & 0.37 \\
\hline hnRNP AB & 36.0 & gi|12803583 & & & & & & & & \\
\hline hnRNP A2/B1 & 37.4 & gi|14043072 & 4.63 & 1.96 & 6 & 3.60 & 0.18 & 4 & 4.11 & 0.73 \\
\hline hnRNP C & 33.3 & gi|4758544 & 1.72 & 0.47 & 23 & 1.97 & 0.30 & 16 & 1.84 & 0.17 \\
\hline hnRNP D & 38.4 & gi|14110420 & & & & & & & & \\
\hline hnRNP F & 45.7 & gi|148470406 & & & & 2.94 & & 1 & 2.94 & \\
\hline hnRNP G & 47.4 & gi|56699409 & 1.52 & 0.59 & 32 & 2.21 & 0.35 & 6 & 1.87 & 0.49 \\
\hline hnRNP G-T & 42.7 & gi|153252068 & & & & & & & & \\
\hline hnRNP H1 & 49.1 & gi|5031753 & 1.27 & 0.30 & 4 & 1.84 & 0.27 & 13 & 1.56 & 0.40 \\
\hline hnRNP H3 & 36.9 & gi|14141157 & & & & & & & & \\
\hline hnRNP K & 51.0 & gi|14165435 & 2.97 & & 1 & 4.01 & 0.73 & 2 & 3.49 & 0.74 \\
\hline hnRNP M & 77.5 & gi|14141152 & 1.32 & 0.31 & 8 & 1.37 & 0.38 & 4 & 1.34 & 0.04 \\
\hline hnRNP Q & 69.6 & gi|15809590 & & & & & & & & \\
\hline hnRNP R & 70.9 & gi|5031755 & 0.88 & 0.32 & 8 & 1.09 & & 1 & 0.98 & 0.15 \\
\hline hnRNP U & 90.6 & gi|14141161 & 3.86 & 2.17 & 10 & & & & 3.86 & \\
\hline PCBP1 & 37.5 & gi|5453854 & 5.02 & 1.56 & 5 & 4.66 & 1.45 & 10 & 4.84 & 0.26 \\
\hline РCBP2 & 38.1 & gi|14141166 & 2.58 & 0.29 & 4 & 3.71 & 1.04 & 16 & 3.15 & 0.80 \\
\hline RALY & 32.5 & gi|8051631 & 0.80 & 0.20 & 4 & 2.48 & 0.68 & 3 & 1.64 & 1.19 \\
\hline
\end{tabular}

Several proteins that are more abundant in $\mathrm{B}$ or $\mathrm{C}$ complexes, i.e. they show a protein ratio $>1$ (high abundance in $\mathrm{B}$ complexes) or $<1$ (high abundance in $\mathrm{C}$ complexes), were identified and quantified by iTRAQ. In addition, some proteins show a protein ratio of approximately 1 meaning that they are present in equal amounts within the two complexes compared. The proteins that were used for normalization of this data set show protein ratios of $1.09,1.05,0.82$, and 1.21 (U5-220K, U5-200K, CBP20, and CBP80, respectively) confirming that these proteins are indeed present in a $1: 1$ ratio within $B$ and $C$ complexes and can be used to normalize the protein ratios. Nonetheless, some proteins that have been 
identified during the proteomic analysis of the $\mathrm{B}$ and $\mathrm{C}$ complexes were only quantified in one of the two replicates and few proteins could not be quantified by iTRAQ.

\subsubsection{SILAC quantification of spliceosomal $B$ and $C$ complexes}

Spliceosomal B and C complexes for SILAC quantification were purified as described above (Figure 4.15 B). To this end, heavy and light nuclear extracts (SILAC nuclear extracts) were prepared from metabolically labeled HeLa cells. B complexes were then purified from heavy nuclear extract and $\mathrm{C}$ complexes were purified from light nuclear extract. The purified complexes were pooled in equal amounts and the proteins were separated by gel electrophoresis. After hydrolysis of the proteins, generated peptides were analyzed by LCMS/MS. B and C complexes assembled in metabolically nuclear extracts were purified by J. Deckert and SILAC quantification was performed by M. Grønborg.

To prove that the different metabolically labeled nuclear extracts (i.e. light and heavy SILAC nuclear extracts) show same behavior during pre-mRNA splicing, splicing kinetics, spliceosomal complex formation, and the RNA composition of the purified complexes were monitored for the differently labeled nuclear extracts (Figure 4.19). Analysis from splicing revealed no differences between the two SILAC nuclear extracts (Figure $4.19 \mathrm{~A}$ ). In both cases, i.e. using light or heavy nuclear extract, splicing products first appeared after 10 minutes incubation time and the amount of pre-mRNA was reduced during incubation over 180 minutes. Spliceosomal complex formation was identical for light and heavy nuclear extracts (Figure 4.19 B). H/E complexes were rapidly formed, whereas $A$ and $B$ complexes appeared after 2 to 4 minutes. C complexes were first observed after 10 to 15 minutes of incubation. RNAse $\mathrm{H}$ digestion using DNA oligonucleotides led to degradation of early spliceosomes. The RNA composition of the purified B and C complexes is shown in Figure 14.9 C. Purified B complexes contained equimolar amounts of U1, U2, U4, U5, and U6 snRNA (Figure $14.9 \mathrm{C}$, lane 1), while C complexes contained only U2, U5, and U6 snRNA (Figure $14.9 \mathrm{C}$, lane 3). Splicing products and reduced amounts of pre-mRNA were detected in $\mathrm{C}$ complexes (Figure $14.9 \mathrm{C}$, lane 4), whereas B complexes contained high amounts of pre-mRNA and no splicing products (Figure $14.9 \mathrm{C}$, lane 2). As the light and heavy nuclear extracts show same behavior during pre-mRNA splicing, the proteomes of purified $B$ and $C$ complexes can be relatively compared by SILAC using the tested nuclear extracts. Analysis of the splicing kinetics and the complex formation was performed by J. Deckert. 
A

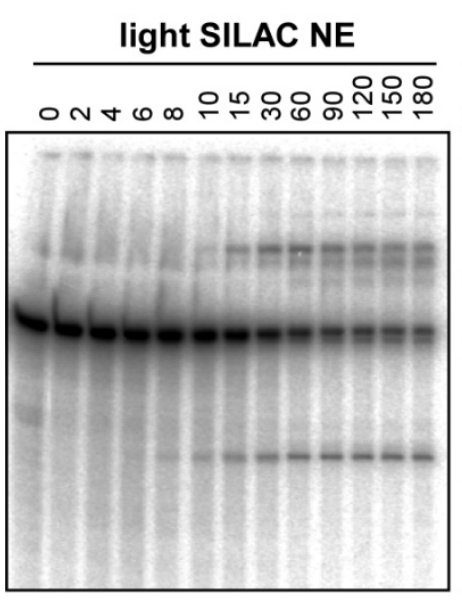

B

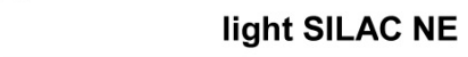

C $\quad \frac{\text { B }}{1 \quad 2} \frac{\text { C }}{3 \quad 4}$ heavy SILAC NE

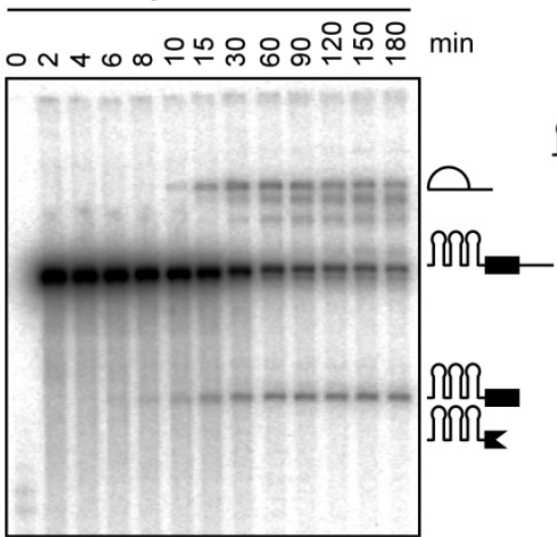

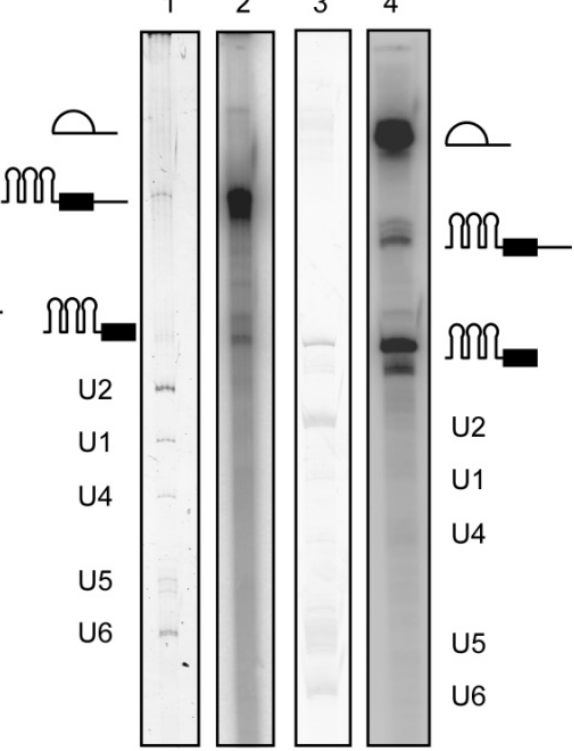

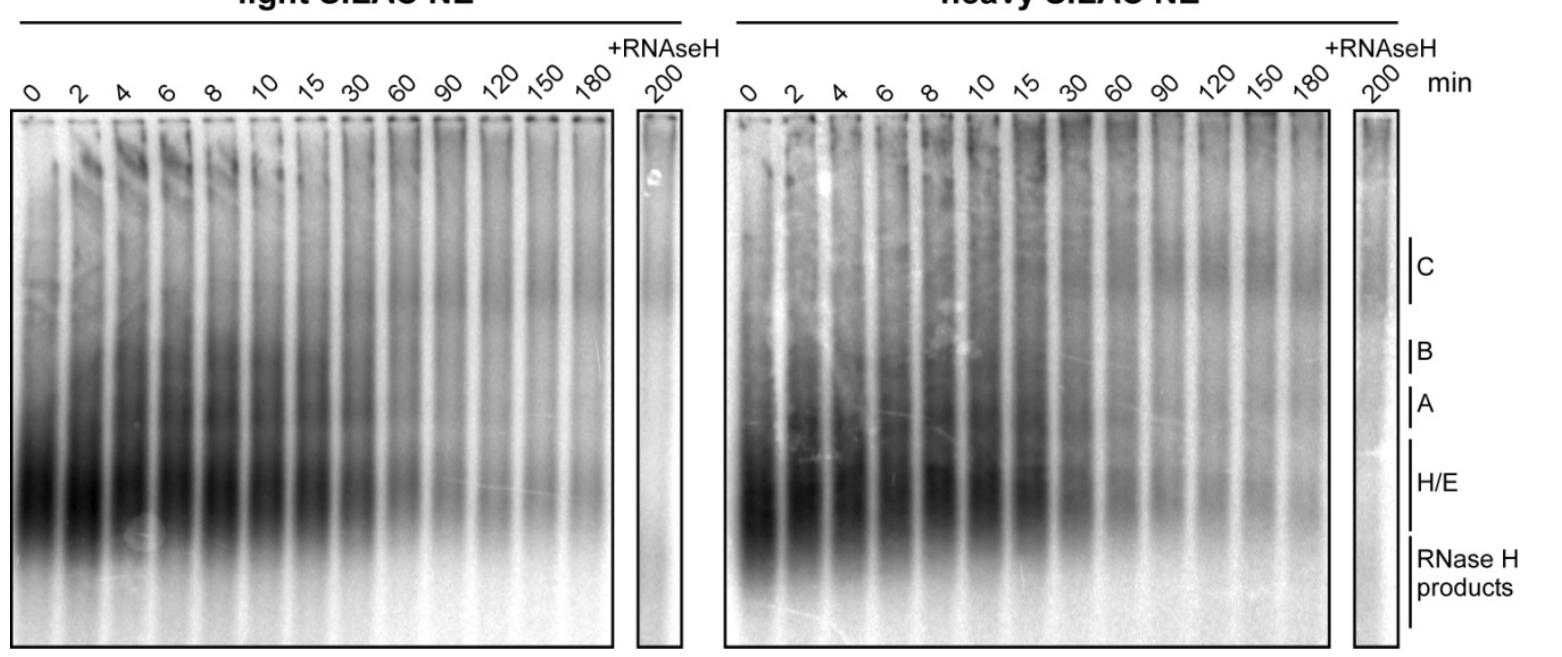

Figure 4.19: Splicing kinetics, complex formation and RNA composition of B and C complexes as obtained using light and heavy SILAC nuclear extracts. The splicing kinetics and the complex formation are identical for light and heavy SILAC nuclear extracts. This figure was kindly provided by J. Deckert. (A) The splicing kinetics were analyzed by denaturing gel electrophoresis of aliquots from splicing rections over $180 \mathrm{~min}$. Pre-mRNA and splicing products were visualized by autoradiography. Splicing products first appeared after 10 min of incubation. (B) The spliceosomal complex formation was assayed by native agarose gel electrophoresis and visualized by autoradiography. $A$ and $B$ complex formation was observed after 2 to 4 min whereas $C$ complexes first appeared after 10 to $15 \mathrm{~min}$. (C) The RNA composition of purified B (heavy nuclear extract) and C (light nuclear extracts) complexes was analyzed by denaturing gel electrophoresis and visualized by silver staining (lanes 1 and 3) or autoradiography (lanes 2 and 4). B complexes contained U1, U2, U4, U5, and U6 snRNA (lane 1) and a high amount of pre-mRNA (lane 2). C complexes contained U2, U5, and U6 snRNA (lane 3 ), splicing products and reduced amounts of pre-mRNA (lane 4). 
Proteins from SILAC labeled $B$ and $C$ complexes were identified by searching peptide and fragment masses against NCBInr database using Mascot as search engine. Peptides generated from the two complexes were quantified by comparing the peak area of the differently labeled peptides in MS spectra. A typical MS spectrum is shown in Figure 4.20. Peptide and resulting protein ratios were obtained using the MS Quant software. In total, 266 proteins could be identified and quantified in the two independent replicates. Of these, 160 proteins were common to both replicates. Obtained protein ratios were normalized as described for iTRAQ (see above) using protein ratios of CBP20, CBP80, U5-220K, and U5$200 \mathrm{~K}$ proteins, which are expected to be present in equal amounts in both complexes. The data are shown in Table 4.11. The protein ratio $(B / C)$, the standard deviation, and the number of peptides used for quantification for the two replicates as well as the average values are given.

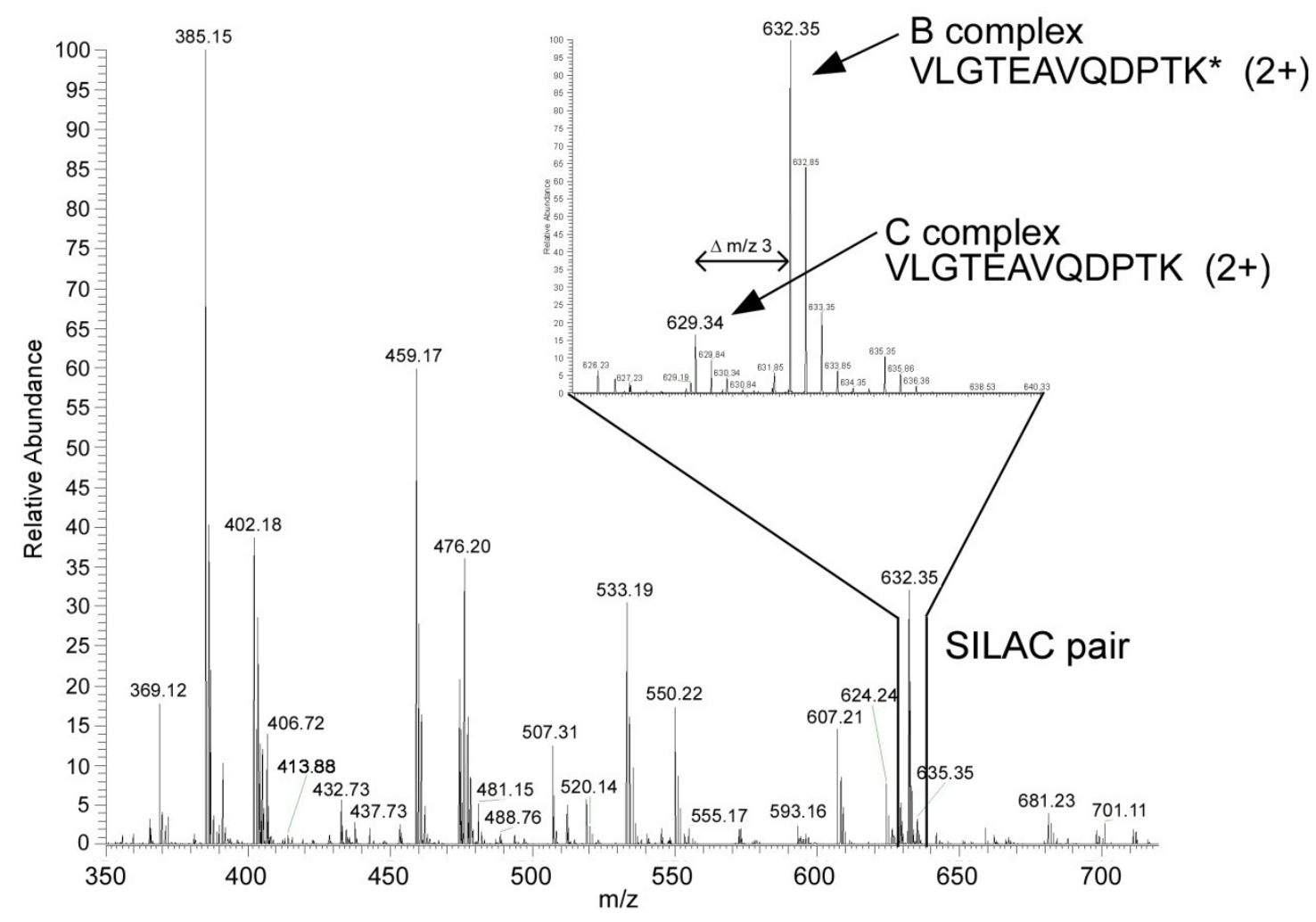

Figure 4.20: Example MS spectrum for SILAC quantification of spliceosomal $B$ and $C$ complexes. The peptide VLGTEAVQDPTK (U4/U6-90K) was metabolically labeled by incorporation of stable isotope labeled lysine $\left(^{*}\right)$. The SILAC pair (i.e. the peptide derived from B and C complexes purified from differently labeled nuclear extracts) is magnified showing different intensities for this particular peptide within the two complexes. The peptide generated from $\mathrm{B}$ and $\mathrm{C}$ complexes was quantified by comparing the peak area of the two differently labeled peptides. 
Table 4.11: Relative quantification of spliceosomal B and C complexes by SILAC. The protein ratio (B/C), the standard deviation (StDev), and the number of peptides used to calculate the protein ratio (\#) are given for two replicates (SILAC 1 and 2). The mean protein ratio and the corresponding standard deviation (SILAC mean) were calculated from the two replicates. Proteins without assigned value could not be quantified.

\begin{tabular}{|c|c|c|c|c|c|c|c|c|c|c|}
\hline \multirow{2}{*}{ Protein } & \multirow{2}{*}{$\begin{array}{c}\text { MW } \\
\text { [kDa] }\end{array}$} & \multirow{2}{*}{$\begin{array}{c}\text { accession } \\
\text { no. }\end{array}$} & \multicolumn{3}{|c|}{ SILAC \#1 } & \multicolumn{3}{|c|}{ SILAC \#2 } & \multicolumn{2}{|c|}{ SILAC mean } \\
\hline & & & $\mathrm{B} / \mathrm{C}$ & StDev & \# & $B / C$ & StDev & \# & $\mathrm{B} / \mathrm{C}$ & StDev \\
\hline $\begin{array}{l}\text { U1 snRNP } \\
\text { U1-A } \\
\text { U1-C } \\
\text { U1-70K }\end{array}$ & $\begin{array}{l}31.3 \\
17.4 \\
51.6 \\
\end{array}$ & $\begin{array}{l}\text { gi|4759156 } \\
\text { gi|4507127 } \\
\text { gi|29568103 }\end{array}$ & $\begin{array}{r}22.18 \\
3.19 \\
\end{array}$ & $\begin{array}{l}3.04 \\
1.40\end{array}$ & 5 & \begin{tabular}{|l|}
29.73 \\
27.15 \\
11.83 \\
\end{tabular} & 0.41 & $\begin{array}{l}1 \\
1 \\
6 \\
\end{array}$ & $\begin{array}{c}25.95 \\
27.15 \\
7.51 \\
\end{array}$ & $\begin{array}{l}5.34 \\
6.11 \\
\end{array}$ \\
\hline $\begin{array}{l}\text { 17S U2 snRNP } \\
\text { U2A' } \\
\text { U2B" } \\
\text { SF3a120 } \\
\text { SF3a66 } \\
\text { SF3a60 } \\
\text { SF3b155 } \\
\text { SF3b145 } \\
\text { SF3b130 } \\
\text { SF3b49 } \\
\text { SF3b14a (p14) } \\
\text { SF3b14b } \\
\text { SF3b10 }\end{array}$ & \begin{tabular}{|c|}
28.4 \\
25.4 \\
88.9 \\
49.3 \\
58.5 \\
145.8 \\
100.2 \\
135.5 \\
44.4 \\
14.6 \\
12.4 \\
10.1
\end{tabular} & $\begin{array}{l}\text { gi|} 50593002 \\
\text { gi|4507123 } \\
\text { gi|5032087 } \\
\text { gi||21361376 } \\
\text { gi|5803167 } \\
\text { gi|54112117 } \\
\text { gi||55749531 } \\
\text { gi|54112121 } \\
\text { gi|5032069 } \\
\text { gi||7706326 } \\
\text { gi||14249398 } \\
\text { gi|| } 13775200\end{array}$ & $\begin{array}{l}1.07 \\
0.96 \\
3.14 \\
3.04 \\
3.16 \\
3.24 \\
3.29 \\
3.40 \\
3.58 \\
5.01\end{array}$ & $\begin{array}{l}0.12 \\
0.45 \\
0.04 \\
0.59 \\
0.77 \\
0.42 \\
0.25 \\
0.35 \\
0.27 \\
0.65\end{array}$ & $\begin{array}{c}24 \\
14 \\
11 \\
13 \\
37 \\
32 \\
25 \\
31 \\
6 \\
6\end{array}$ & $\begin{array}{l}0.78 \\
0.90 \\
3.15 \\
3.30 \\
3.35 \\
4.60 \\
2.95 \\
3.40 \\
2.83 \\
2.80 \\
3.88 \\
3.43 \\
\end{array}$ & $\begin{array}{l}0.03 \\
0.03 \\
0.12 \\
0.01 \\
0.18 \\
1.41 \\
0.20 \\
0.14 \\
0.03 \\
0.09 \\
0.11 \\
0.11\end{array}$ & $\begin{array}{c}25 \\
7 \\
32 \\
2 \\
9 \\
71 \\
42 \\
94 \\
3 \\
10 \\
4 \\
4\end{array}$ & $\begin{array}{l}0.92 \\
0.93 \\
3.15 \\
3.17 \\
3.26 \\
3.92 \\
3.12 \\
3.40 \\
3.20 \\
3.90 \\
3.88 \\
3.43\end{array}$ & $\begin{array}{l}0.21 \\
0.04 \\
0.00 \\
0.19 \\
0.13 \\
0.96 \\
0.24 \\
0.00 \\
0.54 \\
1.56\end{array}$ \\
\hline $\begin{array}{l}\text { 17S U2 related } \\
\text { U2AF65 } \\
\text { U2AF35 } \\
\text { hPRP43 } \\
\text { SPF45 } \\
\text { SR140 } \\
\text { CHERP } \\
\text { SF3b125 } \\
\end{array}$ & \begin{tabular}{|c|}
53.5 \\
27.9 \\
90.9 \\
45.0 \\
118.2 \\
100.0 \\
103.0 \\
\end{tabular} & \begin{tabular}{|l|} 
gi|6005926 \\
gi|5803207 \\
gi|68509926 \\
gi|14249678 \\
gi|122937227 \\
gi|119226260 \\
gi|45446747 \\
\end{tabular} & \begin{tabular}{|c|}
1.51 \\
2.86 \\
4.69 \\
10.09 \\
12.48 \\
4.12 \\
5.15 \\
\end{tabular} & $\begin{array}{l}0.65 \\
0.00 \\
3.02 \\
0.00 \\
0.00 \\
\end{array}$ & $\begin{array}{c}1 \\
1 \\
51 \\
7 \\
52 \\
10 \\
1 \\
\end{array}$ & $\begin{array}{c}4.15 \\
10.78 \\
12.23 \\
10.60 \\
17.58 \\
\end{array}$ & $\begin{array}{l}0.20 \\
0.41 \\
3.32 \\
0.54 \\
1.46 \\
\end{array}$ & $\begin{array}{c}25 \\
5 \\
13 \\
5 \\
3 \\
\end{array}$ & \begin{tabular}{|c|}
1.51 \\
2.86 \\
4.42 \\
10.43 \\
12.35 \\
7.36 \\
11.36 \\
\end{tabular} & $\begin{array}{l}0.38 \\
0.49 \\
0.18 \\
4.58 \\
8.79 \\
\end{array}$ \\
\hline $\begin{array}{l}\text { U5 snRNP } \\
220 \mathrm{~K} \\
200 \mathrm{~K} \\
116 \mathrm{~K} \\
40 \mathrm{~K} \\
102 \mathrm{~K} \\
15 \mathrm{~K} \\
100 \mathrm{~K} \\
52 \mathrm{~K}\end{array}$ & \begin{tabular}{|c|}
273.3 \\
244.5 \\
109.4 \\
39.3 \\
106.9 \\
16.8 \\
95.6 \\
37.6 \\
\end{tabular} & \begin{tabular}{|l|} 
gi|3661610 \\
gi|45861372 \\
gi|41152056 \\
gi|4758560 \\
gi|40807485 \\
gi|5729802 \\
gi|41327771 \\
gi|5174409
\end{tabular} & $\begin{array}{l}0.98 \\
1.00 \\
0.93 \\
0.94 \\
3.32 \\
\\
2.78 \\
2.02 \\
\end{array}$ & $\begin{array}{l}0.02 \\
0.14 \\
0.12 \\
0.13 \\
0.24 \\
\\
\\
0.68 \\
0.54\end{array}$ & $\begin{array}{c}107 \\
128 \\
92 \\
6 \\
18\end{array}$ & \begin{tabular}{|c|}
1.13 \\
1.10 \\
1.08 \\
1.05 \\
3.48 \\
13.85 \\
2.38 \\
2.33 \\
\end{tabular} & $\begin{array}{l}0.07 \\
0.06 \\
0.05 \\
0.07 \\
0.40 \\
1.99 \\
0.18 \\
0.08\end{array}$ & $\begin{array}{c}179 \\
229 \\
65 \\
23 \\
53 \\
2 \\
23 \\
8 \\
\end{array}$ & $\begin{array}{c}1.05 \\
1.05 \\
1.00 \\
0.99 \\
3.40 \\
13.85 \\
2.58 \\
2.17 \\
\end{array}$ & $\begin{array}{l}0.10 \\
0.07 \\
0.11 \\
0.08 \\
0.11 \\
\\
0.29 \\
0.21\end{array}$ \\
\hline $\begin{array}{l}\text { U4/U6 snRNP } \\
90 \mathrm{~K} \\
60 \mathrm{~K} \\
20 \mathrm{~K} \\
61 \mathrm{~K} \\
15.5 \mathrm{~K} \\
\end{array}$ & $\begin{array}{l}77.6 \\
58.4 \\
20.0 \\
55.4 \\
14.2 \\
\end{array}$ & $\begin{array}{l}\text { gi|4758556 } \\
\text { gi|45861374 } \\
\text { gi|5454154 } \\
\text { gi|40254869 } \\
\text { gi|4826860 } \\
\end{array}$ & \begin{tabular}{|c|}
16.28 \\
14.21 \\
7.17 \\
17.30
\end{tabular} & $\begin{array}{l}7.39 \\
5.44 \\
1.90 \\
7.67\end{array}$ & $\begin{array}{c}42 \\
16 \\
5 \\
13\end{array}$ & $\begin{array}{l}19.38 \\
17.08 \\
10.18 \\
21.88 \\
21.50 \\
\end{array}$ & $\begin{array}{l}1.84 \\
0.93 \\
0.36 \\
1.49 \\
1.02 \\
\end{array}$ & $\begin{array}{c}24 \\
4 \\
8 \\
3 \\
2 \\
\end{array}$ & \begin{tabular}{|c|}
17.83 \\
15.64 \\
8.67 \\
19.59 \\
21.50 \\
\end{tabular} & $\begin{array}{l}2.19 \\
2.02 \\
2.12 \\
3.23\end{array}$ \\
\hline $\begin{array}{l}\text { U4/U6.U5 snRNP } \\
\text { 110K } \\
65 \mathrm{~K} \\
\text { 27K (RY1) } \\
\text { hPRP38 } \\
\text { TFIP11 }\end{array}$ & $\begin{array}{l}90.2 \\
65.4 \\
18.9 \\
37.5 \\
96.8\end{array}$ & $\begin{array}{l}\text { gi|13926068 } \\
\text { gi||56550051 } \\
\text { gi|24307919 } \\
\text { gi|24762236 } \\
\text { gi|8393259 }\end{array}$ & $\begin{array}{l}7.25 \\
3.22\end{array}$ & $\begin{array}{l}1.72 \\
0.33\end{array}$ & $\begin{array}{l}51 \\
35\end{array}$ & $\begin{array}{l}10.78 \\
3.28 \\
6.25 \\
2.70 \\
1.45\end{array}$ & $\begin{array}{l}0.67 \\
0.12 \\
0.22 \\
0.67 \\
0.04\end{array}$ & $\begin{array}{c}29 \\
11 \\
3 \\
11 \\
8\end{array}$ & $\begin{array}{l}9.01 \\
3.25 \\
6.25 \\
2.70 \\
1.58\end{array}$ & $\begin{array}{l}2.50 \\
0.04\end{array}$ \\
\hline
\end{tabular}


RESULTS | 105

\begin{tabular}{|c|c|c|c|c|c|c|c|c|c|c|}
\hline \multirow{2}{*}{ Protein } & \multirow{2}{*}{$\begin{array}{c}\text { MW } \\
{[k D a]}\end{array}$} & \multirow{2}{*}{$\begin{array}{c}\text { accession } \\
\text { no. }\end{array}$} & \multicolumn{3}{|c|}{ SILAC \#1 } & \multicolumn{3}{|c|}{ SILAC \#2 } & \multicolumn{2}{|c|}{ SILAC mean } \\
\hline & & & $\mathrm{B} / \mathrm{C}$ & StDev & \# & $B / C$ & StDev & \# & $\mathrm{B} / \mathrm{C}$ & StDev \\
\hline $\begin{array}{l}\text { LSm Proteins } \\
\text { LSM } 2 \\
\text { LSM } 3 \\
\text { LSM } 4 \\
\text { LSM } 5 \\
\text { LSM } 6 \\
\text { LSM } 7 \\
\text { LSM } 8\end{array}$ & \begin{tabular}{|c|}
10.8 \\
11.8 \\
15.4 \\
9.9 \\
9.1 \\
11.6 \\
10.4 \\
\end{tabular} & $\begin{array}{l}\text { gi||10863977 } \\
\text { gi||7657315 } \\
\text { gi|} 6912486 \\
\text { gi||5919153 } \\
\text { gi||7706423 } \\
\text { gi||7706425 }\end{array}$ & $\begin{array}{c}23.42 \\
4.04 \\
9.60 \\
\\
15.80 \\
19.62 \\
10.70 \\
\end{array}$ & $\begin{array}{l}0.00 \\
4.06 \\
\\
0.00 \\
8.34 \\
5.53\end{array}$ & $\begin{array}{l}1 \\
2 \\
3 \\
\\
2 \\
2 \\
5\end{array}$ & $\begin{array}{l}18.35 \\
14.13 \\
16.35 \\
\\
19.85\end{array}$ & $\begin{array}{l}0.77 \\
0.29 \\
0.91 \\
\\
1.58\end{array}$ & $\begin{array}{l}7 \\
3 \\
3 \\
\\
6\end{array}$ & $\begin{array}{c}20.88 \\
9.08 \\
12.98 \\
\\
17.82 \\
19.62 \\
10.70\end{array}$ & $\begin{array}{l}3.58 \\
7.13 \\
4.77 \\
\\
2.87\end{array}$ \\
\hline $\begin{array}{l}\text { Sm Proteins } \\
\text { B } \\
\text { D1 } \\
\text { D2 } \\
\text { D3 } \\
\text { E } \\
\text { F } \\
\text { G }\end{array}$ & $\begin{array}{c}24.6 \\
13.3 \\
13.5 \\
13.9 \\
10.8 \\
9.7 \\
8.5 \\
\end{array}$ & $\begin{array}{l}\text { gi|4507125 } \\
\text { gi|} \mid 5902102 \\
\text { gi|} \mid 29294624 \\
\text { gi|4759160 } \\
\text { gi|4507129 } \\
\text { gi||4507131 } \\
\text { gi|} 4507133\end{array}$ & $\begin{array}{l}1.70 \\
1.96 \\
1.98 \\
1.89 \\
2.03 \\
1.82 \\
1.94 \\
\end{array}$ & $\begin{array}{l}0.09 \\
0.28 \\
0.25 \\
0.24 \\
0.23 \\
0.00 \\
0.00\end{array}$ & $\begin{array}{c}5 \\
11 \\
21 \\
17 \\
8 \\
2 \\
2 \\
\end{array}$ & $\begin{array}{l}1.50 \\
1.70 \\
1.50 \\
1.73 \\
1.73 \\
1.73 \\
1.78 \\
\end{array}$ & $\begin{array}{l}0.15 \\
0.07 \\
0.06 \\
0.06 \\
0.04 \\
0.04 \\
0.03 \\
\end{array}$ & $\begin{array}{c}19 \\
8 \\
21 \\
9 \\
7 \\
7 \\
8\end{array}$ & $\begin{array}{l}1.60 \\
1.83 \\
1.74 \\
1.81 \\
1.88 \\
1.77 \\
1.86 \\
\end{array}$ & $\begin{array}{l}0.14 \\
0.18 \\
0.34 \\
0.12 \\
0.21 \\
0.07 \\
0.12\end{array}$ \\
\hline $\begin{array}{l}\text { hPRP19/CDC5L } \\
\text { complex } \\
\text { hPrp19 } \\
\text { CDC5L } \\
\text { SPF27 } \\
\text { PRL1 } \\
\text { Hsp70 } \\
\text { AD-002 } \\
\text { CTNNBL1 } \\
\text { Npw38BP } \\
\text { Npw38 }\end{array}$ & $\begin{array}{l}55.2 \\
92.2 \\
21.5 \\
57.2 \\
70.4 \\
26.6 \\
65.1 \\
70.0 \\
30.5 \\
\end{array}$ & $\begin{array}{l}\text { gi|7657381 } \\
\text { gi|11067747 } \\
\text { gi|5031653 } \\
\text { gi|4505895 } \\
\text { gi|} 5729877 \\
\text { gi|7705475 } \\
\text { gi| } 18644734 \\
\text { gi|7706501 } \\
\text { gi|74735456 }\end{array}$ & $\begin{array}{l}0.35 \\
0.41 \\
0.33 \\
0.33 \\
0.19 \\
0.21 \\
0.59 \\
3.10\end{array}$ & $\begin{array}{l}0.07 \\
0.13 \\
0.07 \\
0.10 \\
0.12 \\
0.02 \\
0.05 \\
0.49\end{array}$ & $\begin{array}{c}59 \\
38 \\
28 \\
26 \\
21 \\
8 \\
4 \\
2\end{array}$ & $\begin{array}{l}0.38 \\
0.43 \\
0.35 \\
0.33 \\
0.15 \\
0.33 \\
0.75 \\
3.95 \\
3.55 \\
\end{array}$ & $\begin{array}{l}0.06 \\
0.16 \\
0.01 \\
0.01 \\
0.01 \\
0.01 \\
0.02 \\
0.15\end{array}$ & $\begin{array}{c}29 \\
66 \\
17 \\
11 \\
4 \\
9 \\
10 \\
2 \\
1 \\
\end{array}$ & $\begin{array}{l}0.36 \\
0.42 \\
0.34 \\
0.33 \\
0.17 \\
0.27 \\
0.67 \\
3.53 \\
3.55 \\
\end{array}$ & $\begin{array}{l}0.02 \\
0.01 \\
0.01 \\
0.01 \\
0.03 \\
0.08 \\
0.11 \\
0.60\end{array}$ \\
\hline $\begin{array}{l}\text { hPRP19/CDC5L } \\
\text { related } \\
\text { hSYF1 } \\
\text { CRNKL1 } \\
\text { hlsy1 } \\
\text { SKIP } \\
\text { RBM22 } \\
\text { Cyp-E } \\
\text { PPIL1 } \\
\text { KIAA0560 } \\
\text { G10 }\end{array}$ & $\begin{array}{c}100.0 \\
100.6 \\
33.0 \\
51.1 \\
46.9 \\
33.4 \\
18.2 \\
171.3 \\
17.0 \\
\end{array}$ & $\begin{array}{l}\text { gi|55770906 } \\
\text { gi|30795220 } \\
\text { gi|20149304 } \\
\text { gi|6912676 } \\
\text { gi||8922328 } \\
\text { gi|5174637 } \\
\text { gi|7706339 } \\
\text { gi|38788372 } \\
\text { gi|32171175 }\end{array}$ & $\begin{array}{l}0.22 \\
0.27 \\
0.17 \\
0.29 \\
0.28 \\
0.18 \\
0.25 \\
0.22\end{array}$ & $\begin{array}{l}0.04 \\
0.10 \\
0.17 \\
0.05 \\
0.10 \\
0.01 \\
0.03 \\
0.07\end{array}$ & $\begin{array}{c}86 \\
78 \\
\\
85 \\
20 \\
16 \\
8 \\
122\end{array}$ & $\begin{array}{l}0.28 \\
0.25 \\
0.23 \\
0.33 \\
0.30 \\
0.23 \\
0.38 \\
0.28\end{array}$ & $\begin{array}{l}0.05 \\
0.01 \\
0.02 \\
0.03 \\
0.02 \\
0.01 \\
0.02 \\
0.06\end{array}$ & $\begin{array}{l}42 \\
44 \\
15 \\
34 \\
14 \\
10 \\
21 \\
86\end{array}$ & $\begin{array}{l}0.25 \\
0.26 \\
0.20 \\
0.31 \\
0.29 \\
0.20 \\
0.31 \\
0.25\end{array}$ & $\begin{array}{l}0.04 \\
0.02 \\
0.04 \\
0.03 \\
0.02 \\
0.03 \\
0.09 \\
0.04\end{array}$ \\
\hline $\begin{array}{l}\text { hRES complex } \\
\text { proteins } \\
\text { SNIP1 } \\
\text { MGC12135 } \\
\text { CGI-79 } \\
\end{array}$ & \begin{tabular}{|l|}
45.8 \\
70.5 \\
39.7 \\
\end{tabular} & $\begin{array}{l}\text { gi|21314720 } \\
\text { gi|14249338 } \\
\text { gi|4929627 }\end{array}$ & $\begin{array}{l}0.74 \\
0.52 \\
\end{array}$ & $\begin{array}{l}0.07 \\
0.06\end{array}$ & $\begin{array}{c}10 \\
8 \\
\end{array}$ & $\begin{array}{l}0.73 \\
0.75 \\
0.73 \\
\end{array}$ & $\begin{array}{l}0.03 \\
0.03 \\
0.02\end{array}$ & $\begin{array}{c}7 \\
14 \\
6 \\
\end{array}$ & $\begin{array}{l}0.73 \\
0.75 \\
0.62\end{array}$ & $\begin{array}{l}0.01 \\
0.15\end{array}$ \\
\hline $\begin{array}{l}\text { Proteins } \\
\text { recruited to A } \\
\text { complex } \\
\text { RBM39 } \\
\text { p68 (DDX5) } \\
\text { ELAV-like } 1 \text { (HuR) } \\
\text { p72/DDX17 }\end{array}$ & $\begin{array}{l}59.4 \\
69.2 \\
36.1 \\
80.5\end{array}$ & $\begin{array}{l}\text { gi|4757926 } \\
\text { gi|4758138 } \\
\text { gi|38201714 } \\
\text { gi|3122595 }\end{array}$ & $\begin{array}{l}3.32 \\
3.38 \\
9.86 \\
2.90\end{array}$ & $\begin{array}{l}0.75 \\
0.23 \\
4.68\end{array}$ & $\begin{array}{c}17 \\
5 \\
28 \\
1\end{array}$ & $\begin{array}{c}3.65 \\
3.05 \\
10.50\end{array}$ & $\begin{array}{l}0.11 \\
0.39 \\
0.76\end{array}$ & $\begin{array}{c}5 \\
4 \\
16\end{array}$ & $\begin{array}{c}3.48 \\
3.21 \\
10.18 \\
2.90\end{array}$ & $\begin{array}{l}0.24 \\
0.23 \\
0.45\end{array}$ \\
\hline
\end{tabular}




\begin{tabular}{|c|c|c|c|c|c|c|c|c|c|c|}
\hline \multirow{2}{*}{ Protein } & \multirow{2}{*}{$\begin{array}{c}\text { MW } \\
\text { [kDa] }\end{array}$} & \multirow{2}{*}{$\begin{array}{c}\text { accession } \\
\text { no. }\end{array}$} & \multicolumn{3}{|c|}{ SILAC \#1 } & \multicolumn{3}{|c|}{ SILAC \#2 } & \multicolumn{2}{|c|}{ SILAC mean } \\
\hline & & & $B / C$ & StDev & $\#$ & $\mathrm{~B} / \mathrm{C}$ & StDev & $\#$ & $\mathrm{~B} / \mathrm{C}$ & StDev \\
\hline \multicolumn{11}{|l|}{$\begin{array}{l}\text { Proteins } \\
\text { recruited to B } \\
\text { complex }\end{array}$} \\
\hline MFAP1 & 51.9 & gi|50726968 & 2.13 & 0.24 & 10 & 3.18 & 0.16 & 11 & 2.65 & 0.74 \\
\hline RED & 65.6 & gi|10835234 & 5.62 & 0.88 & 11 & 5.40 & 0.16 & 11 & 5.51 & 0.15 \\
\hline hSmu-1 & 57.5 & gi|8922679 & 2.74 & 0.32 & 9 & 9.50 & 0.27 & 8 & 6.12 & 4.78 \\
\hline PPIL2 & 59.5 & gi|7657473 & 0.48 & 0.14 & 12 & 0.80 & 0.03 & 17 & 0.64 & 0.23 \\
\hline hPRP2 (DDX16) & 119.2 & gi|4503293 & 0.04 & & 1 & 0.58 & 0.02 & 23 & 0.31 & 0.38 \\
\hline hPRP4-Kinase & 117.1 & gi|89276756 & 1.41 & 0.24 & 15 & & & & 1.41 & \\
\hline THRAP3 & 108.6 & gi|4827040 & 3.56 & 0.57 & 5 & 16.85 & 1.17 & 11 & 10.21 & 9.39 \\
\hline PABP1 & 70.5 & gi|46367787 & 0.30 & 0.04 & 7 & & & & 0.30 & \\
\hline SKIV2L2 & 117.8 & gi|39930353 & 3.98 & 0.72 & 13 & 2.70 & 0.06 & 7 & 3.34 & 0.91 \\
\hline PABPN1 & 32.6 & gi|4758876 & 0.36 & 0.01 & 4 & 1.08 & 0.03 & 6 & 0.72 & 0.51 \\
\hline RNF113A & 38.8 & gi|5902158 & & & & 0.43 & 0.02 & 8 & 0.43 & \\
\hline NY-CO-10 & 53.8 & gi|64276486 & 0.33 & 0.10 & 8 & 0.70 & 0.04 & 8 & 0.51 & 0.26 \\
\hline KIAA1604 & 105.5 & gi|55749769 & 0.16 & 0.05 & 17 & 0.23 & 0.02 & 26 & 0.19 & 0.04 \\
\hline hsp27 & 22.8 & gi|4504517 & & & & 0.25 & 0.00 & 2 & 0.25 & \\
\hline GCFC & 104.7 & gi|22035565 & 1.60 & 0.26 & 10 & 1.50 & 0.09 & 11 & 1.55 & 0.07 \\
\hline UBL5 & 8.5 & gi|13236510 & & & & & & & & \\
\hline CCDC16 & 42.0 & gi|49472814 & 0.41 & 0.06 & 5 & 0.73 & 0.04 & 5 & 0.57 & 0.22 \\
\hline CCDC12 & 19.2 & gi|21389497 & & & & 0.28 & 0.07 & 13 & 0.28 & \\
\hline HsKin17 & 45.2 & gi|13124883 & 1.25 & 0.14 & 7 & 1.40 & 0.06 & 5 & 1.33 & 0.10 \\
\hline \multicolumn{11}{|l|}{ Step 2 factors } \\
\hline hPRP22 & 139.3 & gi|4826690 & 0.14 & 0.10 & 49 & 0.10 & 0.07 & 48 & 0.12 & 0.03 \\
\hline hPRP18 & 39.9 & gi|4506123 & & & & 0.10 & & 1 & 0.10 & \\
\hline hPRP17 & 65.5 & gi|7706657 & 0.29 & 0.10 & 28 & 0.23 & 0.02 & 29 & 0.26 & 0.04 \\
\hline hPRP16 & 140.5 & gi|17999539 & & & & 0.48 & 0.01 & 2 & 0.48 & \\
\hline hSLU7 & 68.4 & gi|27477111 & 0.24 & 0.08 & 6 & 0.40 & 0.53 & 14 & 0.32 & 0.11 \\
\hline \multicolumn{11}{|l|}{$\begin{array}{l}\text { Proteins } \\
\text { recruited to C } \\
\text { complex }\end{array}$} \\
\hline Abstrakt & 69.8 & gi|21071032 & 0.19 & 0.06 & 25 & 0.13 & 0.15 & 24 & 0.16 & 0.05 \\
\hline GCIP p29 & 28.7 & gi|46371998 & 0.02 & 0.20 & 11 & 0.15 & 0.15 & 18 & 0.09 & 0.09 \\
\hline DDX35 & 78.9 & gi|20544129 & 0.07 & 0.08 & 13 & 0.05 & 0.01 & 15 & 0.06 & 0.01 \\
\hline $\begin{array}{l}\text { Q9BRR8 } \\
\text { c19orf29 (NY- }\end{array}$ & 103.3 & gi|74732921 & & & & 0.05 & 0.01 & 7 & 0.05 & \\
\hline REN-24) & 88.6 & gi|126723149 & & & & 0.05 & 0.02 & 21 & 0.05 & \\
\hline PPlase-like 3b & 18.6 & gi|19557636 & 0.01 & 0.00 & 2 & 0.03 & 0.01 & 7 & 0.02 & 0.01 \\
\hline PPWD1 & 73.6 & gi|24308049 & 0.08 & 0.03 & 18 & 0.08 & 0.05 & 18 & 0.08 & 0.00 \\
\hline MORG1 & 34.3 & gi|153791298 & 0.07 & 0.01 & 2 & 0.05 & 0.00 & 5 & 0.06 & 0.02 \\
\hline FRG1 & 29.2 & gi|4758404 & 0.22 & 0.02 & 3 & 0.23 & 0.01 & 2 & 0.22 & 0.00 \\
\hline NOSIP & 33.2 & gi|7705716 & & & & 0.10 & 0.05 & 2 & 0.10 & \\
\hline GPKOW & 52.1 & gi|15811782 & 0.09 & 0.04 & 5 & 0.33 & 0.04 & 12 & 0.21 & 0.17 \\
\hline C1orf55 & 39.3 & gi|148664216 & 0.19 & 0.00 & 2 & 0.05 & 0.03 & 25 & 0.12 & 0.10 \\
\hline FAM32A & 13.1 & gi|7661696 & 0.12 & & 1 & 0.03 & 0.02 & 4 & 0.07 & 0.07 \\
\hline RACK1 (GNB2L1) & 35.1 & gi|5174447 & & & & & & & & \\
\hline Tip-49 & 50.2 & gi|4506753 & & & & 0.13 & 0.01 & 3 & 0.13 & \\
\hline \multicolumn{11}{|l|}{$\begin{array}{l}\text { Potential C } \\
\text { complex specific } \\
\text { proteins }\end{array}$} \\
\hline PPIG & 88.5 & gi|42560244 & 0.54 & 0.15 & 2 & 0.20 & 0.06 & 5 & 0.37 & 0.24 \\
\hline FAM50A & 40.1 & gi|4758220 & 0.15 & & 1 & 0.03 & 0.00 & 7 & 0.09 & 0.09 \\
\hline FAM50B & 38.6 & gi|6912326 & & & & 0.05 & 0.03 & 2 & 0.05 & \\
\hline
\end{tabular}




\begin{tabular}{|c|c|c|c|c|c|c|c|c|c|c|}
\hline \multirow{2}{*}{ Protein } & \multirow{2}{*}{$\begin{array}{c}\text { MW } \\
{[\mathrm{kDa}]}\end{array}$} & \multirow{2}{*}{$\begin{array}{l}\text { accession } \\
\text { no. }\end{array}$} & \multicolumn{3}{|c|}{ SILAC \#1 } & \multicolumn{3}{|c|}{ SILAC \#2 } & \multicolumn{2}{|c|}{ SILAC mean } \\
\hline & & & $\mathrm{B} / \mathrm{C}$ & StDev & \# & $B / C$ & StDev & \# & $\mathrm{B} / \mathrm{C}$ & StDev \\
\hline C9orf78 & 33.7 & gi|7706557 & 0.04 & 0.01 & 2 & 0.03 & 0.01 & 2 & 0.03 & 0.01 \\
\hline C10orf4 & 37.5 & gi|24432067 & & & & 0.10 & 0.05 & 5 & 0.10 & \\
\hline CXorf56 & 25.6 & gi|11545813 & 0.18 & 0.07 & 3 & 0.08 & 0.06 & 8 & 0.13 & 0.08 \\
\hline DGCR14 & 52.4 & gi|13027630 & 0.25 & 0.07 & 8 & 0.03 & 0.01 & 8 & 0.14 & 0.16 \\
\hline CCDC130 & 44.7 & gi|13540614 & & & & 0.10 & 0.02 & 2 & 0.10 & \\
\hline TOE1 & 56.4 & gi|156564398 & & & & & & & & \\
\hline NKAP & 47.0 & gi|13375676 & 0.10 & & 1 & 0.03 & 0.00 & 5 & 0.06 & 0.05 \\
\hline $\mathrm{ZCCHC} 10$ & 18.4 & gi|8923106 & 0.16 & 0.00 & 2 & 0.08 & 0.03 & 2 & 0.12 & 0.06 \\
\hline CDK10 & 35.4 & gi|16950647 & & & & 0.10 & 0.04 & 6 & 0.10 & \\
\hline TTC14 & 88.2 & gi|33457330 & 0.34 & & 1 & 0.08 & 0.02 & 8 & 0.21 & 0.19 \\
\hline WDR70 & 73.2 & gi|8922301 & & & & & & & & \\
\hline NFKBIL1 & 43.1 & gi|26787991 & & & & 0.08 & 0.01 & 3 & 0.08 & \\
\hline JUP & 81.6 & gi|12056468 & 0.08 & 0.08 & 4 & & & & 0.08 & \\
\hline \multicolumn{11}{|l|}{ EJC/mRNP } \\
\hline elF4A3 & 46.9 & gi|7661920 & 0.26 & 0.08 & 18 & 0.23 & 0.02 & 21 & 0.24 & 0.03 \\
\hline Magoh & 17.2 & gi|4505087 & 0.21 & 0.01 & 6 & 0.28 & 0.00 & 3 & 0.24 & 0.04 \\
\hline Y14 & 19.9 & gi|4826972 & 0.13 & 0.13 & 2 & 0.18 & 0.04 & 2 & 0.15 & 0.03 \\
\hline Pinin & 81.6 & gi|33356174 & 0.31 & & 1 & 1.23 & 0.02 & 4 & 0.77 & 0.65 \\
\hline RNPS1 & 34.2 & gi|6857826 & 0.63 & 0.02 & 3 & 2.28 & 0.08 & 5 & 1.45 & 1.17 \\
\hline Acinus & 151.8 & gi|7662238 & 0.50 & 0.11 & 11 & 2.75 & 0.33 & 13 & 1.63 & 1.59 \\
\hline SAP18 & 17.4 & gi|5032067 & & & & 2.25 & 0.03 & 2 & 2.25 & \\
\hline Aly/REF (THOC4) & 26.9 & gi|55770864 & 0.98 & 0.26 & 2 & 3.20 & 0.07 & 5 & 2.09 & 1.57 \\
\hline UAP56 & 49.1 & gi|18375623 & 0.28 & 0.03 & 5 & 6.08 & 0.07 & 4 & 3.18 & 4.10 \\
\hline \multicolumn{11}{|l|}{ TREX } \\
\hline THOC2 & 169.6 & gi|125656165 & 0.15 & 0.02 & 5 & 3.43 & 0.09 & 3 & 1.79 & 2.31 \\
\hline THOC3 & 38.8 & gi|14150171 & & & & & & & & \\
\hline $\begin{array}{l}\text { KIAA0983 } \\
\text { (THOC5) }\end{array}$ & 78.4 & gi|50959110 & & & & 3.15 & & 1 & 315 & \\
\hline WDR58 (THOC6) & 37.4 & gi|31543164 & & & & 1.25 & 0.11 & 3 & 1.25 & \\
\hline \multicolumn{11}{|l|}{$\begin{array}{l}\text { pre-mRNA/mRNA } \\
\text { binding proteins }\end{array}$} \\
\hline CBP20 & 18.0 & gi|110349727 & 0.88 & 0.06 & 10 & 1.20 & 0.02 & 7 & 1.04 & 0.23 \\
\hline СВР80 & 91.8 & gi|4505343 & 0.89 & 0.11 & 64 & 1.20 & 0.07 & 53 & 1.04 & 0.22 \\
\hline NF45 & 43.0 & gi|24234747 & 2.86 & 0.44 & 12 & 4.90 & 0.22 & 3 & 3.88 & 1.44 \\
\hline ZC3H18 & 104.0 & gi|31377595 & 3.74 & 0.54 & 13 & 4.15 & 0.17 & 7 & 3.95 & 0.29 \\
\hline YB-1 & 35.9 & gi|34098946 & 0.56 & 0.12 & 14 & 0.35 & 0.02 & 11 & 0.45 & 0.15 \\
\hline ELG & 38.9 & gi|8923771 & 1.37 & 0.07 & 5 & 10.50 & 0.06 & 3 & 5.93 & 6.46 \\
\hline DDX3 & 73.3 & gi|87196351 & 4.71 & 1.13 & 4 & & & & 4.71 & \\
\hline ASR2B & 100.0 & gi|33383233 & 3.29 & 0.55 & 51 & 4.18 & 0.32 & 22 & 3.73 & 0.63 \\
\hline BCLAF1 & 107.2 & gi|7661958 & 0.29 & & 1 & 12.80 & 0.33 & 2 & 6.54 & 8.85 \\
\hline DBPA & 40.1 & gi|20070160 & & & & 0.33 & & 1 & 0.33 & \\
\hline RBM7 & 30.5 & gi|4503293 & & & & & & & & \\
\hline HSP70 & 70.0 & gi|5123454 & 0.19 & 0.12 & 21 & 0.15 & 0.01 & 4 & 0.17 & 0.03 \\
\hline \multicolumn{11}{|l|}{$\begin{array}{l}\text { Miscelleneous } \\
\text { proteins }\end{array}$} \\
\hline BAG2 & 23.4 & gi|4757834 & & & & 0.50 & 0.01 & 5 & 0.50 & \\
\hline RBBP6 & 197.2 & gi|33620716 & & & & 1.93 & 0.10 & 2 & 1.93 & \\
\hline RBM42 & 50.3 & gi|21359951 & 12.35 & 12.21 & 2 & & & & 12.35 & \\
\hline
\end{tabular}




\begin{tabular}{|c|c|c|c|c|c|c|c|c|c|c|}
\hline \multirow{2}{*}{ Protein } & \multirow{2}{*}{$\begin{array}{c}\text { MW } \\
{[\mathrm{kDa}]}\end{array}$} & \multirow{2}{*}{$\begin{array}{c}\text { accession } \\
\text { no. }\end{array}$} & \multicolumn{3}{|c|}{ SILAC \#1 } & \multicolumn{3}{|c|}{ SILAC \#2 } & \multicolumn{2}{|c|}{ SILAC mean } \\
\hline & & & $\mathrm{B} / \mathrm{C}$ & StDev & $\#$ & $\mathrm{~B} / \mathrm{C}$ & StDev & $\#$ & $\mathrm{~B} / \mathrm{C}$ & StDev \\
\hline $\begin{array}{l}\text { SR related } \\
\text { proteins }\end{array}$ & & & & & & & & & & \\
\hline SRm160 & 102.5 & gi|42542379 & 0.14 & 0.05 & 3 & 0.50 & 0.06 & 3 & 0.32 & 0.25 \\
\hline $\mathrm{SRm} 300$ & 300.0 & gi|4759098 & 0.26 & 0.12 & 13 & 0.40 & 0.09 & 26 & 0.33 & 0.10 \\
\hline SR proteins & & & & & & & & & & \\
\hline SF2/ASF & 27.8 & gi|5902076 & 1.62 & 0.23 & 44 & 5.43 & 0.73 & 23 & 3.52 & 2.69 \\
\hline $9 G 8$ & 27.4 & gi|72534660 & 1.91 & 0.32 & 14 & 3.98 & 0.98 & 19 & 2.94 & 1.46 \\
\hline SRp20 & 19.4 & gi|4506901 & 2.89 & 0.00 & 2 & 9.15 & 0.00 & 2 & 6.02 & 4.42 \\
\hline SRp30c & 25.5 & gi|4506903 & 1.33 & 0.12 & 16 & 1.30 & 0.25 & 18 & 1.32 & 0.02 \\
\hline SRp38 & 31.3 & gi|5730079 & 0.95 & 0.18 & 6 & & & & 0.95 & \\
\hline SRp40 & 31.3 & gi|3929378 & 0.70 & 0.19 & 10 & 1.90 & 0.58 & 10 & 1.30 & 0.85 \\
\hline SRp46 & 31.2 & gi|15055543 & & & & 4.15 & 0.15 & 2 & 4.15 & \\
\hline SRp55 & 39.6 & gi|20127499 & 1.41 & 0.83 & 4 & 1.58 & 0.44 & 15 & 1.49 & 0.12 \\
\hline SRp75 & 56.8 & gi|21361282 & & & & 5.93 & 0.17 & 2 & 5.93 & \\
\hline SC35 (SFRS2) & 25.5 & gi|47271443 & & & & & & & & \\
\hline hTra-2 alpha & 32.7 & gi|9558733 & 2.90 & & 15 & 4.33 & 0.15 & 6 & 3.61 & 1.01 \\
\hline hTra-2 beta & 33.7 & gi|4759098 & 3.97 & 0.94 & 2 & 5.53 & 0.97 & 16 & 4.75 & 1.10 \\
\hline hnRNP & & & & & & & & & & \\
\hline hnRNP A1 & 38.7 & gi|4504445 & 12.92 & 4.92 & & 12.13 & 1.44 & 12 & 12.52 & 0.56 \\
\hline hnRNP A3 & 39.6 & gi|34740329 & 5.83 & 2.37 & 4 & 5.03 & 0.11 & 3 & 5.43 & 0.57 \\
\hline hnRNP AB & 36.0 & gi|12803583 & 7.38 & 0.05 & 3 & 7.05 & 0.68 & 3 & 7.21 & 0.23 \\
\hline hnRNP A2/B1 & 37.4 & gi|14043072 & 2.93 & & 1 & 9.28 & 1.75 & 8 & 6.10 & 4.49 \\
\hline hnRNP C & 33.3 & gi|4758544 & 1.34 & 0.14 & 41 & 1.68 & 0.11 & 28 & 1.51 & 0.24 \\
\hline hnRNP D & 38.4 & gi|14110420 & 9.75 & 0.00 & 2 & 10.40 & 2.42 & 2 & 10.08 & 0.46 \\
\hline hnRNP F & 45.7 & gi|148470406 & & & & 3.85 & 0.07 & 2 & 3.85 & \\
\hline hnRNP G & 47.4 & gi|56699409 & 3.30 & 0.66 & 19 & 3.75 & 0.12 & 11 & 3.52 & 0.32 \\
\hline hnRNP G-T & 42.7 & gi|153252068 & & & & 0.08 & & 1 & 0.08 & \\
\hline hnRNP H1 & 49.1 & gi|5031753 & 2.63 & 0.89 & 3 & 2.65 & 0.16 & 6 & 2.64 & 0.01 \\
\hline hnRNP H3 & 36.9 & gi|14141157 & & & & & & & & \\
\hline hnRNP K & 51.0 & gi|14165435 & 12.05 & 4.94 & 13 & & & & 12.05 & \\
\hline hnRNP M & 77.5 & gi|14141152 & 4.30 & 1.59 & 8 & 2.83 & & 1 & 3.56 & 1.04 \\
\hline$h n R N P Q$ & 69.6 & gi|15809590 & 2.49 & 0.07 & 3 & & & & 2.49 & \\
\hline hnRNP R & 70.9 & gi|5031755 & 0.87 & 0.15 & 12 & 1.23 & 0.07 & 4 & 1.05 & 0.25 \\
\hline hnRNP U & 90.6 & gi|14141161 & 12.82 & 0.00 & 2 & 9.65 & 1.39 & 2 & 11.24 & 2.24 \\
\hline PCBP1 & 37.5 & gi|5453854 & 5.32 & 1.27 & 11 & 5.53 & 0.08 & 2 & 5.42 & 0.14 \\
\hline РCBP2 & 38.1 & gi|14141166 & 5.44 & 0.81 & 12 & 3.50 & 0.01 & 2 & 4.47 & 1.37 \\
\hline RALY & 32.5 & gi|8051631 & & & & 1.25 & 0.11 & 8 & 1.25 & \\
\hline
\end{tabular}

The proteins used for normalization show a protein ratio of 1 , confirming the suitability of these proteins for normalization. As described for the ITRAQ analysis, several proteins showing a high abundance in $B$ and $C$ complexes and only few proteins present in equal amounts within the two complexes were identified. 


\subsubsection{Relative quantification of spliceosomal B and C complexes}

Several snRNP specific proteins and numerous non-snRNP specific proteins identified in spliceosomal $B$ and $C$ complexes have been quantified by ITRAQ, SILAC, and spectral count and all three approaches give information about the protein abundance within the precatalytic and the catalytic spliceosome (i.e. B and C complexes). In the following paragraphs the results will be compared and discussed more in detail.

\subsubsection{Proteins common to $B$ and $C$ complexes}

Confidence for the quantification approaches was achieved by the observed $1: 1$ ratio of the two 5' pre-mRNA cap binding proteins CBP20 and CBP80 (see Tables 4.9, 4.10, and 4.11). The cap binding proteins interact with the 5' cap structure of the pre-mRNA and should be present in equal amounts in $B$ and $C$ complexes. For all approaches (i.e. iTRAQ, SILAC, and spectral count) a ratio of approximately 1 within $B$ and $C$ complexes was observed for these proteins (except CBP20, which shows a protein ratio of 0.67 for spectral count; see Table 4.9).

The Sm proteins are common to all U snRNP except for U6 snRNP. Therefore, four copies of $\mathrm{Sm}$ proteins are expected in B complex. Upon C complex formation, U1 and U4 are dissociated from the spliceosome and only two copies are left. A comparison of the protein ratios for the Sm proteins obtained by the different methods is shown in Figure 4.21. For all seven Sm proteins the ITRAQ and SILAC procedures yield protein ratios close to the expected value of 2 . In contrast, spectral count yielded the correct value only for SmF and SmG and clearly gave a wrong result for the other Sm proteins.

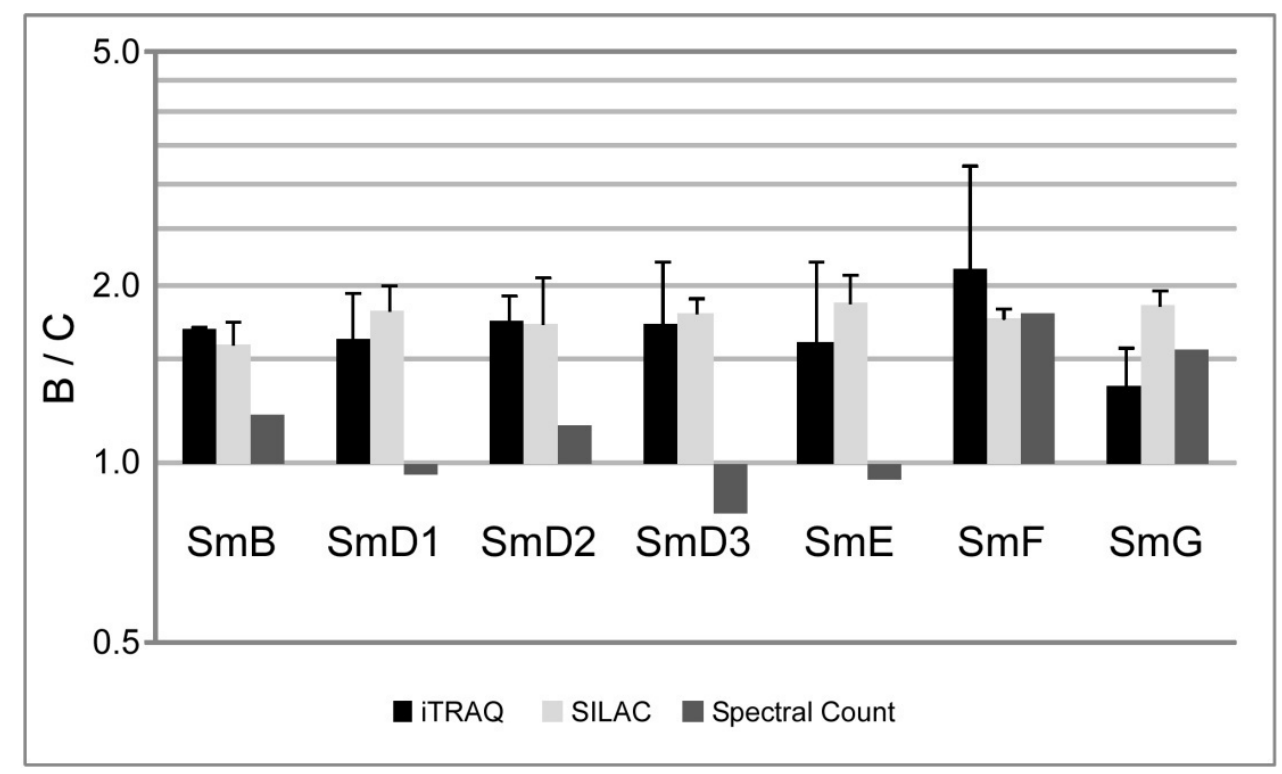

Figure 4.21: Relative abundances of the Sm proteins in the B and C complexes determined by iTRAQ, SILAC, and spectral count. ITRAQ and SILAC quantification reveal an average protein ratio of 1.75 for the $\mathrm{Sm}$ proteins, which is very close to the expected protein ratio of 2 . 
Although the U2 and U5 snRNP are stable associated to B and C complexes, the obtained protein ratios show that only selected members of $\mathrm{U} 2$ and $\mathrm{U} 5$ are present in equal amounts in $B$ and $C$ complexes (Figure 4.22). U2-A' and U2-B" show a protein ratio of 1 for iTRAQ and SILAC analysis and a slightly higher value (approximately 1.3) for spectral count (Figure $4.22 \mathrm{~A}$ ). In contrast, the two U2 snRNP associated splicing factors SF3a and SF3b show a much higher abundance in B complex. SF3a and SF3b likely dissociate from the spliceosome during transition from $B$ to $C$ complex. As they were found to be present in the activated spliceosome (Makarov et al., 2002), our data thus give compelling evidence for their dissociation upon activation of the spliceosome. Comparing ITRAQ, SILAC, and spectral count, the obtained protein ratios are consistent for all SF3a and SF3b proteins (except SF3b49 and SF3b14a).

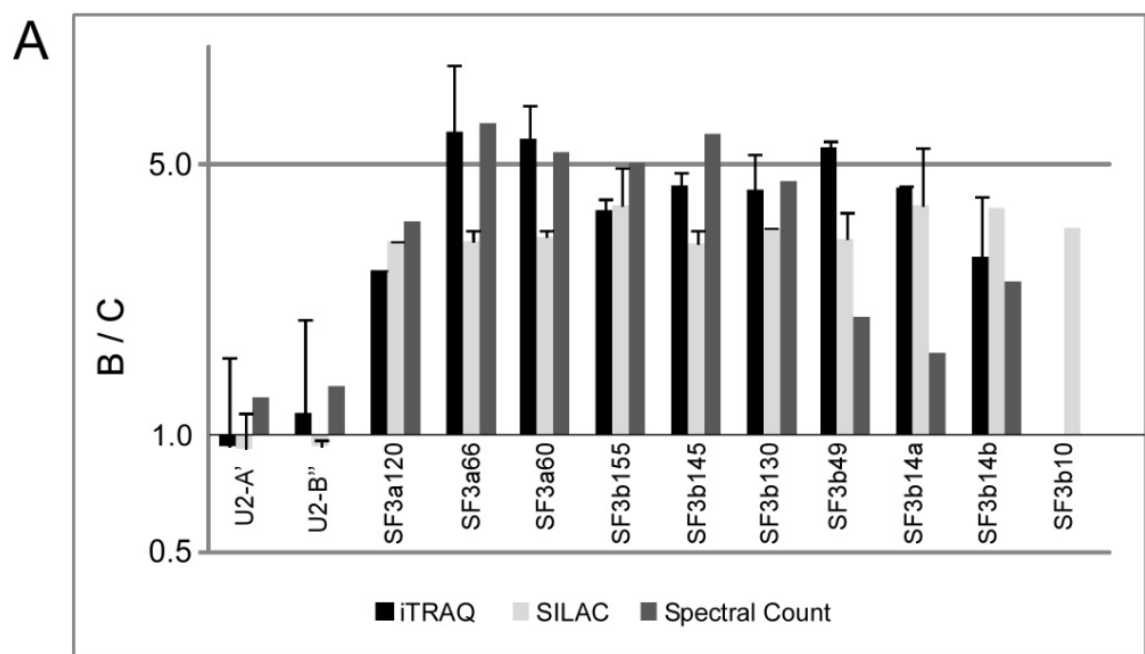

B

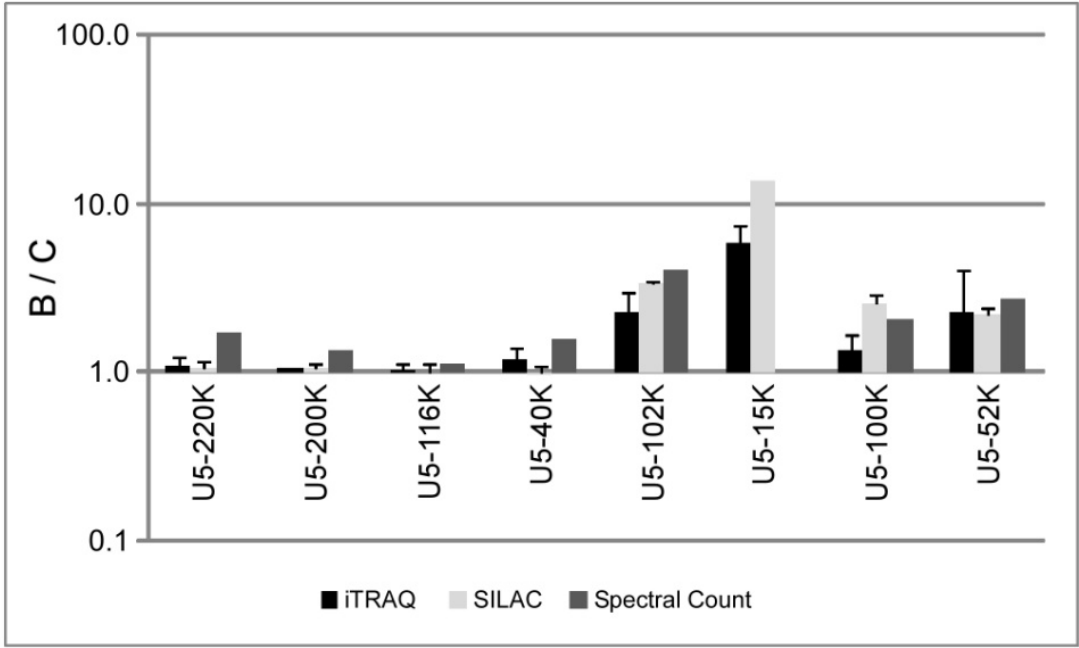

Figure 4.22: Relative protein abundances of the U2 and U5 snRNP specific proteins obtained by iTRAQ, SILAC, and spectral. (A) Protein ratios for U2 snRNP specific proteins. (B) Protein ratios for U5 snRNP proteins. A protein ratio of $U 5-15 \mathrm{~K}$ could not be determined from the spectral count. 
Although U5 snRNA is stably associated with both the B and C complexes, only four of the eight U5 snRNP proteins were found to be present in a 1:1 ratio (U5-220K, U5-200K, U5$116 \mathrm{~K}$, and U5-40K; Figure 4.22 B). The U5 snRNP proteins $102 \mathrm{~K}, 100 \mathrm{~K}, 52 \mathrm{~K}$, and $15 \mathrm{~K}$ show higher ratios (from 2 to 8.5 ) indicating their dissociation from the spliceosome during transition from $B$ to $C$ complex. These findings are in agreement with previous studies (Makarov et al., 2002), where the absence of U5 snRNP proteins $100 \mathrm{~K}, 52 \mathrm{~K}$, and $15 \mathrm{~K}$ in the activated spliceosome is already discussed. Interestingly, the $15 \mathrm{~K}$ protein shows a very high enrichment in $B$ complexes as compared to $C$ complexes. A protein ratio for this particular protein could not be determined from the spectral count as it was totally absent from the $C$ complex (Table 4.9).

The RES complex, which binds to the spliceosome before the first step of splicing, was found to be necessary for efficient intron removal and nuclear pre-mRNA retention (Dziembowski et al., 2004). Our data are consistent with these findings and association with $B$ and $C$ complexes without much change in relative quantification was obtained by all three quantification techniques (see Tables 4.9, 4.10, and 4.11).

Most of the proteins were either specific for B or C complex, underlying the highly dynamic nature of the spliceosome during its assembly pathway.

\subsubsection{Proteins predominantly associated with B complex}

$\mathrm{U} 1$ and U4 snRNP dissociate from the spliceosome during transition from B to C complex. This is clearly observed in our analysis, where all the $\mathrm{U} 1$ and $\mathrm{U} 4$ proteins show high protein ratios indicating their specificity to B complex (see Figure 4.23). For U1-A, U1-C, and U1-70K proteins high protein ratios were obtained by ITRAQ and SILAC indicating their high abundance in B complex (Figure 4.23 A). Furthermore, these proteins were totally absent in C complex and no protein ratios could be determined from spectral count (see Table 4.9).

Also U4/U6 snRNP specific proteins show high protein ratios for all three quantification approaches (Figure 4.23 B) showing that these proteins together with the U4 snRNA dissociate from the spliceosome. In addition, tri-snRNP (U4/U6.U5) specific proteins show (with few exceptions) high protein ratios (Figure $4.23 \mathrm{~B}$ ). This observation reveals that, consistent with previous studies (Makarov et al., 2002), some tri-snRNP specific proteins dissociate from the spliceosome during transition from $B$ to $C$ complex.

The LSm proteins are associated with U6 snRNP and the protein ratios for these proteins corroborate their dissociation from the spliceosome during transition from $\mathrm{B}$ to $\mathrm{C}$ complex, although the U6 snRNA remains associated (Figure $4.23 \mathrm{C}$ ). These protein ratios were high, 
irrespective of the method used. Consistent with Chan et al., 2003 association of the NTC (nineteen complex, the yeast homologue of the human hPrp19/CDC5L complex) during activation of the spliceosome leads to destabilization of LSm proteins and U6 snRNA, what further supports this observation.

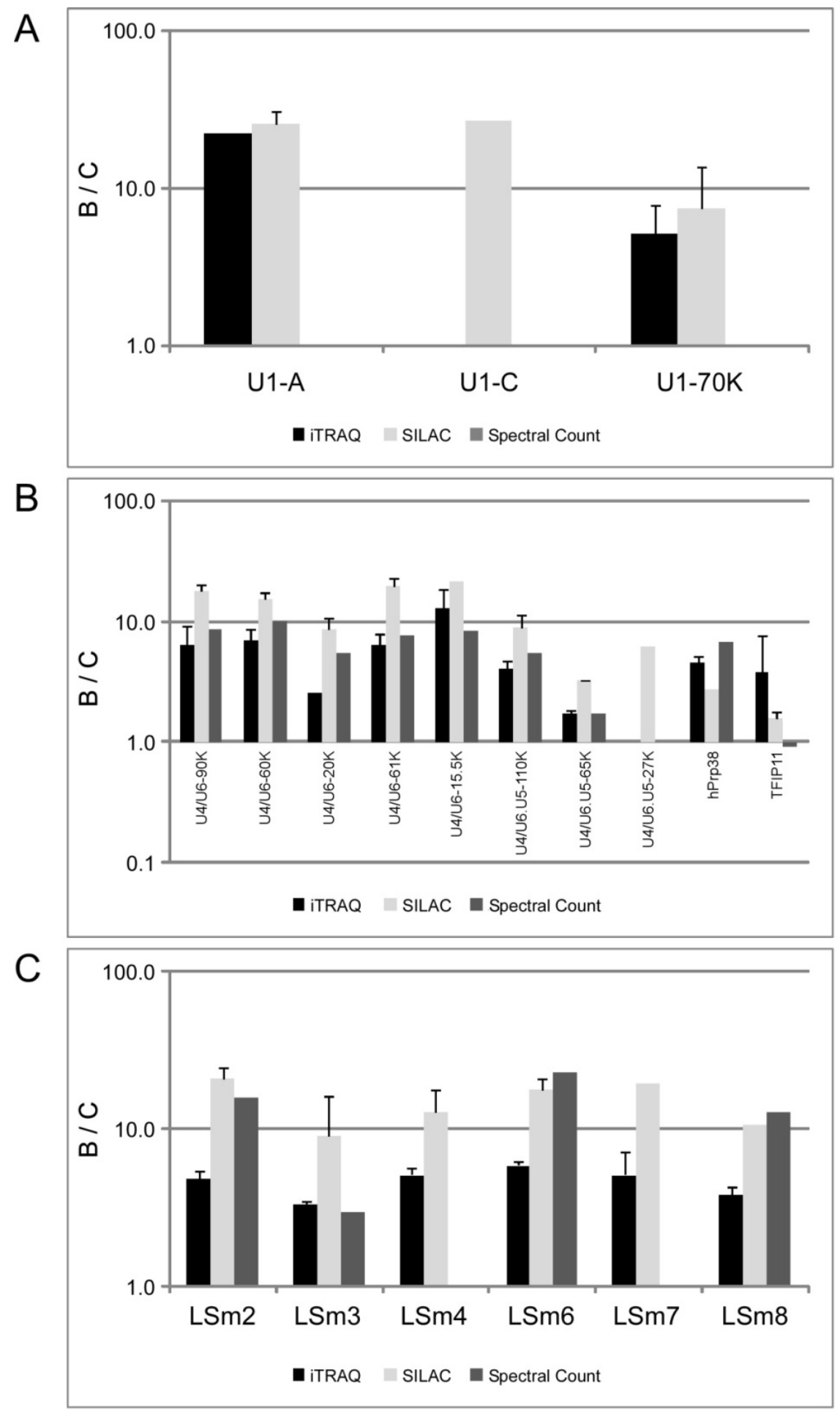

Figure 4.23: Relative protein abundances of the U1, U4/U6, U4/U6.U5 snRNP specific proteins, and LSm proteins obtained by iTRAQ, SILAC, and spectral count. (A) Protein ratios for U1 snRNP specific proteins. No protein ratios were obtained from spectral count as these proteins were totally absent in $C$ complex. (B) Protein ratios for U4/U6 and U4/U6.U5 snRNP specific proteins. (C) Protein ratios for LSm proteins. For LSm7, no protein ratio was obtained from spectral count as this particular protein was totally absent in $\mathrm{C}$ complex. 
The U2 snRNP related proteins are without exceptions highly enriched in B complex. Protein ratios obtained by ITRAQ and SILAC are between 1.5 and 12 indicating their high abundance in B complexes (Tables 4.10 and 4.11). During proteomic analysis, U2 snRNP related proteins were only identified in B complex (with the exception of hPrp43, Table 4.9).

In addition to the snRNP specific proteins, several other proteins are known to be highly enriched in B complex. One group of proteins combined as "proteins recruited to $B$ complexes" were identified in both, B and C complexes (Table 4.9). Of these, only few were highly abundant in B complexes. For MFAP1, RED, hSmu-1, THRAP3 and UBL5 all three methods yielded similarly abundance for the B complex. However, all other proteins show no preferential association to either B or C complexes (see Tables 4.9, 4.10, and 4.11). Furthermore, some other proteins, which were previously detected in A complex (Behzadnia et al., 2007) are found to be highly abundant in B complex. These proteins were never found in $\mathrm{C}$ complex.

The SR and hnRNP proteins are found to be more abundant in B complex. However, according to iTRAQ and spectral count, the SR proteins do not show high protein ratios (Table 4.9 and 4.10 ) whereas SILAC analysis yielded relatively high protein ratios (between 3 and 6) for some of the SR proteins (see Table 4.11). Most of the hnRNP proteins are according to all three quantification approaches highly abundant in B complexes. Exceptions are hnRNP R and RALY, which show a $1: 1$ ratio for $B$ and $C$ complexes obtained by iTRAQ, SILAC, and spectral count.

\subsubsection{Proteins predominantly associated with C complex}

The hPrp19/CDC5L protein complex is essential for pre-mRNA splicing (Ajuh et al., 2000). It associates with the spliceosome during its activation prior to the first catalytic step of splicing. Together with U5 snRNP it forms a remodeled 35S U5 complex (Makarov et al., 2002). In previous studies characterizing the precatalytic A and B complexes (Behzadnia et al., 2007; Deckert et al., 2006), the hPrp19/CDC5L complex proteins have been already detected in A and B complexes. In this study, most of the hPrp19/CDC5L proteins show a higher abundance in C complex (Figure 4.24). An exception is CTNNBL1, which does not show a clear protein ratio. For this particular protein, an enrichment in the $\mathrm{C}$ complex was found by SILAC whereas ITRAQ and spectral count revealed a slightly higher abundance in $B$ complex. The two proteins Npw38 and Npw38BP appear to be present predominantly in B complex. This is evidenced by the high protein ratios obtained by ITRAQ and SILAC (Figure 4.24). During proteomic analysis, these two proteins were only detected in B complex, making it impossible to determine the protein ratios for spectral count. Both these proteins 
were co-isolated with the hPrp19/CDC5L complex but were found on top when applied to a glycerol gradient (Makarova et al., 2004). Their enrichment in B complex thus corroborates their dissociation from the hPrp19/CDC5L complex. The proteins related to the hPrp19/CDC5L complex are no exception and show a high abundance to C complex (Tables $4.9,4.10$, and 4.11).

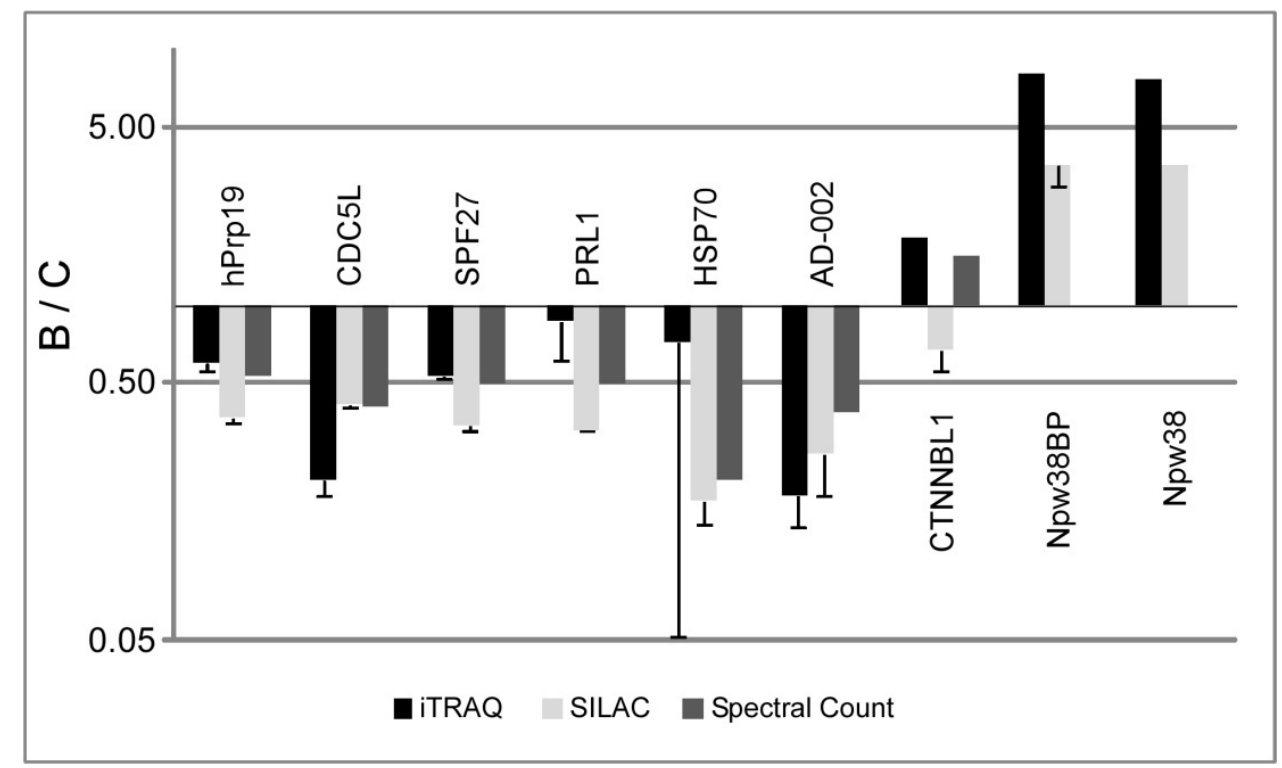

Figure 4.24: Protein ratios for hPrp19/CDC5L complex proteins obtained by ITRAQ, SILAC, and spectral count. Almost all proteins show a clear enrichment in C complex (protein < 1). For CTNNBL1 no clear association to $B$ or $C$ complexes could be determined. Npw38BP and Npw38 show a high abundance in the B complex. For these proteins no protein ratio for spectral count could be calculated as both proteins were solely detected in $B$ complex during proteomic analysis.

All proteins required for the second step of splicing (so-called Step 2 factors, namely hPrp22, hPrp18, hPrp17, hPrp16 and hSLU7) show a B/C ratio of less than 0.5 suggesting their association to the catalytically active spliceosome ( $\mathrm{C}$ complex). This is in agreement with their function in the second catalytic step during pre-mRNA splicing, which occurs in the $C$ complex. Comparing protein ratios obtained by ITRAQ, SILAC, and spectral count, iTRAQ and SILAC revealed protein ratios between 0.5 and 0.1 (Figure 4.25). The spectral count yielded very low B/C ratios (close to 0 ) for the step 2 factors, consistent with their function in C complex (see also Table 4.9). 


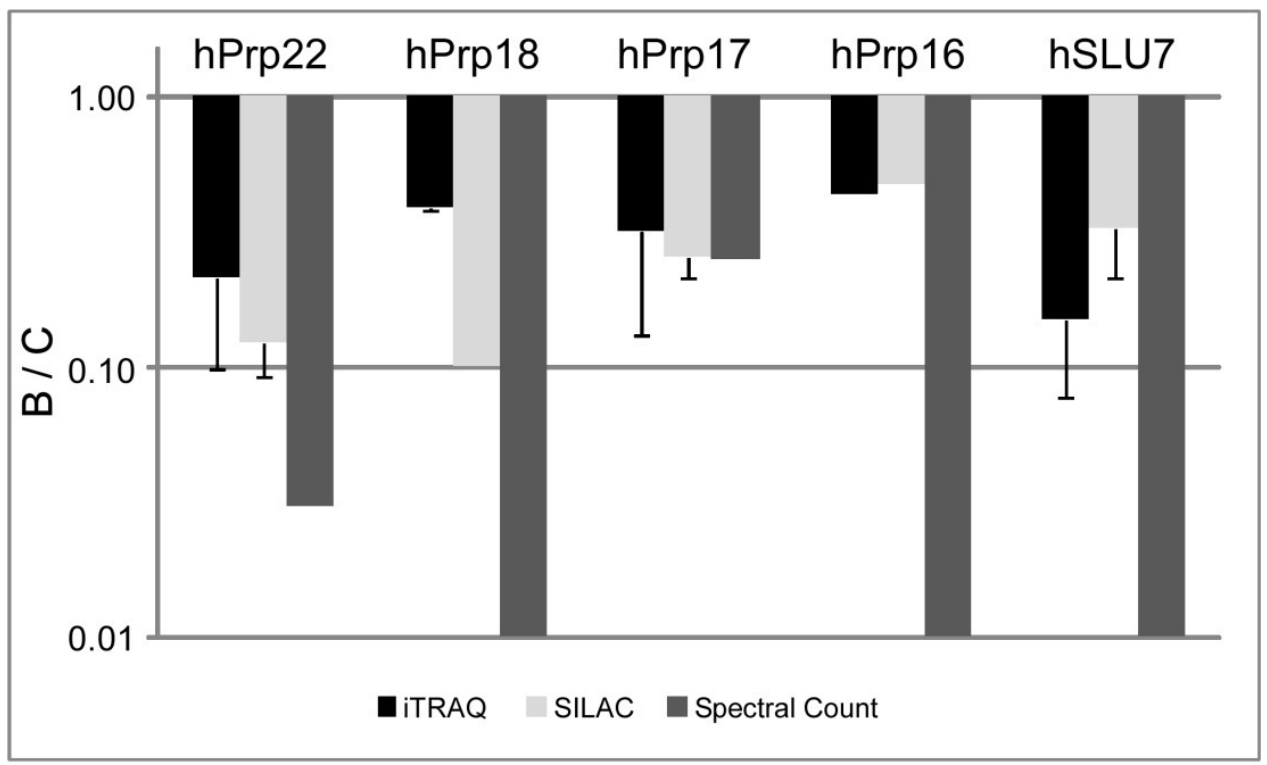

Figure 4.25: Protein ratios of step 2 factors obtained by ITRAQ, SILAC, and spectral count. ITRAQ and SILAC protein ratios are between 0.5 and 0.1 , whereas spectral count yielded very low protein ratios close to 0 .

The EJC proteins elF4A3, Magoh, and $\mathrm{Y} 14$ show B/C ratios of less than 0.4 with all three quantification approaches (iTRAQ, SILAC, and spectral count) indicating that they are recruited to the spliceosome during $C$ complex formation. However, the other EJC/mRNP proteins (Pinin, RNPS1, Acinus, SAP18, and Aly) show a B/C ratio of approximately 1 or slightly higher, implying that they are present in both B and C complexes. UAP56 yielded a protein ratio of approximately 3 by ITRAQ and SILAC, indicating that it is specific for $B$ complex (Tables 4.9, 4.10, and 4.11).

In addition to the above discussed protein groups previously classified as "recruited to $\mathrm{C}$ complex" and "potential C complex specific proteins" (Bessonov et al., 2008) were quantified in this study. These proteins display $\mathrm{B} / \mathrm{C}$ ratios below 0.7 in all three quantification methods (Tables 4.9, 4.10, and 4.11). Comparing the two stable isotope labeling quantification methods (iTRAQ and SILAC), ITRAQ protein ratios are slightly higher (compare Tables 4.10 and 4.11). Spectral count from proteomic analysis clearly shows, that "potential C specific proteins" are only detected in C complex (with the exception of one peptide generated of PPIG identified in one replicate of the B complex analyses). Some representatives of the "proteins recruited to C complex" are represented with few peptides also in B complex (Table 4.9), but still show a clear association with $C$ complex.

For all other groups of proteins (e.g. TREX, miscellaneous proteins etc.) no clear association with either the $B$ or the $C$ complex could be found. Proteins within these groups differ in their abundances in B or C complexes. Interestingly, the DDX34 protein has not been found in any spliceosomal complex before, but was in this study clearly identified to be highly enriched in 
C complex by ITRAQ (see Tables A.5 and A.6) and SILAC. During proteomic analysis of the C complex, DDX34 was identified with 14 and 3 tandem MS spectra within the two replicates, respectively, whereas no DDX34 peptides were found in the B complex.

\subsubsection{Comparison of the three quantification methods - ITRAQ, SILAC, and spectral count}

In general, quantitative information about protein abundances within spliceosomal B and C complexes could be obtained from all three quantification approaches. The protein ratios achieved by ITRAQ and SILAC yielded for all proteins comparable B/C ratios with only few exceptions (e.g. CTNNBL1, Figure 4.24). However, the B/C ratios obtained by SILAC are in most cases slightly higher or slightly lower for proteins enriched in B or C complexes, respectively, when compared to iTRAQ. This phenomenon can be explained by background signals of ITRAQ reporter ions due to the width of the precursor selection window for MS/MS fragmentation. This is not $100 \%$ selective for one precursor and co-eluting peptides might contribute to the ITRAQ reporter signals (Bantscheff et al., 2007). Background signals for iTRAQ reporter ions thus cause slightly lower protein ratios for proteins that are enriched in $B$ complexes and slightly higher values for proteins that are enriched in $\mathrm{C}$ complexes.

Comparing ITRAQ and SILAC to semi-quantitative spectral count, in almost all cases the correct abundance trend was already observed by the spectral count procedure. Most of the $\mathrm{B} / \mathrm{C}$ ratios fit reasonably to the values obtained by the stable isotope labeling procedures (i.e. iTRAQ and SILAC). However, spectral count appears to be limited to proteins showing major changes within the two states that were compared. For proteins that are present in equal amounts in the two complexes, SILAC and ITRAQ yielded accurate protein ratios of 1 in most cases, whereas protein ratios obtained by spectral count deviated within a narrow range. In addition, accurate quantification of the Sm proteins could not be achieved by spectral count. The correct protein ratios were, however, readily obtained by ITRAQ and SILAC. For proteins that were only detected in either the $B$ or the $C$ complexes, spectral count cannot yield the $\mathrm{B} / \mathrm{C}$ ratios. This suggests total absence of a particular protein in one of the compared complexes but might not reflect the actual association of the protein with the analyzed complex. Thus a peptide is not selected for sequencing during MS/MS, it is not necessarily absent in the sample to be analyzed. Therefore some of the extreme values obtained by spectral count might be misleading in terms of relative protein abundances in the different samples. 
Finally, a correlation analysis of ITRAQ and SILAC was performed to see, if the obtained protein ratios are comparable. To this end, protein ratios achieved by ITRAQ and SILAC were $\log _{2}$ transformed and plotted against one another. The scatter plot confirms the observation that iTRAQ shows lower or higher protein ratios for proteins enriched in $B$ or $C$ complexes, respectively. The correlation of SILAC and ITRAQ protein ratios was tested by calculating the Pearson correlation coefficient using software R. A correlation factor of 0.815 and a p-value of $2.2 \cdot 10^{-16}$ were determined showing that a correlation between these two data sets exists.

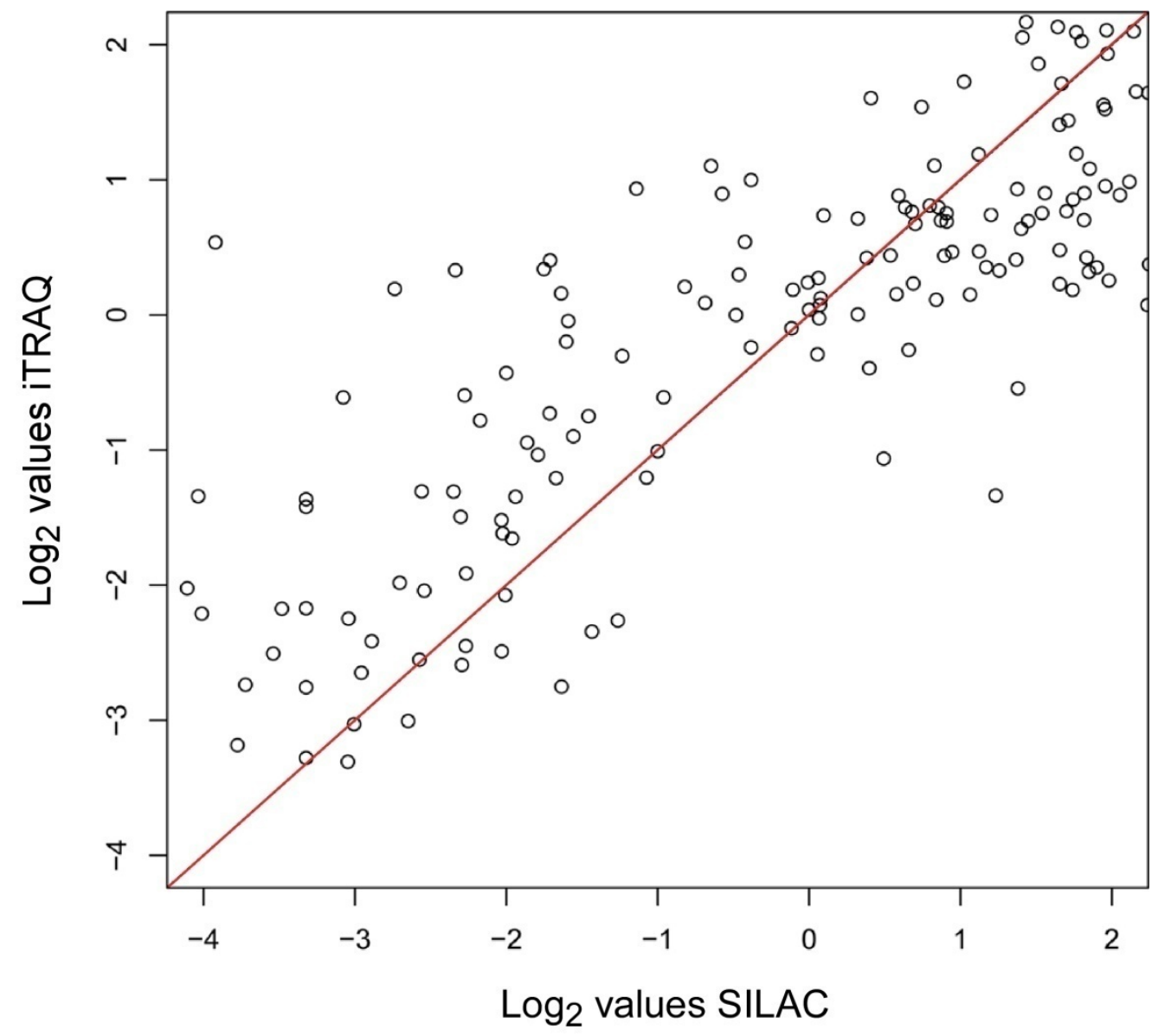

Figure 4.26: Scatter plot of SILAC and ITRAQ protein ratios. The $\log _{2}$ values of ITRAQ and SILAC protein ratios, respectively, were plotted in a scatter plot. The red line shows optimal correlation between the two quantification methods. As described above, lower or higher iTRAQ ratios for proteins enriched in B or C complexes, respectively, were obtained when compared to SILAC values.

For the lack of meaningful $B / C$ ratios in cases where the protein was missing in either the $B$ or the $\mathrm{C}$ complex, the spectral count data could not be included in the statistical analysis. 


\subsection{Protein assembly time line for spliceosomes by relative quantification}

During pre-mRNA splicing the spliceosome assembles on the pre-mRNA and passes sequentially through functional states that differ in their protein and RNA compositions (E, A, $B$, and $\mathrm{C}$ complexes). The relative quantification of the isolated spliceosomal complexes shows differences in the protein abundances between the different assembly states, but does not reveal how the transition from one complex to the other takes place. Mass spectrometry-based relative quantification can also be applied to monitor dynamic protein changes. Triple SILAC allows comparison of three different samples in one MS experiment and thus provides the opportunity to compare three different time points. Performing multiple experiments using the zero time point as a reference allows the construction of dynamic profiles for single proteins. In this study, the protein assembly on splicing-active and splicinginactive pre-mRNAs was analyzed and the protein compositions at different time points were compared. Two approaches were followed: (i) Comparison of the time-dependence of protein assembly on splicing-active or splicing-inactive pre-mRNAs, and (ii) the direct comparison of the protein composition on a splicing-active to a splicing-inactive pre-mRNA at different time points during pre-mRNA splicing.

\subsubsection{Generation of splicing inactive pre-mRNAs}

The protein assembly was investigated on splicing-active and splicing-inactive pre-mRNAs. The PM5 pre-mRNA was used as splicing-active pre-mRNA. It lacks the 3' splice site and the 3' exon and has been shown to be well-suited to isolate catalytically active step $1 \mathrm{C}$ complexes (Bessonov et al., 2008). When incubated under splicing conditions with HeLa nuclear extract, PM5 undergoes 5' splice site cleavage and formation of the intron lariat but no exon ligation takes place.

Two splicing-inactive pre-mRNAs were considered for comparison of the protein assembly on splicing-active (PM5) and splicing-inactive pre-mRNAs: (i) PM5 pre-mRNA lacking the 5' splice site (5'ss) and, (ii) PM5 pre-mRNA lacking the branch point site (BPS). As the U1 and U2 snRNP bind to the pre-mRNA's 5'ss and BPS, respectively, during the formation of A complex, the use of pre-mRNAs from which the 5'ss and BPS have been deleted will hamper the spliceosomal assembly. These pre-mRNAs are therefore splicing-inactive and may be expected to prevent spliceosomal complex formation.

The sequence of the PM5 plasmid is shown in Figure 4.27. The nucleotide sequence AGGTATGT or ACTGA (highlighted in red) was deleted to generate 5'ss- or BPS-deleted PM5 pre-mRNAs, respectively. Generation of the pre-mRNAs was performed as outlined in 
section 3.2.1.11 using the PM5 plasmid as template. Two PM5 pre-mRNAs, one lacking the 5'ss and one lacking the BPS, respectively, were obtained.

\section{MS2}

GAATACAAGCTCATCCGATATCCGTACACCATCAGGGTACGAGCTAGCCCATGG

MS2

MS2

$\mathrm{ECOR} 1$

CGTACACCATCAGGGTACGACTAGTAGATCTCGTACACCATCAGGGTACGGAAT

Exon1

TCTCTAGAGTCGAGGAGGACATCTCAGCAAAAGAGAAGCTGCTGCGGGCGTCGG

AGGACGAGCGGGACCGGGTGCTGGAGGAGCTGCACAAGGCAGAGGACAGCCTGC
\[ 5^{\prime} \text { ss Hind3 } \]
TGGCTGCCGACGAGACCGCCGCCAAGGTATGTATCAAGCTTACAAGACAGCTTT

$\mathrm{Xho1}$

AAGGAGACCAATAGAAACTGGGCATGTGGAGACAGAGAAGACTCTTGGCCTCGA

GAAACCTGTAACTGGAATGTGTGTGGAGTGTGACTGATAGAACACTACCTGATT

BPS

$\left(Y_{n}\right)$

CTTATGTATTACTGACCTGTGTTTTTTGCTACTTTTTTCTTTTCTCCCстT

BamH1

СССстTсCСтAтTTTTTTстTGCCCTGATCCGGAATTGGATCC

Figure 4.27: Sequence of the PM5 plasmid. MS2 binding sites (MS2), exon 1, branch point site (BPS) and polypytimidine tract $\left(\mathrm{Y}_{\mathrm{n}}\right)$ are shown in bold. Restriction sites of DNA restriction enzymes are shown in blue. Deleted nucleotide sequences are shown in red.

The kinetics of splicing and spliceosomal complex formation were investigated for PM5 premRNA and 5'ss- and BPS-deleted PM5 pre-mRNAs (Figure 4.28). When PM5 pre-mRNA was used, splicing products (intron lariat and MS2-exon) first appeared after 10 minutes. The amount of pre-mRNA was reduced during incubation. As RNAseH digestion leads to degradation of early spliceosomes, the pre-mRNA disappeared and only the splicing products and an RNAseH digestion product were visible after RNAseH digestion. For PM5 5'ss-deleted pre-mRNA, no splicing products were formed, and the amount of pre-mRNA remained constant. After 180 minutes incubation followed by RNAseH digestion a digestion product was observed. However, using BPS-deleted PM5 pre-mRNA the appearance of splicing products after 30 minutes and 180 minutes followed by RNAseH digestion was indicated (Figure 4.28 A). Spliceosomal complex formation was assayed by native agarose gel electrophoresis, and the complexes formed were visualized by autoradiography. On PM5 
pre-mRNA, H/E complexes were rapidly formed, whereas $A$ and $B$ complexes first appeared after 2-4 minutes. C complex formation was first observed after 10 minutes. Surprisingly, $A$ and B complex formation was also observed on PM5 5'ss deleted pre-mRNA, although B complex formation seemed to be delayed about 2 minutes. However, $\mathrm{C}$ complex formation was not observed on the 5'ss-deleted PM5 pre-mRNA. Complex formation on BPS-deleted PM5 pre-mRNA suggests also formation of $A$ and $B$ complexes. Strikingly, after 180 minutes incubation followed by RNAseH digestion the presence of $\mathrm{C}$ complexes was indicated, whereas this is not clearly seen (Figure $4.28 \mathrm{~B}$ ).

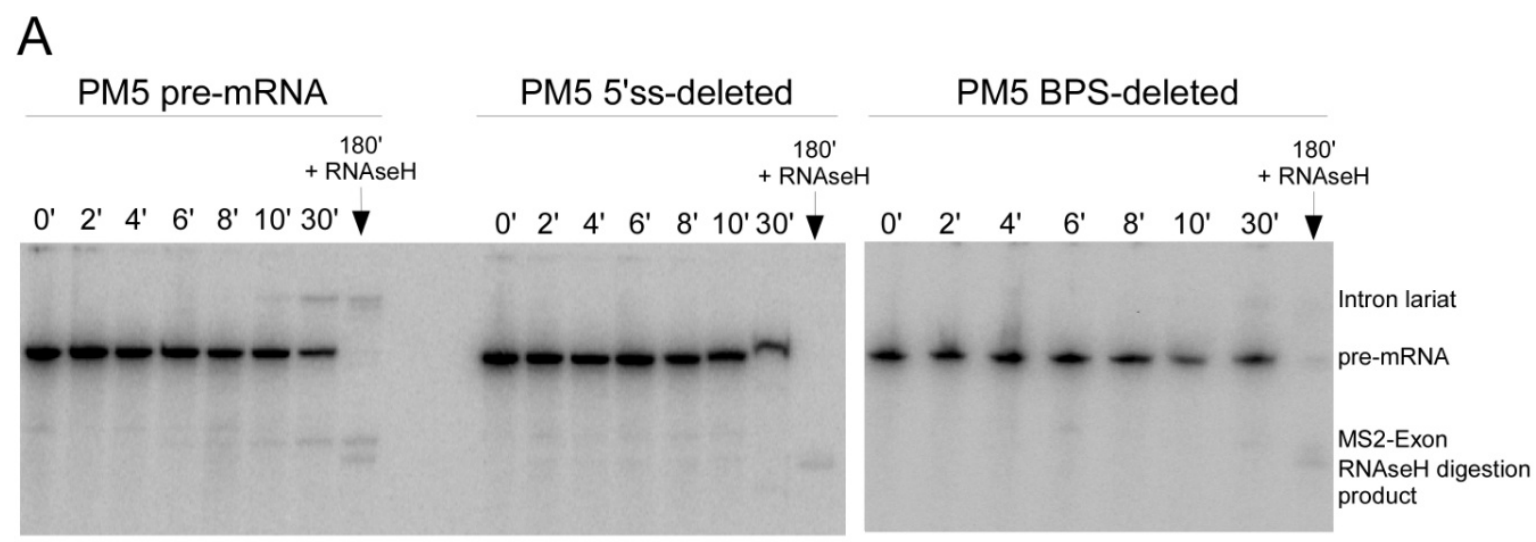

B
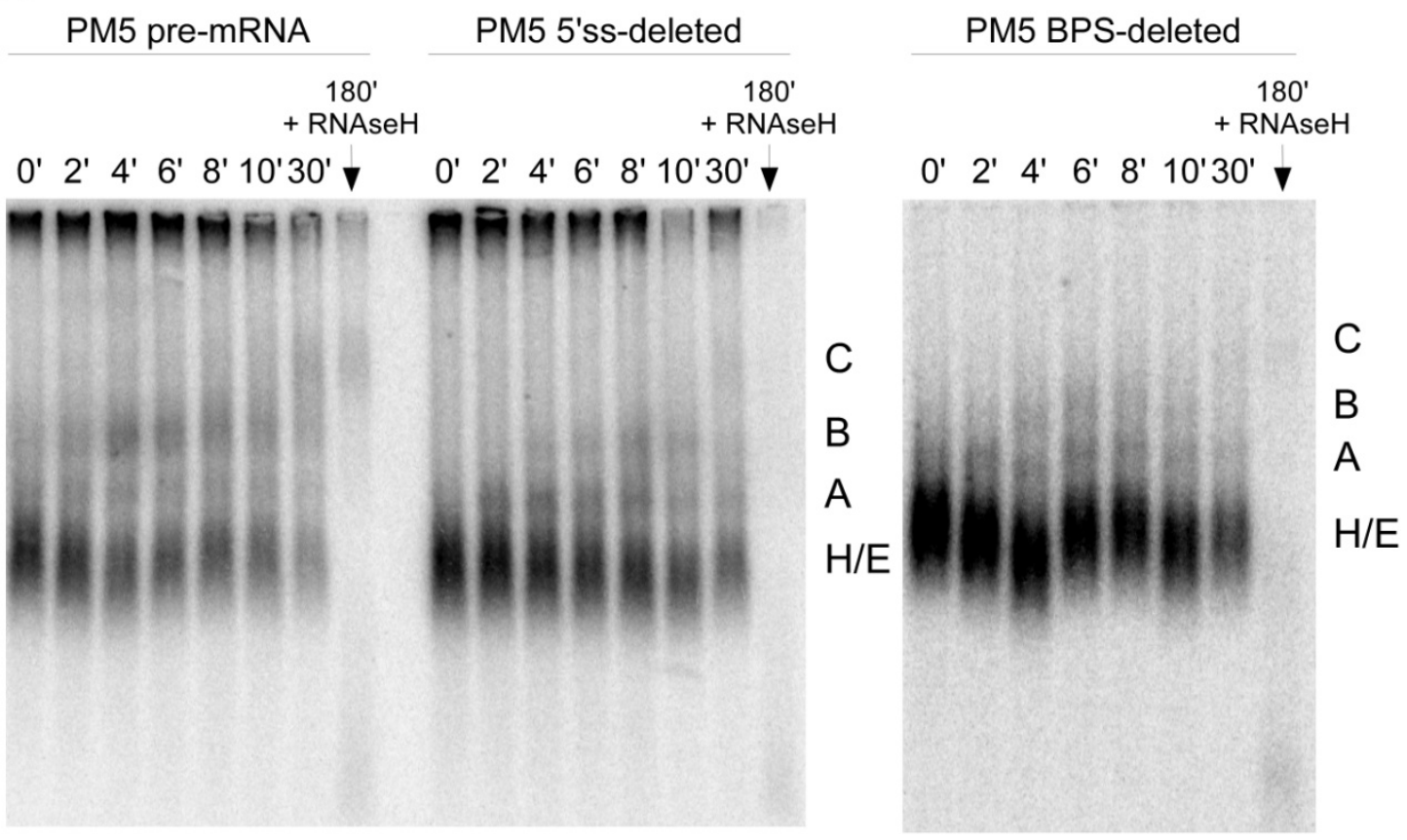

Figure 4.28: The splicing kinetics and the spliceosomal complex formation using PM5 pre-mRNA and 5'ss- and BPS-deleted PM5 pre-mRNA. (A) Splicing kinetics were followed by denaturing gel electrophoresis. Radioactively labeled pre-mRNA and splicing products were visualized by autoradiography. With PM5 pre-mRNA, splicing products first appeared after 10 minutes and the amount of pre-mRNA was reduced during incubation. With 5'ss-deleted PM5 pre-mRNA, no splicing products were observed, whereas the appearance of splicing products after 30 minutes was indicated for BPS-deleted PM5 pre-mRNA. (B) Spliceosomal complex formation was assayed by native agarose gel electrophoresis, and the complexes formed were visualized by autoradiography. On PM5 pre-mRNA, the formation of $\mathrm{H} / \mathrm{E}, \mathrm{A}, \mathrm{B}$ and $\mathrm{C}$ complexes was observed. Surprisingly, A and $B$ complex formation was also observed on 5'ss-deleted PM5 pre-mRNA. On BPS-deleted PM5 pre-mRNA, A and $\mathrm{B}$ complex formation was indicated and after 180 minutes incubation followed by RNAseH digestion $\mathrm{C}$ complex formation was suggested. 
The splicing kinetics revealed that no pre-mRNA splicing occurred when 5'ss-deleted PM5 pre-mRNA was used. Surprisingly, A and B complex formation was observed, even though the 5'ss, with which the U1 snRNP is known to make contact during A complex formation, was deleted. Nonetheless, $\mathrm{C}$ complex formation was not detected, confirming that this premRNA is indeed splicing-inactive. Strikingly, splicing products appeared after 30 and 180 minutes when using BPS-deleted PM5 pre-mRNA. During complex formation the presence of $\mathrm{C}$ complex after RNAseH digestion is indicated, although this is not clearly visible. Inspection of the PM5 plasmid sequence revealed that the deleted nucleotide sequence (ACTGA) exists once more in the PM5 plasmid. This duplicate is located 28 nucleotides downstream of the branch point site within the intron of the PM5 pre-mRNA (see Figure 4.27) and might serve as an alternative branch point. This would explain the presence of splicing products that were observed when the splicing kinetics and complex formation were analyzed.

For this reason, an additional pre-mRNA was produced in which the duplicate BPS sequence was also deleted (in the following referred to as "BPS-ACTGA-deleted PM5 pre-mRNA"). The splicing kinetics and the spliceosomal complex formation were then investigated for the BPSand the BPS-ACTGA-deleted pre-mRNA (Figure 4.29). As described above, when PM5 premRNA was used, splicing products were observed after 10 minutes and the amount of premRNA present decreased during the incubation. The splice assay for BPS-deleted PM5 premRNA again revealed the presence of splicing products after 30 and 180 minutes. The "double-deleted" pre-mRNA (the BPS-ACTGA-deleted PM5 pre-mRNA) did not show splicing products, confirming that this pre-mRNA mutant is splicing-inactive (Figure $4.29 \mathrm{~A}$ ). Spliceosomal complex formation for PM5 pre-mRNA again showed H/E, A, B and C complex formation. As described above, A and B complex formation was also observed for the BPSdeleted PM5 pre-mRNA; moreover $C$ complex formation after 30 and 180 minutes was suggested, although it was not clearly visible. For the BPS-ACTGA-deleted PM5 pre-mRNA no comparable complex formation was achieved. However, native agarose gel electrophoresis indicated the formation of some complexes on this particular pre-mRNA (Figure $4.29 \mathrm{~B}$ ). The complexes formed differed in their migration behavior in native agarose gel electrophoresis and thus appear to have a different RNA and/or protein composition as compared with the spliceosomal A, B and C complexes. Nonetheless, as the "doubledeleted" pre-mRNA (BPS-ACTGA-deleted PM5 pre-mRNA) did not lead to splicing products and did not show $\mathrm{C}$ complex formation, it is splicing-inactive. Therefore, in this study the PM5 pre-mRNA and the 5'ss- and the BPS-ACTGA-deleted PM5 pre-mRNAs were used for comparison of the protein assembly on splicing-active and splicing-inactive pre-mRNAs. 
A

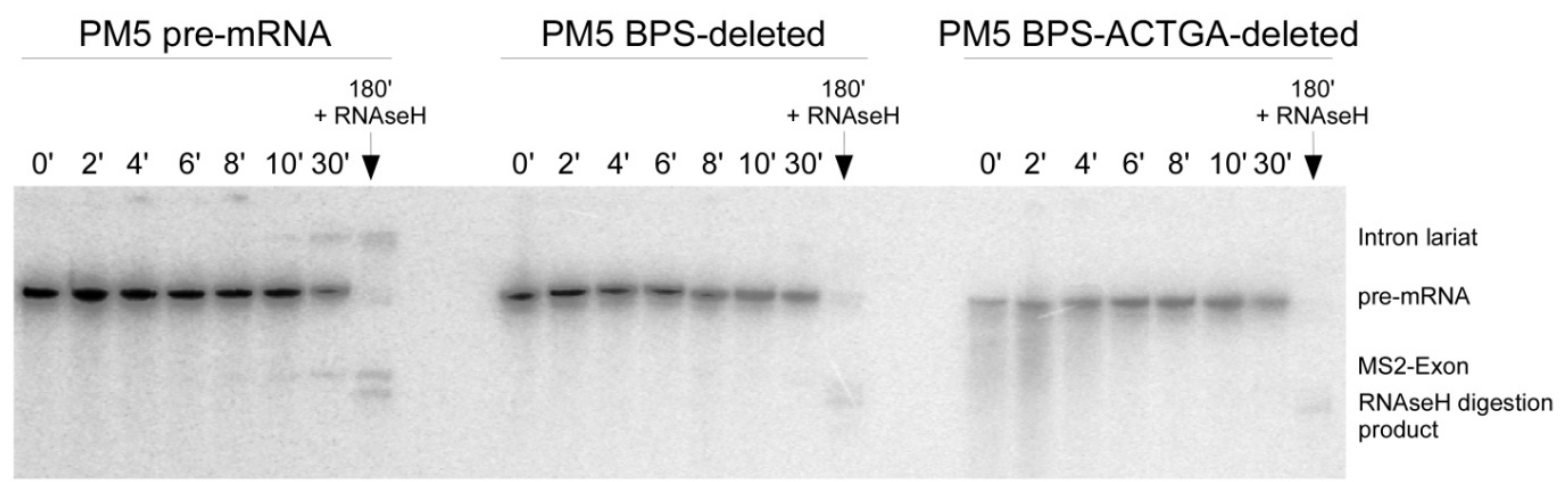

B

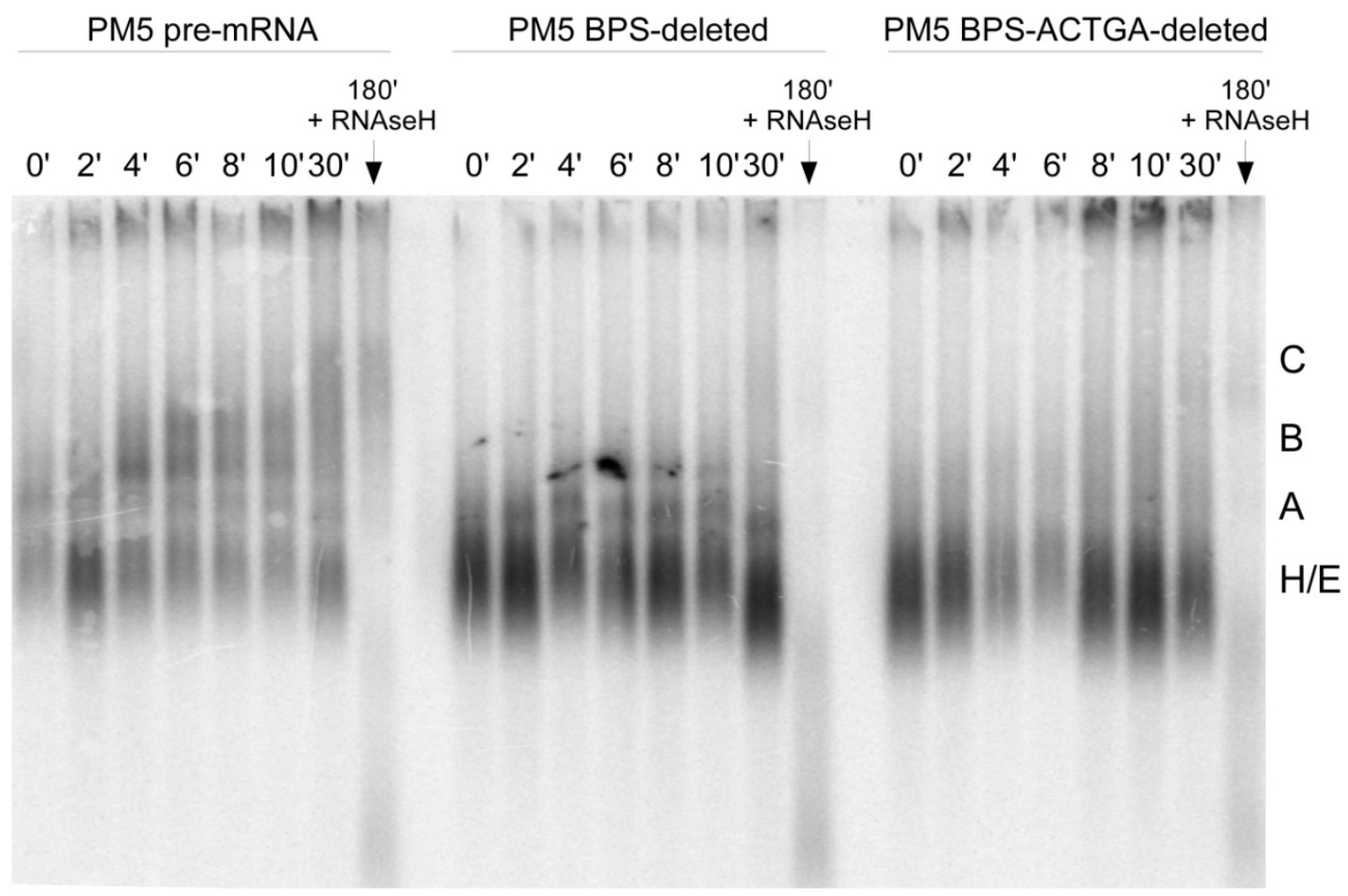

Figure 4.29: The splicing kinetics and the spliceosomal complex formation using PM5 pre-mRNA and BPS and BPS-ACTGA deleted PM5 pre-mRNAs. (A) Splicing kinetics were followed by denaturing gel electrophoresis. Pre-mRNA and splicing products were visualized by autoradiography. With PM5 pre-mRNA, splicing products first appeared after 10 minutes and the amount of pre-mRNA was reduced during incubation. With the BPS-deleted pre-mRNA splicing products had appeared after 30 minutes. For the "double-deleted" BPSACTGA-deleted PM5 pre-mRNA no splicing products were observed. (B) Spliceosomal complex formation was assayed by native agarose gel electrophoresis and the complexes formed were visualized by autoradiography. On PM5 pre-mRNA, the formation of $\mathrm{H} / \mathrm{E}, \mathrm{A}, \mathrm{B}$ and $\mathrm{C}$ complexes was observed. On BPS-deleted PM5 premRNA, A and B complex formation was detected, and after 180 minutes incubation followed by RNAseH digestion some $\mathrm{C}$ complex formation was detected. For BPS-ACTGA-deleted PM5 pre-mRNA no comparable complex formation was observed, whereas formation of some complexes is indicated. 


\subsubsection{Triple SILAC to monitor dynamic protein changes}

Triple SILAC allows quantitative comparison of three samples in one MS experiment and thus comparison of three time points during dynamic changes of protein composition. Using the zero time point as a reference point allows the construction of assembly timelines when performing multiple experiments. To make possible triple SILAC experiments for the investigation of the spliceosomal dynamic protein changes, triple SILAC nuclear extracts were prepared. To this end, HeLa cells were grown in the presence of isotope-labeled lysine and arginine. The combination of different isotope-labeled lysine and arginine allows generation of three differentially labeled cells (light, medium and heavily labeled cells; for experimental details see section 3.2.3.1). Nuclear extracts were then prepared and tested for their splicing activity. Figure 4.30 shows the splicing kinetics and the spliceosomal complex formation for PM5 pre-mRNA using light, medium and heavy SILAC nuclear extracts.

For all nuclear extracts (i.e. light, medium and heavy SILAC nuclear extracts) splicing products were first observed after 10 minutes. The intensity of the splicing products in heavy SILAC nuclear extracts is very low compared to that of the splicing products generated in light and medium extracts. After RNAseH digestion, pre-mRNA had been degraded and the splicing products - as well as an RNAseH digestion product - were visible (Figure 4.30 A). The formation of spliceosomal complexes was assayed by native agarose gel electrophoresis. The yields of complex formation for the three SILAC nuclear extracts were comparable. H/E complexes were formed rapidly, and A complexes first appeared after approximately 2 minutes. B complexes were formed after 2-4 minutes, and C complex formation was first observed after 10 minutes. After RNAseH digestion, early splieceosomes were degraded and only C complexes remained. However, B complex formation seemed to be delayed by about 2 minutes when medium and heavy nuclear extracts were compared to light nuclear extract (Figue $4.30 \mathrm{~B}$ ).

Although the intensity of the splicing products was very low when heavy SILAC nuclear extract was used, the three nuclear extracts were considered to be identical in splicing activity, as C complex formation was clearly observed. The low intensity of the splicing products might have been caused by sample loss during sample preparation, or by sample loading for gel electrophoresis. Nonetheless, it was inferred the delayed B complex formation should be kept in mind when performing time-dependent experiments. 
A

\begin{tabular}{|c|c|c|}
\hline light SILAC nuclear extract & medium SILAC nuclear extract & heavy SILAC nuclear extract \\
\hline $\begin{array}{c}180^{\prime} \\
+ \text { RNAseH }\end{array}$ & $\begin{array}{c}180^{\prime} \\
+ \text { RNAseH }\end{array}$ & $\begin{array}{c}180^{\prime} \\
+ \text { RNAseH }\end{array}$ \\
\hline
\end{tabular}

$\begin{array}{lllllll}0^{\prime} & 2^{\prime} & 4^{\prime} & 6^{\prime} & 8^{\prime} & 10^{\prime} & 30^{\prime} \mathbf{\nabla}\end{array}$

$\begin{array}{lllllll}0^{\prime} & 2^{\prime} & 4^{\prime} & 6^{\prime} & 8^{\prime} & 10^{\prime} 30^{\prime} & \boldsymbol{1}\end{array}$

$\begin{array}{llllllll}0^{\prime} & 2^{\prime} & 4^{\prime} & 6^{\prime} & 8^{\prime} & 10 & 30^{\prime} \mathbf{\nabla}\end{array}$

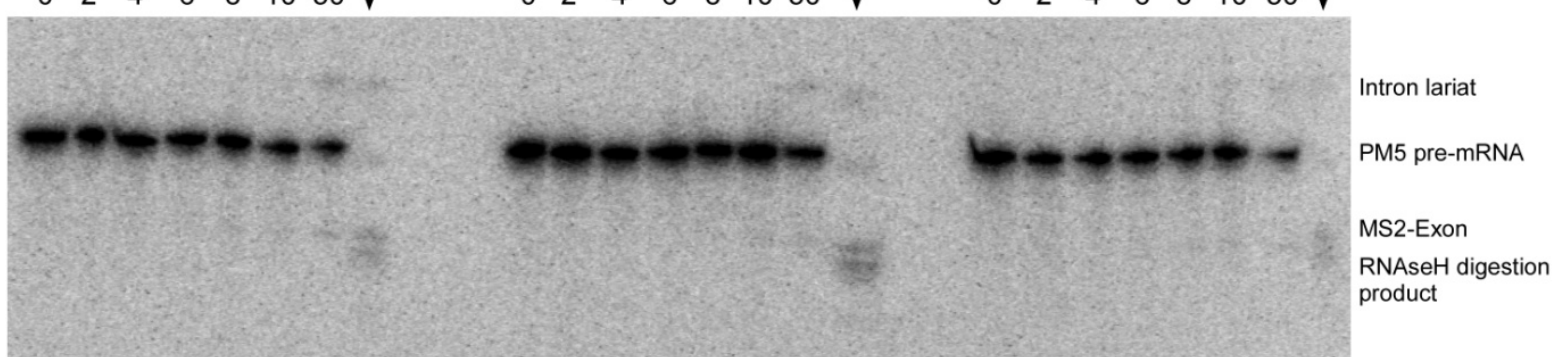

B

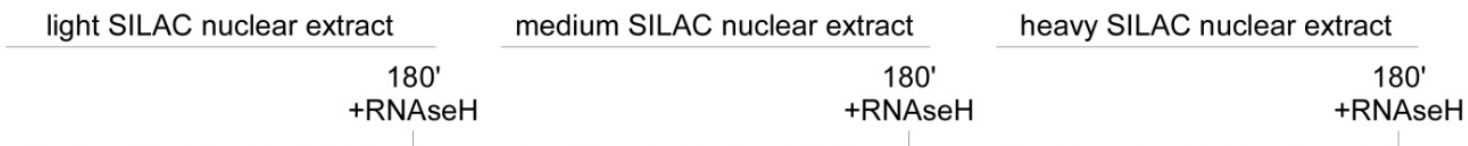

$0^{\prime} \quad 2^{\prime} \quad 4^{\prime} \quad 6^{\prime} \quad 8^{\prime} \quad 10^{\prime} 30^{\prime}$

$\begin{array}{lllllll}0^{\prime} & 2^{\prime} & 4^{\prime} & 6^{\prime} & 8^{\prime} & 10^{\prime} & 30\end{array}$

$\begin{array}{lllllll}0^{\prime} & 2^{\prime} & 4^{\prime} & 6^{\prime} & 8^{\prime} & 10^{\prime} & 30^{\prime}\end{array}$

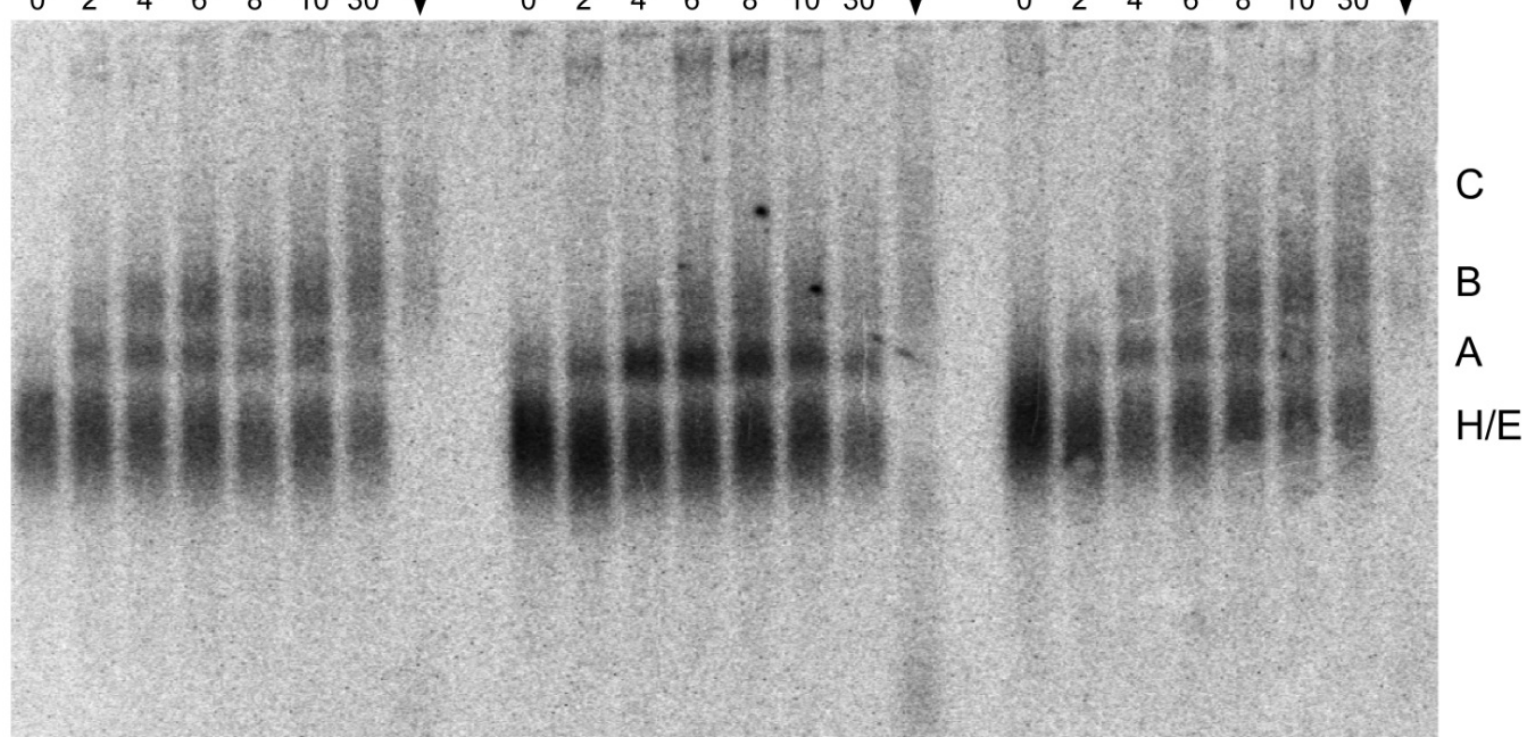

Figure 4.30: Splicing kinetics and spliceosomal complex formation using light, medium and heavy SILAC nuclear extracts. The splicing kinetics and the complex formation are comparable for light, medium and heavy SILAC nuclear extracts. (A) The splicing kinetics were followed by denaturing gel electrophoresis. Pre-mRNA and splicing products were visualized by autoradiography. Splicing products first appeared after 10 minutes incubation for all SILAC nuclear extracts. However, the intensity of the splicing products obtained from heavy SILAC nuclear extracts was very low compared with those from light and medium nuclear extracts. (B) Spliceosomal complex formation was assayed by native agarose gel electrophoresis and visualized by autoradiography. A and B complex formation was observed after 2-4 minutes, whereas $\mathrm{C}$ complexes were first observed after 10 minutes. $\mathrm{B}$ complex formation seems to be delayed about 2 minutes in medium and heavy nuclear extracts compared with light nuclear extract.

As the protein dynamics during pre-mRNA splicing were investigated in this study, the nuclear extracts also had to be compared regarding their protein content. The protein content within the nuclear extracts should be comparable, in order to exclude influences on the spliceosomal protein assembly during pre-mRNA splicing. 
A

B

medium vs. light

\section{SILAC \\ nuclear extracts \\ 1:1:1}

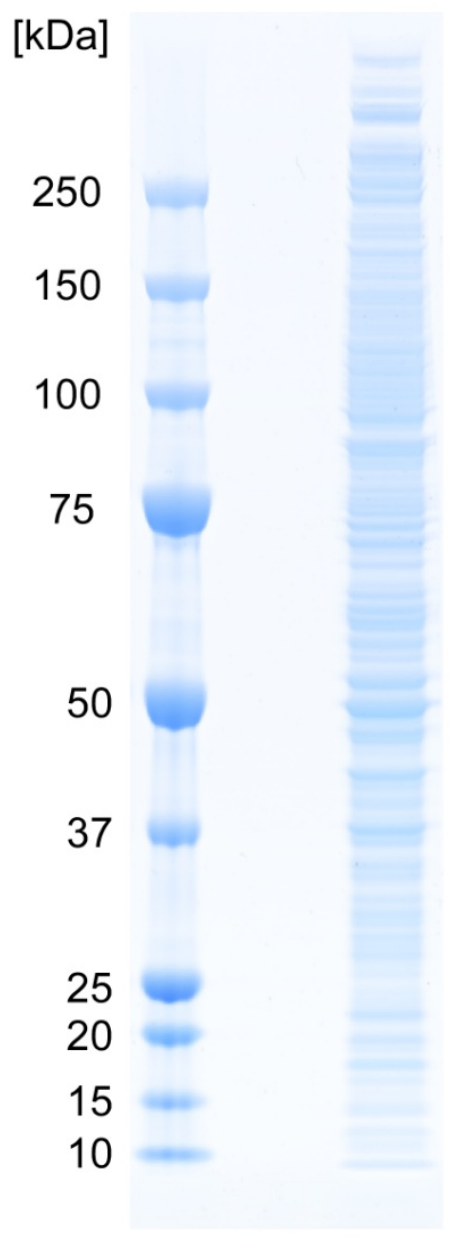

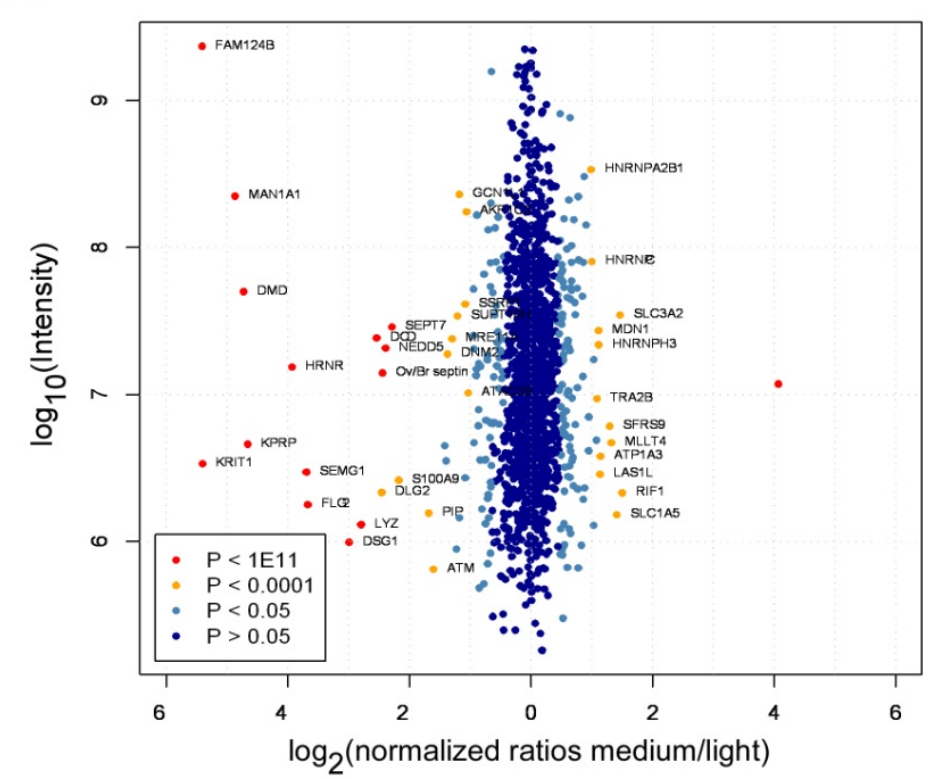

C

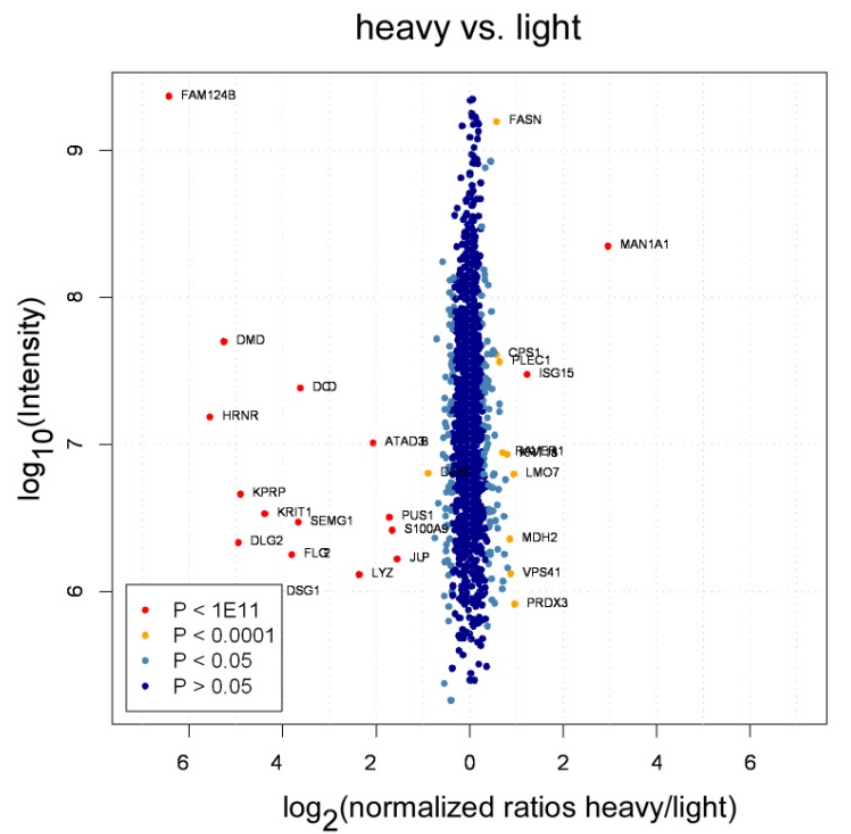

Figure 4.31: Comparison of the protein content within light, medium and heavy SILAC nuclear extracts. (A) Equal amounts of SILAC nuclear extracts (light, medium and heavy) were pooled and proteins were separated by PAGE. (B) Comparison of medium and light nuclear extract. Intensities were plotted against the $\log _{2}$ protein ratios showing that most of the proteins are of similar abundance within the two nuclear extracts and only few proteins were enriched in light nuclear extract. (C) Comparison of heavy and light nuclear extract. As described for comparison of medium and light nuclear extracts, only few proteins were enriched in light nuclear extract.

To compare the protein content of the different SILAC nuclear extracts, the total protein concentrations were obtained by using the Bradford protein assay. Protein concentrations of $16.14,15.08$ and $14.05 \mu \mathrm{g} / \mu \mathrm{l}$ were found for the light, medium and heavy nuclear extract, respectively. Equal amounts of proteins from the light, medium and heavy nuclear extracts were pooled, and the proteins were separated by PAGE (Figure $4.31 \mathrm{~A}$ ). The entire gel lane 
was excised and the proteins were hydrolyzed with trypsin. The peptides thus generated were analyzed by LC-MS/MS and peptides and proteins were subjected to relative quantification quantified using MaxQuant software (Cox and Mann, 2008). The protein ratios obtained (medium/light and heavy/light) were log2 transformed and the intensities $\left(\log _{10}\right)$ were plotted against the $\log _{2}$ protein ratios (Figure $4.31 \mathrm{~B}$ and $\mathrm{C}$ ). $\mathrm{A} \log _{2}$ protein ratio of 0 represents no difference in the protein abundance between the samples compared. $A \log _{2}$ rartio of 1 shows a twofold enrichment, whereas a $\log _{2}$ ratio of -1 shows that the protein amount is halved compared with the other sample. Figure $4.31 \mathrm{~B}$ shows the comparison of the medium with the light nuclear extract. Most of the proteins show a $\log _{2}$ protein ratio of 0 , i.e. most of the proteins are present in equal amounts in the two nuclear extracts. Only few proteins were found to be enriched in the light nuclear extract. However, these proteins are in most cases contaminating proteins (e.g. Hornerin, Filaggrin) and they are not involved in premRNA splicing. Figure $4.31 \mathrm{C}$ shows the comparison of $\log _{2}$ protein ratios between heavy and light nuclear extracts. As described for comparison of medium and light nuclear extracts, most of the proteins were of similar abundance in the heavy and the light nuclear extract. Again, proteins that were enriched in the light nuclear extract are not involved in pre-mRNA splicing and are therefore not expected to affect the incorporation of proteins into the spliceosome. An overview of the proteins that were enriched in one of the SILAC nuclear extracts is given in Table A7 in the Appendix.

In summary, the SILAC nuclear extracts show no differences in their protein content and show the same splicing activity when incubated with pre-mRNA under splicing conditions. They are therefore expected to show the same response when analyzing the spliceosomal protein assembly at different time points. Changes between the different time points to be compared can thus be concluded to reflect differences in the spliceosomal assembly and are not due to differences between the different SILAC nuclear extracts.

\subsubsection{Time-dependent protein assembly on splicing-active and splicing-inactive pre- mRNAs}

\subsubsection{Experimental setup}

To investigate the time-dependent protein assembly on different splicing-active and splicinginactive pre-mRNAs, triple SILAC was used according to the experimental setup described below (Figure 4.32). SILAC nuclear extracts were prepared from differentially labeled SILAC HeLa cells (light, medium and heavy) and spliceosomal assembly was performed by incubation of a MS2-tagged and radioactively labeled pre-mRNA (PM5 pre-mRNA and 5'ssand BPS-ACTGA-deleted PM5 pre-mRNA) with different SILAC nuclear extracts under 
splicing conditions. The splicing reactions were assembled for different time intervals and the assembled complexes were affinity purified (see section 3.2.3.8 for details). According to the radioactivity of the pre-mRNA, the assembled complexes from two different time points were mixed in equal molar amounts with complexes assembled at time point zero (Figure 4.32). To allow monitoring of the time-dependent protein assembly on the different pre-mRNAs, assembled complexes were not further purified by glycerol gradient centrifugation as described in previous studies.

$\begin{array}{lcc}\text { HeLa } & \text { nuclear } & \text { pre-mRNA splicing } \\ \text { cells } & \text { extract } & + \text { affinity purification }\end{array}$

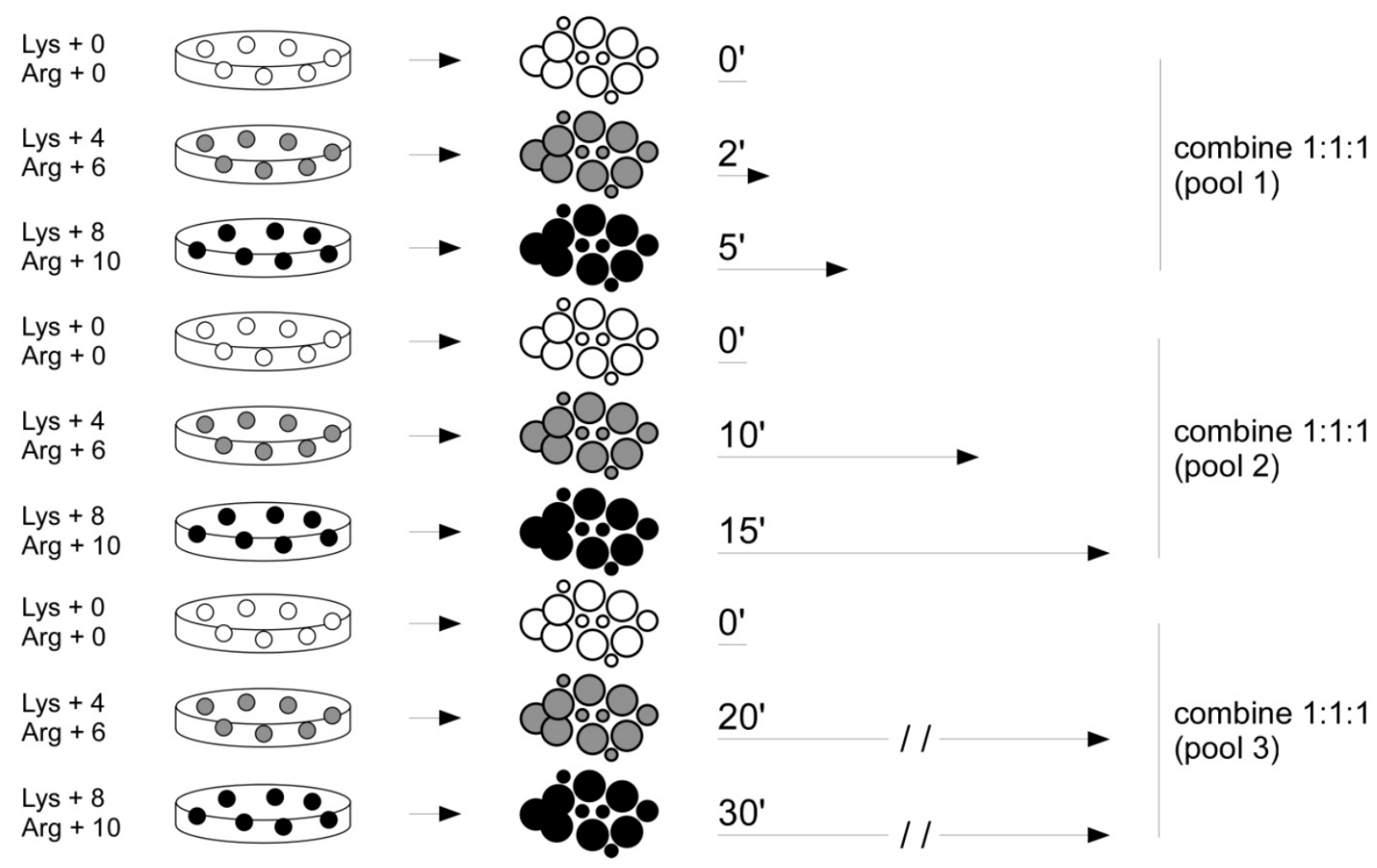

Figure 4.32: Experimental setup to monitor the time-dependent protein assembly during pre-mRNA splicing. SILAC nuclear extracts were prepared from differentially labeled HeLa cells (light, medium, heavy). Spliceosomal protein assembly was performed by incubation of a pre-mRNA (splicing-active or splicing-inactive) with the different nuclear extracts under splicing conditions. Several splicing reactions were assembled for different time intervals. Assembled complexes from two time points were pooled in equal amounts with complexes assembled at time point zero.

Assembled proteins within the combined samples (pools 1-3) were separated by PAGE (Figure 4.33). The MS2-MBP protein which was used for affinity purification shows equal intensity within the combined samples (pools 1-3) for assembly on the different pre-mRNAs confirming the mixing procedure based on the radioactively labeled pre-mRNA. However, significant differences in the protein assembly on the splicing-active and splicing-inactive premRNAs were not observed in the Coomassie stained gels (Figure 4.33). 
After separation by PAGE the proteins were hydrolyzed with trypsin, and the peptides generated were subsequently analyzed by LC-MS/MS (see section 3.2.4.12). The peptides and finally the proteins were quanified by using MaxQuant software (Cox and Mann, 2008). Protein ratios for the time point of analysis versus the reference time point were calculated and used for the construction of assembly time lines for individual proteins.

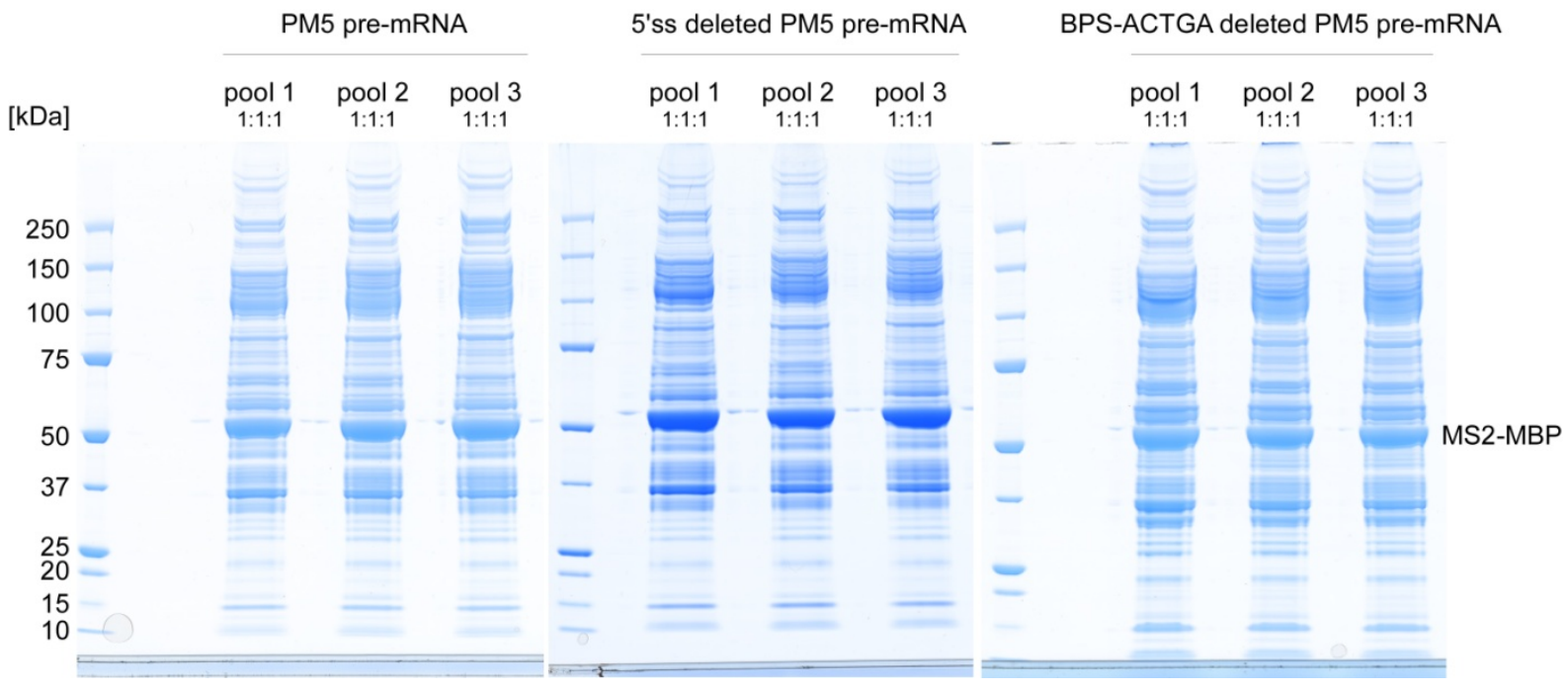

Figure 4.33: Coomassie stained gels of combined samples (pools 1-3) from assembly studies on PM5 pre-mRNA, 5'ss-deleted PM5 pre-mRNA and BPS-ACTGA-deleted PM5 pre-mRNA. Assembled complexes taken from the reaction at different time points were pooled in equal molar amounts with complexes assembled at time point zero and proteins were separated by PAGE.

\subsubsection{Normalization of the data}

Before constructing time-dependent assembly timelines for proteins that assemble on the analyzed pre-mRNAs, the data was normalized to compensate for errors that occurred owing to mixing of the samples or at any other step during sample-handling. The protein ratios obtained were normalized to those found for ribosomal proteins, which are contaminating proteins co-purified during the affinity purification of assembled complexes, and which are expected to be present in equal amounts within all samples. All ribosomal proteins were checked for enrichment in one of the extracts when the different SILAC nuclear extracts were mixed in equal amounts (see above) and only ribosomal proteins that were present in equal amounts within the light, medium and heavy nuclear extract were chosen for normalization (see Table A.8 for a complete list of the ribosomal proteins used for data normalization in this work). The normalization is exemplified for the assembly of proteins on PM5 pre-mRNA (Figure 4.34). 
The protein ratios of 19 ribosomal proteins were plotted for every time point (using zero minutes as reference time point) showing the abundance of the ribosomal proteins in protein complexes assembled on PM5 pre-mRNA as a function of time over the interval investigated (Figure $4.34 \mathrm{~A}$ ). The abundance of the ribosomal proteins is expected to be the same at every time point. Thus, a normalization factor for every time point could be calculated from the average value of the ribosomal protein ratios (Figure $4.34 \mathrm{C}$ ). The calculated normalization factors for the different time points were then applied to the protein ratios. Figure 4.34 B shows the assembly of the normalized ribosomal protein ratios, which are the same at every time point. The calculated normalization factors were used to normalize the protein ratios of every protein quantified in the data set. The normalization factors determined for the assembly studies on different pre-mRNAs are listed in Table A9.

A

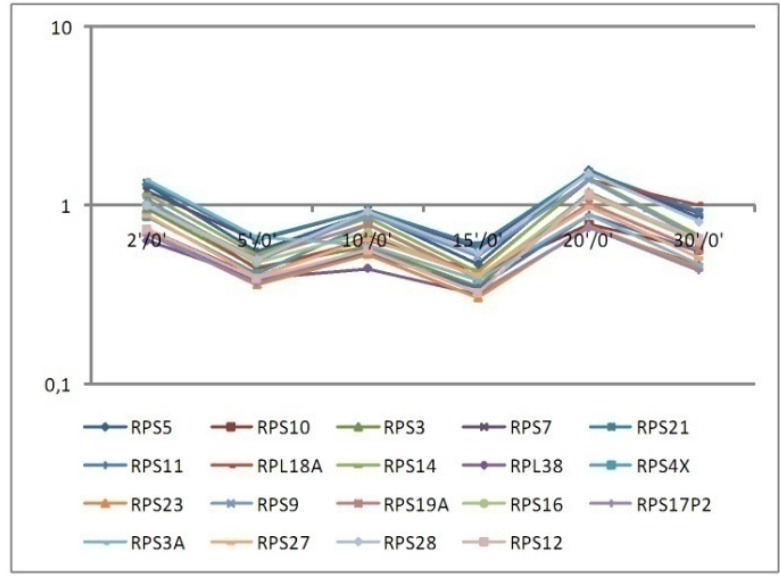

C
B

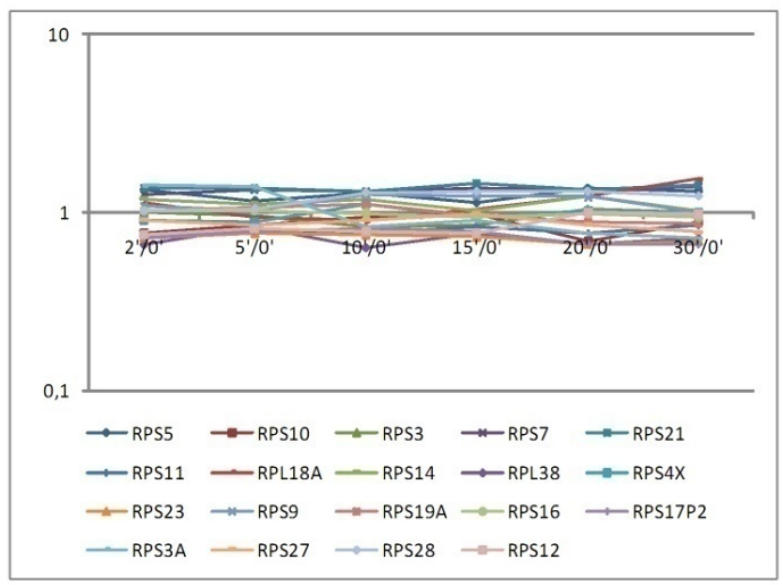

$\begin{array}{llll}10^{\prime} / 0^{\prime} & 15^{\prime} / 0^{\prime} & 20^{\prime} / 0^{\prime} & 30^{\prime} / 0^{\prime} \\ 0.715 & 0.418 & 1.152 & 0.655 \\ 1.399 & 2.395 & 0.868 & 1.526\end{array}$

Figure 4.34: Normalization of the obtained protein ratios on the ribosomal proteins. (A) Obtained protein ratios for 19 ribosomal proteins were plotted for every time point using the zero time point as a reference. (B) The normalized protein ratios for the 19 ribosomal proteins were plotted for every time point. (C) For every time point the average protein ratios for the 19 ribosomal proteins and the calculated normalization factors are given.

\subsubsection{Protein assembly on splicing-active and splicing-inactive pre-mRNAs}

The protein assembly during pre-mRNA splicing was investigated on various splicing-active and splicing-inactive pre-mRNAs: (i) PM5 pre-mRNA, (ii) 5'ss-deleted PM5 pre-mRNA, and (iii) BPS-ACTGA-deleted PM5 pre-mRNA (see above). Several splicing reactions were started on the different pre-mRNAs for different time intervals, and the assembled complexes were mixed in equal molar amounts using the time point zero as a reference value (see Figure 4.32 for an overview). After normalization of the protein ratios (see above), time lines 
for the different proteins were constructed. In the following section the protein assembly of the $\mathrm{U} 1$ and $\mathrm{U} 2$ snRNP specific and some $\mathrm{C}$ specific proteins, for which an effect during the assembly on the splicing-inactive pre-mRNAs is expected, will be described.

Figure 4.35 shows the protein assembly of the U1 snRNP specific proteins on PM5, 5'ssdeleted PM5 and BPS-ACTGA-deleted PM5 pre-mRNAs over a time of 30 minutes. Inspection of the protein assembly on PM5 pre-mRNA reveals that the U1-A, U1-C and U1$70 \mathrm{~K}$ protein ratios showed a maximum at 5 minutes (Figure $4.35 \mathrm{~A}$ ). After 5 minutes the U1-A and the U1-70K proteins showed nearly constant protein ratios, whereas the U1-C assembly time line decreased. On the 5'ss-deleted PM5 pre-mRNA (Figure 4.35 B), the U1-A and U1$70 \mathrm{~K}$ protein ratios again showed a maximum at 5 minutes and are constant thereafter. However, the U1-C protein, which is known to bind the 5'ss of the pre-mRNA (Heinrichs et al., 1990; Pomeranz Krummel et al., 2009), was only detected at few time points and did not show a time-dependent assembly on this pre-mRNA. The protein ratios obtained for the three U1 snRNP specific proteins during assembly on the BPS-ACTGA-deleted pre-mRNA again showed a maximum at 5 minutes, but thereafter varied substantially with time.
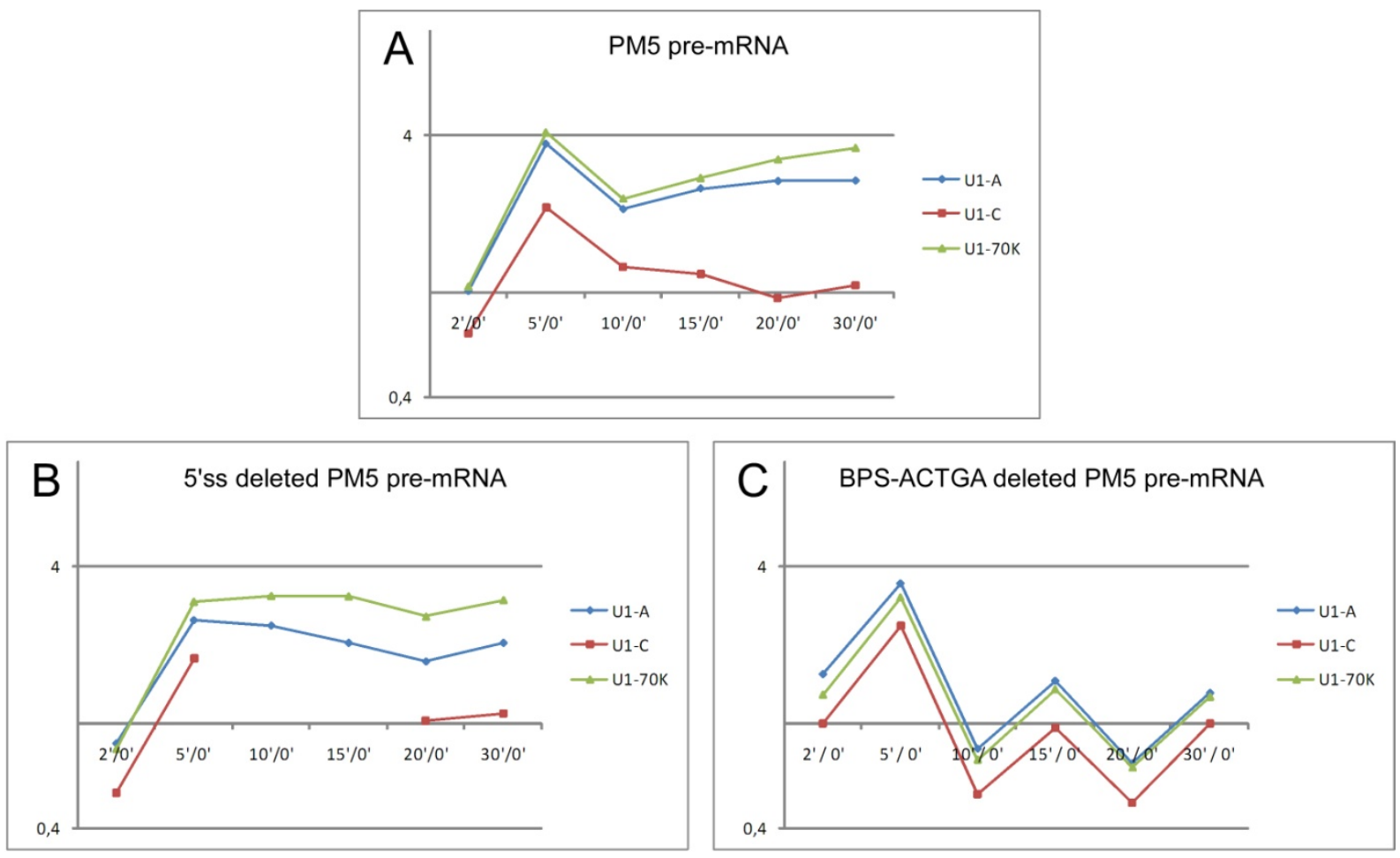

Figure 4.35: Assembly of U1 snRNP specific proteins on PM5 pre-mRNA, 5'ss-deleted PM5 pre-mRNA, and BPS-ACTGA-deleted PM5 pre-mRNA over 30 minutes. (A) Assembly of U1-A, U1-C and U1-70K proteins on PM5 pre-mRNA. (B) Assembly of U1-A, U1-C and U1-70K proteins on5'ss deleted PM5 pre-mRNA. (C) Assembly of U1-A, U1-C and U1-70K proteins on BPS-ACTGA deleted PM5 pre-mRNA. 
The U2 snRNP is known to bind the BPS of the pre-mRNA during pre-mRNA splicing. As the BPS was deleted in one of the splicing-inactive pre-mRNAs, the assembly of the U2 snRNP specific proteins on the different pre-mRNAs was investigated. Figure 4.36 A shows the protein assembly of U2 snRNP specific proteins on the PM5 pre-mRNA. Constructed time lines for all proteins show the same time course. All proteins were enriched on the PM5 premRNA at the different time points compared with at zero minutes. The protein ratios reached a maximum at 5 minutes and were nearly constant afterwards (Figure $4.36 \mathrm{~A}$ ). The protein assembly on the 5'ss-deleted PM5 pre-mRNA showed a time course comparable to that of the splicing-active PM5 pre-mRNA. The protein ratios showed a maximum at 5 minutes and were constant thereafter (Figure $4.36 \mathrm{~B}$ ). Surprisingly, the assembly time course of the U2 snRNP specific proteins on the BPS-ACTGA-deleted PM5 pre-mRNA resembled that of the U1 snRNP specific proteins on this particular pre-mRNA (see above). The protein ratios varied substantially with time and no effect on the binding of the U2 snRNP proteins was observed (Figure $4.36 \mathrm{C}$ ). However, the time lines constructed for protein assembly on this pre-mRNA differed from that of the PM5 and 5'ss-deleted PM5 pre-mRNA.
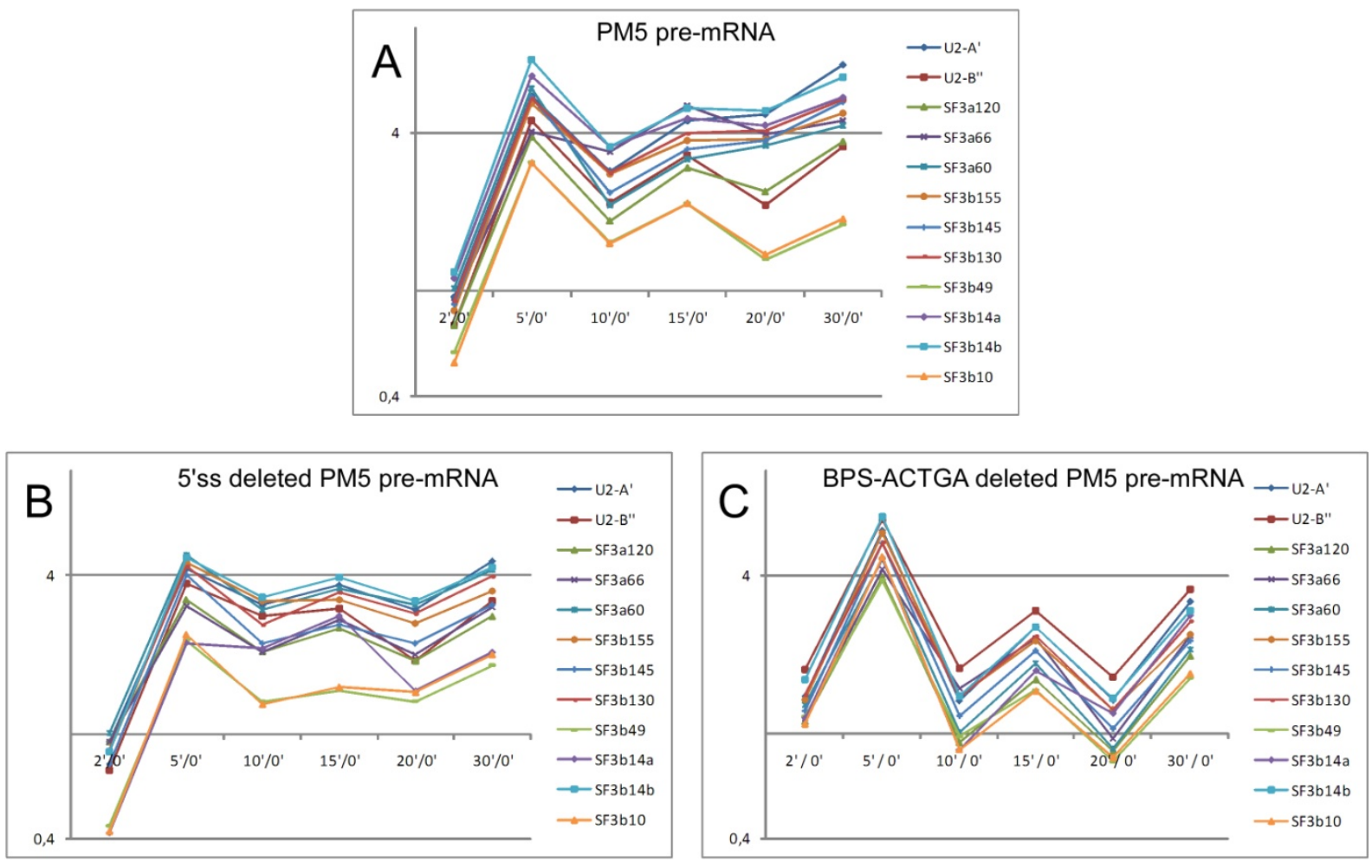

Figure 4.36: Assembly of U2 snRNP specific proteins on PM5 pre-mRNA (A), 5'ss-deleted PM5 pre-mRNA (B) and BPS-ACTGA-deleted PM5 pre-mRNA (C) monitored over 30 minutes.

The hPrp19/CDC5L complex is recruited during spliceosomal activation and its proteins have been found to be enriched in spliceosomal $C$ complex (see section 4.3). However, in earlier studies hPrp19/CDC5L complex proteins were already detected in spliceosomal A and B 
complexes (Behzadnia et al., 2007; Deckert et al., 2006). The protein assembly of the hPrp19/CDC5L complex on the splicing-active pre-mRNA is shown in Figure 4.37 A. Indeed, the time courses of all hPrp19/CDC5L proteins showed a maximum at 15 minutes when the spliceosomal C complex was assembled (see above). However, consistently with previous studies, the proteins were found to be enriched at an earlier time point, i.e. the protein ratios showed an initial increase after 5 minutes. Surprisingly, after 15 minutes the protein ratios decreased followed by an increase at 30 minutes. The protein assembly on 5'ss-deleted PM5 pre-mRNA was found to differ for these proteins (Figure $4.37 \mathrm{~B}$ ). The proteins showed no temporary maximum at 5 minutes but increased continously over 30 minutes. However, a maximum at 15 minutes was also indicated. In contrast to the protein assembly on PM5 premRNA two proteins, namely AD-002 and CTNNBL1, were first observed on the 5'ss-deleted pre-mRNA after 10 minutes. The protein assembly on the BPS-ACTGA-deleted PM5 premRNA resembled the assembly of the $U 1$ and $U 2$ snRNP specific proteins on this particular pre-mRNA variant (see above, Figure $4.35 \mathrm{C}$ and $4.36 \mathrm{C}$ ). It again showed a decreasing and increasing time course with two maxima at 5 and 15 minutes. However, in contrast to the assembly of the U2 snRNP proteins, a maximum was observed at 15 minutes instead of 5 minutes, suggesting that this specific protein group is more highly enriched on the pre-mRNA after 15 minutes (Figure $4.37 \mathrm{C}$ ). Proteins ratios for $\mathrm{Hsp} 70$, which is an additional component of the hPrp19/CDC5L complex (see above), were not monitored, as for this particular protein several isoforms have been detected and quantified.
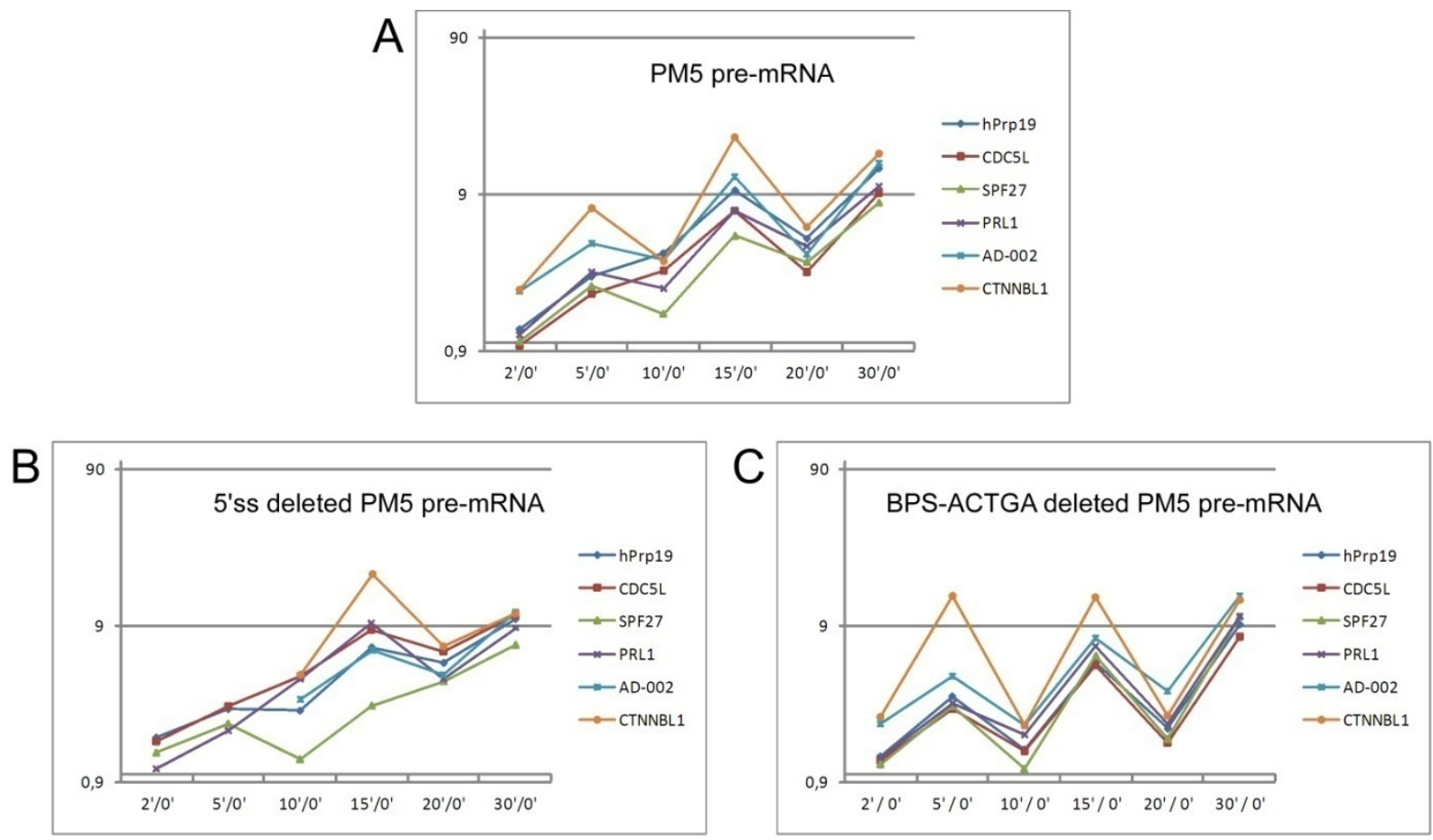

Figure 4.37: Assembly of hPrp19/CDC5L complex proteins on PM5 pre-mRNA (A), 5'ss-deleted PM5 premRNA (B) and BPS-ACTGA-deleted PM5 pre-mRNA (C) monitored over 30 minutes. 
The step 2 factors are essential for the second catalytic step of pre-mRNA splicing. By relative quantification of $B$ versus $C$ complexes (see section 4.3 ), they were found to be highly enriched in $\mathrm{C}$ complex. The time course for the assembly of these proteins onto PM5 pre-mRNA indeed shows a maximum at 15 minutes. Before 10 minutes the protein ratios showed almost no increase. As for the hPrp19/CDC5L complex proteins, the protein ratios showed a decrease at 20 minutes that was followed by an increase at 30 minutes. The hPrp17 protein showed a slightly different time course, with protein ratios continously increasing over 30 minutes (Figure $4.38 \mathrm{~A}$ ). The protein assembly of the step 2 factors on the 5'ss-deleted pre-mRNA differs from that of the splicing-active PM5 pre-mRNA. The protein ratios (except that for hPrp17) showed almost no increase (at most twofold) before 30 minutes. hPrp17 again showed a continuous increase over 30 minutes. The hPrp16 protein was only detected at two time points (Figure $4.38 \mathrm{~B}$ ). The assembly of the step 2 factors on the BPS-ACTGA-deleted pre-mRNA was found to be similar to that of the splicing-active PM5 pre-mRNA. A maximum was observed at 15 minutes followed by a minimum at 20 minutes and a second maximum at 30 minutes (Figure $4.38 \mathrm{C}$ ). The hPrp18 protein, which also belongs to the step 2 factors, was not detected and is therefore not included in these analyses.
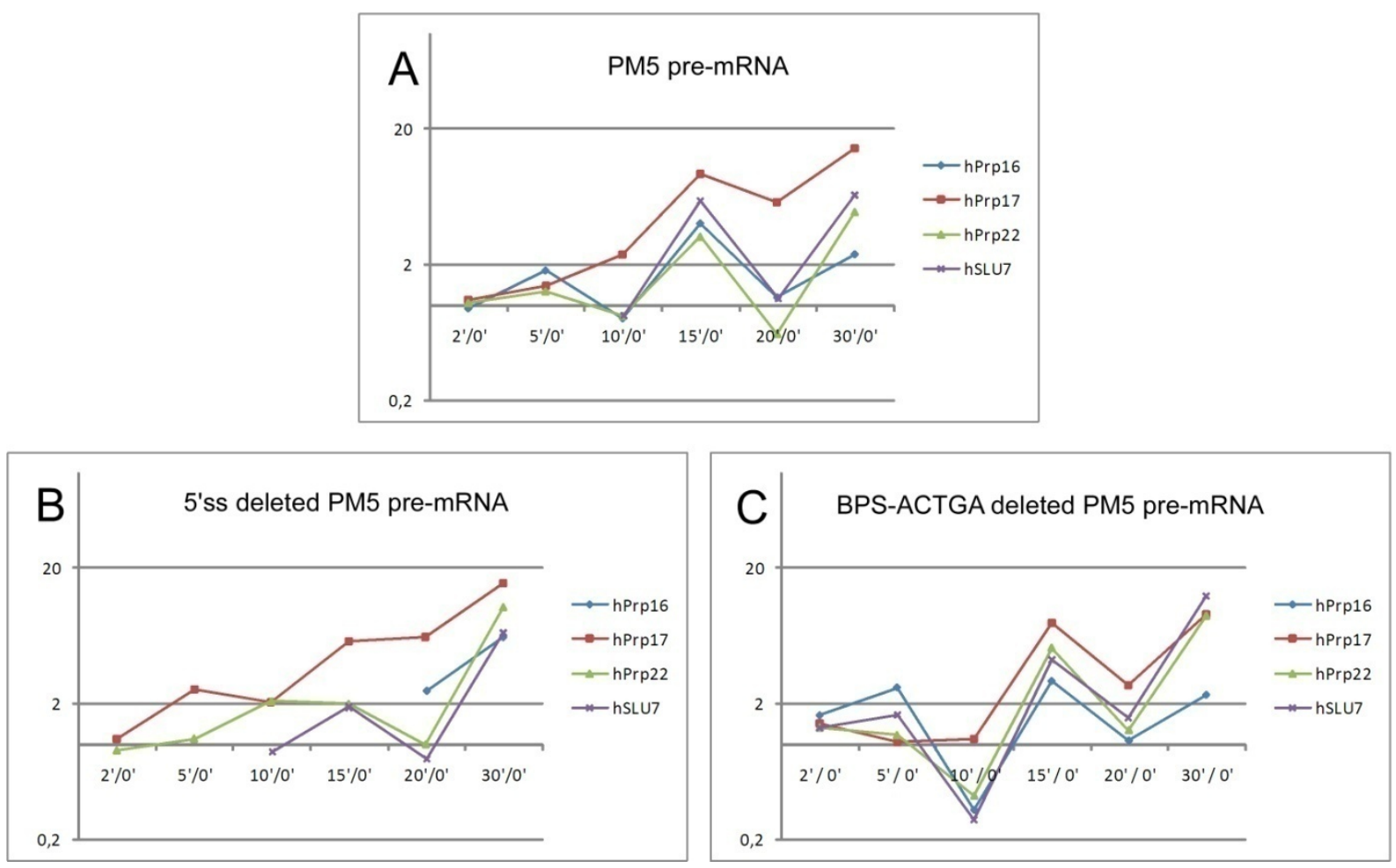

Figure 4.38: Assembly of step 2 factors on PM5 pre-mRNA (A), 5'ss-deleted PM5 pre-mRNA (B), and BPSACTGA-deleted PM5 pre-mRNA (C) monitored over 30 minutes. 


\subsubsection{Direct comparison of the protein assembly on splicing-active PM5 pre-mRNA and splicing-inactive 5'ss deleted PM5 pre-mRNA}

\subsubsection{Experimental setup}

In addition to the time-dependent protein assembly on different splicing-active and splicinginactive pre-mRNAs, the protein compositions at different time points on the splicing-active (PM5 pre-mRNA) and on a splicing-inactive pre-mRNA were compared directly. As the 5'ssdeleted PM5 pre-mRNA showed differences in the assembly of the U1 snRNP specific proteins, hPrp19/CDC5L complex proteins and step 2 factors, this pre-mRNA was chosen for direct comparison of the protein assembly. SILAC nuclear extracts (light and heavy) were prepared from differentially labeled HeLa cells (light and heavy), and spliceosomal assembly was performed in these two extracts in parallel by incubation of an MS2-tagged and radioactively labeled PM5 pre-mRNA and 5'ss-deleted PM5 pre-mRNA with light and heavy SILAC nuclear extract under splicing conditions. Splicing reactions were assembled for different time intervals and affinity purified complexes from the same time points assembled on PM5 or 5'ss-deleted PM5 pre-mRNA, respectively, were mixed in equal molar amounts (Figure 4.39).

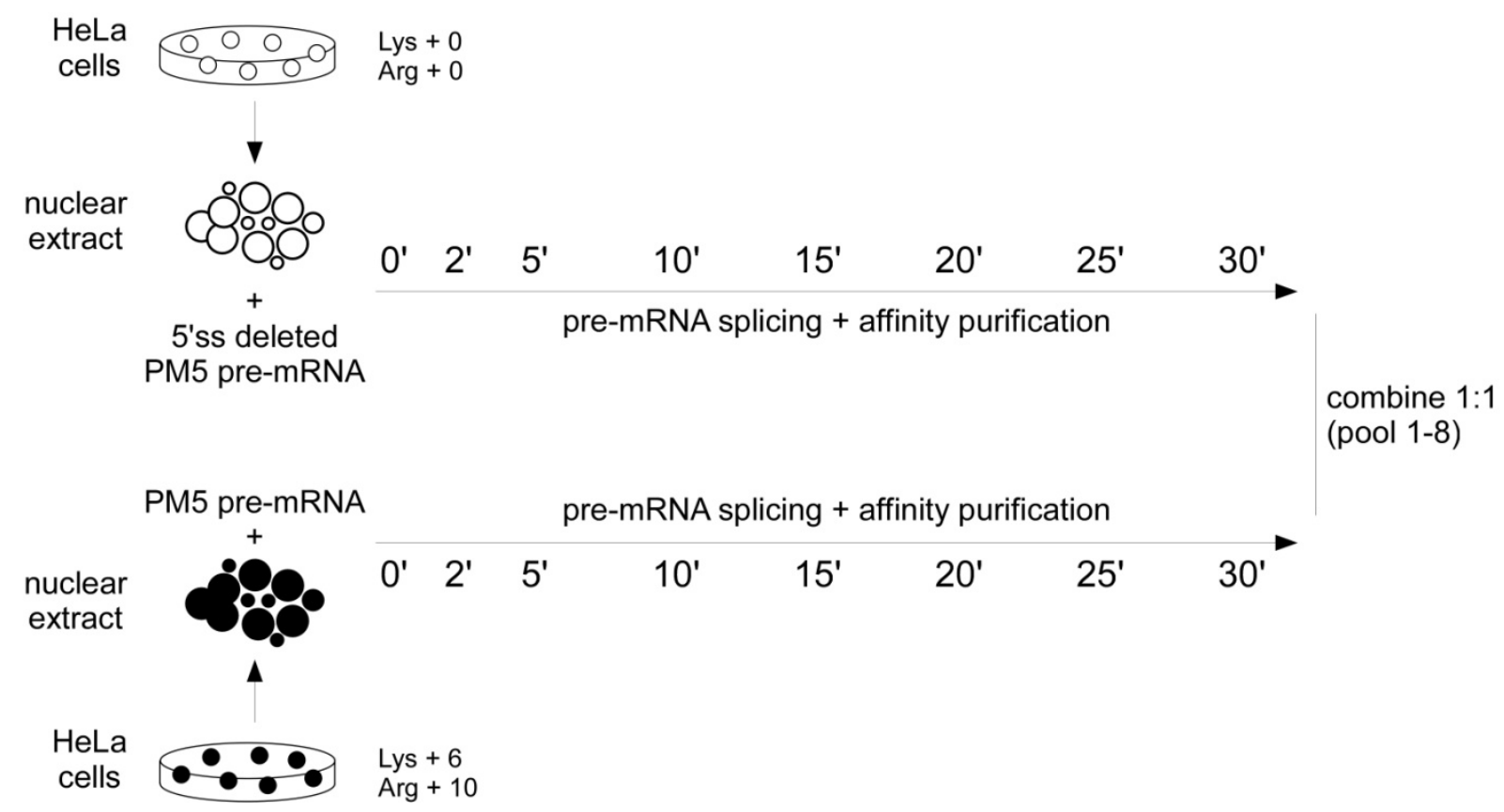

Figure 4.39: Experimental setup to compare the protein compositions of the sets of proteins assembled on PM5 and 5'ss-deleted PM5 pre-mRNA at different time points during pre-mRNA splicing. Splicing reactions were assembled on PM5 and 5'ss deleted PM5 pre-mRNA using light and heavy nuclear extracts, respectively. Assembled complexes from the same time point but assembled on the different pre-mRNAs were pooled in equal amounts. 
The proteins within the assembled complexes purified at the same time point but assembled on different pre-mRNAs were separated by PAGE (Figure 4.40). The MS2-MBP protein, which was used for affinity purification showed nearly equal intensity in the combined samples (pools 1-8) for the protein assembly on the different pre-mRNAs confirming the mixing procedure based on the radioactively labeled pre-mRNA. Minor differences in the protein amounts and in the abundances of individual protein bands were observed in the various samples, i.e. at different time points during spliceosomal assembly (compare pools 1-8, Figure 4.40).

Entire gel lanes were excised and the proteins were hydrolyzed with trypsin. The peptides generated were subsequently analyzed by LC-MS/MS. The peptides and finally the proteins were quantified by using MaxQuant software (Cox and Mann, 2008). Protein ratios for the assembly on the splicing-active versus the splicing-inactive pre-mRNA were calculated for every time point and assembly time lines for individual proteins were plotted.

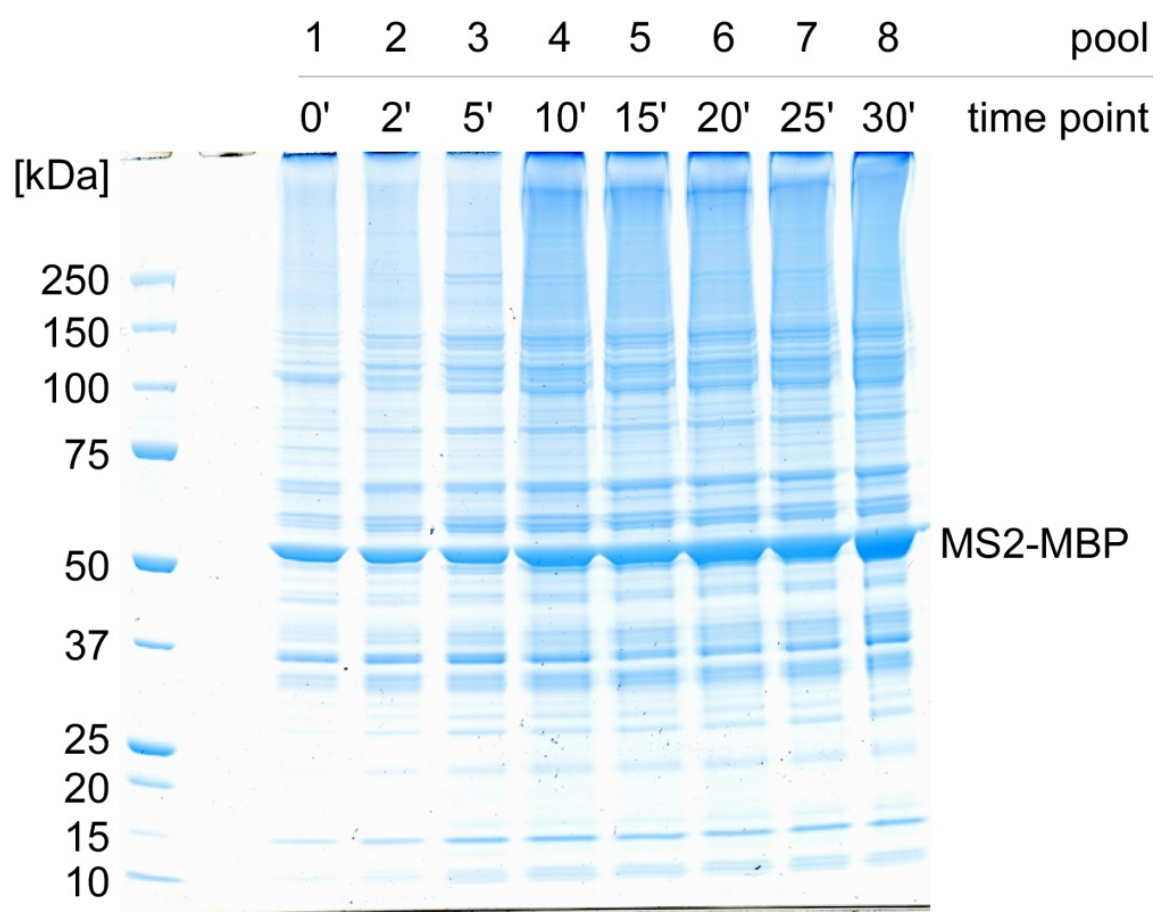

Figure 4.40: Coomassie stained gel of combined samples (pools 1-8) from comparison of the protein assembly on PM5 and 5'ss-deleted PM5 pre-mRNA at different time points during pre-mRNA splicing. Affinity purified complexes assembled for the same time intervals on PM5 and 5'ss-deleted PM5 pre-mRNA were pooled in equal amounts and the proteins were separated by PAGE.

\subsubsection{Normalization of the data}

To compensate for errors that occurred in the mixing of the samples or in any other step during sample handling the protein ratios obtained were normalized. For normalization of 
these ratios, two proteins were chosen that are expected to bind to both pre-mRNAs in equal amounts. These are the cap binding proteins CBP20 and CBP80, which bind to the premRNA cap and are not involved in pre-mRNA splicing.

The protein ratios of CBP20 and CBP80 obtained for every time point were plotted to show their assembly on the PM5 pre-mRNA in comparison with 5'ss-deleted PM5 pre-mRNA over the time investigated (Figure $4.41 \mathrm{~A}$ ). As expected, the two proteins showed the same abundance on both pre-mRNAs, as verified by protein ratios close to 1 . However, some fluctuations were observed and normalization of the data is necessary to compensate for these fluctuations. For this purpose, the average protein ratio of the two proteins was calculated for every time point. These values were then used to calculate the different normalization factors (Figure $4.41 \mathrm{C}$ ). Figure $4.41 \mathrm{~B}$ shows the normalized protein ratios for CBP20 and CBP80. The normalization factors obtained for the different time points were applied to the protein ratios of individual proteins quantified in the data set.
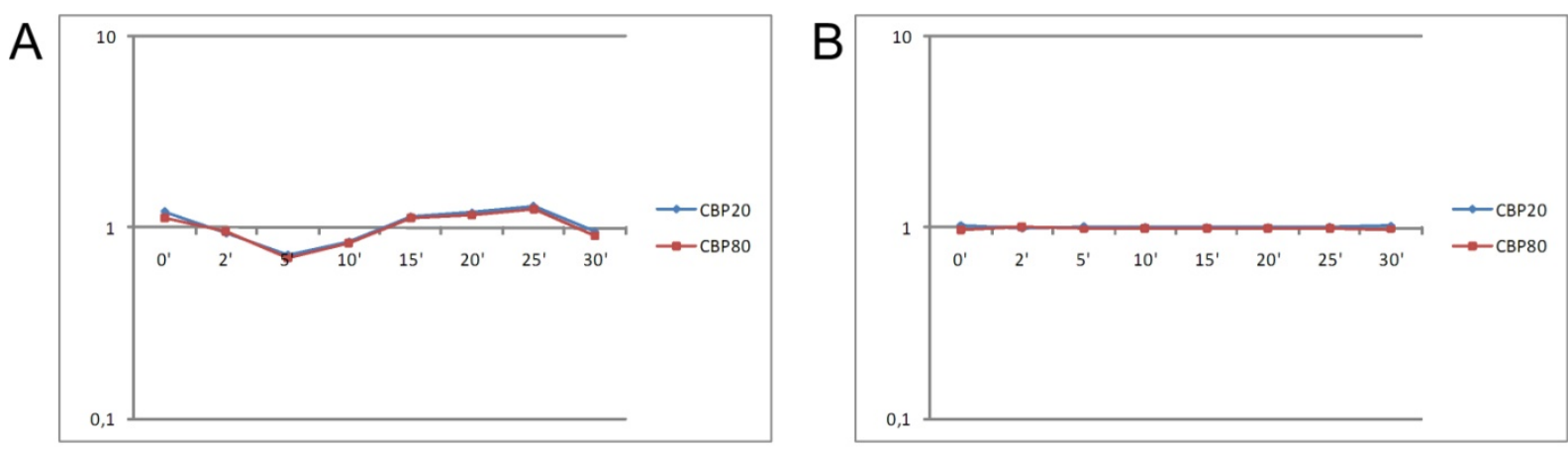

\section{C}

$\begin{array}{rccc}\text { time point } & 0^{\prime} & 2^{\prime} & 5^{\prime} \\ \text { average value } & 1.174 & 0.948 & 0.711\end{array}$
normalization factor

0.852

1.054 $5^{\prime}$
0.711
1.407

$10^{\prime}$
0.836
1.196

0.836

1.196 $20^{\prime}$

0.843

\section{$25^{\prime}$}

1.276

0.784
1.130

0.885

0.932

1.073

Figure 4.41: Normalization of the protein ratios found for the cap binding proteins. (A) Protein ratios obtained for CBP20 and CBP80 were plotted for every time point. (B) The normalized protein ratios for CBP20 and CBP80 were plotted for every time point. (C) For every time point the average protein ratio of the two cap binding proteins and the calculated normalization factor is given.

\subsubsection{Protein assembly on PM5 pre-mRNA versus 5'ss-deleted PM5 pre-mRNA}

Protein assembly on PM5 and 5'ss-deleted PM5 pre-mRNAs was compared. Several splicing reactions were assembled for different time intervals in light and heavy SILAC nuclear extracts (Figure 4.39). Assembled complexes from the same time points were pooled and analyzed as described above. Protein ratios obtained for the different time intervals were plotted to show the protein assembly on PM5 pre-mRNA in comparison with the 5'ss-deleted PM5 pre-mRNA. 
The U1 snRNP is known to bind to the 5' splice site during spliceosomal assembly (Heinrichs et al., 1990; Pomeranz Krummel et al., 2009). Differences in protein abundances on the two pre-mRNAs investigated were therefore expected for U1 snRNP specific proteins. The protein ratios obtained showed clearly that between zero and five minutes the U1-A, U1-C and U1-70K protein were highly enriched on the PM5 pre-mRNA as compared with the 5'ssdeleted PM5 pre-mRNA (Figure 4.42 A). At zero minutes, the proteins are 5-6 times enriched on the PM5 pre-mRNA, whereas protein ratios decreased during incubation. After 10 minutes the protein ratios of the $U 1$ snRNP specific proteins were constant but showed a continuing enrichment on the PM5 pre-mRNA as compared with the 5'ss-deleted PM5 pre-mRNA (Figure $4.42 \mathrm{~A}$ ). Interestingly, the assembly time line found for the $\mathrm{Sm}$ proteins resembles the one found for the U1 snRNP proteins (Figure $4.42 \mathrm{~B}$ ). At zero minutes the Sm proteins are enriched threefold on the PM5 pre-mRNA compared with on the 5'ss-deleted PM5 premRNA. This can be explained by the binding of U2 snRNP on the 5'ss-deleted PM5 premRNA. Two sets of Sm proteins (belonging to U1 and U2 snRNPs) bind to the PM5 premRNA, whereas only one set of Sm proteins (belonging to U2 snRNP) assembles on the 5'ss-deleted PM5 pre-mRNA. The protein ratio of the Sm proteins at zero minutes is thus halved as compared with the U1 snRNP specific proteins. Nonetheless, the protein ratios decreased between zero and 10 minutes and were constant after 10 minutes (Figure $4.42 \mathrm{~B}$ ). The effect of the 5' splice site deletion upon the binding of U1 snRNP is thus clearly demonstrated, although binding of $\mathrm{U} 1$ proteins on the 5'ss-deleted pre-mRNA was also observed.
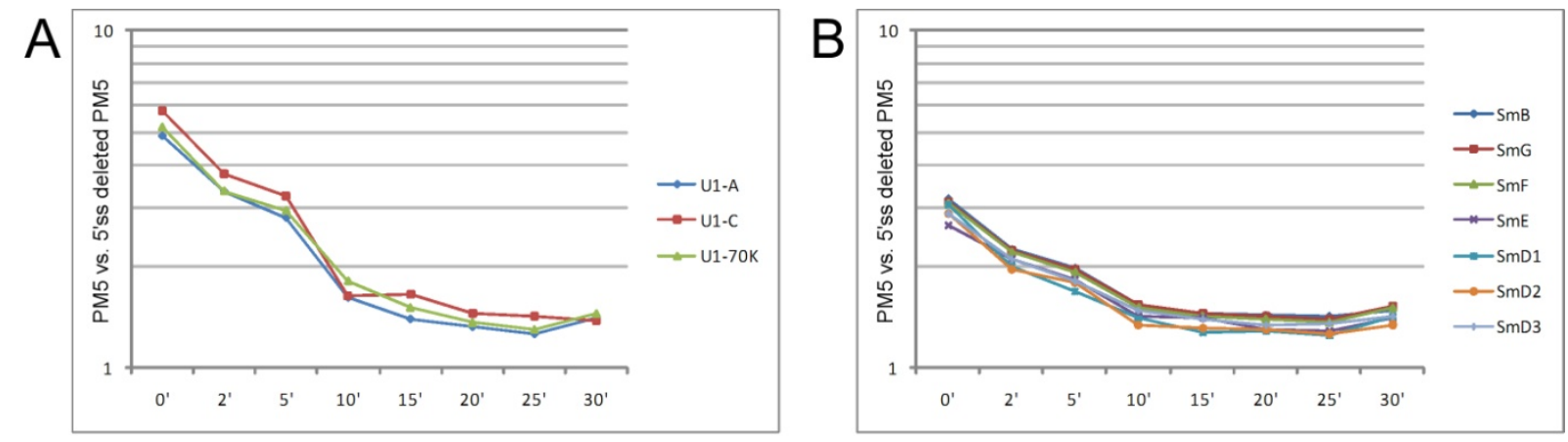

Figure 4.42: Direct comparison of protein assembly on the PM5 pre-mRNA compared with corresponding assembly on the 5'ss-deleted PM5 pre-mRNA for U1 snRNP specific proteins (A) and Sm proteins (B).

As a next step, the effect of the 5'ss deletion on other proteins essential for pre-mRNA splicing was studied. The hPrp19/CDC5L complex and the step 2 factors are enriched in the C complex compared with the B complex (see section 4.3). These proteins are essential for pre-mRNA splicing and are therefore of great interest. Constructed time lines for the direct 
comparison of the protein assembly showed that these proteins were highly enriched on the splicing-active PM5 pre-mRNA as compared with the splicing-inactive variant of the premRNA (Figure 4.43). The hPrp19/CDC5L complex protein ratios showed a substantial increase at 5 minutes and were then nearly constant over the whole period (Figure $4.43 \mathrm{~A}$ ). They are thus highly enriched on the PM5 pre-mRNA from the time point when they predominantly associate with the pre-mRNA. In contrast, assembly time lines of the step 2 factors reach a maximum at 15 minutes but decrease again after 15 minutes. These proteins thus show a time-dependent assembly on the splicing-active compared with the splicinginactive pre-mRNA. It is noteworthy that, hSLU7 and hPrp22 are first observed after 10 and 15 minutes, respectively. hPrp18 was only detected at two time points, but it too shows high enrichment on the PM5 pre-mRNA compared with the 5'ss-deleted pre-mRNA. hPrp16, which also belongs to the step 2 factors, was not detected in these analyses.
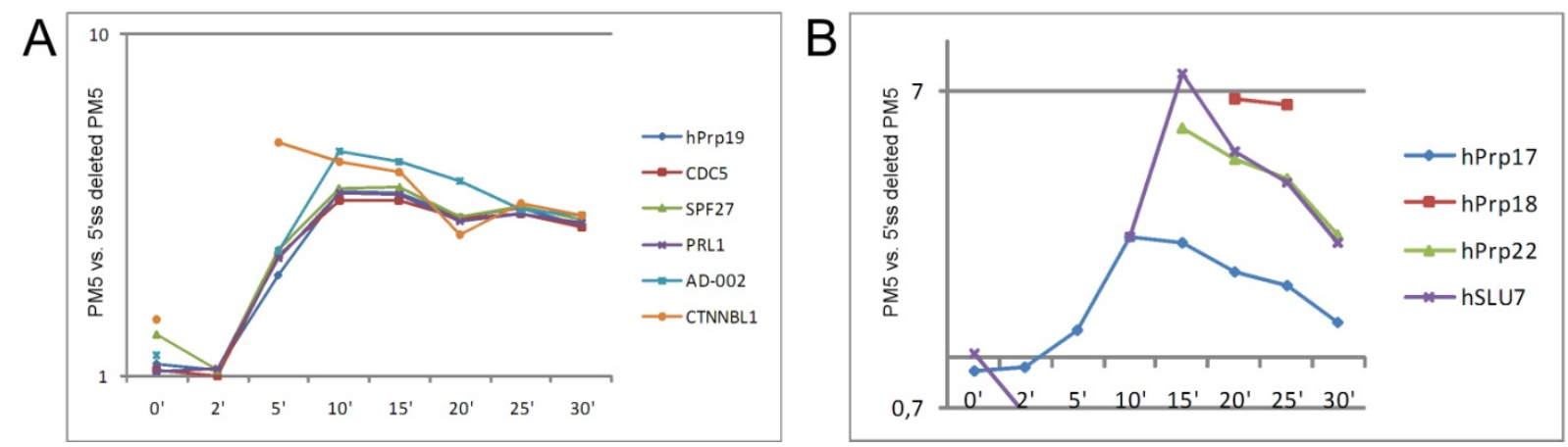

Figure 4.43: Protein assembly of the hPrp19/CDC5L complex (A) and the step 2 factors (B) on the PM5 pre-mRNA compared with corresponding assembly on the 5'ss-deleted PM5 pre-mRNA.

The direct comparison of the protein assembly on the splicing-active (PM5) and the splicinginactive (5'ss deleted PM5) pre-mRNA yields information about the abundance of proteins on these two pre-mRNAs. Although binding of the groups of proteins investigated on the splicing-inactive pre-mRNA was observed (see section 4.4.3), a clear difference between this and the splicing-active pre-mRNA was found. Proteins that had been expected to be affected by deletion of the 5'ss are highly enriched on the splicing-active pre-mRNA, and an effect of the 5 'ss deletion is thus clearly observed. 


\section{Discussion}

\subsection{Determination of the protein stoichiometry within the hPrp19/CDC5L complex by absolute quantification (AQUA)}

This study has shown that for determination of the protein stoichiometry within a protein complex using synthetic standard peptides and mass spectrometry it is crucial to achieve complete enzymatic hydrolysis of all the proteins within the complex. The choice of conditions for complete hydrolysis strongly affected the results for the stoichiometry (Figure 4.10). The consistency of the results obtained in all the analyses after the hydrolysis of the complex in the presence of acetonitrile led to the conclusion that the Prp19/CDC5L protein complex has a stoichiometry of $4 \times \operatorname{Prp} 19,2 \times$ CDC5L, $1 \times$ SPF27, $1 \times$ PRL1, $1 \times$ CTNNBL1 (Figure $4.10 \mathrm{~B}$ ). Two other proteins found in the same fraction of the complex were not quantified: AD-002, because of the lack of suitable standard peptides for this protein, and Hsp70, because of initial results obtained by MS with suitable standard peptides suggesting that this protein is not represented in most copies of the complex (Table A.4 in the Appendix).

For similar studies with other protein complexes, there is thus an absolute requirement for initial experiments to determine (i) the solubility and elution profile of the standard peptides and (ii) the optimum hydrolysis conditions of the complex. Recent studies in absolute quantification using standard peptides address the question of the determination of the absolute amount of the synthesized standard peptides before analysis. Standard peptides, whose total amounts have not been correctly determined lead to false results. Amino acid analysis (AAA) is therefore widely recommended for the determination of the total amount of standard peptides in such experiments. The experiments in this study have shown that the solubility of the peptides is the critical issue, rather than putative false amounts of synthesized peptides. The results revealed that once the various synthesized standard peptides are brought into solution the results are highly consistent for the different proteins. Therefore, the quantity itself does not represent the bottleneck of the analysis.

The analyses demonstrate clearly that the digestion conditions are indeed the most critical issue. By a change in the denaturing conditions, the apparent number of copies of the CDC5L protein in the hPrp19/CDC5L complex could be made to vary from one to two. This result was unexpected and cannot easily be explained. As less endogenous peptides of CDC5L were identified after hydrolysis in the presence of urea than after hydrolysis in the 
presence of acetonitrile, incomplete digestion of CDC5L in the presence of urea is presumed. Indeed, protection of the protein against proteolysis was observed in particular within those regions that harbor the endogenous counterparts of the selected standard peptides (Grote et al., 2010; Figure 5.1), suggesting that these regions are highly structured and therefore cannot be completely denatured by urea.

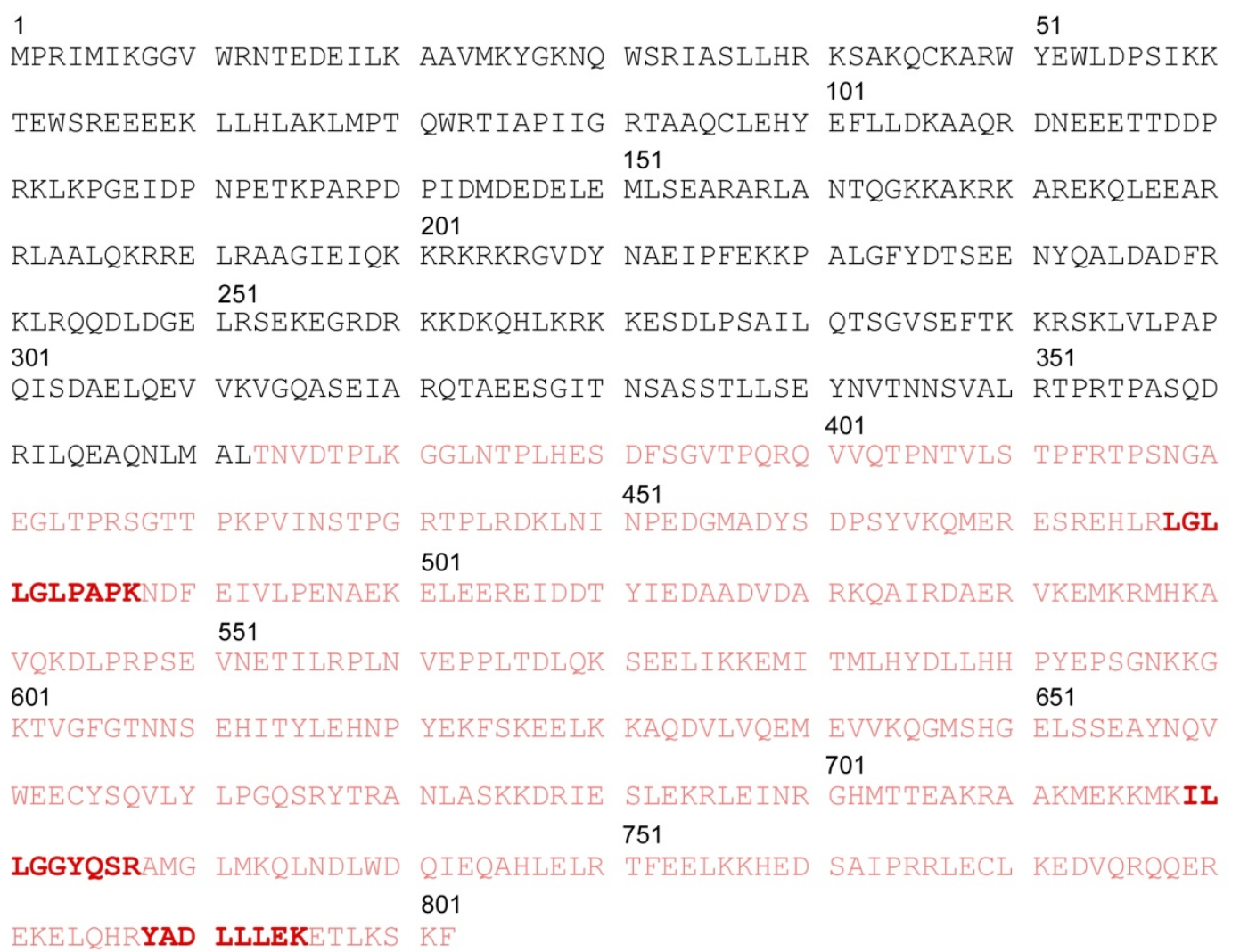

Figure 5.1: Endogenous counterparts of the selected standard peptides for CDC5L protein are located in highly structured regions of CDC5L. The amino acid sequence of CDC5L is shown. Regions that are protected against proteolysis (Grote et al., 2010) are labeled in light red. Endogenous counterparts of selected standard peptides are highlighted in bold red. All peptide sequences selected as standard peptides are located in a highly structured region of CDC5L.

In fact, only a few limited methods are available to determine the complete hydrolysis of a complex. Denaturing PAGE, even at the highest possible resolution, as achieved by Schägger gels (Schagger and von Jagow, 1987), reveals incompletely digested fragments only up to a size of $1 \mathrm{kDa}$. Moreover, PAGE conditions are not necessarily compatible with the digestion of proteins in the presence of urea, so that the risk of sample loss during desalting cannot be excluded. Accordingly, larger residual protein fragments after hydrolysis of the Prp19/CDC5L complex, either in the presence of acetonitrile or in the presence of urea, cannot be monitored reliably. MS per se can monitor larger fragments, but the sensitivity is dependent on the fragments' size, and multiply charged ions in ESI can be 
suppressed by double and triply charged ones. An alternative might be the use of monolithic columns that allow one to separate and elute intact proteins. However, not all proteins elute even under organic and denaturing conditions from these columns.

The amount of peptides containing missed cleavage sites can influence the absolute quantification. For this reason, the use of more than one standard peptide for each protein is highly recommended to provide a confirmatory control and to detect any deviations that might arise. On the basis of miscleavage, two peptides were excluded from the quantification (TGYNFQR derived from PRL1 for hydrolysis in acetonitrile and EAAAALVEEETR derived from SPF27 for hydrolysis in urea; Figure 4.8). The amount of a miscleaved peptide can ultimately only be determined if one also has standard peptides for this peptide and adds these to the sample. However, the generation of isotope labeled standards is expensive and the generation of standard peptides and additional standard peptides containing possible missed cleavage sites might not be justified.

Importantly, the different accessibilities of certain protein regions within proteins are a further critical issue in the determination of protein stoichiometry when the investigated protein complex is, under different cellular conditions, an integral part of another complex comprising even more proteins. Integration into another complex might lead to structural rearrangement of the proteins and therefore to changes in the accessibility of the proteins' regions against which standard peptides have been generated for absolute quantification. Previous proteome analyses have demonstrated that the human Prp19/CDC5L complex associates with the premRNA splicing machinery throughout the various steps of splicing. This complex is present within the so-called pre-catalytic spliceosomal B complex, the catalytically activated B complex $\left(\mathrm{B}^{*}\right)$, and the step $1 \mathrm{C}$ complex. Furthermore, it was shown that it is part of a postspliceosomal 35S U5 complex. Although the hydrolysis conditions for the isolated Prp19/CDC5L complex have been established and, on this basis, proteotypic standard peptides have been selected, the possibility that the major changes in protein-protein interactions that take place during the transition of the spliceosomal $\mathrm{B}$ complex to the $\mathrm{C}$ complex also affect the proteins of the Prp19/CDC5L complex, cannot be ruled out. For instance, the proteotypic peptides selected for the protein PRL1 are located in a region that is readily accessible toward proteases and has therefore been suggested to be highly flexible and unstructured in the Prp19/CDC5L complex (Grote et al., 2010). These regions might become structured upon additional protein-protein interactions in larger spliceosomal complexes. Thus, when extending the studies on the above mentioned spliceosomal complexes, complete hydrolysis conditions must be established for all of the complexes $(B$, $B^{*}$, and $C$ complex), which consist no longer of seven proteins but generally of about 125 proteins (see Wahl et al., 2009 for review). Therefore, the key question to be asked is 
whether, although the strategy applied here produces highly reproducible results under the improved experimental conditions (hydrolysis in acetonitrile and analysis by MRM) for a complex of moderate complexity, the cost in time and financial resources is justified when such studies are extended to complexes of much higher complexity.

Another critical issue for the determination of the protein stoichiometry within a complex is its homogeneity. Here, the affinity-purified hPrp19/CDC5L complex was subjected to glyceroldensity centrifugation in order to obtain the highest possible purity and homogeneity. However, Hsp70 has been identified in the gradient fraction of the hPrp19/CDC5L complex, but was found to be of very low abundance. This raises the question whether gradient centrifugation under these conditions is not sufficient to shift a minor portion of the hPrp19/CDC5L complex that contains Hsp70 toward a different sedimentation coefficient or, alternatively, whether Hsp70 forms a complex by itself that was co-purified and co-migrates with Prp19/CDC5L complex. Alternatively, on the assumption that the very low amount of Hsp70 represents the lowest possible numbers of copies of a single protein with the hPrp19/CDC5L complex, then the copy numbers of all other proteins must be adapted accordingly, thus suggesting that several copies of the hPrp19/CDC5L complex with the stoichiometry reported here are assembled on a single copy of Hsp70. This question cannot be answered unequivocally by the applied method. Rather, the determination of the entire mass of the complex would be required. In addition, different Hsp70 isoforms were detected during proteomic analysis of the hPrp19/CDC5L complex. The composition of these isoforms in the complex has not been investigated so far and might also influence the quantification of this particular protein.

Very recently, the use of standard peptides for absolute quantification of affinity-tagged proteins within protein interaction networks was reported (Wepf et al., 2009). Isotopically labeled standard peptides encompassing a peptide derived from the introduced tag were designed and used for the quantification of several proteins in various affinity-purified protein complexes. In this manner, labeled standard peptides were used to calibrate for any other protein within the complexes. However, since the authors compared the stoichiometry of different affinity-purified complexes without any further purification, no conclusion was drawn about the protein stoichiometry within a single protein complex.

In a similar way, differing only in technical details, absolute quantification is frequently used in clinical proteomics. In such studies, standard peptides have been used to determine by MS the absolute amount of defined proteins (mass or mole number) in samples (Abbatiello et al., 2008; Langenfeld et al., 2009). However, even though within these studies samples from different sources were compared in terms of their absolute protein amount, the quantification is actually a relative one. Thus, when comparing the absolute amount of protein and/or 
protein complexes in a sample, hydrolysis conditions are not crucial as long as they are consistent among the different samples and as long as the proteins in question are not present in different complexes in the samples of different origin.

In summary, the complete hydrolysis of the proteins under investigation and the complete solubility of the standard peptides have been proven to be the major prerequisites for successful absolute quantification. The protein stoichiometry within the hPrp19/CDC5L complex could be determined although discrepancies between different hydrolysis conditions arised. Hydrolysis in the presence of acetonitrile in combination with MRM analysis revealed the most consistent results, thus representing optimal experimental conditions. However, when transferring this method to other (possibly larger) protein complexes, testing of different hydrolysis conditions is mandatory. In addition, selection of suitable standard peptides emerged as not trivial. The protein sequences as well as structural information about the proteins under investigation (if available) should be taken into account to successfully select suitable standard peptides for absolute quantification.

\subsection{Relative quantification by iTRAQ-labeling of in-gel digested proteins}

The traditional iTRAQ workflow comprises in-solution digestion of proteins, labeling of the generated peptides, removal of excess reagent by strong cation exchange chromatography (SCX) and finally the analysis by LC-MS/MS (Ross et al., 2004). To reduce the sample complexity before protein hydrolysis and the possibility of sample loss during SCX, a modified protocol for iTRAQ labeling of in-gel digested proteins was established. This has the advantage that even of small protein amounts can be quantified. The modified workflow was tested by relative quantification of selected proteins of spliceosomal tri-snRNP particles.

$5.0 \mu \mathrm{g}$ and $2.5 \mu \mathrm{g}$ of tri-snRNP was separated by PAGE and quantified using the modified iTRAQ-labeling workflow. The established method showed consistent results for the selected tri-snRNP specific proteins. However, the expected protein ratio of 2.0 was not achieved. The obtained ratio for different tri-snRNP amounts is 2.5 instead. As all quantified proteins show the same protein ratio of 2.5 , this deviation might be due to sample loading or sample preparation for gel electrophoresis and is a matter of normalization of the sample. The blank sample that was processed along with the other samples showed a ratio of 0.1 . The observation that background noise is often obtained during chemical labeling was recently discussed (Bantscheff et al., 2007). This background noise does not depend on the mass resolution of the mass spectrometer but rather on the size of the $\mathrm{m} / \mathrm{z}$ window chosen for isolation of peptides for fragmentation (typically between 2-6 $\mathrm{m} / \mathrm{z}$ ). All peptides present in this 
window will contribute to the obtained signal. In addition, the stable isotopes incorporated to the used ITRAQ reagents do not show $100 \%$ purity. This is typically $98-99 \%$ and contributes to the obtained signal intensities of the lower reporter ions.

During pilot experiments the labeling efficiency was found to be one of the major issues for reliable relative quantification. It can be calculated from the total number of peptides and the number of labeled peptides identified in the same analysis. Using chemical labeling approaches complete labeling of the peptides or proteins is rarely observed (Bantscheff et al., 2007). However, for successful relative quantification, the labeling efficiency should be as high as possible (i.e. nearly $100 \%$ ). In this study, only for experiments with a labeling efficiency higher than $90 \%$ accurate quantification was achieved (i.e. expected protein ratios were obtained). For the proteins identified and quantified within this proof of principle experiment the labeling efficiency was $92 \%$ and the resulting protein ratios were very consistent among the different proteins.

\subsection{Relative quantification of spliceosomal B and C complexes - a comparative study}

\subsubsection{Methodical considerations}

This work is the first proteome study that compares directly iTRAQ and SILAC for relative quantification of proteins. We performed relative quantification of proteins derived from spliceosomal B and C complexes and found that ITRAQ and SILAC yielded similar result. However, when comparing the obtained protein ratios, iTRAQ showed in general slightly lower values for proteins that are enriched in the $B$ complex and slightly higher values for proteins that are enriched in the $\mathrm{C}$ complex. This might be due to the fact that the precursor selection for MS/MS is not $100 \%$ selective and co-eluting peptides thus might contribute to the iTRAQ reporter ion intensity as it has been recently discussed (Bantscheff et al., 2007). Therefore, SILAC protein ratios show extremely high values or low values for proteins that are enriched or underrepresented, respectively, in one of the samples when compared to iTRAQ.

Protein ratios obtained from the SILAC experiments showed a lower standard deviation per se as compared to iTRAQ. Indeed, metabolic labeling should be a more reliable method for labeling proteins as compared to chemical labeling, as it should guarantee a $100 \%$ labeling efficiency of proteins and thus of the peptides. Moreover, samples can be pooled at an earlier stage and therefore variation in the quantification ratios by e.g. sample losses, can be 
neglected. Nonetheless, depending on the cellular system, sometimes complete labeling cannot or can only hardly be achieved by SILAC. In particular for those cells grown in culture that do not rapidly divide (e.g. embryonic stem cells; Graumann et al., 2008) or only proliferate in cell culture without dividing (e.g. primary neurons; Liao et al., 2008). Normally in such cells, a labeling efficiency of maximal $80 \%$ is achieved. However, such incomplete labeling can be handled by novel computational methods (Liao et al., 2008).

Of course, chemical labeling always involves the risk of incomplete labeling. In our initial experiments a low labeling efficiency $(<80 \%$ ) was found to drastically influence the quantification results. Therefore, the labeling efficiency is one of the major issues for obtaining accurate quantification when using chemical labeling approaches and the initial experiments were performed to achieve the highest possible labeling efficiency. Nonetheless, the great advantage of all chemical labeling approaches is that proteins from almost every source (cells, tissue, body fluids etc.) can be quantified.

An alternative chemical labeling approach to iTRAQ labeling has recently been introduced by Boersema et al., 2008. Dimethylation of the peptides' N-termini and lysine side chains using different stable isotope labeled reagents allows relative quantification of three samples in one MS analysis. Dimethyl labeling is based on a simple chemical reaction without any observed byproducts and provides a $100 \%$ labeling efficiency in almost all cases. In addition, it uses inexpensive reagents and is thus a cost-effective labeling technique in comparison to other stable isotope reagents. However, we have not performed this particular labeling strategy.

Among the different chemical labeling strategies, isobaric reagents, such as iTRAQ reagents or TMTs (Thompson et al., 2003), have the advantage that quantification is performed during MS/MS analysis so that sample complexity is not enhanced, as the differently labeled peptides show the same mass in the MS. As the reporter ions, which are used for quantification of the different samples, are released during MS/MS, the analytical depth of the analysis is higher as compared to analysis of differently labeled samples that show peak pairs in the MS (e.g. SILAC or dimethyl labeling).

Until now, isolated spliceosomal complexes have been compared by peptide count after LCMS/MS analysis (Behzadnia et al., 2007; Bessonov et al., 2008; Deckert et al., 2006; Kuhn et al., 2009). The correlation between the relative protein abundances and the number of acquired tandem MS spectra (spectral count), the number of identified peptides (peptide count) and the obtained sequence coverage has recently been compared by Liu et al., 2004 in a study with defined standard proteins. A linear correlation was found between the relative protein amount and the number of acquired MS/MS spectra (spectral count), but not between the relative protein amount and the number of identified peptides (peptide count) or the 
sequence coverage. In addition to ITRAQ and SILAC, we therefore evaluated spectral count for relative quantification of spliceosomal B and C complexes. Spectral count is a simple quantification technique that enables the comparison of almost every sample from a proteome study without additional sample preparation. As it does not require labeling of the peptides or proteins, no expensive labeling reagents are needed and as many samples as desired can be quantified relative to each other. However, it requires highly reproducible LCMSMS analyses (see also below).

In our study, we found a good overall agreement between spectral count and quantification with ITRAQ and SILAC. In all three quantitative analyses, several proteins were clearly identified to be more abundant in spliceosomal $\mathrm{B}$ or $\mathrm{C}$ complexes and some proteins were found to be present in equal amounts within both complexes (see Tables $4.9-4.11$ ). As discussed above, ITRAQ and SILAC yielded consistent results for relative quantification of $B$ and $\mathrm{C}$ complexes. Strikingly, for most of the quantified proteins, the same quantitative trend, i.e. enrichment in one of the complexes or same abundance in both complexes, was also obtained by spectral count. Importantly, spectral count exhibits some discrepancies compared to iTRAQ or SILAC. In particular for small proteins $(<20 \mathrm{kDa})$, accurate quantification could not at all or only roughly be achieved by spectral count, as only a limited number of peptides were generated. For example, the relative quantification of the Sm proteins within both, the spliceosomal B and C complexes, using spectral count did not unambiguously reveal the expected ratios, i.e. a two-fold difference of Sm proteins in the B vs. C complex.

As the $\mathrm{Sm}$ proteins are common to all $\mathrm{U}$ snRNPs except for U6 snRNP, four copies of $\mathrm{Sm}$ proteins are expected in the $\mathrm{B}$ complex. Upon transition from the $\mathrm{B}$ to the $\mathrm{C}$ complex, only two copies of Sm proteins are left, because U1 and U4 snRNPs are destabilized/dissociated. For all seven Sm proteins, protein ratios close to 2 are obtained by ITRAQ and SILAC, whereas spectral count yielded the correct value only for two of the Sm proteins (SmF and $\mathrm{SmG})$. The other Sm proteins show protein ratios of approximately 1 by spectral count.

Another example for the lower accuracy of spectral count is the quantification of proteins that should be present in a 1:1 ratio or where ITRAQ and SILAC clearly showed such a ratio. These are, for example, U5-220K, U5-40K, and CBP20 (for a complete list, see Tables 4.9 4.11). With spectral count, higher or lower protein ratios (approximately between 0.6 and 1.7) were obtained. These results are consistent with the observations of Liu et al., 2004 that small quantitative changes among proteins in different samples cannot be accurately monitored by spectral counting. The quantification of large proteins within the $B$ and $C$ complex by spectral count yielded results very similar to ITRAQ and SILAC (e.g. with the 
SF3a and SF3b proteins), except when proteins are present in nearly equimolar amounts in both samples (e.g. U5-220K and U5-200K).

Another discrepancy between spectral count and labeling approaches is observed with the protein ratios obtained for proteins that are pre-dominantly associated with one of the two complexes. These, like the hPrp18, hPrp22, and DDX35 proteins, show extreme values after spectral count, thus suggesting their complete absence in one of the complexes. Such extreme protein ratios might be misleading regarding the presence or absence of proteins within the different samples. If a peptide is not selected for sequencing it does not necessarily mean that the peptide is not present in the sample. Such low abundance peptides, which might escape detection by spectral count, are still detectable by ITRAQ and SILAC quantification. During ITRAQ analysis, samples are pooled and the differently labeled peptides are isobaric and are thus selected for sequence analysis irrespective of whether the actual non-labeled peptides are of low or high abundance. Quantification is then performed on the MS/MS level, where even low abundance peptides produce the corresponding reporter ions. SILAC quantification is based on the correct assignment of the mass pairs that were generated upon the incorporation of stable isotopes. In this manner, the low abundance peptides - if present - will be recognized by the software (e.g. MSQuant, Schulze and Mann, 2004; or MaxQuant, Cox and Mann, 2008), through the assignment of the corresponding highly abundance peptide.

Spectral count has further limitations that are due to the following technical requirements: (i) A high reproducibility of the chromatography system is required to obtain comparable elution profiles of the peptides during sample separation. (ii) The spectral count response for every protein is not the same, i.e. due to the protein's amino acid sequence and the different properties of the generated peptides (e.g. chromatographic behavior) the number of detectable spectra varies for every protein. As discussed above, smaller proteins generate only few peptides and relative quantification by spectral count is limited for these proteins. (iii) Different co-eluting peptides in the respective samples can affect the acquisition of distinct MS/MS spectra and thus influence the quantification process. (iv) Dynamic exclusion of precursor masses - that is selection of a precursor that already has been selected for fragmentation before and is thus subsequently not selected again for fragmentation (within a certain time window) - is usually used during LC-MS/MS analyses. Although the analytical depth is enhanced by using dynamic exclusion, dynamic exclusion negatively influences accurate quantification by spectral count, because different peptides are always selected for MS/MS fragmentation. It is important to note that spectral count not only takes the number of unique peptides into account, but also the overall number of spectra, i.e. the same peptide is selected several times for fragmentation. Consequently, when working with dynamic 
exclusion, the quantification of highly abundant peptides that show a longer retention time during their elution from the LC is not considered adequately. When working without dynamic exclusion, the spectral count is more reliable but, on the other hand, the analytic depth is drastically reduced and therefore only a limited number of proteins can be quantified.

The same holds true for SILAC and other labeling procedures that are based on peptide intensities on the MS level. Incorporation of stable isotopes (by metabolic or chemical labeling) generates peptides of different masses and the sample complexity is consequently increased. The analytical depth for the analysis of complex samples is thus reduced and only a limited number of proteins can be quantified.

Importantly, heavily modified proteins, protein isoforms and truncated proteins escape quantification. As the modified and the unmodified peptides show different masses, these proteins might yield false quantification values, in particular, when a protein becomes significantly modified during transition from the $B$ to the $C$ complex. Protein ratios of different protein isoforms within the quantified complexes might also not be correctly assigned and might thus affect the quantification for these proteins.

There are several reasons why (semi-quantitative) spectral count in our study, i.e. the comparison of highly purified spliceosomal complexes, is still applicable for relative comparison: (i) The analyzed spliceosomal complexes were highly purified under stringent conditions, thus minimizing the number of contaminating proteins during the analyses; (ii) the complexes are of moderate complexity and consist of only a limited number of proteins; (iii) The use of always the same LC system coupled front-end to the ESI Q-ToF mass spectrometer (Q-ToF Ultima, Waters) in all of the performed previous studies (Behzadnia et al., 2007; Bessonov et al., 2008; Deckert et al., 2006; Kuhn et al., 2009) fulfilled the prerequisite to generate comparable results and ensures high reproducibility such that analyses can be compared. However, when adapting the above described relative quantification approaches to biological systems other than spliceosomal complexes, the critical aspects discussed above have to be taken into account.

\subsubsection{Functional considerations}

Our results are in general very consistent with the findings from Bessonov et al., 2008. In this study, they compared the proteomes of $B$ and $C$ complexes using the number of peptides identified during LC-MS/MS analysis. In addition, the abundances of individual proteins in the respective complexes were monitored by immunoblotting. Peptide count and immunoblotting revealed that both complexes (i.e. B and $\mathrm{C}$ complexes) contained $\mathrm{U} 2$ and U5 snRNP proteins, the hPrp19/CDC5L complex and related proteins and the RES complex. U1 and U4 
snRNP proteins, U6 LSm proteins and several non-snRNP proteins were found to be underrepresented in the $\mathrm{C}$ complexes. Other proteins like the second-step factors, DEADbox helicases Abstrakt and DDX35 and several peptidyl-prolyl isomerases (e.g. PPWD1, PPIG, and PPIL3b) were found solely or predominantly in the C complex. Strikingly, immunoblotting showed that various SF3a/b proteins were less abundant in C complex.

Our relative quantification approaches applied to isolated $B$ and $C$ complexes clearly show that U1 and U4/U6 snRNP specific proteins, as well as LSm proteins are underrepresented in the $\mathrm{C}$ complex. However, quantification by stable isotope-labeling turned out to be more reliable as exemplified by the stoichiometry of the Sm proteins, the cap binding proteins and, as previously suggested, a stable complex of U5 snRNP specific proteins (Achsel et al., 1998). The Sm proteins show a two-fold enrichment in B vs. C complex (see above), and the cap binding proteins CBP20 and CBP80 and various U5 snRNP proteins (namely U5-220K, U5-200K, U5-116K, and U5-40K) were unambiguously found in equimolar amounts within both complexes. This was also shown to be the case for U5-116K by immunoblotting (Bessonov et al., 2008).

Comparison of the canonical U-snRNP specific proteins in the analysis of Bessonov et al., 2008 revealed that U2 snRNP specific SF3a and SF3b proteins are less abundant in the C complex as compared to the B complex. Our data are clearly consistent with their previous analysis and thus support strongly the hypothesis that the SF3b and SF3a proteins either partially dissociate from the U2 snRNA during the transition of $B$ to $C$ complex, or that structural rearrangements cause a destabilization of these proteins so that they, upon the purification of the complexes, partially dissociate. Importantly, the U2-snRNP specific proteins U2-A' and U2-B" show in our analysis a clear equimolar ratio between both complexes. This proves that both of these U2 snRNP specific proteins, together with the Sm proteins, remain stably bound to the U2 snRNA. It remains to be elucidated whether U2 snRNP within the $C$ complex indeed consists only of the so-called 12S U2-snRNP (U2 snRNA, Sm proteins and U2-A' and U2-B"; Behrens et al., 1993) or not.

The hPrp19/CDC5L complex (Ajuh et al., 2000; Makarova et al., 2004) was found to be associated already with the B complex, but was shown to be more abundant in the $\mathrm{C}$ complex. Our results are consistent with this observation and protein ratios (B vs. C) of approximately 0.5 show a higher abundance in the $C$ complex. A loose association of these proteins with the $\mathrm{B}$ complex and a more stable association with the $\mathrm{C}$ complex is therefore suggested.

Several other proteins like hSLU7, Abstrakt, and DDX35 show significantly low B/C protein ratios suggesting that they are only present in the $\mathrm{C}$ complex. Among these $\mathrm{C}$ complex 
specific proteins, we identified by our methods a hitherto unknown protein associated with the C complex, namely DDX34. This protein is a probable ATP-dependent RNA helicase (gi|38158022), but has so far not been functionally described, neither in yeast nor in human. In yeast, several proteins (PRP43, PRP22, PRP16, PRP2, DHR2, YLR419W, and ECM16) with sequences homologous to DDX34 have been identified; however, the sequence homology is limited to the DEAD-box motifs. In addition, core components of the exon junction complex, namely elF4A3, Magoh, and $Y 14$, were also found to be clearly associated with the C complex.

The RES (retention and splicing) complex was found to be present in equimolar amounts within $\mathrm{B}$ and $\mathrm{C}$ complexes. In a study from yeast, this complex was shown to be essential for pre-mRNA splicing (Dziembowski et al., 2004). Its inactivation caused pre-mRNA leakage from the nucleus, confirming our results that RES complex proteins are present in equal amounts in both, B and C complexes.

\subsection{Protein assembly time line for spliceosomes by relative quantification}

Metabolic labeling using SILAC is widely accepted as a technique for monitoring quantitative changes between different cellular states (for review see Ong and Mann, 2005). Here, we have used SILAC to monitor quantitatively the assembly over time of spliceosomal proteins on distinct pre-mRNAs.

To date, only a very few studies have investigated dynamic changes in protein composition by taking quantitative mass spectrometric approaches. All these also used metabolic labeling to follow such protein changes over time. The first study of this kind was the analysis of proteome dynamics in the nucleoli of eukaryotic cells (Andersen et al., 2005), where the response of the nucleolar proteome to transcription inhibition was observed by the SILAC approach. Making use of three differentially labeled HeLa cell lines, Andersen et al., 2005 were able to describe the turnover kinetics of several nucleolar factors influenced by inhibitors of transcription.

Later on, the same group modified the quantitative mass spectrometric approach to protein turnover within the nucleolus by introducing a "pulsed" incorporation of stable isotopes (Lam et al., 2007). For this purpose, unlabeled cells were transferred to SILAC media and cells were labeled for different time intervals. Nucleoli were isolated and the protein changes within the nucleoli were compared with those in the unlabeled cells (i.e., cells taken from the same preparation at the moment the reaction was started). By this approach, ribosomal 
proteins were found to be synthesized and to accumulate in nucleoli more rapidly than any other nucleolar factors.

This approach has been further pursued by Selbach et al., 2008 to study changes in protein translation under the influence of certain microRNAs. It is termed pulsed SILAC (pSILAC). In their study, non-labeled cells were transfected with miRNAs and transferred to cell-culture media containing amino acids labeled with stable isotopes. Subsequently, newly synthesized proteins were labeled with heavy stable isotopes, and thus changes in protein composition could be monitored by LC-MS/MS. A similar approach with pSILAC, by the same group, was applied to investigate the translational response upon cell stimulation (Schwanhausser et al., 2009). However, all these approaches addressed solely the question of which proteins are newly synthesized and which are degraded over time.

The only study so far to address the question of protein assembly by quantitative mass spectrometry was performed by Williamson and co-workers, who analyzed the assembly of the $30 \mathrm{~S}$ ribosomal subunit in vitro by pulse-chase mass spectrometry (Talkington et al., 2005; Williamson, 2005). The assembly of the proteins on $16 \mathrm{~S}$ ribosomal RNA to give a functionally active $30 \mathrm{~S}$ ribosomal subunit was investigated by using ${ }^{15} \mathrm{~N}$ - and ${ }^{14} \mathrm{~N}$-containing ribosomal proteins. In pulse-chase experiments, the $16 \mathrm{~S}$ ribosomal RNA was pulsed with ${ }^{15} \mathrm{~N}$-labeled ribosomal proteins which were then chased with an excess of ${ }^{14} \mathrm{~N}$-labeled proteins. After a certain period of time, the fully assembled 30S ribosomal subunits were isolated by density-gradient centrifugation and the degrees of incorporation of ${ }^{14} \mathrm{~N}$ - and ${ }^{15} \mathrm{~N}$ labeled proteins were measured by mass spectrometry. By varying the pulse over time and keeping the assembling time constant they were able to observe the kinetics of assembly of the various ribosomal proteins into 305 ribosomal subunit.

A similar approach, but with nuclear extract containing differently labeled amino acids lysine and arginine (see section 4.4.2) would have been of benefit, to monitor the kinetics of assembly of the spliceosomal proteins into complex A, B and/or C.

However, there are some restrictions that might have complicated the adoption of the abovementioned strategy for observing the assembly of spliceosomal complexes and thus such (more complex) experiments were omitted during the period of this work. (i) Assembly of the spliceosomal proteins and a pre-mRNA to yield spliceosomes is a process in which the spliceosome passes through different functional states to generate finally a catalytically active spliceosome (see Introduction). Once the pre-mRNA is spliced and the mature mRNA is formed, the intron lariat is released and the spliceosome dissociates; a new spliceosome is formed on each pre-mRNA to start a new round of splicing (for review, see Wahl et al., 2009). In this manner, complexes are continuously assembled on a new pre-mRNA in 
contrast to the studies of the $30 \mathrm{~S}$ ribosomal subunit where the assembly on the 16S rRNA is complete once the subunit has formed. Re-assembly of the spliceosome can be avoided when a pre-mRNA is used that lacks the 3' splice site, like the PM5 pre-mRNA (Anderson and Moore, 1997; Bessonov et al., 2008) and the assembly of the spliceosome halts at the state of the $\mathrm{C}$ complex. (ii) Pulse-chase experiments require the isolation of formed complexes, e.g. by density-gradient centrifugation, to allow elucidation of the ratios of differently labeled proteins. If the PM5 pre-mRNA is used, assembly of the $C$ complex requires 180 min plus time for the additional RNAseH cleavage step. The $\mathrm{C}$ complexes generated are then subjected to density-gradient centrifugation for preparative purification. For such experiments and the subsequent mass spectrometry-based protein analysis, a relatively large amount of nuclear extract is required, and in the case of pulse-chase experiments the amount would be multiplied by the number of time points chosen for pulsing. The same is true for monitoring the formation of $A$ and/or $B$ complexes.

For these reasons - i.e. the dynamic nature of the assembly pathway of spliceosomes and the fact that for preparative purification of assembled spliceosomal complexes a large quantity of nuclear-extracted (labeled and non-labeled) material is needed - together with constraints of time, this strategy was not followed.

Instead, it was decided to compare quantitatively the assembly of spliceosomal proteins on different pre-mRNAs. In the first round of experiments we compared quantitatively the assembly of the proteins on the different pre-mRNAs at distinct time points in a triple-label SILAC experiment (see Results, section 4.4.3). By using a double-labeling SILAC approach, in the second round of experiments we directly compared quantitatively the assembly of the proteins over time on two pre-mRNAs (30 min; see Results, section 4.4.4). Moreover, experiments of this type did not require the purification of assembled spliceosomal complexes by density-gradient centrifugation, but simply an affinity purification through the MS2-tag on the pre-mRNA. In this manner we obtained first quantitative insights into the assembly of various proteins on pre-mRNA.

Our initial results on this system will be discussed briefly in the following paragraphs:

In general these results show that relative mass spectrometry-based quantification is indeed suitable for displaying protein changes during the spliceosomal assembly pathway over time. Although we observed assembly of spliceosomal proteins on splicing-active (PM5) as well as on splicing-inactive pre-mRNAs (5'ss- and BPS-deleted PM5 pre-mRNAs), our studies clearly reveal differences between the splicing-active and -inactive pre-mRNAs. The observed quantitative differences in the assembly of the proteins on different pre-mRNAs will help in subsequent studies aimed at a complete understanding of protein assembly during 
the process of pre-mRNA splicing in vitro. We further believe that this approach is more straightforward than fluorescence techniques such as FRET (Ohrt et al., 2006) and - once all kinetic studies including the above-mentioned pulse-chase experiments have been performed - will yield dissociation rate constants for a multitude of spliceosomal proteins.

We observed quantitative differences in the association of U1 snRNP specific proteins with the three different pre-mRNAs (see Results, section 4.4.3). While in all three experiments a significant amount of U1 snRNP specific proteins was found to be associated with the pre-mRNA after 5 min under splicing conditions, the amount of U1 snRNP specific proteins only drastically decreases over time on the BPS-deleted PM5 pre-mRNA (Results 4.4.3), whereas on the PM5 and on the 5'ss-deleted PM5 pre-mRNA the amount of proteins seems to remain constant over time. Strikingly, the deletion of the 5'ss within the pre-mRNA shows the most significant effect on the protein assembly. The U1 snRNP specific protein $\mathrm{C}$ was absent after 2 min on the 5'ss-deleted PM5 pre-mRNA; however, even on the PM5 premRNA its amount was clearly lowered during assembly time. Protein $\mathrm{U} 1-\mathrm{C}$ is crucial for $\mathrm{E}$ complex formation (Heinrichs et al., 1990) and it has been suggested that this protein stabilizes base-pairing between U1 snRNA and the $5^{\prime}$ splice site of the pre-mRNA (Pomeranz Krummel et al., 2009; and references therein). Its absence on the 5'ss-deleted PM5 pre-mRNA - despite the fact that the other U1 snRNP proteins are present on the 5'ssdeleted pre-mRNA - sheds light on its important function in correct 5'ss recognition and perhaps in the subsequent formation of "correct" $E$ and A complexes. However, the observation that the amount of associated U1-C protein also decreases on PM5 pre-mRNA is not easily explained. We suggest that a part of U1 snRNP also associates unspecifically or loosely with pre-mRNA, irrespective of whether or not a canonical 5'ss is present. This would explain the similar assembly pattern over time of the U1 snRNP-specific proteins (except for U1-C) between PM5 and 5'ss-deleted PM5 pre-mRNA. Importantly, the overall assembly kinetics of the BPS-deleted PM5 pre-mRNA clearly reveals less (or no) association of the U1 snRNP specific proteins. This is consistent with the previous observation that interaction of U2 snRNP with the branch point sequence also stabilizes significantly the binding of $U 1$ snRNP to the pre-mRNA.

We observed very similar association kinetics of U2 snRNP specific proteins on the three different pre-mRNAs as compared with $U 1$ snRNP specific proteins. The association of the U2 snRNP specific proteins does not seem to be altered by the 5'ss-deletion, but it is affected significantly - as expected - by the BPS-deletion (see above).

At the current state of the analysis, the results for the assembly of the proteins of hPrp19/CDC5L complex and second-step splicing factors are difficult to interpret in a comparison of the three different pre-mRNA constructs. On all three pre-mRNAs we 
observed a significant amount of Prp19/CDC5L complex proteins and step 2 factors associated with the RNA after 30 min of incubation under splicing conditions. Overall, the association kinetics of all these proteins show a saw-tooth-like pattern with a maximum at $5 \mathrm{~min}$, a minimum at $10 \mathrm{~min}$, a maximum at 15 and so forth (see Results). An exception is clearly the step 2 factor hPrp17 (Neer et al., 1994), the amount of which associated with PM5 and 5'ss-deleted PM5 steadily increases over time. However, on the basis of the current literature surprisingly little is known about the biological function of Prp17, except for a role in the late splicing process and more recently also in the cell cycle (Ben Yehuda et al., 1998). Mutational analysis of the 3'ss of pre-mRNA in yeast demonstrated that the association of Prp17 and Prp16 with the spliceosome is not affected and suggested that both these factors function at the second catalytic step of splicing but prior to 3'ss recognition (Zhou and Reed, 1998). Proteins Prp16 and Prp17 associate with the spliceosome independently of one another, and cross-linking experiments have suggested that Prp17 acts subsequently to the association of Prp16 with the spliceosome. Indeed, our data suggest that the behavior of Prp17 differs from that of the other step II factors.

In our second quantitative analysis we have compared the amounts of proteins associated on the different pre-mRNAs over time (see Results, section 4.4.4). This comparison reflects more drastically the differences in the association of spliceosomal proteins with the different pre-mRNAs. Strikingly, U1 snRNP-specific proteins readily associate with PM5 pre-mRNA even upon very short incubation under splicing conditions ( $0 \mathrm{~min}$ ) but much less with the 5'ss-deleted PM5 pre-mRNA. As expected, the amount of U1 snRNP on the PM5 pre-mRNA decreases over time, owing the fact that over time the first step of splicing takes place and, concomitantly with it, there occur structural changes that release U1 snRNPs from the spliceosome (for review see Wahl et al., 2009). Importantly, the same pattern is observed with the Sm proteins; this is consistent with the dissociation of $\mathrm{U} 1$ (see above) and U4 snRNP from the spliceosome upon rearrangement of the U4/U6 di-snRNP to form new base pairs between U6 and U2 snRNP (Hausner et al., 1990; Sashital et al., 2004; Sun and Manley, 1995).

Even more impressive is the association of the Prp19/CDC5L proteins and the step 2 factors on the PM5 pre-mRNA as compared with the 5'ss-deleted PM5 pre-mRNA. After 5 min under splicing condition we observe a dramatic increase in the amounts of those proteins associated with the PM5 but not with 5'ss-deleted PM5 pre-mRNA.

When comparing these quantitative results with those obtained for single pre-mRNA, where we observed some minor differences in the assembly of the proteins on the various pre-mRNAs, we have to conclude that - at least under the conditions that we describe here (i.e. incubation of nuclear extract with pre-mRNAs under splicing conditions and affinity 
purification without further density-gradient purification of the spliceosomal complexes formed) - the kinetics of assembly of the proteins on the different pre-mRNAs are surprisingly similar but that proteins assemble in much higher yield on splicing-active premRNA than on the splicing-inactive mutants.

This can be explained by the observation that both the mutants (5'ss and BPS-deleted PM5 pre-mRNA) show a very low remaining activity in splicing (see Results, section 4.4.1). Indeed, mass spectrometry is such a sensitive technique that it is able to monitor those proteins on the pre-mRNA mutants that reflect the residual splicing activity.

However, final conclusions can only been drawn after the evaluation of the remaining other spliceosomal proteins, e.g. the U5 snRNP-specific proteins and the A and B complex specific proteins and - more importantly - after having performed the above-mentioned pulse-chase experiment with the final isolation and mass spectrometric analysis of the assembled A, B, and $\mathrm{C}$ complexes. 


\section{References}

Abbatiello, S.E., Pan, Y.X., Zhou, M., Wayne, A.S., Veenstra, T.D., Hunger, S.P., Kilberg, M.S., Eyler, J.R., Richards, N.G., and Conrads, T.P. (2008). Mass spectrometric quantification of asparagine synthetase in circulating leukemia cells from acute lymphoblastic leukemia patients. J Proteomics 71, 61-70.

Achsel, T., Ahrens, K., Brahms, H., Teigelkamp, S., and Luhrmann, R. (1998). The human U5-220kD protein (hPrp8) forms a stable RNA-free complex with several U5-specific proteins, including an RNA unwindase, a homologue of ribosomal elongation factor EF-2, and a novel WD-40 protein. Mol Cell Biol 18, 6756-6766.

Achsel, T., Brahms, H., Kastner, B., Bachi, A., Wilm, M., and Luhrmann, R. (1999). A doughnut-shaped heteromer of human Sm-like proteins binds to the 3'-end of U6 snRNA, thereby facilitating U4/U6 duplex formation in vitro. EMBO J 18, 5789-5802.

Ajuh, P., Kuster, B., Panov, K., Zomerdijk, J.C., Mann, M., and Lamond, A.I. (2000). Functional analysis of the human CDC5L complex and identification of its components by mass spectrometry. EMBO J 19, 6569-6581.

Andersen, J.S., Lam, Y.W., Leung, A.K., Ong, S.E., Lyon, C.E., Lamond, A.I., and Mann, M. (2005). Nucleolar proteome dynamics. Nature 433, 77-83.

Anderson, K., and Moore, M.J. (1997). Bimolecular exon ligation by the human spliceosome. Science 276, 1712-1716.

Anderson, L., and Hunter, C.L. (2006). Quantitative mass spectrometric multiple reaction monitoring assays for major plasma proteins. Mol Cell Proteomics 5, 573-588.

Bach, M., Winkelmann, G., and Luhrmann, R. (1989). 20 S small nuclear ribonucleoprotein U5 shows a surprisingly complex protein composition. Proc Natl Acad Sci U S A 86, 6038-6042.

Bach, M., Bringmann, P., and Luhrmann, R. (1990). Purification of small nuclear ribonucleoprotein particles with antibodies against modified nucleosides of small nuclear RNAs. Methods Enzymol 181, 232-257.

Bantscheff, M., Schirle, M., Sweetman, G., Rick, J., and Kuster, B. (2007). Quantitative mass spectrometry in proteomics: a critical review. Anal Bioanal Chem 389, 1017-1031.

Bearden, J.C., Jr. (1978). Quantitation of submicrogram quantities of protein by an improved protein-dye binding assay. Biochim Biophys Acta 533, 525-529.

Behrens, S.E., and Luhrmann, R. (1991). Immunoaffinity purification of a [U4/U6.U5] tri-snRNP from human cells. Genes Dev 5, 1439-1452.

Behrens, S.E., Tyc, K., Kastner, B., Reichelt, J., and Luhrmann, R. (1993). Small nuclear ribonucleoprotein (RNP) U2 contains numerous additional proteins and has a bipartite RNP structure under splicing conditions. Mol Cell Biol 13, 307-319.

Behzadnia, N., Golas, M.M., Hartmuth, K., Sander, B., Kastner, B., Deckert, J., Dube, P., Will, C.L., Urlaub, H., Stark, H., and Luhrmann, R. (2007). Composition and three- 
dimensional EM structure of double affinity-purified, human prespliceosomal A complexes. EMBO J 26, 1737-1748.

Ben Yehuda, S., Dix, I., Russell, C.S., Levy, S., Beggs, J.D., and Kupiec, M. (1998). Identification and functional analysis of hPRP17, the human homologue of the PRP17/CDC40 yeast gene involved in splicing and cell cycle control. RNA 4, 1304-1312.

Bessonov, S., Anokhina, M., Will, C.L., Urlaub, H., and Luhrmann, R. (2008). Isolation of an active step I spliceosome and composition of its RNP core. Nature 452, 846-850.

Bienvenut, W.V., Deon, C., Pasquarello, C., Campbell, J.M., Sanchez, J.C., Vestal, M.L., and Hochstrasser, D.F. (2002). Matrix-assisted laser desorption/ionization-tandem mass spectrometry with high resolution and sensitivity for identification and characterization of proteins. Proteomics 2, 868-876.

Birney, E., Kumar, S., and Krainer, A.R. (1993). Analysis of the RNA-recognition motif and RS and RGG domains: conservation in metazoan pre-mRNA splicing factors. Nucleic Acids Res 21, 5803-5816.

Black, D.L., and Pinto, A.L. (1989). U5 small nuclear ribonucleoprotein: RNA structure analysis and ATP-dependent interaction with U4/U6. Mol Cell Biol 9, 3350-3359.

Boersema, P.J., Aye, T.T., van Veen, T.A., Heck, A.J., and Mohammed, S. (2008). Triplex protein quantification based on stable isotope labeling by peptide dimethylation applied to cell and tissue lysates. Proteomics 8, 4624-4632.

Boersema, P.J., Raijmakers, R., Lemeer, S., Mohammed, S., and Heck, A.J. (2009). Multiplex peptide stable isotope dimethyl labeling for quantitative proteomics. Nat Protoc 4 , 484-494.

Bringmann, P., Rinke, J., Appel, B., Reuter, R., and Luhrmann, R. (1983). Purification of snRNPs U1, U2, U4, U5 and U6 with 2,2,7-trimethylguanosine-specific antibody and definition of their constituent proteins reacting with anti-Sm and anti-(U1)RNP antisera. EMBO J 2, 1129-1135.

Brosi, R., Hauri, H.P., and Kramer, A. (1993). Separation of splicing factor SF3 into two components and purification of SF3a activity. J Biol Chem 268, 17640-17646.

Brun, V., Dupuis, A., Adrait, A., Marcellin, M., Thomas, D., Court, M., Vandenesch, F., and Garin, J. (2007). Isotope-labeled protein standards: toward absolute quantitative proteomics. Mol Cell Proteomics 6, 2139-2149.

Burge, C.B., Tuschl, T., and Sharp, P.A. (1999). Splicing of precursors to mRNA by the Spliceosome. In The RNA world, R.F. Gesteland, T.R. Cech, and T.R. Atkins, eds. (Cold Spring Harbor, New York, Cold Spring Harbor Laboratory Press), pp. 525-560.

Chan, S.P., Kao, D.I., Tsai, W.Y., and Cheng, S.C. (2003). The Prp19p-associated complex in spliceosome activation. Science 302, 279-282.

Choi, Y.D., Grabowski, P.J., Sharp, P.A., and Dreyfuss, G. (1986). Heterogeneous nuclear ribonucleoproteins: role in RNA splicing. Science 231, 1534-1539.

Collins, C.A., and Guthrie, C. (1999). Allele-specific genetic interactions between Prp8 and RNA active site residues suggest a function for Prp8 at the catalytic core of the spliceosome. Genes Dev 13, 1970-1982. 
Conrads, T.P., Alving, K., Veenstra, T.D., Belov, M.E., Anderson, G.A., Anderson, D.J., Lipton, M.S., Pasa-Tolic, L., Udseth, H.R., Chrisler, W.B., Thrall, B.D., and Smith, R.D. (2001). Quantitative analysis of bacterial and mammalian proteomes using a combination of cysteine affinity tags and 15N-metabolic labeling. Anal Chem 73, 2132-2139.

Cox, J., and Mann, M. (2008). MaxQuant enables high peptide identification rates, individualized p.p.b.-range mass accuracies and proteome-wide protein quantification. Nat Biotechnol 26, 1367-1372.

Das, R., Zhou, Z., and Reed, R. (2000). Functional association of U2 snRNP with the ATP-independent spliceosomal complex E. Mol Cell 5, 779-787.

Deckert, J., Hartmuth, K., Boehringer, D., Behzadnia, N., Will, C.L., Kastner, B., Stark, H., Urlaub, H., and Luhrmann, R. (2006). Protein composition and electron microscopy structure of affinity-purified human spliceosomal B complexes isolated under physiological conditions. Mol Cell Biol 26, 5528-5543.

Desiderio, D.M., and Kai, M. (1983). Preparation of stable isotope-incorporated peptide internal standards for field desorption mass spectrometry quantification of peptides in biologic tissue. Biomed Mass Spectrom 10, 471-479.

Dignam, J.D., Lebovitz, R.M., and Roeder, R.G. (1983). Accurate transcription initiation by RNA polymerase II in a soluble extract from isolated mammalian nuclei. Nucleic Acids Res 11, 1475-1489.

Dönmez, G. (2006). Investigation of the higher order structure of the spliceosomal RNA network (Göttingen, Georg-August-Universität Göttingen).

Doherty, M.K., Whitehead, C., McCormack, H., Gaskell, S.J., and Beynon, R.J. (2005). Proteome dynamics in complex organisms: using stable isotopes to monitor individual protein turnover rates. Proteomics 5, 522-533.

Dreyfuss, G., Matunis, M.J., Pinol-Roma, S., and Burd, C.G. (1993). hnRNP proteins and the biogenesis of mRNA. Annu Rev Biochem 62, 289-321.

Dziembowski, A., Ventura, A.P., Rutz, B., Caspary, F., Faux, C., Halgand, F., Laprevote, O., and Seraphin, B. (2004). Proteomic analysis identifies a new complex required for nuclear pre-mRNA retention and splicing. EMBO J 23, 4847-4856.

Edman, P. (1949). A method for the determination of amino acid sequence in peptides. Arch Biochem 22, 475.

Elias, J.E., Haas, W., Faherty, B.K., and Gygi, S.P. (2005). Comparative evaluation of mass spectrometry platforms used in large-scale proteomics investigations. Nat Methods 2, 667-675.

Fabrizio, P., Dannenberg, J., Dube, P., Kastner, B., Stark, H., Urlaub, H., and Luhrmann, R. (2009). The evolutionarily conserved core design of the catalytic activation step of the yeast spliceosome. Mol Cell 36, 593-608.

Fenn, J.B., Mann, M., Meng, C.K., Wong, S.F., and Whitehouse, C.M. (1989). Electrospray ionization for mass spectrometry of large biomolecules. Science 246, 64-71.

Gerber, S.A., Rush, J., Stemman, O., Kirschner, M.W., and Gygi, S.P. (2003). Absolute quantification of proteins and phosphoproteins from cell lysates by tandem MS. Proc Natl Acad Sci U S A 100, 6940-6945. 
Gilar, M., Olivova, P., Daly, A.E., and Gebler, J.C. (2005). Two-dimensional separation of peptides using RP-RP-HPLC system with different $\mathrm{pH}$ in first and second separation dimensions. J Sep Sci 28, 1694-1703.

Gilchrist, A., Au, C.E., Hiding, J., Bell, A.W., Fernandez-Rodriguez, J., Lesimple, S., Nagaya, H., Roy, L., Gosline, S.J., Hallett, M., Paiement, J., Kearney, R.E., Nilsson, T., and Bergeron, J.J. (2006). Quantitative proteomics analysis of the secretory pathway. Cell 127, $1265-1281$.

Gozani, O., Feld, R., and Reed, R. (1996). Evidence that sequence-independent binding of highly conserved U2 snRNP proteins upstream of the branch site is required for assembly of spliceosomal complex A. Genes Dev 10, 233-243.

Graub, R., Lancero, H., Pedersen, A., Chu, M., Padmanabhan, K., Xu, X.Q., Spitz, P., Chalkley, R., Burlingame, A.L., Stokoe, D., Bernstein, H.S. (2008). Cell cycle-dependent phosphorylation of human CDC5 regulates RNA processing. Cell Cycle 7, 1795-1803.

Graumann, J., Hubner, N.C., Kim, J.B., Ko, K., Moser, M., Kumar, C., Cox, J., Scholer, H., and Mann, M. (2008). Stable isotope labeling by amino acids in cell culture (SILAC) and proteome quantitation of mouse embryonic stem cells to a depth of 5,111 proteins. Mol Cell Proteomics 7, 672-683.

Graveley, B.R. (2000). Sorting out the complexity of SR protein functions. RNA 6, 1197-1211.

Green, M.R. (1991). Biochemical mechanisms of constitutive and regulated premRNA splicing. Annu Rev Cell Biol 7, 559-599.

Grillari, J., Ajuh, P., Stadler, G., Loscher, M., Voglauer, R., Ernst, W., Chusainow, J., Eisenhaber, F., Pokar, M., Fortschegger, K., Grey, M, Lamond, A.I., and Katinger, H. (2005). SNEV is an evolutionarily conserved splicing factor whose oligomerization is necessary for spliceosome assembly. Nucleic Acids Res 33, 6868-6883.

Grote, M., Wolf, E., Will, C.L., Lemm, I., Agafonov, D.E., Schomburg, A., Fischle, W., Urlaub, H., and Luhrmann, R. (2010). The Molecular Architecture of the Human Prp19/CDC5L Complex. Mol Cell Biol 30, 2105-2119

Gruhler, A., Olsen, J.V., Mohammed, S., Mortensen, P., Faergeman, N.J., Mann, M., and Jensen, O.N. (2005). Quantitative phosphoproteomics applied to the yeast pheromone signaling pathway. Mol Cell Proteomics 4, 310-327. 387-419.

Guthrie, C., and Patterson, B. (1988). Spliceosomal snRNAs. Annu Rev Genet 22,

Gygi, S.P., Rist, B., Gerber, S.A., Turecek, F., Gelb, M.H., and Aebersold, R. (1999). Quantitative analysis of complex protein mixtures using isotope-coded affinity tags. Nat Biotechnol 17, 994-999.

Hanke, S., Besir, H., Oesterhelt, D., and Mann, M. (2008). Absolute SILAC for accurate quantitation of proteins in complex mixtures down to the attomole level. J Proteome Res 7, 1118-1130.

Hartinger, J., Stenius, K., Hogemann, D., and Jahn, R. (1996). 16-BAC/SDS-PAGE: a two-dimensional gel electrophoresis system suitable for the separation of integral membrane proteins. Anal Biochem 240, 126-133. 
Hartmuth, K., Urlaub, H., Vornlocher, H.P., Will, C.L., Gentzel, M., Wilm, M., and Luhrmann, R. (2002). Protein composition of human prespliceosomes isolated by a tobramycin affinity-selection method. Proc Natl Acad Sci U S A 99, 16719-16724.

Hausner, T.P., Giglio, L.M., and Weiner, A.M. (1990). Evidence for base-pairing between mammalian U2 and U6 small nuclear ribonucleoprotein particles. Genes Dev 4, 2146-2156.

Heinrichs, V., Bach, M., Winkelmann, G., and Luhrmann, R. (1990). U1-specific protein $\mathrm{C}$ needed for efficient complex formation of U1 snRNP with a 5' splice site. Science 247, 69-72.

Hochleitner, E.O., Kastner, B., Frohlich, T., Schmidt, A., Luhrmann, R., Arnold, G., and Lottspeich, F. (2005). Protein stoichiometry of a multiprotein complex, the human spliceosomal U1 small nuclear ribonucleoprotein: absolute quantification using isotope-coded tags and mass spectrometry. J Biol Chem 280, 2536-2542.

Holzmann, J., Pichler, P., Madalinski, M., Kurzbauer, R., and Mechtler, K. (2009). Stoichiometry determination of the MP1-p14 complex using a novel and cost-efficient method to produce an equimolar mixture of standard peptides. Anal Chem 81, 10254-10261.

Hsu, J.L., Huang, S.Y., Chow, N.H., and Chen, S.H. (2003). Stable-isotope dimethyl labeling for quantitative proteomics. Anal Chem 75, 6843-6852.

Hu, Q., Noll, R.J., Li, H., Makarov, A., Hardman, M., and Graham Cooks, R. (2005). The Orbitrap: a new mass spectrometer. J Mass Spectrom 40, 430-443.

Ishihama, Y., Oda, Y., Tabata, T., Sato, T., Nagasu, T., Rappsilber, J., and Mann, M. (2005). Exponentially modified protein abundance index (emPAl) for estimation of absolute protein amount in proteomics by the number of sequenced peptides per protein. Mol Cell Proteomics 4, 1265-1272.

Johnson, K.L., and Muddiman, D.C. (2004). A method for calculating 160/180 peptide ion ratios for the relative quantification of proteomes. J Am Soc Mass Spectrom 15, 437-445.

Karas, M., Bachmann, D., Bahr, U., and Hillenkamp, F. (1987). Matrix-assisted ultraviolet laser desorption of non-volatile compounds. Int J Mass Spectrom Ion Proc 78, 53-58.

Karas, M., and Hillenkamp, F. (1988). Laser desorption ionization of proteins with molecular masses exceeding 10,000 daltons. Anal Chem 60, 2299-2301.

Kastner, B., and Luhrmann, R. (1999). Purification of $\mathrm{U}$ small nuclear ribonucleoprotein particles. Methods Mol Biol 118, 289-298.

Kirkpatrick, D.S., Gerber, S.A., and Gygi, S.P. (2005). The absolute quantification strategy: a general procedure for the quantification of proteins and post-translational modifications. Methods 35, 265-273.

Klose, J. (1975). Protein mapping by combined isoelectric focusing and electrophoresis of mouse tissues. A novel approach to testing for induced point mutations in mammals. Humangenetik 26, 231-243.

Klose, J. (1999). Large-gel 2-D electrophoresis. Methods Mol Biol 112, 147-172. 
Knochenmuss, R., Stortelder, A., Breuker, K., and Zenobi, R. (2000). Secondary ionmolecule reactions in matrix-assisted laser desorption/ionization. J Mass Spectrom 35, 12371245.

Koehler, C.J., Strozynski, M., Kozielski, F., Treumann, A., and Thiede, B. (2009). Isobaric peptide termini labeling for MS/MS-based quantitative proteomics. J Proteome Res 8, 4333-4341.

Kramer, A., Gruter, P., Groning, K., and Kastner, B. (1999). Combined biochemical and electron microscopic analyses reveal the architecture of the mammalian U2 snRNP. J Cell Biol 145, 1355-1368.

Krijgsveld, J., Gauci, S., Dormeyer, W., and Heck, A.J. (2006). In-gel isoelectric focusing of peptides as a tool for improved protein identification. J Proteome Res 5, 17211730.

Krijgsveld, J., Ketting, R.F., Mahmoudi, T., Johansen, J., Artal-Sanz, M., Verrijzer, C.P., Plasterk, R.H., and Heck, A.J. (2003). Metabolic labeling of C. elegans and D. melanogaster for quantitative proteomics. Nat Biotechnol 21, 927-931.

Kruger, M., Moser, M., Ussar, S., Thievessen, I., Luber, C.A., Forner, F., Schmidt, S., Zanivan, S., Fassler, R., and Mann, M. (2008). SILAC mouse for quantitative proteomics uncovers kindlin-3 as an essential factor for red blood cell function. Cell 134, 353-364.

Kuhn, A.N., van Santen, M.A., Schwienhorst, A., Urlaub, H., and Luhrmann, R. (2009). Stalling of spliceosome assembly at distinct stages by small-molecule inhibitors of protein acetylation and deacetylation. RNA 15, 153-175.

Kuhn, E., Wu, J., Karl, J., Liao, H., Zolg, W., and Guild, B. (2004). Quantification of Creactive protein in the serum of patients with rheumatoid arthritis using multiple reaction monitoring mass spectrometry and 13C-labeled peptide standards. Proteomics 4, 11751186.

Kunkel, G.R., Maser, R.L., Calvet, J.P., and Pederson, T. (1986). U6 small nuclear RNA is transcribed by RNA polymerase III. Proc Natl Acad Sci U S A 83, 8575-8579.

Kuster, B., Schirle, M., Mallick, P., and Aebersold, R. (2005). Scoring proteomes with proteotypic peptide probes. Nat Rev Mol Cell Biol 6, 577-583.

Laemmli, U.K. (1970). Cleavage of structural proteins during the assembly of the head of bacteriophage T4. Nature 227, 680-685.

Laggerbauer, B., Liu, S., Makarov, E., Vornlocher, H.P., Makarova, O., Ingelfinger, D., Achsel, T., and Luhrmann, R. (2005). The human U5 snRNP 52K protein (CD2BP2) interacts with U5-102K (hPrp6), a U4/U6.U5 tri-snRNP bridging protein, but dissociates upon trisnRNP formation. RNA 11, 598-608.

Lam, Y.W., Lamond, A.I., Mann, M., and Andersen, J.S. (2007). Analysis of nucleolar protein dynamics reveals the nuclear degradation of ribosomal proteins. Curr Biol 17, 749760.

Langenfeld, E., Zanger, U.M., Jung, K., Meyer, H.E., and Marcus, K. (2009). Mass spectrometry-based absolute quantification of microsomal cytochrome P450 2D6 in human liver. Proteomics 9, 2313-2323. 
Liao, L., Park, S.K., Xu, T., Vanderklish, P., and Yates, J.R., 3rd (2008). Quantitative proteomic analysis of primary neurons reveals diverse changes in synaptic protein content in fmr1 knockout mice. Proc Natl Acad Sci U S A 105, 15281-15286.

Liautard, J.P., Sri-Widada, J., Brunel, C., and Jeanteur, P. (1982). Structural organization of ribonucleoproteins containing small nuclear RNAs from HeLa cells. Proteins interact closely with a similar structural domain of $\mathrm{U} 1, \mathrm{U} 2, \mathrm{U} 4$ and $\mathrm{U} 5$ small nuclear RNAs. $\mathrm{J}$ Mol Biol 162, 623-643.

Liu, H., Sadygov, R.G., and Yates, J.R., 3rd (2004). A model for random sampling and estimation of relative protein abundance in shotgun proteomics. Anal Chem 76, 41934201.

Macfarlane, D.E. (1989). Two dimensional benzyldimethyl-n-hexadecylammonium chloride----sodium dodecyl sulfate preparative polyacrylamide gel electrophoresis: a high capacity high resolution technique for the purification of proteins from complex mixtures. Anal Biochem 176, 457-463.

Makarov, E.M., Makarova, O.V., Urlaub, H., Gentzel, M., Will, C.L., Wilm, M., and Luhrmann, R. (2002). Small nuclear ribonucleoprotein remodeling during catalytic activation of the spliceosome. Science 298, 2205-2208.

Makarova, O.V., Makarov, E.M., and Luhrmann, R. (2001). The 65 and 110 kDa SRrelated proteins of the U4/U6.U5 tri-snRNP are essential for the assembly of mature spliceosomes. EMBO J 20, 2553-2563.

Makarova, O.V., Makarov, E.M., Urlaub, H., Will, C.L., Gentzel, M., Wilm, M., and Luhrmann, R. (2004). A subset of human 35S U5 proteins, including Prp19, function prior to catalytic step 1 of splicing. EMBO J 23, 2381-2391.

Malca, H., Shomron, N., and Ast, G. (2003). The U1 snRNP base pairs with the $5^{\prime}$ splice site within a penta-snRNP complex. Mol Cell Biol 23, 3442-3455.

Mallick, P., Schirle, M., Chen, S.S., Flory, M.R., Lee, H., Martin, D., Ranish, J., Raught, B., Schmitt, R., Werner, T., Kuster, B., and Aebersold, R. (2007). Computational prediction of proteotypic peptides for quantitative proteomics. Nat Biotechnol 25, 125-131. 1569-1579.

Manley, J.L., and Tacke, R. (1996). SR proteins and splicing control. Genes Dev 10,

Massenet, S., Mougin, A., and Branlant, C. (1998). Posttranscriptional modifications in the $U$ small nuclear RNAs. In The Modification and Editing of RNA, H. Grosjean, and R. Benne, eds. (Washington D.C., ASM Press), pp. 201-227.

Mayeda, A., and Krainer, A.R. (1992). Regulation of alternative pre-mRNA splicing by hnRNP A1 and splicing factor SF2. Cell 68, 365-375.

Mintz, M., Vanderver, A., Brown, K.J., Lin, J., Wang, Z., Kaneski, C., Schiffmann, R., Nagaraju, K., Hoffman, E.P., and Hathout, Y. (2008). Time series proteome profiling to study endoplasmic reticulum stress response. J Proteome Res 7, 2435-2444.

Mirgorodskaya, O.A., Kozmin, Y.P., Titov, M.I., Korner, R., Sonksen, C.P., and Roepstorff, P. (2000). Quantitation of peptides and proteins by matrix-assisted laser desorption/ionization mass spectrometry using (18)O-labeled internal standards. Rapid Commun Mass Spectrom 14, 1226-1232. 
Moore, M.J., Query, C.C., and Sharp, P.A. (1993). Splicing of precursors to mRNA by the spliceosome. In RNA world, R.F. Gesteland, and T.R. Atkins, eds. (Cold Spring Harbor, New York, Cold Spring Harbor Laboratory Press), pp. 303-357.

Moore, M.J., and Sharp, P.A. (1993). Evidence for two active sites in the spliceosome provided by stereochemistry of pre-mRNA splicing. Nature 365, 364-368.

Neer, E.J., Schmidt, C.J., Nambudripad, R., and Smith, T.F. (1994). The ancient regulatory-protein family of WD-repeat proteins. Nature 371, 297-300.

Nelissen, R.L., Will, C.L., van Venrooij, W.J., and Luhrmann, R. (1994). The association of the U1-specific $70 \mathrm{~K}$ and $\mathrm{C}$ proteins with $\mathrm{U} 1$ snRNPs is mediated in part by common U snRNP proteins. EMBO J 13, 4113-4125.

Neuhoff, V., Arold, N., Taube, D., and Ehrhardt, W. (1988). Improved staining of proteins in polyacrylamide gels including isoelectric focusing gels with clear background at nanogram sensitivity using Coomassie Brilliant Blue G-250 and R-250. Electrophoresis 9, 255-262.

Niggeweg, R., Kocher, T., Gentzel, M., Buscaino, A., Taipale, M., Akhtar, A., and Wilm, M. (2006). A general precursor ion-like scanning mode on quadrupole-TOF instruments compatible with chromatographic separation. Proteomics 6, 41-53.

Nilsen, T.W. (1998). RNA-RNA interactions in nuclear pre-mRNA splicing. In RNA structure and function, R.W. Simmons, and M. Grunerg-Manaro, eds. (Cold Spring Harbor, New York, Cold Spring Harbor Laboratory Press), pp. 1793-1309.

Oda, Y., Huang, K., Cross, F.R., Cowburn, D., and Chait, B.T. (1999). Accurate quantitation of protein expression and site-specific phosphorylation. Proc Natl Acad Sci U S A 96, 6591-6596.

Oellerich, T., Gronborg, M., Neumann, K., Hsiao, H.H., Urlaub, H., and Wienands, J. (2009). SLP-65 phosphorylation dynamics reveals a functional basis for signal integration by receptor-proximal adaptor proteins. Mol Cell Proteomics 8, 1738-1750.

O'Farrell, P.H. (1975). High resolution two-dimensional electrophoresis of proteins. J Biol Chem 250, 4007-4021.

Ohi, M.D., Vander Kooi, C.W., Rosenberg, J.A., Ren, L., Hirsch, J.P., Chazin, W.J., Walz, T., and Gould, K.L. (2005). Structural and functional analysis of essential pre-mRNA splicing factor Prp19p. Mol Cell Biol 25, 451-460.

Ohrt, T., Merkle, D., Birkenfeld, K., Echeverri, C.J., and Schwille, P. (2006). In situ fluorescence analysis demonstrates active siRNA exclusion from the nucleus by Exportin 5 . Nucleic Acids Res 34, 1369-1380.

Old, W.M., Meyer-Arendt, K., Aveline-Wolf, L., Pierce, K.G., Mendoza, A., Sevinsky, J.R., Resing, K.A., and Ahn, N.G. (2005). Comparison of label-free methods for quantifying human proteins by shotgun proteomics. Mol Cell Proteomics 4, 1487-1502.

Olsen, J.V., Ong, S.E., and Mann, M. (2004). Trypsin cleaves exclusively C-terminal to arginine and lysine residues. Mol Cell Proteomics 3, 608-614.

Olsen, J.V., de Godoy, L.M., Li, G., Macek, B., Mortensen, P., Pesch, R., Makarov, A., Lange, O., Horning, S., and Mann, M. (2005). Parts per million mass accuracy on an Orbitrap mass spectrometer via lock mass injection into a C-trap. Mol Cell Proteomics 4, 2010-2021. 
Olsen, J.V., Vermeulen, M., Santamaria, A., Kumar, C., Miller, M.L., Jensen, L.J., Gnad, F., Cox, J., Jensen, T.S., Nigg, E.A., Brunak, S., and Mann, M. (2010). Quantitative phosphoproteomics reveals widespread full phosphorylation site occupancy during mitosis. Sci Signal 3, ra3.

Ong, S.E., Blagoev, B., Kratchmarova, I., Kristensen, D.B., Steen, H., Pandey, A., and Mann, M. (2002). Stable isotope labeling by amino acids in cell culture, SILAC, as a simple and accurate approach to expression proteomics. Mol Cell Proteomics 1, 376-386.

Ong, S.E., Foster, L.J., and Mann, M. (2003). Mass spectrometric-based approaches in quantitative proteomics. Methods 29, 124-130.

Ong, S.E., and Mann, M. (2005). Mass spectrometry-based proteomics turns quantitative. Nat Chem Biol 1, 252-262.

Pan, C., Olsen, J.V., Daub, H., and Mann, M. (2009a). Global effects of kinase inhibitors on signaling networks revealed by quantitative phosphoproteomics. Mol Cell Proteomics 8, 2796-2808.

Pan, S., Aebersold, R., Chen, R., Rush, J., Goodlett, D.R., Mclntosh, M.W., Zhang, J., and Brentnall, T.A. (2009b). Mass spectrometry based targeted protein quantification: methods and applications. J Proteome Res 8, 787-797.

Pan, S., Zhang, H., Rush, J., Eng, J., Zhang, N., Patterson, D., Comb, M.J., and Aebersold, R. (2005). High throughput proteome screening for biomarker detection. Mol Cell Proteomics 4, 182-190.

Patton, J.G., Mayer, S.A., Tempst, P., and Nadal-Ginard, B. (1991). Characterization and molecular cloning of polypyrimidine tract-binding protein: a component of a complex necessary for pre-mRNA splicing. Genes Dev 5, 1237-1251.

Patton, J.R., Habets, W., van Venrooij, W.J., and Pederson, T. (1989). U1 small nuclear ribonucleoprotein particle-specific proteins interact with the first and second stemloops of U1 RNA, with the A protein binding directly to the RNA independently of the $70 \mathrm{~K}$ and Sm proteins. Mol Cell Biol 9, 3360-3368.

Patton, J.R., and Pederson, T. (1988). The Mr 70,000 protein of the U1 small nuclear ribonucleoprotein particle binds to the $5^{\prime}$ stem-loop of U1 RNA and interacts with Sm domain proteins. Proc Natl Acad Sci U S A 85, 747-751.

Pomeranz Krummel, D.A., Oubridge, C., Leung, A.K., Li, J., and Nagai, K. (2009). Crystal structure of human spliceosomal U1 snRNP at 5.5 A resolution. Nature $458,475-480$.

Pratt, J.M., Simpson, D.M., Doherty, M.K., Rivers, J., Gaskell, S.J., and Beynon, R.J. (2006). Multiplexed absolute quantification for proteomics using concatenated signature peptides encoded by QconCAT genes. Nat Protoc 1, 1029-1043.

Query, C.C., Bentley, R.C., and Keene, J.D. (1989). A specific 31-nucleotide domain of U1 RNA directly interacts with the $70 \mathrm{~K}$ small nuclear ribonucleoprotein component. Mol Cell Biol 9, 4872-4881.

Query, C.C., Moore, M.J., and Sharp, P.A. (1994). Branch nucleophile selection in pre-mRNA splicing: evidence for the bulged duplex model. Genes Dev 8, 587-597.

Raker, V.A., Hartmuth, K., Kastner, B., and Luhrmann, R. (1999). Spliceosomal U snRNP core assembly: Sm proteins assemble onto an Sm site RNA nonanucleotide in a specific and thermodynamically stable manner. Mol Cell Biol 19, 6554-6565. 
Raker, V.A., Plessel, G., and Luhrmann, R. (1996). The snRNP core assembly pathway: identification of stable core protein heteromeric complexes and an snRNP subcore particle in vitro. EMBO J 15, 2256-2269.

Ramos-Fernandez, A., Lopez-Ferrer, D., and Vazquez, J. (2007). Improved method for differential expression proteomics using trypsin-catalyzed 180 labeling with a correction for labeling efficiency. Mol Cell Proteomics 6, 1274-1286.

Rao, K.C., Carruth, R.T., and Miyagi, M. (2005). Proteolytic 180 labeling by peptidylLys metalloendopeptidase for comparative proteomics. J Proteome Res 4, 507-514.

Rappsilber, J., Ryder, U., Lamond, A.I., and Mann, M. (2002). Large-scale proteomic analysis of the human spliceosome. Genome Res 12, 1231-1245.

Reynolds, K.J., Yao, X., and Fenselau, C. (2002). Proteolytic 180 labeling for comparative proteomics: evaluation of endoprotease Glu-C as the catalytic agent. J Proteome Res 1, 27-33.

Ross, P.L., Huang, Y.N., Marchese, J.N., Williamson, B., Parker, K., Hattan, S., Khainovski, N., Pillai, S., Dey, S., Daniels, S., Purkayastha, S., Juhasz, P., Martin, S., Bartlet-Jones, M., He, F., Jacobson, A., and Pappin, D.J. (2004). Multiplexed protein quantitation in Saccharomyces cerevisiae using amine-reactive isobaric tagging reagents. Mol Cell Proteomics 3, 1154-1169.

Sambrook, J., Fritsch, E.F., and Maniatis, T. (1989). Molecular cloning - a Laboratory Manual (Cold Spring Harbor, New York, Cold Spring Harbor Laboratory Press).

Sanford, J.R., Longman, D., and Caceres, J.F. (2003). Multiple roles of the SR protein family in splicing regulation. Prog Mol Subcell Biol 31, 33-58.

Sashital, D.G., Cornilescu, G., McManus, C.J., Brow, D.A., and Butcher, S.E. (2004). U2-U6 RNA folding reveals a group II intron-like domain and a four-helix junction. Nat Struct Mol Biol 11, 1237-1242.

Schagger, H., and von Jagow, G. (1987). Tricine-sodium dodecyl sulfatepolyacrylamide gel electrophoresis for the separation of proteins in the range from 1 to 100 kDa. Anal Biochem 166, 368-379.

Schagger, H., Aquila, H., and Von Jagow, G. (1988). Coomassie blue-sodium dodecyl sulfate-polyacrylamide gel electrophoresis for direct visualization of polypeptides during electrophoresis. Anal Biochem 173, 201-205.

Schagger, H., and von Jagow, G. (1991). Blue native electrophoresis for isolation of membrane protein complexes in enzymatically active form. Anal Biochem 199, 223-231.

Scherly, D., Boelens, W., van Venrooij, W.J., Dathan, N.A., Hamm, J., and Mattaj, I.W. (1989). Identification of the RNA binding segment of human U1 A protein and definition of its binding site on U1 snRNA. EMBO J 8, 4163-4170.

Schmidt, A., Karas, M., and Dulcks, T. (2003). Effect of different solution flow rates on analyte ion signals in nano-ESI MS, or: when does ESI turn into nano-ESI? J Am Soc Mass Spectrom 14, 492-500.

Schmidt, A., Kellermann, J., and Lottspeich, F. (2005). A novel strategy for quantitative proteomics using isotope-coded protein labels. Proteomics 5, 4-15. 
Schnolzer, M., Jedrzejewski, P., and Lehmann, W.D. (1996). Protease-catalyzed incorporation of 180 into peptide fragments and its application for protein sequencing by electrospray and matrix-assisted laser desorption/ionization mass spectrometry. Electrophoresis 17, 945-953.

Schoenle, E.J., Adams, L.D., and Sammons, D.W. (1984). Insulin-induced rapid decrease of a major protein in fat cell plasma membranes. J Biol Chem 259, 12112-12116.

Schulze, W.X., and Mann, M. (2004). A novel proteomic screen for peptide-protein interactions. J Biol Chem 279, 10756-10764.

Schwanhausser, B., Gossen, M., Dittmar, G., and Selbach, M. (2009). Global analysis of cellular protein translation by pulsed SILAC. Proteomics 9, 205-209.

Schwer, B. (2001). A new twist on RNA helicases: DExH/D box proteins as RNPases. Nat Struct Biol 8, 113-116.

Selbach, M., Schwanhausser, B., Thierfelder, N., Fang, Z., Khanin, R., and Rajewsky, N. (2008). Widespread changes in protein synthesis induced by microRNAs. Nature $455,58-$ 63.

Shevchenko, A., Wilm, M., Vorm, O., and Mann, M. (1996). Mass spectrometric sequencing of proteins silver-stained polyacrylamide gels. Anal Chem 68, 850-858.

Shi, R., Kumar, C., Zougman, A., Zhang, Y., Podtelejnikov, A., Cox, J., Wisniewski, J.R., and Mann, M. (2007). Analysis of the mouse liver proteome using advanced mass spectrometry. J Proteome Res 6, 2963-2972.

Silva, J.C., Gorenstein, M.V., Li, G.Z., Vissers, J.P., and Geromanos, S.J. (2006). Absolute quantification of proteins by LCMSE: a virtue of parallel MS acquisition. Mol Cell Proteomics 5, 144-156.

Singh, R., and Reddy, R. (1989). Gamma-monomethyl phosphate: a cap structure in spliceosomal U6 small nuclear RNA. Proc Natl Acad Sci U S A 86, 8280-8283.

Singh, S., Springer, M., Steen, J., Kirschner, M.W., and Steen, H. (2009). FLEXIQuant: a novel tool for the absolute quantification of proteins, and the simultaneous identification and quantification of potentially modified peptides. J Proteome Res 8, 22012210.

Stahl-Zeng, J., Lange, V., Ossola, R., Eckhardt, K., Krek, W., Aebersold, R., and Domon, B. (2007). High sensitivity detection of plasma proteins by multiple reaction monitoring of N-glycosites. Mol Cell Proteomics 6, 1809-1817.

Staley, J.P., and Guthrie, C. (1998). Mechanical devices of the spliceosome: motors, clocks, springs, and things. Cell 92, 315-326.

Stevens, S.W., Ryan, D.E., Ge, H.Y., Moore, R.E., Young, M.K., Lee, T.D., and Abelson, J. (2002). Composition and functional characterization of the yeast spliceosomal penta-snRNP. Mol Cell 9, 31-44.

Sun, J.S., and Manley, J.L. (1995). A novel U2-U6 snRNA structure is necessary for mammalian mRNA splicing. Genes Dev 9, 843-854.

Talkington, M.W., Siuzdak, G., and Williamson, J.R. (2005). An assembly landscape for the 30 S ribosomal subunit. Nature $438,628-632$. 
Tanaka, K., Waki, H., Ido, Y., Akita, S., Yoshida, Y., and Yoshida, T. (1988). Laser ionization Time-of-flight mass spectrometry. Rapid Comm Mass Spectrom 2, 151-153.

Tarn, W.Y., Hsu, C.H., Huang, K.T., Chen, H.R., Kao, H.Y., Lee, K.R., and Cheng, S.C. (1994). Functional association of essential splicing factor(s) with PRP19 in a protein complex. EMBO J 13, 2421-2431.

Thompson, A., Schafer, J., Kuhn, K., Kienle, S., Schwarz, J., Schmidt, G., Neumann, T., Johnstone, R., Mohammed, A.K., and Hamon, C. (2003). Tandem mass tags: a novel quantification strategy for comparative analysis of complex protein mixtures by MS/MS. Anal Chem 75, 1895-1904.

Umen, J.G., and Guthrie, C. (1995). The second catalytic step of pre-mRNA splicing. RNA 1, 869-885.

Urlaub, H., Hartmuth, K., Kostka, S., Grelle, G., and Luhrmann, R. (2000). A general approach for identification of RNA-protein cross-linking sites within native human spliceosomal small nuclear ribonucleoproteins (snRNPs). Analysis of RNA-protein contacts in native U1 and U4/U6.U5 snRNPs. J Biol Chem 275, 41458-41468.

Urlaub, H., Raker, V.A., Kostka, S., and Luhrmann, R. (2001). Sm protein-Sm site RNA interactions within the inner ring of the spliceosomal snRNP core structure. EMBO J 20, 187-196.

Valcarcel, J., Gaur, R.K., Singh, R., and Green, M.R. (1996). Interaction of U2AF65 RS region with pre-mRNA branch point and promotion of base pairing with U2 snRNA [corrected]. Science 273, 1706-1709.

Vidal, V.P., Verdone, L., Mayes, A.E., and Beggs, J.D. (1999). Characterization of U6 snRNA-protein interactions. RNA 5, 1470-1481.

Waanders, L.F., Hanke, S., and Mann, M. (2007). Top-down quantitation and characterization of SILAC-labeled proteins. J Am Soc Mass Spectrom 18, 2058-2064.

Wahl, M.C., Will, C.L., and Luhrmann, R. (2009). The spliceosome: design principles of a dynamic RNP machine. Cell 136, 701-718.

Washburn, M.P., Wolters, D., and Yates, J.R., 3rd (2001). Large-scale analysis of the yeast proteome by multidimensional protein identification technology. Nat Biotechnol 19, 242-247.

Wepf, A., Glatter, T., Schmidt, A., Aebersold, R., and Gstaiger, M. (2009). Quantitative interaction proteomics using mass spectrometry. Nat Methods 6, 203-205.

Will, C.L., and Luhrmann, R. (2005). Spliceosome structure and function. In RNA world III, R.F. Gesteland, and T.R. Cech, eds. (CSH laboratory press), pp. 369-400.

Will, C.L., Rumpler, S., Klein Gunnewiek, J., van Venrooij, W.J., and Luhrmann, R. (1996). In vitro reconstitution of mammalian U1 snRNPs active in splicing: the U1-C protein enhances the formation of early (E) spliceosomal complexes. Nucleic Acids Res 24, 46144623.

Will, C.L., Urlaub, H., Achsel, T., Gentzel, M., Wilm, M., and Luhrmann, R. (2002). Characterization of novel SF3b and 17S U2 snRNP proteins, including a human Prp5p homologue and an SF3b DEAD-box protein. EMBO J 21, 4978-4988. 

397-403.

Williamson, J.R. (2005). Assembly of the 30 S ribosomal subunit. Q Rev Biophys 38,

Wilm, M. (2009). Quantitative proteomics in biological research. Proteomics 9, 45904605.

Wolf-Yadlin, A., Hautaniemi, S., Lauffenburger, D.A., and White, F.M. (2007). Multiple reaction monitoring for robust quantitative proteomic analysis of cellular signaling networks. Proc Natl Acad Sci U S A 104, 5860-5865.

Yao, X., Freas, A., Ramirez, J., Demirev, P.A., and Fenselau, C. (2001). Proteolytic 180 labeling for comparative proteomics: model studies with two serotypes of adenovirus. Anal Chem 73, 2836-2842.

Zhou, Z., and Reed, R. (1998). Human homologs of yeast prp16 and prp17 reveal conservation of the mechanism for catalytic step II of pre-mRNA splicing. EMBO J 17, 20952106. 


\section{Appendix}

\section{Additional information}

Table A.1: Additional proteins identified in the hPrp19/CDC5L complex by LC-MS/MS. The number of unique peptides for every protein identified after hydrolysis in $80 \%(\mathrm{v} / \mathrm{v})$ acetonitrile and $8 \mathrm{M} / 2 \mathrm{M}$ urea, respectively, is given for three replicates.

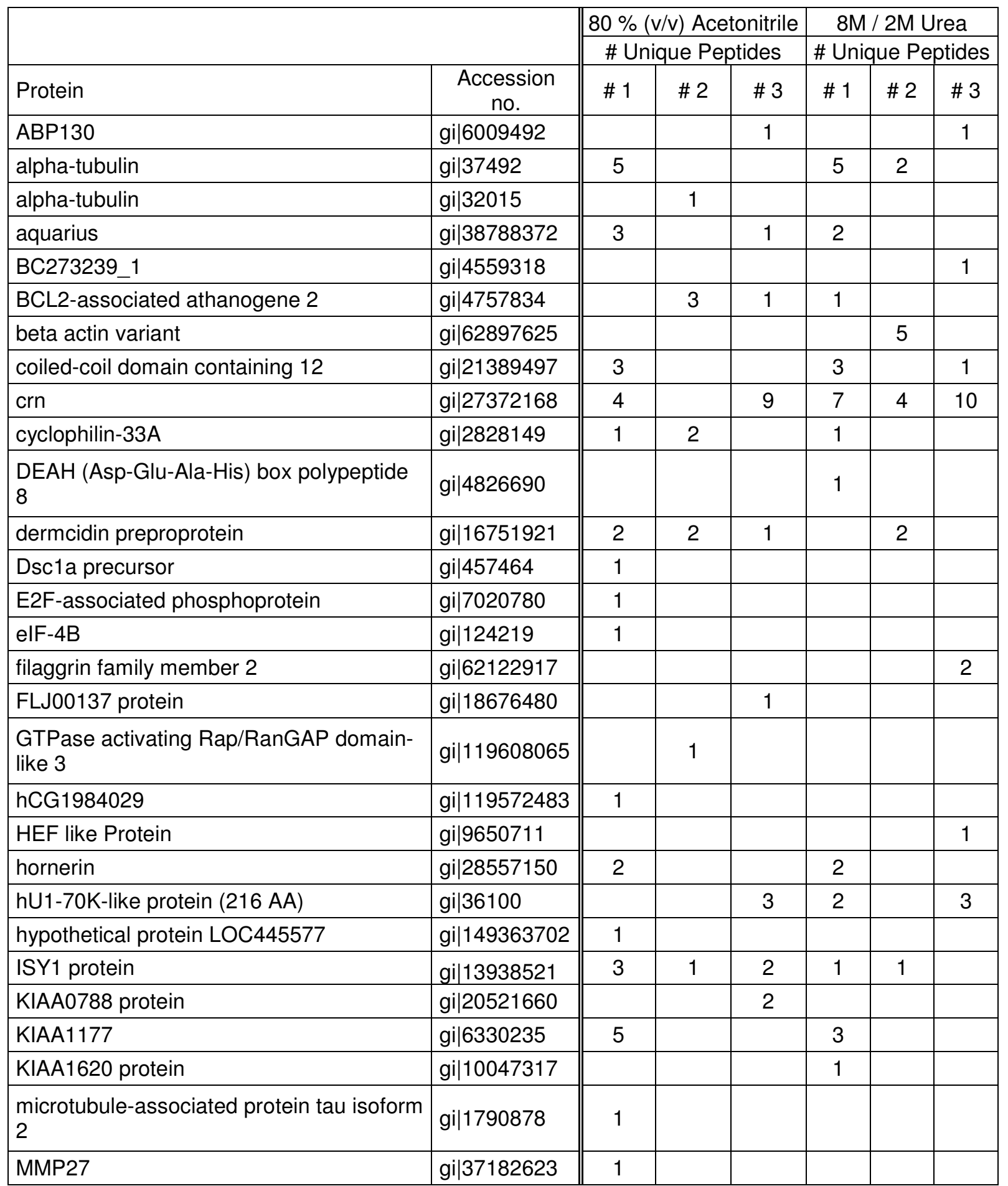




\begin{tabular}{|l|l||l|l|l|l|l|l|}
\hline mutant beta-actin & gi|28336 & 5 & 4 & & 5 & & \\
\hline Myomesin-2 & gi|1709093 & 1 & & & & & \\
\hline Nogo-A protein & gi|9408096 & & & & & 1 & \\
\hline prostate differentiation factor & gi|2290972 & & & 1 & & & \\
\hline PRP8 protein & gi|91208426 & 7 & 5 & 3 & 3 & 5 & 5 \\
\hline putative ORF & gi|763429 & 1 & & & & & \\
\hline sirtuin 1 & gi|7657575 & 3 & & 1 & & & 1 \\
\hline Skb 1 Hs & gi|2323410 & 5 & 6 & & 5 & & \\
\hline SKI-interacting protein & gi|6912676 & 1 & 2 & & 4 & & \\
\hline $\begin{array}{l}\text { small nuclear ribonucleoprotein } \\
\text { polypeptide A }\end{array}$ & gi|4759156 & & & & 1 & & \\
\hline SmD2 & gi|4759158 & & & 2 & & & 1 \\
\hline SmF & gi|4507131 & 1 & & & & & 1 \\
\hline SmG & gi|4507133 & 1 & & & 2 & & \\
\hline $\begin{array}{l}\text { SYF2 homolog, RNA splicing factor } \\
\text { isoform 1 }\end{array}$ & gi|7661636 & & & & 1 & 2 & \\
\hline tubulin 5-beta & gi|35959 & & 1 & & & & \\
\hline tubulin beta & gi|223429 & & & & 3 & & \\
\hline U5-116K & gi|24474791 & 3 & 3 & 3 & 8 & 4 & 3 \\
\hline U5-200K & gi|40217847 & 3 & 3 & & 4 & 1 & 2 \\
\hline WD repeat domain 77 & gi|13129110 & & & & 4 & & \\
\hline $\begin{array}{l}\text { Williams-Beuren syndrome chromosome } \\
\text { region 27 }\end{array}$ & gi|30795190 & & & 1 & & & \\
\hline ZNF461 protein & gi|20306351 & & & 1 & & & \\
\hline
\end{tabular}


Table A.2: Spectral Count for peptides seleceted for quantification and their miscleaved versions. The number of spectra is given for different replicates for hydrolysis in $8 \mathrm{M} / 2 \mathrm{M}$ urea and $80 \%(\mathrm{v} / \mathrm{v})$ acetonitrile analyzed by LC-ESI-MS/MS and LC-offline MALDI-ToF/ToF. The number in parentheses represents the mascot score obtained from the different spectra.

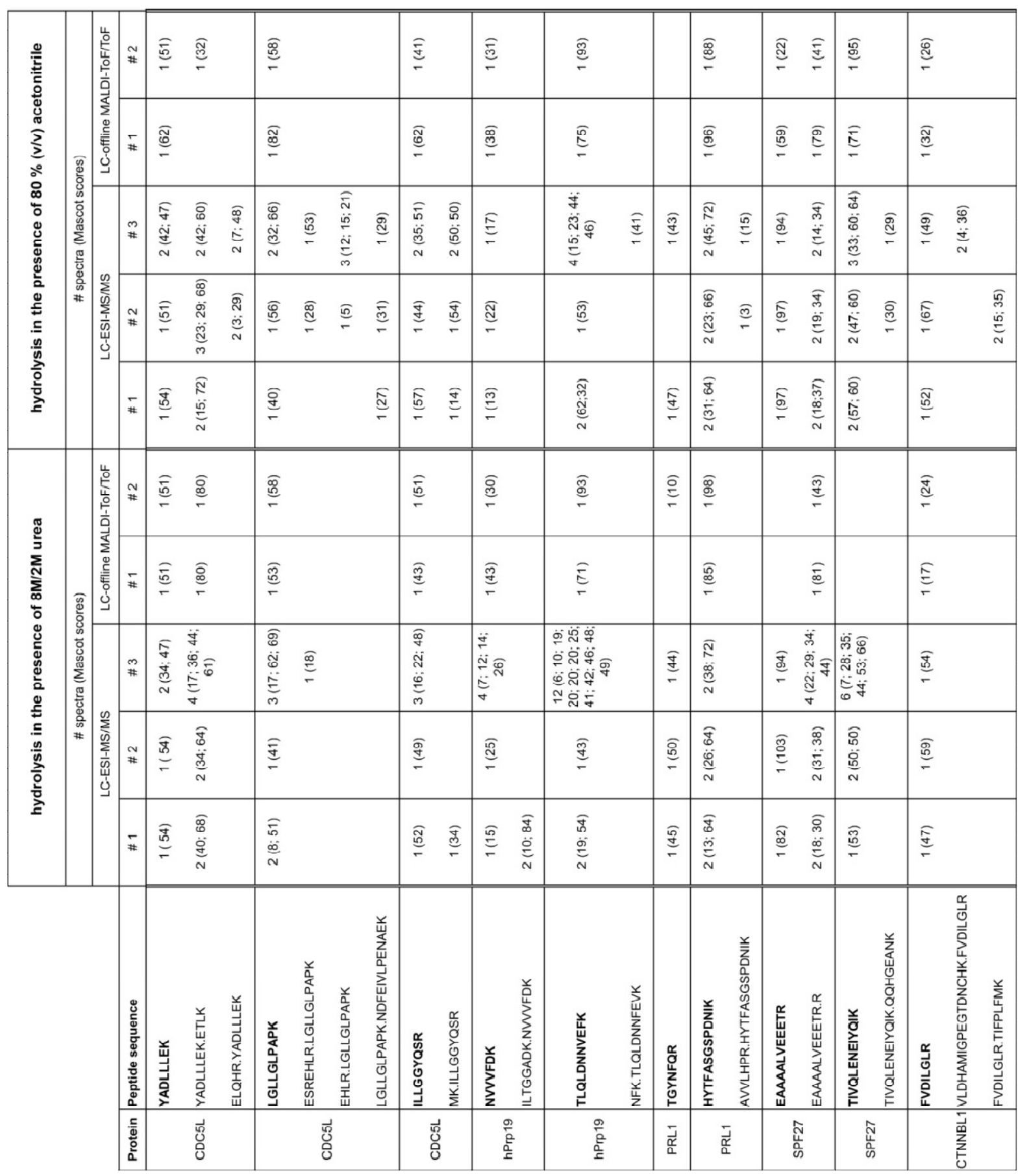


A

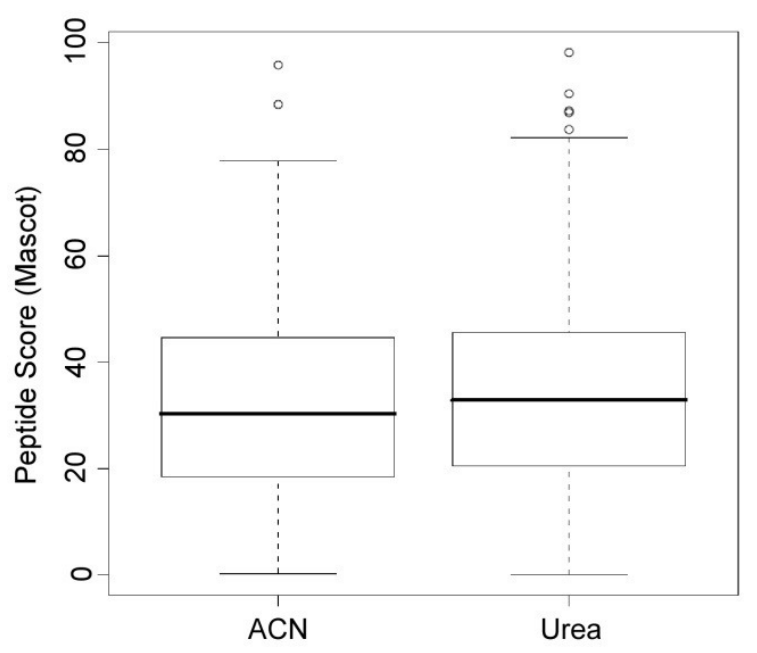

B

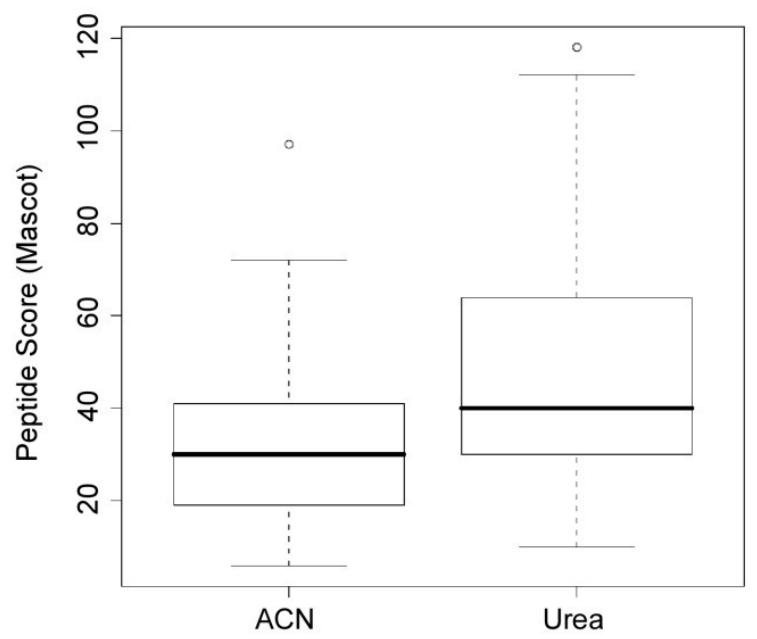

C

\begin{tabular}{|c|c|c|c|}
\hline \multirow{7}{*}{$\begin{array}{l} \\
\text { median } \\
\text { mean } \\
\text { SE.mean } \\
\text { std.dev }\end{array}$} & & & Welch Two Sample t-test \\
\hline & & & data: score by hydrolysis \\
\hline & Urea & $\mathrm{ACN}$ & $\mathrm{t}=-2.7896, \mathrm{df}=54.842, \mathrm{p}$-value $=0.003622$ \\
\hline & 40.00 & 30.00 & alternative hypothesis: true difference in means is less than 0 \\
\hline & 48.91 & 33.30 & 95 percent confidence interval: \\
\hline & 4.758 & 2.950 & $-\operatorname{Inf}-6.250057$ \\
\hline & 27.333 & 18.890 & $\begin{array}{l}\text { sample estimates: } \\
\text { mean in group } A C N \text { mean in group Urea }\end{array}$ \\
\hline & & & 48.90909 \\
\hline
\end{tabular}

Figure A.1: Miscleaved peptides generated in the presence of urea show higher peptide scores for the peptide sequences selected for quantification. (A) The boxplot does not show any significant difference in the Mascot peptide scores for miss cleavage site-containing peptides generated in the presence of urea or acetonitrile. (B) Strikingly, the boxplot for Mascot peptide scores for miscleaved peptides selected as standard peptides for quantification generated in the presence of urea and acetonitrile shows that Mascot peptide scores for miscleaved peptides obtained by hydrolysis in urea are higher. (C) Output of the statistical analysis. Differences of mean peptide scores for miscleaved peptides selected for quantification were tested for statistical significance by applying Welch's two sample t-test ( $R$ 2.8.0). The observed difference is statistically significant ( $p$ value $<0.5)$.

$\begin{array}{lll} & \frac{A C N}{123} & \frac{\text { Urea }}{167} \\ \text { nbr.val } & 6 & 6 \\ \text { min } & 36 & 33 \\ \text { max } & 12.000 & 12.000 \\ \text { median } & 14.073 & 12.760 \\ \text { mean } & 0.597 & 0.405 \\ \text { SE.mean } & 43.790 & 27.448 \\ \text { var } & 6.617 & 5.239 \\ \text { std.dev } & & \end{array}$

Welch Two Sample t-test

data: length by Hydrolysis

$\mathrm{t}=1.8197, \mathrm{df}=225.351, \mathrm{p}$-value $=0.03506$

alternative hypothesis: true difference in means is greater than 0 95 percent confidence interval:

0.1212455 Inf

sample estimates:

mean in group $\mathrm{ACN}$ mean in group Urea
14.07317

Figure A.2: Incompletely cleaved peptides obtained by hydrolysis in the presence of acetonitrile are significantly longer than incompletely cleaved peptides obtained by hydrolysis in the presence of urea. Output of the statistical analysis. 
Table A.3: Three MRM transitions for each endogenous and standard peptide were designed. In all cases, the doubly charged precursor was chosen as Q1 mass, and the three most intense fragment ions with an $\mathrm{m} / \mathrm{z}$ above that of the precursor were chosen as Q3 masses. For the selected MRM transitions declustering potential (DP), entrance potential (EP), collision energy (CE), and collision cell exit potential (CXP) were optimized.

\begin{tabular}{|c|c|c|c|c|c|c|c|c|}
\hline Protein & Peptide sequence & Q1 & Q3 & Fragment & $\mathrm{DP}[\mathrm{V}]$ & $\mathrm{EP}[\mathrm{V}]$ & $\mathrm{CE}[\mathrm{V}]$ & $\mathrm{CXP}[\mathrm{V}]$ \\
\hline \multirow{6}{*}{ CDC5L } & ILLGGYQSR light & 503.79 & 610.29 & y5 & 95 & 14 & 29 & 18 \\
\hline & ILLGGYQSR light & 503.79 & 667.32 & y6 & 95 & 14 & 30 & 19 \\
\hline & ILLGGYQSR light & 503.79 & 780.4 & y7 & 95 & 14 & 28 & 7 \\
\hline & ILLGGYQSR heavy & 508.79 & 620.3 & y5 & 95 & 14 & 29 & 18 \\
\hline & ILLGGYQSR heavy & 508.79 & 677.32 & y6 & 95 & 14 & 30 & 19 \\
\hline & ILLGGYQSR heavy & 508.79 & 790.41 & y7 & 95 & 14 & 28 & 7 \\
\hline \multirow{6}{*}{ CDC5L } & LGLLGLPAPK light & 489.82 & 582.36 & y6 & 105 & 13 & 25 & 17 \\
\hline & LGLLGLPAPK light & 489.82 & 695.45 & y7 & 105 & 13 & 26 & 21 \\
\hline & LGLLGLPAPK light & 489.82 & 865.55 & y9 & 105 & 13 & 28 & 23 \\
\hline & LGLLGLPAPK heavy & 493.82 & 590.38 & y6 & 105 & 13 & 25 & 17 \\
\hline & LGLLGLPAPK heavy & 493.82 & 703.46 & $y 7$ & 105 & 13 & 26 & 21 \\
\hline & LGLLGLPAPK heavy & 493.82 & 873.56 & y9 & 105 & 13 & 28 & 23 \\
\hline \multirow{6}{*}{ CDC5L } & YADLLLEK light & 482.77 & 615.41 & y5 & 97 & 13 & 28 & 17 \\
\hline & YADLLLEK light & 482.77 & 730.43 & y6 & 97 & 13 & 24 & 21 \\
\hline & YADLLLEK light & 482.77 & 801.47 & y7 & 97 & 13 & 26 & 23 \\
\hline & YADLLLEK heavy & 486.28 & 622.42 & y5 & 97 & 13 & 28 & 17 \\
\hline & YADLLLEK heavy & 486.28 & 737.45 & y6 & 97 & 13 & 24 & 21 \\
\hline & YADLLLEK heavy & 486.28 & 808.49 & $y 7$ & 97 & 13 & 26 & 23 \\
\hline \multirow{6}{*}{ CTNNBL1 } & FVDILGLR light & 466.78 & 571.39 & y5 & 73 & 11 & 32 & 15 \\
\hline & FVDILGLR light & 466.78 & 686.42 & y6 & 73 & 11 & 25 & 15 \\
\hline & FVDILGLR light & 466.78 & 785.49 & y7 & 73 & 11 & 27 & 15 \\
\hline & FVDILGLR heavy & 470.21 & 578.41 & y5 & 73 & 11 & 32 & 15 \\
\hline & FVDILGLR heavy & 470.21 & 693.44 & y6 & 73 & 11 & 25 & 15 \\
\hline & FVDILGLR heavy & 470.21 & 792.51 & y7 & 73 & 11 & 27 & 15 \\
\hline \multirow{6}{*}{ PRL1 } & HYTFASGSPDNIK light & 718.84 & 817.41 & y8 & 135 & 10 & 41 & 30 \\
\hline & HYTFASGSPDNIK light & 718.84 & 888.44 & y9 & 135 & 10 & 41 & 30 \\
\hline & HYTFASGSPDNIK light & 718.84 & 1136.56 & $y 11$ & 135 & 10 & 39 & 30 \\
\hline & HYTFASGSPDNIK heavy & 722.35 & 824.42 & y8 & 135 & 10 & 41 & 30 \\
\hline & HYTFASGSPDNIK heavy & 722.35 & 895.46 & y9 & 135 & 10 & 41 & 30 \\
\hline & HYTFASGSPDNIK heavy & 722.35 & 1143.58 & $\mathrm{y} 11$ & 135 & 10 & 39 & 30 \\
\hline \multirow{6}{*}{ PRL1 } & TGYNFQR light & 443.21 & 564.29 & y4 & 80 & 11 & 27 & 16 \\
\hline & TGYNFQR light & 443.21 & 727.35 & y5 & 80 & 11 & 25 & 16 \\
\hline & TGYNFQR light & 443.21 & 784.37 & y6 & 80 & 11 & 28 & 16 \\
\hline & TGYNFQR heavy & 448.23 & 574.32 & y4 & 80 & 11 & 27 & 16 \\
\hline & TGYNFQR heavy & 448.23 & 737.38 & y5 & 80 & 11 & 25 & 16 \\
\hline & TGYNFQR heavy & 448.23 & 794.4 & y6 & 80 & 11 & 28 & 16 \\
\hline \multirow{6}{*}{ hPrp19 } & TLQLDNNFEVK light & 660.84 & 865.41 & $y 7$ & 123 & 8 & 34 & 8 \\
\hline & TLQLDNNFEVK light & 660.84 & 978.49 & y8 & 123 & 8 & 33 & 9.4 \\
\hline & TLQLDNNFEVK light & 660.84 & 1106.55 & y9 & 123 & 8 & 32 & 11 \\
\hline & TLQLDNNFEVK heavy & 664.85 & 873.42 & y7 & 123 & 8 & 34 & 8 \\
\hline & TLQLDNNFEVK heavy & 664.85 & 986.5 & y8 & 123 & 8 & 33 & 9.4 \\
\hline & TLQLDNNFEVK heavy & 664.85 & 1114.56 & y9 & 123 & 8 & 32 & 11 \\
\hline \multirow{6}{*}{ hPrp19 } & NVVVFDK light & 410.73 & 508.28 & y4 & 85 & 10 & 23 & 19 \\
\hline & NVVVFDK light & 410.73 & 607.35 & y5 & 85 & 10 & 20 & 18 \\
\hline & NVVVFDK light & 410.73 & 706.41 & y6 & 85 & 10 & 23 & 20 \\
\hline & NVVVFDK heavy & 415.75 & 518.3 & y4 & 85 & 10 & 23 & 19 \\
\hline & NVVVFDK heavy & 415.75 & 617.37 & y5 & 85 & 10 & 20 & 18 \\
\hline & NVVVFDK heavy & 415.75 & 716.44 & y6 & 85 & 10 & 23 & 20 \\
\hline
\end{tabular}




\begin{tabular}{|l|l|c|c|c|c|c|c|c|}
\hline & EAAAALVEEETR light & 644.82 & 663.29 & $\mathrm{y} 5$ & 110 & 10 & 31 & 20 \\
& EAAAALVEEETR light & 644.82 & 762.36 & $\mathrm{y} 6$ & 110 & 10 & 34 & 23 \\
SPF27 & EAAAALVEEETR light & 644.82 & 875.45 & $\mathrm{y} 7$ & 110 & 10 & 34 & 25 \\
& EAAAALVEEETR heavy & 649.83 & 673.3 & $\mathrm{y} 5$ & 110 & 10 & 31 & 20 \\
& EAAAALVEEETR heavy & 649.83 & 772.37 & $\mathrm{y} 6$ & 110 & 10 & 34 & 23 \\
& EAAAALVEEETR heavy & 649.83 & 885.46 & $\mathrm{y} 7$ & 110 & 10 & 34 & 25 \\
\hline \multirow{5}{*}{ SPF27 } & TIVQLENEIYQIK light & 795.94 & 907.49 & $\mathrm{y} 7$ & 145 & 10 & 40 & 8.2 \\
& TIVQLENEIYQIK light & 795.94 & 1036.53 & $\mathrm{y} 8$ & 145 & 10 & 40 & 9.5 \\
& TIVQLENEIYQIK light & 795.94 & 1149.62 & $\mathrm{y} 9$ & 145 & 10 & 40 & 11 \\
& TIVQLENEIYQIK heavy & 799.45 & 914.51 & $\mathrm{y} 7$ & 145 & 10 & 40 & 8.2 \\
& TIVQLENEIYQIK heavy & 799.45 & 1043.55 & $\mathrm{y} 8$ & 145 & 10 & 40 & 9.5 \\
& TIVQLENEIYQIK heavy & 799.45 & 1156.63 & $\mathrm{y} 9$ & 145 & 10 & 40 & 11 \\
\hline
\end{tabular}

Table A.4: Absolute amounts of proteins of the hPrp19/CDC5L complex. The protein complex was hydrolyzed in the presence of acetonitrile and a mix of standard peptides (100 fmol each) was added to the sample. Absolute amounts were then obtained by generating XICs of the endogenous and standard peptides. Hsp70 was found in reduced amounts when compared with other proteins of the hPrp19/CDC5L complex.

\begin{tabular}{|l|c|c|c|}
\hline hPrp19/CDC5L complex [ng] & 70 & 70 & 35 \\
\hline & [fmol] & [fmol] & [fmol] \\
hPrp19 & 252.0 & 255.0 & 128.9 \\
CDC5 & 85.8 & 84.2 & 42.7 \\
SPF27 & 31.9 & 38.2 & 16.5 \\
PRL1 & 38.6 & 33.1 & 19.6 \\
CTNNBL1 & 34.2 & 28.7 & 13.9 \\
Hsp70 & 13.9 & 7.65 & 3.7 \\
\hline
\end{tabular}


Table A.5: Proteins identified in the first replicate of the relative quantification of spliceosomal B and C complexes by iTRAQ. The accession number, the protein database description, the obtained protein score (Mascot), the protein mass, the determined protein ratio $(\mathrm{B} / \mathrm{C})$, the standard deviation (StDev), and the number of peptides used for quantification (\#) is given for all proteins found. Proteins used for normalization are highlighted in red.

\begin{tabular}{|c|c|c|c|c|c|c|c|}
\hline Protein & $\begin{array}{l}\text { accession } \\
\text { number }\end{array}$ & $\begin{array}{l}\text { Protein database } \\
\text { description }\end{array}$ & $\begin{array}{c}\text { Protein } \\
\text { score }\end{array}$ & $\begin{array}{c}\text { Protein } \\
\text { mass } \\
{[\mathrm{Da}]}\end{array}$ & $\mathrm{B} / \mathrm{C}$ & StDev & $\#$ \\
\hline 9G8 & gi|72534660 & $\begin{array}{l}\text { splicing factor, } \\
\text { arginine/serine-rich } 7 \\
\text { [Homo sapiens] }\end{array}$ & 171 & 28687 & 1.16 & 0.32 & 24 \\
\hline abstrakt & gi|21071032 & $\begin{array}{l}\text { DEAD-box protein } \\
\text { abstrakt [Homo sapiens] } \\
\text { PREDICTED: apoptotic }\end{array}$ & 604 & 77694 & 0.08 & 0.06 & 48 \\
\hline Acinus & gi|109082928 & $\begin{array}{l}\text { chromatin condensation } \\
\text { inducer } 1 \text { isoform } 2 \\
\text { [Macaca mulatta] }\end{array}$ & 148 & 77764 & 1.35 & 0.42 & 13 \\
\hline Acinus & gi|7662238 & $\begin{array}{l}\text { apoptotic chromatin } \\
\text { condensation inducer } 1 \\
\text { [Homo sapiens] }\end{array}$ & 218 & 166097 & 1.03 & 0.23 & 9 \\
\hline Aquarius & gi|58257729 & $\begin{array}{l}\text { KIAA0560 protein [Homo } \\
\text { sapiens] }\end{array}$ & 380 & 187968 & 2.24 & 0.64 & 23 \\
\hline Aquarius & gi|38788372 & aquarius [Homo sapiens] & 2120 & 183588 & 0.09 & 0.05 & 96 \\
\hline ASF/SF2 & gi|179074 & alternative & 209 & 33984 & 0.80 & 0.19 & 14 \\
\hline ASR2B & gi|13383501 & $\begin{array}{l}\text { ASR2B [Homo sapiens] } \\
\text { HLA-B associated }\end{array}$ & 182 & 110669 & 1.00 & 0.27 & 15 \\
\hline UAP56 & gi|4758112 & $\begin{array}{l}\text { transcript } 1 \text { [Homo } \\
\text { sapiens] }\end{array}$ & 182 & 53651 & 2.01 & 0.44 & 11 \\
\hline BCLAF1 & gi|7661958 & $\begin{array}{l}\text { BCL2-associated } \\
\text { transcription factor } 1 \\
\text { isoform } 1 \text { [Homo sapiens] }\end{array}$ & 84 & 119552 & 1.61 & 0.24 & 23 \\
\hline C10orf4 & gi|24432067 & $\begin{array}{l}\text { FRA10AC1 protein [Homo } \\
\text { sapiens] } \\
\text { hypothetical protein }\end{array}$ & 61 & 44908 & 0.10 & 0.06 & 2 \\
\hline C1orf55 & gi|40255125 & $\begin{array}{l}\text { LOC163859 [Homo } \\
\text { sapiens] } \\
\text { chromosome } 9 \text { open }\end{array}$ & 73 & 54043 & 0.08 & 0.04 & 19 \\
\hline C9orf78 & gi|7706557 & $\begin{array}{l}\text { reading frame } 78 \text { [Homo } \\
\text { sapiens] }\end{array}$ & 66 & 38613 & 0.14 & 0.05 & 6 \\
\hline Cactin (C19orf29) & gi|3253120 & $\begin{array}{l}\text { R31449_3 [Homo } \\
\text { sapiens] } \\
\text { nuclear cap binding }\end{array}$ & 465 & 101398 & 0.17 & 0.13 & 21 \\
\hline CBP20 & gi|19923387 & $\begin{array}{l}\text { nuclear cap binding } \\
\text { protein subunit } 2,20 \mathrm{kDa} \\
\text { isoform } 1 \text { [Homo sapiens] }\end{array}$ & 110 & 20001 & 0.71 & 0.16 & 8 \\
\hline CBP80 & gi|24987336 & $\begin{array}{l}\text { Chain C, Structure Of The } \\
\text { Human Nuclear Cap- } \\
\text { Binding-Complex (Cbc) }\end{array}$ & 805 & 97287 & 1.41 & 0.41 & 33 \\
\hline CCAP2 & gi|6841518 & HSPC148 [Homo sapiens] & 75 & 30099 & 0.22 & 0.08 & 6 \\
\hline CCNK & gi|8980825 & $\begin{array}{l}\text { cyclin K [Homo sapiens] } \\
\text { similar to KIAA0904; }\end{array}$ & 29 & 44722 & 2.56 & & 1 \\
\hline CDC2L5 & gi|5870326 & $\begin{array}{l}\text { similar to AAA58424 } \\
\text { (PID:g180492) [Homo } \\
\text { sapiens] }\end{array}$ & 23 & 86103 & 1.23 & 0.29 & 2 \\
\hline CDC5L & gi|11067747 & CDC5-like [Homo sapiens] & 703 & 103618 & 2.26 & 0.75 & 30 \\
\hline CDC5L & gi|11067747 & CDC5-like [Homo sapiens] & 361 & 103618 & 0.23 & 0.14 & 45 \\
\hline CLK1 & gi|67551261 & $\begin{array}{l}\text { CDC-like kinase } 1 \text { [Homo } \\
\text { sapiens] }\end{array}$ & 117 & 63472 & 1.08 & 0.45 & 4 \\
\hline CLK4 & gi|10190706 & $\begin{array}{l}\text { CDC-like kinase } 4 \text { [Homo } \\
\text { sapiens] }\end{array}$ & 42 & 62901 & 0.85 & & 1 \\
\hline
\end{tabular}




\begin{tabular}{|c|c|c|c|c|c|c|c|}
\hline Protein & $\begin{array}{l}\text { accession } \\
\text { number }\end{array}$ & $\begin{array}{l}\text { Protein database } \\
\text { description }\end{array}$ & $\begin{array}{l}\text { Protein } \\
\text { score }\end{array}$ & $\begin{array}{c}\text { Protein } \\
\text { mass } \\
\text { [Da] }\end{array}$ & $\mathrm{B} / \mathrm{C}$ & StDev & \# \\
\hline CDK10(PISSLRE) & gi|6226784 & $\begin{array}{l}\text { Cell division protein } \\
\text { kinase } 10 \\
\text { (Serine/threonine-protein } \\
\text { kinase PISSLRE) }\end{array}$ & 66 & 45362 & 0.20 & 0.09 & 3 \\
\hline CHERP & gi|1770394 & DAN26 [Homo sapiens] & 51 & 55656 & 5.36 & 0.05 & 2 \\
\hline CIP29 & gi|119617243 & $\begin{array}{l}\text { hCG2016179, isoform } \\
\text { CRA d [Homo sapiens] }\end{array}$ & 28 & 25459 & 3.57 & & 1 \\
\hline CRNKL1/hSYF3 & gi|50949465 & $\begin{array}{l}\text { hypothetical protein } \\
\text { [Homo sapiens] }\end{array}$ & 1070 & 96523 & 0.47 & 0.52 & 88 \\
\hline CTNNBL1 & gi|18644734 & $\begin{array}{l}\text { beta catenin-like } 1 \text { [Homo } \\
\text { sapiens] }\end{array}$ & 83 & 70832 & 1.44 & 0.46 & 4 \\
\hline CUG & gi|5729794 & $\begin{array}{l}\text { CUGG triplet repeat, RNA- } \\
\text { binding protein } 1 \text { isoform } 1 \\
\text { [Homo sapiens] } \\
\text { hypothetical protein }\end{array}$ & 31 & 55437 & 1.97 & 0.02 & 2 \\
\hline CXorf56 & gi|11545813 & $\begin{array}{l}\text { LOC63932 [Homo } \\
\text { sapiens] } \\
\text { hypothetical protein }\end{array}$ & 416 & 29821 & 0.08 & 0.08 & 12 \\
\hline CyclinM & gi|24308243 & $\begin{array}{l}\text { LOC92002 [Homo } \\
\text { sapiens] }\end{array}$ & 65 & 26090 & 0.09 & & 1 \\
\hline СурЕ & gi|45439318 & $\begin{array}{l}\text { peptidylprolyl isomerase } E \\
\text { isoform } 3 \text { [Homo sapiens] }\end{array}$ & 113 & 29821 & 0.13 & 0.05 & 8 \\
\hline DDX21 & gi|2135315 & $\begin{array}{l}\text { RNA helicase Gu - human } \\
\text { (fragment) }\end{array}$ & 144 & 103155 & 1.67 & 0.58 & 7 \\
\hline DDX3 & gi|2580550 & $\begin{array}{l}\text { dead box, } \mathrm{X} \text { isoform } \\
\text { [Homo sapiens] }\end{array}$ & 51 & 78159 & 1.05 & 0.16 & 2 \\
\hline DDX35 & gi|10439270 & $\begin{array}{l}\text { unnamed protein product } \\
\text { [Homo sapiens] }\end{array}$ & 380 & 84743 & 0.32 & 0.17 & 28 \\
\hline DDX9 & gi|33878473 & $\begin{array}{l}\text { DHX9 protein [Homo } \\
\text { sapiens] } \\
\text { Similar to expressed }\end{array}$ & 54 & 73628 & 1.34 & & 1 \\
\hline DGCR14 & gi|12804313 & $\begin{array}{l}\text { sequence } 2 \text { embryonic } \\
\text { lethal [Homo sapiens] } \\
\text { DEAH (Asp-Glu-Ala-His) }\end{array}$ & 147 & 56774 & 0.09 & 0.03 & 3 \\
\hline DHX34 & gi|38158022 & $\begin{array}{l}\text { box polypeptide } 34 \text { [Homo } \\
\text { sapiens] }\end{array}$ & 90 & 135588 & 0.49 & 0.33 & 7 \\
\hline EEF1A1 & gi|31092 & $\begin{array}{l}\text { unnamed protein product } \\
\text { [Homo sapiens] }\end{array}$ & 80 & 57288 & 0.32 & 0.15 & 4 \\
\hline elF4A3 & gi|496902 & $\begin{array}{l}\text { translation initiation factor } \\
\text { [Homo sapiens] }\end{array}$ & 495 & 50492 & 0.15 & 0.07 & 31 \\
\hline ELAV & gi|1022961 & HuR RNA binding protein & 312 & 38915 & 4.76 & 2.29 & 27 \\
\hline ELG & gi|8923771 & $\begin{array}{l}\text { ELG protein [Homo } \\
\text { sapiens] }\end{array}$ & 92 & 43508 & 3.11 & 0.54 & 5 \\
\hline NOSIP & gi|7705716 & $\begin{array}{l}\text { eNOS interacting protein } \\
\text { [Homo sapiens] }\end{array}$ & 217 & 37167 & 0.11 & 0.08 & 9 \\
\hline $\mathrm{ERH}$ & gi|4758302 & $\begin{array}{l}\text { enhancer of rudimentary } \\
\text { homolog [Homo sapiens] }\end{array}$ & 154 & 13542 & 2.04 & 0.53 & 5 \\
\hline EXOSC2 & gi|19923403 & $\begin{array}{l}\text { exosome component } 2 \\
\text { [Homo sapiens] }\end{array}$ & 41 & 35402 & 0.40 & 0.17 & 3 \\
\hline EXOSC5 & gi|14043511 & $\begin{array}{l}\text { Exosome component } 5 \\
\text { [Homo sapiens] } \\
\text { hypothetical protein }\end{array}$ & 30 & 27406 & 0.52 & & 1 \\
\hline FAM32A & gi|7661696 & $\begin{array}{l}\text { LOC26017 [Homo } \\
\text { sapiens] }\end{array}$ & 57 & 16773 & 0.11 & 0.06 & 2 \\
\hline FAM50A & gi|4758220 & $\begin{array}{l}\text { XAP-5 protein [Homo } \\
\text { sapiens] }\end{array}$ & 158 & 46701 & 0.15 & 0.08 & 15 \\
\hline
\end{tabular}




\begin{tabular}{|c|c|c|c|c|c|c|c|}
\hline Protein & $\begin{array}{l}\text { accession } \\
\text { number }\end{array}$ & $\begin{array}{l}\text { Protein database } \\
\text { description }\end{array}$ & $\begin{array}{c}\text { Protein } \\
\text { score }\end{array}$ & $\begin{array}{c}\text { Protein } \\
\text { mass } \\
\text { [Da] }\end{array}$ & $B / C$ & StDev & \# \\
\hline FAM50B & gi|6912326 & $\begin{array}{l}\text { family with sequence } \\
\text { similarity } 50, \text { member B } \\
\text { [Homo sapiens] } \\
\text { Pre-mRNA-processing } \\
\text { factor } 40 \text { homolog A } \\
\text { (Formin-binding protein } 3 \text { ) } \\
\text { (Formin-binding protein }\end{array}$ & 55 & 42766 & 0.13 & 0.03 & 2 \\
\hline FBP11 & gi|34222504 & $\begin{array}{l}\text { 11) (Huntingtin-interacting } \\
\text { protein HYPA) (Huntingtin } \\
\text { yeast partner A) (Fas } \\
\text { ligand-associated factor 1) } \\
\text { (NY-REN-6 antigen) }\end{array}$ & 121 & 123665 & 1.26 & 0.21 & 4 \\
\hline FUSE3 & gi|1575609 & $\begin{array}{l}\text { FUSE binding protein } 3 \\
\text { [Homo sapiens] } \\
\text { FUS interacting protein }\end{array}$ & 72 & 67621 & 3.18 & 1.54 & 4 \\
\hline FUSIP1/SRp38 & gi|5730079 & $\begin{array}{l}\text { (serine-arginine rich) } 1 \\
\text { isoform } 1 \text { [Homo sapiens] }\end{array}$ & 114 & 23166 & 0.91 & 0.52 & 15 \\
\hline $\begin{array}{l}\text { Q9BRR8 } \\
\text { (GPATC1) }\end{array}$ & gi|21361684 & $\begin{array}{l}\text { G patch domain } \\
\text { containing } 1 \text { [Homo } \\
\text { sapiens] }\end{array}$ & 119 & 116769 & 0.40 & 0.09 & 3 \\
\hline G10 & gi|32171175 & $\begin{array}{l}\text { G10 protein [Homo } \\
\text { sapiens] }\end{array}$ & 166 & 19898 & 0.39 & 0.19 & 14 \\
\hline GCIP p29 & gi|7661636 & $\begin{array}{l}\text { SYF2 homolog, RNA } \\
\text { splicing factor isoform } 1 \\
\text { [Homo sapiens] }\end{array}$ & 219 & 32930 & 0.18 & 0.14 & 11 \\
\hline GPKOW & gi|15811782 & $\begin{array}{l}\text { G patch domain and KOW } \\
\text { motifs [Homo sapiens] } \\
\text { guanine nucleotide }\end{array}$ & 107 & 55983 & 0.66 & 0.21 & 6 \\
\hline GU2 & gi|5174447 & $\begin{array}{l}\text { binding protein (G } \\
\text { protein), beta polypeptide } \\
\text { 2-like } 1 \text { [Homo sapiens] }\end{array}$ & 50 & 38016 & 0.85 & & 1 \\
\hline $\mathrm{H} 2 \mathrm{~A}$ & gi|32111 & $\begin{array}{l}\text { unnamed protein product } \\
\text { [Homo sapiens] }\end{array}$ & 0 & 16181 & 1.23 & & 1 \\
\hline hECM2 (RBM22) & gi|8922328 & $\begin{array}{l}\text { RNA binding motif protein } \\
22 \text { [Homo sapiens] }\end{array}$ & 419 & 51786 & 0.45 & 0.13 & 35 \\
\hline hISY1 & gi|20149304 & $\begin{array}{l}\text { ISY1 splicing factor } \\
\text { homolog [Homo sapiens] } \\
\text { A0=heterogeneous }\end{array}$ & 247 & 37197 & 0.20 & 0.07 & 16 \\
\hline hnRNP A0 & gi|1911429 & $\begin{array}{l}\text { nuclear ribonucleoprotein } \\
\text { [human, placenta, } \\
\text { Peptide, } 305 \text { aa] }\end{array}$ & 33 & 33758 & 1.25 & 0.67 & 2 \\
\hline hnRNP A1 & gi|36102 & $\begin{array}{l}\text { unnamed protein product } \\
\text { [Homo sapiens] } \\
\text { heterogeneous nuclear }\end{array}$ & 127 & 37105 & 3.44 & 1.81 & 6 \\
\hline hnRNP A2/B1 & gi|119614244 & $\begin{array}{l}\text { ribonucleoprotein A2/B1, } \\
\text { isoform CRA_d [Homo } \\
\text { sapiens] } \\
\text { heterogeneous nuclear }\end{array}$ & 56 & 33161 & 4.63 & 1.96 & 6 \\
\hline hnRNP A3 & gi|34740329 & $\begin{array}{l}\text { ribonucleoprotein A3 } \\
\text { [Homo sapiens] }\end{array}$ & 104 & 42925 & 4.62 & 1.12 & 6 \\
\hline hnRNP C1/C2 & gi|306875 & $\begin{array}{l}\mathrm{C} \text { protein } \\
\text { heterogeneous nuclear }\end{array}$ & 90 & 36316 & 1.72 & 0.47 & 23 \\
\hline hnRNP G & gi|542850 & $\begin{array}{l}\text { ribonucleoprotein G - } \\
\text { human } \\
\text { heterogeneous nuclear }\end{array}$ & 322 & 50301 & 1.52 & 0.59 & 32 \\
\hline hnRNP H1 & gi|5031753 & $\begin{array}{l}\text { ribonucleoprotein H1 } \\
\text { [Homo sapiens] }\end{array}$ & 160 & 51878 & 1.27 & 0.30 & 4 \\
\hline
\end{tabular}




\begin{tabular}{|c|c|c|c|c|c|c|c|}
\hline Protein & $\begin{array}{l}\text { accession } \\
\text { number }\end{array}$ & $\begin{array}{l}\text { Protein database } \\
\text { description }\end{array}$ & $\begin{array}{l}\text { Protein } \\
\text { score }\end{array}$ & $\begin{array}{c}\text { Protein } \\
\text { mass } \\
\text { [Da] }\end{array}$ & $\mathrm{B} / \mathrm{C}$ & StDev & \# \\
\hline hnRNP K & gi|460789 & $\begin{array}{l}\text { transformation } \\
\text { upregulated nuclear } \\
\text { protein [Homo sapiens] }\end{array}$ & 30 & 54441 & 2.97 & & 1 \\
\hline hnRNP L & gi|133274 & $\begin{array}{l}\text { Heterogeneous nuclear } \\
\text { ribonucleoprotein L } \\
\text { (hnRNP L) }\end{array}$ & 25 & 64788 & 5.84 & & 1 \\
\hline hnRNP M & gi|187281 & M4 protein & 72 & 83739 & 1.32 & 0.31 & 8 \\
\hline hnRNP Q2 & gi|15809588 & $\begin{array}{l}\text { hnRNP Q2 [Homo } \\
\text { sapiens] } \\
\text { PREDICTED: similar to }\end{array}$ & 68 & 72196 & 0.84 & 0.12 & 3 \\
\hline hnRNP R & gi|73950226 & $\begin{array}{l}\text { heterogeneous nuclear } \\
\text { ribonucleoprotein } \mathrm{R} \\
\text { isoform } 1 \text { [Canis familiaris] }\end{array}$ & 85 & 65593 & 0.88 & 0.32 & 8 \\
\hline hnRNP U & gi|39645240 & $\begin{array}{l}\text { HNRPU protein [Homo } \\
\text { sapiens] }\end{array}$ & 44 & 87577 & 3.86 & 2.17 & 10 \\
\hline hPRP17 & gi|7706657 & $\begin{array}{l}\text { cell division cycle } 40 \\
\text { homolog [Homo sapiens] } \\
\text { PRP18 pre-mRNA }\end{array}$ & 654 & 73381 & 0.19 & 0.08 & 32 \\
\hline hPRP18 & gi|4506123 & $\begin{array}{l}\text { processing factor } 18 \\
\text { homolog [Homo sapiens] } \\
\text { PRP19/PSO4 pre-mRNA }\end{array}$ & 32 & 45161 & 0.38 & & 1 \\
\hline hPRP19 & gi|7657381 & $\begin{array}{l}\text { processing factor } 19 \\
\text { homolog [Homo sapiens] } \\
\text { DEAH (Asp-Glu-Ala-His) }\end{array}$ & 674 & 59981 & 0.62 & 0.36 & 61 \\
\hline hPRP2 & gi|4503293 & $\begin{array}{l}\text { box polypeptide } 16 \text { [Homo } \\
\text { sapiens] } \\
\text { DEAH (Asp-Glu-Ala-His) }\end{array}$ & 283 & 128919 & 0.54 & 0.16 & 19 \\
\hline hPRP22 & gi|4826690 & $\begin{array}{l}\text { box polypeptide } 8 \text { [Homo } \\
\text { sapiens] } \\
\text { PRP38 pre-mRNA } \\
\text { processing factor } 38\end{array}$ & 924 & 153030 & 0.13 & 0.12 & 55 \\
\hline hPRP38 & gi|24762236 & $\begin{array}{l}\text { (yeast) domain containing } \\
\text { A isoform } 2 \text { [Homo } \\
\text { sapiens] } \\
\text { DEAH (Asp-Glu-Ala-His) }\end{array}$ & 137 & 41240 & 4.95 & 1.88 & 11 \\
\hline hPRP43 & gi|68509926 & $\begin{array}{l}\text { box polypeptide } 15 \text { [Homo } \\
\text { sapiens] } \\
\text { serine/threonine-protein }\end{array}$ & 554 & 98436 & 5.98 & 2.21 & 28 \\
\hline hPRP4-Kinase & gi|89276756 & $\begin{array}{l}\text { kinase PRP4K [Homo } \\
\text { sapiens] }\end{array}$ & 390 & 135781 & 0.59 & 0.19 & 25 \\
\hline HsKin17 & gi|13124883 & $\begin{array}{l}\text { HsKin17 protein [Homo } \\
\text { sapiens] }\end{array}$ & 78 & 52728 & 3.10 & 1.36 & 6 \\
\hline hSLU7 & gi|4249705 & $\begin{array}{l}\text { step II splicing factor } \\
\text { SLU7 [Homo sapiens] }\end{array}$ & 339 & 79345 & 0.10 & 0.10 & 19 \\
\hline hSmu-1 & gi|7023065 & $\begin{array}{l}\text { unnamed protein product } \\
\text { [Homo sapiens] }\end{array}$ & 815 & 62752 & 5.67 & 2.36 & 44 \\
\hline hSnu23 & gi|13385046 & $\begin{array}{l}\text { zinc finger, matrin type } 2 \\
\text { [Mus musculus] }\end{array}$ & 34 & 28778 & 5.48 & 1.95 & 2 \\
\hline Hsp27 & gi|662841 & $\begin{array}{l}\text { heat shock protein } 27 \\
\text { [Homo sapiens] } \\
\text { heat shock } 70 \mathrm{kDa} \text { protein }\end{array}$ & 0 & 23414 & 1.02 & & 1 \\
\hline Hsp70 & gi|5729877 & $\begin{array}{l}8 \text { isoform } 1 \text { [Homo } \\
\text { sapiens] }\end{array}$ & 314 & 78964 & 0.25 & 0.14 & 15 \\
\hline hSYF1 & gi|55770906 & $\begin{array}{l}\text { XPA binding protein } 2 \\
\text { [Homo sapiens] }\end{array}$ & 1201 & 106931 & 0.27 & 0.09 & 62 \\
\hline hTra-2-alpha & gi|9558733 & $\begin{array}{l}\text { transformer-2 alpha } \\
\text { [Homo sapiens] }\end{array}$ & 201 & 33724 & 1.44 & 0.70 & 9 \\
\hline
\end{tabular}




\begin{tabular}{|c|c|c|c|c|c|c|c|}
\hline Protein & $\begin{array}{l}\text { accession } \\
\text { number }\end{array}$ & $\begin{array}{l}\text { Protein database } \\
\text { description }\end{array}$ & $\begin{array}{l}\text { Protein } \\
\text { score }\end{array}$ & $\begin{array}{c}\text { Protein } \\
\text { mass } \\
\text { [Da] }\end{array}$ & $B / C$ & StDev & $\#$ \\
\hline hTra-2-beta & gi|4377849 & $\begin{array}{l}\text { transformer-2-beta } \\
\text { isoform } 3 \text { [Homo sapiens] }\end{array}$ & 245 & 22734 & 1.37 & 1.30 & 22 \\
\hline KIAA0073 & gi|559713 & KIAA0073 [Homo sapiens] & 630 & 80918 & 0.10 & 0.12 & 39 \\
\hline KIAA1604 & gi|10047283 & $\begin{array}{l}\text { KIAA1604 protein [Homo } \\
\text { sapiens] }\end{array}$ & 232 & 120523 & 0.35 & 0.15 & 25 \\
\hline LOC124245 & gi|31377595 & $\begin{array}{l}\text { conserved nuclear protein } \\
\text { NHN1 [Homo sapiens] } \\
\text { GC-rich sequence DNA- }\end{array}$ & 58 & 117864 & 0.71 & 0.42 & 11 \\
\hline LOC51325 & gi|22035565 & $\begin{array}{l}\text { binding factor candidate } \\
\text { isoform } 1 \text { [Homo sapiens] } \\
\text { LSM2 homolog, U6 small }\end{array}$ & 198 & 115914 & 2.12 & 0.42 & 16 \\
\hline Lsm2 & gi|10863977 & $\begin{array}{l}\text { nuclear RNA associated } \\
\text { [Homo sapiens] }\end{array}$ & 184 & 12217 & 5.24 & 1.28 & 4 \\
\hline Lsm3 & gi|7657315 & $\begin{array}{l}\text { Lsm3 protein [Homo } \\
\text { sapiens] } \\
\text { U6 snRNA-associated }\end{array}$ & 46 & 12414 & 3.45 & & 1 \\
\hline Lsm4 & gi|6912486 & $\begin{array}{l}\text { Sm-like protein } 4 \text { [Homo } \\
\text { sapiens] }\end{array}$ & 54 & 17207 & 4.78 & 1.60 & 8 \\
\hline Lsm6 & gi|5901998 & $\begin{array}{l}\text { Sm protein F [Homo } \\
\text { sapiens] } \\
\text { U6 snRNA-associated }\end{array}$ & 47 & 10176 & 5.64 & 2.12 & 4 \\
\hline Lsm7 & gi|7706423 & $\begin{array}{l}\text { Sm-like protein LSm7 } \\
\text { [Homo sapiens] } \\
\text { U6 snRNA-associated }\end{array}$ & 55 & 13272 & 6.61 & 3.62 & 8 \\
\hline Lsm8 & gi|7706425 & $\begin{array}{l}\text { Sm-like protein LSm8 } \\
\text { [Homo sapiens] }\end{array}$ & 113 & 10684 & 3.55 & 1.53 & 3 \\
\hline Magoh & gi|4505087 & $\begin{array}{l}\text { mago-nashi homolog } \\
\text { [Homo sapiens] }\end{array}$ & 135 & 19216 & 0.15 & 0.06 & 10 \\
\hline MFAP1 & gi|50726968 & $\begin{array}{l}\text { microfibrillar-associated } \\
\text { protein } 1 \text { [Homo sapiens] }\end{array}$ & 302 & 58411 & 3.54 & 1.00 & 38 \\
\hline MGC13125 & gi|14249338 & $\begin{array}{l}\text { BUD13 homolog [Homo } \\
\text { sapiens] }\end{array}$ & 132 & 78018 & 1.97 & 0.71 & 10 \\
\hline $\begin{array}{l}\text { MGC20398 } \\
\text { (CCDC16) }\end{array}$ & gi|74732532 & $\begin{array}{l}\text { Coiled-coil domain- } \\
\text { containing protein } 16\end{array}$ & 160 & 47290 & 0.93 & 0.37 & 11 \\
\hline $\begin{array}{l}\text { MGC23918 } \\
(\text { CCDC12) }\end{array}$ & gi|21389497 & $\begin{array}{l}\text { coiled-coil domain } \\
\text { containing } 12 \text { [Homo } \\
\text { sapiens] } \\
\text { mitogen-activated protein }\end{array}$ & 149 & 22674 & 0.53 & 0.17 & 6 \\
\hline MORG1 & gi|14150114 & $\begin{array}{l}\text { kinase organizer } 1 \text { [Homo } \\
\text { sapiens] }\end{array}$ & 72 & 37126 & 0.10 & & 1 \\
\hline NKAP & gi|13375676 & $\begin{array}{l}\text { NF-kappaB activating } \\
\text { protein [Homo sapiens] }\end{array}$ & 85 & 55386 & 0.22 & 0.11 & 6 \\
\hline $\begin{array}{l}\mathrm{N} \text {-myc and STAT } \\
\text { interactor }\end{array}$ & gi|4758814 & $\begin{array}{l}\mathrm{N} \text {-myc and STAT } \\
\text { interactor [Homo sapiens] }\end{array}$ & 43 & 39988 & 4.96 & & 1 \\
\hline Npw38BP & gi|7706501 & $\begin{array}{l}\text { WW domain binding } \\
\text { protein } 11 \text { [Homo sapiens] } \\
\text { nuclear fragile } \mathrm{X} \text { mental }\end{array}$ & 131 & 77303 & 7.31 & 0.72 & 6 \\
\hline NUFIP1 & gi|6912542 & $\begin{array}{l}\text { retardation protein } \\
\text { interacting protein } 1 \\
\text { [Homo sapiens] } \\
\text { serologically defined colon }\end{array}$ & 42 & 64004 & 0.22 & & 1 \\
\hline NY-CO-10 & gi|64276486 & $\begin{array}{l}\text { cancer antigen } 10 \text { [Homo } \\
\text { sapiens] }\end{array}$ & 122 & 61734 & 0.63 & 0.22 & 12 \\
\hline NY-REN-37 & gi|10433149 & $\begin{array}{l}\text { unnamed protein product } \\
\text { [Homo sapiens] }\end{array}$ & 56 & 51438 & 1.16 & & 1 \\
\hline p68(DDX5) & gi|226021 & $\begin{array}{l}\text { growth regulated nuclear } \\
68 \text { protein }\end{array}$ & 114 & 72338 & 1.52 & 0.55 & 8 \\
\hline
\end{tabular}




\begin{tabular}{|c|c|c|c|c|c|c|c|}
\hline Protein & $\begin{array}{l}\text { accession } \\
\text { number }\end{array}$ & $\begin{array}{l}\text { Protein database } \\
\text { description }\end{array}$ & $\begin{array}{l}\text { Protein } \\
\text { score }\end{array}$ & $\begin{array}{c}\text { Protein } \\
\text { mass } \\
\text { [Da] }\end{array}$ & $\mathrm{B} / \mathrm{C}$ & StDev & \# \\
\hline p72/DDX17 & gi|119580652 & $\begin{array}{l}\text { DEAD (Asp-Glu-Ala-Asp) } \\
\text { box polypeptide 17, } \\
\text { isoform CRA_h [Homo } \\
\text { sapiens] }\end{array}$ & 74 & 64210 & 1.07 & & 1 \\
\hline PABP & gi|119612222 & $\begin{array}{l}\text { poly(A) binding protein, } \\
\text { cytoplasmic } 1 \text {, isoform } \\
\text { CRA_c [Homo sapiens] }\end{array}$ & 192 & 52768 & 1.70 & 0.48 & 8 \\
\hline PABPC4 & gi|4504715 & $\begin{array}{l}\text { poly A binding protein, } \\
\text { cytoplasmic } 4 \text { [Homo } \\
\text { sapiens] }\end{array}$ & 46 & 77931 & 1.52 & 0.35 & 4 \\
\hline PABPN1 & gi|4758876 & $\begin{array}{l}\text { poly(A) binding protein, } \\
\text { nuclear } 1 \text { [Homo sapiens] }\end{array}$ & 77 & 34406 & 1.21 & 0.09 & 3 \\
\hline PCBP1 & gi|5453854 & $\begin{array}{l}\text { poly }(\mathrm{rC}) \text { binding protein } 1 \\
\text { [Homo sapiens] }\end{array}$ & 56 & 40077 & 5.02 & 1.56 & 5 \\
\hline РCBP2 & gi|14141166 & $\begin{array}{l}\text { poly }(\mathrm{rC}) \text {-binding protein } 2 \\
\text { isoform b [Homo sapiens] }\end{array}$ & 352 & 41113 & 2.58 & 0.29 & 4 \\
\hline Pinin & gi|3021392 & $\begin{array}{l}\text { nuclear protein SDK3 } \\
\text { [Homo sapiens] } \\
\text { peptidyl-prolyl isomerase }\end{array}$ & 35 & 89790 & 2.11 & 0.58 & 5 \\
\hline PPIG & gi|42560244 & $\begin{array}{l}\text { G (cyclophilin G) [Homo } \\
\text { sapiens] }\end{array}$ & 109 & 104685 & 0.20 & 0.09 & 6 \\
\hline PPIL1 & gi|7706339 & $\begin{array}{l}\text { peptidylprolyl isomerase- } \\
\text { like } 1 \text { [Homo sapiens] } \\
\text { peptidylprolyl isomerase- }\end{array}$ & 116 & 19902 & 0.42 & 0.18 & 10 \\
\hline PPIL2 & gi|7657473 & $\begin{array}{l}\text { like } 2 \text { isoform a [Homo } \\
\text { sapiens] }\end{array}$ & 331 & 66360 & 1.33 & 0.88 & 19 \\
\hline PPIL3 & gi|14043400 & $\begin{array}{l}\text { Peptidylprolyl isomerase } \\
\text { (cyclophilin)-like } 3 \text { [Homo } \\
\text { sapiens] }\end{array}$ & 134 & 20214 & 0.17 & 0.14 & 8 \\
\hline PRCC & gi|14714625 & $\begin{array}{l}\text { Papillary renal cell } \\
\text { carcinoma (translocation- } \\
\text { associated) [Homo }\end{array}$ & 54 & 57721 & 5.20 & 0.41 & 2 \\
\hline & & & & & & & \\
\hline PRKRIP1 & gi|13375901 & $\begin{array}{l}1 \text { (IL11 inducible) [Homo } \\
\text { sapiens] } \\
\text { pleiotropic regulator } 1\end{array}$ & 201 & 24875 & 0.13 & 0.09 & 7 \\
\hline PRL1 & gi|4505895 & $\begin{array}{l}\text { (PRL1 homolog, } \\
\text { Arabidopsis) [Homo } \\
\text { sapiens] }\end{array}$ & 603 & 62478 & 0.69 & 0.22 & 33 \\
\hline PUF60 & gi|1809248 & $\begin{array}{l}\text { siah binding protein } 1 \\
\text { [Homo sapiens] } \\
\text { RNA binding protein } \\
\text { (autoantigenic, hnRNP- }\end{array}$ & 48 & 62853 & 2.34 & & 1 \\
\hline RALY & gi|8051631 & $\begin{array}{l}\text { associated with lethal } \\
\text { yellow) long isoform } \\
\text { [Homo sapiens] } \\
\text { retinoblastoma-binding }\end{array}$ & 90 & 35804 & 0.80 & 0.20 & 4 \\
\hline RBBP6 & gi|33620716 & $\begin{array}{l}\text { protein } 6 \text { isoform } 2 \text { [Homo } \\
\text { sapiens] }\end{array}$ & 33 & 229462 & 1.38 & & 1 \\
\hline RBM10 & gi|34785044 & $\begin{array}{l}\text { RBM10 protein [Homo } \\
\text { sapiens] }\end{array}$ & 48 & 64274 & 6.49 & & 1 \\
\hline RBM14 & gi|5454064 & $\begin{array}{l}\text { RNA binding motif protein } \\
14 \text { [Homo sapiens] }\end{array}$ & 23 & 72469 & 1.26 & & 1 \\
\hline RBM15(OTT) & gi|10433990 & $\begin{array}{l}\text { unnamed protein product } \\
\text { [Homo sapiens] }\end{array}$ & 27 & 108615 & 1.41 & 0.19 & 2 \\
\hline RBM5/LUCA15 & gi|1244404 & putative tumor suppressor & 56 & 100212 & 6.73 & 0.86 & 2 \\
\hline
\end{tabular}




\begin{tabular}{|c|c|c|c|c|c|c|c|}
\hline Protein & $\begin{array}{l}\text { accession } \\
\text { number }\end{array}$ & $\begin{array}{l}\text { Protein database } \\
\text { description }\end{array}$ & $\begin{array}{c}\text { Protein } \\
\text { score }\end{array}$ & $\begin{array}{c}\text { Protein } \\
\text { mass } \\
{[\mathrm{Da}]}\end{array}$ & $\mathrm{B} / \mathrm{C}$ & StDev & $\#$ \\
\hline RBM7 & gi|7023641 & $\begin{array}{l}\text { unnamed protein product } \\
\text { [Homo sapiens] } \\
\text { IK cytokine, down- }\end{array}$ & 0 & 32759 & 1.14 & & 1 \\
\hline RED & gi|119582432 & $\begin{array}{l}\text { regulator of HLA II, } \\
\text { isoform CRA_a [Homo } \\
\text { sapiens] }\end{array}$ & 438 & 75361 & 4.64 & 2.17 & 23 \\
\hline RNF113A & gi|5902158 & $\begin{array}{l}\text { ring finger protein } 113 \mathrm{~A} \\
\text { [Homo sapiens] } \\
\text { RNA binding motif protein }\end{array}$ & 33 & 43684 & 0.43 & 0.15 & 2 \\
\hline RNPC2(CAPER) & gi|4757926 & $\begin{array}{l}39 \text { isoform b [Homo } \\
\text { sapiens] }\end{array}$ & 350 & 64182 & 4.08 & 0.89 & 17 \\
\hline RNPS1 & gi|3253165 & $\begin{array}{l}\text { SR protein [Homo } \\
\text { sapiens] }\end{array}$ & 49 & 38412 & 0.63 & 0.17 & 2 \\
\hline S164 & gi|55741709 & $\begin{array}{l}\text { RNA binding motif protein } \\
25 \text { [Homo sapiens] }\end{array}$ & 50 & 113081 & 0.73 & 0.35 & 3 \\
\hline SAFB-like & gi|62825862 & $\begin{array}{l}\text { SLTM protein [Homo } \\
\text { sapiens] }\end{array}$ & 55 & 86766 & 4.10 & 0.84 & 5 \\
\hline SAP18 & gi|5032067 & $\begin{array}{l}\text { Sin3A-associated protein, } \\
\text { 18kDa [Homo sapiens] } \\
\text { splicing factor } 3 a \text {, subunit }\end{array}$ & 99 & 19469 & 1.37 & 0.21 & 6 \\
\hline SF3a120 & gi|5032087 & $\begin{array}{l}1,120 \mathrm{kDa} \text { isoform } 1 \\
\text { [Homo sapiens] }\end{array}$ & 444 & 97523 & 2.66 & 0.58 & 26 \\
\hline SF3a60 & gi|551450 & $\begin{array}{l}\text { splicing factor SF3a60 } \\
\text { [Homo sapiens] }\end{array}$ & 602 & 65068 & 4.91 & 1.28 & 24 \\
\hline SF3a66 & gi|409219 & spiceosomal protein & 121 & 52572 & 3.94 & 0.71 & 7 \\
\hline SF3b125 & gi|3435312 & $\begin{array}{l}\text { RNA helicase-related } \\
\text { protein [Homo sapiens] }\end{array}$ & 126 & 84712 & 10.06 & 4.64 & 6 \\
\hline SF3b130 & gi|6006515 & $\begin{array}{l}\text { spliceosomal protein SAP } \\
130 \text { [Homo sapiens] }\end{array}$ & 1144 & 144018 & 5.01 & 1.85 & 98 \\
\hline SF3b145 & gi|14043240 & $\begin{array}{l}\text { SF3B2 protein [Homo } \\
\text { sapiens] }\end{array}$ & 403 & 83704 & 4.63 & 1.53 & 21 \\
\hline SF3b14a/p14 & gi|7706326 & $\begin{array}{l}\text { splicing factor 3B, } 14 \mathrm{kDa} \\
\text { subunit [Homo sapiens] }\end{array}$ & 41 & 16541 & 4.25 & 1.31 & 11 \\
\hline SF3b14b & gi|14249398 & $\begin{array}{l}\text { PHD-finger 5A [Homo } \\
\text { sapiens] } \\
\text { splicing factor } 3 b \text {, subunit }\end{array}$ & 84 & 14868 & 3.75 & 1.04 & 10 \\
\hline SF3b155 & gi|54112117 & $\begin{array}{l}1 \text { isoform } 1 \text { [Homo } \\
\text { sapiens] }\end{array}$ & 474 & 158585 & 3.98 & 2.36 & 68 \\
\hline SF3b49 & gi|5032069 & $\begin{array}{l}\text { splicing factor } 3 b \text {, subunit } \\
4 \text { [Homo sapiens] } \\
\text { Similar to expressed }\end{array}$ & 105 & 46421 & 5.67 & 1.26 & 2 \\
\hline SFRS12 & gi|28703790 & $\begin{array}{l}\text { sequence Al450757 } \\
\text { [Homo sapiens] } \\
550 \text { amino acids } \\
\text { MW=61kDa, } \\
\text { glycosylated }=75 \mathrm{kDa} \\
\text { expressed on }\end{array}$ & 35 & 46956 & 6.41 & & 1 \\
\hline SFRS17A & gi|187242 & $\begin{array}{l}\text { endothelium, activated } \\
\text { lymphocytes and } \\
\text { syncytiotrophoblast, } \\
\text { contains leucine zipper } \\
\text { and basic region } \\
\text { homologous to myc; 721P }\end{array}$ & 58 & 71752 & 1.36 & 0.20 & 4 \\
\hline SKIP & gi|69126 & $\begin{array}{l}\text { SKI-interacting protein } \\
\text { [Homo sapiens] }\end{array}$ & 651 & 69284 & 0.58 & 0.17 & 58 \\
\hline SKIV2L2 & gi|6633995 & $\begin{array}{l}\text { KIAA0052 protein [Homo } \\
\text { sapiens] }\end{array}$ & 206 & 131978 & 1.13 & 0.20 & 12 \\
\hline SmB & gi|190247 & snRNP polypeptide B & 192 & 31604 & 1.69 & 0.28 & 13 \\
\hline
\end{tabular}




\begin{tabular}{|c|c|c|c|c|c|c|c|}
\hline Protein & $\begin{array}{l}\text { accession } \\
\text { number }\end{array}$ & $\begin{array}{l}\text { Protein database } \\
\text { description }\end{array}$ & $\begin{array}{l}\text { Protein } \\
\text { score }\end{array}$ & $\begin{array}{c}\text { Protein } \\
\text { mass } \\
\text { [Da] }\end{array}$ & $B / C$ & StDev & \# \\
\hline SmD1 & gi|5902102 & \begin{tabular}{|l} 
small nuclear \\
ribonucleoprotein D1 \\
polypeptide 16kDa [Homo \\
sapiens] \\
small nuclear
\end{tabular} & 205 & 14858 & 1.39 & 0.26 & 6 \\
\hline SmD2 & gi|4759158 & $\begin{array}{l}\text { ribonucleoprotein } \\
\text { polypeptide D2 [Homo } \\
\text { sapiens] } \\
\text { small nuclear }\end{array}$ & 361 & 16204 & 1.62 & 0.66 & 35 \\
\hline SmD3 & gi|4759160 & $\begin{array}{l}\text { ribonucleoprotein } \\
\text { polypeptide D3 [Homo } \\
\text { sapiens] } \\
\text { small nuclear }\end{array}$ & 249 & 15584 & 1.40 & 0.32 & 32 \\
\hline SmE & gi|4507129 & $\begin{array}{l}\text { ribonucleoprotein } \\
\text { polypeptide E [Homo } \\
\text { sapiens] } \\
\text { small nuclear }\end{array}$ & 292 & 11851 & 1.20 & 0.17 & 11 \\
\hline SmF & gi|4507131 & $\begin{array}{l}\text { ribonucleoprotein } \\
\text { polypeptide F [Homo } \\
\text { sapiens] } \\
\text { small nuclear }\end{array}$ & 114 & 10629 & 2.91 & 0.93 & 2 \\
\hline SmG & gi|4507133 & $\begin{array}{l}\text { ribonucleoprotein } \\
\text { polypeptide G [Homo } \\
\text { sapiens] }\end{array}$ & 122 & 9545 & 1.52 & 0.44 & 4 \\
\hline SNIP & gi|21314720 & $\begin{array}{l}\text { Smad nuclear interacting } \\
\text { protein [Homo sapiens] }\end{array}$ & 58 & 49687 & 0.80 & 0.11 & 10 \\
\hline SON3 & gi|17046381 & $\begin{array}{l}\text { SON DNA binding protein } \\
\text { isoform } \mathrm{E} \text { [Homo sapiens] } \\
\text { spen homolog, }\end{array}$ & 144 & 240773 & 1.13 & 0.22 & 5 \\
\hline Spen & gi|14790190 & $\begin{array}{l}\text { transcriptional regulator } \\
\text { [Homo sapiens] } \\
\text { breast carcinoma }\end{array}$ & 251 & 440442 & 4.77 & 1.53 & 16 \\
\hline SPF27 & gi|5031653 & $\begin{array}{l}\text { amplified sequence } 2 \\
\text { [Homo sapiens] } \\
\text { survival motor neuron }\end{array}$ & 305 & 28080 & 0.53 & 0.13 & 18 \\
\hline SPF30 & gi|5032113 & $\begin{array}{l}\text { domain containing } 1 \\
\text { [Homo sapiens] }\end{array}$ & 37 & 31300 & 30.16 & & 1 \\
\hline SPF45 & gi|14249678 & $\begin{array}{l}\text { RNA binding motif protein } \\
17 \text { [Homo sapiens] }\end{array}$ & 64 & 50450 & 5.38 & 1.25 & 5 \\
\hline SR140 & gi|2224605 & KIAA0332 [Homo sapiens] & 73 & 134163 & 3.04 & 0.17 & 3 \\
\hline SRm160 & gi|23274133 & $\begin{array}{l}\text { Serine/arginine repetitive } \\
\text { matrix } 1 \text { [Homo sapiens] }\end{array}$ & 89 & 117116 & 1.12 & 0.16 & 5 \\
\hline SRm300 & gi|5821153 & $\begin{array}{l}\text { RNA binding protein } \\
\text { [Homo sapiens] }\end{array}$ & 133 & 318973 & 0.31 & 0.13 & 7 \\
\hline SRp20 & gi|2125864 & $\begin{array}{l}\text { Srp20 [Mus musculus] } \\
\text { splicing factor, }\end{array}$ & 101 & 14955 & 1.65 & 0.44 & 8 \\
\hline SRp30c & gi|4506903 & $\begin{array}{l}\text { arginine/serine-rich } 9 \\
\text { [Homo sapiens] } \\
\text { PREDICTED: splicing }\end{array}$ & 186 & 26915 & 0.99 & 0.20 & 16 \\
\hline SRp40 & gi|55640963 & $\begin{array}{l}\text { factor, arginine/serine-rich } \\
5 \text { isoform } 6 \text { [Pan } \\
\text { troglodytes] }\end{array}$ & 70 & 33844 & 0.85 & 0.07 & 4 \\
\hline SRp46 & gi|14141216 & $\begin{array}{l}\text { SRp46 splicing factor } \\
\text { [Homo sapiens] } \\
\text { arginine/serine-rich }\end{array}$ & 65 & 33004 & 1.85 & 1.08 & 2 \\
\hline SRp55 & gi|62087532 & $\begin{array}{l}\text { splicing factor } 6 \text { variant } \\
\text { [Homo sapiens] }\end{array}$ & 87 & 33759 & 1.35 & 0.23 & 17 \\
\hline
\end{tabular}




\begin{tabular}{|c|c|c|c|c|c|c|c|}
\hline Protein & $\begin{array}{l}\text { accession } \\
\text { number }\end{array}$ & $\begin{array}{l}\text { Protein database } \\
\text { description }\end{array}$ & $\begin{array}{c}\text { Protein } \\
\text { score }\end{array}$ & $\begin{array}{c}\text { Protein } \\
\text { mass } \\
\text { [Da] }\end{array}$ & $\mathrm{B} / \mathrm{C}$ & StDev & \# \\
\hline TARDBP & gi|6678271 & $\begin{array}{l}\text { TAR DNA binding protein } \\
\text { [Homo sapiens] } \\
\text { transcription elongation }\end{array}$ & 0 & 48013 & 0.99 & 0.33 & 3 \\
\hline TCERG1 & gi|21327715 & $\begin{array}{l}\text { regulator } 1 \text { isoform } 1 \\
\text { [Homo sapiens] }\end{array}$ & 81 & 138273 & 2.10 & 0.26 & 3 \\
\hline TCP1 & gi|1800303 & $\begin{array}{l}\text { HIV-1 Nef interacting } \\
\text { protein [Homo sapiens] }\end{array}$ & 0 & 50858 & 0.36 & 0.13 & 3 \\
\hline TFIP11 & gi|8393259 & $\begin{array}{l}\text { tuftelin interacting protein } \\
11 \text { [Homo sapiens] }\end{array}$ & 150 & 106591 & 6.50 & 1.91 & 12 \\
\hline TFIP11 & gi|8393259 & $\begin{array}{l}\text { tuftelin interacting protein } \\
11 \text { [Homo sapiens] } \\
\text { THO complex subunit } 1\end{array}$ & 257 & 106591 & 0.66 & 0.15 & 16 \\
\hline THOC1 & gi|37999906 & $\begin{array}{l}\text { (Tho1) (Nuclear matrix } \\
\text { protein p84) }\end{array}$ & 136 & 83277 & 1.13 & 0.42 & 4 \\
\hline THOC2 & gi|125656165 & $\begin{array}{l}\text { THO complex } 2 \text { isoform } 1 \\
\text { [Homo sapiens] }\end{array}$ & 148 & 211268 & 0.62 & 0.17 & 13 \\
\hline Aly/REF (THOC4) & gi|55770864 & $\begin{array}{l}\text { THO complex } 4 \text { [Homo } \\
\text { sapiens] }\end{array}$ & 183 & 28601 & 1.11 & 0.27 & 4 \\
\hline $\begin{array}{l}\text { KIAA0983 } \\
\text { (THOC5) }\end{array}$ & gi|40789009 & $\begin{array}{l}\text { KIAA0983 protein [Homo } \\
\text { sapiens] }\end{array}$ & 59 & 88032 & 1.44 & 0.46 & 5 \\
\hline WDR58 (THOC6) & gi|22761350 & $\begin{array}{l}\text { unnamed protein product } \\
\text { [Homo sapiens] } \\
\text { PREDICTED: thyroid } \\
\text { hormone receptor }\end{array}$ & 55 & 35141 & 0.51 & 0.25 & 2 \\
\hline THRAP3 & gi|114555524 & $\begin{array}{l}\text { associated protein } 3 \\
\text { isoform } 1 \text { [Pan } \\
\text { troglodytes] }\end{array}$ & 207 & 123201 & 2.04 & 0.67 & 30 \\
\hline TOE1 & gi|10436256 & $\begin{array}{l}\text { unnamed protein product } \\
\text { [Homo sapiens] } \\
\text { tetratricopeptide repeat }\end{array}$ & 35 & 61544 & 0.84 & 0.37 & 3 \\
\hline TPR14 (TTC14) & gi|33457330 & $\begin{array}{l}\text { domain } 14 \text { isoform a } \\
\text { [Homo sapiens] }\end{array}$ & 99 & 99198 & 0.22 & 0.14 & 8 \\
\hline tubulin alpha-2 & gi|34740335 & $\begin{array}{l}\text { tubulin, alpha } 2 \text { [Mus } \\
\text { musculus] }\end{array}$ & 614 & 53554 & 0.73 & 0.11 & 2 \\
\hline tubulin beta & gi|18088719 & $\begin{array}{l}\text { Tubulin, beta [Homo } \\
\text { sapiens] }\end{array}$ & 381 & 52313 & 0.93 & 0.23 & 2 \\
\hline tubulin beta-2 & gi|5174735 & $\begin{array}{l}\text { tubulin, beta, } 2 \text { [Homo } \\
\text { sapiens] }\end{array}$ & 255 & 52473 & 1.15 & & 1 \\
\hline U1-70K & gi|36100 & $\begin{array}{l}\text { unnamed protein product } \\
\text { [Homo sapiens] } \\
\text { small nuclear }\end{array}$ & 83 & 73878 & 3.24 & 1.42 & 12 \\
\hline U2-A' & gi|50593002 & $\begin{array}{l}\text { ribonucleoprotein } \\
\text { polypeptide A [Homo } \\
\text { sapiens] } \\
\text { U2 small nuclear RNA }\end{array}$ & 227 & 31660 & 0.48 & 0.18 & 23 \\
\hline U2AF35 & gi|5803207 & $\begin{array}{l}\text { auxillary factor } 1 \text { isoform a } \\
\text { [Homo sapiens] }\end{array}$ & 49 & 29853 & 3.63 & 0.80 & 2 \\
\hline U2AF65 & gi|228543 & $\begin{array}{l}\text { splicing factor } \\
\text { U2AF:SUBUNIT=large } \\
\text { small nuclear }\end{array}$ & 107 & 57879 & 5.59 & 2.69 & 8 \\
\hline U2-B" & gi|4507123 & $\begin{array}{l}\text { ribonucleoprotein } \\
\text { polypeptide B [Homo } \\
\text { sapiens] } \\
\text { squamous cell carcinoma }\end{array}$ & 194 & 29217 & 0.53 & 0.25 & 14 \\
\hline U4/U6.U5-110K & gi|10863889 & $\begin{array}{l}\text { antigen recognized by }{ }^{\top} \\
\text { cells } 1 \text { [Homo sapiens] }\end{array}$ & 629 & 101434 & 4.50 & 1.96 & 23 \\
\hline
\end{tabular}




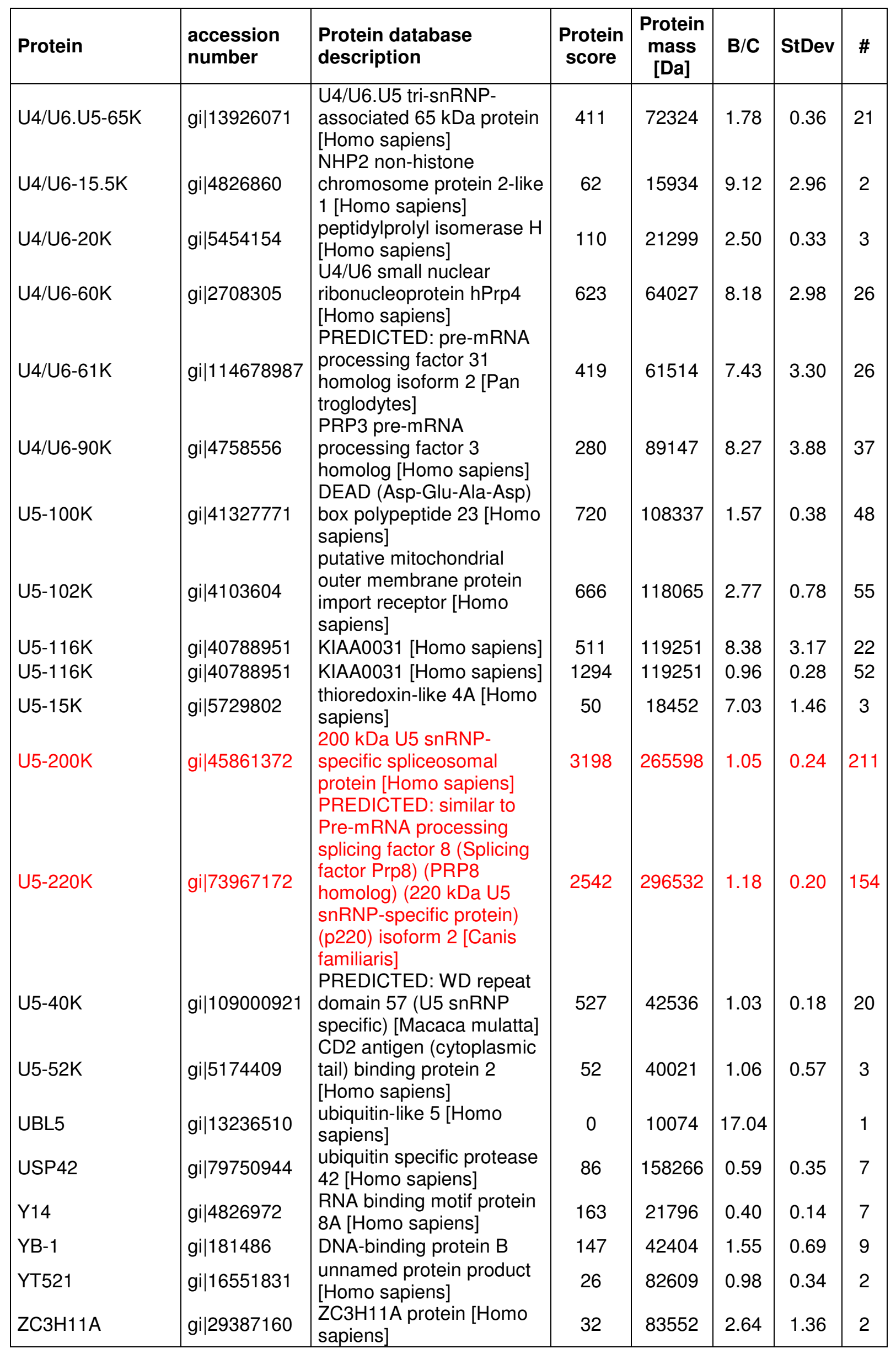




\begin{tabular}{|l|l|l|c|c|c|c|c|}
\hline Protein & $\begin{array}{l}\text { accession } \\
\text { number }\end{array}$ & $\begin{array}{l}\text { Protein database } \\
\text { description }\end{array}$ & $\begin{array}{l}\text { Protein } \\
\text { score }\end{array}$ & $\begin{array}{l}\text { Protein } \\
\text { mass } \\
\text { [Da] }\end{array}$ & B/C & StDev & \# \\
\hline ZCCHC10 & gi|8923106 & $\begin{array}{l}\text { zinc finger, CCHC domain } \\
\text { containing 10 [Homo } \\
\text { sapiens] }\end{array}$ & 60 & 21419 & 0.66 & 0.71 & 2 \\
ZCCHC finger CCHC-type \\
and RNA binding motif 1 \\
ZCCHC8
\end{tabular}


Table A.6: Proteins identified in the second replicate of the relative quantification of spliceosomal B and C complexes by iTRAQ. The accession number, the protein database description, the obtained protein score (Mascot), the protein mass, the determined protein ratio $(B / C)$, the standard deviation (StDev), and the number of peptides used for quantification (\#) is given for all proteins found. Proteins used for normalization are highlighted in red.

\begin{tabular}{|c|c|c|c|c|c|c|c|}
\hline Protein & $\begin{array}{l}\text { accession } \\
\text { number }\end{array}$ & $\begin{array}{l}\text { Protein database } \\
\text { description }\end{array}$ & $\begin{array}{c}\text { Protein } \\
\text { score }\end{array}$ & $\begin{array}{c}\text { Protein } \\
\text { mass } \\
\text { [Da] }\end{array}$ & $\mathrm{B} / \mathrm{C}$ & StDev & $\#$ \\
\hline $9 \mathrm{G} 8$ & gi|72534660 & $\begin{array}{l}\text { splicing factor, } \\
\text { arginine/serine-rich } 7 \\
\text { [Homo sapiens] }\end{array}$ & 146 & 28687 & 2.58 & 0.38 & 9 \\
\hline Abstrakt & gi|21071032 & $\begin{array}{l}\text { DEAD-box protein } \\
\text { abstrakt [Homo sapiens] } \\
\text { PREDICTED: apoptotic }\end{array}$ & 382 & 77694 & 0.17 & 0.14 & 90 \\
\hline Acinus & gi|109082928 & $\begin{array}{l}\text { chromatin condensation } \\
\text { inducer } 1 \text { isoform } 2 \\
\text { [Macaca mulatta] } \\
\text { hypothetical protein }\end{array}$ & 164 & 77764 & 1.84 & 0.38 & 17 \\
\hline Acinus & gi|7513059 & $\begin{array}{l}\text { KIAA0670 - human } \\
\text { (fragment) }\end{array}$ & 99 & 158783 & 1.76 & 0.37 & 8 \\
\hline ALB & gi|28592 & $\begin{array}{l}\text { serum albumin [Homo } \\
\text { sapiens] }\end{array}$ & 79 & 79864 & 7.91 & 0.97 & 3 \\
\hline alpha tubulin & gi|340021 & alpha-tubulin & 363 & 53554 & 1.14 & 0.20 & 26 \\
\hline Aquarius & gi|38788372 & aquarius [Homo sapiens] & 1599 & 183588 & 0.39 & 0.13 & 186 \\
\hline ASR2B & gi|19879862 & $\begin{array}{l}\text { arsenite-resistant protein } \\
\text { ASR2 [Homo sapiens] } \\
\text { BCL2-associated }\end{array}$ & 357 & 99344 & 1.55 & 0.20 & 32 \\
\hline BAG2 & gi|4757834 & $\begin{array}{l}\text { athanogene } 2 \text { [Homo } \\
\text { sapiens] }\end{array}$ & 0 & 26057 & 0.50 & & 1 \\
\hline BCLAF1 & gi|7582386 & $\begin{array}{l}\text { Bcl-2-associated } \\
\text { transcription factor short } \\
\text { form [Homo sapiens] } \\
\text { Chain A, Crystal }\end{array}$ & 47 & 112929 & 2.23 & 0.31 & 6 \\
\hline BCR & gi|116666695 & $\begin{array}{l}\text { Structure Of Full-Length } \\
\text { 3 -Exonuclease }\end{array}$ & 0 & 45012 & 1.38 & & 1 \\
\hline beta-tubulin & gi|1297274 & $\begin{array}{l}\text { beta-tubulin [Homo } \\
\text { sapiens] }\end{array}$ & 90 & 53303 & 0.67 & 0.10 & 5 \\
\hline BRIX & gi|55770900 & BRIX [Homo sapiens] & 0 & 47945 & 1.39 & 0.31 & 2 \\
\hline c10orf4/FRA10AC1 & gi|24432067 & $\begin{array}{l}\text { FRA10AC1 protein } \\
\text { [Homo sapiens] } \\
\text { hypothetical protein }\end{array}$ & 49 & 44908 & 0.11 & 0.05 & 6 \\
\hline C1orf55 & gi|40255125 & $\begin{array}{l}\text { LOC163859 [Homo } \\
\text { sapiens] }\end{array}$ & 178 & 54043 & 0.13 & 0.06 & 14 \\
\hline c9orf78 & gi|6808233 & $\begin{array}{l}\text { hypothetical protein } \\
\text { [Homo sapiens] } \\
\text { Uncharacterized protein }\end{array}$ & 40 & 32417 & 0.26 & 0.01 & 2 \\
\hline Cactin & gi|91208260 & $\begin{array}{l}\text { C19orf29 (Renal } \\
\text { carcinoma antigen NY- } \\
\text { REN-24) } \\
\text { Cas-Br-M (murine) }\end{array}$ & 571 & 87199 & 0.17 & 0.08 & 35 \\
\hline Cas-Br-M & gi|13376204 & $\begin{array}{l}\text { ecotropic retroviral } \\
\text { transforming sequence- } \\
\text { like } 1 \text { [Homo sapiens] } \\
\text { nuclear cap binding }\end{array}$ & 66 & 57712 & 1.94 & 0.08 & 2 \\
\hline CBP20 & gi|19923387 & $\begin{array}{l}\text { protein subunit 2, 20kDa } \\
\text { isoform } 1 \text { [Homo } \\
\text { sapiens] } \\
\text { nuclear cap binding }\end{array}$ & 123 & 20001 & 0.93 & 0.16 & 10 \\
\hline CBP80 & gi|4505343 & $\begin{array}{l}\text { protein subunit } 1,80 \mathrm{kDa} \\
{[\text { Homo sapiens] }}\end{array}$ & 580 & 99715 & 1.01 & 0.12 & 32 \\
\hline
\end{tabular}




\begin{tabular}{|c|c|c|c|c|c|c|c|}
\hline Protein & $\begin{array}{l}\text { accession } \\
\text { number }\end{array}$ & $\begin{array}{l}\text { Protein database } \\
\text { description }\end{array}$ & $\begin{array}{c}\text { Protein } \\
\text { score }\end{array}$ & $\begin{array}{c}\text { Protein } \\
\text { mass } \\
\text { [Da] }\end{array}$ & $\mathrm{B} / \mathrm{C}$ & StDev & \# \\
\hline CCAP2 & gi|6841518 & $\begin{array}{l}\text { HSPC148 [Homo } \\
\text { sapiens] } \\
\text { death inducer with SAP }\end{array}$ & 78 & 30099 & 0.15 & 0.08 & 3 \\
\hline CCAR1 & gi|27497118 & $\begin{array}{l}\text { domain DIS [Homo } \\
\text { sapiens] } \\
\text { coiled-coil domain }\end{array}$ & 38 & 147586 & 5.02 & 1.24 & 2 \\
\hline CCDC55 & gi|14149807 & $\begin{array}{l}\text { containing } 55 \text { isoform } 1 \\
\text { [Homo sapiens] }\end{array}$ & 0 & 76765 & 1.17 & & 1 \\
\hline CCNK & gi|8980825 & cyclin K [Homo sapiens] & 46 & 44722 & 2.33 & 1.10 & 2 \\
\hline CDC5L & gi|11067747 & $\begin{array}{l}\text { CDC5-like [Homo } \\
\text { sapiens] }\end{array}$ & 716 & 103618 & 1.42 & 0.39 & 53 \\
\hline CDC5L & gi|11067747 & $\begin{array}{l}\text { CDC5-like [Homo } \\
\text { sapiens] } \\
\text { Cell division protein }\end{array}$ & 360 & 103618 & 0.19 & 0.12 & 30 \\
\hline CDK10(PISSLRE) & gi|6226784 & $\begin{array}{l}\text { kinase } 10 \\
\text { (Serine/threonine-protein } \\
\text { kinase PISSLRE) }\end{array}$ & 69 & 45362 & 0.24 & 0.10 & 4 \\
\hline CGI-79 & gi|4929627 & $\begin{array}{l}\text { CGI-79 protein [Homo } \\
\text { sapiens] }\end{array}$ & 44 & 46601 & 1.36 & 0.10 & 2 \\
\hline CHERP & gi|2058691 & $\begin{array}{l}\text { ERPROT 213-21 [Homo } \\
\text { sapiens] }\end{array}$ & 73 & 105842 & 4.27 & 1.06 & 5 \\
\hline CRNKL1 & gi|50949465 & $\begin{array}{l}\text { hypothetical protein } \\
\text { [Homo sapiens] }\end{array}$ & 757 & 96523 & 0.32 & 0.07 & 82 \\
\hline CTNNBL1 & gi|18644734 & $\begin{array}{l}\text { beta catenin-like } 1 \\
\text { [Homo sapiens] } \\
\text { CUG triplet repeat, RNA- }\end{array}$ & 120 & 70832 & 2.29 & 0.34 & 11 \\
\hline CUG & gi|5729794 & $\begin{array}{l}\text { binding protein } 1 \text { isoform } \\
1 \text { [Homo sapiens] } \\
\text { hypothetical protein }\end{array}$ & 76 & 55437 & 3.42 & 0.53 & 7 \\
\hline CXorf56 & gi|11545813 & $\begin{array}{l}\text { LOC63932 [Homo } \\
\text { sapiens] } \\
\text { PREDICTED: similar to }\end{array}$ & 167 & 29821 & 0.24 & 0.13 & 11 \\
\hline Cyclin M & gi|88989669 & $\begin{array}{l}\text { CG31232-PA, isoform A } \\
\text { [Homo sapiens] } \\
\text { peptidylprolyl isomerase }\end{array}$ & 39 & 27359 & 0.18 & 0.03 & 2 \\
\hline СурЕ & gi|45439318 & $\begin{array}{l}\mathrm{E} \text { isoform } 3 \text { [Homo } \\
\text { sapiens] } \\
\text { DEAH (Asp-Glu-Ala-His) }\end{array}$ & 71 & 29821 & 0.58 & 0.14 & 5 \\
\hline DDX34 & gi|38158022 & $\begin{array}{l}\text { box polypeptide } 34 \\
\text { [Homo sapiens] } \\
\text { DEAH (Asp-Glu-Ala-His) }\end{array}$ & 460 & 135588 & 0.26 & 0.17 & 20 \\
\hline DDX35 & gi|20544129 & $\begin{array}{l}\text { box polypeptide } 35 \\
\text { [Homo sapiens] }\end{array}$ & 505 & 84842 & 0.17 & 0.07 & 22 \\
\hline DDX9 & gi|307383 & $\begin{array}{l}\text { RNA helicase A } \\
\text { Similar to expressed }\end{array}$ & 69 & 152784 & 1.43 & 0.14 & 3 \\
\hline DGCR14 & gi|12804313 & $\begin{array}{l}\text { sequence } 2 \text { embryonic } \\
\text { lethal [Homo sapiens] }\end{array}$ & 105 & 56774 & 0.29 & 0.16 & 8 \\
\hline E1B-AP5 & gi|3319956 & $\begin{array}{l}\text { E1B-55kDa-associated } \\
\text { protein [Homo sapiens] }\end{array}$ & 55 & 101542 & 1.62 & 0.10 & 4 \\
\hline Ecm29 & gi|2224677 & $\begin{array}{l}\text { KIAA0368 [Homo } \\
\text { sapiens] }\end{array}$ & 22 & 175362 & 1.77 & 0.17 & 2 \\
\hline eEF1A1 & gi|31092 & $\begin{array}{l}\text { unnamed protein product } \\
\text { [Homo sapiens] } \\
\text { ELAV (embryonic lethal, } \\
\text { abnormal vision, }\end{array}$ & 35 & 57288 & 1.07 & 0.51 & 2 \\
\hline ELAV & gi|119589356 & $\begin{array}{l}\text { Drosophila)-like } 1 \text { (Hu } \\
\text { antigen R), isoform } \\
\text { CRA_b [Homo sapiens] }\end{array}$ & 305 & 53703 & 5.65 & 0.82 & 18 \\
\hline
\end{tabular}




\begin{tabular}{|c|c|c|c|c|c|c|c|}
\hline Protein & $\begin{array}{l}\text { accession } \\
\text { number }\end{array}$ & $\begin{array}{l}\text { Protein database } \\
\text { description }\end{array}$ & $\begin{array}{l}\text { Protein } \\
\text { score }\end{array}$ & $\begin{array}{c}\text { Protein } \\
\text { mass } \\
\text { [Da] }\end{array}$ & $\mathrm{B} / \mathrm{C}$ & StDev & \# \\
\hline elF4A3 & gi|496902 & \begin{tabular}{|l} 
translation initiation \\
factor [Homo sapiens]
\end{tabular} & 166 & 50492 & 0.55 & 0.06 & 9 \\
\hline ELG & gi|8923771 & $\begin{array}{l}\text { ELG protein [Homo } \\
\text { sapiens] }\end{array}$ & 0 & 43508 & 1.08 & & 1 \\
\hline EXOSC4 & gi|9506689 & $\begin{array}{l}\text { exosome component } 4 \\
\text { [Homo sapiens] }\end{array}$ & 63 & 27317 & 1.67 & & 1 \\
\hline EXOSC5 & gi|14043511 & $\begin{array}{l}\text { Exosome component } 5 \\
\text { [Homo sapiens] }\end{array}$ & 30 & 27406 & 0.86 & & 1 \\
\hline EXOSC7 & gi|473949 & $\begin{array}{l}\text { KIAA0116 [Homo } \\
\text { sapiens] }\end{array}$ & 0 & 34928 & 2.51 & & 1 \\
\hline Fam50A(XAP-5) & gi|4758220 & $\begin{array}{l}\text { XAP-5 protein [Homo } \\
\text { sapiens] }\end{array}$ & 44 & 46701 & 0.29 & 0.12 & 5 \\
\hline FAM50B & gi|6912326 & $\begin{array}{l}\text { similarity } 50, \text { member B } \\
\text { [Homo sapiens] }\end{array}$ & 35 & 42766 & 0.36 & 0.05 & 3 \\
\hline FBP11 & gi|5360087 & $\begin{array}{l}\text { NY-REN-6 antigen } \\
\text { [Homo sapiens] }\end{array}$ & 88 & 59273 & 2.70 & 0.50 & 4 \\
\hline FNBP4 & gi|6808095 & $\begin{array}{l}\text { hypothetical protein } \\
\text { [Homo sapiens] }\end{array}$ & 86 & 119110 & 2.71 & 0.29 & 4 \\
\hline FRG1 & gi|4758404 & $\begin{array}{l}\text { FSHD region gene } 1 \\
\text { [Homo sapiens] } \\
\text { far upstream element }\end{array}$ & 100 & 35148 & 0.58 & 0.17 & 6 \\
\hline FUSE3 & gi|100816392 & $\begin{array}{l}\text { (FUSE) binding protein } 3 \\
\text { [Homo sapiens] } \\
\text { FUS interacting protein }\end{array}$ & 214 & 65481 & 3.78 & 0.65 & 17 \\
\hline FUSIP1/SRp38 & gi|5730079 & $\begin{array}{l}\text { (serine-arginine rich) } 1 \\
\text { isoform } 1 \text { [Homo } \\
\text { sapiens] }\end{array}$ & 54 & 23166 & 1.44 & 0.32 & 7 \\
\hline G10 & gi|32171175 & $\begin{array}{l}\text { G10 protein [Homo } \\
\text { sapiens] } \\
\text { SYF2 homolog, RNA }\end{array}$ & 85 & 19898 & 0.88 & 0.02 & 4 \\
\hline GCIP p29 & gi|7661636 & $\begin{array}{l}\text { splicing factor isoform } 1 \\
\text { [Homo sapiens] }\end{array}$ & 104 & 32930 & 0.18 & 0.07 & 7 \\
\hline hECM2 (RBM22) & gi|8922328 & $\begin{array}{l}\text { RNA binding motif } \\
\text { protein } 22 \text { [Homo } \\
\text { sapiens] }\end{array}$ & 380 & 51786 & 0.53 & 0.11 & 39 \\
\hline hISY1 & gi|6330157 & $\begin{array}{l}\text { KIAA1160 protein [Homo } \\
\text { sapiens] }\end{array}$ & 261 & 43813 & 0.61 & 0.10 & 17 \\
\hline hnRNP A1 & gi|36102 & $\begin{array}{l}\text { unnamed protein product } \\
\text { [Homo sapiens] } \\
\text { heterogeneous nuclear }\end{array}$ & 56 & 37105 & 5.21 & 0.13 & 2 \\
\hline hnRNP A2/B1 & gi|4504447 & $\begin{array}{l}\text { ribonucleoprotein A2/B1 } \\
\text { isoform A2 [Homo } \\
\text { sapiens] } \\
\text { heterogeneous nuclear }\end{array}$ & 77 & 38480 & 3.60 & 0.18 & 4 \\
\hline hnRNP A3 & gi|34740329 & $\begin{array}{l}\text { ribonucleoprotein A3 } \\
\text { [Homo sapiens] }\end{array}$ & 0 & 42925 & 5.14 & & 1 \\
\hline hnRNP C1/C2 & gi|306875 & $\begin{array}{l}\text { C protein } \\
\text { Chain A, Nmr Structure }\end{array}$ & 193 & 36316 & 1.97 & 0.30 & 16 \\
\hline hnRNP F & gi|112491343 & $\begin{array}{l}\text { Of The Third Qrrm } \\
\text { Domain Of Human } \\
\text { Hnrnp F }\end{array}$ & 47 & 15509 & 2.94 & & 1 \\
\hline hnRNP G & gi|3256007 & $\begin{array}{l}\text { hnRNP G protein [Homo } \\
\text { sapiens] } \\
\text { heterogeneous nuclear }\end{array}$ & 80 & 44396 & 2.21 & 0.35 & 6 \\
\hline hnRNP H1 & gi|5031753 & $\begin{array}{l}\text { ribonucleoprotein } \mathrm{H} 1 \\
\text { [Homo sapiens] }\end{array}$ & 123 & 51878 & 1.84 & 0.27 & 13 \\
\hline
\end{tabular}




\begin{tabular}{|c|c|c|c|c|c|c|c|}
\hline Protein & $\begin{array}{l}\text { accession } \\
\text { number }\end{array}$ & $\begin{array}{l}\text { Protein database } \\
\text { description }\end{array}$ & $\begin{array}{c}\text { Protein } \\
\text { score }\end{array}$ & $\begin{array}{c}\text { Protein } \\
\text { mass } \\
\text { [Da] }\end{array}$ & $B / C$ & StDev & \# \\
\hline hnRNP K & gi|55958547 & $\begin{array}{l}\text { heterogeneous nuclear } \\
\text { ribonucleoprotein K } \\
\text { [Homo sapiens] }\end{array}$ & 31 & 44558 & 4.01 & 0.73 & 2 \\
\hline hnRNP M & gi|187281 & M4 protein & 33 & 83739 & 1.37 & 0.38 & 4 \\
\hline hnRNP Q2 & gi|15809588 & $\begin{array}{l}\text { hnRNP Q2 [Homo } \\
\text { sapiens] } \\
\text { heterogeneous nuclear }\end{array}$ & 41 & 72196 & 0.96 & 0.44 & 2 \\
\hline hnRNP R & gi|5031755 & $\begin{array}{l}\text { ribonucleoprotein R } \\
\text { [Homo sapiens] } \\
\text { DEAH (Asp-Glu-Ala-His) }\end{array}$ & 71 & 77614 & 1.09 & & 1 \\
\hline hPRP16 & gi|17999539 & $\begin{array}{l}\text { box polypeptide } 38 \\
\text { [Homo sapiens] }\end{array}$ & 84 & 152201 & 0.43 & 0.11 & 12 \\
\hline hPRP17 & gi|7706657 & $\begin{array}{l}\text { cell division cycle } 40 \\
\text { homolog [Homo sapiens] } \\
\text { PRP18 pre-mRNA }\end{array}$ & 535 & 73381 & 0.45 & 0.15 & 57 \\
\hline hPRP18 & gi|4506123 & $\begin{array}{l}\text { processing factor } 18 \\
\text { homolog [Homo sapiens] } \\
\text { PRP19/PSO4 pre-mRNA }\end{array}$ & 55 & 45161 & 0.39 & 0.18 & 4 \\
\hline hPRP19 & gi|7657381 & $\begin{array}{l}\text { processing factor } 19 \\
\text { homolog [Homo sapiens] } \\
\text { DEAH (Asp-Glu-Ala-His) }\end{array}$ & 226 & 59981 & 0.57 & 0.12 & 62 \\
\hline hPRP2 & gi|14250712 & $\begin{array}{l}\text { box polypeptide } 16 \\
\text { [Homo sapiens] } \\
\text { DEAH (Asp-Glu-Ala-His) }\end{array}$ & 266 & 129236 & 2.10 & 0.29 & 20 \\
\hline hPRP22 & gi|4826690 & $\begin{array}{l}\text { box polypeptide } 8 \text { [Homo } \\
\text { sapiens] } \\
\text { PRP38 pre-mRNA } \\
\text { processing factor } 38\end{array}$ & 863 & 153030 & 0.29 & 0.15 & 89 \\
\hline hPRP38 & gi|24762236 & $\begin{array}{l}\text { (yeast) domain } \\
\text { containing A isoform } 2 \\
\text { [Homo sapiens] } \\
\text { DEAH (Asp-Glu-Ala-His) }\end{array}$ & 107 & 41240 & 4.04 & 0.85 & 7 \\
\hline hPRP43 & gi|68509926 & $\begin{array}{l}\text { box polypeptide } 15 \\
\text { [Homo sapiens] }\end{array}$ & 566 & 98436 & 2.59 & 0.35 & 37 \\
\hline HsKin17 & gi|13124883 & $\begin{array}{l}\text { HsKin17 protein [Homo } \\
\text { sapiens] }\end{array}$ & 76 & 52728 & 2.99 & 0.57 & 8 \\
\hline hSLU7 & gi|4249705 & $\begin{array}{l}\text { step II splicing factor } \\
\text { SLU7 [Homo sapiens] }\end{array}$ & 286 & 79345 & 0.20 & 0.09 & 38 \\
\hline hSnu23 & gi|13385046 & $\begin{array}{l}\text { zinc finger, matrin type } 2 \\
\text { [Mus musculus] }\end{array}$ & 0 & 28778 & 12.22 & 3.17 & 4 \\
\hline Hsp27 & gi|4504517 & $\begin{array}{l}\text { heat shock } 27 \mathrm{kDa} \\
\text { protein } 1 \text { [Homo sapiens] } \\
\text { heat shock } 70 \mathrm{kDa}\end{array}$ & 54 & 23967 & 0.47 & 0.02 & 2 \\
\hline Hsp70 & gi|5729877 & $\begin{array}{l}\text { protein } 8 \text { isoform } 1 \\
\text { [Homo sapiens] } \\
\text { heat shock } 70 \mathrm{kDa}\end{array}$ & 156 & 78964 & 1.19 & 0.22 & 10 \\
\hline Hsp70 & gi|5729877 & $\begin{array}{l}\text { protein } 8 \text { isoform } 1 \\
\text { [Homo sapiens] }\end{array}$ & 314 & 78964 & 0.24 & 0.11 & 18 \\
\hline hSYF1 & gi|55770906 & $\begin{array}{l}\text { XPA binding protein } 2 \\
\text { [Homo sapiens] }\end{array}$ & 1131 & 106931 & 0.38 & 0.08 & 65 \\
\hline hTra-2-alpha & gi|9558733 & $\begin{array}{l}\text { transformer-2 alpha } \\
\text { [Homo sapiens] } \\
\text { transformer-2-beta }\end{array}$ & 59 & 33724 & 2.80 & 0.47 & 2 \\
\hline hTra-2-beta & gi|4377849 & $\begin{array}{l}\text { isoform } 3 \text { [Homo } \\
\text { sapiens] }\end{array}$ & 179 & 22734 & 1.22 & 0.37 & 9 \\
\hline KIAA0073 & gi|559713 & $\begin{array}{l}\text { KIAA0073 [Homo } \\
\text { sapiens] }\end{array}$ & 588 & 80918 & 0.20 & 0.09 & 53 \\
\hline
\end{tabular}




\begin{tabular}{|c|c|c|c|c|c|c|c|}
\hline Protein & $\begin{array}{l}\text { accession } \\
\text { number }\end{array}$ & $\begin{array}{l}\text { Protein database } \\
\text { description }\end{array}$ & $\begin{array}{l}\text { Protein } \\
\text { score }\end{array}$ & $\begin{array}{c}\text { Protein } \\
\text { mass } \\
{[\mathrm{Da}]}\end{array}$ & $\mathrm{B} / \mathrm{C}$ & StDev & $\#$ \\
\hline $\begin{array}{l}\text { KIAA0983 } \\
\text { (THOC5) }\end{array}$ & gi|40789009 & $\begin{array}{l}\text { KIAA0983 protein [Homo } \\
\text { sapiens] }\end{array}$ & 49 & 88032 & 1.35 & 0.26 & 5 \\
\hline KIAA1604 & gi|10047283 & $\begin{array}{l}\text { KIAA1604 protein [Homo } \\
\text { sapiens] } \\
\text { conserved nuclear }\end{array}$ & 310 & 120523 & 0.46 & 0.23 & 27 \\
\hline LOC124245 & gi|31377595 & $\begin{array}{l}\text { protein NHN1 [Homo } \\
\text { sapiens] } \\
\text { hypothetical protein }\end{array}$ & 134 & 117864 & 1.68 & 0.36 & 27 \\
\hline LOC150383 & gi|49533621 & $\begin{array}{l}\text { LOC150383 [Homo } \\
\text { sapiens] } \\
\text { GC-rich sequence DNA- }\end{array}$ & 34 & 15480 & 0.07 & & 1 \\
\hline LOC51325 & gi|22035565 & $\begin{array}{l}\text { binding factor candidate } \\
\text { isoform } 1 \text { [Homo } \\
\text { sapiens] }\end{array}$ & 284 & 115914 & 1.36 & 0.26 & 24 \\
\hline LOC540543 & gi|84000355 & $\begin{array}{l}\text { hypothetical protein } \\
\text { LOC540543 [Bos taurus] } \\
\text { leukocyte receptor }\end{array}$ & 43 & 38096 & 2.45 & 1.21 & 2 \\
\hline LRC & gi|24308289 & $\begin{array}{l}\text { cluster (LRC) member } 1 \\
\text { [Homo sapiens] } \\
\text { LSM2 homolog, U6 }\end{array}$ & 0 & 33537 & 0.37 & & 1 \\
\hline LSm2 & gi|10863977 & $\begin{array}{l}\text { small nuclear RNA } \\
\text { associated [Homo } \\
\text { sapiens] }\end{array}$ & 129 & 12217 & 4.47 & 0.39 & 8 \\
\hline LSm3 & gi|7657315 & $\begin{array}{l}\text { Lsm3 protein [Homo } \\
\text { sapiens] } \\
\text { U6 snRNA-associated }\end{array}$ & 38 & 12414 & 3.21 & & 1 \\
\hline Lsm4 & gi|6912486 & $\begin{array}{l}\text { Sm-like protein } 4 \text { [Homo } \\
\text { sapiens] }\end{array}$ & 43 & 17207 & 5.52 & 2.31 & 2 \\
\hline LSm6 & gi|5901998 & $\begin{array}{l}\text { Sm protein F [Homo } \\
\text { sapiens] } \\
\text { U6 snRNA-associated }\end{array}$ & 56 & 10176 & 6.16 & 1.02 & 3 \\
\hline LSm7 & gi|7706423 & $\begin{array}{l}\text { Sm-like protein LSm7 } \\
\text { [Homo sapiens] } \\
\text { U6 snRNA-associated }\end{array}$ & 0 & 13272 & 3.70 & 0.08 & 2 \\
\hline LSm8 & gi|7706425 & $\begin{array}{l}\text { Sm-like protein LSm8 } \\
\text { [Homo sapiens] }\end{array}$ & 57 & 10684 & 4.14 & 0.70 & 2 \\
\hline LUC7A & gi|8922297 & $\begin{array}{l}\text { LUC7-like isoform a } \\
\text { [Homo sapiens] }\end{array}$ & 34 & 42306 & 4.20 & 0.88 & 2 \\
\hline Matrin3 & gi|57162250 & $\begin{array}{l}\text { novel protein [Homo } \\
\text { sapiens] }\end{array}$ & 0 & 16315 & 1.09 & & 1 \\
\hline Magoh & gi|4505087 & $\begin{array}{l}\text { mago-nashi homolog } \\
\text { [Homo sapiens] }\end{array}$ & 65 & 19216 & 0.20 & 0.05 & 5 \\
\hline MFAP1 & gi|50726968 & $\begin{array}{l}\text { microfibrillar-associated } \\
\text { protein } 1 \text { [Homo sapiens] }\end{array}$ & 216 & 58411 & 4.77 & 0.56 & 11 \\
\hline MGC13125 & gi|14249338 & $\begin{array}{l}\text { BUD13 homolog [Homo } \\
\text { sapiens] }\end{array}$ & 121 & 78018 & 0.94 & 0.38 & 10 \\
\hline $\begin{array}{l}\text { MGC20398 } \\
\text { (CCDC16) }\end{array}$ & gi|74732532 & $\begin{array}{l}\text { Coiled-coil domain- } \\
\text { containing protein } 16\end{array}$ & 333 & 47290 & 1.38 & 0.25 & 22 \\
\hline $\begin{array}{l}\text { MGC23918 } \\
\text { (CCDC12) }\end{array}$ & gi|21389497 & $\begin{array}{l}\text { coiled-coil domain } \\
\text { containing } 12 \text { [Homo } \\
\text { sapiens] } \\
\text { mitogen-activated }\end{array}$ & 92 & 22674 & 0.51 & 0.05 & 4 \\
\hline MORG1 & gi|14150114 & $\begin{array}{l}\text { protein kinase organizer } \\
1 \text { [Homo sapiens] }\end{array}$ & 120 & 37126 & 0.69 & 0.09 & 3 \\
\hline NCL & gi|189306 & nucleolin & 0 & 89313 & 1.30 & & 1 \\
\hline NF45 & gi|532313 & NF45 protein & 63 & 47880 & 1.94 & 0.32 & 4 \\
\hline
\end{tabular}




\begin{tabular}{|c|c|c|c|c|c|c|c|}
\hline Protein & $\begin{array}{l}\text { accession } \\
\text { number }\end{array}$ & $\begin{array}{l}\text { Protein database } \\
\text { description }\end{array}$ & $\begin{array}{l}\text { Protein } \\
\text { score }\end{array}$ & $\begin{array}{c}\text { Protein } \\
\text { mass } \\
\text { [Da] }\end{array}$ & $B / C$ & StDev & \# \\
\hline $\begin{array}{l}\mathrm{N}-\mathrm{myc} \text { and STAT } \\
\text { inetractor }\end{array}$ & gi|4758814 & $\begin{array}{l}\mathrm{N} \text {-myc and STAT } \\
\text { interactor [Homo } \\
\text { sapiens] }\end{array}$ & 43 & 39988 & 4.03 & 1.19 & 4 \\
\hline NOSIP & gi|7705716 & $\begin{array}{l}\text { eNOS interacting protein } \\
\text { [Homo sapiens] }\end{array}$ & 79 & 37167 & 0.19 & 0.09 & 3 \\
\hline Npw38 & gi|5031957 & $\begin{array}{l}\text { polyglutamine binding } \\
\text { protein } 1 \text { [Homo sapiens] } \\
\text { WW domain binding }\end{array}$ & 69 & 33814 & 7.68 & 1.60 & 3 \\
\hline Npw38BP & gi|7706501 & $\begin{array}{l}\text { protein } 11 \text { [Homo } \\
\text { sapiens] } \\
\text { nuclear fragile } X \text { mental }\end{array}$ & 236 & 77303 & 8.77 & 2.32 & 17 \\
\hline NUFIP1 & gi|6912542 & $\begin{array}{l}\text { retardation protein } \\
\text { interacting protein } 1 \\
\text { [Homo sapiens] }\end{array}$ & 48 & 64004 & 0.33 & 0.02 & 2 \\
\hline Numa 1 & gi|35119 & $\begin{array}{l}\text { NuMA protein [Homo } \\
\text { sapiens] }\end{array}$ & 30 & 260907 & 3.11 & & 1 \\
\hline NY-CO-10 & gi|3170184 & $\begin{array}{l}\text { antigen NY-CO-10 } \\
\text { [Homo sapiens] }\end{array}$ & 27 & 49863 & 0.68 & 0.05 & 2 \\
\hline p68(DDX5) & gi|226021 & $\begin{array}{l}\text { growth regulated nuclear } \\
68 \text { protein } \\
\text { DEAD (Asp-Glu-Ala- }\end{array}$ & 151 & 72338 & 2.30 & 0.45 & 15 \\
\hline p72/DDX17 & gil119580652 & $\begin{array}{l}\text { Asp) box polypeptide 17, } \\
\text { isoform CRA_h [Homo } \\
\text { sapiens] }\end{array}$ & 49 & 64210 & 2.30 & 0.73 & 2 \\
\hline PABP & gi|35570 & $\begin{array}{l}\text { unnamed protein product } \\
\text { [Homo sapiens] }\end{array}$ & 55 & 76372 & 4.72 & 2.46 & 4 \\
\hline PABP & gi|35570 & $\begin{array}{l}\text { unnamed protein product } \\
\text { [Homo sapiens] } \\
\text { polv A bindina protein }\end{array}$ & 42 & 76372 & 0.83 & 0.25 & 2 \\
\hline PABPC4 & gi|4504715 & $\begin{array}{l}\text { cytoplasmic } 4 \text { [Homo } \\
\text { sapiens] } \\
\text { polv(A) binding protein. }\end{array}$ & 0 & 77931 & 0.99 & & 1 \\
\hline PABPN1 & gi|4758876 & $\begin{array}{l}\text { nuclear } 1 \text { [Homo } \\
\text { sapiens] }\end{array}$ & 33 & 34406 & 0.79 & 0.00 & 2 \\
\hline PCBP1 & gi|5453854 & $\begin{array}{l}\text { poly }(\mathrm{rC}) \text { binding protein } \\
1 \text { [Homo sapiens] } \\
\text { poly }(\mathrm{rC}) \text {-binding protein }\end{array}$ & 133 & 40077 & 4.66 & 1.45 & 10 \\
\hline PCBP2 & gi|14141166 & $\begin{array}{l}2 \text { isoform b [Homo } \\
\text { sapiens] }\end{array}$ & 121 & 41113 & 3.71 & 1.04 & 16 \\
\hline Pinin & gi|3021392 & $\begin{array}{l}\text { nuclear protein SDK3 } \\
\text { [Homo sapiens] }\end{array}$ & 23 & 89790 & 1.89 & 0.06 & 3 \\
\hline plakophilin & gi|6005830 & $\begin{array}{l}\text { plakophilin } 3 \text { [Homo } \\
\text { sapiens] }\end{array}$ & 0 & 91432 & 2.49 & & 1 \\
\hline PPIL1 & gi|7706339 & $\begin{array}{l}\text { peptidylprolyl isomerase- } \\
\text { like } 1 \text { [Homo sapiens] } \\
\text { peptidylprolyl isomerase- }\end{array}$ & 68 & 19902 & 0.45 & 0.08 & 8 \\
\hline PPIL2 & gi|7657473 & $\begin{array}{l}\text { like } 2 \text { isoform a [Homo } \\
\text { sapiens] } \\
\text { Peptidylprolyl isomerase }\end{array}$ & 477 & 66360 & 2.96 & 0.74 & 32 \\
\hline PPIL3b & gi|14043400 & $\begin{array}{l}\text { (cyclophilin)-like } 3 \\
\text { [Homo sapiens] }\end{array}$ & 58 & 20214 & 0.31 & 0.19 & 3 \\
\hline PPIL4 & gi|20911035 & $\begin{array}{l}\text { peptidylprolyl isomerase- } \\
\text { like } 4 \text { [Homo sapiens] } \\
\text { Papillary renal cell } \\
\text { carcinoma }\end{array}$ & 42 & 65817 & 2.91 & & 1 \\
\hline PRCC & gi|14714625 & $\begin{array}{l}\text { (translocation- } \\
\text { associated) [Homo } \\
\text { sapiens] }\end{array}$ & 98 & 57721 & 2.94 & 0.18 & 4 \\
\hline
\end{tabular}




\begin{tabular}{|c|c|c|c|c|c|c|c|}
\hline Protein & $\begin{array}{l}\text { accession } \\
\text { number }\end{array}$ & $\begin{array}{l}\text { Protein database } \\
\text { description }\end{array}$ & $\begin{array}{l}\text { Protein } \\
\text { score }\end{array}$ & $\begin{array}{c}\text { Protein } \\
\text { mass } \\
\text { [Da] }\end{array}$ & $B / C$ & StDev & $\#$ \\
\hline PRKRIP1 & gi|13375901 & $\begin{array}{l}\text { PRKR interacting protein } \\
1 \text { (IL11 inducible) [Homo } \\
\text { sapiens] } \\
\text { pleiotropic regulator } 1\end{array}$ & 36 & 24875 & 0.11 & 0.06 & 4 \\
\hline PRL1 & gi|4505895 & $\begin{array}{l}\text { (PRL1 homolog, } \\
\text { Arabidopsis) [Homo } \\
\text { sapiens] } \\
\text { PRP4 pre-mRNA }\end{array}$ & 26 & 62478 & 1.06 & 0.22 & 6 \\
\hline hPRP4-Kinase & gi|23271009 & $\begin{array}{l}\text { processing factor } 4 \\
\text { homolog B (yeast) }\end{array}$ & 268 & 135785 & 0.37 & 0.07 & 18 \\
\hline PUF60 & gi|6176532 & $\begin{array}{l}\text { poly-U binding splicing } \\
\text { factor PUF60 [Homo } \\
\text { sapiens] }\end{array}$ & 331 & 64428 & 4.26 & 1.35 & 19 \\
\hline Q9BRR8 & gi|21361684 & $\begin{array}{l}\text { G patch domain } \\
\text { containing } 1 \text { [Homo } \\
\text { sapiens] }\end{array}$ & 141 & 116769 & 0.19 & 0.07 & 9 \\
\hline RALY & gi|3334899 & $\begin{array}{l}\text { e1b-55kDa-associated } \\
\text { protein [Homo sapiens] }\end{array}$ & 45 & 33780 & 2.48 & 0.68 & 3 \\
\hline RBM10 & gi|1469167 & $\begin{array}{l}\text { KIAA0122 [Homo } \\
\text { sapiens] }\end{array}$ & 98 & 120110 & 5.09 & 1.05 & 4 \\
\hline RBM15(OTT) & gi|10433990 & $\begin{array}{l}\text { unnamed protein product } \\
\text { [Homo sapiens] } \\
\text { RNA binding motif }\end{array}$ & 108 & 108615 & 1.91 & 0.26 & 9 \\
\hline RBM18 & gi|14916461 & $\begin{array}{l}\text { protein } 18 \text { [Homo } \\
\text { sapiens] }\end{array}$ & 62 & 25042 & 0.42 & & 1 \\
\hline RBM5/LUCA & gi|1244404 & $\begin{array}{l}\text { putative tumor } \\
\text { suppressor } \\
\text { IK cytokine, down- }\end{array}$ & 28 & 100212 & 4.99 & 0.36 & 2 \\
\hline RED & gi|119582433 & $\begin{array}{l}\text { regulator of HLA II, } \\
\text { isoform CRA_b [Homo } \\
\text { sapiens] }\end{array}$ & 280 & 75577 & 7.21 & 2.03 & 20 \\
\hline RNF 113A & gi|5902158 & $\begin{array}{l}\text { ring finger protein 113A } \\
\text { [Homo sapiens] } \\
\text { RNA binding motif }\end{array}$ & 50 & 43684 & 1.20 & 0.13 & 3 \\
\hline RNPC2(CAPER) & gi|4757926 & $\begin{array}{l}\text { protein } 39 \text { isoform b } \\
\text { [Homo sapiens] }\end{array}$ & 295 & 64182 & 4.07 & 0.73 & 13 \\
\hline RNPS1 & gi|3253165 & $\begin{array}{l}\text { SR protein [Homo } \\
\text { sapiens] }\end{array}$ & 41 & 38412 & 2.09 & & 1 \\
\hline RSRC1 & gi|14714462 & $\begin{array}{l}\text { RSRC1 protein [Homo } \\
\text { sapiens] } \\
\text { S100 calcium-binding }\end{array}$ & 75 & 35833 & 1.69 & 0.54 & 4 \\
\hline $\mathrm{S} 100 \mathrm{~A} 9$ & gi|4506773 & $\begin{array}{l}\text { protein A9 [Homo } \\
\text { sapiens] } \\
\text { RNA binding motif }\end{array}$ & 0 & 15009 & 89.37 & & 1 \\
\hline S164 & gi|55741709 & $\begin{array}{l}\text { protein } 25 \text { [Homo } \\
\text { sapiens] }\end{array}$ & 47 & 113081 & 3.22 & 0.48 & 4 \\
\hline SAP18 & gi|5032067 & $\begin{array}{l}\text { Sin3A-associated } \\
\text { protein, 18kDa [Homo } \\
\text { sapiens] }\end{array}$ & 33 & 19469 & 1.19 & 0.17 & 6 \\
\hline SEMG I & gi|487420 & SEMGI & 133 & 58184 & 14.26 & 5.79 & 4 \\
\hline SEMG II & gi|4506885 & $\begin{array}{l}\text { semenogelin II precursor } \\
\text { [Homo sapiens] }\end{array}$ & 48 & 73999 & 28.49 & & 1 \\
\hline SF2/ASF & gi|179074 & $\begin{array}{l}\text { alternative } \\
\text { splicing factor } 3 a \text {, }\end{array}$ & 138 & 33984 & 2.46 & 0.37 & 8 \\
\hline SF3a120 & gi|5032087 & $\begin{array}{l}\text { subunit 1, 120kDa } \\
\text { isoform } 1 \text { [Homo } \\
\text { sapiens] }\end{array}$ & 465 & 97523 & 2.64 & 0.44 & 29 \\
\hline
\end{tabular}




\begin{tabular}{|c|c|c|c|c|c|c|c|}
\hline Protein & $\begin{array}{l}\text { accession } \\
\text { number }\end{array}$ & $\begin{array}{l}\text { Protein database } \\
\text { description }\end{array}$ & $\begin{array}{c}\text { Protein } \\
\text { score }\end{array}$ & $\begin{array}{l}\text { Protein } \\
\text { mass } \\
\text { [Da] }\end{array}$ & $B / C$ & StDev & \# \\
\hline SF3a60 & gi|551450 & $\begin{array}{l}\text { splicing factor SF3a60 } \\
\text { [Homo sapiens] }\end{array}$ & 277 & 65068 & 6.68 & 0.65 & 14 \\
\hline SF3a66 & gi|409219 & spiceosomal protein & 162 & 52572 & 8.09 & 0.43 & 6 \\
\hline SF3b125 & gi|3435312 & $\begin{array}{l}\text { RNA helicase-related } \\
\text { protein [Homo sapiens] } \\
\text { spliceosomal protein }\end{array}$ & 83 & 84712 & 6.19 & 2.07 & 6 \\
\hline SF3b130 & gi|6006515 & $\begin{array}{l}\text { SAP } 130 \text { [Homo } \\
\text { sapiens] } \\
\text { splicing factor } 3 b \text {, }\end{array}$ & 294 & 144018 & 7.46 & 1.45 & 20 \\
\hline SF3b130 & gi|54112121 & $\begin{array}{l}\text { subunit } 3 \text { [Homo } \\
\text { sapiens] } \\
\text { Splicing factor 3B } \\
\text { subunit } 2 \text { (Spliceosome- } \\
\text { associated protein 145) }\end{array}$ & 498 & 144003 & 3.52 & 0.72 & 49 \\
\hline SF3b145 & gi|2498883 & $\begin{array}{l}\text { (SAP 145) (SF3b150) } \\
\text { (Pre-mRNA-splicing } \\
\text { factor SF3b 145 kDa } \\
\text { subunit) } \\
\text { splicing factor 3B, } 14\end{array}$ & 441 & 109792 & 4.13 & 0.93 & 34 \\
\hline SF3b14a/p14 & gi|7706326 & $\begin{array}{l}\text { kDa subunit [Homo } \\
\text { sapiens] }\end{array}$ & 179 & 16541 & 4.37 & 0.60 & 14 \\
\hline SF3b14b & gi|14249398 & $\begin{array}{l}\text { PHD-finger } 5 \mathrm{~A} \text { [Homo } \\
\text { sapiens] } \\
\text { splicing factor } 3 \mathrm{~b},\end{array}$ & 44 & 14868 & 2.00 & 0.25 & 2 \\
\hline SF3b155 & gi|54112117 & $\begin{array}{l}\text { subunit } 1 \text { isoform } 1 \\
\text { [Homo sapiens] } \\
\text { splicing factor } 3 \mathrm{~b}\end{array}$ & 765 & 158585 & 3.66 & 2.15 & 118 \\
\hline SF3b49 & gi|5032069 & $\begin{array}{l}\text { subunit } 4 \text { [Homo } \\
\text { sapiens] } \\
\text { splicing factor, }\end{array}$ & 63 & 46421 & 5.33 & 2.56 & 4 \\
\hline SFRS11 & gi|4759100 & $\begin{array}{l}\text { arginine/serine-rich } 11 \\
\text { [Homo sapiens] } \\
\text { Similar to expressed }\end{array}$ & 50 & 61240 & 3.21 & & 1 \\
\hline SFRS12 & gi|28703790 & $\begin{array}{l}\text { sequence Al450757 } \\
\text { [Homo sapiens] } \\
550 \text { amino acids } \\
\text { MW=61kDa, } \\
\text { glycosylated=75 kDa; } \\
\text { expressed on } \\
\text { endothelium, activated }\end{array}$ & 95 & 46956 & 2.80 & 0.59 & 6 \\
\hline SFRS17A & gi|187242 & $\begin{array}{l}\text { lymphocytes and } \\
\text { syncytiotrophoblast, } \\
\text { contains leucine zipper } \\
\text { and basic region } \\
\text { homologous to myc; } \\
721 \mathrm{P}\end{array}$ & 33 & 71752 & 0.41 & 0.01 & 2 \\
\hline SKIP & gi|6912676 & $\begin{array}{l}\text { SKI-interacting protein } \\
\text { [Homo sapiens] }\end{array}$ & 268 & 69284 & 0.63 & 0.10 & 42 \\
\hline SKIV2L2 & gi|6633995 & $\begin{array}{l}\text { KIAA0052 protein [Homo } \\
\text { sapiens] }\end{array}$ & 78 & 131978 & 1.15 & 0.19 & 8 \\
\hline SmB & gi|190247 & $\begin{array}{l}\text { snRNP polypeptide B } \\
\text { small nuclear }\end{array}$ & 84 & 31604 & 1.70 & 0.29 & 15 \\
\hline SmD1 & gi|5902102 & $\begin{array}{l}\text { ribonucleoprotein D1 } \\
\text { polypeptide 16kDa } \\
\text { [Homo sapiens] }\end{array}$ & 111 & 14858 & 1.86 & 0.51 & 7 \\
\hline
\end{tabular}




\begin{tabular}{|c|c|c|c|c|c|c|c|}
\hline Protein & $\begin{array}{l}\text { accession } \\
\text { number }\end{array}$ & $\begin{array}{l}\text { Protein database } \\
\text { description }\end{array}$ & $\begin{array}{l}\text { Protein } \\
\text { score }\end{array}$ & $\begin{array}{c}\text { Protein } \\
\text { mass } \\
\text { [Da] }\end{array}$ & $\mathrm{B} / \mathrm{C}$ & StDev & \# \\
\hline SmD2 & gi|4759158 & \begin{tabular}{|l|} 
small nuclear \\
ribonucleoprotein \\
polypeptide D2 [Homo \\
sapiens] \\
small nuclear
\end{tabular} & 94 & 16204 & 1.88 & 0.30 & 22 \\
\hline SmD3 & gi|4759160 & $\begin{array}{l}\text { ribonucleoprotein } \\
\text { polypeptide D3 [Homo } \\
\text { sapiens] } \\
\text { small nuclear }\end{array}$ & 118 & 15584 & 2.08 & 0.44 & 19 \\
\hline SmE & gi|4507129 & $\begin{array}{l}\text { ribonucleoprotein } \\
\text { polypeptide E [Homo } \\
\text { sapiens] } \\
\text { small nuclear }\end{array}$ & 179 & 11851 & 2.03 & 0.47 & 7 \\
\hline SmF & gi|4507131 & $\begin{array}{l}\text { ribonucleoprotein } \\
\text { polypeptide F [Homo } \\
\text { sapiens] }\end{array}$ & 126 & 10629 & 1.40 & 0.37 & 6 \\
\hline SmG & gi|4507133 & $\begin{array}{l}\text { small nuclear } \\
\text { ribonucleoprotein } \\
\text { polypeptide G [Homo } \\
\text { sapiens] }\end{array}$ & 145 & 9545 & 1.19 & 0.09 & 5 \\
\hline hSmu-1 & gi|7023065 & $\begin{array}{l}\text { unnamed protein product } \\
\text { [Homo sapiens] }\end{array}$ & 93 & 62752 & 4.00 & 0.65 & 4 \\
\hline SNIP1 & gi|21314720 & $\begin{array}{l}\text { Smad nuclear interacting } \\
\text { protein [Homo sapiens] }\end{array}$ & 26 & 49687 & 1.66 & 0.15 & 2 \\
\hline SON3 (DBP-5) & gi|17046381 & $\begin{array}{l}\text { SON DNA binding } \\
\text { protein isoform E [Homo } \\
\text { sapiens] } \\
\text { spen homolog }\end{array}$ & 44 & 240773 & 1.38 & 0.06 & 2 \\
\hline Spen & gi|14790190 & $\begin{array}{l}\text { spen nomolog, } \\
\text { transcriptional regulator } \\
\text { [Homo sapiens] } \\
\text { breast carcinoma }\end{array}$ & 55 & 440442 & 7.31 & 2.57 & 4 \\
\hline SPF27 & gi|5031653 & $\begin{array}{l}\text { amplified sequence } 2 \\
\text { [Homo sapiens] } \\
\text { RNA binding motif }\end{array}$ & 202 & 28080 & 0.55 & 0.12 & 15 \\
\hline SPF45 & gi|14249678 & $\begin{array}{l}\text { protein } 17 \text { [Homo } \\
\text { sapiens] }\end{array}$ & 70 & 50450 & 6.22 & 0.84 & 6 \\
\hline SR140 & gi|2224605 & $\begin{array}{l}\text { KIAA0332 [Homo } \\
\text { sapiens] }\end{array}$ & 55 & 134163 & 4.82 & 0.80 & 5 \\
\hline SRm300 & gi|71891780 & $\begin{array}{l}\text { KIAA0324 protein [Homo } \\
\text { sapiens] }\end{array}$ & 150 & 323720 & 1.63 & 0.85 & 4 \\
\hline SRp20 & gi|2125864 & $\begin{array}{l}\text { Srp20 [Mus musculus] } \\
\text { splicing factor, }\end{array}$ & 40 & 14955 & 2.53 & & 1 \\
\hline SRp30c & gi|4506903 & $\begin{array}{l}\text { arginine/serine-rich } 9 \\
\text { [Homo sapiens] } \\
\text { PREDICTED: splicing }\end{array}$ & 21 & 26915 & 0.53 & & 1 \\
\hline SRp40 & gi|55640963 & $\begin{array}{l}\text { factor, arginine/serine- } \\
\text { rich } 5 \text { isoform } 6 \text { [Pan } \\
\text { troglodytes] } \\
\text { arginine/serine-rich }\end{array}$ & 40 & 33844 & 1.83 & 0.26 & 5 \\
\hline SRp55 & gi|20127499 & $\begin{array}{l}\text { splicing factor } 6 \text { [Homo } \\
\text { sapiens] }\end{array}$ & 87 & 43546 & 0.88 & 0.18 & 7 \\
\hline SRp75 & gi|2914669 & $\begin{array}{l}\text { SRP0001LB [Homo } \\
\text { sapiens] }\end{array}$ & 25 & 24207 & 1.16 & 0.17 & 2 \\
\hline TARDBP & gi|6678271 & $\begin{array}{l}\text { TAR DNA binding } \\
\text { protein [Homo sapiens] }\end{array}$ & 33 & 48013 & 5.26 & 1.78 & 2 \\
\hline tat SF1 & gi|1667611 & Tat-SF1 [Homo sapiens] & 30 & 96255 & 1.13 & & 1 \\
\hline
\end{tabular}




\begin{tabular}{|c|c|c|c|c|c|c|c|}
\hline Protein & $\begin{array}{l}\text { accession } \\
\text { number }\end{array}$ & $\begin{array}{l}\text { Protein database } \\
\text { description }\end{array}$ & $\begin{array}{l}\text { Protein } \\
\text { score }\end{array}$ & $\begin{array}{c}\text { Protein } \\
\text { mass } \\
\text { [Da] }\end{array}$ & $B / C$ & StDev & $\#$ \\
\hline TCERG1 & gi|21327715 & $\begin{array}{l}\text { transcription elongation } \\
\text { regulator } 1 \text { isoform } 1 \\
\text { [Homo sapiens] }\end{array}$ & 27 & 138273 & 4.52 & 0.71 & 6 \\
\hline TCP1 & gi|1800303 & $\begin{array}{l}\text { HIV-1 Nef interacting } \\
\text { protein [Homo sapiens] }\end{array}$ & 31 & 50858 & 0.64 & 0.09 & 2 \\
\hline TF IIB & gi|8392875 & $\begin{array}{l}\text { transcription factor IIB } \\
\text { [Homo sapiens] }\end{array}$ & 25 & 25348 & 3.61 & & 1 \\
\hline TFIP11 & gi|8393259 & $\begin{array}{l}\text { tuftelin interacting } \\
\text { protein } 11 \text { [Homo } \\
\text { sapiens] }\end{array}$ & 341 & 106591 & 1.01 & 0.08 & 22 \\
\hline THOC2 & gi|52486999 & $\begin{array}{l}\text { THO complex } 2 \text { isoform } \\
2 \text { [Homo sapiens] }\end{array}$ & 57 & 196891 & 1.54 & 0.26 & 7 \\
\hline THOC1 & gi|37999906 & $\begin{array}{l}\text { (Tho1) (Nuclear matrix } \\
\text { protein p84) }\end{array}$ & 74 & 83277 & 1.77 & 0.17 & 3 \\
\hline THOC7 & gi|34783006 & $\begin{array}{l}\text { THOC7 protein [Homo } \\
\text { sapiens] }\end{array}$ & 31 & 26105 & 1.81 & 0.47 & 2 \\
\hline THRAP3 & gi|4827040 & $\begin{array}{l}\text { thyroid hormone } \\
\text { receptor associated } \\
\text { protein } 3 \text { [Homo sapiens] }\end{array}$ & 86 & 123229 & 1.19 & 0.08 & 4 \\
\hline TPR14 (TTC14) & gi|37182643 & $\begin{array}{l}\text { DRDL5813 [Homo } \\
\text { sapiens] }\end{array}$ & 130 & 82362 & 0.32 & 0.10 & 11 \\
\hline tubulin alpha-2 & gi|34740335 & $\begin{array}{l}\text { tubulin, alpha } 2 \text { [Mus } \\
\text { musculus] }\end{array}$ & 341 & 53554 & 1.09 & 0.22 & 33 \\
\hline tubulin beta-5 & gi|7106439 & $\begin{array}{l}\text { tubulin, beta } 5 \text { [Mus } \\
\text { musculus] }\end{array}$ & 372 & 52313 & 0.94 & 0.25 & 29 \\
\hline U1-70K & gi|36100 & $\begin{array}{l}\text { unnamed protein product } \\
\text { [Homo sapiens] } \\
\text { small nuclear }\end{array}$ & 61 & 73878 & 7.08 & 1.10 & 7 \\
\hline U1-A & gi|4759156 & $\begin{array}{l}\text { ribonucleoprotein } \\
\text { polypeptide A [Homo } \\
\text { sapiens] } \\
\text { small nuclear }\end{array}$ & 117 & 34574 & 22.35 & 7.32 & 5 \\
\hline U2-A' & gi|50593002 & $\begin{array}{l}\text { ribonucleoprotein } \\
\text { polypeptide A [Homo } \\
\text { sapiens] } \\
\text { small nuclear }\end{array}$ & 259 & 31660 & 1.39 & 0.20 & 24 \\
\hline U2-B" & gi|4507123 & $\begin{array}{l}\text { ribonucleoprotein } \\
\text { polypeptide B [Homo } \\
\text { sapiens] } \\
\text { squamous cell }\end{array}$ & 122 & 29217 & 1.74 & 0.42 & 12 \\
\hline U4/U6.U5-110K & gi|10863889 & $\begin{array}{l}\text { carcinoma antigen } \\
\text { recognized by T cells } 1 \\
\text { [Homo sapiens] } \\
\text { U4/U6.U5 tri-snRNP. }\end{array}$ & 338 & 101434 & 3.60 & 1.09 & 19 \\
\hline U4/U6.U5-65K & gi|13926071 & $\begin{array}{l}\text { associated } 65 \mathrm{kDa} \\
\text { protein [Homo sapiens] } \\
\text { NHP2 non-histone }\end{array}$ & 353 & 72324 & 1.63 & 0.21 & 19 \\
\hline U4/U6-15.5K & gi|4826860 & $\begin{array}{l}\text { chromosome protein 2- } \\
\text { like } 1 \text { [Homo sapiens] }\end{array}$ & 107 & 15934 & 16.96 & 0.28 & 2 \\
\hline U4/U6-20K & gi|5454154 & $\begin{array}{l}\text { peptidylprolyl isomerase } \\
\text { H [Homo sapiens] } \\
\text { U4/U6 small nuclear }\end{array}$ & 83 & 21299 & 2.55 & 0.40 & 4 \\
\hline U4/U6-60K & gi|2708305 & $\begin{array}{l}\text { ribonucleoprotein hPrp4 } \\
\text { [Homo sapiens] }\end{array}$ & 364 & 64027 & 5.83 & 0.85 & 20 \\
\hline
\end{tabular}




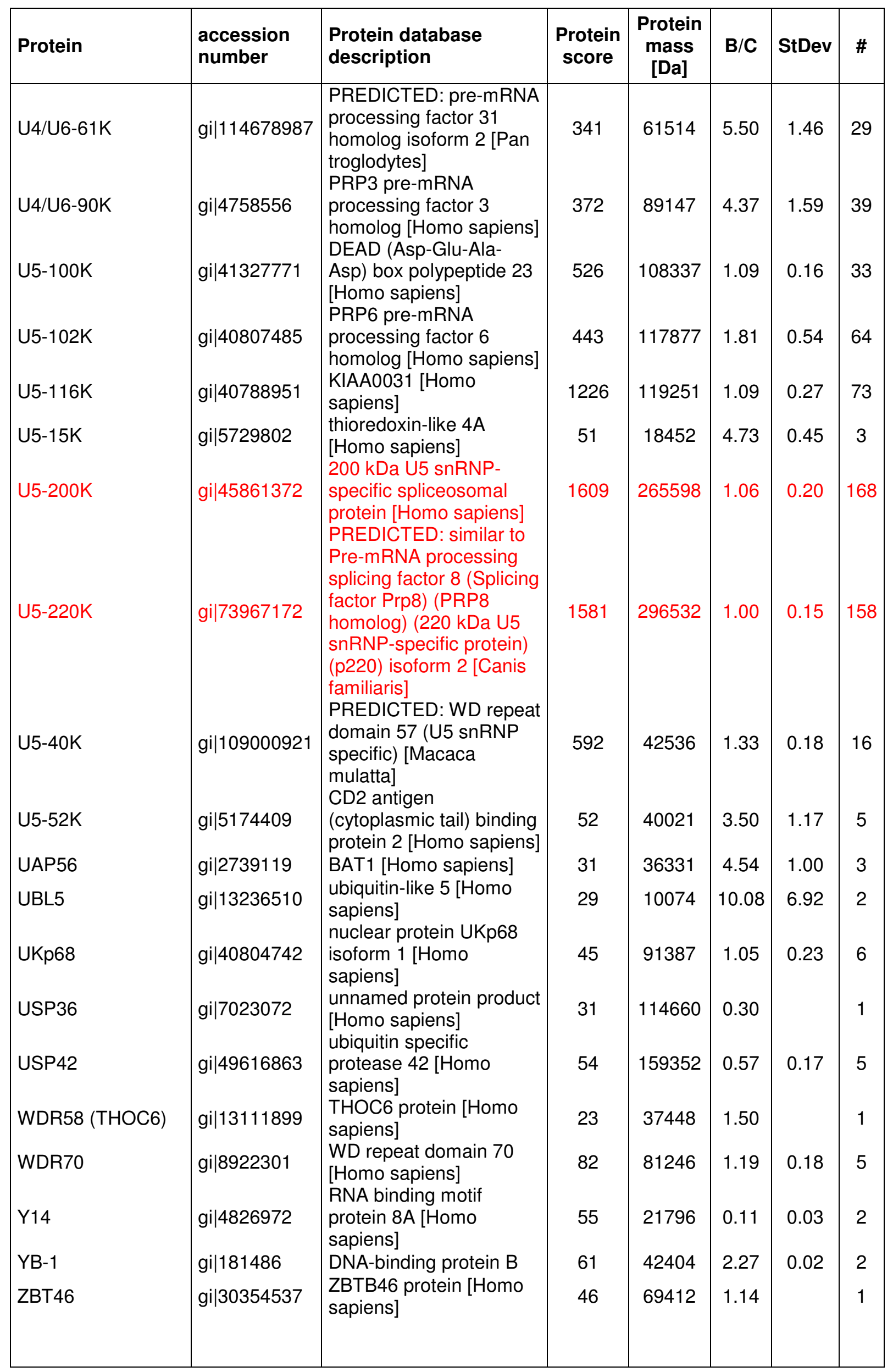




\begin{tabular}{|c|c|c|c|c|c|c|c|}
\hline Protein & $\begin{array}{l}\text { accession } \\
\text { number }\end{array}$ & $\begin{array}{l}\text { Protein database } \\
\text { description }\end{array}$ & $\begin{array}{l}\text { Protein } \\
\text { score }\end{array}$ & $\begin{array}{c}\text { Protein } \\
\text { mass } \\
{[\mathrm{Da}]}\end{array}$ & $\mathrm{B} / \mathrm{C}$ & StDev & \# \\
\hline ZCCHC19 & gi|51243065 & $\begin{array}{l}\text { zinc finger CCHC-type } \\
\text { and RNA binding motif } 1 \\
\text { [Homo sapiens] }\end{array}$ & 32 & 29083 & 1.38 & 0.01 & 2 \\
\hline $\mathrm{ZCCHC8}$ & gi|14042579 & $\begin{array}{l}\text { unnamed protein product } \\
\text { [Homo sapiens] }\end{array}$ & 79 & 85705 & 0.94 & 0.09 & 6 \\
\hline ZNF & gi|14330434 & $\begin{array}{l}\text { putative zinc finger } \\
\text { protein [Homo sapiens] }\end{array}$ & 0 & 127231 & 0.57 & & 1 \\
\hline ZNF432 & gi|40788368 & $\begin{array}{l}\text { KIAA0798 protein [Homo } \\
\text { sapiens] }\end{array}$ & 0 & 89866 & 1.19 & & 1 \\
\hline
\end{tabular}


Table A.7: Proteins that were found to be enriched in light (L), medium (M) or heavy (H) nuclear extracts, respectively. The protein ratios obtained for comparison of medium to light nuclear extract $(\mathrm{M} / \mathrm{L})$ and heavy to light nuclear extract $(\mathrm{H} / \mathrm{L})$ are given.

\begin{tabular}{|c|c|c|c|c|c|}
\hline \multirow[t]{2}{*}{ Protein name } & \multirow[t]{2}{*}{ Gene name } & \multicolumn{2}{|c|}{$\begin{array}{c}\text { Protein } \\
\text { ratio }\end{array}$} & \multicolumn{2}{|c|}{$\begin{array}{c}\text { enriched } \\
\text { in }\end{array}$} \\
\hline & & $M / L$ & $H / L$ & L $\quad M$ & $\mathbf{H}$ \\
\hline ATPase family AAA domain-containing protein 3B & ATAD3B & 0.490 & 0.239 & - & \\
\hline Dermcidin & DCD & 0.173 & 0.081 & $\bullet$ & \\
\hline Disks large homolog 2 & DLG2 & 0.183 & 0.032 & $\bullet$ & \\
\hline Dystrophin & DMD & 0.038 & 0.026 & $\bullet$ & \\
\hline Desmoglein-1 & DSG1 & 0.126 & 0.055 & $\bullet$ & \\
\hline Protein FAM124B & FAM124B & 0.023 & 0.012 & $\bullet$ & \\
\hline Filaggrin-2 & FLG2 & 0.079 & 0.071 & $\bullet$ & \\
\hline Hornerin & HRNR & 0.066 & 0.021 & $\bullet$ & \\
\hline Interferon-induced $17 \mathrm{kDa}$ protein & ISG15 & 1.424 & 2.343 & & • \\
\hline Junction plakoglobin & JUP & 0.574 & 0.342 & - & \\
\hline Keratinocyte proline-rich protein & KPRP & 0.039 & 0.033 & $\bullet$ & \\
\hline Krev interaction trapped protein 1 & KRIT1 & 0.024 & 0.048 & - & \\
\hline Lysozyme C & LYZ & 0.145 & 0.194 & $\bullet$ & \\
\hline Mannosidase, alpha, class $1 \mathrm{~A}$, member 1 , isoform CRA_a & MAN1A1 & 0.034 & 7.796 & - & • \\
\hline highly similar to Septin-2 & NEDD5 & 0.190 & 0.691 & $\bullet$ & \\
\hline highly similar to Septin-9 & Ov/Br septin & 0.184 & 0.695 & $\bullet$ & \\
\hline tRNA pseudouridine synthase A & PUS1 & 1.341 & 0.304 & $\bullet$ & \\
\hline Protein S100-A9 & S100A9 & 0.222 & 0.317 & $\bullet$ & \\
\hline Semenogelin-1 & SEMG1 & 0.078 & 0.079 & $\bullet$ & \\
\hline Septin-7 & SEPT7 & 0.205 & 0.701 & $\bullet$ & \\
\hline
\end{tabular}


Table A.8: List of ribosomal proteins used for normalization. The protein ratios obtained for comparison of medium to light nuclear extract $(\mathrm{M} / \mathrm{L})$ and heavy to light nuclear extract $(\mathrm{H} / \mathrm{L})$ are given. The significance for the proteins to be up- or down regulated within the different nuclear extracts is listed.

\begin{tabular}{|l|cc|cc|}
\hline $\begin{array}{l}\text { Ribosomal } \\
\text { Protein }\end{array}$ & $\begin{array}{c}\text { Protein Ratio } \\
\mathrm{M} / \mathrm{L}\end{array}$ & $\begin{array}{c}\text { Significance } \\
\mathrm{M} / \mathrm{L}\end{array}$ & $\begin{array}{c}\text { Protein Ratio } \\
\mathrm{H} / \mathrm{L}\end{array}$ & $\begin{array}{c}\text { Significance } \\
\mathrm{H} / \mathrm{L}\end{array}$ \\
\hline & & & & \\
RPS7 & 1.076 & 0.3603 & 0.881 & 0.2092 \\
RPS27 & 1.076 & 0.3588 & 0.845 & 0.1410 \\
RPS20 & 1.088 & 0.3389 & 0.781 & 0.0569 \\
RPS14 & 1.090 & 0.3352 & 0.948 & 0.3668 \\
RPS19 & 1.102 & 0.3134 & 0.815 & 0.0952 \\
RPS11 & 1.109 & 0.3027 & 0.859 & 0.1658 \\
RPS3 & 1.115 & 0.2933 & 0.811 & 0.0898 \\
RPS21 & 1.130 & 0.2684 & 0.825 & 0.1097 \\
RPS16 & 1.137 & 0.2587 & 0.834 & 0.1231 \\
RPS4X & 1.141 & 0.2526 & 0.828 & 0.1138 \\
RPS17 & 1.141 & 0.2517 & 0.870 & 0.1862 \\
RPS25 & 1.142 & 0.2512 & 0.849 & 0.1475 \\
RPS10 & 1.148 & 0.2420 & 0.832 & 0.1194 \\
RPS23 & 1.169 & 0.2133 & 0.884 & 0.2161 \\
RPS15A & 1.177 & 0.2028 & 0.843 & 0.1376 \\
RPS28 & 1.183 & 0.1955 & 0.926 & 0.3111 \\
RPS5 & 1.190 & 0.1874 & 0.830 & 0.1173 \\
RPS3A & 1.207 & 0.1680 & 0.915 & 0.2844 \\
RPS12 & 1.211 & 0.1635 & 0.831 & 0.1176 \\
RPS9 & 1.264 & 0.1144 & 0.898 & 0.2466 \\
RPL8 & 1.274 & 0.1066 & 0.828 & 0.1144 \\
RPL18A & 1.299 & 0.0890 & 0.864 & 0.1745 \\
RPL38 & 1.328 & 0.0717 & 0.863 & 0.1732 \\
\hline
\end{tabular}

Table A.9: List of normalization factors calculated for the different assembly studies. For every time point of the protein assembly on different pre-mRNAs a normalization factor was calculated. The normalization factors were applied to the protein ratios obtained.

\begin{tabular}{|c|c|c|c|c|c|c|}
\hline protein assembly on: & $2^{\prime} / 0^{\prime}$ & $5^{\prime} / 0^{\prime}$ & $10^{\prime} / 0^{\prime}$ & $15^{\prime} / 0^{\prime}$ & $20^{\prime} / 0^{\prime}$ & $30^{\prime} / 0^{\prime}$ \\
\hline PM5 pre-mRNA & 1.023 & 2.108 & 1.399 & 2.395 & 0.868 & 1.526 \\
\hline 5'ss-deleted PM5 pre-mRNA & 1.139 & 1.484 & 1.171 & 1.249 & 1.114 & 2.031 \\
\hline BPS-deleted PM5 pre-mRNA & 2.244 & 3.448 & 1.116 & 1.753 & 0.986 & 2.171 \\
\hline
\end{tabular}




\section{List of abbreviations}

$\%$

1D

2D

3'ss

5'ss

AAA

ACN

approx.

APS

AQUA

ATP

16BAC

BPS

BSA

${ }^{\circ} \mathrm{C}$

C.elegans

CE

$\mathrm{Ci}$

CICAT

$\mathrm{cm}$

cpm

CTP

CXP

D.melanogaster

$\mathrm{Da}$

DDA

DMEM

DMSO

DNA

DP

dpm

DTE

DTT

e.g.

EDTA

emPAl

EP

EPI

ER

ESI

et al.

FA

FBS

FLEXIQuant

fmol

FT

FT ICR

FWHM

g

GTP

$\mathrm{h}$

$\mathrm{H}$

HEPES

$\mathrm{HIC}$ percent

one dimensional

two dimensional

3 ' splice site

5' splice site

amino acid analysis

acetonitrile

Approximatelya

Ammonium peroxodisulfate

absolute quantification

adenosine 5'-triphosphate

benzyldimethyl-n-hexadecylammonium chloride

branch point site

Bovine serum albumin

degree Celsius

Caenorhabditis elegans

collision energy

Curie

Cleavable ICAT

centimeter

counts per minute

cytidine 5'-triphosphate

collision cell exit potential

Drosophila melanogaster

Dalton

data-dependent acquisition

Dulbecco's Modified Eagle's Medium

Dimethylsulfoxide

deoxyribonucleic acid

declustering potential

disintegrations per minute

Dithioerythrol

Dithiothreitol

for example, exempli gratia

Ethylenediaminetetraacetic acid

Exponentially modified PAI

entrance potential

Enhanced product ion

enhanced resolution

electrospray ionization

and others, et alii

formic acid

foetal bovine serum

Full-length expressed stable isotope-labeled proteins for quantification femtomole

fourier transformation

Fourier transform ion cyclotron resonance

full width at half maximum intensity

gram / gravitational acceleration

guanosine 5'-triphosphate

Hours

heavy SILAC nuclear extract

4-(2-hydroxyethyl)-1-piperazinethanesulfonic acid

hydrophobic interaction chromatography 


\begin{tabular}{|c|c|}
\hline HILIC & hydrophilic interaction chromatography \\
\hline HPLC & high pressure liquid chromatography \\
\hline i.d. & inner diameter \\
\hline i.e. & that is, id est \\
\hline ICAT & isotope-coded affinity tag \\
\hline ICPL & isotope-coded protein label \\
\hline IEC & ion exchange chromatography \\
\hline IEF & isoelectric focussing \\
\hline IPG & immobilized $\mathrm{pH}$ gradient \\
\hline IPI & International Protein Index \\
\hline IPTG & Isopropyl-b-D-1-thiogalactopyranoside \\
\hline iTRAQ & isobaric tags for relative and absolute quantification \\
\hline $\mathrm{kDa}$ & Kilodalton \\
\hline $\mathrm{kV}$ & kilovolts \\
\hline I & liter \\
\hline $\mathrm{L}$ & light SILAC nuclear extract \\
\hline LB & Luria-Bertani \\
\hline LC & liquid chromatography \\
\hline$\mu g$ & microgram \\
\hline$\mu \mathrm{M}$ & micromole \\
\hline$\mu \mathrm{m}$ & Micrometer \\
\hline M & molar / medium SILAC nuclear extract \\
\hline$m / z$ & mass-to-charge ratio \\
\hline MALDI & Matrix-assisted laser desorption/ionization \\
\hline MC & missed cleavages \\
\hline $\mathrm{MDHI}$ & 2-methoxy-4,5-dihydro- $1 \mathrm{H}$-imidazole \\
\hline $\mathrm{mg}$ & milligram \\
\hline $\min$ & minutes \\
\hline $\mathrm{ml}$ & milliliter \\
\hline $\mathrm{mM}$ & millimolar \\
\hline $\mathrm{mm}$ & Millimeter \\
\hline $\mathrm{mmol}$ & millimole \\
\hline MOPS & 3-(N-morpholino)propanesulfonic acid \\
\hline MRM & multiple reaction monitoring \\
\hline MS & mass spectrometry \\
\hline $\mathrm{ms}$ & milliseconds \\
\hline MS/MS & tandem mass spectrometry \\
\hline MuD-PIT & multidimensional protein identification technology \\
\hline MW & molecular weight \\
\hline NCBI & National Center for Biotechnology Information \\
\hline NE & nuclear extract \\
\hline ng & nanogram \\
\hline NHS & N-hydroxysuccinimide \\
\hline $\mathrm{nl}$ & nanoliter \\
\hline $\mathrm{nm}$ & nanometer \\
\hline $\mathrm{nM}$ & nanomole \\
\hline no. & number \\
\hline OD & optical density \\
\hline PAGE & polyacrylamide gel electrophoresis \\
\hline PAl & protein abundance index \\
\hline PBS & Phosphate buffered saline \\
\hline PC/QMS & pulse-chase/quantitative mass spectrometry \\
\hline $\mathrm{PCl}$ & Phenol/Chloroform/Isoamylalcohol \\
\hline pmol & picomole \\
\hline PMSF & Phenylmethylsulfonyl fluoride \\
\hline PNK & polynucleotide kinase \\
\hline
\end{tabular}




\begin{tabular}{|c|c|}
\hline ppm & parts per million \\
\hline pre-mRNA & pre-messenger RNA \\
\hline PSAQ & Protein Standard Absolute Quantification \\
\hline$Q / q$ & quandrupole \\
\hline QconCAT & concatenated signature peptides encoded by QconCAT genes \\
\hline Q-ToF & Quadrupole-time-of-flight \\
\hline RNA & ribonucleic acid \\
\hline RNP & ribonucleoprotein \\
\hline $\mathrm{RP}$ & reversed phase \\
\hline rpm & revolutions per minute \\
\hline RRM & RNA recognition motif \\
\hline rRNA & Ribosomal RNA \\
\hline RT & retention time \\
\hline S & Svedberg \\
\hline $\mathrm{s}$ & seconds \\
\hline SA & succinic anhydride \\
\hline SC & Sequence coverage \\
\hline SCX & strong cation-exchange \\
\hline SDS & sodium dodecylsulfate \\
\hline SDS & Dodecyl sulfate sodium salt \\
\hline SEC & size exclusion chromatography \\
\hline SILAC & stable isotope labeling with amino acids in cell culture \\
\hline snRNA & small-nuclear RNA \\
\hline SRM & selected reaction monitoring \\
\hline StDev & standard deviation \\
\hline TBE & Tris-Borate-EDTA buffer \\
\hline TEAB & triethylammonium bicorbonate \\
\hline TEMED & $\mathrm{N}, \mathrm{N}, \mathrm{N}^{\prime}, \mathrm{N}$ '-Tetramethylethylendiamid \\
\hline TFA & trifluoroacetic acid \\
\hline TIC & total ion current/count \\
\hline TMTs & tandem mass tags \\
\hline ToF & time-of-flight \\
\hline Tris & Tris-(hydroxymethyl) aminoethane \\
\hline TTP & thymidine-5'-triphosphate \\
\hline$U$ & units \\
\hline U snRNA & uridine-rich small-nuclear RNA \\
\hline UPS & unique peptide sequences \\
\hline UTP & uridine 5'-triphosphate \\
\hline UV & ultraviolet \\
\hline V & volts \\
\hline vol. & volumes \\
\hline vs. & versus \\
\hline $\mathrm{w} / \mathrm{o}$ & without \\
\hline $\mathrm{XIC}$ & extracted ion chromatogram \\
\hline$Y_{n}$ & Polypyrimidine tract \\
\hline
\end{tabular}




\section{Acknowledgements}

I would like to thank many people, who have helped and supported me during the last years.

First of all, I would like to thank Dr. Henning Urlaub for supervision of this work, and my thesis and examination committee - Prof. Dr. Ralf Ficner, Prof. Dr. Ivo Feußner, Prof. Dr. Ulf Diederichsen, Prof. Dr. Kai Tittmann, and Prof. Dr. Dirk Görlich - for support, thesis committee meetings, and review of this work.

I thank Prof. Dr. Reinhard Lührmann for the opportunity to work on these interesting projects, and Sergey Bessonov, Jochen Deckert, Mads Grønborg and Michael Grote for collaboration on the absolute and relative quantification projects - in addition, Sergey Bessonov and Jochen Deckert for introducing me to the field of complex purification.

I am thankful to Dr. Christof Lenz for help, support, and introducing me to the world of MRM!

My very special thanks go to the Bioanalytical Mass Spectrometry Group. Thanks for the great atmosphere, the nice time in and outside the lab, help, support, ... ! Monika, Johanna and Uwe - thanks for excellent (!!) technical assistance and help with diverse problems! I would like to thank He-Hsuan for help in solving different problems and Miro for discussions about data analysis and statistics. I would also like to thank the former members of our group; in particular, Mads for the nice time, fun and support. Last but not least, Henning - for continuous support, never ending enthusiasm, encouragement, discussions, ..., biking, first aid, Summer Schools, conferences!

I am more than grateful to Klaus Hartmuth for reading (almost) every word I wrote during the last year, for discussions, advice and small talk.

Many thanks are addressed to...

...Thomas Conrad for fermentation of SILAC cells and answering all my questions.

...Peter Odenwälder, Norbert Rigo and Thomas Oellerich for help with HeLa cells.

...Paul Woolley and Cindy Will for reading parts of this thesis.

...Juliane Moses for solving all kinds of problems.

...Hossein Kohansal for help in preparing nuclear extracts.

...Gabi Heyne, Peter Kempkes and Irene Öchsner for help and technical assistance. 
...Ursula Drössler and Gertrud Nowak for support with daily needs.

...Julia and Ramazan for answering diverse questions, help, support.

...the whole Lührmann department for help with various problems and the nice atmosphere.

...the GGNB office for help in solving all problems.

...the GGNB for a travel grant and a bridging fund.

At last, I would like to thank my family and friends - my parents, who always supported me during the last years, my brother Carsten and my sister Cornelia for the nice meetings, fun, support, encouragement and help; all my friends for continuous help and support, especially Simone and Jennifer for reading this thesis, interest, sympathy, endless discussions - and Simone for the nice time in Göttingen (and Leipzig)! 


\section{Curriculum Vitae}

\section{Personal data}

Name

Date of birth

Place of birth

\section{Education}

$11 / 2006$ - present

$11 / 2006$

$01 / 2006-10 / 2006$

03/2005-06/2005

09/2004

09/2003

10/2001 - 10/2006

06/2001

08/1994 - 06/2001

08/1992 - 07/1994

08/1988-07/1992
Carla Schmidt

August 15, 1981

Wolfenbüttel
PhD project, Bioanalytical Mass Spectrometry Group, Max Planck Institute for Biophysical Chemistry, Göttingen;

Diploma

"Charakterisierung hirnspezifischer Proteine im Zusammenhang mit der Alzheimer-Krankheit"

Diploma project; Group of Bioanalytics, Center for Biotechnology and Biomedicine, University Leipzig;

Exchange student (Erasmus) at the University of Uppsala, Sweden;

"Protein structure and function"

Examination of the basic study period

Intermediate diploma

Studies of chemistry, University Leipzig

Abitur

Gymnasium im Schloß, Wolfenbüttel

Werla-Schule Schladen

Grundschule Börßum

\section{Publications}

Schmidt, C., Lenz, C., Grote, M., Lührmann, R., and Urlaub, H. (2010). Determination of protein stoichiometry within protein complexes using absolute quantification and multiple reaction monitoring. Anal Chem 82, 2784-96.

Schmidt, C.*, Gronbørg, M. ${ }^{*}$ Deckert, J., Bessonov, S., Lemm, I., Conrad, T., Lührmann, R., and Urlaub, H. Quantification of spliceosomal B and C complexes by mass spectrometry, in preparation 


\section{Invited publications}

Marcus, K., Schmidt, C., and Urlaub, H. (2009). Proteomic basics - Quantification and posttranslational modifications of proteins: The 3rd European summer school in Kloster Neustift. J Proteomics [Epub ahead of print]

Schmidt, C. and Urlaub, H. (2009). iTRAQ-labeling of in-gel digested proteins for relative quantification. Methods Mol Biol 564, 207-26.

Marcus, K., Kühn-Hölsken, E., Schmidt, C., Schulenborg, T., and Urlaub, H. (2008).

'Proteomic basics--sample preparation and separation': the 1st European Summer School in Kloster Neustift, 12-18 August, 2007 Brixen/Bressanone, South Tyrol, Italy. Proteomics 8, 230-3.

Schmidt, C., Urlaub, H., and Lenz, C. (2008). Protein-Quantifizierung nach In-Gel Verdau und iTRAQ-Markierung, Laborwelt, 9.Jahrgang, Nr4/2008.

Schmidt, C. and Urlaub, H. Analysis of protein complexes by (quantitative) mass spec analysis. Methods Mol Biol, submitted.

\section{Conference contributions}

Schmidt, C., Grote, M., Lenz, C., Lührmann, R., Urlaub, H. (2009) Evaluation of Protein Stoichiometry within Protein-RNA Complexes by Multiple Reaction Monitoring in Comparison to MS Standard Methods; 57th ASMS Conference on Mass Spectrometry and Allied Topics, Philadelphia, Pennsylvania

Schmidt, C., Grote, M., Lenz, C., Lührmann, R., Urlaub, H. (2009) Establishing the protein stoichiometry in Protein Complexes by Multiple Reaction Monitoring, Proteomic Forum 2009, Berlin

Schmidt, C., Grote, M., Lenz, C., Lührmann, R., Urlaub, H. (2009) Bestimmung der Stöchiometrie von Proteinen in Protein-Komplexen mittels Multiple Reaction Monitoring; 42. Jahrestagung der Deutschen Gesellschaft für Massenspektrometrie, Konstanz

Schmidt, C., Grønborg, M., Deckert, J., Bessonov, S., Lemm, I., Lührmann, R., Urlaub, H. (2008) Quantitation of native catalytically active spliceosomal B and C complexes by chemical (iTRAQ) and metabolic (SILAC) labeling; 2nd European Summer School "Proteomic Basics", Brixen/Bressanone, South Tyrol, Italy

Schmidt, C., Grønborg, M., Deckert, J., Bessonov, S., Lemm, I., Lührmann, R., Urlaub, H. (2008) Quantitation of native catalytically active spliceosomal B and C complexes by chemical (iTRAQ) and metabolic (SILAC) labeling; 56th ASMS Conference on Mass Spectrometry and Allied Topics, Denver, Colorado

Schmidt, C., Grønborg, M., Karaduman, R., Deckert, J., Bessonov, S., Kastner, B., Stark, H., Lührmann, R., Urlaub, H. (2007) Relative and Absolute Quantitation of Spliceosomal Proteins by Mass Spectrometry; 1st European Summer School "Proteomic Basics", Brixen/Bressanone, South Tyrol, Italy 


\section{Invited oral presentations}

Absolute Quantitation of Proteins in Protein Complexes by nano LC-MRM, QTRAP Pro Workshop 2009, Applied Biosystems, Darmstadt

Relative und absolute Quantifizierung spleißosomaler Komplexe mittels Massenspektrometrie, Seminar am Medizinischen Proteom Center, Bochum

\section{Stipends}

Proteomic Forum 2009, Berlin; Travel Grant, Deutsche Gesellschaft für Proteom Forschung 57th ASMS Conference on Mass Spectrometry and Allied Topics, Philadelphia, Pennsylvania, USA; Travel Grant, GGNB

Bridging Fund, 03/2010 - 05/2010; GGNB

\section{Memberships}

American Society for Mass Spectrometry (ASMS)

Deutsche Gesellschaft für Massenspektrometrie (DGMS)

Deutsche Gesellschaft für Proteomforschung (DGPF)

Gesellschaft für Biochemie und Molekularbiologie (GBM)

\section{Conference organization}

Co-organization of the

$1^{\text {st }}$ European Summer School "Proteomic Basics", 2007

$2^{\text {nd }}$ European Summer School "Proteomic Basics", 2008

$3^{\text {rd }}$ European Summer School "Proteomic Basics", 2009

in Brixen, Bressanone, South Tyrol, Italy. 